\title{
Interventions for Substance Use Disorders in Adolescents: A Systematic Review
}




\title{
Comparative Effectiveness Review
}

Number 225

\section{Interventions for Substance Use Disorders in Adolescents: A Systematic Review}

\author{
Prepared for: \\ Agency for Healthcare Research and Quality \\ U.S. Department of Health and Human Services \\ 5600 Fishers Lane \\ Rockville, MD 20857 \\ www.ahrq.gov
}

Contract No. 290-2015-00002-I

Prepared by:

Brown Evidence-based Practice Center

Providence, RI

Investigators:

Dale W. Steele, M.D., M.Sc.

Sara J. Becker, Ph.D.

Kristin J. Danko, Ph.D.

Ethan M. Balk, M.D., M.P.H.

Ian J. Saldanha, M.B.B.S., M.P.H., Ph.D.

Gaelen P. Adam, M.L.I.S., M.P.H.

Sarah M. Bagley, M.D., M.Sc.

Catherine Friedman, M.D.

Anthony Spirito, M.D.

Kelli Scott, Ph.D.

Evangelia E. Ntzani, M.D.

Iman Saeed, Sc.M.

Bryant Smith, M.S., C.P.H.

Jonah Popp. Ph.D.

Thomas A. Trikalinos, M.D., Ph.D.

AHRQ Publication No. 20-EHC014

May 2020 
This report is based on research conducted by the Brown Evidence-based Practice Center (EPC) under contract to the Agency for Healthcare Research and Quality (AHRQ), Rockville, MD (Contract No. 290-2015-00002-I). The findings and conclusions in this document are those of the authors, who are responsible for its contents; the findings and conclusions do not necessarily represent the views of AHRQ. Therefore, no statement in this report should be construed as an official position of AHRQ or of the U.S. Department of Health and Human Services.

\section{None of the investigators have any affiliations or financial involvement that conflicts with the material presented in this report.}

The information in this report is intended to help health care decision makers-patients and clinicians, health system leaders, and policymakers, among others-make well-informed decisions and thereby improve the quality of health care services. This report is not intended to be a substitute for the application of clinical judgment. Anyone who makes decisions concerning the provision of clinical care should consider this report in the same way as any medical reference and in conjunction with all other pertinent information, i.e., in the context of available resources and circumstances presented by individual patients.

This report is made available to the public under the terms of a licensing agreement between the author and the Agency for Healthcare Research and Quality. This report may be used and reprinted without permission except those copyrighted materials that are clearly noted in the report. Further reproduction of those copyrighted materials is prohibited without the express permission of copyright holders.

AHRQ or U.S. Department of Health and Human Services endorsement of any derivative products that may be developed from this report, such as clinical practice guidelines, other quality enhancement tools, or reimbursement or coverage policies may not be stated or implied.

This report may periodically be assessed for the currency of conclusions. If an assessment is done, the resulting surveillance report describing the methodology and findings will be found on the Effective Health Care Program Web site at www.effectivehealthcare.ahrq.gov. Search on the title of the report.

AHRQ appreciates appropriate acknowledgment and citation of its work. Suggested language for acknowledgment: This work was based on an evidence report, [INSERT TITLE], by the Evidence-based Practice Center Program at the Agency for Healthcare Research and Quality (AHRQ).

Suggested citation: Steele DW, Becker SJ, Danko KJ, Balk EM, Saldanha IJ, Adam GP, Bagley SM, Friedman C, Spirito A, Scott K, Ntzani EE, Saeed I, Smith B, Popp J, Trikalinos TA. Interventions for Substance Use Disorders in Adolescents: A Systematic Review. Comparative Effectiveness Review No. 225. (Prepared by the Brown Evidence-based Practice Center under Contract No. 290-2015-00002-I.) AHRQ Publication No. 20-EHC014. Rockville, MD: Agency for Healthcare Research and Quality. May 2020. Posted final reports are located on the Effective Health Care Program search page. DOI: https://doi.org/10.23970/AHRQEPCCER225. 


\section{Preface}

The Agency for Healthcare Research and Quality (AHRQ), through its Evidence-based Practice Centers (EPCs), sponsors the development of systematic reviews to assist public- and private-sector organizations in their efforts to improve the quality of health care in the United States. These reviews provide comprehensive, science-based information on common, costly medical conditions, and new health care technologies and strategies.

Systematic reviews are the building blocks underlying evidence-based practice; they focus attention on the strength and limits of evidence from research studies about the effectiveness and safety of a clinical intervention. In the context of developing recommendations for practice, systematic reviews can help clarify whether assertions about the value of the intervention are based on strong evidence from clinical studies. For more information about AHRQ EPC systematic reviews, see https://effectivehealthcare.ahrq.gov/about/epc/evidence-synthesis.

AHRQ expects that these systematic reviews will be helpful to health plans, providers, purchasers, government programs, and the health care system as a whole. Transparency and stakeholder input are essential to the Effective Health Care Program. Please visit the Web site (www.effectivehealthcare.ahrq.gov) to see draft research questions and reports or to join an email list to learn about new program products and opportunities for input.

If you have comments on this systematic review, they may be sent by mail to the Task Order Officer named below at: Agency for Healthcare Research and Quality, 5600 Fishers Lane, Rockville, MD 20857, or by email to epc@ahrq.hhs.gov.

Gopal Khanna, M.B.A.

Director

Agency for Healthcare Research and Quality

Stephanie Chang, M.D., M.P.H.

Director

Evidence-based Practice Center Program

Center for Evidence and Practice Improvement Agency for Healthcare Research and Quality
Arlene Bierman, M.D., M.S.

Director

Center for Evidence and Practice

Improvement

Agency for Healthcare Research and Quality

Suchitra Iyer, Ph.D.

Task Order Officer

Center for Evidence and Practice

Improvement

Agency for Healthcare Research and Quality 


\section{Acknowledgments}

The authors gratefully acknowledge the following individuals for their contributions to this project: Jenni Quiroz, B.S., Khamden Soch, M.S., and Anya Wallack, Ph.D.

\section{Key Informants}

In designing the study questions, the EPC consulted several Key Informants who represent the end-users of research. The EPC sought the Key Informant input on the priority areas for research and synthesis. Key Informants are not involved in the analysis of the evidence or the writing of the report. Therefore, in the end, study questions, design, methodological approaches, and/or conclusions do not necessarily represent the views of individual Key Informants.

Key Informants must disclose any financial conflicts of interest greater than $\$ 5,000$ and any other relevant business or professional conflicts of interest. Because of their role as end-users, individuals with potential conflicts may be retained. The TOO and the EPC work to balance, manage, or mitigate any conflicts of interest.

The list of Key Informants who provided input to this report follows:

David “Joel” Beckstead, Ph.D.

Indian Health Service (IHS)

Rockville, MD

Kirsten Beronio, J.D.

The Center for Medicaid and CHIP Services

Washington, DC

Kevin M. Gray, M.D.* †

Medical University of South Carolina

Charleston, SC

Aaron Hogue, Ph.D.* +

Center on Addiction

New York, NY

Danica Kalling Knight, Ph.D.

Texas Christian University

Fort Worth, TX

Ellen Kreida, M.P.H., L.I.C.S.W.

MA Department of Children and Families

Boston, MA

Justine Larson, M.D., M.P.H., M.H.S.* +

The Substance Abuse and Mental Health

Services Administration (SAMHSA)

Rockville, MD
Sharon Levy, M.D., M.P.H.* +

Boston Children's Hospital

Boston, MA

Frederick Muench, Ph.D.

Center on Addiction

New York, NY

Ruth Potee, M.D.

Valley Medical Group

Greenfield, MA

Sheryl Ryan, M.D.*†

Penn State Children's Hospital

Hershey, PA

Audra Stock, L.P.C., MAC

Substance Abuse and Mental Health

Services Administration (SAMHSA)

Rockville, MD

Geetha Subramaniam, M.D.

National Institute on Drug Abuse (NIDA)

Rockville, MD 
* Also Peer Reviewer

† Also Technical Expert Panel member

\section{Technical Expert Panel}

In designing the study questions and methodology at the outset of this report, the EPC consulted several technical and content experts. Broad expertise and perspectives were sought. Divergent and conflicting opinions are common and perceived as healthy scientific discourse that results in a thoughtful, relevant systematic review. Therefore, in the end, study questions, design, methodologic approaches, and/or conclusions do not necessarily represent the views of individual technical and content experts.

Technical Experts must disclose any financial conflicts of interest greater than $\$ 5,000$ and any other relevant business or professional conflicts of interest. Because of their unique clinical or content expertise, individuals with potential conflicts may be retained. The TOO and the EPC work to balance, manage, or mitigate any potential conflicts of interest identified.

The list of Technical Experts who provided input to this report follows:

Craig Henderson, Ph.D.*

Sam Houston State University

Huntsville, TX

Emily Tanner-Smith, Ph.D.*

University of Oregon

Eugene, OR

* Also Peer Reviewer

\section{Peer Reviewers}

Prior to publication of the final evidence report, EPCs sought input from independent Peer Reviewers without financial conflicts of interest. However, the conclusions and synthesis of the scientific literature presented in this report does not necessarily represent the views of individual reviewers.

Peer Reviewers must disclose any financial conflicts of interest greater than $\$ 5,000$ and any other relevant business or professional conflicts of interest. Because of their unique clinical or content expertise, individuals with potential nonfinancial conflicts may be retained. The TOO and the EPC work to balance, manage, or mitigate any potential nonfinancial conflicts of interest identified.

The list of Peer Reviewers follows:

Rongwei (Rochelle) Fu, Ph.D. Oregon Health \& Science University Portland, OR 
Scott Hadland, M.D., M.P.H., M.S.

Boston University School of Medicine/Boston Medical Center

Boston, MA

Veronika Mesheriakova, M.D.

University of California, San Francisco

San Francisco, CA 


\section{Interventions for Substance Use Disorders in Adolescents: A Systematic Review}

\section{Structured Abstract}

Objectives. This systematic review (SR) synthesizes the literature on behavioral, pharmacologic, and combined interventions for adolescents ages 12 to 20 years with problematic substance use or substance use disorder. We included interventions designed to achieve abstinence, reduce use quantity and frequency, improve functional outcomes, and reduce substance-related harms.

Data sources. We conducted literature searches in MEDLINE, the Cochrane CENTRAL Trials Registry, Embase, CINAHL, and PsycINFO to identify primary studies meeting eligibility criteria through November 1, 2019.

Review methods. Studies were extracted into the Systematic Review Data Repository. We categorized interventions into seven primary intervention components: motivational interviewing (MI), family focused therapy (Fam), cognitive behavioral therapy (CBT), psychoeducation, contingency management (CM), peer group therapy, and intensive case management. We conducted meta-analyses of comparative studies and evaluated the strength of evidence (SoE). The PROSPERO protocol registration number is CRD42018115388.

Results. The literature search yielded 33,272 citations, of which 118 studies were included. Motivational interviewing reduced heavy alcohol use days by 0.7 days/month, alcohol use days by 1.2 days/month, and overall substance use problems by a standardized mean difference of 0.5 , compared with treatment as usual. Brief MI did not reduce cannabis use days (net mean difference of 0). Across multiple intensive interventions, Fam was most effective, reducing alcohol use days by 3.5 days/month compared with treatment as usual. No intensive interventions reduced cannabis use days. Pharmacologic treatment of opioid use disorder led to a more than 4 times greater likelihood of abstinence with extended courses ( 2 to 3 months) of buprenorphine compared to short courses (14 to 28 days).

Conclusions. Brief interventions: MI reduces heavy alcohol use (low SoE), alcohol use days (moderate SoE), and substance use-related problems (low SoE) but does not reduce cannabis use days (moderate SoE). Nonbrief interventions: Fam may be most effective in reducing alcohol use (low SoE). More research is needed to identify other effective intensive behavioral interventions for alcohol use disorder. Intensive interventions did not appear to decrease cannabis use (low SoE). Some interventions (CBT, CBT+MI, and CBT+MI+CM) were associated with increased cannabis use (low SoE). Both MI and CBT reduce combined alcohol and other drug use (low SoE). Combined CBT+MI reduces illicit drug use (low SoE). Subgroup analyses of interest (male vs. female, racial and ethnic minorities, socioeconomic status, and family characteristics) were sparse, precluding conclusions regarding differential effects. Pharmacological interventions: longer courses of buprenorphine (2-3 months) are more effective than shorter courses (14-28 days) to reduce opioid use and achieve abstinence (low SoE). SRs in the college settings support use of brief interventions for students with any use, heavy or problematic use. More research is needed to identify the most effective combinations of behavioral and pharmacologic treatments for opioid, alcohol, and cannabis use disorders. 


\section{Contents}

Evidence Summary ........................................................................................................................................ 1

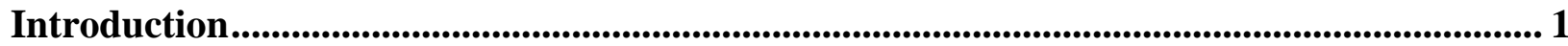

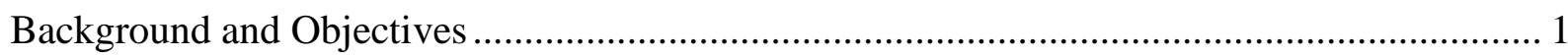

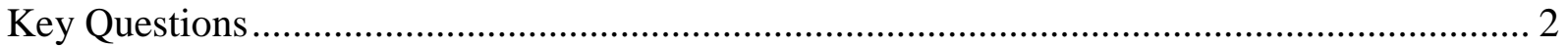

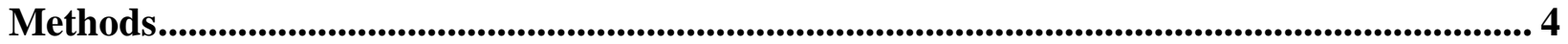

Searching for the Evidence ............................................................................................. 4

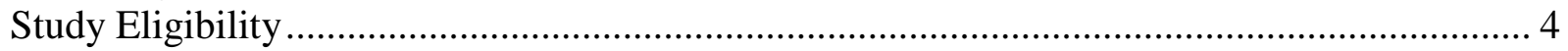

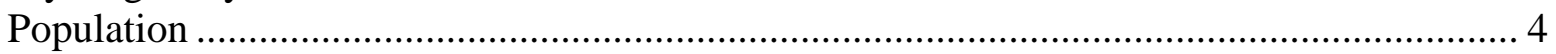

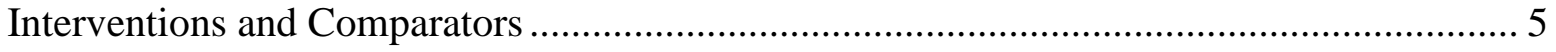

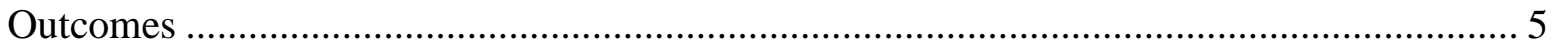

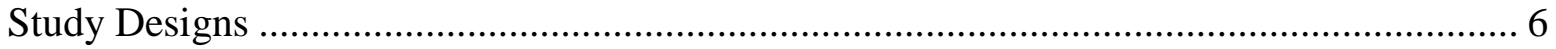

Screening Studies for Eligibility................................................................................... 9

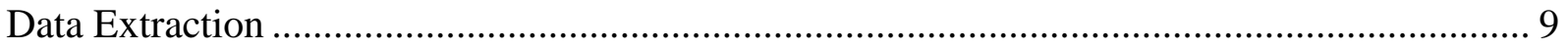

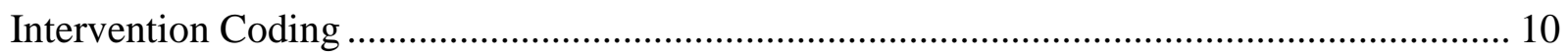

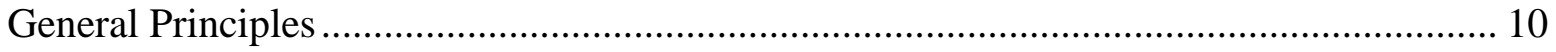

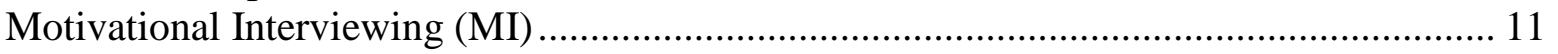

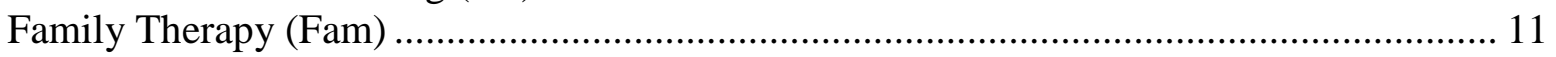

Cognitive Behavioral Therapy (CBT) ........................................................................ 11

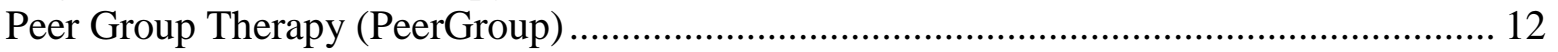

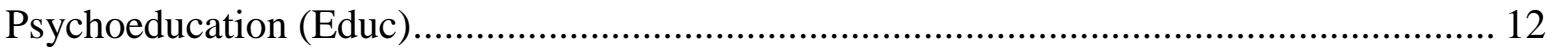

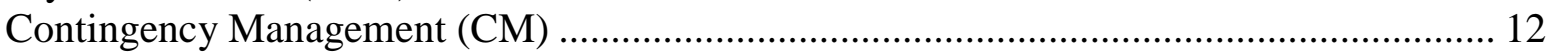

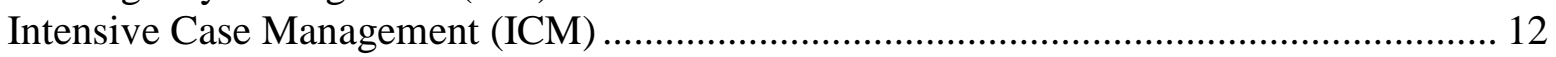

Treatment as Usual (TAU)............................................................................................... 13

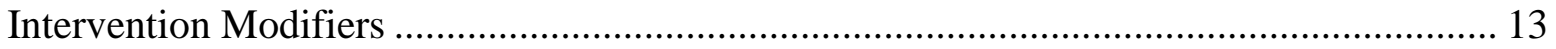

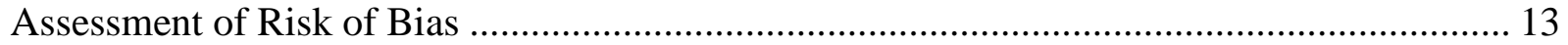

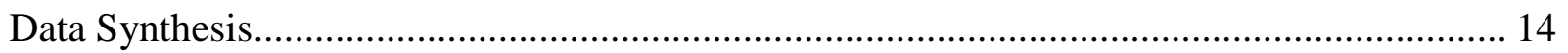

Qualitative Evidence Synthesis............................................................................................. 15

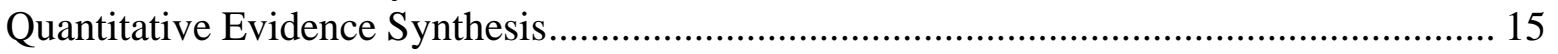

Grading the Strength of Evidence for Major Comparisons and Outcomes ............................... 17

Assessing Applicability ………………............................................................................. 18

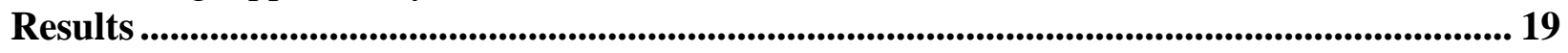

Qualitative Categorization ................................................................................................ 19

Brief Behavioral Interventions................................................................................... 20

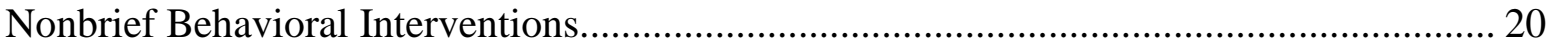

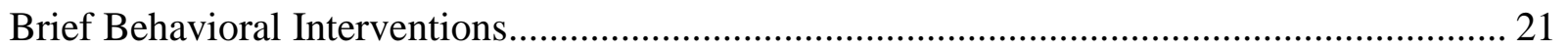

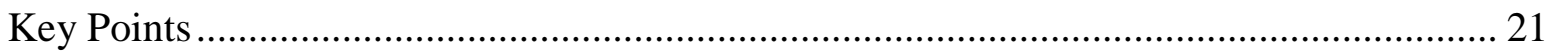

Studies Not Included in Meta-Analyses........................................................................ 21

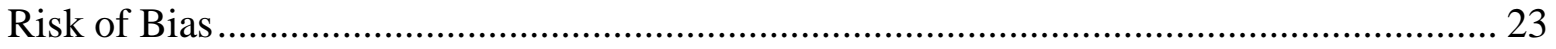

Nonbrief Behavioral Interventions ........................................................................... 36

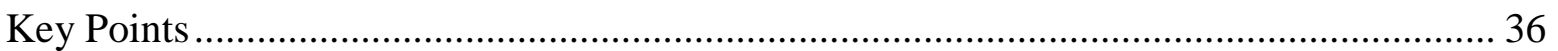

Studies Including Arms With Nondistinguished Intervention Codes ................................... 36

Substances Other Than Alcohol or Cannabis ................................................................... 37

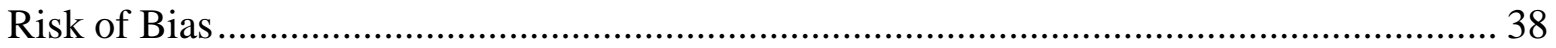

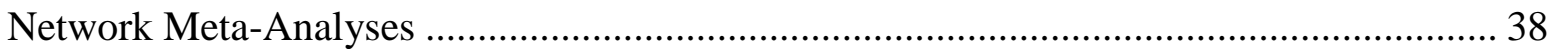

Systematic Reviews of Treatments for Alcohol Use in the College Setting ............................. 54 


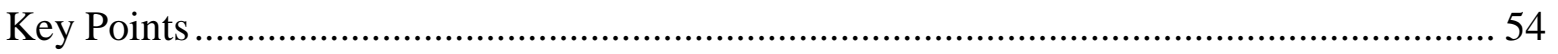

General Population of College Students Who Drink Alcohol ................................................ 55

College Students Mandated To Receive Interventions for Alcohol Use ................................. 56

College Students Who Engaged in Heavy or Hazardous Levels of Alcohol Use.................. 56

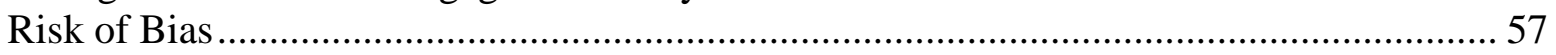

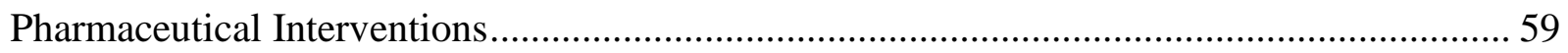

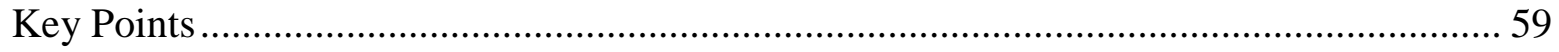

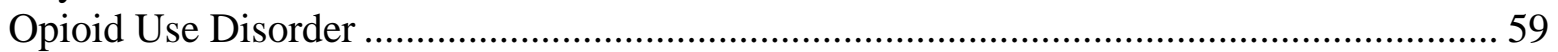

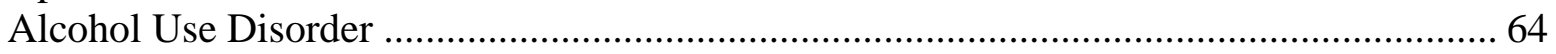

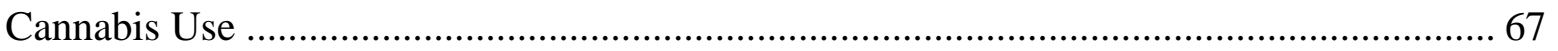

Comorbid Psychiatric Disorders in Adolescents With SUD ...................................................... 68

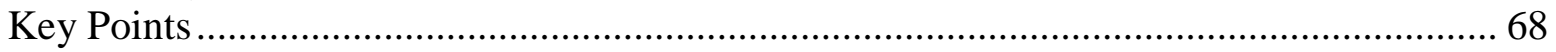

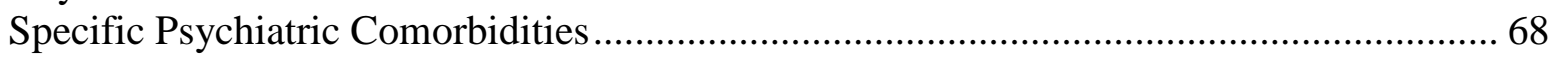

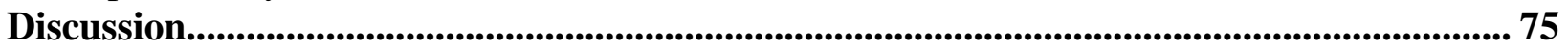

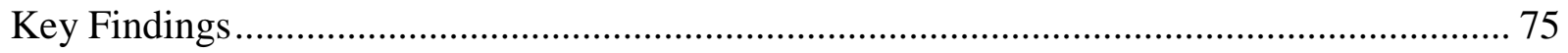

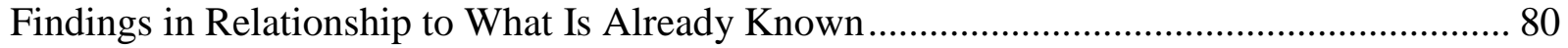

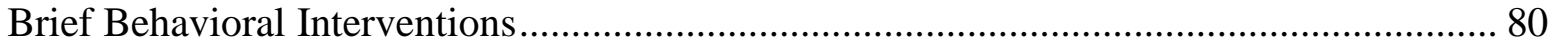

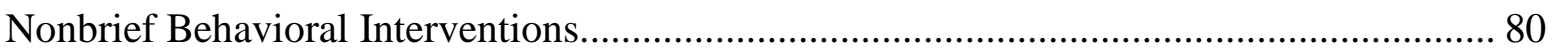

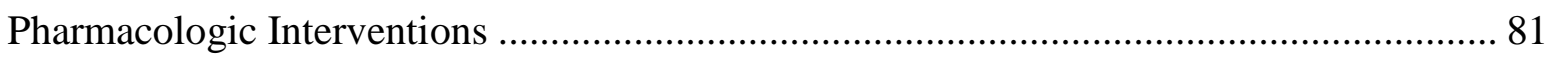

Pharmacologic Treatment of Psychiatric Comorbidities in Adolescents With Substance Use

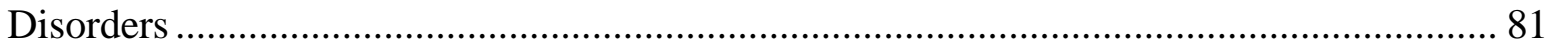

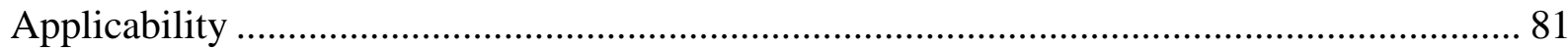

Implications for Clinical and Policy Decision Making .............................................................. 83

Limitations of the Systematic Review Process ........................................................................ 83

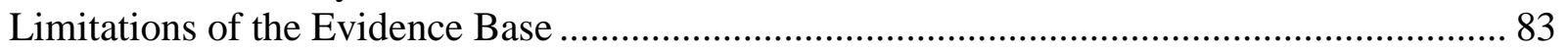

Recommendations for Future Research ................................................................................. 84

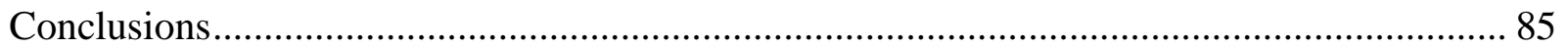

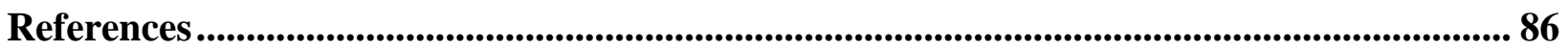

Abbreviations and Acronyms .......................................................................................................... 108

\section{Tables}

Table 1. Eligibility criteria .................................................................................................. 6

Table 2. Results: Brief behavioral interventions for substances other than alcohol or cannabis.. 22

Table 3. Brief behavioral intervention studies with two treatment arms with nondistinguishable components ......................................................................................................................... 23

Table 4. Brief behavioral intervention studies with three treatment arms with two nondistinguishable components that were pooled in MA......................................................... 23 Table 5. Brief behavioral interventions and alcohol abstinence: Net mean difference of days per month of abstinence between all interventions ....................................................................... 27

Table 6. Brief behavioral interventions and alcohol abstinence: Odds ratios for abstinence between all interventions .................................................................................................... 29

Table 7. Brief behavioral interventions and cannabis abstinence: Odds ratios for abstinence between all interventions ....................................................................................................... 32

Table 8. Legal outcomes with brief behavioral interventions.......................................................... 35

Table 9. Nonbrief behavioral intervention studies with two treatment arms with nondistinguishable components 
Table 10. Nonbrief behavioral intervention studies with multiple treatment arms with nondistinguishable components

Table 11. Results: Nonbrief behavioral interventions for substances other than alcohol or cannabis.....

Table 12. Nonbrief behavioral interventions and alcohol use days: Net mean difference of use days per month between all interventions.....

Table 13. Probabilities of nonbrief behavioral interventions ranking in top third, middle third and bottom third to reduce alcohol use days

Table 14. Nonbrief behavioral interventions and cannabis use days: Net mean difference of use days per month between all interventions.................................................................... 45 Table 15. Probabilities of nonbrief behavioral interventions ranking in top third, middle third and bottom third to reduce cannabis use days 47 Table 16. Nonbrief behavioral interventions and aggregate alcohol and other drug use: Net mean difference of use days per month between all interventions. 50 Table 17. Probabilities of nonbrief behavioral interventions ranking in top third, middle third and bottom third to reduce aggregate alcohol and other drug use days....................................... 51 Table 18. Nonbrief behavioral interventions and illicit drug use: Net mean difference of use days per month between all interventions 53 Table 19. Probabilities of nonbrief behavioral interventions ranking in top third, middle third and

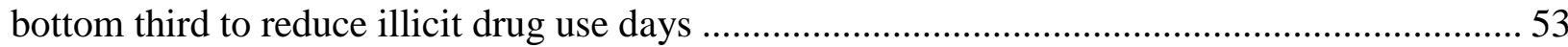

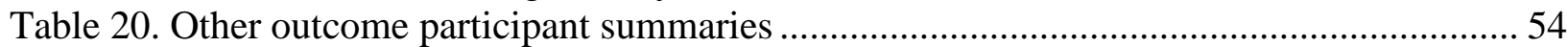
Table 21. Risk of bias in college alcohol intervention systematic reviews ............................... 58 Table 22. Baseline data and Interventions: Pharmacologic interventions for opioid use............ 59 Table 23. Results: Pharmacologic interventions for opioid use disorder .................................. 62 Table 24. Baseline data and interventions: Pharmacologic treatments of alcohol use ................ 64 Table 25. Results: Pharmacologic treatments for alcohol use .............................................. 66 Table 26. Baseline data and interventions: Pharmacologic treatments for cannabis ................... 67 Table 27. Results: Pharmacologic treatments for cannabis use .............................................. 68 Table 28. Baseline data and interventions: Pharmacologic interventions for psychiatric comorbidities in adolescents with substance use disorders 69 Table 29. Results: Pharmacologic interventions for ADHD in adolescents with substance use disorders

Table 30. Results: Pharmacologic interventions for depression in adolescents with substance use disorders

Table 31. Results: Pharmacologic interventions for bipolar disorder in adolescents with substance use disorders

Table 32. Evidence profile for interventions for substance use disorders and problematic use in adolescents ....

Table 33. Number of meta-analyzed studies reporting outcomes for alcohol only, cannabis only, or both

\section{Figures}

Figure 1. Analytic framework for the Key Questions.......................................................... 3

Figure 2. Literature flow diagram ............................................................................ 19

Figure 3. Meta-analyzed brief behavioral intervention studies: Percentage of studies in each risk

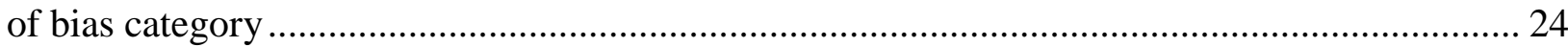


Figure 4. Evidence graph for brief behavioral intervention studies reporting heavy alcohol use

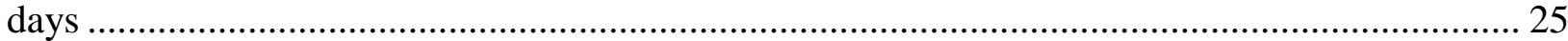

Figure 5. Heavy alcohol use: Forest plot depicting individual study effects with summary estimates of the relative effect of MI versus TAU.............................................................. 25 Figure 6. Evidence graph for brief behavioral intervention studies reporting alcohol use days .. 26 Figure 7. Alcohol use days: forest plot depicting individual study effects with summary estimates

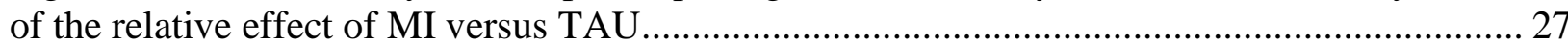

Figure 8. Evidence graph for studies reporting alcohol abstinence ...................................... 28

Figure 9. Alcohol abstinence: MI (brief intervention) versus TAU ......................................... 28

Figure 10. Evidence graph for brief behavioral intervention studies reporting cannabis use

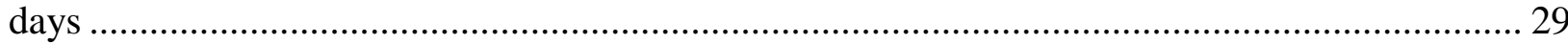

Figure 11. Cannabis use days: Forest plot of net mean difference for MI (brief intervention)

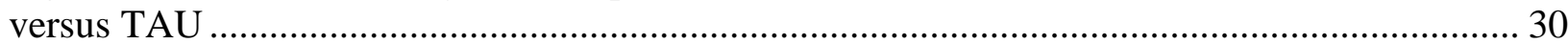

Figure 12. Cannabis use days: Forest plot of net mean difference for the brief interventions MI

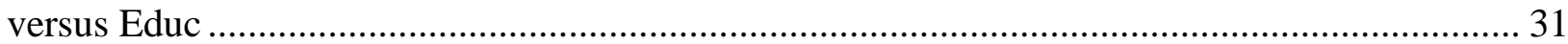

Figure 13. Evidence graph for brief behavioral intervention studies reporting cannabis

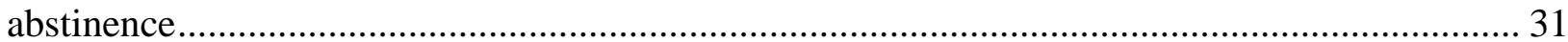

Figure 14. Cannabis abstinence: Forest plot of log odds ratio for MI (brief intervention) compared with TAU ............................................................................................ 32

Figure 15. Evidence graph of brief behavioral intervention studies reporting a substance use

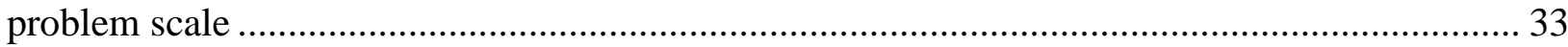

Figure 16. Substance use problem scales: Forest plot of standardized net mean difference of the brief interventions MI versus TAU .............................................................................. 34 Figure 17. Substance use problem scales: Forest plot of standardized net mean difference of the brief interventions MI versus Educ ......................................................................................... 35 Figure 18. Meta-analyzed nonbrief behavioral intervention studies: Percentage of studies in each

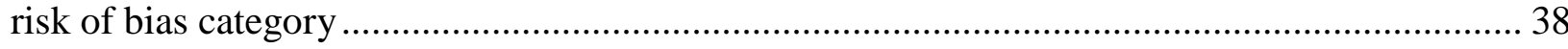
Figure 19. Evidence graph for nonbrief behavioral intervention studies reporting mean alcohol

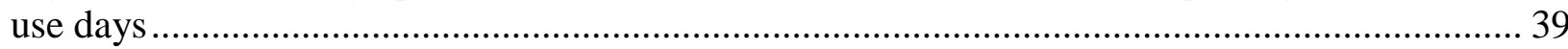
Figure 20. Alcohol use days: Summary forest plot of meta-analyzed net mean difference for all

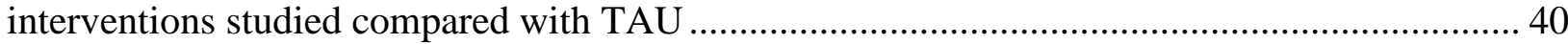
Figure 21. Evidence graph for studies reporting cannabis use days ........................................ 43 Figure 22. Cannabis use days: Summary forest plot of meta-analyzed net mean differences for all

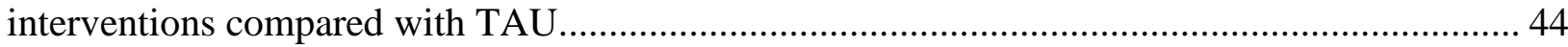
Figure 23. Evidence graph for studies reporting aggregate alcohol and other drug use days ...... 48 Figure 24. Aggregate alcohol and other drug use: Summary forest plot of meta-analyzed net mean differences for all interventions compared with TAU ................................................ 49 Figure 25. Evidence graph for studies reporting illicit drug use days ..................................... 51 Figure 26. Illicit drug use days: Summary forest plot of interventions reporting meta-analyzed illicit drug use days compared to TAU .............................................................................. 52 Figure 27. Pharmacologic intervention studies for opioid use: Percentage of studies in each risk

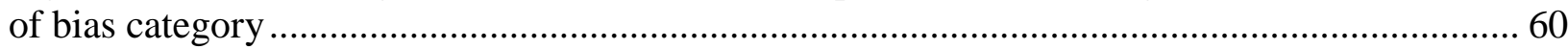
Figure 28. Pharmacologic Interventions for alcohol use: Percentage of studies in each risk of bias

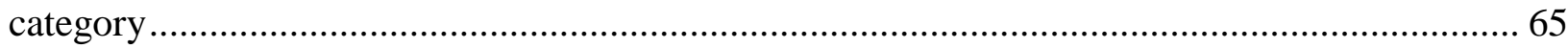
Figure 29. Pharmacologic interventions for cannabis use disorder: Percentage of studies in each risk of bias category. 
Figure 30. Pharmacologic interventions for psychiatric comorbidities in adolescents with substance use disorders: Percentage of studies in each risk of bias category ............................ 70

\section{Appendixes}

Appendix A. Search Strategies

Appendix B. Excluded Studies

Appendix C. Intervention Coding Manual

Appendix D. Baselines

Appendix E. Outcomes Extracted by Study

Appendix F. Brief Interventions: Detailed Results

Appendix G. Nonbrief Interventions: Detailed Results

Appendix H. Risk of Bias Assessments

Appendix I. Technical Appendix 


\section{Evidence Summary}

\section{Main Points}

- Adolescents, 12 to 20 years of age, with problematic alcohol and/or cannabis use or use disorder

o Brief behavioral interventions (that involve 1 or 2 encounters only)

- Motivational interviewing decreases days of heavy alcohol use and overall alcohol use.

- Motivational interviewing has not been found to decrease cannabis use. Further research is needed to identify if any other brief interventions may decrease cannabis use.

- Motivational interviewing decreases problems associated with substance use.

o Intensive behavioral interventions (that involve more than 2 encounters)

- Family-focused therapies reduce alcohol use.

- None of the interventions have been found to decrease cannabis use.

- Motivational interviewing decreases combined alcohol and other drug use.

- Combined cognitive behavioral therapy and motivational interviewing decrease illicit drug use.

- Adolescents, 12 to 25 years of age, with substance use disorders

o Pharmacological interventions

- In opioid use disorder, longer courses (2-3 months) of buprenorphine/buprenorphine-naloxone are more effective than shorter courses (14-28 days) to reduce days of opioid use and achieve abstinence.

- More research is needed to understand the role of medications in treatment of alcohol and cannabis use disorders and of pharmacological treatments typically used for comorbid psychiatric illnesses.

- College students with problematic alcohol use

o Behavioral interventions

- Mandated alcohol programs decrease alcohol use in the medium term, regardless of intervention. Four commercially available interventions are more effective in the short term than no intervention.

- Brief behavioral interventions, particularly those based on motivational interviewing, reduce alcohol use compared to no intervention in college students with heavy or hazardous alcohol use.

\section{Background and Purpose}

In 2017, an estimated 992,000 adolescents aged 12 to 17 in the United States (4\% of the adolescent population) and 5.1 million young adults aged 18 to 25 (14.8\% of the young adult population) met diagnostic criteria for a substance use disorder. When left untreated or ineffectively treated, adolescents with substance use disorders are at risk of experiencing a cascade of far-reaching adverse outcomes that often persist into adulthood. The pervasive negative consequences associated with untreated or ineffectively treated adolescent substance use, and the high lethality of opioid misuse, underscore the importance of identifying effective interventions to treat adolescent substance users. 
The review aims to inform health care providers, policymakers, and a clinical practice guideline update from the American Academy of Child and Adolescent Psychiatry (AACAP) about the currently available evidence on interventions for adolescents to reduce or cease substance use. The review addresses both behavioral and pharmacological interventions used for adolescents or young adults with problematic substance use or a diagnosis of a substance use disorder (SUD), excluding tobacco.

\section{Methods}

We employed methods consistent with those outlined in the AHRQ EPC Program Methods Guidance (https://effectivehealthcare.ahrq.gov/products/cer-methods-guide/overview). We describe these in the full report. Our searches covered reports published from database inception to April 11, 2019. Behavioral interventions were described based on their inclusion of seven primary intervention components: motivational interviewing, family-focused therapy, cognitive behavioral therapy, psychoeducation, contingency management, peer group therapy, and intensive case management. Pharmacologic interventions were divided into those used primarily for problematic substance use (or use disorder) or primarily to manage psychiatric comorbidities. The PROSPERO registration number is CRD42018115388.

\section{Results}

We found 118 randomized controlled trials that evaluated treatment of adolescents or young adults with problematic substance use or substance use disorders. Most studies enrolled adolescents with some combination of alcohol and cannabis use. The most commonly reported outcomes included frequency of use and abstinence. We describe evidence about five major categories of interventions: (1) brief behavioral interventions (consisting of one or two encounters), typically targeted at adolescents with problematic use; (2) intensive (nonbrief) behavioral interventions; (3) pharmacological treatments for psychiatric comorbidities in adolescents with concurrent substance use disorder; (4) pharmacological treatments used to treat use disorders; and (5) interventions of any kind for alcohol use in the college setting.

Our meta-analyses of brief interventions found that motivational interviewing reduced heavy alcohol use days by up to 0.7 days per month, alcohol use days by up to 1.2 days per month and overall substance use problems by a standardized mean difference of 0.5 , compared to treatment as usual. However, brief motivational interviewing did not reduce cannabis use days (net mean difference of 0 ).

Of the multiple intensive interventions, family-focused therapies were most effective; they reduced alcohol use days by 3.5 days per month compared to treatment as usual. None of the intensive interventions were found to reduce cannabis use days.

For the subgroups of interest (male versus female, racial and ethnic minorities, socioeconomic status, and family characteristics), data within or between studies of brief and intensive interventions were sparse or not available. Therefore, no conclusions regarding differential effects in these subgroups is possible.

Pharmacologic treatment of opioid use disorder led to a more than 4 times greater likelihood of abstinence with an extended (2 to 3 month) course of buprenorphine compared to short courses (14 to 28 days). Similarly, a slow buprenorphine taper (over 56 days) was more effective than a 28-day taper.

A review of existing systematic reviews found that treatment of problematic alcohol use among college student with behavioral interventions resulted in small improvements in alcohol 
use. In students with heavy or hazardous use, single-session interventions resulted in a small reduction in alcohol consumption. In students mandated to treatment, there were small improvements in heavy drinking frequency and alcohol-related problems in the medium term.

\section{Limitations}

For many topics, evidence was sparse or entirely absent. Most studies enrolled some combination of adolescents with mixed use of alcohol, cannabis, and occasionally other drugs. Very few studies evaluated users of opioids, methamphetamines, or substances other than alcohol or cannabis. Studies often combined different types of interventions, making comparisons of specific interventions difficult. The available studies did not consistently report a common set of outcomes, which limited our ability to combine information from potentially relevant studies. For most outcomes, individual studies were deemed to have moderate risk of bias, most commonly due to incomplete outcome data, poor compliance, and a lack of blinding of participants, study personnel, and outcome assessors.

The existing systematic reviews addressing treatments for alcohol use in the college setting were inadequate in their assessment and reporting of risk of bias and did not discuss the consistency of results.

\section{Implications and Conclusions}

Compared with treatment as usual (e.g., brief advice and a handout), brief motivational interviewing for adolescents with problematic substance use reduces both heavy alcohol use and overall days of use and may decrease problems related to substance use, such as missing school or work or getting into trouble. Among intensive interventions, family therapy (with a focus on intervening in the entire family system) was the most effective in reducing alcohol use.

Neither brief motivational interviewing nor intensive interventions have been demonstrated to reduce cannabis use. For opioid use disorder, buprenorphine and buprenorphine-naloxone are more effective for the short-term management of opioid withdrawal if they are tapered over longer periods of time.

Further research is needed to identify: (1) effective brief and intensive interventions for problematic cannabis use and cannabis use disorder and (2) effective combinations of behavioral treatments and medication to treat alcohol and cannabis use disorder(s). In addition, (3) studies of longer term pharmacological treatment of opioid use disorder are needed in this population. Future studies should evaluate outcomes that are most meaningful to adolescents, such as better functioning in school and improved relationships with peers and parents. 


\section{Introduction}

\section{Background and Objectives}

In 2017, in the United States, an estimated 992,000 adolescents aged 12 to 17 (4\% of the adolescent population) and 5.1 million young adults aged 18 to 25 (14.8\% of the young adult population) met diagnostic criteria for a substance use disorder (SUD). ${ }^{1}$ Thus, about 1 in 25 adolescents and 1 in 7 young adults had a diagnosable SUD. The vast majority were untreated, with fewer than 1 in 10 adolescents or young adults with a diagnosable condition receiving specialty care. ${ }^{1}$ When left untreated or ineffectively treated, adolescents with problematic substance use are at risk of experiencing a cascade of far-reaching adverse outcomes that often persist into adulthood, including sexually transmitted infections, ${ }^{2}$ unintended pregnancy, ${ }^{3}$ criminal involvement, ${ }^{4}$ school truancy, ${ }^{5}$ psychiatric disorders, ${ }^{6}$ and physical health problems. ${ }^{7}$ Adolescent substance use is also associated with the leading causes of death in this age cohort: suicide, unintentional injury, and violence. ${ }^{8,9}$

Alcohol, marijuana, and tobacco are the most commonly misused substances, followed by prescription and over-the-counter medications, among twelfth graders; ${ }^{10}$ with 1 percent of youth between the ages of 12 and 17 reporting current opioid misuse. ${ }^{11}$ Youth who use opioids are more likely to use other substances. ${ }^{10}$ Among youth under 21 who initiate heroin use, 80 percent misused prescription and/or over-the-counter medication before the age of $18 .{ }^{12}$ National concerns about opioid misuse, encompassing nonmedical use of prescription opioid-based medications (e.g., morphine, fentanyl) and the use of illegal opiates (e.g., heroin), have brought heightened attention to the significant risk of drug overdose death in adolescents. ${ }^{13}$

The pervasive negative consequences associated with untreated or ineffectively treated adolescent substance use (SU), and the high lethality of opioid misuse in particular, underscore the importance of identifying effective interventions for substance use in adolescents.

In 2005, the American Academy of Child and Adolescent Psychiatry (AACAP) created a Practice Parameter (PP) for the Assessment and Treatment of Children and Adolescents with substance use disorders. The 2005 Practice Parameters made eight recommendations pertaining to treatment. For behavioral treatments, AACAP concluded that family therapy models "have the most supporting evidence" and "individual approaches such as cognitive-behavioral therapy, both alone and with motivational enhancement therapy, have been shown to be efficacious.” AACAP recommended that "medication can be used when indicated," noting that this recommendation was "not based on empirical research in adolescents but rather on research and experience with adults." "14 The AACAP also recommended that psychiatrists consider cooccurring mental health disorders, since the majority of adolescents with substance use problems present with a co-occurring mental health diagnosis. Recommendations made in the $2005 \mathrm{PP}$ were limited by a relative lack of rigorous trials at the time.

Since the publication of the 2005 PP, there has been a proliferation of adolescent substance use treatment trials, many of which have employed more rigorous designs, larger samples, random assignment, direct comparisons of two or more active treatments, improved measures of substance use and other variables, newer interventions (e.g., manual-guided interventions), and longer-term outcome assessments. This systematic review (SR) will inform a Clinical Update and Clinical Practice Guideline to update the 2005 AACAP PP for the Assessment and Treatment of Children and Adolescents with SUDs. Given the high co-occurrence of substance use and other mental illnesses, and the increased focus on integrated treatment, there is a great $\backslash$ 
need to evaluate the evidence, and to engage researchers and clinicians, including primary care physicians, regarding the most effect treatments for substance use in adolescents. ${ }^{15}$

In 2014, a guide developed by the National Institute of Drug Abuse (NIDA) identified multiple approaches to treating adolescent SUDs, which were divided into behavioral approaches, family-based approaches, addiction medicine, and recovery support services, but this report did not synthesize evidence on comparative effectiveness. ${ }^{16}$ The American Academy of Pediatrics (AAP) Committee on Substance Use and Prevention recently recommended consideration of pharmacotherapy for adolescent and young adult patients with severe opioid use disorders or co-occurring alcohol use disorders. ${ }^{17}$ Thus, there is a significant need for a rigorous and comprehensive synthesis of the adolescent substance use treatment literature that addresses both pharmacologic and psychological treatments.

The overarching goal of this review is to evaluate the available evidence for the treatment effects (and comparative effects) of available behavioral and pharmacologic interventions to manage SUD and problematic use (not including tobacco) in adolescents and young adults. The review evaluates treatment effects across population subgroups and identifies evidence (or gaps in evidence) regarding the key ingredients of successful interventions for problematic substance use in adolescents and young adults. For most specific topics, we conducted de novo systematic review, but for treatment of alcohol use disorders/problematic alcohol use in the college setting, we summarized existing SRs, since this literature is large, highly contextual, and has been extensively reviewed.

\section{Key Questions}

Key Question 1. What are the effects of behavioral, pharmacologic, and combined interventions compared with placebo or no active treatment for substance use disorders and problematic substance use in adolescents to achieve abstinence, reduce quantity and frequency of use, improve functional outcomes, and reduce substance-related harms?
a. How do benefits and adverse outcomes of interventions vary by subpopulations?
b. How do benefits and adverse outcomes of interventions vary by intervention characteristics

Key Question 2. What are the comparative effects of active interventions for substance use disorders and problematic substance use in adolescents to achieve abstinence, reduce quantity and frequency of use, improve functional outcomes, and reduce harms?
a. How do comparative benefits and adverse outcomes of interventions vary by subpopulations?




\section{b. How do comparative benefits and adverse outcomes of interventions vary by intervention characteristics?}

The analytic framework for the key questions is shown in Figure 1.

Figure 1. Analytic framework for the Key Questions

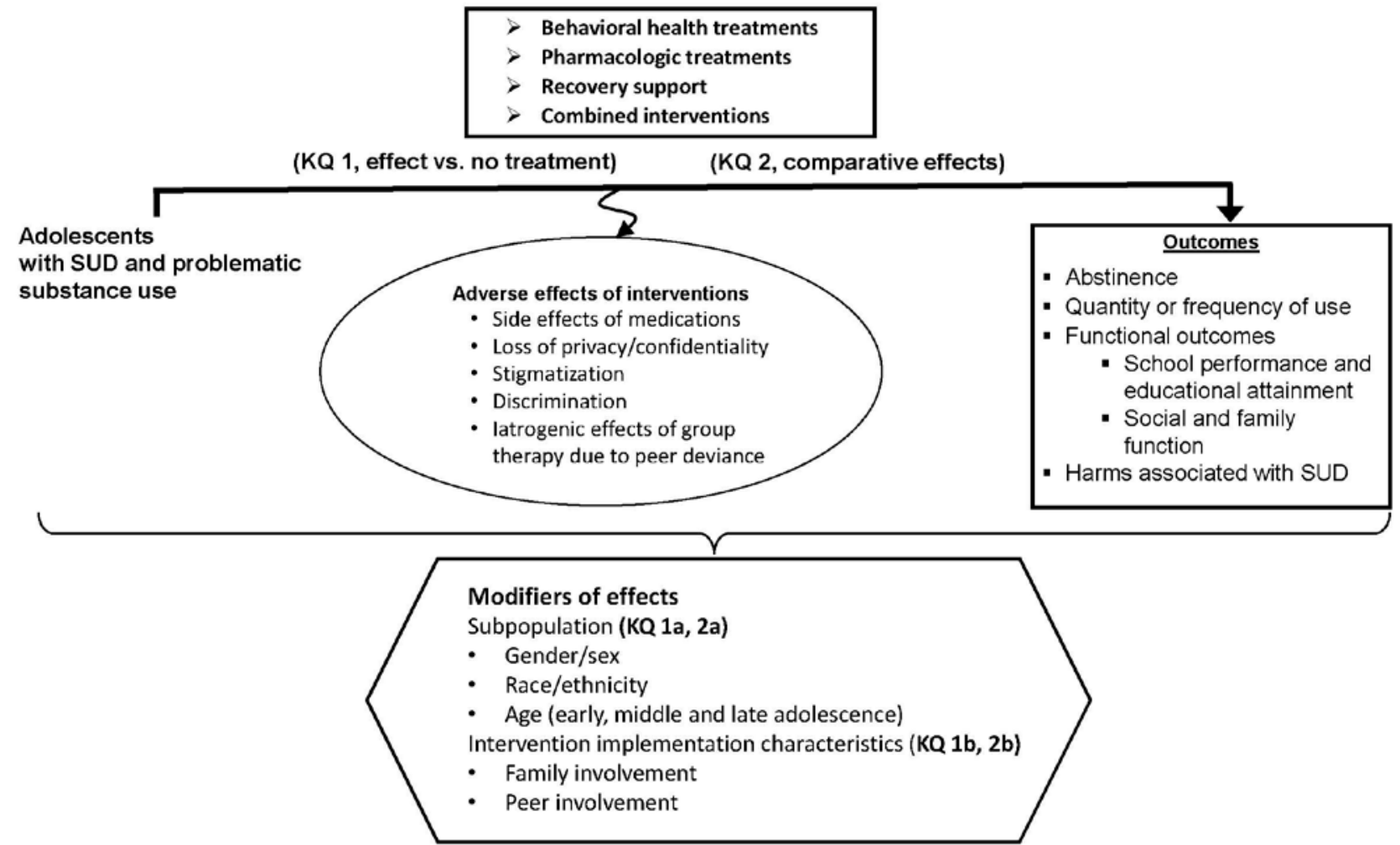




\section{Methods}

The Evidence-based Practice Center conducted the review based on a systematic review (SR) of the scientific literature, using established methodologies as outlined in the Agency for Healthcare Research and Quality (AHRQ) Methods Guide for Effectiveness and Comparative Effectiveness Reviews. ${ }^{18}$ The PROSPERO registration number is CRD42018115388.

\section{Searching for the Evidence}

We conducted literature searches in MEDLINE, the Cochrane CENTRAL Trials Registry, Embase, CINAHL, and PsycINFO databases (all from inception) to identify primary studies meeting our criteria through April 11, 2019. As a part of an independent methods project, an interim search of MEDLINE was undertaken using text mining tools on October 30, 2018. A separate search for SRs of interventions for alcohol disorders/problematic alcohol use in the college setting was conducted in MEDLINE, Cochrane Database of Systematic Reviews, and Epistemonikos also through April 11, 2019; after discussion with the Technical Expert Panel (TEP), it was decided to restrict the review of this topic to existing SRs because the literature is vast and has been extensively reviewed. All search strategies are detailed in Appendix A. The search strategies were peer reviewed by an independent, experienced information specialist/librarian. We asked the TEP to provide citations of potentially relevant articles. We also perused the reference lists of published clinical practice guidelines and relevant existing SRs for eligible studies. We also searched ClinicalTrials.gov on October 30, 2018 to identify unpublished and ongoing studies, and the U.S. Food and Drug Administration (FDA) Web site on October 30, 2018 for pharmacologic trials.

Peer and public review provided an additional opportunity for the TEP and other experts in the field to ensure that no key publications were missed. Finally, a Supplemental Evidence and Data for Systematic review (SEADS) portal and Federal Register Notice was posted for this review.

\section{Study Eligibility}

Table 1 and the following paragraphs detail the eligibility criteria.

\section{Population}

\section{Adolescents}

We included studies of adolescents and young adults. Our a priori definition of this population's age range was 12 to 20 years inclusive. Our search was designed to identify studies whose lower inclusion ages overlapped our age range of interest. Thus, we screened the full text of otherwise eligible studies that also included transition age youth (age 21 to 25) and adults (age 18 and above) for reported subgroups. For studies of behavioral interventions, we excluded studies that enrolled more than 20 percent of subjects older than our a priori upper age of 20 years. Because of the relative sparseness of studies of pharmacologic interventions, we expanded the upper inclusion age to 25 years inclusive.

\section{Substance Use-Related Eligibility Criteria}

We included studies that enrolled patients with substance use disorder(s) or problematic use of all substances except for tobacco. 
We included studies that enrolled participants with at least one substance use disorder diagnosis, or subjects with problematic use.

\section{Substance Use Disorder}

Studies were considered to have enrolled subjects with substance use disorder if subjects met DSM-III, DSM-IV or DSM-V criteria for one or more substance use disorders (except tobacco). ${ }^{19-21}$

\section{Problematic Use}

A designation of problematic use was applied if a study applied one or more of the following inclusion criteria: (1) subjects were referred for treatment by self, parent, school, other professional, or the juvenile justice system; (2) subjects were screened using a validated tool, such as the AUDIT-C (Alcohol Use Disorders Identification Test-Consumption) ${ }^{22}$ and an intervention was given to those who met a prespecified threshold; (3) subjects were asked about amount and frequency of use, and a threshold of at least monthly substance use was defined or; (4) subjects were identified after a consequence of substance use, such as an alcohol related emergency department visit.

\section{Interventions and Comparators}

Each study of behavioral interventions was categorized as either brief (if the intervention consisted of one or two sessions), or nonbrief (defined as 3 or more sessions). The broad range of behavioral interventions were consolidated into combinations of seven primary intervention components and four intervention modifiers, based on components identified in prior SRs (see Intervention Coding below for details).

Two distinct categories of pharmacologic interventions were considered: (1) medications used specifically to reduce and/or eliminate substance use and to prevent relapse, and (2) medications to treat co-occurring psychiatric disorders in patients with concurrent problematic substance use or substance use disorder. The latter set of medications were considered substance use treatments only if their effects on substance use were explicitly examined.

\section{Outcomes}

Use-related outcomes for specific substances (e.g., alcohol, cannabis) and outcomes that aggregated multiple substances were eligible for synthesis. We extracted continuous measures reflecting frequency of use and categorical measures of abstinence. For alcohol, we considered both frequency of heavy alcohol use (e.g., mean days of heavy use per 30 days) and frequency of any use (e.g., percent days of alcohol use per 30 days).

Aggregate outcomes of use or abstinence for multiple substances were classified into one of three categories: (1) alcohol and other drugs, (2) illicit drug use (excludes alcohol, but includes cannabis and other drugs, regardless of local laws), (3) other drugs (which explicitly excludes alcohol and cannabis).

A variety of substance use related problem scales were encountered, and they are detailed in Appendix H. When a study reported multiple problem scales that reflected problems associated with use of a specific substance, we chose the scale with the highest mean severity in each study. 


\section{Study Designs}

For studies of behavioral interventions and of pharmacologic treatment of psychiatric comorbidities, we included only randomized controlled trials with a minimum of 10 patients per arm.

For pharmacologic interventions of medications specifically to reduce and/or eliminate substance use and to prevent relapse, we included randomized controlled trials with a minimum of 10 patients per arm, and nonrandomized comparative studies or single group studies enrolling at least 100 patients per arm.

Table 1. Eligibility criteria

\begin{tabular}{|c|c|c|}
\hline PICOTS & Inclusion & Exclusion \\
\hline Population & $\begin{array}{l}\text { Adolescents } \\
\text { - Nonpharmacologic }(12-20 \text { years, inclusive) } \\
\text { - Pharmacologic }(12-25 \text { years, inclusive) } \\
\text { Substance use disorder or problematic use of: } \\
\text { - Alcohol } \\
\text { - Cannabis } \\
\text { - Opioids, including nonmedical prescription and illicit } \\
\text { - Sedatives, hypnotics, or anxiolytics } \\
\text { - Stimulants, including nonmedical prescription and illicit } \\
\text { - Inhalants } \\
\text { - Hallucinogens } \\
\text { - Unspecified or polysubstance use } \\
\text { Subpopulations of particular interest } \\
\text { - Psychiatric comorbidities } \\
\text { - Age subgroups (12-14, } 15-17,18-20 \text { years) } \\
\text { - Sex, gender, and sexuality } \\
\text { o Male/female, cis/transgender, orientation } \\
\text { - Racial/ethnic minority } \\
\quad \text { o White } \\
\quad \text { o African American/Other } \\
\text { o Hispanic } \\
\text { - Socioeconomic status and related characteristics } \\
\text { - Pregnancy, postpartum, parenting } \\
\text { Family characteristics } \\
\text { Demographics, family dynamics, involvement with } \\
\text { child protection services }\end{array}$ & $\begin{array}{l}\text { For nonpharmacologic studies, } \\
\text { if }>20 \% \text { of study sample (or } \\
\text { identifiable subgroup) is }<12 \text { or } \\
>20 \text { years, combined } \\
\text { For pharmacologic studies, } \\
\text { if }>20 \% \text { of study sample (or } \\
\text { identifiable subgroup) is }<12 \text { or } \\
>25 \text { years, combined } \\
\text { Tobacco/nicotine use (including if } \\
\text { the polysubstance use is } \\
\text { predominantly tobacco/nicotine) } \\
\text { Substance use not meeting } \\
\text { definition of at least } \\
\text { "problematic use" }\end{array}$ \\
\hline
\end{tabular}




\begin{tabular}{|c|c|c|c|}
\hline PICOTS & \multicolumn{2}{|c|}{ Inclusion } & Exclusion \\
\hline Interventions & 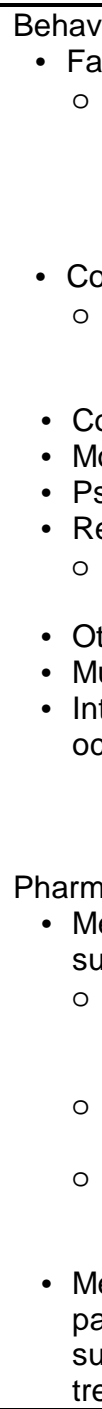 & $\begin{array}{l}\text { ral health treatments } \\
\text { ily therapies } \\
\text { Family behavioral therapy, family systems therapy, } \\
\text { brief strategic family therapy, functional family } \\
\text { therapy, ecological family therapy, multidimensional } \\
\text { family therapy, family systems network, educational } \\
\text { family therapy, multisystemic therapy, others } \\
\text { nitive behavioral therapy } \\
\text { Adolescent community reinforcement approach, } \\
\text { dialectical behavioral therapy, cognitive therapy, } \\
\text { others } \\
\text { tingency management } \\
\text { tivational interviewing or enhancement therapy } \\
\text { choeducation (aimed at substance use) } \\
\text { covery support } \\
\text { 12-step programs, peer-based and/or peer supports, } \\
\text { assertive continuing care, others } \\
\text { er (e.g., culturally sensitive interventions) } \\
\text { ticomponent interventions ( } 2 \text { or more models) } \\
\text { grated interventions (for substance use and a co- } \\
\text { urring disorder) } \\
\text { - Behavioral interventions are divided by duration } \\
\quad \text { o Brief interventions ( } 1 \text { or } 2 \text { sessions only) } \\
\text { o Nonbrief interventions ( } \geq 3 \text { sessions) } \\
\text { cologic interventions } \\
\text { dications used specifically to reduce and/or eliminate } \\
\text { stance use and to prevent relapse } \\
\text { For alcohol } \\
\text { - Gabapentin, naltrexone, acamprosate, } \\
\text { disulfiram, topiramate, ondansetron } \\
\text { For cannabis } \\
\text { Foracetylcysteine } \\
\text { - Methadone, buprenorphine, combination } \\
\text { buprenorphine and naloxone, naltrexone } \\
\text { dications to treat co-occurring psychiatric disorders in } \\
\text { sents with concurrent problematic substance use or } \\
\text { ttance use disorder (regardless of primary goal of } \\
\text { thent with the drug) }\end{array}$ & $\begin{array}{l}\text { Preventive interventions (i.e., } \\
\text { interventions among nonusers } \\
\text { to prevent future substance use) } \\
\text { Interventions not aimed at } \\
\text { reducing substance use (e.g., } \\
\text { needle exchange, condom } \\
\text { promotion) } \\
\text { Medications to treat overdose } \\
\text { Pharmacologic management of } \\
\text { acute withdrawal symptoms }\end{array}$ \\
\hline Comparators & $\begin{array}{r}\text { Key Q } \\
-\mathrm{Nc} \\
-\mathrm{Tr} \\
\text { - Ec } \\
\text { us } \\
\text { Key Q } \\
\text { - Ar } \\
\text { wi } \\
\text { co }\end{array}$ & $\begin{array}{l}\text { estion } 1 \\
\text { active treatment, including waitlist and placebo } \\
\text { atment as usual (including if poorly defined) } \\
\text { Ication or other materials (not aimed at substance } \\
\text { estion } 2 \\
\text { other active intervention, including interventions both } \\
\text { in a given category (e.g., comparing two types of } \\
\text { nitive behavioral interventions) }\end{array}$ & $\begin{array}{l}\text { For Key Question 2, within- } \\
\text { category comparisons, if differ } \\
\text { only in personnel (e.g., years of } \\
\text { therapist training) }\end{array}$ \\
\hline
\end{tabular}




\begin{tabular}{|c|c|c|}
\hline PICOTS & Inclusion & Exclusion \\
\hline Outcomes & $\begin{array}{l}\text { Use outcomes } \\
\text { - Frequency of use (self report) } \\
\text { o Days of use over specified time period } \\
\text { o Heavy alcohol use days over specified time } \\
\text { - Abstinence (objective or self-reported) } \\
\text { - Severity of use } \\
\text { o Substance-related problems or symptoms counts or } \\
\text { Functional outcomes } \\
\text { - School performance and educational attainment, } \\
\text { including attendance, academic performance, } \\
\text { graduation, entering higher education, and others } \\
\text { - Social relationships, including family functioning, peer } \\
\text { relationships, and others } \\
\text { Harmful consequences of substance use } \\
\text { - Serious mental health events, including suicidal ideation } \\
\text { and behavior } \\
\text { - Physical health, including mortality, substance-use- } \\
\text { related morbidities, infections (e.g., HIV, hepatitis C, } \\
\text { other sexually transmitted diseases) } \\
\text { - Serious legal events, including arrest, recidivism, contact } \\
\text { with juvenile justice system } \\
\text { Adverse events of interventions } \\
\text { - Side effects of pharmacologic interventions } \\
\text { - Loss of privacy or confidentiality } \\
\text { - Stigmatization or discrimination } \\
\text { - latrogenic effects of group therapy due to peer deviance } \\
\text { - Other reported adverse effects ascribed to interventions }\end{array}$ & None \\
\hline Timing & $\begin{array}{l}\text { Minimum } 1 \text { month followup (since the start of the } \\
\text { intervention) }\end{array}$ & None \\
\hline Settings & $\begin{array}{l}\text { Any, including community, residential, jail/prison, court- } \\
\text { mandated, etc. } \\
\text { Any country or geographic area }\end{array}$ & College setting (for alcohol)* \\
\hline $\begin{array}{l}\text { Study } \\
\text { designs }\end{array}$ & $\begin{array}{l}\text { All studies: } \\
\text { - Published, peer-reviewed articles or unpublished data } \\
\text { from the FDA or from the Results reported on } \\
\text { ClinicalTrials.gov web site } \\
\text { - Any publication date } \\
\text { For any intervention and outcome (except alcohol use among } \\
\text { college students): } \\
\text { - Randomized controlled trials } \\
\text { - N } \geq 10 \text { per study group } \\
\text { For pharmacologic studies reporting adverse events, as } \\
\text { above and: } \\
\text { - Nonrandomized comparative studies, prospective or } \\
\text { retrospective, N } \geq 100 \text { per study group } \\
\text { For studies of alcohol use among college students: } \\
\text { - Systematic reviews of randomized controlled trials } \\
\end{array}$ & $\begin{array}{l}\text { Case control studies } \\
\text { Cross-sectional studies } \\
\text { Case reports or series }\end{array}$ \\
\hline $\begin{array}{l}\text { Publication } \\
\text { language }\end{array}$ & Any & $\begin{array}{l}\text { Unable to read, translate, or } \\
\text { retrieve }\end{array}$ \\
\hline
\end{tabular}

Abbreviations: FDA = Food and Drug Administration; $\mathrm{N}=$ sample size; $\mathrm{PICOTS}=$ populations, interventions, comparators, outcomes, timing, setting

*Because we did a review of reviews for alcohol in the college setting, we excluded primary studies thereof. 


\section{Screening Studies for Eligibility}

For citation screening, we initially conducted a series of pilot training sessions to achieve a satisfactory level of agreement among researchers regarding the nuances of the eligibility criteria for title and abstract screening. Because abstracts sometimes do not mention all outcomes that are reported in the full-text, we did not exclude titles and abstracts based on outcomes. We conducted all abstract screening using the open-source, online software Abstrackr (http://abstrackr.cebm.brown.edu/). To assist with screening, we used the predictive algorithm capabilities of Abstrackr, which frontloads more-likely-to-be-relevant citations. We began with double, independent screening of abstracts. Conflicts were resolved during full-group meetings. Using the labels (accept, reject) given to screened abstracts, Abstrackr determines a prediction value for all remaining unscreened citations and sorts these such that the most-likely-to-beaccepted abstracts are screened first. Based on empirical research on Abstrackr (soon to be submitted for publication), when all remaining unscreened abstracts have a prediction value $<0.40$ (on a scale of 0 to 1 ), these abstracts are highly likely (>99\% probability) to be rejected. We, thus, double screened abstracts until this threshold was met; thereafter, we switched to single screening of remaining abstracts.

We obtained the full-texts of all citations that were screened in (accepted) during abstract screening. The reference lists from SRs were reviewed for the presence of additional primary studies. We evaluated these articles using an evidence map structure, in which we gathered basic data on each article (i.e., study design, sample size, confirmation of substance use disorder/problematic use, age data, intervention(s), confirmation of outcomes of interest). Articles derived from the same studies (multiple publications, secondary analyses) were grouped. Using this process, we determined final eligibility status for each study.

\section{Data Extraction}

Studies with multiple publications or secondary analyses were extracted as one study. Multiple studies reported in a single publication were extracted separately. Small teams of researchers focused on extraction of different elements from each of the studies. One team extracted study design, population characteristics, and baseline data; one team extracted and categorized interventions (as described in the next section); one team extracted outcome descriptions and study results; one team extracted risk of bias information. Each section of each study was extracted by one researcher and then verified by at least one other experienced researcher. Discrepancies were discussed between them, as needed.

Data were extracted into customized forms in the Systematic Review Data Repository (SRDR) online system (http://srdr.ahrq.gov) or into separate spreadsheets designed to capture all elements relevant to the Key Questions (KQs). Upon completion of the review, all data were uploaded to SRDR, and the SRDR database was made accessible to the public, with capacity to read, download, and comment on data.

The basic elements and design of these forms are similar to those we have used for other comparative effectiveness reviews and include elements that address population characteristics; descriptions of the interventions and comparators; outcome definitions; intervention modifiers; sample sizes; study design features; funding source; results; and risk of bias.

We did not contact study authors for additional data. 


\section{Intervention Coding}

Each active intervention was categorized as either a brief (defined as 1 or 2 sessions) or nonbrief (defined as 3 or more sessions) intervention. To be classified as a "session" the adolescent had to connect directly with a therapy provider either in-person, by phone, or on the Web. Text message prompts or queries were not considered sessions.

A codebook (Appendix C) with definitions for seven primary intervention components and intervention modifiers was developed by the Scientific Lead based on the most commonly reported approaches in prior SRs. ${ }^{23-25}$ The intervention components were:

- Motivational interviewing (MI)

- Family focused therapy (Fam)

- Cognitive behavioral therapy (CBT)

- Psychoeducation (Educ)

- Contingency management (CM)

- Peer group therapy (PeerGroup)

- Intensive case management (ICM).

Each study was independently coded by two investigators, one with expertise in adolescent substance use interventions and the other with expertise in the analysis of multi-component health service and behavior interventions. After assigning one or more intervention components to each study arm, the investigators reviewed and compared intervention codes and identified discrepant codes, which were discussed in detail with the goal of obtaining consensus. In cases when discrepancies were not resolved via discussion, a third senior investigator with expertise in adolescent substance use interventions reviewed the codes and served as the tie breaker.

The coders assigned four intervention modifiers: delivery of therapy in groups, additional parent involvement, culturally accommodated treatment, and integrated interventions targeting both substance use and mental health. The definitions for the intervention components and modifiers are described below.

\section{General Principles}

The following principles guided intervention coding:

a. Intervention components had to be unique and distinct from one another (e.g., if a CBT intervention described educating adolescents about skills, we did not code education unless there was a distinct psychoeducational session or module);

b. Intervention components had to be sufficiently well described to ensure that all adolescents consistently received the treatment (e.g., if usual services was referral to an intervention or host of potential interventions but was not standardized to ensure all adolescents received it, we did not code the presence of the intervention component). Related, vaguely described intervention components lacking reference to a specific manual or another form of fidelity monitoring were not coded as having a component (e.g., intervention described as "motivational interviewing informed" or "cognitive behavioral therapy inspired”); and

c. Study arms that were reported as "treatment as usual" (TAU) were coded as having specific intervention components if the description treatment met the definition of the components and threshold criteria above. 


\section{Motivational Interviewing (MI)}

We coded an intervention as containing MI if it explicitly described at least one session focused on building the adolescent's motivation to reduce substance use and/or attain abstinence. Motivation enhancement therapy (MET), a more structured and specific approach to building the adolescent's motivation, was also categorized as MI. MET typically includes techniques, such as a decisional balance and personalized feedback on substance use patterns with normative comparisons, which are specifically designed to enhance motivation to change.

Family, cognitive behavioral, and educational therapy models that generally referenced a goal to build the adolescent's motivation to change were not coded as containing MI unless there was a stand-alone, manual-guided intervention MI component. If MI was delivered to the parent either instead of or in addition to the adolescent sessions, this was recorded and assigned a parent involvement qualifier.

\section{Family Therapy (Fam)}

We coded an intervention as containing family therapy if sessions were predominantly delivered with the entire family present and if the focus of the therapy was on changing the adolescent's substance use by intervening with the entire family system. Interventions that were delivered predominantly to the adolescent and that contained parent-only sessions or periodic family check-ins were not categorized as family therapy. Building upon the classifications used in prior SRs (Becker \& Curry, 2008; Hogue et al., 2018), ${ }^{26,27}$ we assigned qualitative descriptors to describe family therapy models within five broad categories: ecological, systems/structural, behavioral, functional, and educational. These categories were assigned to enhance the qualitative description of distinct models but were not included in the quantitative synthesis.

Ecological models explicitly targeted adolescent substance use in the context of extrafamilial influences across multiple interrelated, nested systems. Example intervention models included multidimensional family therapy, ecological family therapy, and multisystemic therapy.

Systems/structural approaches attempted to restructure problematic family interaction patterns associated with the adolescent's substance use. Systems/structural models included brief strategic family therapy, family systems therapy, and family structural therapy.

Behavioral approaches were those that applied principles of operant conditioning and social learning within the family context in order to encourage healthy behavior and discourage substance use.

Functional approaches integrated principles of both systems and behavioral approaches, such as functional family therapy.

Educational approaches explicitly aimed to address the adolescent's substance use through the provision of psychoeducation to the entire family.

\section{Cognitive Behavioral Therapy (CBT)}

We coded an intervention as containing CBT if it explicitly described using a manual or protocol focused on providing the adolescent with either cognitive (e.g., thought identification, thought modification) or behavioral (e.g., peer refusal, communication, problem solving) skills needed to reduce substance use and/or attain abstinence. CBT was only coded in adolescentfocused intervention components: family models that focused on building skills to change were coded as family behavioral therapy. Because all CBT models involve some degree of parent involvement, an intervention was coded as CBT if sessions were primarily delivered to the 
adolescent alone (even if there were some parental involvement in the form of updates, parent only sessions, or parent check-ins) and categorized as family behavioral therapy if sessions were primarily delivered to the entire family.

\section{Peer Group Therapy (PeerGroup)}

Adolescent peer group therapy models were nondirective therapy interventions delivered to adolescents in group format, which aimed to reduce the adolescent's substance use by having adolescents interact and provide social support. Interventions were coded as containing adolescent peer group therapy if the following two conditions were met: a) clear reference to therapy sessions delivered to adolescents in group format; and b) sessions were described as interactive, process-oriented, and/or following a self-help approach. Group therapy sessions that explicitly referenced a CBT or psychoeducational manual were not coded as peer group therapy: such approaches were coded as CBT or psychoeducation, respectively, and assigned the group therapy intervention modifier.

\section{Psychoeducation (Educ)}

Psychoeducational interventions were interventions explicitly designed to reduce the adolescent's substance use through the provision of education about the harms of alcohol and illicit drugs. An intervention was coded as containing psychoeducation if it had an explicit standalone module focused on the provision of education. Because the majority of intervention models designed for the target population involve some degree of education about adolescent substance use, we only coded an intervention as containing psychoeducation if there was explicit reference to a stand-alone psychoeducation module or intervention. For example, family therapy, MI, and CBT models that made general reference to providing education to parents or teens were not coded as educational unless they had a clearly specified independent psychoeducation component.

\section{Contingency Management (CM)}

CM interventions explicitly described the provision of external, consistent reinforcement for the adolescent's attainment of pre-defined goals. An intervention was coded as containing CM if it described a specific protocol (e.g., manual, prize schedule) for positive reinforcement of the adolescent's behavior. Family therapy models that taught the parent how to monitor and reinforce the adolescent's behavior through household contracts were not coded as containing CM unless the reinforcement was explicitly provided as a part of the therapy sessions: intervention approaches in which parents learned to provide reinforcement were coded as CBT or family behavior therapy. Additionally, we only coded an intervention as CM if the reinforcement was positive in nature: enforcement of negative consequences for missed sessions or positive urine screens (e.g., as part of family or drug court) were not considered CM.

\section{Intensive Case Management (ICM)}

Intensive case management interventions were interventions in which the primary focus was on linking the adolescent to supportive services. Interventions were coded as intensive case management if they identified specific protocols focused on promoting continuity of care (e.g. assertive continuing care). 


\section{Treatment as Usual (TAU)}

Interventions designed to be comparators to active intervention and were not directed at treating substance use were categorized as TAU. Examples included waitlists or pamphlets regarding issues other than substance use. In addition, interventions that were not adequately described, actively monitored for fidelity, or in which it was not possible to determine whether all adolescents received the same intervention, were coded as TAU.

\section{Intervention Modifiers}

In addition to coding primary intervention components, we coded several intervention modifiers, as follows:

Group involvement. Therapy models were coded as group if any of the intervention elements were delivered in group format. This modifier encompassed a broader range of studies than the peer group therapy code. Studies that described parent-only groups and family groups were captured by this effect modifier, as were studies that described manual-driven CBT delivered in a group format or psychoeducational group therapy.

Parent Involvement. Nonfamily models were coded as having substantial parent involvement if they specifically described delivering intervention elements to parents only (i.e., parentfocused intervention) or if they described frequent parent check-ins.

Culturally accommodated. Therapy models that were specifically designed as adaptations for specific cultural groups were classified as culturally accommodated. Models had to explicitly reference being adapted for specific cultural groups or using formative work with specific cultural populations to receive this designation.

Integrated interventions. Interventions that were specifically designed to target co-occurring substance use and mental health diagnoses were coded as integrated. This modifier was only coded if the intervention was explicitly designed to address dual substance use and mental health diagnoses. If the sample had high proportions of dual diagnosis patients, but the intervention did not specify a specific focus on diagnoses of interest, then this modifier was not coded. Similarly, if the intervention targeted substance use and a co-occurring physical health concern such as HIV or sexual risk, then this modifier was not coded.

\section{Assessment of Risk of Bias}

Two senior investigators, highly experienced in SR and risk of bias assessment, assessed the risk of bias for all studies. After two rounds of double, independent risk of bias assessment, with adjudication of 10 studies per round, the remaining studies were assessed by one investigator and verified by the other (each was the primary assessor for about half the studies).

We assessed the risk of bias (methodological quality) of each study based on predefined criteria. For all studies, we used the Cochrane risk of bias tool, ${ }^{28}$ which examines methodological items, such as random sequence generation; allocation concealment; blinding of participants, care providers, and outcome assessors; incomplete outcome data; and selective reporting, to inform judgments about various sources of bias and overall risk of bias assessments. We also assessed whether intention-to-treat analyses were conducted. In addition, we used relevant questions from the Newcastle Ottawa Scale, ${ }^{29}$ including similarity of groups at baseline, whether any cointerventions differed between groups, absolute and comparative compliance, timing of outcome assessments (between groups), and any additional biases. 
If a randomized trial used an "urn method" for randomization (used to balance groups among prespecified participant characteristics) we assumed that randomization method and allocation concealment were low risk of bias (since randomization would need to be done centrally by computer). For outcome assessor blinding of nonpharmaceutical interventions, we assessed whether the outcome assessors were blinded to intervention group; if so, we determined these studies were low risk of bias for outcome assessor blinding (even though the adolescent users reporting substance use to the outcome assessor may not have been blinded). For incomplete outcome data (attrition bias) and compliance, we deemed studies to be high risk of bias if more than 20 percent of participants dropped out or did not comply with the intervention, regardless of whether intention-to-treat analyses were conducted. Regarding selective outcome reporting, we captured information from available protocols (including from ClinicalTrials.gov) on planned outcomes. For group similarity, we captured information about the statistical significance of differences between groups at baseline; if there were differences, but these were statistically accounted for in analyses, we deemed these to be low risk of bias.

For SRs of interventions for alcohol use disorder or problematic alcohol use in the college setting, we assessed SR quality using specific items from AMSTAR 2 (A Measurement Tool to Assess Systematic Reviews, version 2) ${ }^{30}$ We omitted questions about SR protocol timing, justification of excluded studies, study funding sources, and assessment of publication bias. Also, two questions about description of eligibility criteria were combined as were two questions about assessment of risk of bias in their analyses were combined. Thus, the risk of bias questions (with corresponding item numbers in AMSTAR 2) included: description of eligibility criteria (item 1), comprehensive search strategy (item 4), duplicate study screening (item 5), duplicate data extraction (or with verification) (item 6), adequate description of details of included studies (item 8), use of a satisfactory technique for assessing risk of bias in included studies (item 9), appropriate meta-analysis methods (if applicable) (item 11), assessment of potential impact of risk of bias (item 12), explanation and discussion of any heterogeneity (item 14), and reporting of SR conflict of interest (item 16). We deemed that SRs that meta-analyzed (standardized) "effect sizes" across disparate outcomes did not address the KQs of our systematic review because they indiscriminately combined highly heterogeneous outcomes. Furthermore, some SRs included multiple outcomes from the same underlying trials without correction for correlation or for double-counting.

For all studies, any quality issues pertinent to specific outcomes within a study were noted and applied to those outcomes. Quality issues pertinent to specific outcomes within a study were noted and considered when determining the overall strength of evidence (SoE) for conclusions related to those outcomes.

Detailed risk of bias assessments for each study are listed in Appendix H. A summary of the risk of bias for the studies in each group eligible for meta-analysis is displayed in a stacked bar chart.

\section{Data Synthesis}

Frequency of use outcomes were reported in multiple forms. If use was reported as days of use per time interval (e.g., days of use per month, percent use days per 90 days), mean use was converted to a common metric of mean use days per 30 days, despite the acknowledged caveat that this metric assumes that use was constant over the various reporting intervals.

We preferentially included outcomes evaluated at 4 months after baseline assessment. If outcomes were not reported at 4 months, we accepted the closest followup time in the range from 
1 to 6 months (in the case of ties, e.g. data available for both 3 months and 5 months, we chose the earlier time). Abstinence outcomes were summarized as odds ratios.

For continuous outcomes with an available baseline data scale, we evaluated the "net mean difference" (NMD) of the outcome, the difference between arms of the within-arm changes in outcome.

When necessary, standard errors (SE) of the differences were estimated from reported standard deviations (or SEs) of baseline and final values. For parallel trials, we assumed a correlation of 0.5 between baseline and final values in patients receiving a given intervention. Thus, we used the following equation to estimate the $\mathrm{SE}$ :

$$
\mathrm{SE}_{\text {difference }}^{2}\left(\mathrm{SE}_{\mathrm{A}}\right)^{2}+\left(\mathrm{SE}_{\mathrm{B}}\right)^{2}-2 \cdot \mathrm{r} \cdot\left(\mathrm{SE}_{\mathrm{A}}\right) \cdot\left(\mathrm{SE}_{\mathrm{B}}\right)
$$

where $r=0.5$ (the assumed correlation) and A and B index the correlated measurements (baseline and final time points).

Standardized net mean differences (SNMD) were calculated for substance use problem scales. In a sensitivity analysis, we calculated standardized net mean differences (SNMD) to enable combined analyses of: 1) scales that reflected (intensity of) substance use, and 2) nonlinear transformations, e.g. square root of mean use days, and substance specific days of use outcomes.

A minority of studies did not report either standard continuous or categorical outcome metrics, but instead either summarized models with metrics that could not be converted to net difference, odds ratio, or risk ratio, or reported only statistical significance (with or without directionality). These were not included in the quantitative analysis and are not explicitly summarized in the review text. Their results are included in the Evidence Tables and, electronically, in the SRDR project file.

\section{Qualitative Evidence Synthesis}

Prior to meta-analysis, with input from subject matter experts, we qualitatively evaluated whether populations were sufficiently comparable for quantitative synthesis.

We assessed population comparability in two ways. First, we identified the study inclusion criteria to determine whether each study targeted alcohol, cannabis, another drug, or a combination of substances. Next, we assessed substance use reported in the recruited samples. Although study eligibility criteria were heterogeneous in terms of targeted substances; the final samples were predominantly comprised of adolescents with some combination of alcohol and cannabis use, with a minority using other drugs. Studies that specified substances other than alcohol and cannabis in their eligibility criteria (e.g. ecstasy or cocaine, ${ }^{31}$ methamphetamine, ${ }^{32}$ inhalants ${ }^{33}$ ) were excluded from meta-analyses.

The vast majority of studies reported overlapping substance specific outcomes (i.e., a given study might report cannabis outcomes, alcohol outcomes or both). We analyzed these outcomes separately by substance. Thus, a given study might contribute to a cannabis analysis, to an alcohol analysis, or to both. Some studies only reported an aggregate use measure, e.g., alcohol and other drug use or illicit drug use.

Substance use problem scales were combined and pooled across substances.

\section{Quantitative Evidence Synthesis}

We conducted pairwise meta-analyses (MA) using both frequentist and Bayesian frameworks, and network meta-analyses (NMA) in the Bayesian framework. Analyses were done using $\mathrm{R}^{34}$ with the metafor ${ }^{35}$ and gemtc packages. ${ }^{36}$ 
MAs used a random effects model assuming that within-study estimates and between studies true effects are normally distributed.

NMA is an extension of pairwise meta-analyses that simultaneously combines direct (when interventions are compared head-to-head) and indirect (when interventions are compared through other reference interventions) evidence. We performed NMA when more than three studies formed a connected network. Combining the direct and indirect evidence not only improves precision of estimates, but also provides estimates for all pairwise comparisons, including those missing from the direct evidence. The key assumption of the network meta-analysis is that of consistency of direct and indirect effects. Consistency is likely to hold when the distribution of effect modifiers is (equivalently, patient characteristics are) similar across trials. If this assumption is violated, there may be inconsistency between the direct evidence and indirect evidence of treatment comparisons. ${ }^{37}$

Our NMA used a hierarchical model with a within-study level and a between-studies level that models responses at the arm level and nests arms within studies. We ran two sets of analyses, one assuming consistency of treatment effects and one examining this assumption. The models are shown in Appendix I Briefly, the analysis assuming consistency parameterizes treatment effects as linear combinations of $T-1$ parameters, where $T$ is the number of treatments in the network. Treatment effects are assumed to be normally distributed across studies with a common variance (i.e., are homoscedastic random effects). We used noninformative default priors on study-level mean treatment effects. Specifically, priors on the means were zerocentered normal distributions, with standard errors 15 times larger than the observed scatter of study effect estimates.

We used empirical prior distributions for the between-study heterogeneity variance. For outcomes modeled on the log odds ratio scale, we assigned a log-normal hyperprior for the between-study heterogeneity variance based on empirical results from meta-epidemiological analyses of nonpharmacologic trials with subjective outcomes. ${ }^{38,39}$ For continuous outcomes (NMD and SNMD) we used inverse gamma priors based on analogous empirical results nonpharmacologic studies of mental health outcomes. ${ }^{40}$ We performed a sensitivity analysis using alternative priors for the between-study variance.

In gemtc, estimation is done with MCMC via the $\mathrm{JAGS}^{41}$ sampler, using initial values drawn randomly from the marginal distributions of the priors of respective parameters. We fit four MCMC chains. After a burn in of 5000 iterations, we monitored convergence of random effects means and variances automatically, by checking every 10,000 iterations whether the Gelman Rubin diagnostic was less than 1.05 with 95 percent probability for all monitored parameters. After convergence was reached, an extra 10,000 iterations were run. All models converged within 10000 iterations. Model fit was assessed by comparing the posterior mean of the residual deviance to the number of data points. The ratio of residual deviance to number of data points in the various models was very close to 1 (within 5\%), suggesting adequate model fit.

For each analysis, we empirically assessed if the network meta-analysis consistency assumption was violated by comparing the direct and indirect evidence using a node-splitting approach. ${ }^{42}$ To this end, for each comparison that is informed by both direct and indirect data, we separately parameterized the direct and indirect effects, and compared the estimates of the two. Although these analyses were not suggestive of inconsistency (not shown), in sparse networks, like the ones in this report, they can be underpowered. 
Results are presented in terms of net mean or standardized net mean differences and corresponding 95 percent credible intervals (CrI). We preferentially report net mean differences for substance specific and aggregated (over multiple substances) use days.

Using the sampled posterior distribution of effects, we estimated the probability that a treatment is the most effective, second most effective, and so on, based on the results of the network meta-analyses. We report the surface under the cumulative ranking curve (SUCRA), which represents a single number ranging from 0 to 100 percent associated with each intervention. The higher the SUCRA value (closer to 100\%), the higher the likelihood that an intervention is in the top rank or one of the top ranks. As SUCRA values approach 0 percent, it is more likely that an intervention is in the bottom rank, or one of the bottom ranks. ${ }^{43}$

We performed an additional sensitivity analyses by comparing of an additional analysis of standardized net mean differences which combined use days with scales and nonlinear transformations relating to use days. Results were similar with the main analysis (not shown).

Statistical heterogeneity was explored qualitatively. Because of the relatively small number of studies, and the little variability in characteristics, meta-regression and subgroup analyses were not performed.

\section{Grading the Strength of Evidence for Major Comparisons and Outcomes}

We graded the strength of the body of evidence (SoE) as per the AHRQ methods guide on assessing the SoE. ${ }^{44}$ For conclusions based on NMA of sparse networks, we provided a qualification, and downgraded the SoE due to lack of precision and directness, as applicable.

We assessed the SoE for comparisons of major interventions (i.e., behavioral intervention methods, pharmacologic interventions, and combinations) to no treatment (TAU) and to each other.

To our knowledge, there is no information on the minimal clinically importance differences for the outcomes we consider. The commonly used conventions for standardized mean differences similarly do not translate to minimally importance differences. ${ }^{45}$

For each evaluated comparison, we assessed the number of studies, their study designs, the study limitations (i.e., risk of bias and overall methodological quality), the directness of the evidence to the KQs, the consistency of study results, the likelihood of reporting bias, in addition to the precision and magnitude of the effect estimated across studies using NMA. When at least 3 direct comparisons were available, we compared effects estimated from direct comparisons (using both frequentist and Bayesian random effect models), with effects obtained from Bayesian NMA.

If the Bayesian NMA random effect models (direct and indirect evidence) were consistent with the pairwise (direct), the effect size and precision from the NMA informed SoE ratings. For sparse networks with few direct comparisons, we downgraded the SoE by one category. In these cases consistency is rated as unclear.

Outcomes with highly imprecise estimates, highly inconsistent findings across studies, or with data from only one study were deemed to have insufficient evidence to allow for a conclusion (with the exception that particularly large, generalizable single studies could provide at least low SoE). This approach is consistent with the concept that for imprecise evidence "any estimate of effect is very uncertain," the definition of Very Low quality evidence per GRADE. ${ }^{46}$

Based on these multidimensional assessments, we assigned a SoE rating as being either high, moderate, low, or insufficient. 


\section{Assessing Applicability}

We assessed the applicability within and across studies with reference to adolescents in the populations of interest (i.e., type and severity of abuse and setting). ${ }^{47}$ 


\section{Results}

As illustrated by the flow diagram in Figure 2, we found 118 randomized controlled studies that evaluated treatment of adolescents with problematic substance use or substance use disorders. Excluded studies, along with reasons for exclusion, are listed in Appendix B.

Figure 2. Literature flow diagram

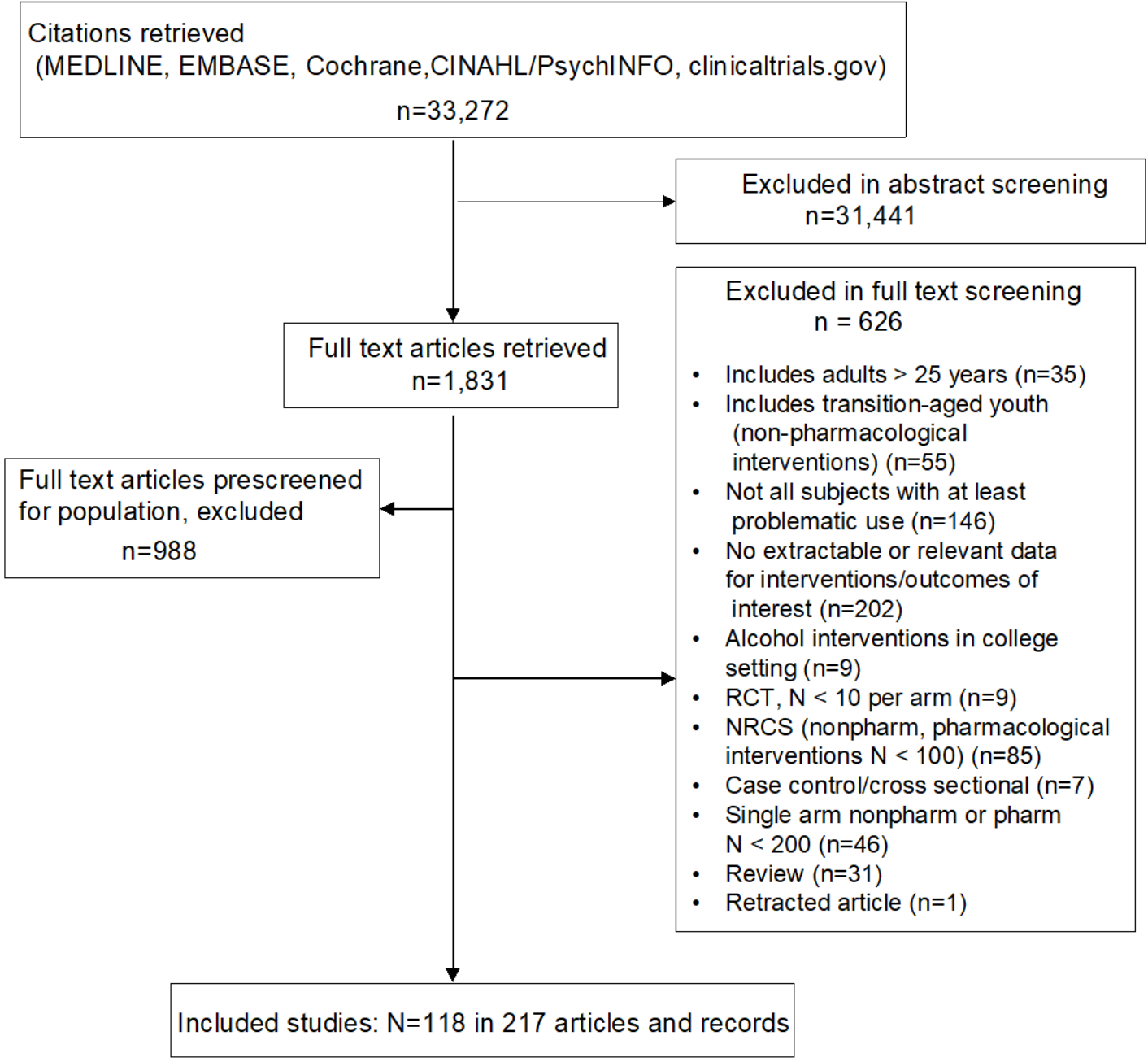

In a separate search for systematic reviews (SR) of interventions for problematic alcohol use in the college setting, we screened 401 abstracts, of these we screened 42 papers in full text, of which 36 were excluded (18 were reviews of preventive interventions, 10 were not in college students, 6 were not SRs, and 2 were not focused on alcohol users). This left 6 SRs that were deemed informative for our narrative summary.

\section{Qualitative Categorization}

Among studies of behavioral interventions, brief ( 2 or fewer sessions) and nonbrief ( 3 or more sessions) were qualitatively distinct. On initial review of the eligible studies, we found that participants in the brief intervention studies had problematic use, whereas nonbrief behavioral intervention studies enrolled adolescents with diagnosed substance disorder(s). In addition, the 
interventions provided in nonbrief studies were much more intensive. Therefore, we considered these groups of studies separately in our quantitative synthesis.

Despite superficial heterogeneity of study inclusion criteria with respect to the targeted substance(s), most studies enrolled adolescents using some combination of alcohol and cannabis, with a minority using other drugs.

The 3 studies that specified use of substances other than alcohol and cannabis in their eligibility criteria are briefly described in brief ${ }^{31,32}$ and nonbrief ${ }^{33}$ sections, but were excluded from meta-analyses.

The following substance-specific outcomes were evaluated for possible meta-analysis: heavy alcohol use days, alcohol use days, alcohol abstinence, cannabis use days and cannabis abstinence. In addition, studies reported aggregate outcomes reflecting abstinence for, or use of multiple substances. These outcomes included: alcohol and other drug use (AOD), illicit drug use and other drug use. In addition, we considered substance use problem scales within brief and nonbrief categories. Appendix D lists baseline and interventions. Appendix E identifies the outcomes reported by each study and Appendices $\mathrm{F}$ and $\mathrm{G}$ report detailed results for brief and nonbrief interventions, respectively. Outcomes with sufficient data for an outcome (bulleted below) were meta-analyzed and reported.

\section{Brief Behavioral Interventions}

- Alcohol

o Heavy alcohol use days

o Alcohol use days

o Abstinence from alcohol

- Cannabis

o Cannabis use days

o Cannabis Abstinence

- $\quad$ Substance use problem scales (Legal outcomes)

\section{Nonbrief Behavioral Interventions}

- Alcohol

$$
\text { o Alcohol use days }
$$

- Cannabis

$$
\text { o Cannabis use days }
$$

- Aggregate drug use

o Alcohol and other drug use

o Illicit drug use days

(Other outcomes: school performance and education attainment, family-related, peerrelated, mental health events, physical health events and legal outcomes)

The results in subsequent sections describe less commonly reported outcomes and are not meta-analyzed. We first briefly review of systematic reviews of interventions for alcohol use in the college setting.

Finally, we separately describe two categories of pharmacologic interventions.

1. Studies of medications to reduce and/or eliminate and/or to prevent relapse in adolescents with opioid, alcohol, and cannabis use disorders. In studies that combined pharmacologic 
and behavioral interventions, the behavioral interventions were often less completely described, and therefore not easily compared to the detailed manual based interventions typical in behavioral trials. Drug trials included placebo arms, which due to the likelihood of a placebo effect, were not deemed comparable to TAU arms in studies of behavioral interventions. Thus, we did not jointly synthesize studies of behavioral interventions with studies of pharmacologic interventions and summarize these studies separately by use disorder.

2. Studies of medications targeting specific co-occurring psychiatric disorders in patients with a substance use disorder(s). Given that effects on substance use may depend on how effectively the underlying psychiatric disorder was treated, we reported scales reflecting the severity of the psychiatric disorder in addition to substance use related outcomes.

\section{Brief Behavioral Interventions}

\section{Key Points}

Key points from the meta-analyses are summarized below.

- Motivational interviewing (MI)

o Reduces days of heavy alcohol use compared to TAU (low SoE)

o Reduces days of overall alcohol use compared to TAU (moderate SoE)

o Does not reduce days of cannabis use compared to TAU (moderate SoE)

o Reduces substance use problems compared to TAU (low SoE)

Thirty-six studies (in 64 papers; sample size range, 33 to 1449) 31, 32, 48-109,31, 32, 49-52, 54-69, 71-111 published between 1982 and 2019, evaluated effects of brief behavioral interventions in adolescents (mean age range, 14.8-18.9 years). Thirty-three studies enrolled participants with problematic use of alcohol, cannabis, and/or other drugs and three enrolled adolescents with a diagnosed substance use disorder. Appendix D (Table D-1) provides baseline and intervention details. Each of these studies each had methodological concerns including lack of outcome assessor blinding and incomplete outcome data. Most of the studies evaluated interventions we have coded as MI. Within-study descriptions varied, the intervention most commonly would be classified as Motivational Enhancement Therapy (MET). There was some variation in the number of sessions, the length of individual sessions, and the background and training of the interventionalist. Detailed results are presented by outcome in Appendix F.

\section{Studies Not Included in Meta-Analyses}

\section{Enrolled for Use of Substances Other Than Alcohol or Cannabis}

Two two-arm studies, published between 2006 and 2011, assessed adolescents with problematic use of specific substances other than alcohol or cannabis and evaluated brief behavioral interventions (Table 2) ${ }^{31,32}$ Adolescents in the studies were on average 15 to 18 years of age (range across studies, 14-22). The studies each had methodological concerns including lack of outcome assessor (or other) blinding, incomplete outcome data, poor compliance with the interventions, and others.

Each study was unique regarding substance used. The primary substances of misuse under study were ecstasy and cocaine ${ }^{31}$ (problematic use: at least four times over the past month) and methamphetamine ${ }^{32}$ (use disorder per DSM-IV). 
Neither study reported significant differences in mean number of use days or abstinence between adolescents who received active behavioral interventions and those who received treatment as usual or non-substance use disorder-related education. In the Marsden 2006 study, those receiving the brief intervention had slightly higher rates of abstinence, but it did not exclude the null effect - for cocaine (RR 1.17, 95\% CI 0.94 to 1.46) and for abstinence from crack cocaine (RR 1.12, 95\% CI 0.99, 1.26). ${ }^{31}$

In the small study of methamphetamine users, adolescents in both the brief MI and education groups used methamphetamine on average about 1 or 2 days per 30 days with no statistically significant difference between groups. About 50 to 60 percent of adolescents were abstinent at 1 and 2 months, with no statistically significant difference between groups. ${ }^{32}$

Table 2. Results: Brief behavioral interventions for substances other than alcohol or cannabis

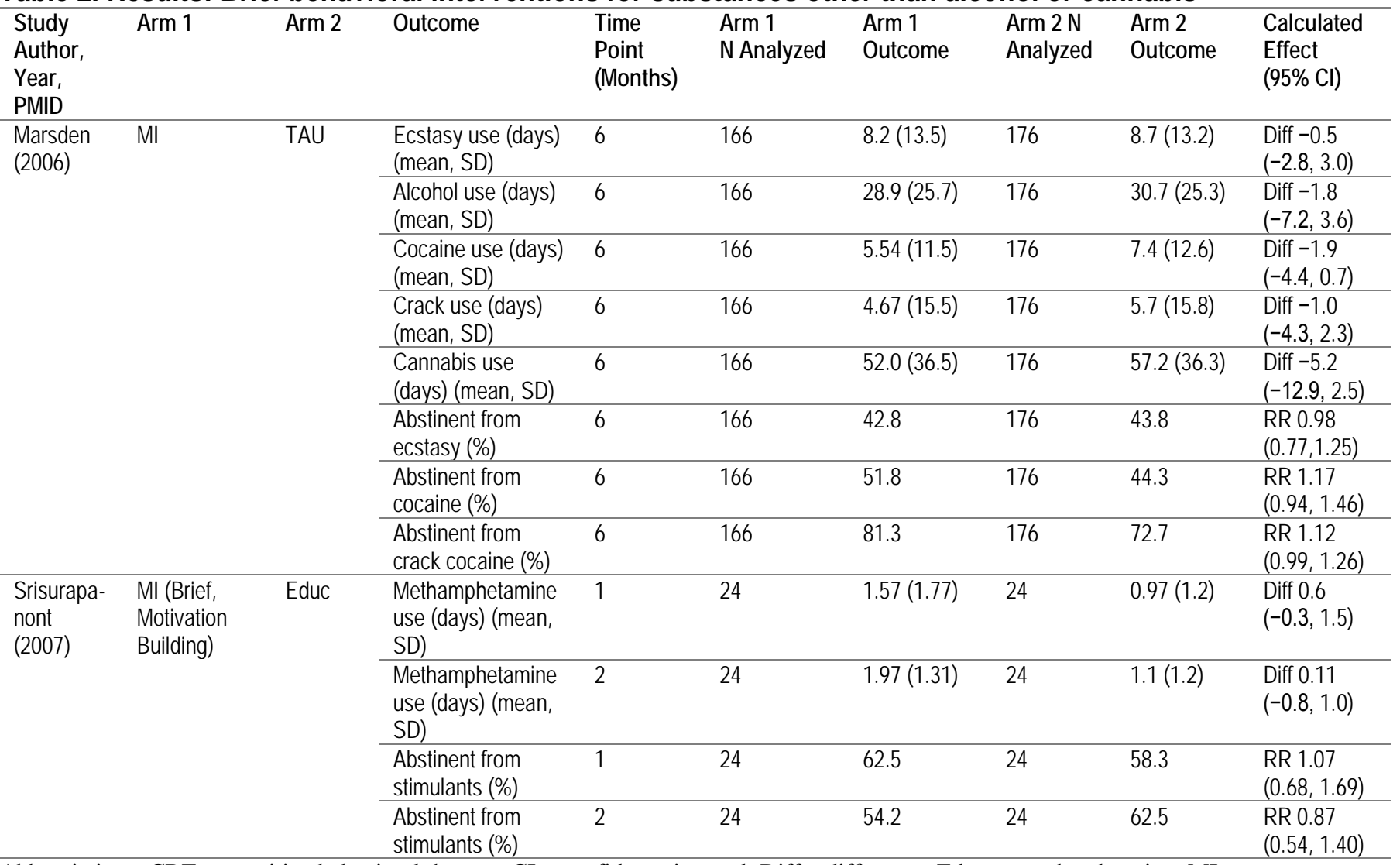

Abbreviations: CBT = cognitive behavioral therapy; $\mathrm{CI}$ = confidence interval; Diff = difference; Educ = psychoeducation; $\mathrm{MI}=$ motivational interviewing; $\mathrm{RR}$ = risk ratio; $\mathrm{SD}$ = standard deviation; TAU = treatment as usual

\section{Studies with Nondistinguishable Arms Excluded From Meta-Analysis}

Three two-arm studies (in 7 papers) of brief behavioral interventions (Table 3) ${ }^{84,87,88,101-104}$ compared interventions that were not distinguishable by our taxonomy, precluding inclusion in the meta-analysis. 
Table 3. Brief behavioral intervention studies with two treatment arms with nondistinguishable components

\begin{tabular}{llll}
\hline Author, Year & $\begin{array}{l}\text { Nondistinguished } \\
\text { Intervention } \\
\text { Component(s) }\end{array}$ & $\begin{array}{l}\text { No. } \\
\text { Arms }\end{array}$ & Distinguishing Intervention Features \\
\hline Spirito, 2011 87,88 & Ml & 2 & $\begin{array}{l}\text { Ml with separate family-focused Ml session } \\
\text { vs. } \\
\text { Ml with youth only }\end{array}$ \\
\hline $\begin{array}{l}\text { Smith, 2015 } 84 \\
\text { Walker, 2016 101- }\end{array}$ & CBT+Ml & 2 & $\begin{array}{l}\text { Ml with normative feedback vs. } \\
\text { Ml without normative feedback }\end{array}$ \\
\hline 04 & 2 & $\begin{array}{l}\text { Motivational check-in vs. } \\
\text { Assessment only check in }\end{array}$ \\
\hline
\end{tabular}

Abbreviations: $\mathrm{CBT}$ = cognitive behavioral therapy; $\mathrm{MI}=$ motivational interviewing; No. = number study arms

\section{Studies Eligible for Meta-Analysis}

There were 31 studies $^{48-83,85,86,89-100,105-109}$ eligible for meta-analysis, of which 24 were twoarm studies and seven were three-arm studies. One study, ${ }^{70}$ reported 12 -month outcomes only, and was not included in the meta-analyses.

In six three-arm studies (Table 4), two arms were not distinguishable using our coding schema. The nondistinguished treatment arms were pooled and included in meta-analyses.

Table 4. Brief behavioral intervention studies with three treatment arms with two nondistinguishable components that were pooled in MA

\begin{tabular}{|c|c|c|c|}
\hline Author, Year & $\begin{array}{l}\text { Nondistinguished } \\
\text { Component }\end{array}$ & \# Arms & $\begin{array}{l}\text { Distinguishing Intervention or Control } \\
\text { Features }\end{array}$ \\
\hline Winters, 2007105 & $\mathrm{Ml}$ & 3 & $\begin{array}{l}\text { MI with separate family-focused MI } \\
\text { session vs. MI with youth only vs. TAU }\end{array}$ \\
\hline $\begin{array}{l}\text { Winters, } 2012^{106-} \\
109\end{array}$ & $\mathrm{Ml}$ & 3 & $\begin{array}{l}\text { MI with separate family-focused MI } \\
\text { session vs. MI with youth only vs. TAU }\end{array}$ \\
\hline $\begin{array}{l}\text { Spijkerman, } 2010 \\
85\end{array}$ & $\mathrm{Ml}$ & 3 & $\begin{array}{l}\text { MI with normative feedback vs. MI } \\
\text { without normative feedback vs. TAU }\end{array}$ \\
\hline Dembo, 2014 66-69 & $\mathrm{Ml}$ & 3 & $\begin{array}{l}\text { MI with separate family-session vs. MI } \\
\text { with youth only vs. TAU }\end{array}$ \\
\hline $\begin{array}{l}\text { Cunningham, } 2015 \\
58-62\end{array}$ & $\mathrm{Ml}$ & 3 & $\begin{array}{l}\text { Computer-delivered MI vs. therapist- } \\
\text { delivered MI vs. TAU }\end{array}$ \\
\hline Peterson, $2006{ }^{83}$ & TAU & 3 & $\begin{array}{l}\text { Assessment only (TAU) vs. Assessment } \\
\text { (TAU) followed by MI }\end{array}$ \\
\hline
\end{tabular}

The variations in active interventions (MI or MI+CBT) compared in the nine studies with duplicate arm codes included delivery method (computer vs. therapist), ${ }^{58-62}$ post-intervention check-ins (motivational check-in vs. no check-in), ${ }^{101-104}$ target recipient (parents/family vs. adolescents only), ${ }^{87,88,105-109}$, or content (MI with normative feedback vs. without) ${ }^{84}$.

\section{Risk of Bias}

Risk of bias summaries are presented graphically in Figure 3 for the 30 studies that we considered eligible for meta-analysis. The most common methodological concerns involved lack of blinding of participants, personnel, and outcome assessors. 
Figure 3. Meta-analyzed brief behavioral intervention studies: Percentage of studies in each risk of bias category

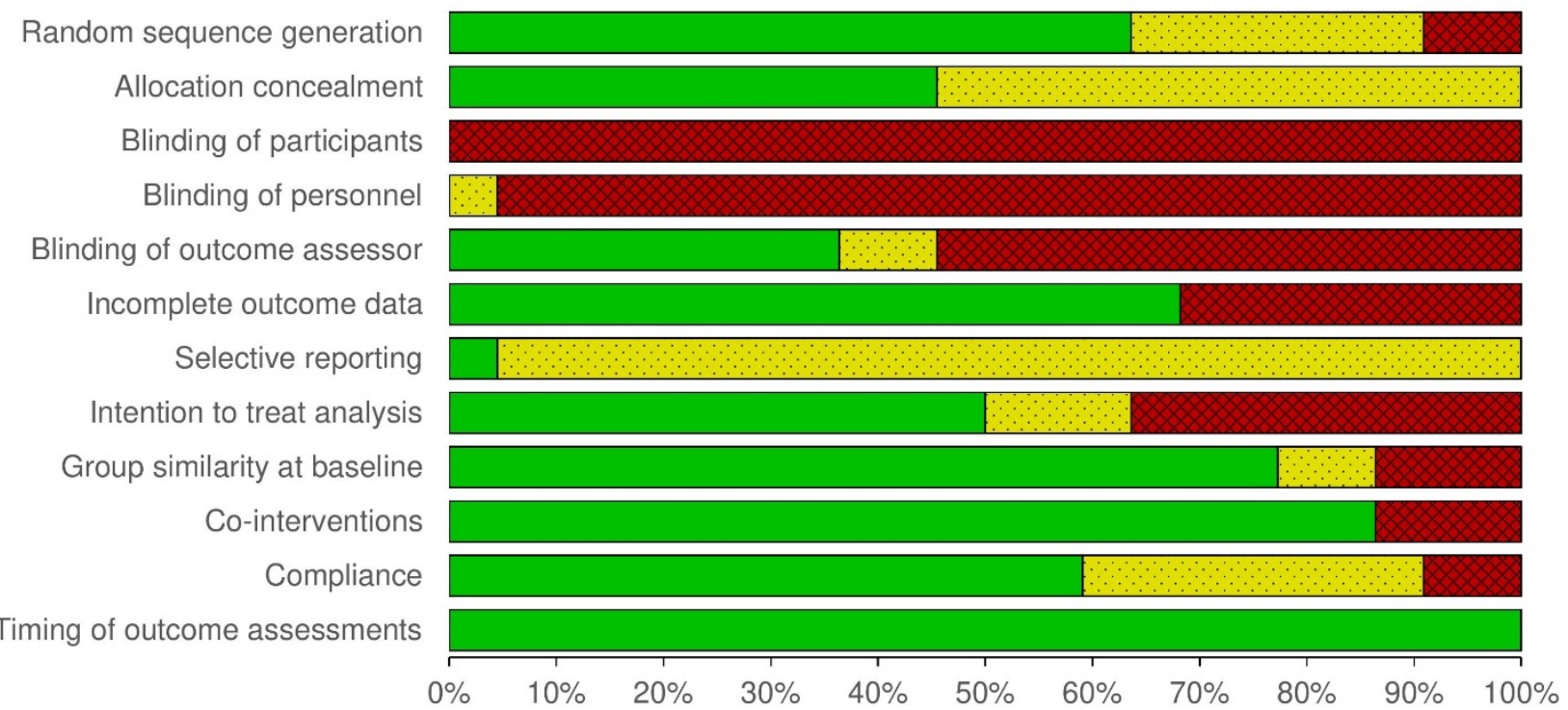

$\square$ Low risk of bias $\square$ Unclear risk of bias $\quad$ High risk of bias

\section{Alcohol Outcomes}

\section{Heavy Alcohol Use Days}

Seven studies compared MI with TAU $48-50,52,55-57,64,86,105$ and reported a measure of heavy alcohol use in 2,821 participants. Of these, five studies ${ }^{50,52,55-57,64,105}$ (1,248 subjects), reported heavy use days and two studies ${ }^{48,49,86}$ (1,573 subjects), reported a scale (Figure 4). ${ }^{48,86}$ 
Figure 4. Evidence graph for brief behavioral intervention studies reporting heavy alcohol use days

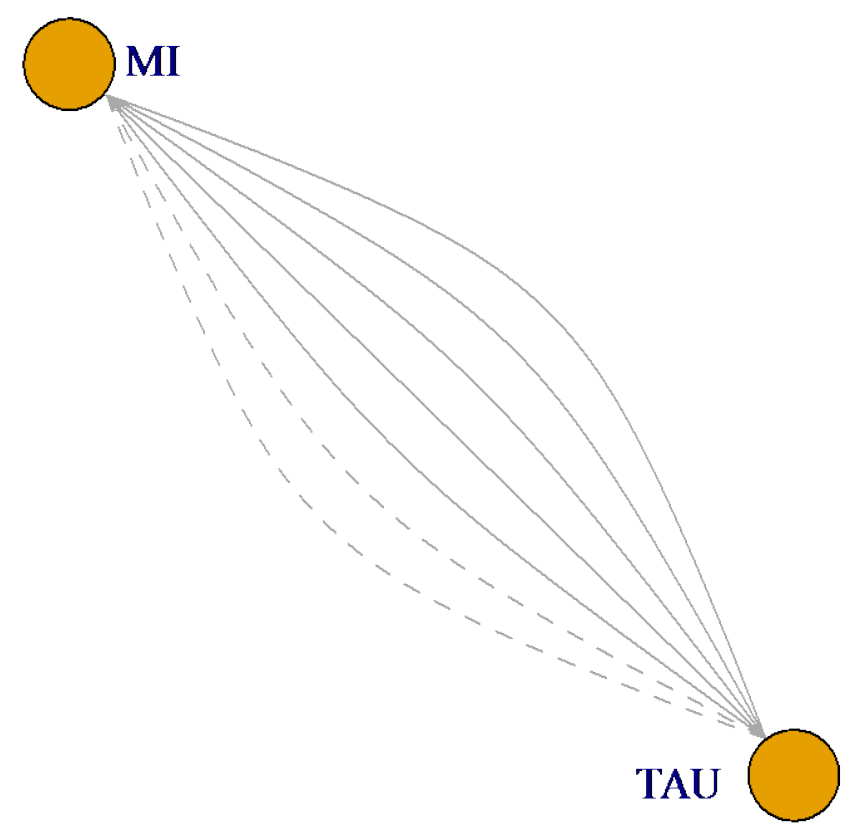

The network plot consists of nodes (yellow circles) representing the interventions being compared and edges (connecting lines) representing the available direct comparisons between interventions. Each edge represents a within study comparison. Dotted edges represent comparisons reported as scales. Abbreviations: TAU = treatment as usual; $\mathrm{MI}=$ motivational interview

\section{Key Question 1: Heavy Alcohol Use Days - MI Compared With TAU}

As shown in Figure 5, MI relative to TAU has a net mean difference (NMD) of -0.7 (95\% CrI -1.6, 0.02) days/month of heavy alcohol use. These results correspond to a Bayesian posterior probability that MI is better than TAU is 97.3 percent.

$\mathrm{MI}$ is more effective than TAU in reducing heavy alcohol use days. We rated the strength of evidence (SoE) as low.

Figure 5. Heavy alcohol use: Forest plot depicting individual study effects with summary estimates of the relative effect of MI versus TAU

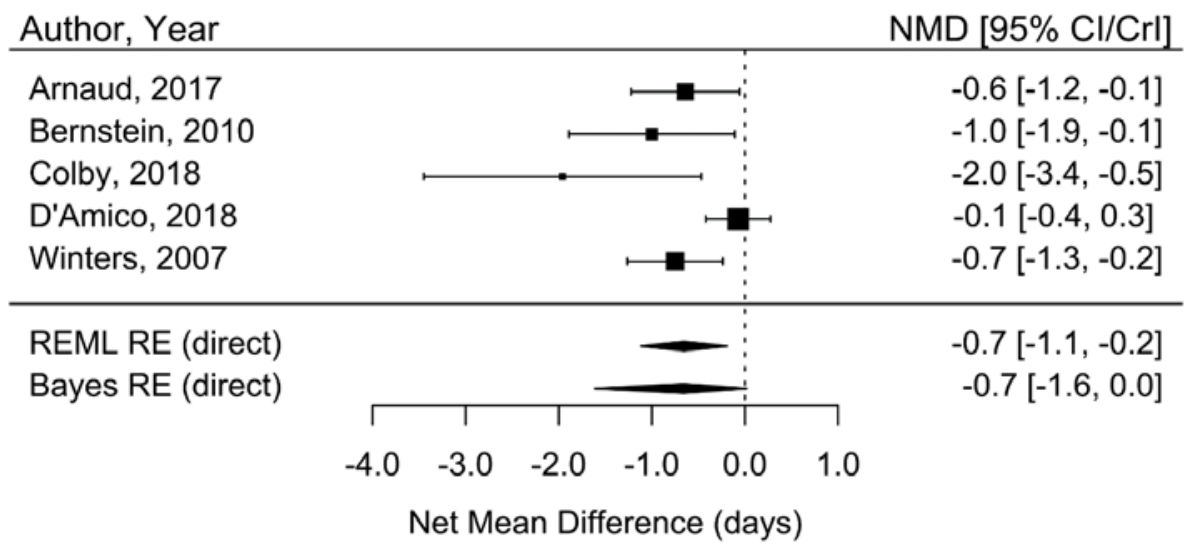

NMD < 1 favors MI. Abbreviations: NMD = net mean difference; REML = restricted maximum likelihood, RE = random effect; $\mathrm{CI} / \mathrm{CrI}=$ credible interval (for Bayes RE Model); direct = direct (pairwise) comparisons. 


\section{Alcohol Use Days}

Ten dual-arm studies enrolled a total of 3,726 subjects and reported a measure of the frequency of alcohol use (Figure 6). Of these eight studies ${ }^{52,54-57,64,78-81,105-109}$ (2,153 subjects) reported use days and two studies ${ }^{48,49,86}$ (1573 subjects) reported a scale. One trial, that enrolled 326 subjects compared MI with Educ and reported use days. ${ }^{78-80}$

\section{Figure 6. Evidence graph for brief behavioral intervention studies reporting alcohol use days}

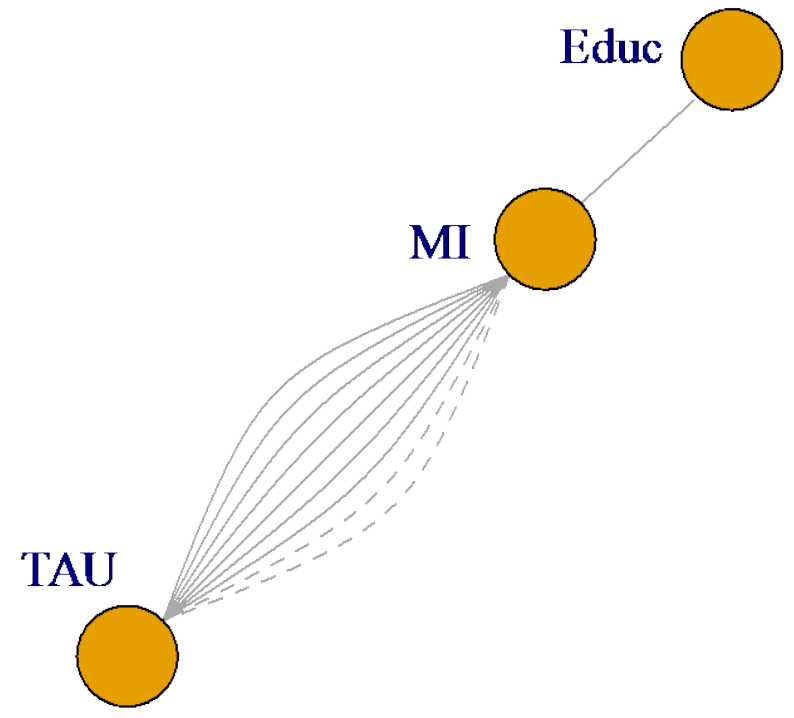

The network plot consists of nodes (yellow circles) representing the interventions being compared and edges (connecting lines) representing the available direct comparisons between interventions. Each edge represents a within study comparison. Dotted edges represent comparisons reported as scales. Abbreviations: TAU = treatment as usual; $\mathrm{MI}=$ motivational interview; Educ = education

\section{Key Question 1: Alcohol Use Days - MI Compared With TAU}

The studies contributing direct evidence for the MI versus TAU comparison are illustrated in Figure 7. The pooled NMD of direct comparisons of MI vs TAU was -1.2 (95\% CrI, $-2.2,-0.2$ ) days/month of alcohol use, compared to those in TAU groups.

In the NMAs, the pooled NMD for the MI versus TAU comparison was -1.1 (95\% CrI -2.2 , $-0.3)$ days/month of alcohol use.

MI is more effective than TAU in reducing overall alcohol use days. We rated the overall SoE as moderate. 
Figure 7. Alcohol use days: forest plot depicting individual study effects with summary estimates of the relative effect of MI versus TAU

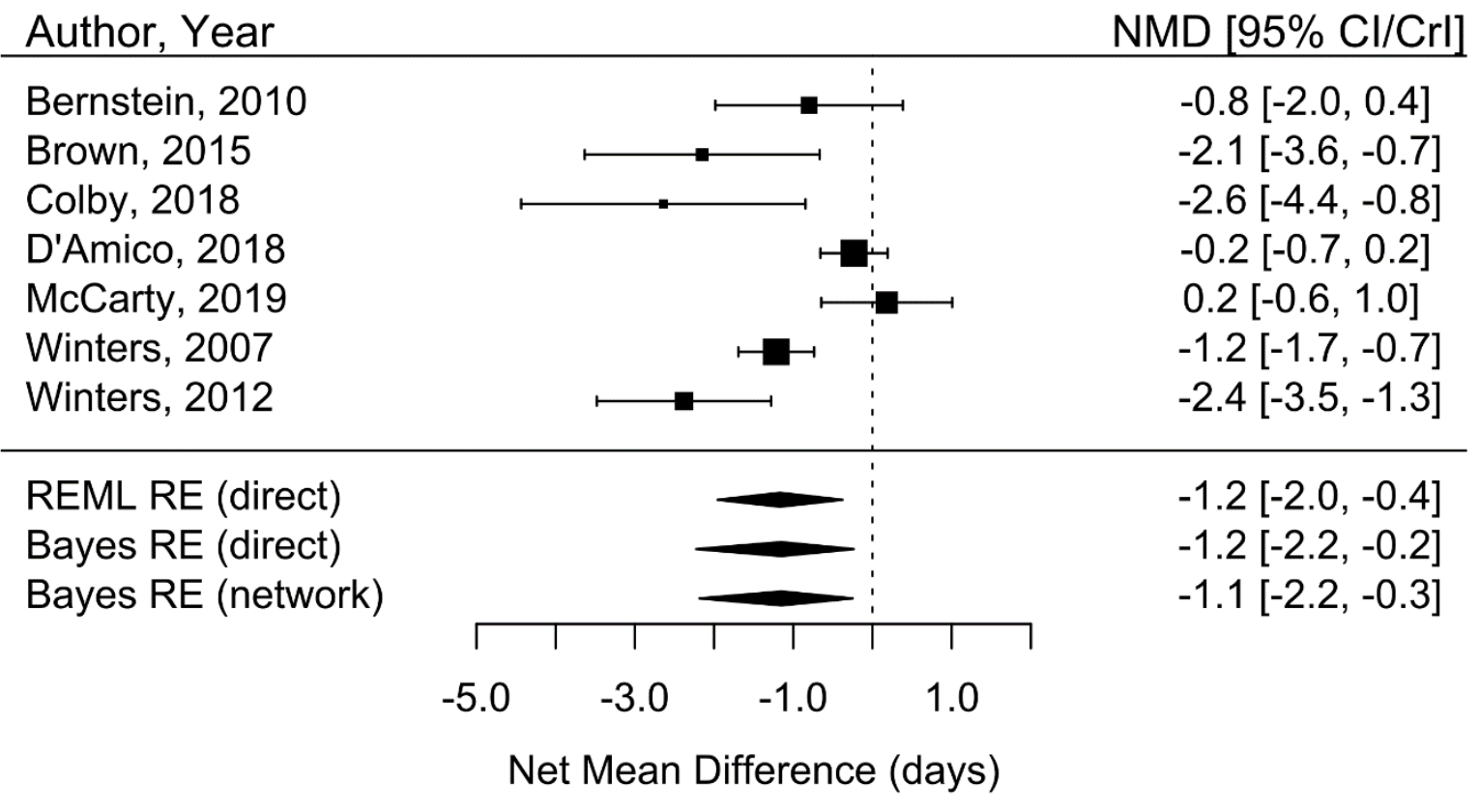

NMD < 1 favors MI. Abbreviations: MI = motivational interviewing; TAU = treatment as usual, REML = restricted maximum likelihood, $\mathrm{RE}$ = random effect; $\mathrm{CI}$ = confidence interval, $\mathrm{CrI}$ = credible interval (for Bayes estimates).

\section{Key Question 2: Alcohol Use Days - Comparative Effect of MI Versus Educ}

As shown in Table 5, the estimated NMD for MI versus Educ was 0.3 (95\% CrI -2.5 to 3.1) days/month (insufficient SoE).

Table 5. Brief behavioral interventions and alcohol abstinence: Net mean difference of days per month of abstinence between all interventions

\begin{tabular}{lrrr}
\hline Intervention & Educ & MI & TAU \\
\hline Educ & Educ & 0.3 & 1.4 \\
& & $(-2.5,3.1)$ & $(-1.4,4.4)$ \\
\hline MI & -0.3 & MI & 1.1 \\
& $(-3.1,2.5)$ & & $(0.3,2.2)$ \\
\hline TAU & -1.4 & -1.1 & TAU \\
& $(-4.4,1.4)$ & $(-2.2,-0.3)$ & \\
\hline
\end{tabular}

Gray cells display comparisons for which there is indirect evidence only. Effects are expressed as NMD (days/month), with 95\% credible intervals in parentheses.

Abbreviations: Educ = psychoeducation; $\mathrm{MI}=$ motivational interviewing; $\mathrm{TAU}=$ treatment as usual.

\section{Alcohol Abstinence}

Abstinence for alcohol was reported by seven studies with outcomes for 2,482 participants (Figure 8). ${ }^{48,49,54,72,76-80,89,106-109}$ 
Figure 8. Evidence graph for studies reporting alcohol abstinence

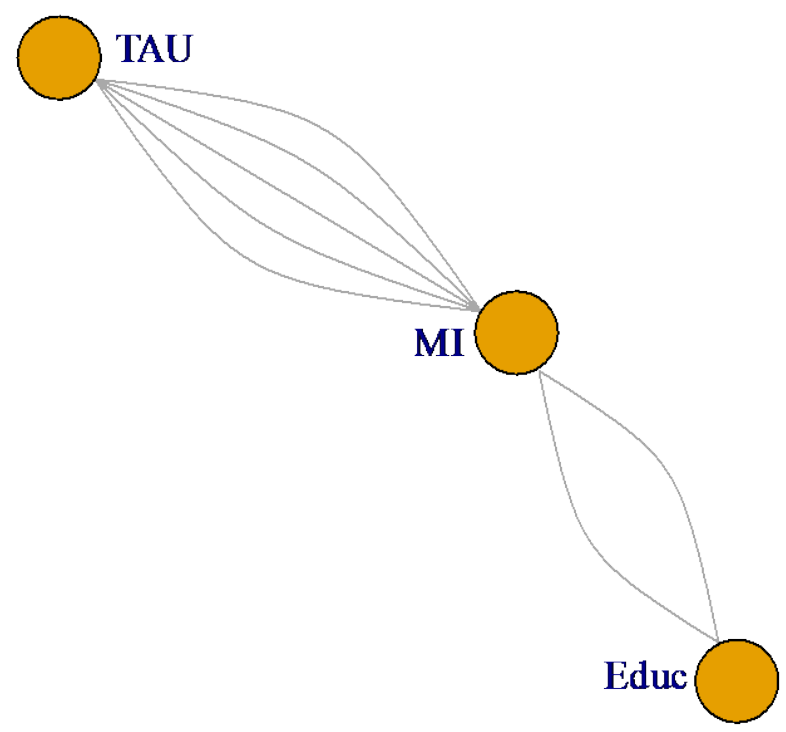

The network plot consists of nodes (yellow circles) representing the interventions being compared and edges (connecting lines) representing the available direct comparisons between interventions. Each edge represents a within study comparison.

Abbreviations: $\mathrm{MI}=$ motivational interviewing; $\mathrm{TAU}=$ treatment as usual; Educ = education

\section{Key Question 1: Alcohol Abstinence - MI Versus TAU}

The studies contributing direct evidence for the MI versus TAU comparison for the odds ratio of attaining abstinence are illustrated in Figure 9. When direct comparisons of MI vs TAU were considered, the odds of abstinence were 2.0 (95\% CrI 0.9, 7.8) fold higher for MI than for TAU. In the NMA, the pooled odds ratio 1.9 (95\% CrI 0.9, 6.).

MI may be more effective than TAU. However, the credible intervals for both the pairwise NMA are wide, and also compatible with no effect (insufficient SoE).

Figure 9. Alcohol abstinence: MI (brief intervention) versus TAU

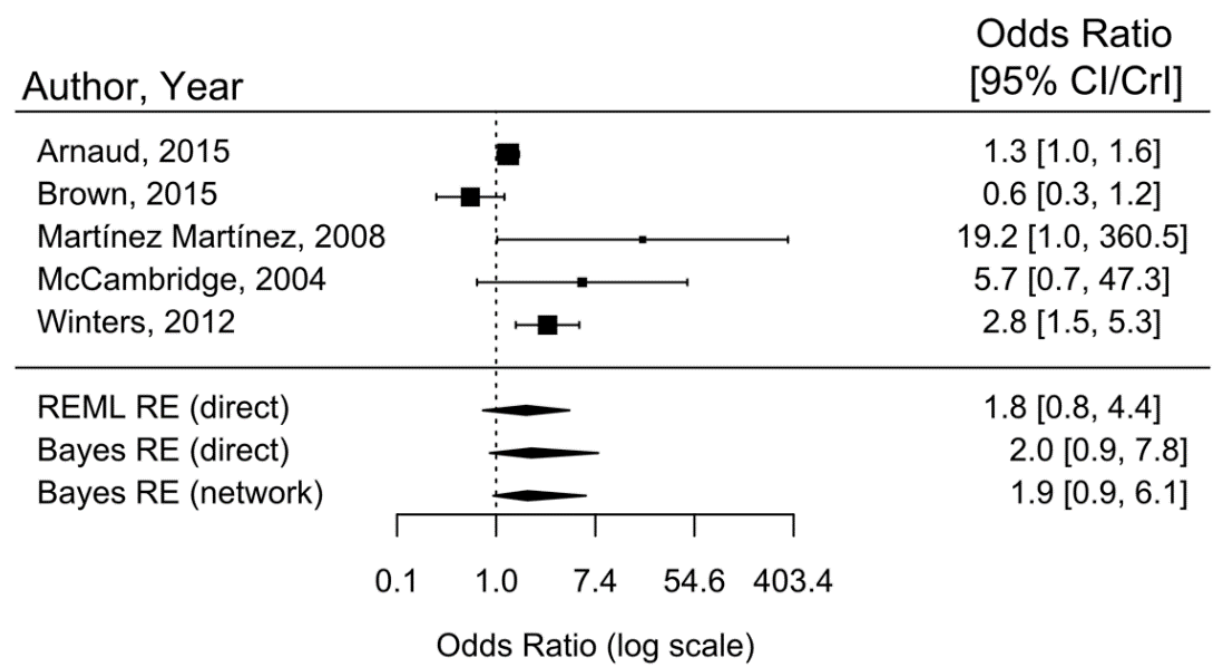

Odds ratio > 1 favors MI. Abbreviations: $\mathrm{MI}=$ motivational interviewing; $\mathrm{TAU}=$ treatment as usual, $\mathrm{REML}=$ restricted maximum likelihood, $\mathrm{RE}$ = random effect; $\mathrm{CI}$ = confidence interval; CrI = credible interval (for Bayesian RE Model) 


\section{Key Question 2: Comparative Effects of MI and Educ}

The comparative effects, as log odds ratios are shown in Table 6.

The estimated effect for Educ versus MI is highly imprecise, with direct evidence from 2 studies only (insufficient SoE).

Table 6. Brief behavioral interventions and alcohol abstinence: Odds ratios for abstinence between all interventions

\begin{tabular}{lrrr}
\hline Intervention & Educ & MI & TAU \\
\hline \multirow{2}{*}{ Educ } & Educ & 1.3 & 0.7 \\
& & $(0.4,4.6)$ & $(0.1,2.6)$ \\
\hline \multirow{2}{*}{ Ml } & 0.8 & $\mathrm{Ml}$ & 0.5 \\
\hline \multirow{2}{*}{ TAU } & $(0.2,2.8)$ & & $(0.2,1.1)$ \\
\hline
\end{tabular}

Gray cells display comparisons for which there is indirect evidence only. Effects are expressed as odds ratios with $95 \%$ credible intervals in parentheses.

Abbreviations: Educ = psychoeducation; $\mathrm{MI}=$ motivational interviewing; TAU $=$ treatment as usual

\section{Cannabis Outcomes}

\section{Cannabis Use Days}

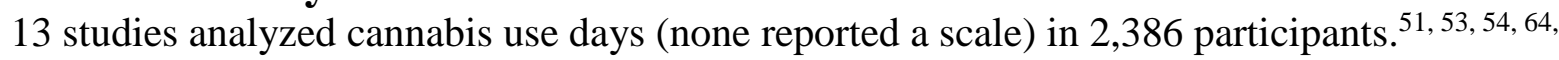
65, 71, 78-81, 83, 89, 99, 100, 106-109 The network geometry is shown in Figure 10.

Figure 10. Evidence graph for brief behavioral intervention studies reporting cannabis use days

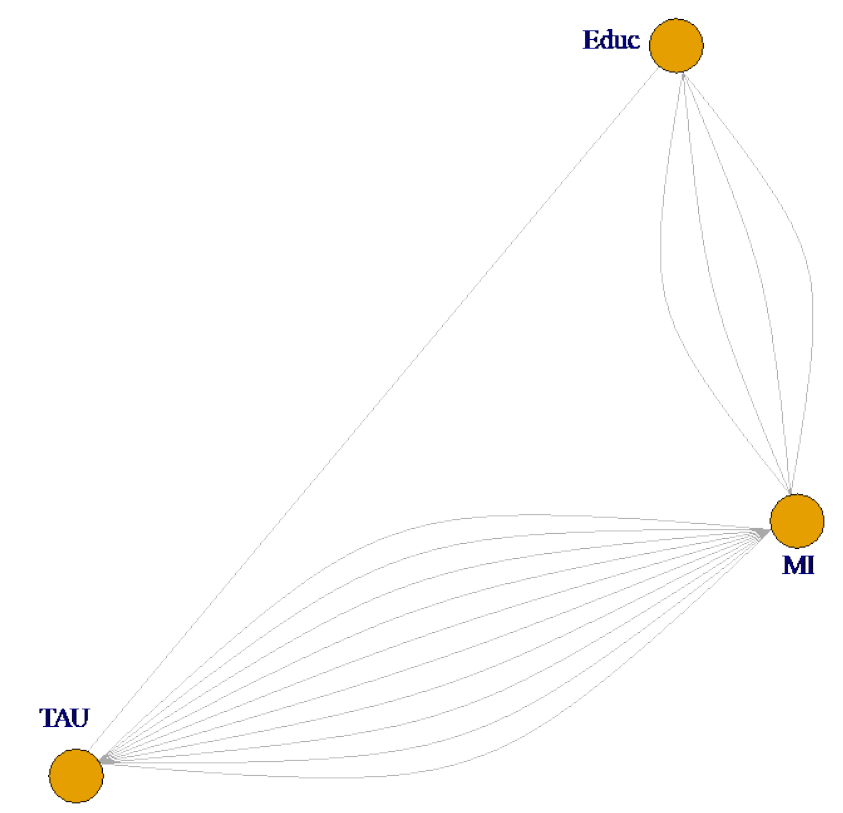

The network plot consists of nodes (yellow circles) representing the interventions being compared and edges (connecting lines) representing the available direct comparisons between interventions. Each edge represents a within study comparison.

Abbreviations: $\mathrm{MI}=$ motivational interviewing; $\mathrm{TAU}=$ treatment as usual; Educ = education 


\section{Key Question 1: Cannabis Use Days}

\section{Compared With TAU}

Figure 11 is a forest plot of the 10 studies that performed direct comparisons between MI and TAU. Based on pairwise comparisons only, the direct estimate of NMD was -0.2 (95\% CrI -1.4, $0.5)$ days/month. The effect estimate from the NMA was -0.05 (95\% CrI $-0.3,0.1)$ days/month.

$\mathrm{MI}$ is not more effective than TAU. We rated the SoE as moderate.

Figure 11. Cannabis use days: Forest plot of net mean difference for MI (brief intervention) versus TAU

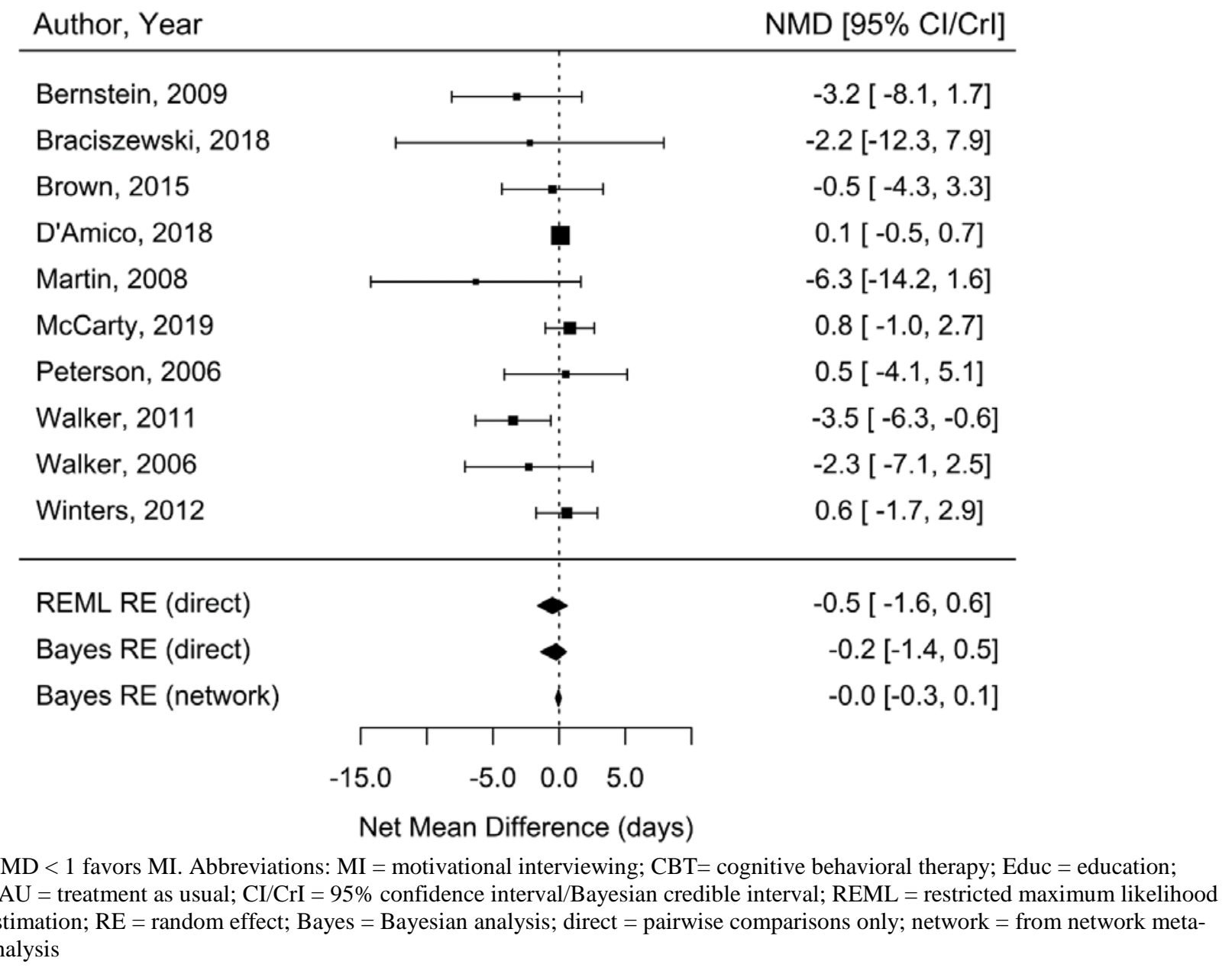

\section{Cannabis Use Days - Educ Compared With TAU}

The effect estimates for Educ versus TAU are based on a single direct comparison and have wide credible intervals (insufficient SoE).

\section{Key Question 2: Cannabis Use Days - MI Versus Educ}

Based on pairwise comparisons only, the direct estimate of NMD was -0.2 (95\% CrI -2.4, 1.2) days/month. The effect estimate from the NMA was -0.2 ( $95 \% \mathrm{CrI}:-2.2,1.7)$ days/month. As shown in Figure 12, the credible intervals are wide for the effect of MI versus Educ (insufficient SoE). 
Figure 12. Cannabis use days: Forest plot of net mean difference for the brief interventions MI versus Educ

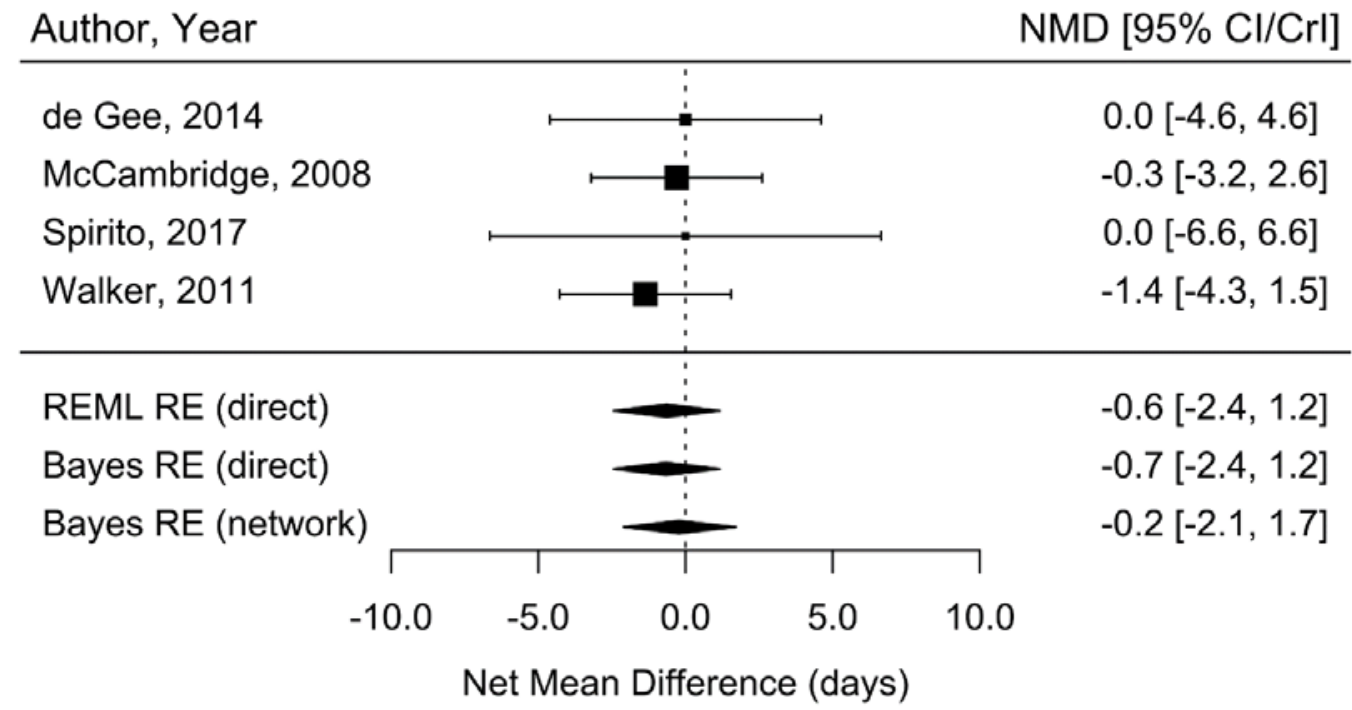

NMD < 1 favors MI. Abbreviations: MI = motivational interviewing; Educ = education; $\mathrm{TAU}=$ treatment as usual; $\mathrm{CI} / \mathrm{CrI}=95 \%$ confidence interval/Bayesian credible interval; NMD = net mean difference; REML = restricted maximum likelihood estimation; $\mathrm{RE}=$ random effect; Bayes = Bayesian analysis; direct = pairwise comparisons only; network = from network meta-analysis

\section{Cannabis Abstinence}

Six studies reported the cannabis abstinence outcomes in 1,119 participants (Figure 13). ${ }^{51,54 \text {, }}$ 76-80, 89, 106-109 Of these, two studies compared MI with a control group who received Educ. ${ }^{78-80,89}$

Figure 13. Evidence graph for brief behavioral intervention studies reporting cannabis abstinence

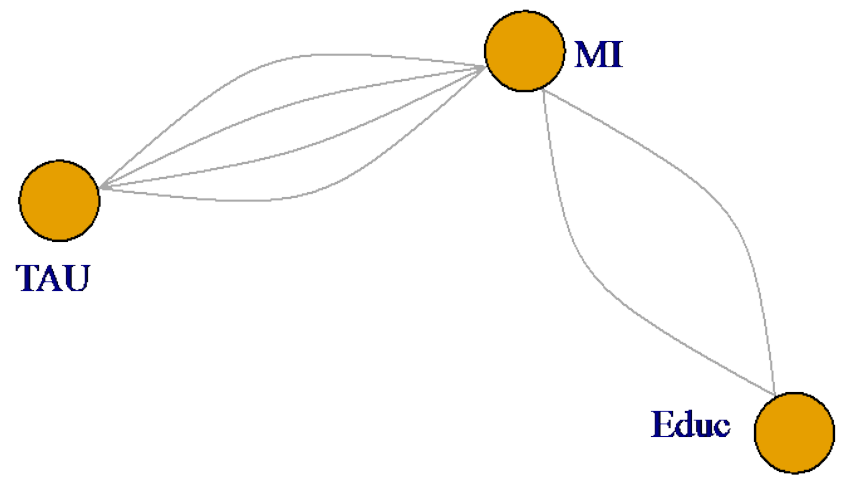

The network plot consists of nodes (yellow circles) representing the interventions being compared and edges (connecting lines) representing the available direct comparisons between interventions. Each edge represents a within study comparison.

Abbreviations: Educ = education, $\mathrm{MI}=$ motivational interviewing, $\mathrm{TAU}=$ treatment as usual

\section{Key Question 1: Cannabis Abstinence}

\section{Versus TAU}

Figure 14 illustrates the study level effects for each of the 4 studies that compared MI and TAU. The summary estimate from the NMA was 1.5 (95\% CrI: 0.7 to 3.4). 
The credible interval for this estimate is wide and does not exclude no effect or an adverse effect. Therefore, we rated the SoE as insufficient.

Figure 14. Cannabis abstinence: Forest plot of log odds ratio for MI (brief intervention) compared with TAU

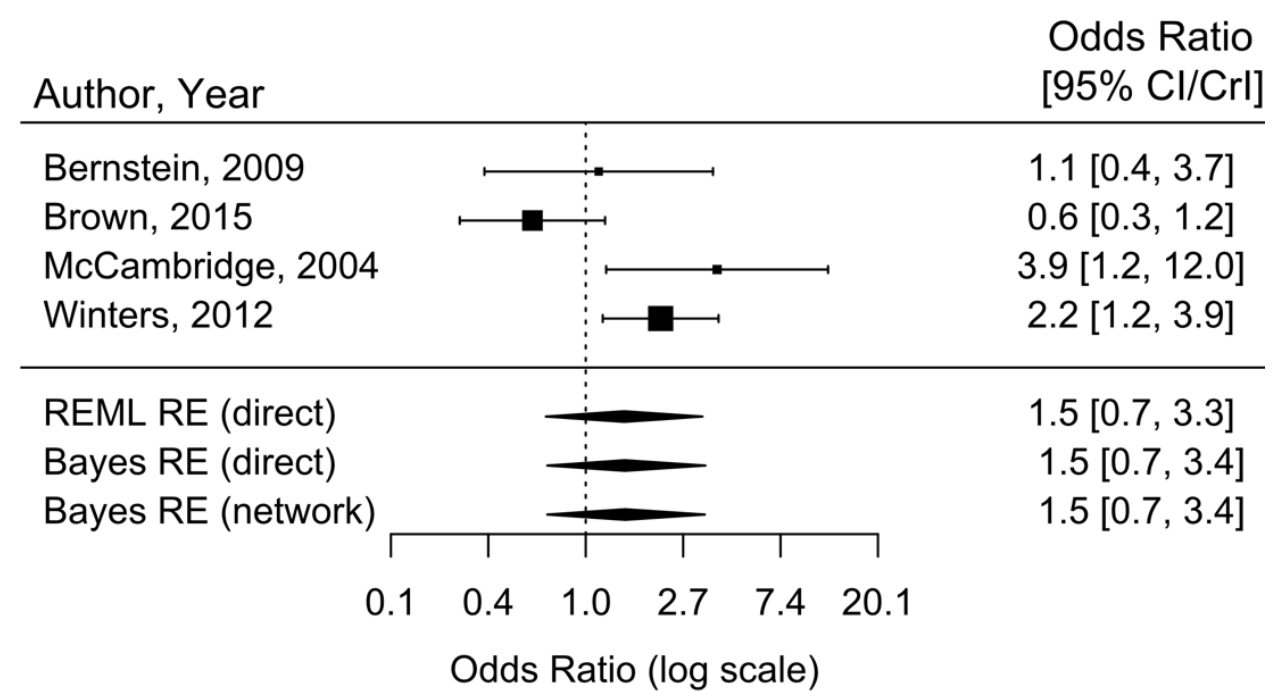

Odds ratio > 1 favors MI. Abbreviations: $\mathrm{MI}=$ motivational interviewing; $\mathrm{Educ}=$ education; $\mathrm{TAU}=$ treatment as usual; $\mathrm{CI} / \mathrm{CrI}=$ 95\% confidence interval/Bayesian credible interval; REML = restricted maximum likelihood estimation; RE = random effect;

Bayes = Bayesian analysis; direct $=$ pairwise comparisons only; network $=$ from network meta-analysis

\section{Key Question 2: Cannabis Abstinence - Comparative Effects of MI Versus Educ}

The credible interval for the indirect estimate of the Educ vs TAU effect was similarly imprecise.

As shown in Table 7, the odds ratio for abstinence between MI versus Educ is 2.0 (95\% CrI $0.7,7.4)$. We rated the $\mathrm{SoE}$ as insufficient due to imprecision.

Table 7. Brief behavioral interventions and cannabis abstinence: Odds ratios for abstinence between all interventions

\begin{tabular}{lrrr}
\hline Intervention & Educ & Ml & TAU \\
\hline \multirow{2}{*}{ Educ } & Educ & 2 & $\begin{array}{r}1.4 \\
(0.7,7.5)\end{array}$ \\
\hline \multirow{2}{*}{ MI } & 0.5 & $\mathrm{Ml}$ & $0.4,6.3)$ \\
\hline \multirow{2}{*}{ TAU } & $(0.1,1.4)$ & & $(0.3,1.5)$ \\
\hline
\end{tabular}

Gray cells display comparisons for which there is indirect evidence only. Effects are expressed as odds ratios with $95 \%$ credible intervals in parentheses. Abbreviations: Educ = psychoeducation; $\mathrm{MI}=$ motivational interviewing; TAU = treatment as usual

\section{Substance Use Problem Scale}

Nine studies, with the comparisons show in Figure 15, reported one of 8 substance use problem scales in 1,854 participants. ${ }^{50,55-57,64,65,71,78-80,100,105-109}$ 
Figure 15. Evidence graph of brief behavioral intervention studies reporting a substance use problem scale

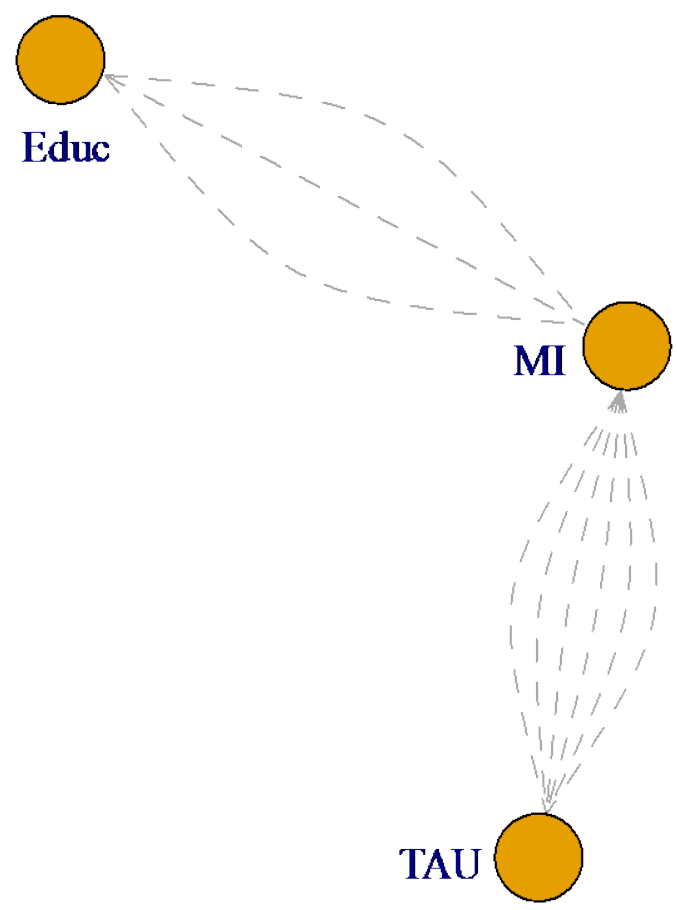

The network plot consists of nodes (yellow circles) representing the interventions being compared and edges (connecting lines) representing the available direct comparisons between interventions. Each edge represents a within study comparison. Abbreviations: Educ = education, $\mathrm{MI}$ = motivational interviewing, $\mathrm{TAU}=$ treatment as usual

\section{Key Question 1: Substance Use Problem Scales}

\section{Compared With TAU}

Figure 16 illustrates the study level effects for each of the 6 studies that compared MI and TAU. Based on pairwise comparisons only, the SNMD was -0.5 (95\% CrI -1.25, 0.2). The pooled estimate from the NMA was -0.5 (95\% CrI -1.0 to 0$)$, similar to the direct estimates that included the 3 studies that compared MI with Educ was -0.4 (95\% CrI -1.0, 0.01).

$\mathrm{MI}$ is better than TAU in reducing substance abuse related problems. We rated the SoE as low. 
Figure 16. Substance use problem scales: Forest plot of standardized net mean difference of the brief interventions MI versus TAU

\begin{tabular}{|c|c|c|c|c|}
\hline \multicolumn{3}{|l|}{ Author, Year } & \multicolumn{2}{|c|}{ SNMD $[95 \% \mathrm{Cl} / \mathrm{Crl}]$} \\
\hline Arnaud, 2017 & & $\mapsto$ & & $-0.2[-0.4,0.1]$ \\
\hline Colby, 2018 & & $\longmapsto$ & & $-0.5[-0.8,-0.2]$ \\
\hline D'Amico, 2018 & & & & $0.0[-0.2,0.2]$ \\
\hline Martin, 2008 & & $\longrightarrow$ & & $-0.5[-1.2,0.1]$ \\
\hline Winters, 2012 & & $\longmapsto$ & & $-0.2[-0.5,0.1]$ \\
\hline Winters, 2007 & $\longmapsto$ & & & $-1.7[-2.2,-1.1]$ \\
\hline REML RE (direct) & & & & $-0.5[-0.9,-0.0]$ \\
\hline Bayes RE (direct) & & & & $-0.5[-1.2,0.2]$ \\
\hline Bayes RE (network) & & - & & $-0.5[-1.0,0.0]$ \\
\hline & 1 & 1 & $\neg$ & \\
\hline & -1.5 & -0.5 & 0.5 & \\
\hline
\end{tabular}

\section{Educ Compared With TAU}

No studies directly compared Educ with TAU. The SNMD for this effect was -0.5 (95\% CrI $-1.4,0.32)$.

We rated the SoE as insufficient, due to imprecision.

\section{Key Question 2: Substance Use Problem Scales - MI Versus Educ}

Figure 17 illustrates the study level effects for each of the 3 studies that directly compared MI with Educ. The pairwise random effect estimate of the SNMD was 0.04 (95\% CrI -0.1, 0.2). The SNMD of 0.04 obtained from the NMA that included all studies was identical to the pairwise estimate. However, this estimate (not shown in Figure 17 had a much wider credible interval, from -0.6 to 0.7 .

We rated the SoE as insufficient, due to imprecision. 
Figure 17. Substance use problem scales: Forest plot of standardized net mean difference of the brief interventions MI versus Educ

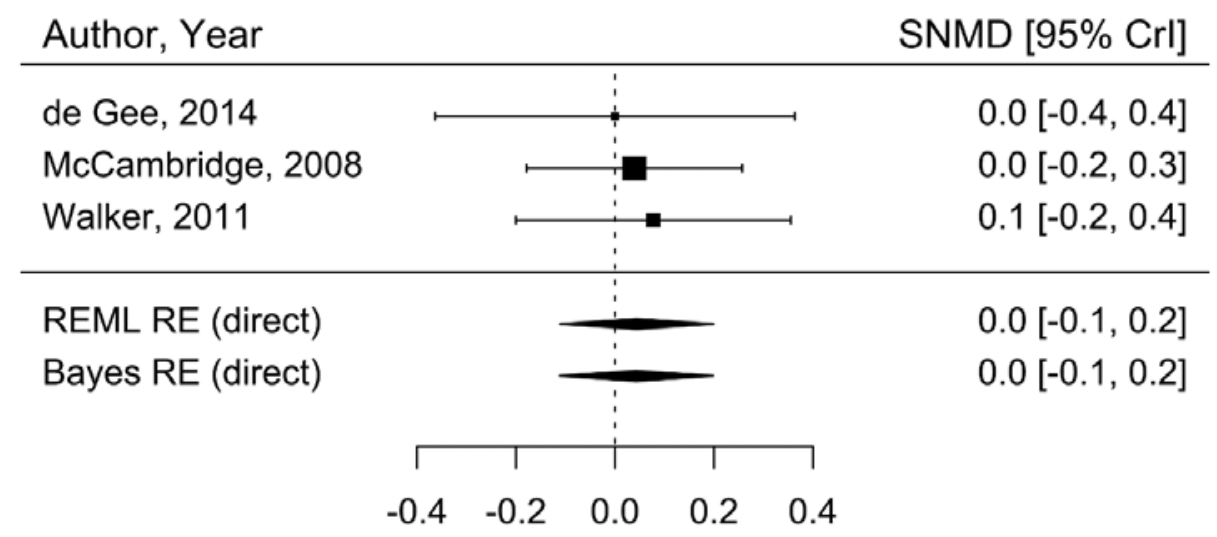

Standardized Net Mean Difference

SNMD < 1 favors MI. Abbreviations: SNMD = standardized net mean difference; CrI = credible interval; REML = restricted maximum likelihood; RE = random effect; direct = estimated from pairwise comparisons only.

\section{Legal Outcomes}

One study (McCambridge 2003) compared Motivational Interviewing (MI) and Treatment As Usual (TAU) and provided data on adolescents' self-reported selling of drugs to their friends and to non-friends. As detailed in Table 8, more adolescents in the MI group than the TAU group sold drugs to their friends ( $40 \%$ vs. $15 \%$; OR=3.7, $95 \%$ CI 1.8 to 7.5 ).

Table 8. Legal outcomes with brief behavioral interventions

\begin{tabular}{|c|c|c|c|c|c|c|c|c|c|}
\hline $\begin{array}{l}\text { Author, Year } \\
\text { PMID }\end{array}$ & $\begin{array}{l}\text { Intervention } \\
\text { Label }\end{array}$ & $\begin{array}{l}\text { Control } \\
\text { Label }\end{array}$ & $\begin{array}{l}\text { Time } \\
\text { (Months) }\end{array}$ & $\begin{array}{l}\text { Sold Drugs } \\
\text { to Friends }\end{array}$ & $\begin{array}{l}\text { Sold Drugs } \\
\text { to Friends }\end{array}$ & $\begin{array}{l}\text { Sold } \\
\text { Drugs to } \\
\text { Friends }\end{array}$ & $\begin{array}{l}\text { Sold } \\
\text { Drugs to } \\
\text { Non- } \\
\text { friends }\end{array}$ & $\begin{array}{l}\text { Sold } \\
\text { Drugs to } \\
\text { Non- } \\
\text { friends }\end{array}$ & $\begin{array}{l}\text { Sold } \\
\text { Drugs to } \\
\text { Non- } \\
\text { friends }\end{array}$ \\
\hline & & & & Int. & Cont. & $\begin{array}{l}\text { Calc. } \\
\text { Effect } \\
(95 \% \mathrm{Cl})\end{array}$ & Int. & Cont. & $\begin{array}{l}\text { Calc. } \\
\text { Effect } \\
(95 \% \mathrm{Cl})\end{array}$ \\
\hline $\begin{array}{l}\text { McCambridge } \\
2003^{76,77}\end{array}$ & $\begin{array}{l}\text { Motivational } \\
\text { Interviewing } \\
(\mathrm{N}=82)\end{array}$ & $\begin{array}{l}\text { Treatment as } \\
\text { usual }(\mathrm{N}=97)\end{array}$ & 3 & $40 \%$ & $15 \%$ & $\begin{array}{l}\text { OR } 3.7 \\
(1.8,7.5)\end{array}$ & $14 \%$ & $7 \%$ & $\begin{array}{l}\text { OR } 2.2 \\
(0.8,5.9)\end{array}$ \\
\hline
\end{tabular}

Abbreviations: Calc. = calculated; $\mathrm{CI}$ = confidence interval; Cont. = control; Int. = intervention; OR = odds ratio 


\section{Nonbrief Behavioral Interventions}

\section{Key Points}

Key points from the meta-analyses of nonbrief behavioral interventions are summarized by outcome below.

\section{Nonbrief Behavioral Interventions by Outcome}

\section{- Days of alcohol use}

o Limited, primarily indirect evidence, suggests that family focused therapy (Fam) may reduce days of alcohol use relative to TAU (low SoE)

o Limited, primarily indirect evidence suggests that family focused therapy (Fam) may be more effective than ICM, CBT and MI in reducing days of alcohol use (low SoE).

- Days of cannabis use

o Limited, primarily indirect evidence, suggests that CBT, CBT+MI and $\mathrm{CBT}+\mathrm{MI}+\mathrm{CM}$, Educ) may result in relative increases relative to TAU in days of cannabis use (low SoE)

- Days of alcohol and other drug use (AOD)

o Limited, primarily indirect evidence suggests that both MI and CBT may reduce days of AOD use relative to TAU (low SoE)

o MI was more effective than PeerGroup, CBT+MI, Fam, CBT+ICM, CBT+MI+ICM, CBT and ICM (low SoE)

\section{- Days of illicit drug use}

o Limited, primarily indirect evidence, suggests that CBT+MI reduces days of illicit drug use relative to TAU (low SoE)

There were 59 studies in 103 studies (sample size, range 26 to 514), which enrolled 8,786

participants with substance use disorders involving alcohol, cannabis and other drugs. ${ }^{18,33,112-210}$ Table 10 provides baseline and arm details.

Of these, there were 44 two-arm studies, 12 three-arm studies and 3 four-arm studies. Across studies, there were a total of 136 arms, of which 97 were coded as a single intervention, including TAU (29 arms), Fam (25 arms), CBT (19 arms), MI (5 arms), PeerGroup (10 arms), Educ (6 arms), ICM (2 arm), and CM (1 arm). The remaining 39 arms were coded as compound interventions (two or more separate components). Details of baselines and interventions are given in Appendix D (Table D-2). Detailed results are presented by outcome in Appendix G.

\section{Studies Including Arms With Nondistinguished Intervention Codes}

Thirteen nonbrief behavioral intervention studies (5 two-arm studies [Table 9]; 8 multi-arm studies [Table 10]) evaluated different variants of CBT, MI, Fam, Peer group, and TAU components (or their combinations) not captured by our taxonomy, and therefore have two arms with the same coding (i.e., they are nondistinguishable in our categorizations). 
Table 9. Nonbrief behavioral intervention studies with two treatment arms with nondistinguishable components

\begin{tabular}{|c|c|c|c|}
\hline Author, Year & $\begin{array}{l}\text { Nondistinguished } \\
\text { Intervention Component(s) }\end{array}$ & No. Studies & Distinguishing Comparison of Interest \\
\hline Amini, $1982{ }^{18}$ & TAU & 2 & $\begin{array}{l}\text { Outpatient vs. inpatient care. Both interventions were too } \\
\text { poorly specified to warrant coding of components. }\end{array}$ \\
\hline Schaeffer, 2013187 & & & $\begin{array}{l}\text { Building apprenticeship program vs. standard vocational } \\
\text { education }\end{array}$ \\
\hline $\begin{array}{l}\text { Burrow-Sanchez, } \\
2012115\end{array}$ & CBT & 2 & $\begin{array}{l}\text { CBT culturally adapted for Latino youth population vs. } \\
\text { standard CBT }\end{array}$ \\
\hline $\begin{array}{l}\text { Burrow-Sanchez, } \\
2015116,117\end{array}$ & & & $\begin{array}{l}\text { CBT culturally adapted for Latino youth population vs. } \\
\text { standard CBT }\end{array}$ \\
\hline Rohde, 2014 182, 183 & CBT+Fam & 1 & $\begin{array}{l}\text { Sequencing and combined effects of CBT and Fam. } \\
\text { Evaluated CBT followed by Fam, Fam followed by CBT, } \\
\text { and CBT combined with Fam concurrently }\end{array}$ \\
\hline
\end{tabular}

Abbreviations: CBT = cognitive behavioral therapy; Fam = family therapy; TAU = treatment as usual

Table 10. Nonbrief behavioral intervention studies with multiple treatment arms with nondistinguishable components

\begin{tabular}{|c|c|c|c|}
\hline $\begin{array}{l}\text { Study Author, Year, } \\
\text { PMID }\end{array}$ & Components Studied & No. Studies & Distinguishing Component of Interest \\
\hline Kaminer, $2008^{146-148}$ & $\begin{array}{l}\text { CBT+MI vs. } \\
\text { CBT+MI vs. } \\
\text { TAU }\end{array}$ & 1 & In-person MI vs. telephone MI vs. TAU \\
\hline Dennis, $2004{ }^{120}$ & $\begin{array}{l}\text { CBT+MI vs. } \\
\text { CBT+MI vs. } \\
\text { CBT+MI+Educ+ICM }\end{array}$ & 1 & $\begin{array}{l}\mathrm{CBT}+\mathrm{Ml} \text { for } 5 \text { sessions vs. CBT+Ml for } 12 \text { sessions vs. CBT+Ml for } \\
12 \text { sessions with parent group education and case management }\end{array}$ \\
\hline Stanger, 2015200 & $\begin{array}{l}\text { CBT+Ml vs. } \\
\text { CBT+MI+CM vs. } \\
\text { CBT+MI+CM }\end{array}$ & 1 & $\begin{array}{l}\text { CBT+MI vs. CBT+MI+CM vs. CBT+MI+CM with additional parent } \\
\text { sessions }\end{array}$ \\
\hline Robbins, $2008^{173}$ & $\begin{array}{l}\text { Fam vs. } \\
\text { Fam vs. } \\
\text { TAU }\end{array}$ & 2 & $\begin{array}{l}\text { Family therapy using a systems/structural model vs. family therapy } \\
\text { using an ecological model vs. TAU }\end{array}$ \\
\hline Slesnick, 2009190 & & & $\begin{array}{l}\text { Family therapy using a functional model vs. family therapy using an } \\
\text { ecological model vs. TAU }\end{array}$ \\
\hline Joanning, 1992138 & $\begin{array}{l}\text { Fam vs. } \\
\text { Fam vs. } \\
\text { PeerGroup }\end{array}$ & 2 & $\begin{array}{l}\text { Family therapy using an educational model vs. family therapy using } \\
\text { a structural model vs. youth-only group therapy }\end{array}$ \\
\hline Liddle, 2001153 & & & $\begin{array}{l}\text { Family therapy using an educational model vs. family therapy using } \\
\text { an ecological model vs. youth-only group therapy }\end{array}$ \\
\hline Henggeler, 2006135 & $\begin{array}{l}\text { Peer group vs. } \\
\text { PeerGroup vs. } \\
\text { Fam+PeerGroup vs. } \\
\text { Fam+CM+PeeerGroup }\end{array}$ & 1 & $\begin{array}{l}\text { Family court with usual community services (including peer group } \\
\text { therapy) vs. drug with usual community services (including peer } \\
\text { group therapy) vs. drug court combined with family therapy using } \\
\text { an ecological model and peer group therapy vs. drug court } \\
\text { combined with family therapy using an ecological model and peer } \\
\text { group therapy and contingency management }\end{array}$ \\
\hline
\end{tabular}

Abbreviations: $\mathrm{CBT}$ = cognitive behavioral therapy; $\mathrm{CM}=$ contingency management; Educ = psychoeducation; Fam = family therapy; ICM = intensive case management; $\mathrm{MI}=$ motivational interviewing; TAU = treatment as usual

\section{Substances Other Than Alcohol or Cannabis}

One 2-arm study ${ }^{33}$ summarized in Table 11, enrolled adolescents with inhalant use and evaluated a 4-session CBT-based intervention with an educational component. The authors concluded that adolescents who received CBT and education were about 3 times more likely to be abstinent at 1 year than those who received education alone (16\% vs. 5\%; RR 3.20, 95\% CI 1.34 to 7.65$).^{33}$ 
Table 11. Results: Nonbrief behavioral interventions for substances other than alcohol or cannabis

\begin{tabular}{|c|c|c|c|c|c|c|c|c|c|}
\hline $\begin{array}{l}\text { Study } \\
\text { Author, } \\
\text { Year, } \\
\text { PMID }\end{array}$ & Arm 1 & Arm 2 & Outcome & $\begin{array}{l}\text { Time Point } \\
\text { (Months) }\end{array}$ & $\begin{array}{l}\text { Arm } 1 \\
\text { N Analyzed }\end{array}$ & $\begin{array}{l}\text { Arm } 1 \\
\text { Outcome }\end{array}$ & $\begin{array}{l}\text { Arm } 2 \text { N } \\
\text { Analyzed }\end{array}$ & $\begin{array}{l}\text { Arm } 2 \\
\text { Outcome }\end{array}$ & $\begin{array}{l}\text { Calculated } \\
\text { Effect } \\
(95 \% \mathrm{Cl})\end{array}$ \\
\hline $\begin{array}{l}\text { Ogel } \\
(2011)^{33}\end{array}$ & $\begin{array}{l}\text { CBT_Educ } \\
\text { (Nonbrief, } \\
\text { Cognitive } \\
\text { Behavioral/E } \\
\text { ducational) }\end{array}$ & Educ & $\begin{array}{l}\text { Abstinent from } \\
\text { inhalants }(\mathrm{N})\end{array}$ & 12 & 31 & 16 & 31 & 5 & $\begin{array}{l}\text { RR 3.20 } \\
(1.34,7.65)\end{array}$ \\
\hline
\end{tabular}

Abbreviations: $\mathrm{CBT}=$ cognitive behavioral therapy; $\mathrm{Cl}=$ confidence interval; Diff = difference; $\mathrm{MI}=$ motivational interviewing; $\mathrm{RR}=$ risk ratio; $\mathrm{SD}=$ standard deviation; $\mathrm{TAU}=$ treatment as usual

\section{Risk of Bias}

Risk of Bias summaries are given in Figure 18 for the 53 studies that were eligible for metaanalysis. Each of these studies had methodological concerns, most prominently lack of blinding of participants and personnel and compliance.

Figure 18. Meta-analyzed nonbrief behavioral intervention studies: Percentage of studies in each risk of bias category

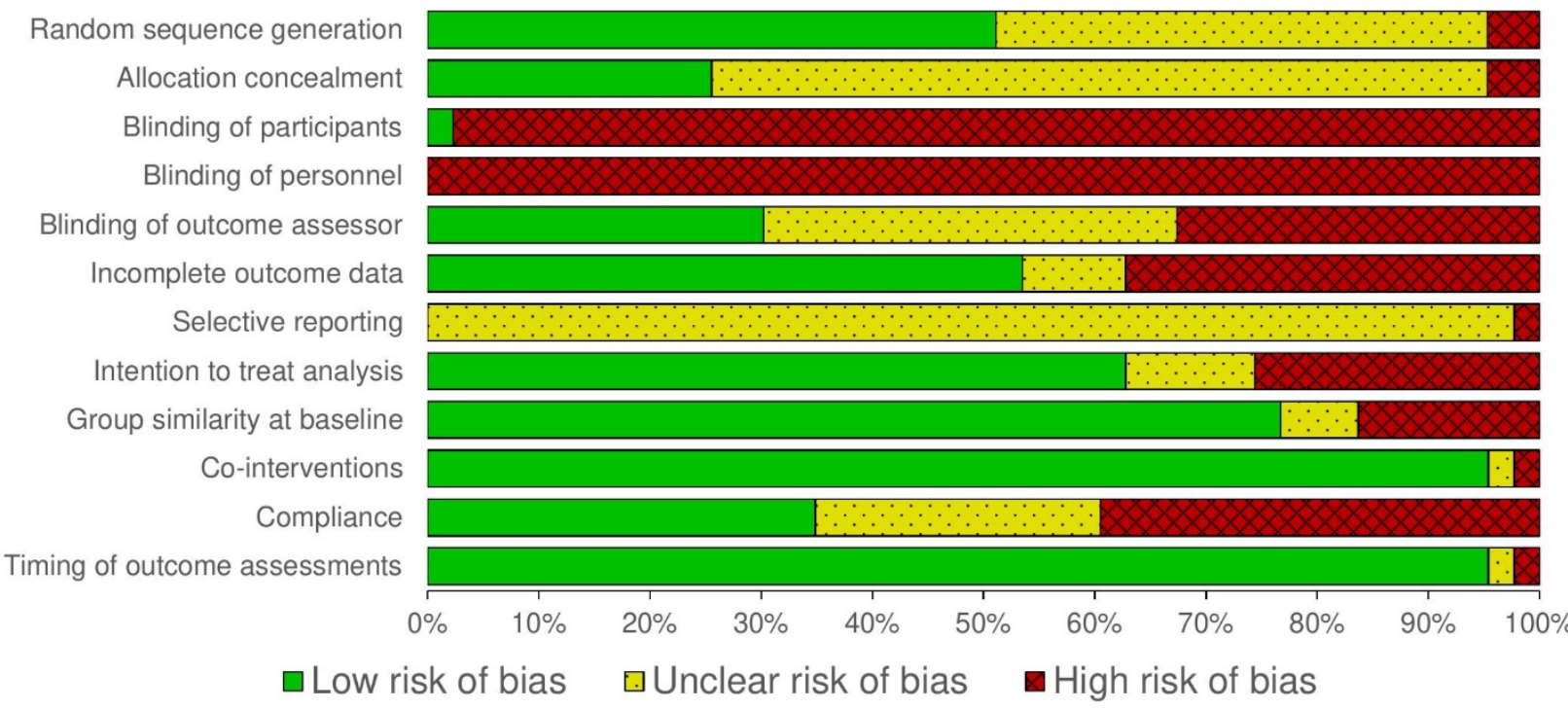

\section{Network Meta-Analyses}

\section{Alcohol Outcomes}

\section{Alcohol Use Days}

Eleven studies, comprised of eight dual-arm and three multi-arm studies as illustrated in Figure 19, enrolled 2,248 subjects and reported a measure of mean alcohol use days. Of these, two studies ${ }^{129,205}$ reported a scale. 
Figure 19. Evidence graph for nonbrief behavioral intervention studies reporting mean alcohol use days

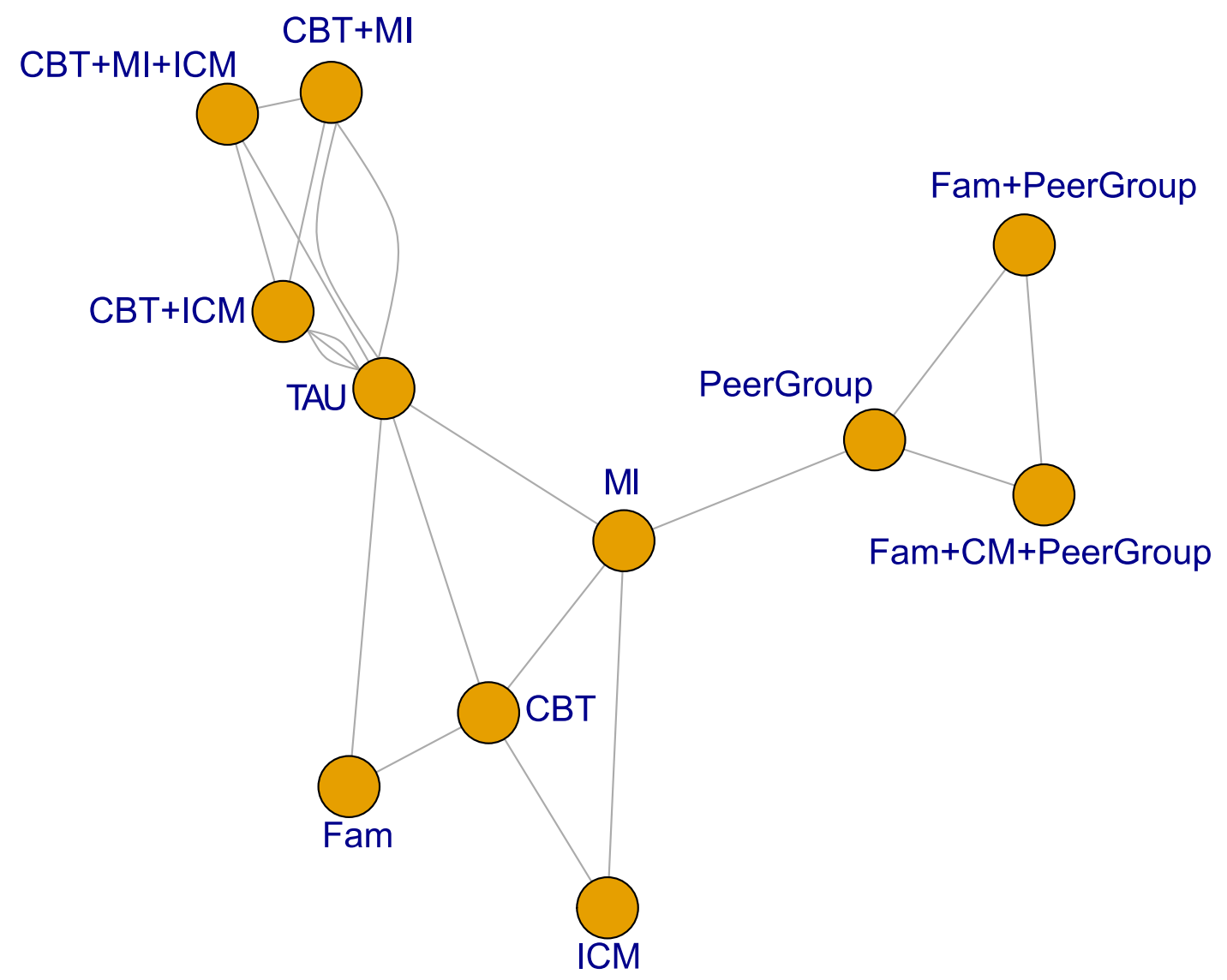

Abbreviations: $\mathrm{MI}$ = motivational interviewing; Fam = family focused therapy; $\mathrm{CBT}=$ cognitive behavioral therapy; $\mathrm{CM}=$ contingency management; PeerGroup = peer group therapy; ICM = intensive case management; TAU = treatment as usual

\section{Key Question 1: Alcohol Use Days - Behavioral Interventions Compared With TAU}

Figure 20 illustrates that net mean differences (NMD) from the network meta-analysis (NMA) for the 10 interventions evaluated. Note, however, that the network is sparse, and the comparisons between different treatments are often based on a series of single-study indirect comparisons. Because the network is not densely connected (most indirect comparisons rely on a small set of RCTs) and because most RCTs are small, the statistical power to detect inconsistency between direct and indirect effects is very limited. Thus, estimates of treatment effectiveness are very imprecise.

Fam was more effective than TAU. Participants who received Fam versus TAU had an NMD of -3.5 (95\% CrI -6.9, -0.4) days of alcohol use per month. We rated the associated SoE for this effect as low.

There is insufficient evidence regarding the relative effects of the other interventions compared with TAU. 
Figure 20. Alcohol use days: Summary forest plot of meta-analyzed net mean difference for all interventions studied compared with TAU

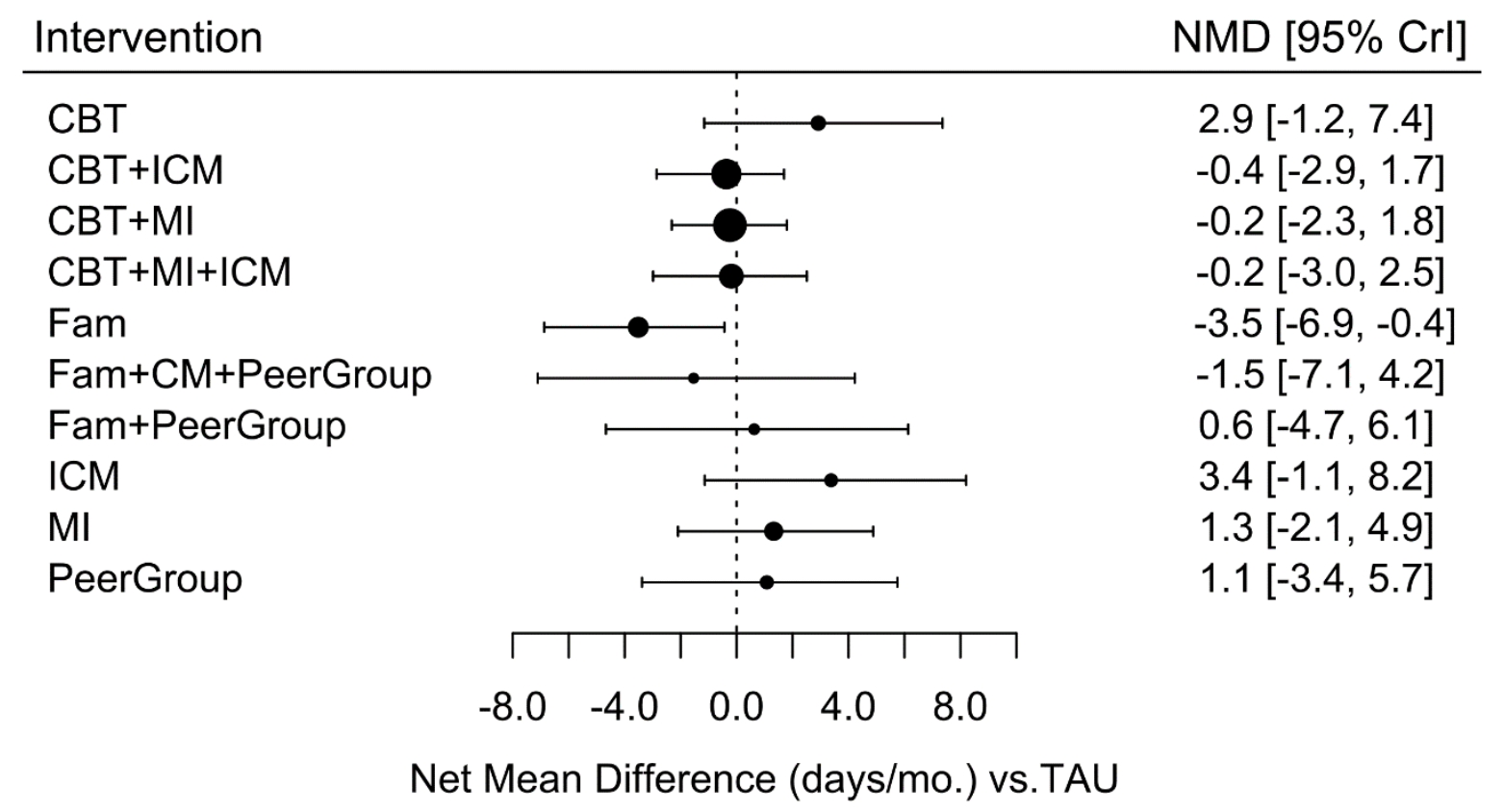

NMD < 1 favors intervention relative to TAU. Abbreviations: NMD = net mean difference; $\mathrm{MI}=$ motivational interviewing; Fam = family focused therapy; $\mathrm{CBT}=$ cognitive behavioral therapy; $\mathrm{CM}=$ contingency management; PeerGroup = peer group therapy; ICM = intensive case management; TAU = treatment as usual; CrI = Bayesian credible interval. 


\section{Key Question 2: Comparative Effects of Behavioral Interventions}

Most of the results in this network are based on indirect data, with direct data limited to only one or two studies. The statistical power to detect inconsistency between direct and indirect effects is very limited. Thus, estimates of treatment effectiveness are very imprecise.

The comparative effects of all interventions are detailed in Table 12. Among single component interventions, suggesting that Fam is better than ICM, CBT and MI. We rated the associated SoE as low.

\begin{tabular}{|c|c|c|c|c|c|c|c|c|c|c|c|}
\hline Intervention(s) & СВT & CBT+ICM & CBT+MI & $\begin{array}{l}\text { CBT+MI+ } \\
\text { ICM }\end{array}$ & Fam & $\begin{array}{l}\text { Fam+CM+ } \\
\text { PeerGroup }\end{array}$ & $\begin{array}{l}\text { Fam+ } \\
\text { PeerGroup }\end{array}$ & ICM & MI & PeerGroup & TAU \\
\hline CBT & СВТ & $\begin{array}{r}-3.3 \\
(-8.5,1.2) \\
\end{array}$ & $\begin{array}{l}-3.1 \\
(-8.1,1.4)\end{array}$ & $\begin{array}{l}-3.1 \\
(-8.4,1.7)\end{array}$ & $\begin{array}{l}-6.5 \\
(-11.2,-2.2)\end{array}$ & $\begin{array}{l}-4.4 \\
(-10.1,0.9)\end{array}$ & $\begin{array}{l}-2.3 \\
(-7.7,2.9)\end{array}$ & $\begin{array}{l}0.5 \\
(-3,3.8)\end{array}$ & $\begin{array}{l}-1.6 \\
(-4.9,1.5)\end{array}$ & $\begin{array}{l}-1.8 \\
(-6.4,2.4)\end{array}$ & $\begin{array}{l}-2.9 \\
(-7.4,1.2)\end{array}$ \\
\hline CBT+ICM & $\begin{array}{r}3.3 \\
(-1.2,8.5)\end{array}$ & CBT+ICM & $\begin{array}{l}0.1 \\
(-2.2,2.9)\end{array}$ & $\begin{array}{l}0.2 \\
(-2.5,3.2)\end{array}$ & $\begin{array}{l}-3.1 \\
(-7,0.8)\end{array}$ & $\begin{array}{l}-1.2 \\
(-7,5.2)\end{array}$ & $\begin{array}{l}1 \\
(-4.5,7.2)\end{array}$ & $\begin{array}{l}3.8 \\
(-1.1,9.2)\end{array}$ & $\begin{array}{l}1.7 \\
(-2.2,6.1)\end{array}$ & $\begin{array}{l}1.5 \\
(-3.4,6.9)\end{array}$ & $\begin{array}{l}0.4 \\
(-1.7,2.9)\end{array}$ \\
\hline $\mathrm{CBT}+\mathrm{MI}$ & $\begin{array}{r}3.1 \\
(-1.4,8.1)\end{array}$ & $\begin{array}{r}-0.1 \\
(-2.9,2.2)\end{array}$ & $\mathrm{CBT}+\mathrm{MI}$ & $\begin{array}{l}0 \\
(-2.8,2.8)\end{array}$ & $\begin{array}{l}-3.3 \\
(-7.2,0.4)\end{array}$ & $\begin{array}{l}-1.3 \\
(-7.2,4.8)\end{array}$ & $\begin{array}{l}0.9 \\
(-4.8,6.8)\end{array}$ & $\begin{array}{l}3.6 \\
(-1.3,8.9)\end{array}$ & $\begin{array}{l}1.6 \\
(-2.4,5.7)\end{array}$ & $\begin{array}{l}1.3 \\
(-3.5,6.5)\end{array}$ & $\begin{array}{l}0.2 \\
(-1.8,2.3)\end{array}$ \\
\hline CBT+MI+ICM & $\begin{array}{r}3.1 \\
(-1.7,8.4)\end{array}$ & $\begin{array}{r}-0.2 \\
(-3.2,2.5)\end{array}$ & $\begin{array}{l}0 \\
(-2.8,2.8)\end{array}$ & $\begin{array}{l}\text { CBT+MI+ } \\
\text { ICM }\end{array}$ & $\begin{array}{l}-3.3 \\
(-7.6,0.7)\end{array}$ & $\begin{array}{l}-1.4 \\
(-7.5,5.1)\end{array}$ & $\begin{array}{l}0.8 \\
(-5.1,7)\end{array}$ & $\begin{array}{l}3.6 \\
(-1.7,9.2)\end{array}$ & $\begin{array}{l}1.5 \\
(-2.8,6)\end{array}$ & $\begin{array}{l}1.2 \\
(-3.9,6.8)\end{array}$ & $\begin{array}{l}0.2 \\
(-2.5,3)\end{array}$ \\
\hline Fam & $\begin{array}{r}6.5 \\
(2.2,11.2) \\
\end{array}$ & $\begin{array}{r}3.1 \\
(-0.8,7) \\
\end{array}$ & $\begin{array}{l}3.3 \\
(-0.4,7.2)\end{array}$ & $\begin{array}{l}3.3 \\
(-0.7,7.6)\end{array}$ & Fam & $\begin{array}{l}2 \\
(-4,8.3)\end{array}$ & $\begin{array}{l}4.1 \\
(-1.5,10.3)\end{array}$ & $\begin{array}{l}6.9 \\
(2.1,12.1)\end{array}$ & $\begin{array}{l}4.8 \\
(0.8,9.2)\end{array}$ & $\begin{array}{l}4.6 \\
(-0.3,10)\end{array}$ & $\begin{array}{l}3.5 \\
(0.4,6.9)\end{array}$ \\
\hline $\begin{array}{l}\text { Fam+CM+ } \\
\text { PeerGroup }\end{array}$ & $\begin{array}{r}4.4 \\
(-0.9,10.1)\end{array}$ & $\begin{array}{r}1.2 \\
(-5.2,7)\end{array}$ & $\begin{array}{l}1.3 \\
(-4.8,7.2)\end{array}$ & $\begin{array}{l}1.4 \\
(-5.1,7.5)\end{array}$ & $\begin{array}{l}-2 \\
(-8.3,4)\end{array}$ & $\begin{array}{l}\text { Fam+CM+ } \\
\text { PeerGroup }\end{array}$ & $\begin{array}{l}2.2 \\
(-1.2,5.5)\end{array}$ & $\begin{array}{l}4.9 \\
(-0.6,10.6)\end{array}$ & $\begin{array}{l}2.8 \\
(-1.6,7.3)\end{array}$ & $\begin{array}{l}2.6 \\
(-0.8,6)\end{array}$ & $\begin{array}{l}1.5 \\
(-4.2,7.1)\end{array}$ \\
\hline $\begin{array}{l}\text { Fam+ } \\
\text { PeerGroup }\end{array}$ & $\begin{array}{r}2.3 \\
(-2.9,7.7)\end{array}$ & $\begin{array}{r}-1 \\
(-7.2,4.5)\end{array}$ & $\begin{array}{l}-0.9 \\
(-6.8,4.8)\end{array}$ & $\begin{array}{l}-0.8 \\
(-7,5.1)\end{array}$ & $\begin{array}{l}-4.1 \\
(-10.3,1.5)\end{array}$ & $\begin{array}{l}-2.2 \\
(-5.5,1.2)\end{array}$ & $\begin{array}{l}\text { Fam+ } \\
\text { PeerGroup }\end{array}$ & $\begin{array}{l}2.7 \\
(-2.6,8.2)\end{array}$ & $\begin{array}{l}0.7 \\
(-3.5,4.9)\end{array}$ & $\begin{array}{l}0.4 \\
(-2.5,3.4)\end{array}$ & $\begin{array}{l}-0.6 \\
(-6.1,4.7)\end{array}$ \\
\hline ICM & $\begin{array}{r}-0.5 \\
(-3.8,3)\end{array}$ & $\begin{array}{r}-3.8 \\
(-9.2,1.1)\end{array}$ & $\begin{array}{l}-3.6 \\
(-8.9,1.3)\end{array}$ & $\begin{array}{l}-3.6 \\
(-9.2,1.7)\end{array}$ & $\begin{array}{l}-6.9 \\
(-12.1,-2.1)\end{array}$ & $\begin{array}{l}-4.9 \\
(-10.6,0.6)\end{array}$ & $\begin{array}{l}-2.7 \\
(-8.2,2.6)\end{array}$ & ICM & $\begin{array}{l}-2.1 \\
(-5.6,1.3)\end{array}$ & $\begin{array}{l}-2.3 \\
(-6.9,2.1)\end{array}$ & $\begin{array}{l}-3.4 \\
(-8.2,1.1)\end{array}$ \\
\hline MI & $\begin{array}{r}1.6 \\
(-1.5,4.9)\end{array}$ & $\begin{array}{r}-1.7 \\
(-6.1,2.2)\end{array}$ & $\begin{array}{l}-1.6 \\
(-5.7,2.4)\end{array}$ & $\begin{array}{l}-1.5 \\
(-6,2.8) \\
\end{array}$ & $\begin{array}{l}-4.8 \\
(-9.2,-0.8)\end{array}$ & $\begin{array}{l}-2.8 \\
(-7.3,1.6)\end{array}$ & $\begin{array}{l}-0.7 \\
(-4.9,3.5)\end{array}$ & $\begin{array}{l}2.1 \\
(-1.3,5.6)\end{array}$ & MI & $\begin{array}{l}-0.2 \\
(-3.2,2.8)\end{array}$ & $\begin{array}{l}-1.3 \\
(-4.9,2.1)\end{array}$ \\
\hline PeerGroup & $\begin{array}{r}1.8 \\
(-2.4,6.4)\end{array}$ & $\begin{array}{r}-1.5 \\
(-6.9,3.4)\end{array}$ & $\begin{array}{l}-1.3 \\
(-6.5,3.5)\end{array}$ & $\begin{array}{l}-1.2 \\
(-6.8,3.9)\end{array}$ & $\begin{array}{l}-4.6 \\
(-10,0.3)\end{array}$ & $\begin{array}{l}-2.6 \\
(-6,0.8)\end{array}$ & $\begin{array}{l}-0.4 \\
(-3.4,2.5)\end{array}$ & $\begin{array}{l}2.3 \\
(-2.1,6.9)\end{array}$ & $\begin{array}{l}0.2 \\
(-2.8,3.2)\end{array}$ & PeerGroup & $\begin{array}{l}-1.1 \\
(-5.7,3.4)\end{array}$ \\
\hline TAU & $\begin{array}{r}2.9 \\
(-1.2,7.4)\end{array}$ & $\begin{array}{r}-0.4 \\
(-2.9,1.7)\end{array}$ & $\begin{array}{l}-0.2 \\
(-2.3,1.8)\end{array}$ & $\begin{array}{l}-0.2 \\
(-3,2.5)\end{array}$ & $\begin{array}{l}-3.5 \\
(-6.9,-0.4)\end{array}$ & $\begin{array}{l}-1.5 \\
(-7.1,4.2)\end{array}$ & $\begin{array}{l}0.6 \\
(-4.7,6.1)\end{array}$ & $\begin{array}{l}3.4 \\
(-1.1,8.2)\end{array}$ & $\begin{array}{l}1.3 \\
(-2.1,4.9)\end{array}$ & $\begin{array}{l}1.1 \\
(-3.4,5.7)\end{array}$ & TAU \\
\hline
\end{tabular}

Bold font indicates $95 \%$ CrI excludes the null effect.

Abbreviations: $\mathrm{CBT}=$ cognitive behavioral therapy; $\mathrm{CM}=$ contingency management; Educ = psychoeducation; Fam = family therapy; ICM = intensive case management; $\mathrm{MI}=$ motivational interviewing; TAU = treatment as usual. 
In Table 13, the interventions are ranked by the surface area under the cumulative ranking curve (SUCRA). The higher the SUCRA value (closer to 100\%), the higher the likelihood that an intervention is in the top rank or one of the top ranks. As SUCRA values approach 0 percent, the more likely that an intervention is in the bottom rank, or one of the bottom ranks. ${ }^{43}$ The last three columns summarize the probability that each intervention ranks in the top third, middle third, and bottom third, respectively. There is a 95 percent chance that Fam ranks in the top third, a 91 percent chance that ICM ranks in the bottom third, and an 88 percent chance that CBT is in the bottom third with respect effects on overall alcohol use days.

Table 13. Probabilities of nonbrief behavioral interventions ranking in top third, middle third and bottom third to reduce alcohol use days

\begin{tabular}{lrrrr}
\hline Intervention(s) & SUCRA & Top third & Middle third & Bottom third \\
\hline Fam & $96 \%$ & 95 & 5 & 0 \\
\hline Fam+CM+PeerGroup & $79 \%$ & 66 & 28 & 6 \\
\hline CBT+ICM & $66 \%$ & 36 & 51 & 14 \\
\hline CBT+Ml & $64 \%$ & 28 & 58 & 15 \\
\hline CBT+MI+ICM & $62 \%$ & 29 & 52 & 19 \\
\hline TAU & $58 \%$ & 12 & 71 & 17 \\
\hline Fam+PeerGroup & $51 \%$ & 22 & 43 & 35 \\
\hline PeerGroup & $44 \%$ & 7 & 38 & 55 \\
\hline MI & $40 \%$ & 3 & 37 & 60 \\
\hline CBT & $22 \%$ & 1 & 11 & 88 \\
\hline ICM & $18 \%$ & 1 & 8 & 91
\end{tabular}

Abbreviations: CBT = cognitive behavioral therapy; CM = contingency management; Fam = family therapy; ICM = intensive case management; MI = motivational interviewing; PeerGroup = peer group therapy; SUCRA = surface area under the cumulative ranking curve; TAU = treatment as usual

\section{Cannabis Outcomes}

\section{Cannabis Use Days}

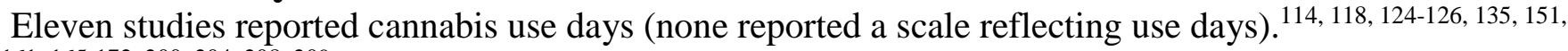
154-161, 165-172, 200, 204, 208, 209 The network of treatment comparisons shown in Figure 21 for the nine dual-arm and two multi-arm, with 1,643 participants included in this network meta-analysis. 
Figure 21. Evidence graph for studies reporting cannabis use days
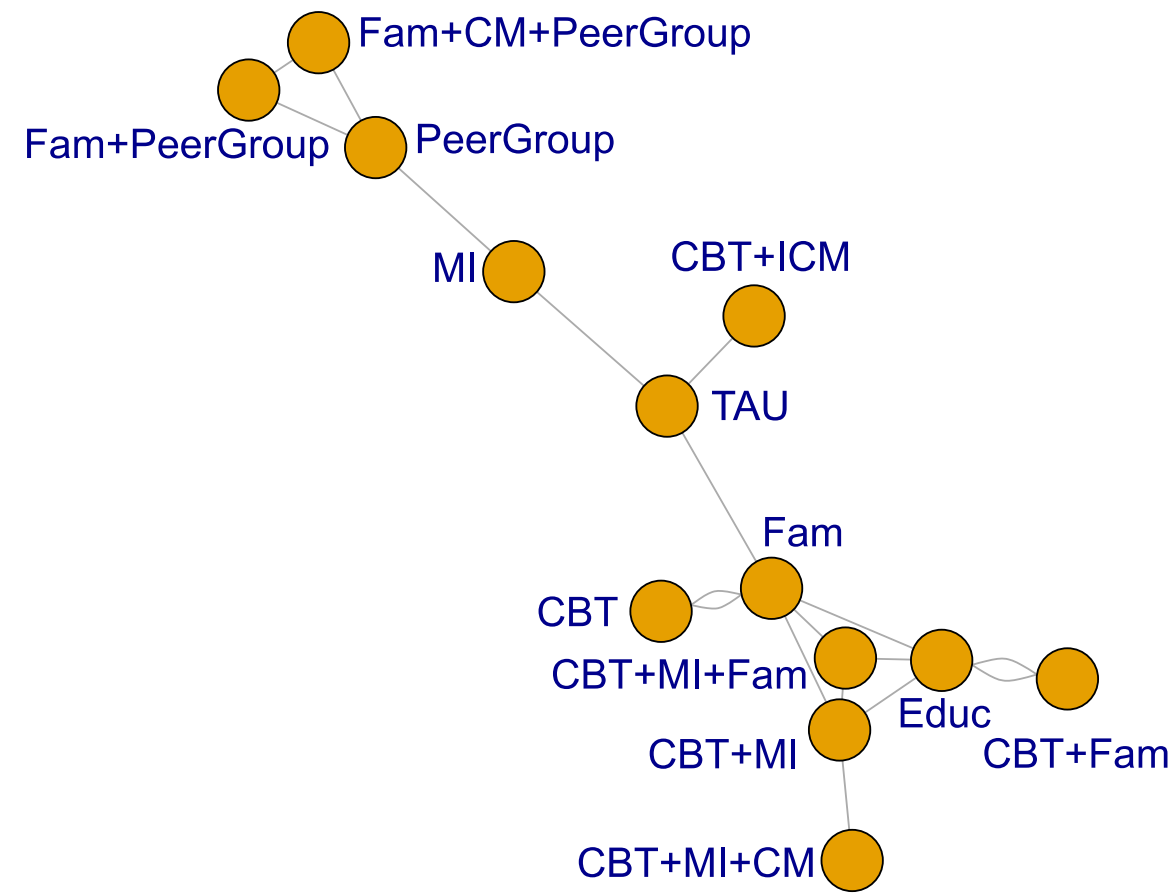

Abbreviations: Fam = family; $\mathrm{CM}=$ contingency management; $\mathrm{TAU}=$ treatment as usual; $\mathrm{CBT}=$ cognitive behavioral therapy; ICM = intensive case management; $\mathrm{MI}=$ motivational interviewing; Educ = education.

\section{Key Question 1: Cannabis Use Days - Behavioral Interventions Compared With TAU}

Compared to TAU, we cannot conclude that any of interventions studied reduced cannabis use days (Figure 22). All point estimates of treatment effects, except for CBT+ICM and Fam+PeerGroup, were positive, consistent with an increase in cannabis use days.

However, the credible intervals were generally wide. As was the case in the previous analysis, the network is very sparse and loosely connected, and because most RCTs are small, the statistical power to detect inconsistency between direct and indirect effects is very limited. Thus, estimates of treatment effectiveness are very imprecise.

One intervention (CBT), and two combined interventions (CBT+MI and CBT+MI+CM) increased cannabis use days relative to TAU. We rated the associated SoE for this conclusion as low. 
Figure 22. Cannabis use days: Summary forest plot of meta-analyzed net mean differences for all interventions compared with TAU

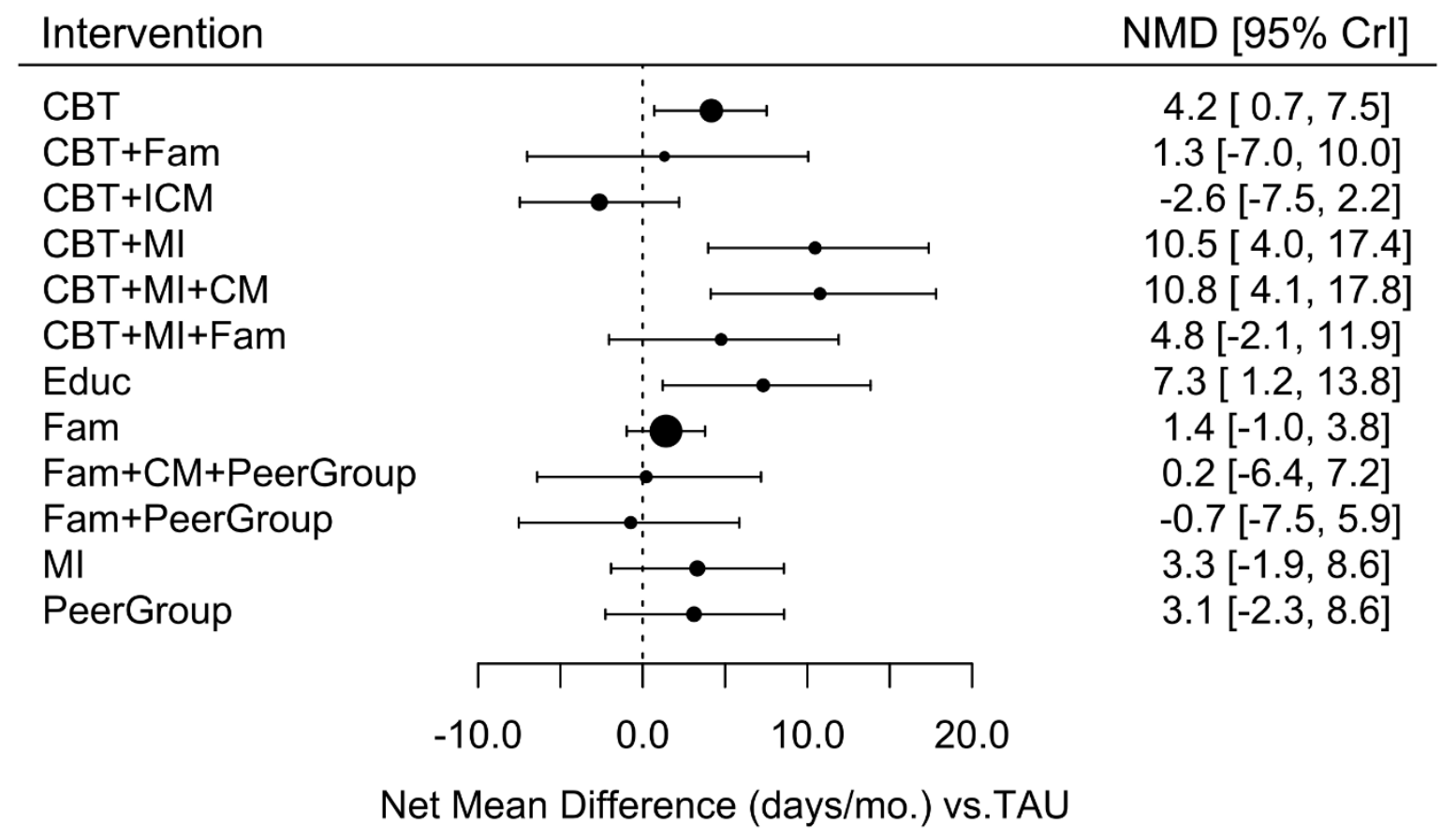

NMD <1 favors intervention versus TAU. Abbreviations: MI = motivational interviewing; Fam = family focused therapy; CBT = cognitive behavioral therapy; $\mathrm{CM}$ = contingency management; PeerGroup = peer group therapy; ICM = intensive case management; TAU = treatment as usual; $\mathrm{CrI}=$ credible interval

\section{Key Question 2: Cannabis Use Days - Comparative Effects of Behavioral Interventions}

Table 14 enumerates the relative effects of all interventions and interventions combinations studied. Most of the results in the table are based on indirect data, with direct data limited to one or two studies. The statistical power to detect inconsistency between direct and indirect effects is very limited. Thus, estimates of treatment effectiveness are imprecise.

Given that we cannot conclude that any of interventions studied reduced cannabis use days compared to TAU, and the limitations noted above, we rated the SoE for all comparative effects as insufficient. 
Table 14. Nonbrief behavioral interventions and cannabis use days: Net mean difference of use days per month between all interventions

\begin{tabular}{|c|c|c|c|c|c|c|c|c|c|c|c|c|c|}
\hline Intervention(s) & CBT & CBT+Fam & $\mathrm{CBT}+\mathrm{ICM}$ & CBT+MI & $\mathrm{CBT}+\mathrm{MI}+\mathrm{CM}$ & CBT+MI+Fam & Educ & Fam & $\begin{array}{l}\text { Fam+CM+ } \\
\text { PeerGroup }\end{array}$ & Fam+PeerGroup & MI & PeerGroup & TAU \\
\hline CBT & CBT & $\begin{array}{l}-2.9 \\
(-11.1,5.6)\end{array}$ & $\begin{array}{l}-6.7 \\
(-12.7,-1)\end{array}$ & $\begin{array}{l}6.3 \\
(-0.3,13.3)\end{array}$ & $\begin{array}{l}6.7 \\
(-0.1,13.7)\end{array}$ & $\begin{array}{l}0.7 \\
(-6.2,7.7)\end{array}$ & $\begin{array}{l}3.2 \\
(-3,9.6)\end{array}$ & $\begin{array}{l}-2.8 \\
(-5.1,-0.3)\end{array}$ & $\begin{array}{l}-4 \\
(-11.4,3.8)\end{array}$ & $\begin{array}{l}-4.9 \\
(-12.5,2.6)\end{array}$ & $\begin{array}{l}-0.8 \\
(-7.1,5.5)\end{array}$ & $\begin{array}{l}-1 \\
(-7.4,5.5)\end{array}$ & $\begin{array}{l}-4.2 \\
(-7.5,-0.7)\end{array}$ \\
\hline CBT+Fam & $\begin{array}{l}2.9 \\
(-5.6,11.1)\end{array}$ & CBT+Fam & $\begin{array}{l}-3.9 \\
(-14.1,5.7)\end{array}$ & $\begin{array}{l}9.2 \\
(0.5,18)\end{array}$ & $\begin{array}{l}9.5 \\
(0.8,18.4)\end{array}$ & $\begin{array}{l}3.6 \\
(-5.5,11.6)\end{array}$ & $\begin{array}{l}6.1 \\
(0.3,11.5)\end{array}$ & $\begin{array}{l}0.1 \\
(-8.1,8)\end{array}$ & $\begin{array}{l}-1.3 \\
(-12,9.8)\end{array}$ & $\begin{array}{l}-2.1 \\
(-12.9,8.6)\end{array}$ & $\begin{array}{l}1.9 \\
(-8,11.6)\end{array}$ & $\begin{array}{l}1.7 \\
(-8.3,11.5)\end{array}$ & $\begin{array}{l}-1.3 \\
(-10,7)\end{array}$ \\
\hline CBT+ICM & $\begin{array}{l}6.7 \\
(1,12.7)\end{array}$ & $\begin{array}{l}3.9 \\
(-5.7,14.1)\end{array}$ & $\mathrm{CBT}+\mathrm{ICM}$ & $\begin{array}{l}13.3 \\
(4.8,21.9)\end{array}$ & $\begin{array}{l}13.5 \\
(5,22.2)\end{array}$ & $\begin{array}{l}7.4 \\
(-0.8,15.9)\end{array}$ & $\begin{array}{l}10.1 \\
(2.1,18.1)\end{array}$ & $\begin{array}{l}4 \\
(-1.3,9.5)\end{array}$ & $\begin{array}{l}2.8 \\
(-5.5,11.1)\end{array}$ & $\begin{array}{l}1.9 \\
(-6.1,10)\end{array}$ & $\begin{array}{l}5.9 \\
(-0.8,13.3)\end{array}$ & $\begin{array}{l}5.7 \\
(-1.2,12.9)\end{array}$ & $\begin{array}{l}2.6 \\
(-2.2,7.5)\end{array}$ \\
\hline $\mathrm{CBT}+\mathrm{MI}$ & $\begin{array}{l}-6.3 \\
(-13.3,0.3)\end{array}$ & $\begin{array}{l}-9.2 \\
(-18,-0.5)\end{array}$ & $\begin{array}{l}-13.3 \\
(-21.9,-4.8)\end{array}$ & $\mathrm{CBT}+\mathrm{MI}$ & $\begin{array}{l}0.3 \\
(-1,1.6)\end{array}$ & $\begin{array}{l}-5.7 \\
(-13,1.4)\end{array}$ & $\begin{array}{l}-3.1 \\
(-9.9,3.1)\end{array}$ & $\begin{array}{l}-9.1 \\
(-15.6,-2.9)\end{array}$ & $\begin{array}{l}-10.5 \\
(-20.3,-0.5)\end{array}$ & $\begin{array}{l}-11.1 \\
(-21.6,-2)\end{array}$ & $\begin{array}{l}-7.3 \\
(-16.5,1.3)\end{array}$ & $\begin{array}{l}-7.5 \\
(-16.9,1.3)\end{array}$ & $\begin{array}{l}-10.5 \\
(-17.4,-4)\end{array}$ \\
\hline $\mathrm{CBT}+\mathrm{MI}+\mathrm{CM}$ & $\begin{array}{l}-6.7 \\
(-13.7,0.1)\end{array}$ & $\begin{array}{l}-9.5 \\
(-18.4,-0.8)\end{array}$ & $\begin{array}{l}-13.5 \\
(-22.2,-5)\end{array}$ & $\begin{array}{l}-0.3 \\
(-1.6,1)\end{array}$ & $\mathrm{CBT}+\mathrm{MI}+\mathrm{CM}$ & $\begin{array}{l}-6 \\
(-13.4,1.2)\end{array}$ & $\begin{array}{l}-3.4 \\
(-10.3,2.9)\end{array}$ & $\begin{array}{l}-9.4 \\
(-16.1,-3.1)\end{array}$ & $\begin{array}{l}-10.8 \\
(-20.6,-0.8)\end{array}$ & $\begin{array}{l}-11.4 \\
(-22,-2.2)\end{array}$ & $\begin{array}{l}-7.6 \\
(-16.8,1.1)\end{array}$ & $\begin{array}{l}-7.8 \\
(-17.2,1.1)\end{array}$ & $\begin{array}{l}-10.8 \\
(-17.8,-4.1)\end{array}$ \\
\hline CBT+MI+Fam & $\begin{array}{l}-0.7 \\
(-7.7,6.2)\end{array}$ & $\begin{array}{l}-3.6 \\
(-11.6,5.5)\end{array}$ & $\begin{array}{l}-7.4 \\
(-15.9,0.8)\end{array}$ & $\begin{array}{l}5.7 \\
(-1.4,13)\end{array}$ & $\begin{array}{l}6 \\
(-1.2,13.4)\end{array}$ & CBT+MI+Fam & $\begin{array}{l}2.4 \\
(-3.9,9.2)\end{array}$ & $\begin{array}{l}-3.3 \\
(-9.9,3)\end{array}$ & $\begin{array}{l}-4.8 \\
(-13.8,5.3)\end{array}$ & $\begin{array}{l}-5.6 \\
(-15,4)\end{array}$ & $\begin{array}{l}-1.5 \\
(-9.9,7)\end{array}$ & $\begin{array}{l}-1.7 \\
(-10.2,6.9)\end{array}$ & $\begin{array}{l}-4.8 \\
(-11.9,2.1)\end{array}$ \\
\hline Educ & $\begin{array}{l}-3.2 \\
(-9.6,3)\end{array}$ & $\begin{array}{l}-6.1 \\
(-11.5,-0.3)\end{array}$ & $\begin{array}{l}-10.1 \\
(-18.1,-2.1)\end{array}$ & $\begin{array}{l}3.1 \\
(-3.1,9.9)\end{array}$ & $\begin{array}{l}3.4 \\
(-2.9,10.3)\end{array}$ & $\begin{array}{l}-2.4 \\
(-9.2,3.9)\end{array}$ & Educ & $\begin{array}{l}-5.9 \\
(-11.9,-0.3)\end{array}$ & $\begin{array}{l}-7.4 \\
(-16.9,2.6)\end{array}$ & $\begin{array}{l}-8.2 \\
(-17.4,1.2)\end{array}$ & $\begin{array}{l}-4.2 \\
(-12.4,4.6)\end{array}$ & $\begin{array}{l}-4.3 \\
(-12.7,4.3)\end{array}$ & $\begin{array}{l}-7.3 \\
(-13.8,-1.2)\end{array}$ \\
\hline Fam & $\begin{array}{l}2.8 \\
(0.3,5.1)\end{array}$ & $\begin{array}{l}-0.1 \\
(-8,8.1)\end{array}$ & $\begin{array}{l}-4 \\
(-9.5,1.3)\end{array}$ & $\begin{array}{l}9.1 \\
(2.9,15.6)\end{array}$ & $\begin{array}{l}9.4 \\
(3.1,16.1)\end{array}$ & $\begin{array}{l}3.3 \\
(-3,9.9)\end{array}$ & $\begin{array}{l}5.9 \\
(0.3,11.9)\end{array}$ & Fam & $\begin{array}{l}-1.2 \\
(-8.3,6.1)\end{array}$ & $\begin{array}{l}-2.1 \\
(-9.5,4.9)\end{array}$ & $\begin{array}{l}1.9 \\
(-4.1,7.8)\end{array}$ & $\begin{array}{l}1.7 \\
(-4.4,7.8)\end{array}$ & $\begin{array}{l}-1.4 \\
(-3.8,1)\end{array}$ \\
\hline $\begin{array}{l}\text { Fam+CM+ } \\
\text { PeerGroup }\end{array}$ & $\begin{array}{l}4 \\
(-3.8,11.4)\end{array}$ & $\begin{array}{l}1.3 \\
(-9.8,12)\end{array}$ & $\begin{array}{l}-2.8 \\
(-11.1,5.5)\end{array}$ & $\begin{array}{l}10.5 \\
(0.5,20.3)\end{array}$ & $\begin{array}{l}10.8 \\
(0.8,20.6)\end{array}$ & $\begin{array}{l}4.8 \\
(-5.3,13.8)\end{array}$ & $\begin{array}{l}7.4 \\
(-2.6,16.9)\end{array}$ & $\begin{array}{l}1.2 \\
(-6.1,8.3)\end{array}$ & $\begin{array}{l}\text { Fam+CM+ } \\
\text { PeerGroup }\end{array}$ & $\begin{array}{l}-0.9 \\
(-5.6,4.1)\end{array}$ & $\begin{array}{l}3.1 \\
(-1.2,7.7)\end{array}$ & $\begin{array}{l}2.9 \\
(-1.2,7.3)\end{array}$ & $\begin{array}{l}-0.2 \\
(-7.2,6.4)\end{array}$ \\
\hline Fam+PeerGroup & $\begin{array}{l}4.9 \\
(-2.6,12.5)\end{array}$ & $\begin{array}{l}2.1 \\
(-8.6,12.9)\end{array}$ & $\begin{array}{l}-1.9 \\
(-10,6.1)\end{array}$ & $\begin{array}{l}11.1 \\
(2,21.6)\end{array}$ & $\begin{array}{l}11.4 \\
(2.2,22)\end{array}$ & $\begin{array}{l}5.6 \\
(-4,15)\end{array}$ & $\begin{array}{l}8.2 \\
(-1.2,17.4)\end{array}$ & $\begin{array}{l}2.1 \\
(-4.9,9.5)\end{array}$ & $\begin{array}{l}0.9 \\
(-4.1,5.6)\end{array}$ & Fam+PeerGroup & $\begin{array}{l}4 \\
(-0.2,8.3)\end{array}$ & $\begin{array}{l}3.8 \\
(-0.2,7.9)\end{array}$ & $\begin{array}{l}0.7 \\
(-5.9,7.5)\end{array}$ \\
\hline MI & $\begin{array}{l}0.8 \\
(-5.5,7.1)\end{array}$ & $\begin{array}{l}-1.9 \\
(-11.6,8)\end{array}$ & $\begin{array}{l}-5.9 \\
(-13.3,0.8)\end{array}$ & $\begin{array}{l}7.3 \\
(-1.3,16.5)\end{array}$ & $\begin{array}{l}7.6 \\
(-1.1,16.8)\end{array}$ & $\begin{array}{l}1.5 \\
(-7,9.9)\end{array}$ & $\begin{array}{l}4.2 \\
(-4.6,12.4)\end{array}$ & $\begin{array}{l}-1.9 \\
(-7.8,4.1)\end{array}$ & $\begin{array}{l}-3.1 \\
(-7.7,1.2)\end{array}$ & $\begin{array}{l}-4 \\
(-8.3,0.2)\end{array}$ & MI & $\begin{array}{l}-0.2 \\
(-1.6,1.2)\end{array}$ & $\begin{array}{l}-3.3 \\
(-8.6,1.9)\end{array}$ \\
\hline PeerGroup & $\begin{array}{l}1 \\
(-5.5,7.4)\end{array}$ & $\begin{array}{l}-1.7 \\
(-11.5,8.3)\end{array}$ & $\begin{array}{l}-5.7 \\
(-12.9,1.2)\end{array}$ & $\begin{array}{l}7.5 \\
(-1.3,16.9)\end{array}$ & $\begin{array}{l}7.8 \\
(-1.1,17.2)\end{array}$ & $\begin{array}{l}1.7 \\
(-6.9,10.2)\end{array}$ & $\begin{array}{l}4.3 \\
(-4.3,12.7)\end{array}$ & $\begin{array}{l}-1.7 \\
(-7.8,4.4)\end{array}$ & $\begin{array}{l}-2.9 \\
(-7.3,1.2)\end{array}$ & $\begin{array}{l}-3.8 \\
(-7.9,0.2)\end{array}$ & $\begin{array}{l}0.2 \\
(-1.2,1.6)\end{array}$ & PeerGroup & $\begin{array}{l}-3.1 \\
(-8.6,2.3)\end{array}$ \\
\hline TAU & $\begin{array}{l}4.2 \\
(0.7,7.5)\end{array}$ & $\begin{array}{l}1.3 \\
(-7,10)\end{array}$ & $\begin{array}{l}-2.6 \\
(-7.5,2.2)\end{array}$ & $\begin{array}{l}10.5 \\
(4,17.4)\end{array}$ & $\begin{array}{l}10.8 \\
(4.1,17.8)\end{array}$ & $\begin{array}{l}4.8 \\
(-2.1,11.9)\end{array}$ & $\begin{array}{l}7.3 \\
(1.2,13.8)\end{array}$ & $\begin{array}{l}1.4 \\
(-1,3.8)\end{array}$ & $\begin{array}{l}0.2 \\
(-6.4,7.2)\end{array}$ & $\begin{array}{l}-0.7 \\
(-7.5,5.9)\end{array}$ & $\begin{array}{l}3.3 \\
(-1.9,8.6)\end{array}$ & $\begin{array}{l}3.1 \\
(-2.3,8.6)\end{array}$ & TAU \\
\hline
\end{tabular}


Bold font indicates the 95\% CrI for the comparative effect excludes the null effect.

Abbreviations: $\mathrm{CBT}=$ cognitive behavioral therapy; $\mathrm{CM}$ = contingency management; Educ = psychoeducation; Fam = family therapy; ICM = intensive case management; $\mathrm{MI}=$ motivational interviewing; $\mathrm{TAU}=$ treatment as usual 
These patterns are reflected in the rankings, Table 15, which estimates that TAU has a 79 percent chance of being in the top third of all interventions.

Table 15. Probabilities of nonbrief behavioral interventions ranking in top third, middle third and bottom third to reduce cannabis use days

\begin{tabular}{lrrrr}
\hline Intervention(s) & SUCRA & Top Third & Middle Third & Bottom Third \\
\hline CBT+ICM & $91 \%$ & 91 & 8 & 1 \\
\hline Fam+PeerGroup & $81 \%$ & 71 & 26 & 3 \\
\hline TAU & $79 \%$ & 72 & 28 & 0 \\
\hline Fam+CM+PeerGroup & $74 \%$ & 59 & 33 & 8 \\
\hline Fam & $66 \%$ & 33 & 64 & 2 \\
\hline CBT+Fam & $66 \%$ & 44 & 32 & 24 \\
\hline PeerGroup & $51 \%$ & 10 & 59 & 30 \\
\hline Ml & $48 \%$ & 5 & 59 & 36 \\
\hline CBT & $44 \%$ & 2 & 46 & 52 \\
\hline CBT+MI+Fam & $43 \%$ & 10 & 30 & 60 \\
\hline Educ & $27 \%$ & 1 & 12 & 87 \\
\hline CBT+MI & $17 \%$ & 0 & 2 & 98 \\
\hline CBT+Ml+CM & $12 \%$ & 0 & 1 & 99 \\
\hline
\end{tabular}

Abbreviations: $\mathrm{CBT}$ = cognitive behavioral therapy; $\mathrm{CM}=$ contingency management; Educ = psychoeducation; Fam = family therapy; ICM = intensive case management; $\mathrm{MI}=$ motivational interviewing; SUCRA = surface area under the cumulative ranking curve; TAU = treatment as usual 


\section{Alcohol and Other Drug Use}

Eight studies reported aggregate use days for alcohol and other drugs by 1,202 participants, with comparisons illustrated in Figure 23. ${ }^{112,119,127,139,141,142,162,189,194,195}$ One of these studies, with 32 subjects, reported a scale that reflected aggregate alcohol and drug use. ${ }^{141,142}$ There were six dual-arm studies and two multi-arm studies.

Figure 23. Evidence graph for studies reporting aggregate alcohol and other drug use days

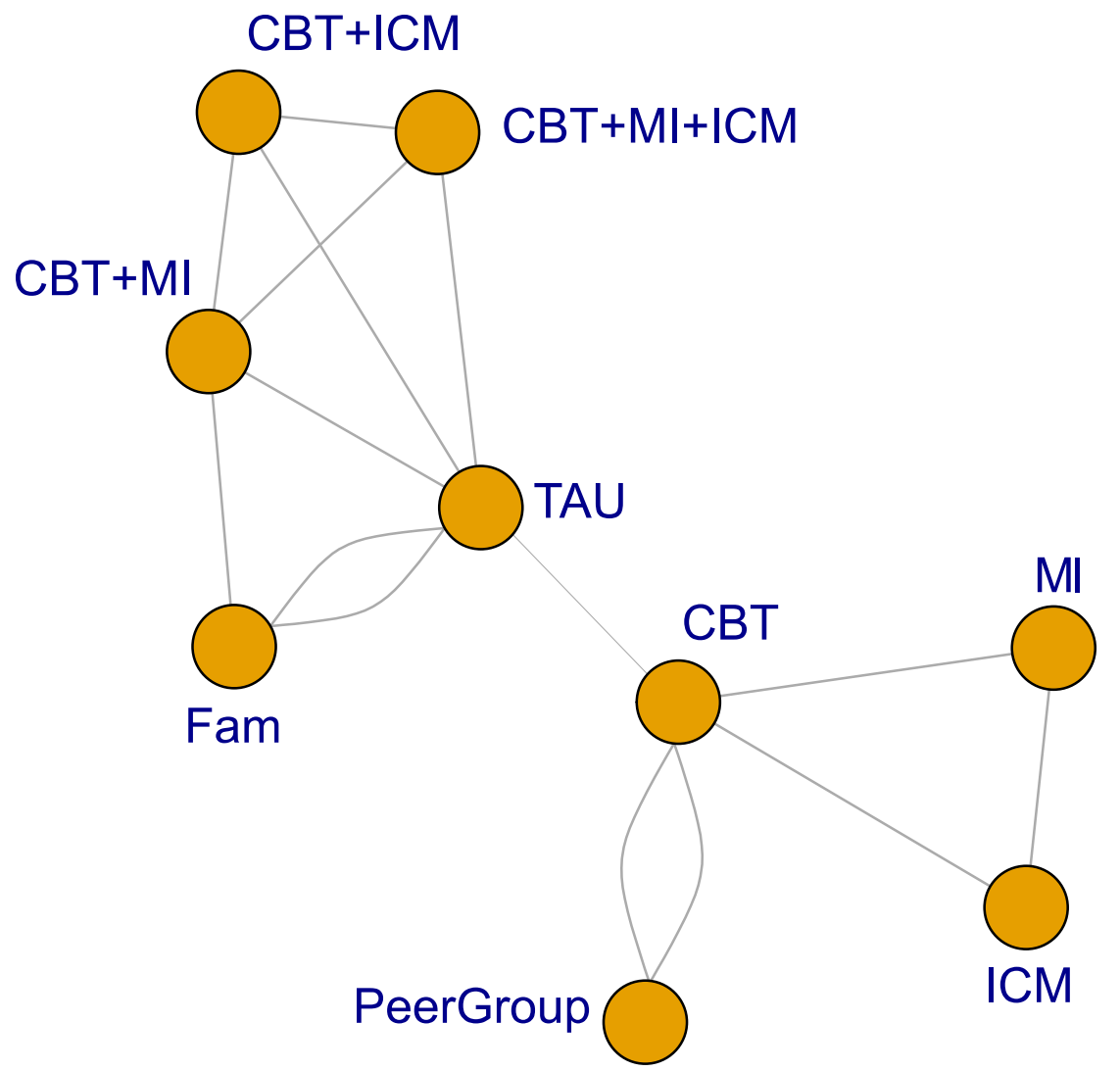

\section{Key Question 1: Aggregate Alcohol and Other Drug Use - Behavioral Interventions Compared to TAU}

Overall the precision of estimates was low (Figure 24). This network is very sparse and loosely connected, and because most RCTs are small, the statistical power to detect inconsistency between direct and indirect effects is very limited. Estimates of treatment effectiveness are very imprecise.

Of the interventions compared, MI and CBT were more effective than TAU. We rated the associated SoE as low. 
Figure 24. Aggregate alcohol and other drug use: Summary forest plot of meta-analyzed net mean differences for all interventions compared with TAU

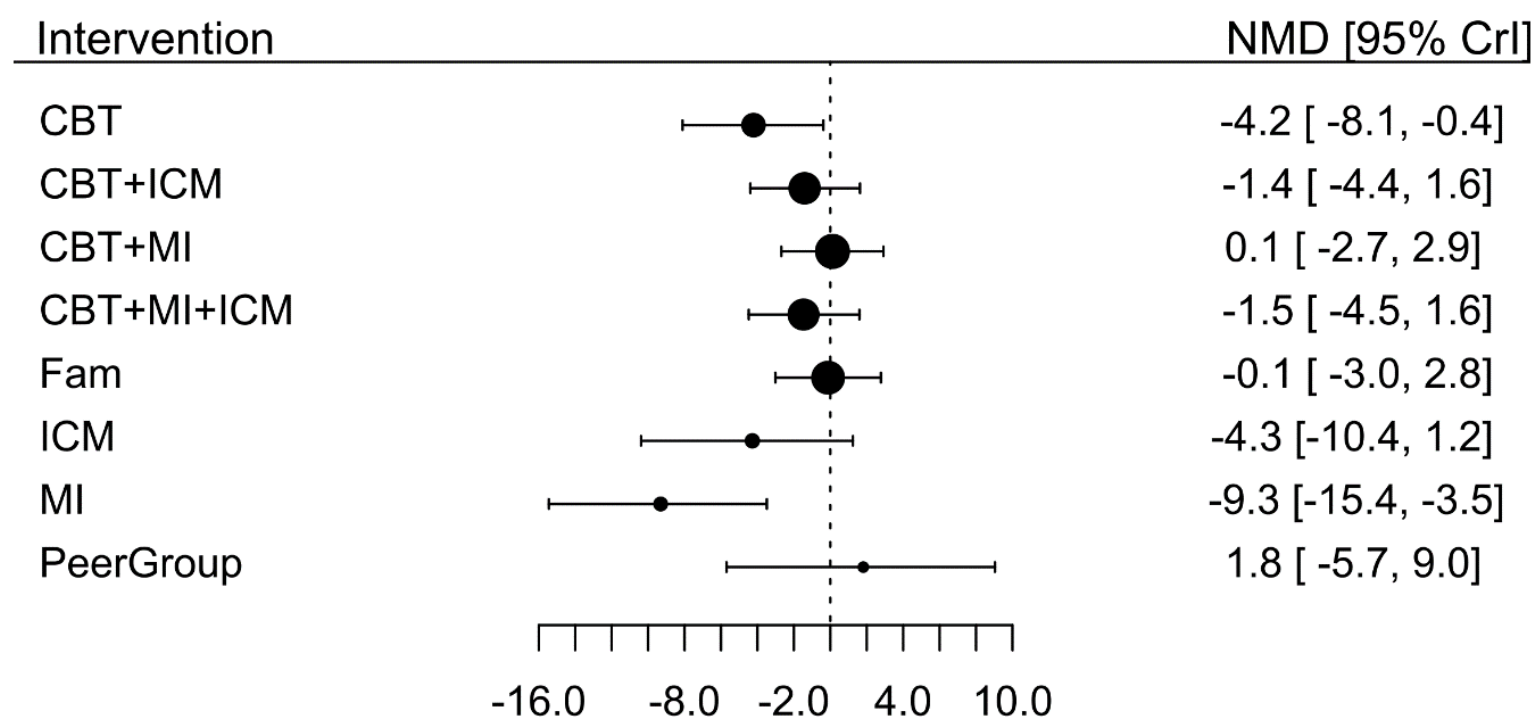

Net Mean Difference (days/mo.) vs.TAU

NMD $<1$ favors intervention versus TAU. Abbreviations: MI = motivational interviewing; Fam = family focused therapy; $\mathrm{CBT}=$ cognitive behavioral therapy; $\mathrm{CM}$ = contingency management; PeerGroup = peer group therapy; $\mathrm{ICM}=$ intensive case management; TAU = treatment as usual; $\mathrm{CrI}=$ credible interval

\section{Key Question 2: Aggregate Alcohol and Other Drug Use - Comparative Effects of Behavioral Interventions}

Table 16 details the comparative effects the studied interventions in studies reporting aggregate alcohol and other drug use outcomes. When the interventions are ranked, MI has the highest probability (0.98) of ranking in the top third (Table 17). Most of the results in the Table are based on indirect data, with direct data limited to only one or two studies. The statistical power to detect inconsistency between direct and indirect effects is very limited. Thus, estimates of treatment effectiveness are very imprecise.

MI was more effect than CBT+MI, Fam, and CBT. We rated the associated SoE as low. 
Table 16. Nonbrief behavioral interventions and aggregate alcohol and other drug use: Net mean difference of use days per month between all

interventions

\begin{tabular}{|c|c|c|c|c|c|c|c|c|c|}
\hline Intervention(s) & CBT & $\mathrm{CBT}+\mathrm{ICM}$ & CBT+MI & $\mathrm{CBT}+\mathrm{MI}+\mathrm{ICM}$ & Fam & ICM & MI & PeerGroup & TAU \\
\hline CBT & CBT & $\begin{array}{l}2.8 \\
(-2.1,7.9)\end{array}$ & $\begin{array}{l}4.3 \\
(-0.4,9.2)\end{array}$ & $\begin{array}{l}2.7 \\
(-2.2,7.7)\end{array}$ & $\begin{array}{l}4.1 \\
(-0.8,8.9)\end{array}$ & $\begin{array}{l}-0.2 \\
(-4.7,4.1)\end{array}$ & $\begin{array}{l}-5.2 \\
(-9.7,-0.8)\end{array}$ & $\begin{array}{l}6 \\
(-0.1,12.2)\end{array}$ & $\begin{array}{l}4.2 \\
(0.4,8.1)\end{array}$ \\
\hline CBT+ICM & $\begin{array}{l}-2.8 \\
(-7.9,2.1)\end{array}$ & $\mathrm{CBT}+\mathrm{ICM}$ & $\begin{array}{l}1.5 \\
(-1.3,4.3)\end{array}$ & $\begin{array}{l}-0.1 \\
(-3,2.7)\end{array}$ & $\begin{array}{l}1.2 \\
(-2.8,5.2)\end{array}$ & $\begin{array}{l}-3 \\
(-9.7,3.4)\end{array}$ & $\begin{array}{l}-8 \\
(-14.9,-1.3)\end{array}$ & $\begin{array}{l}3.2 \\
(-4.9,10.9)\end{array}$ & $\begin{array}{l}1.4 \\
(-1.6,4.4)\end{array}$ \\
\hline $\mathrm{CBT}+\mathrm{MI}$ & $\begin{array}{l}-4.3 \\
(-9.2,0.4)\end{array}$ & $\begin{array}{l}-1.5 \\
(-4.3,1.3)\end{array}$ & $\mathrm{CBT}+\mathrm{MI}$ & $\begin{array}{l}-1.6 \\
(-4.4,1.1)\end{array}$ & $\begin{array}{l}-0.3 \\
(-3.9,3.4)\end{array}$ & $\begin{array}{l}-4.4 \\
(-10.9,1.8)\end{array}$ & $\begin{array}{l}-9.5 \\
(-16.1,-3)\end{array}$ & $\begin{array}{l}1.7 \\
(-6.4,9.4)\end{array}$ & $\begin{array}{l}-0.1 \\
(-2.9,2.7)\end{array}$ \\
\hline $\mathrm{CBT}+\mathrm{MI}+\mathrm{ICM}$ & $\begin{array}{l}-2.7 \\
(-7.7,2.2)\end{array}$ & $\begin{array}{l}0.1 \\
(-2.7,3)\end{array}$ & $\begin{array}{l}1.6 \\
(-1.1,4.4)\end{array}$ & $\mathrm{CBT}+\mathrm{MI}+\mathrm{ICM}$ & $\begin{array}{l}1.3 \\
(-2.7,5.3)\end{array}$ & $\begin{array}{l}-2.9 \\
(-9.5,3.4)\end{array}$ & $\begin{array}{l}-7.8 \\
(-14.8,-1.3)\end{array}$ & $\begin{array}{l}3.3 \\
(-4.8,11.2)\end{array}$ & $\begin{array}{l}1.5 \\
(-1.6,4.5)\end{array}$ \\
\hline Fam & $\begin{array}{l}-4.1 \\
(-8.9,0.8)\end{array}$ & $\begin{array}{l}-1.2 \\
(-5.2,2.8)\end{array}$ & $\begin{array}{l}0.3 \\
(-3.4,3.9)\end{array}$ & $\begin{array}{l}-1.3 \\
(-5.3,2.7)\end{array}$ & Fam & $\begin{array}{l}-4.2 \\
(-10.8,2.1)\end{array}$ & $\begin{array}{l}-9.3 \\
(-15.8,-2.7)\end{array}$ & $\begin{array}{l}1.9 \\
(-6.1,9.8)\end{array}$ & $\begin{array}{l}0.1 \\
(-2.8,3)\end{array}$ \\
\hline ICM & $\begin{array}{l}0.2 \\
(-4.1,4.7)\end{array}$ & $\begin{array}{l}3 \\
(-3.4,9.7)\end{array}$ & $\begin{array}{l}4.4 \\
(-1.8,10.9)\end{array}$ & $\begin{array}{l}2.9 \\
(-3.4,9.5)\end{array}$ & $\begin{array}{l}4.2 \\
(-2.1,10.8)\end{array}$ & ICM & $\begin{array}{l}-5 \\
(-9.3,-0.7)\end{array}$ & $\begin{array}{l}6.1 \\
(-1.1,13.9)\end{array}$ & $\begin{array}{l}4.3 \\
(-1.2,10.4)\end{array}$ \\
\hline MI & $\begin{array}{l}5.2 \\
(0.8,9.7)\end{array}$ & $\begin{array}{l}8 \\
(1.3,14.9)\end{array}$ & $\begin{array}{l}9.5 \\
(3,16.1)\end{array}$ & $\begin{array}{l}7.8 \\
(1.3,14.8)\end{array}$ & $\begin{array}{l}9.3 \\
(2.7,15.8)\end{array}$ & $\begin{array}{l}5 \\
(0.7,9.3)\end{array}$ & MI & $\begin{array}{l}11.3 \\
(3.6,18.7)\end{array}$ & $\begin{array}{l}9.3 \\
(3.5,15.4)\end{array}$ \\
\hline PeerGroup & $\begin{array}{l}-6 \\
(-12.2,0.1)\end{array}$ & $\begin{array}{l}-3.2 \\
(-10.9,4.9)\end{array}$ & $\begin{array}{l}-1.7 \\
(-9.4,6.4)\end{array}$ & $\begin{array}{l}-3.3 \\
(-11.2,4.8)\end{array}$ & $\begin{array}{l}-1.9 \\
(-9.8,6.1)\end{array}$ & $\begin{array}{l}-6.1 \\
(-13.9,1.1)\end{array}$ & $\begin{array}{l}-11.3 \\
(-18.7,-3.6)\end{array}$ & PeerGroup & $\begin{array}{l}-1.8 \\
(-9,5.7)\end{array}$ \\
\hline TAU & $\begin{array}{l}-4.2 \\
(-8.1,-0.4)\end{array}$ & $\begin{array}{l}-1.4 \\
(-4.4,1.6)\end{array}$ & $\begin{array}{l}0.1 \\
(-2.7,2.9)\end{array}$ & $\begin{array}{l}-1.5 \\
(-4.5,1.6)\end{array}$ & $\begin{array}{l}-0.1 \\
(-3,2.8)\end{array}$ & $\begin{array}{l}-4.3 \\
(-10.4,1.2)\end{array}$ & $\begin{array}{l}-9.3 \\
(-15.4,-3.5)\end{array}$ & $\begin{array}{l}1.8 \\
(-5.7,9)\end{array}$ & TAU \\
\hline
\end{tabular}

Bold font indicates the 95\% CrI for the comparative effect excludes the null effect.

Abbreviations: $\mathrm{CBT}=$ cognitive behavioral therapy; $\mathrm{CM}=$ contingency management; Educ = psychoeducation; Fam = family therapy; ICM = intensive case management; $\mathrm{MI}=$ motivational interviewing; TAU = treatment as usual 
Table 17. Probabilities of nonbrief behavioral interventions ranking in top third, middle third and bottom third to reduce aggregate alcohol and other drug use days

\begin{tabular}{lcccr}
\hline Intervention(s) & SUCRA & Top Third & Middle Third & $\begin{array}{r}\text { Bottom } \\
\text { Third }\end{array}$ \\
\hline MI & $99 \%$ & 100 & 0 & 0 \\
\hline CBT & $79 \%$ & 83 & 15 & 2 \\
\hline ICM & $76 \%$ & 75 & 18 & 7 \\
\hline CBT+MI+ICM & $58 \%$ & 17 & 68 & 14 \\
\hline CBT+ICM & $56 \%$ & 15 & 67 & 18 \\
\hline Fam & $37 \%$ & 4 & 37 & 58 \\
\hline TAU & $36 \%$ & 0 & 38 & 61 \\
\hline CBT+MI & $34 \%$ & 2 & 34 & 65 \\
\hline PeerGroup & $26 \%$ & 4 & 22 & 74
\end{tabular}

Abbreviations: $\mathrm{CBT}$ = cognitive behavioral therapy; Fam = family therapy; ICM = intensive case management; $\mathrm{MI}=$ motivational interviewing; SUCRA = surface area under the cumulative ranking curve; TAU = treatment as usual

\section{Illicit Drug Use Outcomes}

Five studies with 1,310 participants ${ }^{113,174-181,190,206,207,210}$ reported illicit drug use days, and two studies with 281 participants ${ }^{153,205}$ reported a scale reflecting illicit drug use. These compare interventions as shown in Figure 25.

Figure 25. Evidence graph for studies reporting illicit drug use days

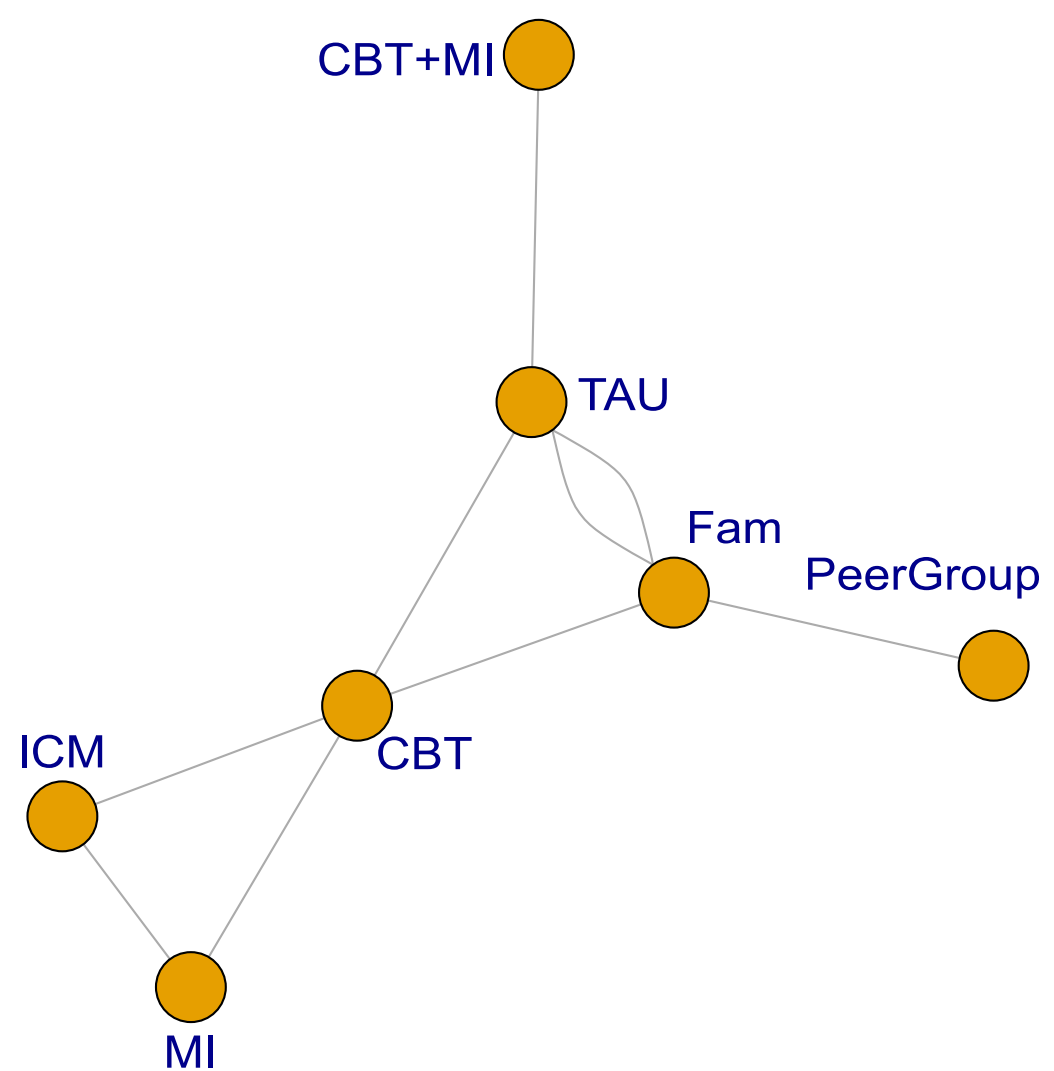

Abbreviations: Fam = family; $\mathrm{CM}=$ contingency management; $\mathrm{TAU}=$ treatment as usual; $\mathrm{CBT}=$ cognitive behavioral therapy; ICM = intensive case management; $\mathrm{MI}=$ motivational interviewing; Educ = education. 


\section{Key Question 1: Illicit Drug Use Days - Interventions Compared to TAU}

$\mathrm{CBT}+\mathrm{MI}$ resulted in a net decrease in overall illicit drug use days, compared to TAU. We rated the associated SoE as low.

The effects for CBT, ICM and MI were imprecisely estimated, as illustrated in Figure 26. This network is also very sparse and loosely connected, and because most RCTs are small, the statistical power to detect inconsistency between direct and indirect effects is very limited. Estimates of treatment effectiveness are very imprecise.

Figure 26. Illicit drug use days: Summary forest plot of interventions reporting meta-analyzed illicit drug use days compared to TAU

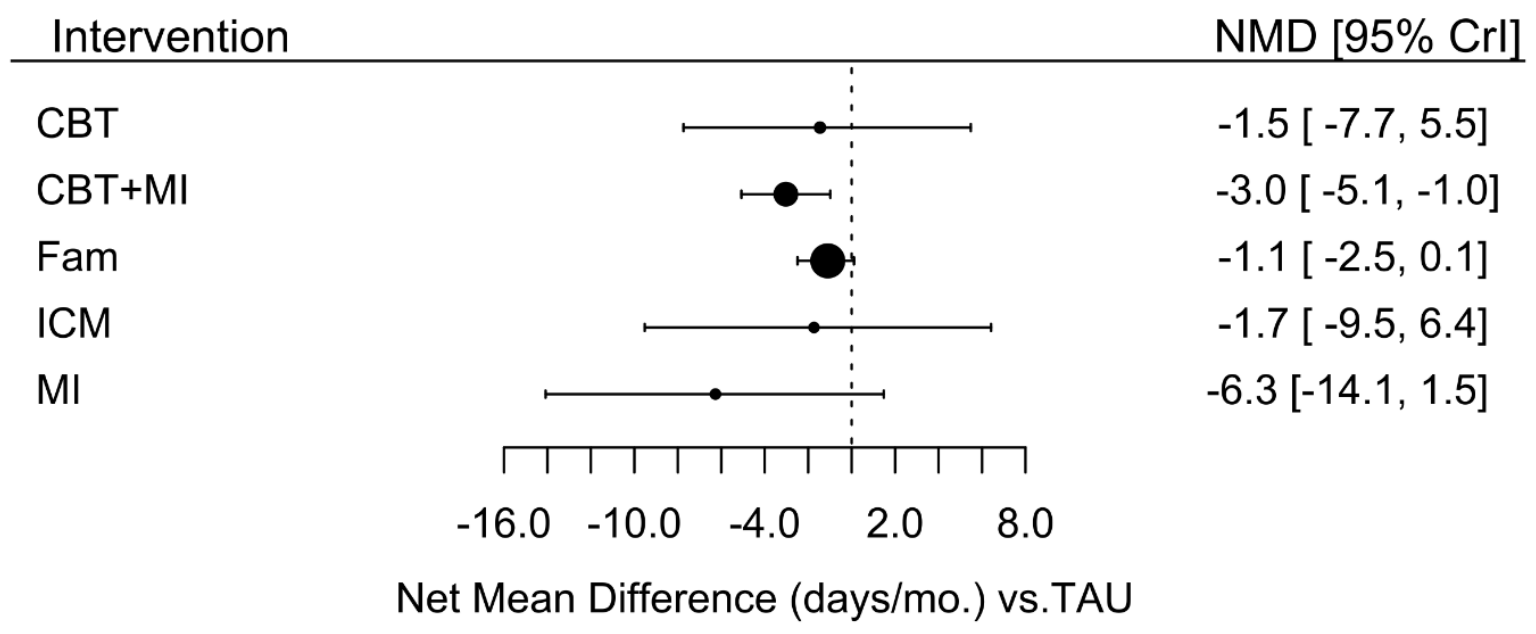

Abbreviations: Fam = family; $\mathrm{CM}=$ contingency management; $\mathrm{TAU}=$ treatment as usual; $\mathrm{CBT}=$ cognitive behavioral therapy; ICM = intensive case management; $\mathrm{MI}=$ motivational interviewing; Educ = education.

\section{Key Question 2: Illicit Drug Use Days - Comparative Effects}

None of the active interventions or combined interventions were different from each other (Table 18). We rated the associated SoE as insufficient.

Most of the results in the Table are based on indirect data, with direct data limited to only one or two studies. The statistical power to detect inconsistency between direct and indirect effects is very limited. Thus, estimates of treatment effectiveness are very imprecise.

As shown in Table 19, MI was most likely to rank in the top third. TAU had a 75 percent probability of ranking in the bottom third. 
Table 18. Nonbrief behavioral interventions and illicit drug use: Net mean difference of use days per month between all interventions

\begin{tabular}{lrrllll}
\hline Intervention(s) & CBT & CBT+MI & Fam & ICM & MI & TAU \\
\hline CBT & CBT & -1.6 & 0.3 & -0.2 & -4.9 & 1.5 \\
& & $(-8.8,5.1)$ & $(-6.5,6.5)$ & $(-4.8,4)$ & $(-9.5,-0.4)$ & $(-5.5,7.7)$ \\
\hline CBT+MI & 1.6 & CBT+MI & 1.9 & 1.3 & -3.2 & 3 \\
& $(-5.1,8.8)$ & & $(-0.5,4.2)$ & $(-6.7,9.6)$ & $(-11.3,4.7)$ & $(1,5.1)$ \\
\hline Fam & -0.3 & -1.9 & Fam & -0.6 & -5.1 & 1.1 \\
& $(-6.5,6.5)$ & $(-4.2,0.5)$ & & $(-8.3,7.4)$ & $(-12.8,2.5)$ & $(-0.1,2.5)$ \\
\hline ICM & 0.2 & -1.3 & 0.6 & ICM & -4.6 & 1.7 \\
& $(-4,4.8)$ & $(-9.6,6.7)$ & $(-7.4,8.3)$ & & $(-9,-0.2)$ & $(-6.4,9.5)$ \\
\hline MI & 4.9 & 3.2 & 5.1 & 4.6 & MI & 6.3 \\
& $(0.4,9.5)$ & $(-4.7,11.3)$ & $(-2.5,12.8)$ & $(0.2,9)$ & & $(-1.5,14.1)$ \\
\hline TAU & -1.5 & -3 & -1.1 & -1.7 & -6.3 & TAU \\
& $(-7.7,5.5)$ & $(-5.1,-1)$ & $(-2.5,0.1)$ & $(-9.5,6.4)$ & $(-14.1,1.5)$ & \\
\hline
\end{tabular}

Bold font indicates statistical significance.

Abbreviations: $\mathrm{CBT}$ = cognitive behavioral therapy; Fam = family therapy; ICM = intensive case management; $\mathrm{MI}=$ motivational interviewing; TAU = treatment as usual

Table 19. Probabilities of nonbrief behavioral interventions ranking in top third, middle third and bottom third to reduce illicit drug use days

\begin{tabular}{lrrrr}
\hline Intervention(s) & SUCRA & Top Third & Middle Third & $\begin{array}{r}\text { Bottom } \\
\text { Third }\end{array}$ \\
\hline MI & $93 \%$ & 90 & 9 & 1 \\
\hline CBT+MI & $74 \%$ & 55 & 42 & 3 \\
\hline ICM & $53 \%$ & 30 & 31 & 39 \\
\hline Fam & $50 \%$ & 10 & 46 & 44 \\
\hline CBT & $50 \%$ & 14 & 47 & 39 \\
\hline TAU & $29 \%$ & 0 & 25 & 75
\end{tabular}

Bold font indicates the highest probability of ranking in top third and bottom third respectively.

Abbreviations: $\mathrm{CBT}$ = cognitive behavioral therapy; Fam = family therapy; $\mathrm{ICM}$ = intensive case management; $\mathrm{MI}=$ motivational interviewing; SUCRA = surface area under the cumulative ranking curve; TAU = treatment as usual

\section{Other Outcomes}

Other outcomes were sparsely reported. The outcomes, number of studies reporting the outcome and number of participants are summarized in Table 20 below. 
Table 20. Other outcome participant summaries

\begin{tabular}{|c|c|c|}
\hline Outcome & No. Studies & No. Participants \\
\hline School Performance and Educational Attainment & $5^{113,141,143,154,187}$ & 353 \\
\hline & $5_{174,185^{141,143,154,}}$ & \\
\hline Family Related & & 709 \\
\hline Peer Related & $3^{141,143,154}$ & 200 \\
\hline Mental Health Events & $2^{121,146}$ & 184 \\
\hline Physical Health Events & $2^{121,184}$ & 194 \\
\hline & $\begin{array}{l}10113,119,121,130 \\
135,139,141,143,187\end{array}$ & \\
\hline Legal Outcomes ${ }^{*}$ & 208 & 1620 \\
\hline
\end{tabular}

These studies evaluated multiple interventions using a variety of outcome metrics. In general, estimated effects were imprecise with no clear trends across studies. Study-specific details are tabulated in Appendix G (Tables G-4 to G-8).

\section{Systematic Reviews of Treatments for Alcohol Use in the College Setting}

\section{Key Points}

- 2 SRs of the general population of college students who drink alcohol found that, on average, compared to no intervention, behavioral interventions resulted in reduced alcohol use for up to about 6 months, but these effects waned in the long term. However, behavioral interventions resulted in fewer alcohol-related problems over the medium to long term. One SR found that, by indirect comparison, face-to-face interventions provide larger and more enduring effects than computer-delivered interventions (SOE not assessed).

- 2 SRs focused on college students mandated to attend alcohol programs. On average, alcohol use decreased in the short- to medium-term regardless of intervention, but mostly did not persist. Four specific commercially available interventions were found to be more effective in the short term than others (SOE not assessed).

- 2 SRs focused on college students who engaged in heavy or hazardous alcohol use. Brief, single-session interventions and the commercially-available BASICS program were found to reduce alcohol use compared with no intervention. Among the brief behavioral interventions, MET/MI had the strongest effect (SOE not assessed).

To evaluate treatment of alcohol use disorders/problematic alcohol use in the college setting, we summarized existing SRs only, since this literature is large, highly contextual, and has been extensively reviewed. The search for SRs addressing interventions for treating problematic alcohol use or alcohol use disorder in the college setting identified six SRs published between 2005 and 2015. These SRs included between 16 and 73 studies each (median 40).

The SRs mostly meta-analyzed standardized effect sizes across multiple outcomes and interventions. This approach requires the strong (and unlikely) assumption that effects are homogeneous across disparate outcomes and interventions. In general these SRs did not adequately perform and report risk of bias assessments and did not discuss the consistency of results. Given these limitations, we have not make SoE assessments. 


\section{General Population of College Students Who Drink Alcohol}

Two SRs did not require any population-specific eligibility criteria for studies other than that the study participants be college students who consume alcohol. ${ }^{211,212}$

Carey 2007 reported a SR of 62 randomized controlled trials (RCTs), with 13,750 participants that compared 98 separate individual-level behavioral interventions (usually multicomponent) with control interventions. ${ }^{211}$ Studies included different subpopulations, such as heavy drinkers, moderate drinkers, and alcohol offenders. Effect sizes (standardized mean differences) were reported in the immediate term ( $\leq 3$ weeks), short term (4 to 13 weeks), intermediate term (14 to 26 weeks), and long term (27 to 195 weeks) for various alcohol use measures and alcohol-related problem measures. The meta-analyses were deemed inadequate due to use of effect sizes comprised of disparate outcomes, inclusion of multiple effect sizes from individual studies, and exclusion of outlier results.

The effect sizes for the alcohol use measures (e.g., alcohol use quantity, frequency of heavy drinking) generally favored the intervention groups until the intermediate term, ranging from 0.11 to 0.41 standard deviation (SD) units. However, these benefits did not persist in the long term; almost all effect sizes diminished over time. In the long term, the only persistent effect of the interventions on alcohol use was on frequency of drinking days (effect size $=0.16$ SD units, 95\% CI 0.03 to 0.30 ).

A different pattern emerged for the alcohol-related problem measures (e.g., drinking and driving, property damage, fights). Although students in the intervention groups had fewer alcohol-related problems than those in the control groups, the beneficial effect of the interventions on alcohol-related problem measures took longer to emerge (no immediate effect, but short-term effect size $=0.15$ SD units, 95\% CI 0.08 to 0.21 ), peaked in the intermediate term (effect size $=0.22$ SD units, $95 \%$ CI 0.12 to 0.32 ), and persisted in the long term (effect size $=0.14$ SD units, $95 \%$ CI 0.06 to 0.22 ). ${ }^{211}$

In a subsequent SR, Carey 2012 primarily examined RCTs (47 RCTs, 1 nonrandomized comparative study [NRCS]), with 5,237 participants, that focused on the mode of delivery of behavioral interventions. ${ }^{212}$ The SR compared face-to-face interventions and computerdelivered behavioral interventions with TAU or no intervention, and with each other. Carey 2012 examined similar outcomes as Carey 2007 that were analyzed using the same effect size measurements at the same follow-up time-periods. The meta-analyses, thus, had similar issues as Carey 2007, except that outlier results were not omitted.

Compared with TAU, students receiving face-to-face interventions drank less per week or month (effect sizes ranged from 0.15 to 0.19 SD units) and per drinking day (effect sizes ranged from 0.17 to 0.23 SD units), drank less frequently (effect sizes ranged from 0.07 to 0.16 SD units), and reported fewer alcohol-related problems (effect sizes ranged from 0.09 to 0.15 SD units) in the short and intermediate term. However, the only persistent effect of faceto-face interventions in the long term was on alcohol use per drinking day (effect size $=0.16$, $95 \%$ CI 0.03 to 0.30 SD units).

Comparing different active interventions, students in the computer-delivered interventions groups had similar benefits as face-to-face interventions in the short term (4 to 13 weeks), but not in the intermediate or long term. Direct comparisons between face-to-face interventions and computer-delivered interventions were infrequent. In the short term, the two modes of delivery had similar effects on alcohol use and alcohol-related problems. However, face-to-face interventions were more effective in reducing peak blood alcohol concentration (BAC) in the intermediate term (weighted sum of squares of group mean effect size $\left.\left[\mathrm{Q}_{\mathrm{b}}\right]=6.74, \mathrm{p}=0.009\right)$ and frequency of heavy drinking in the long term $\left(\mathrm{Q}_{\mathrm{b}}=6.65\right.$, $\mathrm{p}=0.010$ ). Overall, Carey 2012 concluded that face-to-face interventions provide the strongest and most enduring effects in this population. ${ }^{212}$ 


\section{College Students Mandated To Receive Interventions for Alcohol Use}

Two SRs focused on studies of college students who were mandated to attend a program to reduce their alcohol consumption. ${ }^{213,214}$

Carey 2016 included 31 studies (21 RCTs and 10 NRCSs), with 8,621 participants, that compared various group- or individual-level behavioral treatments. A single effect size was selected from each study for inclusion in meta-analysis, but the effect sizes were comprised of disparate outcomes.

In the short term (i.e., $\leq 13$ weeks), all alcohol use and alcohol-related problem measures improved (compared with baseline) in students, regardless of interventions (within-group effect sizes ranged from 0.14 to $0.27 \mathrm{SD}$ units). In the medium term, improvements were observed in frequency of heavy drinking (effect size=0.14 SD units, 95\% CI 0.04 to 0.23 ), peak BAC (effect size $=0.25$ SD units, 95\% CI 0.14 to 0.36), typical BAC (effect size $=0.17$ SD units, 95\% CI 0.04 to 0.29), and alcohol-related problems (effect size=0.13 SD units, $95 \%$ CI 0.06 to 0.21 ). However, the only within-group effect to persist in the long term was on typical BAC (effect size $=0.12$ SD units, 95\% CI 0.01 to 0.25 ).

Four commercially available intervention protocols (Brief Alcohol Screening and Intervention for College Students [BASICS], Electronic Check-Up To Go [e-CHUG], Alcohol 101, and Alcohol Skills Training Program) were shown to be most effective. Carey 2016 reported between-group comparisons, based on short term followup, when one of these 4 interventions was mandated. In the control group, participation in a mandated intervention was associated with lower number of drinks per week (between-group effect size $=0.13 \mathrm{SD}$ units, 95\% CI 0.02 to 0.25 ), peak BAC (effect size $=0.20$ SD units, 95\% CI 0.06 to 0.33 ), and typical BAC (effect size $=0.16$ SD units, 95\% CI 0.01 to 0.31). Alcohol-related problems were similar between the two groups. ${ }^{214}$

Barnett 2005 included 16 studies, but only three of these, all RCTs with a total of 213 participants, were comparative in design. ${ }^{213}$ Due to small numbers of similar studies, metaanalysis was not performed. The three trials randomized mandated college students to three pairs of brief behavioral interventions (MI vs. alcohol education, lifestyle management compared to no intervention, and videotaped expectancy challenge vs. alcohol education). Outcomes were similar and not statistically significant for the first two comparisons/studies, except that MI was more effective than alcohol education in improving alcohol-related problems (between-group effect size $=0.39$ SD units, $\mathrm{p}<0.05$ ). For the third comparison/study, alcohol education was more effective than the alcohol expectancy challenge intervention in improving alcohol knowledge (effect size $=-1.47$ SD units, $\mathrm{p}<0.05$ ). ${ }^{213}$

\section{College Students Who Engaged in Heavy or Hazardous Levels of Alcohol Use}

Two SRs examined studies of college students who engaged in heavy or hazardous levels of alcohol use. ${ }^{215,216}$ Both SRs focused on the comparison between brief behavioral interventions (one or two sessions) with no intervention or TAU.

Samson 2015 included 73 studies ("experimental” or “controlled quasi-experimental” studies) that evaluated a single-session intervention (CBT, MET/MI, personalized feedback, or psychoeducation therapy [PET]). ${ }^{216}$ The meta-analysis combined 662 disparate effect sizes from 73 individual studies. The total number of participants was not reported.

Single-session interventions were found to have a modest effect on reducing alcohol consumption among heavy-drinking college students (effect size=0.18 SD units, 95\% CI 0.12 to 0.24 ). Among the various types of single-session interventions, MET/MI was found to 
have the strongest effect. This suggests that single-session interventions that incorporate aspects of MET/MI are likely the most effective among the single-session interventions in this population. ${ }^{216}$

Fachini 2012 included 18 RCTs, with a total of 6,233 participants, that compared BASICS with TAU or no intervention in students engaged in heavy episodic drinking. ${ }^{215}$ The meta-analyses focused on specific outcomes, and were deemed to be adequate. Overall, BASICS lowered both alcohol consumption and negative consequences in college students. Compared with the control group, students who received BASICS had fewer drinks per week (mean difference $=-1.50,95 \%$ CI -3.24 to -0.29 ) and fewer alcohol-related problems measured using the Rutgers Alcohol Program Index (RAPI) (mean difference in score = $-0.87,95 \%$ CI -1.58 to -0.20$)$.

\section{Risk of Bias}

Based on a modified AMSTAR 2 assessment (Table 21), the SRs mostly adhered to standard design and reporting elements (except for failure to report methods regarding screening citations and articles). Four of the five SRs that conducted meta-analyses were deemed to have used inappropriate methods, only one SR adequately evaluated the summary results based on risk of bias assessments, and only one study reported conflict of interest information (they reported none). The primary concern about the meta-analyses, was that SRs mostly combined standardized effect sizes comprised of disparate sets of outcomes and often included multiple effect sizes from individual studies. 
Table 21. Risk of bias in college alcohol intervention systematic reviews

\begin{tabular}{|c|c|c|c|c|c|c|c|c|c|c|}
\hline $\begin{array}{l}\text { Author } \\
\text { Year } \\
\text { PMID }\end{array}$ & PICODa $^{a}$ & Lit Search ${ }^{b}$ & Dupl Screenc & Dupl Extrnd & Study Details & $\mathrm{RoB}^{f}$ & $M A^{g}$ & RoB Analysis ${ }^{h}$ & Heterogeneityi & $\mathrm{COI}$ \\
\hline $\begin{array}{l}\text { Barnett } \\
2005 \\
16135343\end{array}$ & Sufficient & Yes & NR & NR & Sufficientk & No & $\mathrm{N} / \mathrm{A}$ & No & No & No \\
\hline $\begin{array}{l}\text { Carey } \\
2007 \\
17590277\end{array}$ & Yes & Yes & NR & Yes & Yes & No & Nol & No & Yes & No \\
\hline $\begin{array}{l}\text { Fachini } \\
2012 \\
22967716\end{array}$ & Yes & Yes & Yes & Yes & No & Yes & Yes & No & $\mathrm{No}^{\mathrm{m}}$ & Yes \\
\hline $\begin{array}{l}\text { Carey } \\
2012 \\
23022767\end{array}$ & Yes & Yes & NR & Yes & Yes & Sufficient $^{\mathrm{n}}$ & $\mathrm{No}^{\circ}$ & Yes & Yes & No \\
\hline $\begin{array}{l}\text { Samson } \\
2015 \\
26098028\end{array}$ & Yes & Yes & Yes & Yes & No & Sufficientp & Noq & No & Yes & No \\
\hline $\begin{array}{l}\text { Carey } \\
2016 \\
27100126\end{array}$ & Yes & Yes & NR & Yes & Yes & Sufficientr & $\mathrm{No}^{\mathrm{s}}$ & $\mathrm{No}^{\mathrm{t}}$ & Yes & No \\
\hline
\end{tabular}

Ratings based on AMSTAR 2. Ratings: Yes $=$ item explicitly reported (or done), No = item not reported (or done), Sufficient $=$ reporting of item was adequate but not fully explicit, NR = not reported, N/A = not applicable. Ratings are color coded for emphasis only. Other abbreviations are defined in the footnotes.

a Did the research questions and inclusion criteria for the review include the components of PICOD (population, intervention, comparator, outcomes, study design)? (AMSTAR 2 item 1)

b Did the review authors use a comprehensive literature (Lit) search strategy? (AMSTAR 2 item 4)

c Did the review authors perform study selection (Screen) in duplicate (Dupl)? (AMSTAR 2 item 5)

$\mathrm{d}$ Did the review authors perform data extraction (Extrn) in duplicate (Dupl)? (AMSTAR 2 item 6)

e Did the review authors describe the included studies in adequate detail? (AMSTAR 2 item 8 )

$f$ Did the review authors use a satisfactory technique for assessing the risk of bias (RoB) in individual studies that were included in the review? (AMSTAR 2 item 9 )

$\mathrm{g}$ If meta-analysis (MA) was performed did the review authors use appropriate methods for statistical combination of results? (AMSTAR 2 item 11 ) See subsequent footnotes. Meta-analyses of standardized effect sizes pertaining to disparate outcomes were not deemed to be appropriate for statistical combination.

$\mathrm{h}$ Did the review authors assess the potential impact of risk of bias (RoB) in individual studies on the summary results, interpretation, discussion? (AMSTAR 2 item 13)

$\mathrm{i}$ Did the review authors provide a satisfactory explanation for, and discussion of, any heterogeneity observed in the results of the review? (AMSTAR 2 item 14)

j Did the review authors report any potential sources of conflict of interest (COI) regarding conducting the review? (AMSTAR 2 item 16)

$\mathrm{k}$ Populations of included studies were not well described

I Multiple effect sizes within studies were averaged prior to meta-analysis. In addition, outliers were excluded from meta-analysis.

$\mathrm{m}$ Moderators of effect were included in the discussion, but only qualitatively. 
n A risk of bias score (range $0-17$ ) was used, but not adequately described.

o Multiple effect sizes within studies were averaged prior to meta-analysis.

p Risk of bias assessment was unclear and possibly incomplete.

q 662 effect sizes from 73 studies were included in a single meta-analysis.

r Unclear, possibly incomplete, risk of bias assessment.

s A single effect size was selected from each study for meta-analysis, but effect sizes pertained to disparate outcomes.

t Meta-analyses were adjusted for risk of bias, but not analyzed based on risk of bias.

\section{Pharmaceutical Interventions}

We found only a small number of studies pharmacologic treatments (with or without combined behavioral interventions) for substance use disorder in adolescents. We found no large nonrandomized studies which evaluated medication side-effects in adolescents.

In studies that combined pharmacologic and behavioral interventions, the behavioral interventions were often less completely described, and therefore not easily compared to the detailed manual based interventions typical in behavioral trials. Drug trials included placebo arms, which due to the likelihood of a placebo effect, were not deemed comparable to TAU arms in studies of behavioral interventions. Thus, we did not jointly synthesize studies of behavioral interventions with studies of pharmacologic interventions and summarize these studies separately by use disorder.

\section{Key Points}

- Opioid use disorder

o Longer courses (2 to 3 months) of buprenorphine are more effective than shorter courses (14 to 28 days) to reduce opioid use and achieve abstinence (low SoE)

- Buprenorphine-naloxone (12-week versus 2-week) is more effective in reducing opioid use at 9 and 12 months (1 study)

- Buprenorphine+CBT+CM was more effective than clonidine $+\mathrm{CBT}+\mathrm{CM}$ in increasing odds of opioid abstinence at 1 month (1 study)

\section{Opioid Use Disorder}

Four comparative studies (in 13 publications ${ }^{217-228}$ ) published between 2005 and 2016 assessed pharmacologic or combination pharmacologic and behavioral interventions to reduce opioid use in a total of 330 adolescents, all of whom had SUD. Participants in the studies were on average 17 to 23 years of age (range across studies 14 to 25 years). Baseline and arm details are given in Table 22. Risk of Bias summaries are given in Figure 27.

Table 22. Baseline data and Interventions: Pharmacologic interventions for opioid use

\begin{tabular}{|c|c|c|c|c|c|c|c|c|}
\hline Author, Year & $\mathrm{N}$ & $\begin{array}{l}\text { Substances } \\
\text { Used }\end{array}$ & Severity & $\begin{array}{l}\text { Ages } \\
\text { [Eligible] } \\
\text { Mean } \\
\text { (SD) }\end{array}$ & $\begin{array}{l}\text { Male } \\
\%\end{array}$ & Setting & $\begin{array}{l}\text { Intervention } \\
\text { Delivery }\end{array}$ & Arm Names \\
\hline Gonzalez, 2015219, 229 & 87 & $\begin{array}{l}\text { opioid } \\
\text { cannabis }\end{array}$ & SUD & $\begin{array}{l}{[18,25]} \\
22.6(1.9)\end{array}$ & 66 & $\begin{array}{l}\text { outpatient } \\
\text { research clinic }\end{array}$ & $\begin{array}{l}\text { research staff } \\
\text { (PhD } \\
\text { psychologist) }\end{array}$ & $\begin{array}{l}\text { 1. Buprenorphine- } \\
\text { Naloxone+Placebo+CBT } \\
\text { (group): "Placebo" } \\
\text { 2. Buprenorphine- } \\
\text { Naloxone+Memantine30+CBT } \\
\text { (group): "Memantine 30" } \\
\text { 3. Buprenorphine- } \\
\text { Naloxone+Memantine15+CBT } \\
\text { (group): "Memantine 15" }\end{array}$ \\
\hline
\end{tabular}




\begin{tabular}{|c|c|c|c|c|c|c|c|c|}
\hline Author, Year & $\mathrm{N}$ & $\begin{array}{l}\text { Substances } \\
\text { Used }\end{array}$ & Severity & $\begin{array}{l}\text { Ages } \\
\text { [Eligible] } \\
\text { Mean } \\
\text { (SD) }\end{array}$ & $\begin{array}{l}\text { Male } \\
\%\end{array}$ & Setting & $\begin{array}{l}\text { Intervention } \\
\text { Delivery }\end{array}$ & Arm Names \\
\hline Marsch, 2005221, 224 & 36 & $\begin{array}{l}\text { opioid } \\
\text { cannabis } \\
\text { alcohol } \\
\text { cocaine } \\
\text { amphetamine }\end{array}$ & SUD & $\begin{array}{l}{[13,18]} \\
17.3(0.7)\end{array}$ & 50 & $\begin{array}{l}\text { outpatient } \\
\text { research clinic }\end{array}$ & $\begin{array}{l}\text { no detail (drug } \\
\text { trial) }\end{array}$ & $\begin{array}{l}\text { 1. Clonidine+CBT+CM: } \\
\text { "Clonidine" } \\
\text { 2. Buprenorphine+CBT+CM: } \\
\text { "Buprenorphine" }\end{array}$ \\
\hline Marsch, $2016^{222}$ & 53 & $\begin{array}{l}\text { opioid } \\
\text { alcohol } \\
\text { cocaine } \\
\text { cannabis } \\
\text { amphetamine }\end{array}$ & SUD & $\begin{array}{l}{[16,24]} \\
21(2.5)\end{array}$ & 54 & $\begin{array}{l}\text { outpatient } \\
\text { research clinic }\end{array}$ & $\begin{array}{l}\text { no detail (drug } \\
\text { trial) }\end{array}$ & $\begin{array}{l}\text { 1. Buprenorphine+Placebo+ } \\
\text { CBT+Ml+Educ+ } \\
\text { Fam[systems/structural]+CM: } \\
\text { "Buprenorphine 28-day taper" } \\
\text { 2. Buprenorphine+CBT+Ml+ } \\
\text { Educ+Fam[systems/structural]+ } \\
\text { CM: "Buprenorphine 56-day } \\
\text { taper" }\end{array}$ \\
\hline $\begin{array}{l}\text { Woody, } 2008^{217}, 218,220 \text {, } \\
223,225-228\end{array}$ & 154 & $\begin{array}{l}\text { opioid } \\
\text { cannabis } \\
\text { alcohol } \\
\text { cocaine } \\
\text { injection drugs }\end{array}$ & SUD & $\begin{array}{l}{[14,21]} \\
\mathrm{nr}\end{array}$ & $\mathrm{nr}$ & $\begin{array}{l}\text { outpatient } \\
\text { community }\end{array}$ & $\begin{array}{l}\text { no detail (drug } \\
\text { trial) }\end{array}$ & $\begin{array}{l}\text { 1. Buprenorphine+Naloxone- } \\
\text { short+TAU (group): "Short-term } \\
\text { buprenorphine-naloxone" } \\
\text { 2. Buprenorphine+Naloxone- } \\
\text { extended+TAU (group): } \\
\text { "Extended buprenorphine- } \\
\text { naloxone" }\end{array}$ \\
\hline
\end{tabular}

Abbreviations: $\mathrm{CBT}$ = cognitive behavioral therapy; $\mathrm{CM}$ = contingency management; $\mathrm{ED}$ = emergency department; Educ = psychoeducation; Fam = Family therapy; $\mathrm{MI}=$ motivational interviewing; $\mathrm{N}=$ number randomized; $\mathrm{nr}=$ not reported; $\mathrm{SD}=$ standard deviation; SUD = substance use disorder; TAU = treatment as usual

Arm names = Intervention codes, (intervention modifiers) and [family subclassification]: "study arm name".

Except for Woody 2008, the studies were double blinded. However, studies mostly had high attrition rates and poor compliance, reflecting the challenges of engaging this population.

\section{Figure 27. Pharmacologic intervention studies for opioid use: Percentage of studies in each risk of bias category}

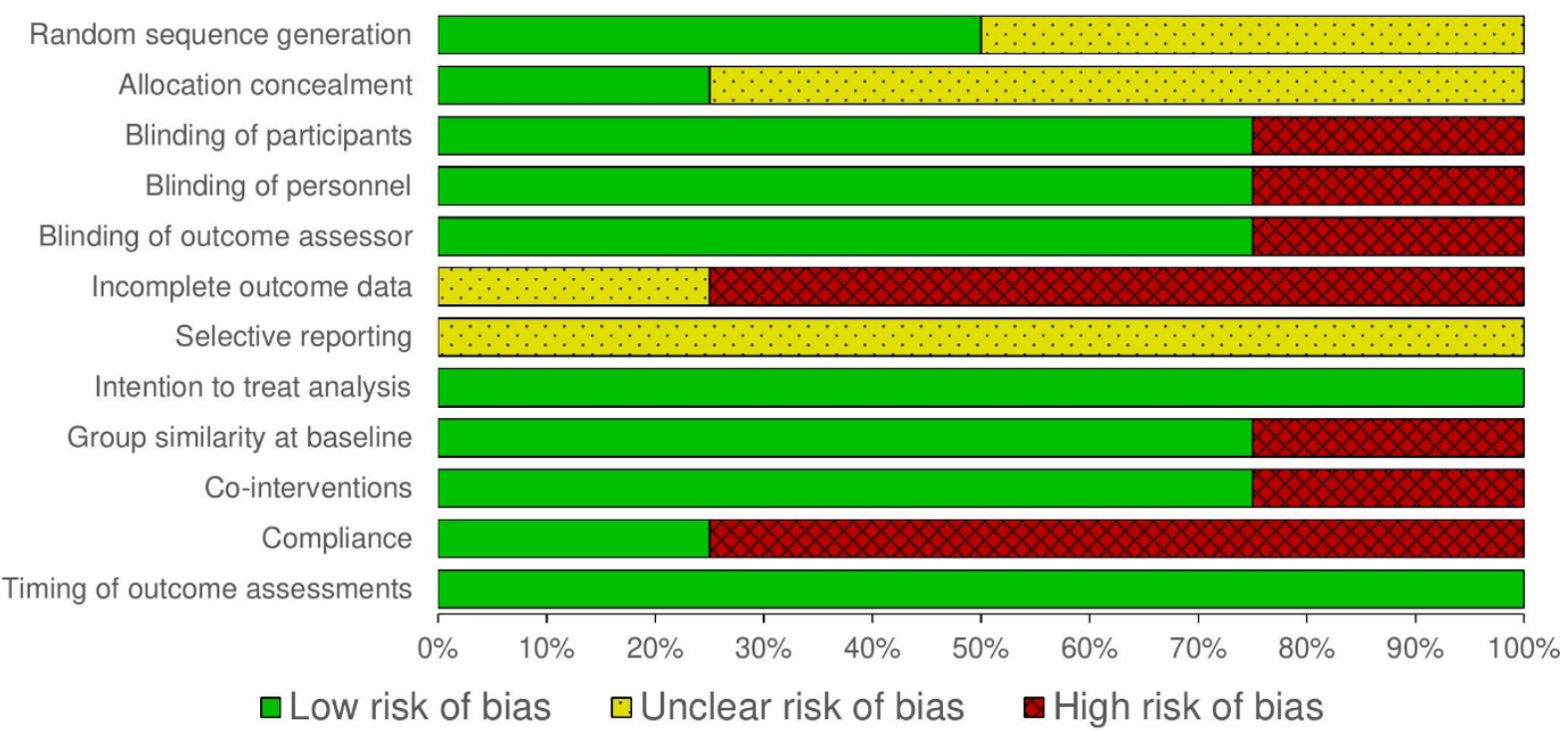

As shown in Table 23, all four studies assessed buprenorphine-naloxone or buprenorphine alone or combined with behavioral interventions. Comparisons were between doses or tapering schedules, with clonidine, or with memantine. 
Woody et al. 2008 reported that adolescents in the extended 12-week buprenorphinenaloxone arm were more likely to report no opioid use in the last month at 9 and 12 months than adolescents in the short term (2 week) buprenorphine-naloxone group, but not at 6 months. Additionally, they report an overall group-by-time interaction odds ratio for any opioid use of 1.34 (95\% CI 0.70 to 2.57), favoring the extended 12-week buprenorphinenaloxone arm. $217,218,220,223,225-228$

Woody et al. 2008 reported no serious adverse events and no loss to follow-up due to adverse events. Adverse events reported were nausea, insomnia, stomach ache, vomiting, and anxiety. ${ }^{217,218,220,223,225-228}$ A secondary analysis found that overall in the sample, those in the buprenorphine arm had a statistically significant decrease in injection drug use compared to the detox arm although there was a decrease in both groups. ${ }^{223,225}$

Marsch et al. 2016 found that among those treated with buprenorphine (in combination with other behavioral interventions), opioid abstinence was higher in the 56-day taper group than in the 28-day taper group (OR 2.59, 95\% CI 0.73 to 9.18). ${ }^{222}$ They reported that there were no serious adverse events related to treatment in either arm.

Marsch et al. 2005 found that buprenorphine (combined with CBT + CM) performed better for abstinence than clonidine (combined with CBT + CM) (OR 4.00, 95\% CI 1.00 to 16.0), although the confidence interval was very wide. HIV risk behavior did not differ between the groups. No information was given on adverse events. ${ }^{221,}{ }^{224} \mathrm{~A}$ secondary analysis focused on emotional and behavioral outcomes found that among youth who were retained in treatment, there were significant reductions in two grouping scales (internalizing problems and total problems) and four syndrome scales (somatic, social, attention, and thought ${ }^{224}$ ). Of note, there were more youth retained in the buprenorphine arm retained $(n=13)$ compared to the clonidine arm ( $\mathrm{n}=7)$.

Gonzales et al. 2015 evaluated buprenorphine-naloxone plus memantine (either $15 \mathrm{mg}$ or $30 \mathrm{mg}$ ) or buprenorphine-naloxone plus placebo in 80 18- to 25-year-old young adults. Each arm also received weekly CBT delivered in a group format. They reported that for abstinence at 3 months, the arm with buprenorphine-naloxone plus $30 \mathrm{mg}$ of memantine performed much better than the $15 \mathrm{mg}$ arm (OR 9.2, 95\% CI 2.62 to 32.28) or the placebo arm (OR 9.2, 95\% CI 2.69 to 31.46), and the $15 \mathrm{mg}$ arm performed slightly worse than placebo (OR 0.78, $95 \%$ CI 0.27 to 2.31). The same general pattern held for opioid use frequency, though the confidence intervals were wide for both abstinence and use outcomes and the $15 \mathrm{mg}$ arm had a lower use frequency than placebo. Reported adverse events included pain, drowsiness, vivid dreams, constipation, upper respiratory infection, nausea, and headaches. Any adverse event was reported in 39 and 30 percent of the memantine $30 \mathrm{mg}$ and $15 \mathrm{mg}$ arms, respectively, as compared to 49 percent of the placebo arms, and there were no serious adverse events. ${ }^{219}$ 


\begin{tabular}{|c|c|c|c|c|c|c|c|c|c|}
\hline $\begin{array}{l}\text { Study Author, } \\
\text { Year, PMID }\end{array}$ & Arm 1 & Arm 2 & Outcome & $\begin{array}{r}\text { Time } \\
\text { (Months) }\end{array}$ & $\begin{array}{r}\text { Arm 1 } \\
\mathrm{N} \text { Analyzed }\end{array}$ & Arm 1 Outcome & $\begin{array}{r}\text { Arm } 2 \mathrm{~N} \\
\text { Analyzed }\end{array}$ & $\begin{array}{r}\text { Arm } 2 \\
\text { Outcome }\end{array}$ & $\begin{array}{r}\text { Calculated Effect } \\
(95 \% \mathrm{Cl})\end{array}$ \\
\hline \multirow[t]{4}{*}{$\begin{array}{l}\text { Woody, } 2008^{217} \text {, } \\
218,220,223,225-228\end{array}$} & $\begin{array}{l}\text { Buprenorphine + } \\
\text { Naloxone + } \\
\text { extended (group) }\end{array}$ & $\begin{array}{l}\text { Buprenorphine + } \\
\text { Naloxone + short } \\
\text { (group) }\end{array}$ & $\begin{array}{l}\text { Abstinence per } 30 \\
\text { days }(n, \%)\end{array}$ & 1 & 47 & $13(28)$ & 46 & $17(37)$ & OR $0.7(0.3,1.6)$ \\
\hline & & & $\begin{array}{l}\text { Abstinence per } 30 \\
\text { days }(n, \%)\end{array}$ & 2 & 45 & $21(47)$ & 45 & $14(30)$ & OR $2.0(0.9,4.9)$ \\
\hline & & & $\begin{array}{l}\text { Abstinence per } 30 \\
\text { days }(n, \%)\end{array}$ & 3 & 49 & $23(47)$ & 42 & $12(28)$ & OR $2.4(1.0,5.8)$ \\
\hline & & & $\begin{array}{l}\text { Serious adverse } \\
\text { events }\end{array}$ & 3 & 78 & 0 & 74 & 0 & No events \\
\hline \multirow[t]{2}{*}{ Marsch, 2016222} & $\begin{array}{l}\text { Buprenorphine + } \\
\text { CBT + Ml + Educ } \\
+ \text { CM ( } 56 \text { day } \\
\text { taper) }\end{array}$ & $\begin{array}{l}\text { Buprenorphine + } \\
\text { Placebo + CBT + } \\
\text { Ml + Educ + CM } \\
\text { (28 day taper) }\end{array}$ & $\begin{array}{l}\text { Mean Opioid } \\
\text { negative urine } \\
\text { screens/28 days } \\
(\text { mean } \%, 95 \% \mathrm{Cl})\end{array}$ & 2 & 25 & $34.6(23.2,50.0)$ & 28 & $17.2(5.8,28.6)$ & $\begin{array}{r}\text { Cohen's d } 0.57 \\
(0.02,1.13)\end{array}$ \\
\hline & & & $\begin{array}{l}\text { Serious adverse } \\
\text { events }\end{array}$ & 2 & 25 & 0 & 28 & 0 & No events \\
\hline $\begin{array}{l}\text { Marsch, } 2005 \text { 221, } \\
224\end{array}$ & $\begin{array}{l}\text { Buprenorphine + } \\
\text { CBT + CM }\end{array}$ & $\begin{array}{l}\text { Clonidine + CBT } \\
+\mathrm{CM}\end{array}$ & Abstinence (n, \%) & 1 & 18 & $12(64)$ & 18 & $6(32)$ & OR $4.0(1.0,16.0)$ \\
\hline \multirow[t]{5}{*}{$\begin{array}{l}\text { Gonzalez, } 2015 \\
219,229\end{array}$} & $\begin{array}{l}\text { Buprenorphine + } \\
\text { naloxone + } \\
\text { memantine30 + } \\
\text { CBT (group) }\end{array}$ & $\begin{array}{l}\text { Buprenorphine + } \\
\text { naloxone + } \\
\text { memantine15 + } \\
\text { CBT(group) }\end{array}$ & Abstinence $(\mathrm{n}, \%)$ & 3 & 28 & $23(82)$ & 27 & $9(32)$ & OR $9.2(2.6,32.3)$ \\
\hline & $\begin{array}{l}\text { Buprenorphine + } \\
\text { naloxone + } \\
\text { memantine30 + } \\
\text { CBT (group) }\end{array}$ & $\begin{array}{l}\text { Buprenorphine + } \\
\text { naloxone + } \\
\text { placebo + CBT } \\
\text { (group) }\end{array}$ & Abstinence (n, \%) & 3 & 28 & $23(82)$ & 32 & $10(30)$ & OR $9.2(2.7,31.5)$ \\
\hline & $\begin{array}{l}\text { Buprenorphine + } \\
\text { naloxone + } \\
\text { memantine15 + } \\
\text { CBT (group) }\end{array}$ & $\begin{array}{l}\text { Buprenorphine + } \\
\text { naloxone + } \\
\text { placebo + CBT } \\
\text { (group) }\end{array}$ & Abstinence $(\mathrm{n}, \%)$ & 3 & 27 & $9(32)$ & 32 & $10(30)$ & OR $0.8(0.3,2.3)$ \\
\hline & $\begin{array}{l}\text { Buprenorphine + } \\
\text { naloxone + } \\
\text { memantine30 + } \\
\text { CBT (group) }\end{array}$ & $\begin{array}{l}\text { Buprenorphine + } \\
\text { naloxone + } \\
\text { memantine15 + } \\
\text { CBT (group) }\end{array}$ & $\begin{array}{l}\text { Opioid use (mean, } \\
\text { SE) }\end{array}$ & 3 & 27 & $0(0)$ & 24 & $0.27(0.10)$ & $\begin{array}{r}\text { Net Mean Diff. } \\
-0.3(-23.9,23.3)\end{array}$ \\
\hline & $\begin{array}{l}\text { Buprenorphine + } \\
\text { naloxone + } \\
\text { memantine30 + } \\
\text { CBT (group) }\end{array}$ & $\begin{array}{l}\text { Buprenorphine + } \\
\text { naloxone + } \\
\text { placebo + CBT } \\
\text { (group) }\end{array}$ & $\begin{array}{l}\text { Opioid use (mean, } \\
\text { SE) }\end{array}$ & 3 & 27 & $0(0)$ & 29 & $0.39(0.14)$ & $\begin{array}{r}\text { Net Mean Diff. } \\
-0.39(-40,39.1)\end{array}$ \\
\hline
\end{tabular}




\begin{tabular}{|c|c|c|c|c|c|c|c|c|c|}
\hline $\begin{array}{l}\text { Study Author, } \\
\text { Year, PMID }\end{array}$ & Arm 1 & Arm 2 & Outcome & $\begin{array}{r}\text { Time } \\
\text { (Months) }\end{array}$ & $\begin{array}{r}\text { Arm } 1 \\
\mathrm{~N} \text { Analyzed }\end{array}$ & Arm 1 Outcome & $\begin{array}{r}\text { Arm } 2 \mathrm{~N} \\
\text { Analyzed }\end{array}$ & $\begin{array}{r}\text { Arm } 2 \\
\text { Outcome }\end{array}$ & $\begin{array}{r}\text { Calculated Effect } \\
(95 \% \mathrm{Cl})\end{array}$ \\
\hline & $\begin{array}{l}\text { Buprenorphine + } \\
\text { naloxone + } \\
\text { memantine15 + } \\
\text { CBT (group) }\end{array}$ & $\begin{array}{l}\text { Buprenorphine + } \\
\text { naloxone + } \\
\text { placebo + CBT } \\
\text { (group) }\end{array}$ & $\begin{array}{l}\text { Opioid use (mean, } \\
\text { SE) }\end{array}$ & 3 & 24 & $0.27(0.10)$ & 29 & $0.39(0.14)$ & $\begin{array}{r}\text { Net Mean Diff. } \\
-0.12(-43.1,42.9)\end{array}$ \\
\hline & $\begin{array}{l}\text { Buprenorphine + } \\
\text { naloxone + } \\
\text { memantine30 + } \\
\text { CBT (group) }\end{array}$ & $\begin{array}{l}\text { Buprenorphine + } \\
\text { naloxone + } \\
\text { memantine15 + } \\
\text { CBT (group) }\end{array}$ & $\begin{array}{l}\text { Any adverse events } \\
(\mathrm{n}, \%)\end{array}$ & 3 & 27 & $11(40.7)$ & 24 & $7(29.3)$ & $\begin{array}{r}\text { OR } 1.7 \\
(0.5,5.4)\end{array}$ \\
\hline & $\begin{array}{l}\text { Buprenorphine + } \\
\text { naloxone + } \\
\text { memantine30 + } \\
\text { CBT (group) }\end{array}$ & $\begin{array}{l}\text { Buprenorphine + } \\
\text { naloxone + } \\
\text { placebo + CBT } \\
\text { (group) }\end{array}$ & $\begin{array}{l}\text { Any adverse events } \\
(\mathrm{n}, \%)\end{array}$ & 3 & 27 & $11(40.1)$ & 29 & $14(48.3)$ & $\begin{array}{r}\text { OR } 0.7 \\
(0.3,2.1)\end{array}$ \\
\hline & $\begin{array}{l}\text { Buprenorphine + } \\
\text { naloxone + } \\
\text { memantine15 + } \\
\text { CBT (group) }\end{array}$ & $\begin{array}{l}\text { Buprenorphine + } \\
\text { naloxone + } \\
\text { placebo + CBT } \\
\text { (group) }\end{array}$ & $\begin{array}{l}\text { Any adverse events } \\
(\mathrm{n}, \%)\end{array}$ & 3 & 24 & $7(29.2)$ & 29 & $14(48.3)$ & $\begin{array}{r}0.4 \\
(0.1,1.4)\end{array}$ \\
\hline & $\begin{array}{l}\text { Buprenorphine + } \\
\text { naloxone + } \\
\text { memantine30 + } \\
\text { CBT (group) }\end{array}$ & $\begin{array}{l}\text { Buprenorphine + } \\
\text { naloxone + } \\
\text { memantine15 + } \\
\text { CBT (group) }\end{array}$ & $\begin{array}{l}\text { Serious adverse } \\
\text { events }(n, \%)\end{array}$ & 3 & 28 & $0(0)$ & 27 & $0(0)$ & No events \\
\hline & $\begin{array}{l}\text { Buprenorphine + } \\
\text { naloxone + } \\
\text { memantine30 + } \\
\text { CBT (group) }\end{array}$ & $\begin{array}{l}\text { Buprenorphine + } \\
\text { naloxone + } \\
\text { placebo + CBT } \\
\text { (group) }\end{array}$ & $\begin{array}{l}\text { Serious adverse } \\
\text { events }(n, \%)\end{array}$ & 3 & 28 & $0(0)$ & 32 & $0(0)$ & No events \\
\hline & $\begin{array}{l}\text { Buprenorphine + } \\
\text { naloxone + } \\
\text { memantine15 + } \\
\text { CBT (group) }\end{array}$ & $\begin{array}{l}\text { Buprenorphine + } \\
\text { naloxone + } \\
\text { placebo + CBT } \\
\text { (group) }\end{array}$ & $\begin{array}{l}\text { Serious adverse } \\
\text { events }(n, \%)\end{array}$ & 3 & 27 & $0(0)$ & 32 & $0(0)$ & No events \\
\hline
\end{tabular}

Bold font indicates statistical significance.

Abbreviations: $\mathrm{CBT}=$ cognitive behavioral therapy; $\mathrm{Cl}=$ confidence interval; $\mathrm{CM}=$ contingency management; Educ = psychoeducation; group = at least one

component of the intervention was delivered in a group format; $\mathrm{MI}=$ motivational interviewing; OR = odds ratio; SE = standard error 


\section{Alcohol Use Disorder}

Seven comparative studies published between 2003 and 2016 assessed pharmaceutical interventions to reduce alcohol ${ }^{230-239}$ use in 543 adolescents, total. Participants in the studies were on average 16 to 21 years of age (range across studies 13 to 21). Baseline and intervention details are given in Table 24.

Five of the seven studies were placebo-controlled evaluations of a single pharmaceutical agent (disulfiram, cyanamide or naltrexone). Two studies compared disulfiram to naltrexone. In addition to a medication, four of the seven studies included a behavioral intervention in both arms (e.g., education, MI, or CM).

Table 24. Baseline data and interventions: Pharmacologic treatments of alcohol use

\begin{tabular}{|c|c|c|c|c|c|c|c|c|}
\hline Author, Year & $\mathrm{N}$ & $\begin{array}{l}\text { Substances } \\
\text { Used }\end{array}$ & Severity & $\begin{array}{l}\text { Ages [Eligible] } \\
\text { Mean (SD) }\end{array}$ & $\begin{array}{l}\text { Male } \\
\% \\
\end{array}$ & Setting & $\begin{array}{l}\text { Intervention } \\
\text { Delivery }\end{array}$ & Arm Names \\
\hline Miranda, $2014^{232}$ & 22 & $\begin{array}{l}\text { alcohol } \\
\text { cannabis }\end{array}$ & PU & $\begin{array}{l}{[15,19]} \\
18(1.2)\end{array}$ & 36 & $\begin{array}{l}\text { outpatient } \\
\text { research clinic }\end{array}$ & $\begin{array}{l}\text { no detail (drug } \\
\text { trial) }\end{array}$ & $\begin{array}{l}\text { 1. Placebo: "Placebo" } \\
\text { 2. Naltrexone: "Naltrexone" }\end{array}$ \\
\hline $\begin{array}{l}\text { Niederhofer, } 2003^{233}, \\
234\end{array}$ & 26 & alcohol & SUD & $\begin{array}{l}{[16,19]} \\
17.1(0.9)\end{array}$ & 38 & hospital & $\begin{array}{l}\text { no detail (drug } \\
\text { trial) }\end{array}$ & $\begin{array}{l}\text { 1. Placebo: "Placebo" } \\
\text { 2. Cyanamide: "Cyanamide" }\end{array}$ \\
\hline Niederhofer, $2003^{235}$ & 49 & alcohol & SUD & $\begin{array}{l}{[16,19]} \\
16.9(0.3)\end{array}$ & 69 & hospital & $\begin{array}{l}\text { no detail (drug } \\
\text { trial) }\end{array}$ & $\begin{array}{l}\text { 1. Placebo: "Placebo" } \\
\text { 2. Disulfiram: "Disulfiram" }\end{array}$ \\
\hline $\begin{array}{l}\text { Niederhofer, } 2003^{233} \text {, } \\
234\end{array}$ & 26 & alcohol & SUD & $\begin{array}{l}{[16,19]} \\
\mathrm{nr}\end{array}$ & $\mathrm{nr}$ & & & $\begin{array}{l}\text { 1. Placebo: "Placebo" } \\
\text { 2. Naltrexone: "Naltrexone" }\end{array}$ \\
\hline O'Malley, 2015²36-239 & 140 & $\begin{array}{l}\text { alcohol } \\
\text { cannabis }\end{array}$ & PU & $\begin{array}{l}{[18,25]} \\
21.5(2.1)\end{array}$ & 69 & $\begin{array}{l}\text { outpatient } \\
\text { research clinic }\end{array}$ & $\begin{array}{l}\text { therapists and } \\
\text { nurse } \\
\text { practitioner } \\
\text { (no detail) }\end{array}$ & $\begin{array}{l}\text { 1. Placebo+Ml: "Placebo+Ml" } \\
\text { 2. Naltrexone+Ml: } \\
\text { "Naltrexone+Ml" }\end{array}$ \\
\hline De Sousa, $2008^{230}$ & 58 & alcohol & SUD & $\begin{array}{l}{[15,18]} \\
17.3(\mathrm{nr})\end{array}$ & $\mathrm{nr}$ & $\begin{array}{l}\text { outpatient } \\
\text { psychiatric } \\
\text { center }\end{array}$ & $\begin{array}{l}\text { no detail (drug } \\
\text { trial) }\end{array}$ & $\begin{array}{l}\text { 1. Naltrexone+Educ (group): } \\
\text { "Naltrexone" } \\
\text { 2. Disulfiram+Educ (group): } \\
\text { "Disulfiram" }\end{array}$ \\
\hline De Sousa, $2014^{231}$ & 52 & alcohol & SUD & $\begin{array}{l}{[15,18]} \\
17.3(\mathrm{nr})\end{array}$ & $\mathrm{nr}$ & $\begin{array}{l}\text { outpatient } \\
\text { psychiatric } \\
\text { center }\end{array}$ & $\begin{array}{l}\text { no detail (drug } \\
\text { trial) }\end{array}$ & $\begin{array}{l}\text { 1. Naltrexone+Educ (group): } \\
\text { "Naltrexone" } \\
\text { 2. Disulfiram+Educ (group): } \\
\text { "Disulfiram" }\end{array}$ \\
\hline
\end{tabular}

Arm names = Intervention codes, (intervention modifiers) and [family subclassification]: "study arm name".

Abbreviations: Educ = psychoeducation; group = at least one component of the intervention was delivered in a group setting; $\mathrm{MI}$ = motivational interviewing; $\mathrm{N}=$ number randomized; $\mathrm{nr}=$ not reported; $\mathrm{PU}$ = problematic use; $\mathrm{SD}=$ standard deviation; $\mathrm{SUD}=$ substance use disorder

Risk of bias summaries are given in Figure 28. With the exception of de Sousa 2008, ${ }^{230,231}$ the studies were double blinded. Most studies did not conduct intention-to-treat analyses. 
Figure 28. Pharmacologic Interventions for alcohol use: Percentage of studies in each risk of bias category

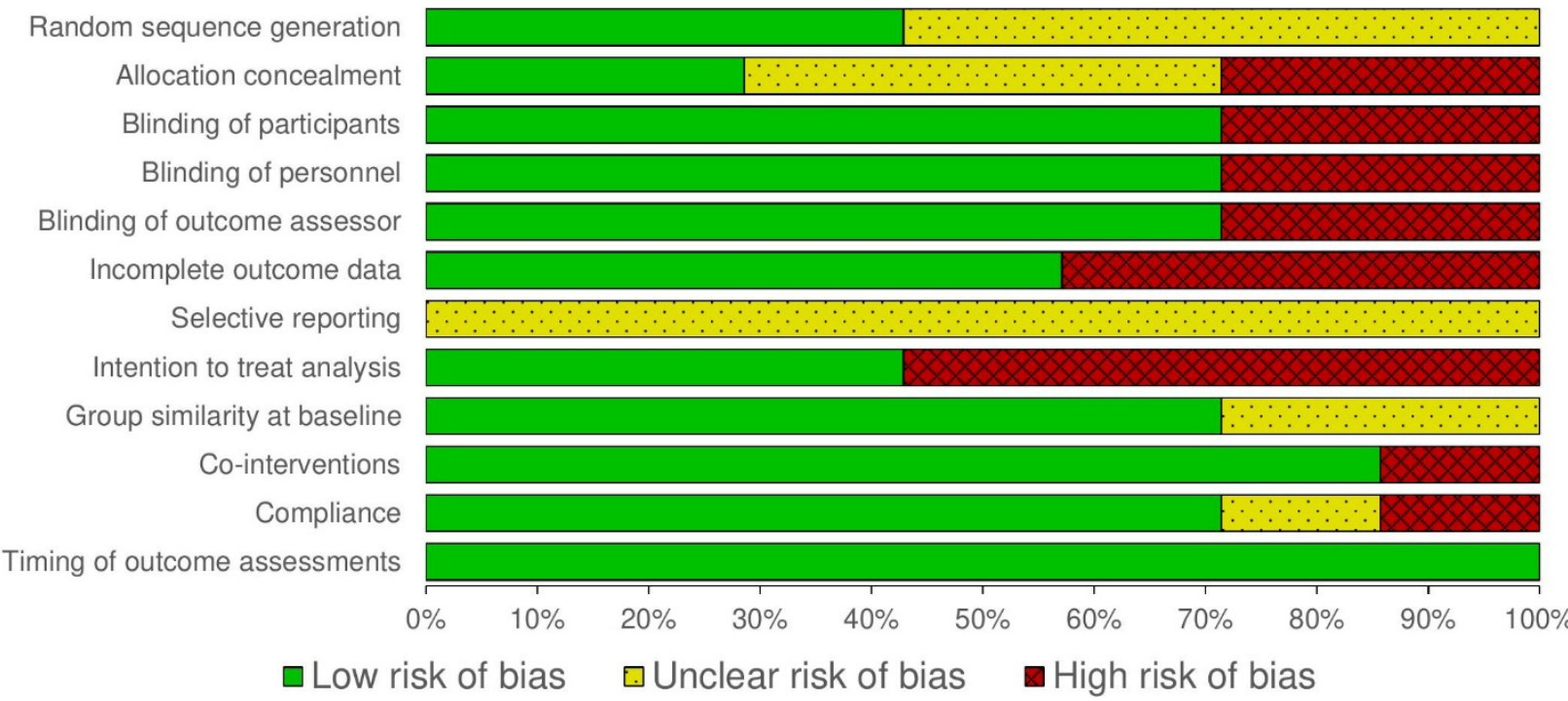

The reported outcomes included mean days of substance use, heavy use, abstinence, consequences, and adverse events. Results for each outcome are given in Table 25.

The six studies that reported on use outcomes in alcohol users reported that active interventions performed better than placebo for abstinence, use days, heavy drinking, or consequence scores in alcohol ${ }^{230-233,235,236}$ or cannabis. ${ }^{232,240-244}$ Two studies compared naltrexone to placebo, and one study compared naltrexone and MI to placebo and MI. Niederhofer 2003 found naltrexone performed better than placebo for increasing days abstinent (mean difference 47.0, 95\% CI 36.6 to 57.4) and abstinence overall (OR 4.00, 95\% CI 1.37 to 11.7) at 3 months Similarly, Miranda 2014 found naltrexone performed better than placebo in reducing alcohol use days (mean difference $-0.70,95 \% \mathrm{CI}-2.13$ to 0.73 ) and heavy drinking days (mean difference $-0.50,95 \% \mathrm{CI}-1.69$ to 0.69 ) at 3 months, although the latter findings were not significant. When MI was combined with naltrexone and placebo, O’Malley 2015 observed similar results in favor of naltrexone for percent heavy drinking days, percent days abstinent, and alcohol consequences, however results were not significant (net mean difference $-2.20,95 \%$ CI -7.43 to 3.03; net mean difference 0.30 , 95\% CI -6.35 to 6.95; net mean difference $-0.90,95 \%$ CI -2.45 to 0.65 , respectively) at 2 months Two studies by Niederhofer 2003 found disulfiram and cyanamide performed better than placebo for abstinence (disulfiram: OR 6.42, 95\% CI 1.00 to 41.2; cyanamide: OR 6.42, 95\% CI 1.00 to 41.2) and days abstinent (disulfiram: mean difference 38.8, 95\% CI 16.0 to 61.7; cyanamide mean difference 43.8 95\% CI 26.3 to 61.3) at 3 months. Finally, De Sousa 2008 found that disulfiram and education performed better than naltrexone and education for abstinence (OR 3.37, 95\% CI 1.10 to 10.3). ${ }^{230,231}$ Across studies, adverse events were rarely reported, and where they were they were generally mild, including nausea, headache, and similar events. One study (O'Malley 2015) reported that there were no serious adverse events with either naltrexone or placebo; a second study (Miranda 2014) reported that there were two adverse events that lead to study discontinuation in the naltrexone arm (gastrointestinal symptoms) and none in the placebo arm. ${ }^{232}$ 
Table 25. Results: Pharmacologic treatments for alcohol use

\begin{tabular}{|c|c|c|c|c|c|c|c|c|c|}
\hline Author, Year & Arm 1 & Arm 2 & Outcome & $\begin{array}{l}\text { Time Point } \\
\text { (Months) }\end{array}$ & $\begin{array}{l}\text { Arm } 1 \\
\mathrm{~N}\end{array}$ & $\begin{array}{l}\text { Arm } 1 \\
\text { Outcome }\end{array}$ & $\begin{array}{l}\text { Arm } 2 \\
\mathrm{~N}\end{array}$ & $\begin{array}{l}\text { Arm } 2 \\
\text { Outcome }\end{array}$ & $\begin{array}{l}\text { Calculated Effect } \\
(95 \% \mathrm{Cl})\end{array}$ \\
\hline \multirow[t]{4}{*}{$\begin{array}{l}\text { O'Malley, 2015, } \\
\text { 236-239 }\end{array}$} & \multirow[t]{4}{*}{ Naltrexone + Ml } & \multirow[t]{4}{*}{ Placebo + MI } & Percent heavy drinking days & 2 & 61 & $21.6(16.1)$ & 67 & $22.9(13.2)$ & $\begin{array}{l}\text { Net mean diff } \\
-2.20 \\
(-7.43,3.03)\end{array}$ \\
\hline & & & $\begin{array}{l}\text { Percent of days abstinent per } 8 \\
\text { weeks (mean, SD) }\end{array}$ & 2 & 61 & $56.6(22.5)$ & 67 & $62.5(15.8)$ & $\begin{array}{l}\text { Net mean diff } 0.30 \\
(-6.35,6.95)\end{array}$ \\
\hline & & & $\begin{array}{l}\text { Alcohol consequences (BYAACS) } \\
\text { (Mean, SD) }\end{array}$ & 2 & 61 & $4.7(3.6)$ & 67 & $5.6(3.9)$ & $\begin{array}{l}\text { Net mean diff } \\
-0.90 \\
(-2.45,0.65)\end{array}$ \\
\hline & & & Serious adverse events $n(\%)$ & 2 & 61 & $0(0)$ & 67 & $0(0)$ & No events \\
\hline \multirow[t]{2}{*}{$\begin{array}{l}\text { Niederhofer, } 2003 \\
235\end{array}$} & \multirow[t]{2}{*}{ Disulfiram } & \multirow[t]{2}{*}{ Placebo } & Abstinent from alcohol (n (\%)) & 3 & 13 & $7(54)$ & 13 & $2(15)$ & $\begin{array}{l}\text { OR } 6.42 \\
(1.00,41.2)\end{array}$ \\
\hline & & & $\begin{array}{l}\text { Days Abstinent from alcohol per } 3 \\
\text { months (Mean, SD) }\end{array}$ & 3 & 13 & $68.5(37.5)$ & 13 & $29.7(19.0)$ & $\begin{array}{l}\text { Diff } 38.8 \\
(16.0,61.7)\end{array}$ \\
\hline \multirow[t]{2}{*}{$\begin{array}{l}\text { Niederhofer,2003 } \\
233,234\end{array}$} & \multirow[t]{2}{*}{ Cyanamide } & \multirow[t]{2}{*}{ Placebo } & Abstinent from alcohol $(\mathrm{n}, \%)$ & 3 & 13 & $7(54)$ & 13 & $2(15)$ & $\begin{array}{l}\text { OR } 6.42 \\
(1.00,41.2)\end{array}$ \\
\hline & & & $\begin{array}{l}\text { Days Abstinent from alcohol per } 3 \\
\text { months (Mean, SD) }\end{array}$ & 3 & 13 & $77.7(24.3)$ & 13 & $33.9(21.0)$ & $\begin{array}{l}\text { Diff } 43.8 \\
(26.3,61.3)\end{array}$ \\
\hline \multirow[t]{2}{*}{$\begin{array}{l}\text { Niederhofer, 2003, } \\
233,234\end{array}$} & \multirow[t]{2}{*}{ Naltrexone } & \multirow[t]{2}{*}{ Placebo } & $\begin{array}{l}\text { Days Abstinent from alcohol per } 3 \\
\text { months (Mean, SD) }\end{array}$ & 3 & 30 & $69.8(27.5)$ & 30 & $22.8(9.0)$ & $\begin{array}{l}\text { Diff } 47.0 \\
(36.6,57.4)\end{array}$ \\
\hline & & & Abstinence from alcohol (n, \%) & 3 & 30 & $20(66.7)$ & 30 & $10(33.3)$ & $\begin{array}{l}\text { OR } 4.00(1.37, \\
11.7)\end{array}$ \\
\hline \multirow[t]{3}{*}{ Miranda, 2014, ${ }^{232}$} & \multirow[t]{3}{*}{ Naltrexone } & \multirow[t]{3}{*}{ Placebo } & $\begin{array}{l}\text { Alcohol use days, per } 3 \text { months } \\
\text { (Mean, SD) }\end{array}$ & 1 & 10 & $2.4(1.4)$ & 12 & $3.1(2.0)$ & $\begin{array}{l}\text { Diff }-0.70 \\
(-2.13,0.73)\end{array}$ \\
\hline & & & Heavy drinking days (Mean, SD) & 1 & 10 & $1.1(1.0)$ & 12 & $1.6(1.8)$ & $\begin{array}{l}\text { Diff }-0.50 \\
(-1.69,0.69)\end{array}$ \\
\hline & & & $\begin{array}{l}\text { Adverse events leading to } \\
\text { withdrawal }(\mathrm{n}, \%)\end{array}$ & 1 & 14 & $2(14)$ & 14 & $0(0)$ & $\begin{array}{l}5.8 \\
(0.25,133.8)\end{array}$ \\
\hline $\begin{array}{l}\text { De Sousa, } 2008, \\
230\end{array}$ & $\begin{array}{l}\text { Disulfiram + } \\
\text { Educ (group) }\end{array}$ & $\begin{array}{l}\text { Naltrexone + } \\
\text { Educ (group) }\end{array}$ & Abstinent from alcohol $(\mathrm{n}, \%)$ & 6 & 29 & $23(80)$ & 29 & $15(52)$ & OR $3.6(1.1,11.4)$ \\
\hline De Sousa, $2014^{231}$ & $\begin{array}{l}\text { Disulfiram+ } \\
\text { Educ (group): }\end{array}$ & $\begin{array}{l}\text { Naltrexone+ } \\
\text { Educ (group) }\end{array}$ & Abstinent from alcohol $(\mathrm{n}, \%)$ & 6 & 29 & $25(86)$ & 29 & $16(54)$ & $\begin{array}{l}\text { OR } 5.0 \\
(1.4,18.3)\end{array}$ \\
\hline
\end{tabular}

Bold font indicates that the 95\% CI does not contain 1 for ORs or 0 for differences.

Abbreviations: BYAACS = Brief Young Adult Alcohol Consequences Scale; CI = confidence interval; Educ = psychoeducation; group = at least one component of the intervention was delivered in a group setting; $\mathrm{MI}=$ motivational interviewing; $\mathrm{OR}$ = odds ratio; $\mathrm{SD}$ = standard deviation 


\section{Cannabis Use}

We found two studies that enrolled 182 subjects (Table 26). Treatment with N-acetylcysteine did not result in over decreases in cannabis use days or cannabis abstinence. Risk of bias information is given in Figure 29. Treatment with topiramate+MI decreased cannabis use days, but not cannabis abstinence, compared to placebo+MI. However, treatment with Topiramate was associated with a higher odds of adverse events leading to withdrawal from treatment (Table 27).

Table 26. Baseline data and interventions: Pharmacologic treatments for cannabis

\begin{tabular}{|c|c|c|c|c|c|c|c|c|}
\hline Author, Year & $\mathbf{N}$ & $\begin{array}{l}\text { Substances } \\
\text { Used }\end{array}$ & Severity & $\begin{array}{l}\text { Ages } \\
\text { [Eligible] } \\
\text { Mean } \\
\text { (SD) }\end{array}$ & $\begin{array}{l}\text { Male } \\
\%\end{array}$ & Setting & $\begin{array}{l}\text { Intervention } \\
\text { Delivery }\end{array}$ & Arm Names \\
\hline Gray, 2012 $241-244$ & 116 & cannabis & SUD & $\begin{array}{l}{[13,21]} \\
18.9(1.5)\end{array}$ & 72 & $\begin{array}{l}\text { outpatient } \\
\text { research clinic }\end{array}$ & $\begin{array}{l}\text { physician, } \\
\text { physician } \\
\text { assistant }\end{array}$ & $\begin{array}{l}\text { 1. Placebo+CM: "Placebo" } \\
\text { 2. N-acetylcysteine+CM: } \\
\text { "N- acetylcysteine" }\end{array}$ \\
\hline $\begin{array}{l}\text { Miranda, } 2017240, \\
245\end{array}$ & 66 & cannabis & PU & $\begin{array}{l}{[15,24]} \\
18.8(2.1)\end{array}$ & 46 & $\begin{array}{l}\text { outpatient } \\
\text { research clinic }\end{array}$ & $\begin{array}{l}\text { research staff } \\
\text { (graduate } \\
\text { students) }\end{array}$ & $\begin{array}{l}\text { 1. Topiramate+MI: } \\
\text { "Topiramate" } \\
\text { 2. Placebo+MI: "Placebo" }\end{array}$ \\
\hline
\end{tabular}

Arm names = Intervention codes, (intervention modifiers) and [family subclassification]: "study arm name".

Abbreviations: $\mathrm{CM}$ = contingency management; $\mathrm{N}=$ number randomized; $\mathrm{PU}=$ problematic use; $\mathrm{SD}=$ standard deviation; $\mathrm{SUD}=$ substance use disorder

Figure 29. Pharmacologic interventions for cannabis use disorder: Percentage of studies in each risk of bias category

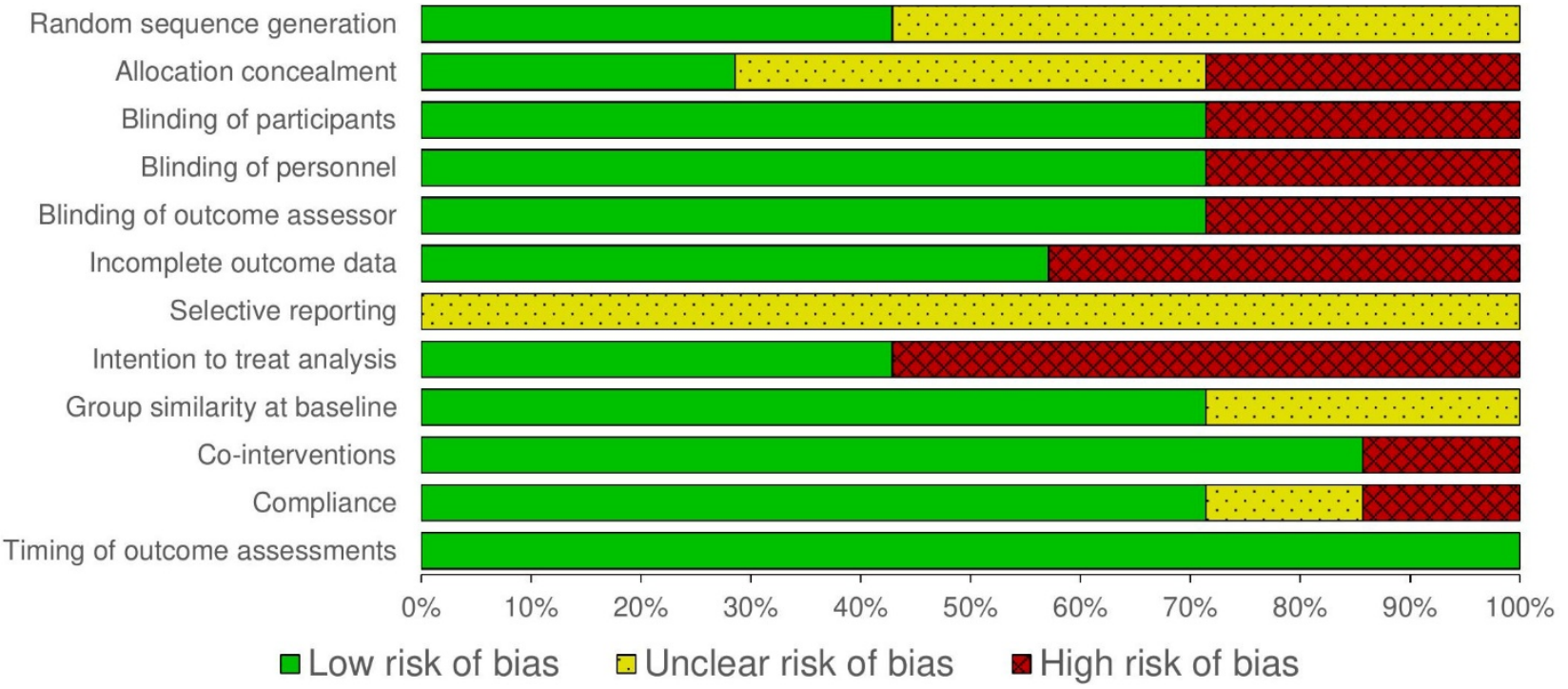


Table 27. Results: Pharmacologic treatments for cannabis use

\begin{tabular}{|c|c|c|c|c|c|c|c|c|c|}
\hline $\begin{array}{l}\text { Study } \\
\text { Author, } \\
\text { Year, PMID }\end{array}$ & Arm 1 & Arm 2 & Outcome & $\begin{array}{l}\text { Time } \\
\text { (mo) }\end{array}$ & $\begin{array}{r}\text { Arm } 1 \\
\mathrm{~N}\end{array}$ & $\begin{array}{r}\text { Arm } 1 \\
\text { Events (\%) }\end{array}$ & $\begin{array}{r}\text { Arm } 2 \\
\mathrm{~N}\end{array}$ & $\begin{array}{r}\text { Arm } 2 \\
\text { Events (\%) }\end{array}$ & $\begin{array}{r}\text { Calculated Effect } \\
(95 \% \mathrm{Cl})\end{array}$ \\
\hline \multirow{4}{*}{$\begin{array}{l}\text { Miranda, } \\
2016,240, \\
245\end{array}$} & \multirow[t]{4}{*}{ Topiramate + MI } & \multirow[t]{4}{*}{$\begin{array}{l}\text { Placebo } \\
+\mathrm{Ml}\end{array}$} & $\begin{array}{l}\text { Abstinent from } \\
\text { cannabis (n, \%) }\end{array}$ & 1 & 40 & $12(30)$ & 26 & $\begin{array}{r}4 \\
(16)\end{array}$ & $\begin{array}{r}\text { OR 2.4 } \\
(0.7,8.3)\end{array}$ \\
\hline & & & $\begin{array}{l}\text { Abstinent from } \\
\text { cannabis (n, \%) }\end{array}$ & 1.5 & 40 & $8(20)$ & 26 & $4(16)$ & $\begin{array}{r}\text { OR } 1.38 \\
(0.4,5.1)\end{array}$ \\
\hline & & & $\begin{array}{l}\text { Cannabis use } \\
\text { days } \\
(\%, 95 \% \mathrm{Cl})\end{array}$ & 1.5 & 40 & $41(34,47)$ & 26 & $\begin{array}{r}56 \\
(47,63)\end{array}$ & $\begin{array}{r}\text { Diff }-15.0 \\
(-24.1,-5.9)\end{array}$ \\
\hline & & & $\begin{array}{l}\text { Serious } \\
\text { adverse events } \\
n(\%)\end{array}$ & 1 & 40 & $0(0)$ & 26 & $0(0)$ & No events \\
\hline \multirow[t]{3}{*}{$\begin{array}{l}\text { Gray, 2012, } \\
\text { 241-244 }\end{array}$} & \multirow[t]{3}{*}{$\begin{array}{l}\text { N-acetylcysteine } \\
+\mathrm{CM}\end{array}$} & \multirow[t]{3}{*}{$\begin{array}{l}\text { Placebo } \\
+\mathrm{CM}\end{array}$} & $\begin{array}{l}\text { Abstinent from } \\
\text { cannabis (n, \%) }\end{array}$ & 2 & 58 & $11(19)$ & 58 & $6(10.3)$ & $\begin{array}{r}\text { OR 2.4 } \\
(0.8,7.5)\end{array}$ \\
\hline & & & $\begin{array}{l}\text { Cannabis use } \\
\text { days (\% } \\
\text { decrease) }\end{array}$ & 2 & 58 & $41.1(4.3)$ & 58 & $37.0(4.4)$ & $\begin{array}{r}\text { Diff }-4.0 \\
(-15.8,7.9)\end{array}$ \\
\hline & & & $\begin{array}{l}\text { Serious } \\
\text { adverse events } \\
n(\%)\end{array}$ & 2 & 58 & $0(0)$ & 58 & $0(0)$ & No events \\
\hline
\end{tabular}

Bold font indicates that the 95\% CI does not contain 1 for ORs or 0 for differences.

Abbreviations: $\mathrm{CI}$ = confidence interval; $\mathrm{CM}$ = contingency management; group = at least one component of the intervention was delivered in a group setting; $\mathrm{MI}=$ motivational interviewing; $\mathrm{OR}$ = odds ratio

\section{Comorbid Psychiatric Disorders in Adolescents With SUD}

\section{Key Points}

- In studies of combined pharmacological and behavioral treatments for ADHD, bipolar disorder and depression for adolescents with SUD, the various interventions did not have consistent effects on the severity of the target psychiatric disorder.

- No study found significant increases or decreases in substance use outcomes. However, substance use outcomes were imprecisely estimated.

\section{Specific Psychiatric Comorbidities}

We found 10 RCTs that described treatments for specific psychiatric comorbidities in adolescents with a concurrent substance use disorder. ${ }^{246-263}$ Studies were included if they reported the effects of pharmacologic treatment (with or without integrated behavioral interventions) on the severity of the comorbid mental health disorder and at least one substance use outcome. Given that substance use outcomes may depend on successful treatment of the psychiatric disorder, we have summarized both psychiatric and substance use outcomes.

Sample size per study ranged from 34 to 303 participants. The studies enrolled patients with one of four psychiatric diagnoses: Attention-deficit/hyperactivity disorder (ADHD; 3 studies 
total; 1 with associated conduct disorder), depression (4 studies) and bipolar disorder (3 studies) who had concurrent substance use disorders for alcohol and/or cannabis, or other unspecified substances. Many adolescents in all studies used multiple substances, most commonly alcohol and cannabis and reported use of opioids, stimulants, sedatives, hallucinogens, and inhalants less commonly ( $<10 \%$ of participants).

Baseline and arm details are given in Table 28. Risk of bias summaries are given in Figure 30.

Table 28. Baseline data and interventions: Pharmacologic interventions for psychiatric comorbidities in adolescents with substance use disorders

\begin{tabular}{|c|c|c|c|c|c|c|c|c|}
\hline Comorbidity & Author, Year & $\mathrm{N}$ & $\begin{array}{l}\text { Substances } \\
\text { Used }\end{array}$ & Severity & $\begin{array}{l}\text { Ages } \\
\text { [Eligible] } \\
\text { Mean } \\
\text { (SD) }\end{array}$ & $\begin{array}{l}\text { Male } \\
\%\end{array}$ & Setting & Arm Names \\
\hline ADHD & $\begin{array}{l}\text { Riggs, } 2011^{252,255,} \\
257-259,262,263\end{array}$ & 303 & $\begin{array}{l}\text { cannabis } \\
\text { alcohol } \\
\text { other drugs }\end{array}$ & SUD & $\begin{array}{l}{[13,18]} \\
16.5(1.3)\end{array}$ & 79 & outpatient & $\begin{array}{l}\text { 1. Placebo+CBT+MI } \\
\text { (integrated): "Placebo + CBT" } \\
\text { 2. Methylphenidate+CBT+MI } \\
\text { (integrated): "Osmotic-release } \\
\text { methylphenidate + CBT" }\end{array}$ \\
\hline ADHD & Thurstone, $2010^{260}$ & 70 & $\begin{array}{l}\text { cannabis } \\
\text { alcohol } \\
\text { other drugs }\end{array}$ & SUD & $\begin{array}{l}{[13,19]} \\
16.1(1.8)\end{array}$ & 79 & $\begin{array}{l}\text { outpatient } \\
\text { (medications } \\
\text { prepared by } \\
\text { research } \\
\text { pharmacist) }\end{array}$ & $\begin{array}{l}\text { 1. Placebo+CBT+MI } \\
\text { (integrated): "Placebo + } \\
\text { CBT/MI" } \\
\text { 2. Atomoxetine+CBT+MI } \\
\text { (integrated): "Atomoxetine + } \\
\text { CBT/Ml" }\end{array}$ \\
\hline $\begin{array}{l}\text { ADHD, } \\
\text { conduct } \\
\text { disorder }\end{array}$ & Riggs, $2004^{264}$ & 69 & $\begin{array}{l}\text { cannabis } \\
\text { alcohol } \\
\text { other drugs }\end{array}$ & SUD & $\begin{array}{l}{[13,19]} \\
15.8(1.4)\end{array}$ & 83 & $\begin{array}{l}\text { outpatient } \\
\text { research } \\
\text { clinic }\end{array}$ & $\begin{array}{l}\text { 1. Placebo (integrated): } \\
\text { "Placebo" } \\
\text { 2. Pemoline (integrated): } \\
\text { "Pemoline" }\end{array}$ \\
\hline $\begin{array}{l}\text { bipolar } \\
\text { disorder }\end{array}$ & Delbelo, $2017^{253}$ & 39 & alcohol & SUD & $\begin{array}{l}{[12,25]} \\
18(3.1)\end{array}$ & 38 & outpatient & $\begin{array}{l}\text { 1. Quetiapine+Topiramate } \\
\text { (integrated): } \\
\text { "Quetiapine+Topiramate" } \\
\text { 2. Quetiapine+Placebo } \\
\text { (integrated): } \\
\text { "Quetiapine+Placebo" }\end{array}$ \\
\hline $\begin{array}{l}\text { bipolar } \\
\text { disorder }\end{array}$ & Geller, $1998^{251}$ & 25 & $\begin{array}{l}\text { cannabis } \\
\text { alcohol } \\
\text { other drugs }\end{array}$ & SUD & $\begin{array}{l}{[12,18]} \\
16.3(1.2)\end{array}$ & 64 & outpatient & $\begin{array}{l}\text { 1. Placebo (integrated): } \\
\text { "Placebo" } \\
\text { 2. Lithium (integrated): "Active" }\end{array}$ \\
\hline $\begin{array}{l}\text { bipolar } \\
\text { disorder in a } \\
\text { current } \\
\text { manic or } \\
\text { mixed } \\
\text { episode }\end{array}$ & Delbelo, $2017^{254}$ & 75 & cannabis & PU & $\begin{array}{l}{[12,18]} \\
17.4(0.2)\end{array}$ & 49 & & $\begin{array}{l}\text { 1. Quetiapine+Topiramate } \\
\text { (integrated): } \\
\text { "Quetiapine+topiramate" } \\
\text { 2. Quetiapine+Placebo } \\
\text { (integrated): } \\
\text { "Quetiapine+placebo" }\end{array}$ \\
\hline depression & Cornelius, $2009^{247}$ & 50 & alcohol & SUD & $\begin{array}{l}{[15,20]} \\
\mathrm{nr}\end{array}$ & 44 & $\begin{array}{l}\text { outpatient } \\
\text { research } \\
\text { clinic }\end{array}$ & $\begin{array}{l}\text { 1. Placebo+CBT+MI } \\
\text { (integrated): "Placebo" } \\
\text { 2. Fluoxetine+CBT+Ml } \\
\text { (integrated): "Fluoxetine" }\end{array}$ \\
\hline
\end{tabular}




\begin{tabular}{|c|c|c|c|c|c|c|c|c|}
\hline Comorbidity & Author, Year & $\mathrm{N}$ & $\begin{array}{l}\text { Substances } \\
\text { Used }\end{array}$ & Severity & $\begin{array}{l}\text { Ages } \\
\text { [Eligible] } \\
\text { Mean } \\
\text { (SD) }\end{array}$ & $\begin{array}{l}\text { Male } \\
\%\end{array}$ & Setting & Arm Names \\
\hline depression & $\begin{array}{l}\text { Cornelius, 2010246, } \\
249,265\end{array}$ & 70 & $\begin{array}{l}\text { cannabis } \\
\text { alcohol }\end{array}$ & SUD & $\begin{array}{l}{[14,25]} \\
21.1(2.4)\end{array}$ & 61 & $\begin{array}{l}\text { outpatient } \\
\text { research } \\
\text { clinic }\end{array}$ & $\begin{array}{l}\text { 1. Placebo+CBT+MI } \\
\text { (integrated): "Placebo" } \\
\text { 2. Fluoxetine+CBT+MI } \\
\text { (integrated): "Fluoxetine" }\end{array}$ \\
\hline depression & Findling, $2009^{250}$ & 34 & $\begin{array}{l}\text { cannabis } \\
\text { alcohol }\end{array}$ & SUD & $\begin{array}{l}{[12,17]} \\
16.5(1.1)\end{array}$ & 85 & $\begin{array}{l}\text { outpatient } \\
\text { research } \\
\text { clinic }\end{array}$ & $\begin{array}{l}\text { 1. Placebo (integrated): } \\
\text { "Placebo" } \\
\text { 2. Fluoxetine (integrated): } \\
\text { "Fluoxetine" }\end{array}$ \\
\hline depression & Riggs, 2007256, 261 & 126 & $\begin{array}{l}\text { cannabis } \\
\text { alcohol } \\
\text { other drugs }\end{array}$ & SUD & $\begin{array}{l}{[13,19]} \\
17.2(1.7)\end{array}$ & 67 & $\begin{array}{l}\text { outpatient } \\
\text { research } \\
\text { clinic }\end{array}$ & $\begin{array}{l}\text { 1. Placebo+CBT: "Placebo + } \\
\text { CBT" } \\
\text { 2. Fluoxetine+CBT: "Fluoxetine } \\
\text { + CBT" }\end{array}$ \\
\hline
\end{tabular}

Arm names = Intervention codes, (intervention modifiers) and [family subclassification]: "study arm name".

Abbreviations: ADHD = attention-deficit/hyperactivity disorder; CBT = cognitive behavioral therapy; integrated = intervention as a whole was designed to treat substance use disorder/problematic use and at least one other diagnosis (e.g., mental health); MI = motivational interviewing; $\mathrm{N}=$ number randomized; $\mathrm{nr}=$ not reported; $\mathrm{PU}=$ problematic use; $\mathrm{SD}=$ standard deviation; $\mathrm{SUD}=$ substance use disorder

The most commonly observed risk of bias concerns related to incomplete outcome data, group similarity at baseline and compliance (Figure 30).

Figure 30. Pharmacologic interventions for psychiatric comorbidities in adolescents with substance use disorders: Percentage of studies in each risk of bias category

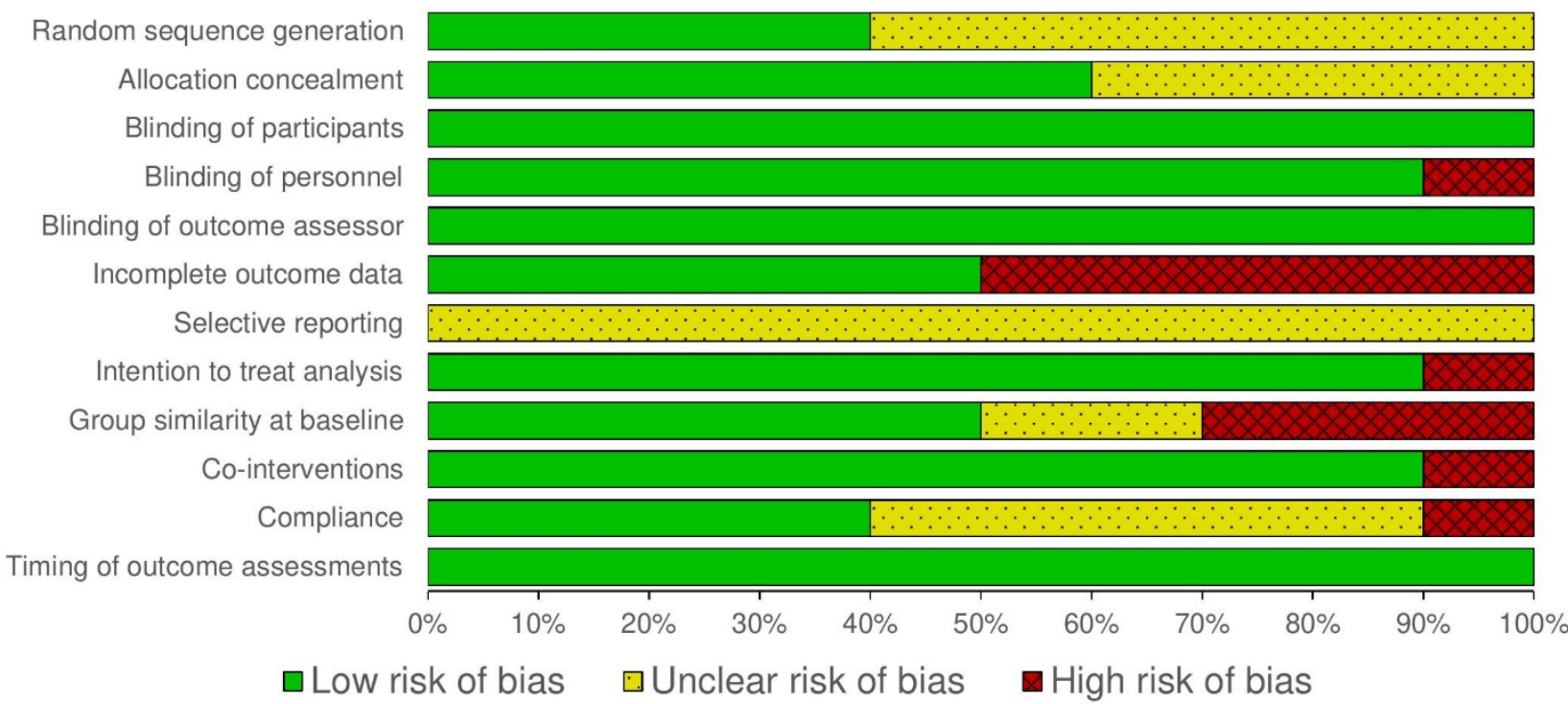

The reported outcomes included severity of comorbidity condition, mean days of substance use, heavy use, abstinence, consequences, and adverse events. Full results for each outcome in studies treated for co-existing ADHD, depression, and bipolar disorder are given in Tables 29, 30, and 31, respectively. 
The three studies that reported on outcomes in not otherwise specified substance abuse populations with comorbid ADHD found no impact of pharmacologic agents pemoline, atomoxetine, or fluoxetine (with or without behavioral interventions) on ADHD symptoms, use days, or adverse events.

Four studies that reported on outcomes in populations defined by alcohol, cannabis, or not otherwise specified substance abuse populations with comorbid depression. Three studies found no impact of fluoxetine (with or without CBT + MI) on symptoms of depression, use days, problem scores (e.g., abuse symptoms and dependence symptoms). The exception is Riggs et al., 2007. ${ }^{256}$ This study enrolled patients with substance use and depression and compared fluoxetine $+\mathrm{MI}+\mathrm{CBT}$, with placebo+MI+CBT. They found a net mean difference of -4.2 (95\% CI -9.7 , -2.0) suggesting an improvement in depression symptoms as measured by the Children's Depression Rate Scale, Revised (CDRS-R).

The three studies that reported outcomes in populations defined by alcohol and cannabis use with comorbid bipolar disorder found no impact of lithium or quetiapine + topiramate on symptoms of mental health and of adverse events observed, most either occurred rarely, or where presented, were comparable between groups.

Table 29. Results: Pharmacologic interventions for ADHD in adolescents with substance use disorders

\begin{tabular}{|c|c|c|c|c|c|c|c|c|c|}
\hline $\begin{array}{l}\text { Study } \\
\text { Author, } \\
\text { Year, PMID }\end{array}$ & Arm 1 & Arm 2 & Outcome & Time (mo) & $\begin{array}{r}\text { Arm } \\
1 \mathrm{~N}\end{array}$ & $\begin{array}{r}\text { Arm-1 } \\
\text { Outcome }\end{array}$ & $\begin{array}{r}\text { Arm- } \\
2 \mathrm{~N}\end{array}$ & $\begin{array}{r}\text { Arm-2 } \\
\text { Outcome }\end{array}$ & $\begin{array}{r}\text { Effect } \\
(95 \% \\
\mathrm{Cl})\end{array}$ \\
\hline $\begin{array}{l}\text { Riggs, } \\
2004264\end{array}$ & Pemoline & Placebo & $\mathrm{CHIS}$ & 4 & 34 & $\begin{array}{r}-12.2 \\
(-18.5 \\
-5.9)\end{array}$ & 34 & $\begin{array}{r}-7.2 \\
(-12.0 \\
-2.4)\end{array}$ & $\begin{array}{r}\mathrm{MD} \\
-5.0 \\
(-12.9 \\
2.9)\end{array}$ \\
\hline \multirow[t]{2}{*}{$\begin{array}{l}\text { Riggs, } \\
2011 \\
252,255,257- \\
259,262,263\end{array}$} & $\begin{array}{l}\text { Methylphenidate } \\
+\mathrm{CBT}+\mathrm{MI}\end{array}$ & $\begin{array}{l}\text { Placebo + } \\
\text { CBT + MI }\end{array}$ & ADHD-RS & 4 & 151 & $\begin{array}{r}-19.2 \\
(-21.2 \\
-17.2)\end{array}$ & 152 & $\begin{array}{r}-21.2 \\
(-23.2 \\
-19.2)\end{array}$ & $\begin{array}{r}\text { MD 2.0 } \\
(-0.9, \\
4.9)\end{array}$ \\
\hline & & & $\begin{array}{l}\text { Uses days (past } \\
28 \text { days non- } \\
\text { tobacco) }\end{array}$ & 4 & 151 & $\begin{array}{l}-5.7 \\
(-7.2 \\
-4.1)\end{array}$ & 152 & $\begin{array}{l}-5.2 \\
(-6.8 \\
-3.6)\end{array}$ & $\begin{array}{r}\text { MD-0. } \\
5 \\
(-2.7 \\
1.7)\end{array}$ \\
\hline \multirow[t]{7}{*}{$\begin{array}{l}\text { Thurstone, } \\
2010^{260}\end{array}$} & $\begin{array}{l}\text { Ato + CBT + Ml } \\
+ \text { Fam }\end{array}$ & $\begin{array}{l}\text { Placebo + } \\
\text { CBT + MI } \\
+ \text { Fam }\end{array}$ & $\begin{array}{l}\text { ADE - Vomiting } \\
(\%)\end{array}$ & 3 & 32 & 16 & 33 & 7 & $\begin{array}{r}\text { OR } 3.7 \\
(1.3, \\
11.0)\end{array}$ \\
\hline & & & $\begin{array}{l}\text { ADE - } \\
\text { Drowsiness (\%) }\end{array}$ & 3 & 32 & 16 & 33 & 14 & $\begin{array}{r}\text { OR } 1.4 \\
(0.51 \\
3.6) \\
\end{array}$ \\
\hline & & & $\begin{array}{l}\text { ADE - Dif staying } \\
\text { asleep (\%) }\end{array}$ & 3 & 32 & 16 & 33 & 20 & $\begin{array}{r}\text { OR } 0.6 \\
(0.4 \\
1.7)\end{array}$ \\
\hline & & & $\begin{array}{l}\text { ADE - } \\
\text { Abdominal Pain } \\
(\%)\end{array}$ & 3 & 32 & 18 & 33 & 15 & $\begin{array}{r}\text { OR } 1.5 \\
(0.6 \\
4.1) \\
\end{array}$ \\
\hline & & & $\begin{array}{l}\text { ADE - Nasal } \\
\text { Congestion (\%) }\end{array}$ & 3 & 32 & 19 & 33 & 17 & $\begin{array}{r}\text { OR } 1.4 \\
(0.5 \\
3.7)\end{array}$ \\
\hline & & & $\begin{array}{l}\text { ADE - Difficulty } \\
\text { falling asleep (\%) }\end{array}$ & 3 & 32 & 19 & 33 & 23 & $\begin{array}{r}\text { OR } 0.6 \\
(0.2 \\
1.8)\end{array}$ \\
\hline & & & $\begin{array}{l}\text { ADE - Appetite } \\
\text { decrease (\%) }\end{array}$ & 3 & 32 & 19 & 33 & 12 & $\begin{array}{r}\text { OR } 2.6 \\
(0.4 \\
7.0)\end{array}$ \\
\hline
\end{tabular}




\begin{tabular}{|c|c|c|c|c|c|c|c|c|c|}
\hline $\begin{array}{l}\text { Study } \\
\text { Author, } \\
\text { Year, PMID }\end{array}$ & Arm 1 & Arm 2 & Outcome & Time (mo) & $\begin{array}{r}\text { Arm } \\
1 \mathrm{~N}\end{array}$ & $\begin{array}{r}\text { Arm-1 } \\
\text { Outcome }\end{array}$ & $\begin{array}{r}\text { Arm- } \\
2 \mathrm{~N}\end{array}$ & $\begin{array}{r}\text { Arm-2 } \\
\text { Outcome }\end{array}$ & $\begin{array}{r}\text { Effec } \\
(95 \% \\
\text { Cl) }\end{array}$ \\
\hline & & & $\begin{array}{l}\text { ADE - Difficulty } \\
\text { concentrating (\%) }\end{array}$ & 3 & 32 & 21 & 33 & 15 & $\begin{array}{r}\text { OR } 2.3 \\
(0.4 \\
6.2)\end{array}$ \\
\hline
\end{tabular}

Bold font indicates that the $95 \% \mathrm{Cl}$ does not contain 1 for ORs or 0 for differences.

Abbreviations: $A D E=$ adverse event; $A D H D-R S=$ Attention-Deficit/Hyperactivity Disorder Rating Scale; Ato = Atomoxetine; CBT = cognitive behavioral therapy; CHIS = Conners Hyperactivity-Impulsivity scale (parent related); $\mathrm{Cl}$ = confidence interval; Fam = family based therapy; $\mathrm{MD}=$ mean difference; $\mathrm{MI}=$ motivational interviewing; $\mathrm{N}=$ number of participants analyzed; OR = odds ratio 
Table 30. Results: Pharmacologic interventions for depression in adolescents with substance use disorders

\begin{tabular}{|c|c|c|c|c|c|c|c|c|c|}
\hline $\begin{array}{l}\text { Study Author, Year, } \\
\text { PMID }\end{array}$ & Arm 1 & Arm 2 & Outcome & $\begin{array}{l}\text { Time } \\
(\mathrm{mo})\end{array}$ & $\begin{array}{r}\text { Arm } 1 \\
\mathrm{~N}\end{array}$ & $\begin{array}{r}\text { Arm } 1 \\
\text { Outcome }\end{array}$ & $\begin{array}{r}\text { Arm } \\
2 \mathrm{~N}\end{array}$ & Arm 2 Outcome & Effect $(95 \% \mathrm{Cl})$ \\
\hline \multirow[t]{2}{*}{$\begin{array}{l}\text { Cornelius } \\
2009 \\
247\end{array}$} & $\begin{array}{l}\text { Fluoxetine } \\
+\mathrm{CBT}+ \\
\mathrm{Ml}\end{array}$ & $\begin{array}{l}\text { Placebo } \\
+ \\
\mathrm{CBT}+ \\
\mathrm{Ml}\end{array}$ & $\begin{array}{l}\text { Depression } \\
\text { (BDI) }\end{array}$ & 3 & 24 & $\begin{array}{r}M D \\
-10.46 \\
(-13.8 \\
-7.1) \\
\end{array}$ & 26 & $\begin{array}{r}M D-11.7 \\
(-15.3,-8.0)\end{array}$ & $\begin{array}{r}\text { NMD 1.2 } \\
(-3.8,6.2)\end{array}$ \\
\hline & & & $\begin{array}{l}\text { Problems } \\
\text { (alcohol } \\
\text { sxs) } \\
\end{array}$ & 3 & 24 & $\begin{array}{r}\mathrm{MD}-2.66 \\
(-3.5 \\
-1.8) \\
\end{array}$ & 26 & $\begin{array}{r}M D-2.5 \\
(-3.6,-1.5)\end{array}$ & $\begin{array}{r}\text { NMD -0.1 } \\
(-1.5,1.2)\end{array}$ \\
\hline \multirow[t]{6}{*}{ Cornelius, 2010 246, 249, 265} & $\begin{array}{l}\text { Fluoxetine } \\
+\mathrm{CBT}+ \\
\mathrm{Ml}\end{array}$ & $\begin{array}{l}\text { Placebo } \\
+ \\
\text { CBT + } \\
\mathrm{MI}\end{array}$ & $\begin{array}{l}\text { Use days } \\
\text { (heavy } \\
\text { drinking } \\
\text { days/wk) }\end{array}$ & 3 & 34 & $\begin{array}{l}M D-0.03 \\
(-0.3,0.3)\end{array}$ & 36 & $\begin{array}{r}M D-0.12 \\
(-0.4,0.2)\end{array}$ & $\begin{array}{r}\text { NMD } 0.09 \\
(-0.4,0.5)\end{array}$ \\
\hline & & & $\begin{array}{l}\text { Problems } \\
\text { (alcohol } \\
\text { abuse sxs) }\end{array}$ & 3 & 34 & $\begin{array}{r}\mathrm{MD}-0.1 \\
(-0.3,0.1)\end{array}$ & 36 & $\begin{array}{r}\mathrm{MD}-0.19 \\
(-0.4,0.01)\end{array}$ & $\begin{array}{r}\text { NMD } 0.11 \\
(-0.2,0.4\end{array}$ \\
\hline & & & $\begin{array}{l}\text { Problems } \\
\text { (dep sxs) }\end{array}$ & 3 & & $\begin{array}{c}\text { MD }-0.6 \\
(-1.1 \\
-0.1)\end{array}$ & & $\begin{array}{l}M D-0.25 \\
(-0.7,0.2)\end{array}$ & $\begin{array}{r}\text { NMD } \\
-0.3(-1.0,0.3)\end{array}$ \\
\hline & & & $\begin{array}{l}\text { Use days } \\
\text { (per week) }\end{array}$ & 3 & 34 & $\begin{array}{r}\text { MD }-0.7 \\
(-1.54 \\
0.08) \\
\end{array}$ & 36 & $\begin{array}{r}\mathrm{MD}-1.2 \\
(-1.9,-0.6)\end{array}$ & $\begin{array}{r}\text { NMD } 0.5 \\
(-0.6,1.6)\end{array}$ \\
\hline & & & $\begin{array}{l}\text { Problems } \\
\text { (abuse sxs) }\end{array}$ & 3 & 34 & $\begin{array}{c}\mathrm{MD}-0.8 \\
(-1.1 \\
-0.4) \\
\end{array}$ & 36 & $\begin{array}{r}M D-0.7 \\
(-1.0,-0.5)\end{array}$ & $\begin{array}{r}\text { NMD }-0.04 \\
(-0.4,0.4)\end{array}$ \\
\hline & & & $\begin{array}{l}\text { Problems } \\
\text { (dep sxs) }\end{array}$ & 3 & 34 & $\begin{array}{r}\mathrm{MD}-1.59 \\
(-2.2 \\
-1.0) \\
\end{array}$ & 36 & $\begin{array}{r}M D-2.0 \\
(-2.6,-1.5)\end{array}$ & $\begin{array}{r}\text { NMD } 0.5 \\
(-0.4,1.3)\end{array}$ \\
\hline \multirow[t]{2}{*}{$\begin{array}{l}\text { Riggs } \\
2007 \\
256,261\end{array}$} & $\begin{array}{l}\text { Fluoxetine } \\
+\mathrm{CBT}+ \\
\mathrm{Ml}\end{array}$ & $\begin{array}{l}\text { Placebo } \\
+ \\
\text { CBT + } \\
\mathrm{Ml}\end{array}$ & $\begin{array}{l}\text { Depression } \\
\text { CDRS-R }\end{array}$ & 4 & 63 & $\begin{array}{c}\mathrm{MD}-24.8 \\
(-27.5 \\
-22.0)\end{array}$ & 63 & $\begin{array}{r}M D-18.9 \\
(-21.6,-16.2)\end{array}$ & $\begin{array}{r}N M D-5.9 \\
(-9.7,-2.0)\end{array}$ \\
\hline & & & $\begin{array}{l}\text { AOD use } \\
\text { days } \\
\text { (per month) }\end{array}$ & 4 & 63 & $\begin{array}{r}\mathrm{MD}-3.94 \\
(-6.8 \\
-1.1) \\
\end{array}$ & 63 & $\begin{array}{r}M D-4.7 \\
(-7.6,-1.8)\end{array}$ & $\begin{array}{r}\text { NMD } 0.8 \\
(-3.3,4.8)\end{array}$ \\
\hline \multirow[t]{2}{*}{$\begin{array}{l}\text { Findling } \\
2009 \\
250\end{array}$} & Fluoxetine & Placebo & $\begin{array}{l}\text { Depression } \\
\text { CDRS-R }\end{array}$ & 2 & 18 & $\begin{array}{r}\text { MD }-18.4 \\
(-19.7 \\
-17.1) \\
\end{array}$ & 16 & $\begin{array}{r}M D-22.6 \\
(-24.1,-21.1)\end{array}$ & $\begin{array}{l}\text { NMD 4.2 } \\
(2.2,6.2)\end{array}$ \\
\hline & & & $\begin{array}{l}\text { AOD } \\
\text { abstinence }\end{array}$ & 2 & 12 & 8 & 13 & 10 & $\begin{array}{r}\text { OR } 0.6 \\
(0.1,3.5)\end{array}$ \\
\hline
\end{tabular}

Bold font indicates that the 95\% CI does not contain 1 for ORs or 0 for differences.

Abbreviations: AOD = alcohol and other drugs; BDI = Beck Depression Inventory; CBT = cognitive behavioral therapy; CDRS-R = Children's Depression Rating Scale-Revised; $\mathrm{CI}=$ confidence interval; dep = dependence; $\mathrm{MD}=$ mean difference; $\mathrm{MI}=$ motivational interviewing; $\mathrm{N}=$ number of participants analyzed; NMD = net mean difference; $\mathrm{OR}=$ odds ratio; sxs = symptoms 
Table 31. Results: Pharmacologic interventions for bipolar disorder in adolescents with substance use disorders

\begin{tabular}{|c|c|c|c|c|c|c|c|c|c|}
\hline $\begin{array}{l}\text { Study } \\
\text { Author, Year, } \\
\text { PMID }\end{array}$ & Arm 1 & Arm 2 & Outcome & $\begin{array}{l}\text { Time } \\
\text { (mo) }\end{array}$ & $\begin{array}{r}\text { Arm } 1 \\
\mathrm{~N}\end{array}$ & $\begin{array}{r}\text { Arm 1 } \\
\text { No. } \\
\text { events }\end{array}$ & $\begin{array}{r}\text { Arm } \\
2 \mathrm{~N}\end{array}$ & $\begin{array}{r}\text { Arm } 2 \\
\text { No. } \\
\text { Events }\end{array}$ & $\begin{array}{r}\text { OR } \\
(95 \% \\
\mathrm{Cl})\end{array}$ \\
\hline $\begin{array}{l}\text { Geller, } 1998 \\
251\end{array}$ & Lithium & Placebo & $\begin{array}{l}\text { CGAS >=65 } \\
(\%)\end{array}$ & 1.5 & 13 & 6 & 12 & 1 & $\begin{array}{r}9.4 \\
(0.9, \\
95.9) \\
\end{array}$ \\
\hline \multirow[t]{4}{*}{$\begin{array}{l}\text { Delbelo } 2017 \\
253\end{array}$} & Quetiapine/Topiramate & $\begin{array}{l}\text { Quetiapine } \\
+ \text { Placebo }\end{array}$ & $\begin{array}{l}\text { ADE - } \\
\text { Suicidal } \\
\text { Ideation (N) }\end{array}$ & 4 & 18 & 2 & 21 & 8 & $\begin{array}{r}0.2 \\
(0.04, \\
1.1)\end{array}$ \\
\hline & & & $\begin{array}{l}\text { ADE - } \\
\text { Suicidal } \\
\text { attempt (N) }\end{array}$ & 4 & 18 & 0 & 21 & 1 & $\begin{array}{r}0.6 \\
(0.02, \\
18.1)\end{array}$ \\
\hline & & & $\begin{array}{l}\text { ADE - } \\
\text { Sedation (N) }\end{array}$ & 4 & 18 & 11 & 21 & 4 & $\begin{array}{r}6.7 \\
(1.6, \\
28.3)\end{array}$ \\
\hline & & & $\begin{array}{l}\text { ADE - Dif } \\
\text { Arousing (N) }\end{array}$ & 4 & 18 & 9 & 21 & 3 & $\begin{array}{r}6.0 \\
(1.3, \\
27.8)\end{array}$ \\
\hline \multirow[t]{8}{*}{$\begin{array}{l}\text { Delbelo } 2017 \\
254\end{array}$} & Quetiapine/Topiramate & $\begin{array}{l}\text { Quetiapine } \\
\text { + placebo }\end{array}$ & $\begin{array}{l}\text { ADE- Dry } \\
\text { mouth (N) }\end{array}$ & 4 & 38 & 23 & 37 & 30 & $\begin{array}{r}0.4 \\
(0.1 \\
1.0)\end{array}$ \\
\hline & & & $\begin{array}{l}\text { ADE- } \\
\text { Excitement (N) }\end{array}$ & 4 & 38 & 5 & 37 & 0 & $\begin{array}{r}11.1 \\
(0.6, \\
210.3)\end{array}$ \\
\hline & & & $\begin{array}{l}\text { ADE-Dif } \\
\text { Staying } \\
\text { Asleep (N) }\end{array}$ & 4 & 38 & 12 & 37 & 20 & $\begin{array}{r}0.4 \\
(0.2 \\
1.0) \\
\end{array}$ \\
\hline & & & $\begin{array}{l}\text { ADE-Dif } \\
\text { Falling Asleep } \\
\text { (N) }\end{array}$ & 4 & 38 & 12 & 37 & 20 & $\begin{array}{r}0.4 \\
(0.15, \\
1.0)\end{array}$ \\
\hline & & & $\begin{array}{l}\text { ADE- } \\
\text { Pregnancy (N) }\end{array}$ & 4 & 38 & 0 & 37 & 1 & $\begin{array}{r}0.5 \\
(0.02, \\
14.8)\end{array}$ \\
\hline & & & $\begin{array}{l}\text { ADE- Suicidal } \\
\text { Ideation (N) }\end{array}$ & 4 & 38 & 1 & 37 & 0 & $\begin{array}{r}2.0 \\
(0.06, \\
60.6)\end{array}$ \\
\hline & & & $\begin{array}{l}\text { ADE- } \\
\text { Hospitalization } \\
\text { (N) }\end{array}$ & 4 & 38 & 4 & 37 & 5 & $\begin{array}{r}0.8 \\
(0.2, \\
3.1) \\
\end{array}$ \\
\hline & & & ADE- Any (N) & 4 & 38 & 23 & 37 & 30 & $\begin{array}{r}0.4 \\
(0.1 \\
1.0)\end{array}$ \\
\hline
\end{tabular}

Bold font indicates that the $95 \%$ CI does not contain 1 for ORs or 0 for difference.

Abbreviations: $\mathrm{ADE}=$ adverse event; CGAS = Children's Global Assessment Scale; $\mathrm{CI}$ = confidence interval; $\mathrm{N}$ = number of participants analyzed; OR = odds ratio 


\section{Discussion}

\section{Key Findings}

Most studies of brief behavioral interventions evaluated motivational interviewing (MI) compared to treatment as usual (TAU). These studies enrolled adolescents who often had problematic use of both alcohol and cannabis. Some heterogeneity in effects between studies was found, but MI resulted in overall decreases in heavy alcohol use days (i.e., binge drinking), overall alcohol use days and may reduce all substance use related problems compared to TAU. However, brief MI does not reduce days of cannabis use compared to TAU.

Longer term (i.e., "nonbrief”) behavioral interventions were diverse and often combined multiple intervention components. This diversity severely limited our ability to evaluate their efficacy compared to TAU and to each other. Nevertheless, our analyses did suggest that familybased therapies (Fam) may most effectively decrease alcohol use days, and may be more effective than ICM, CBT and MI in reducing days of alcohol use. We found no evidence of efficacy for any intervention in decreasing days of cannabis use. Indeed, summary estimates suggest that some interventions (CBT, CBT+MI, CBT+MI+CM and Educ) increase cannabis use relative to TAU.

Both MI and CBT reduce days of alcohol and other drug use relative to TAU, with MI more effective than PeerGroup, CBT+MI, Fam, CBT+ICM, CBT+MI+ICM, CBT and ICM. Illicit drug use decreased for CBT+MI compared to TAU.

Overall, our findings suggest that that effects may vary by substance for both brief and nonbrief interventions. However, these conclusions are based on separate analyses (of overlapping groups of studies) that are not directly comparable.

Existing systematic reviews of the general population of college students who drink alcohol found that, on average, compared to no intervention, behavioral interventions resulted in reduced alcohol use for up to about 6 months, but these effects waned in the long term. However, behavioral interventions resulted in fewer alcohol-related problems over the medium to long term. One SR found that, by indirect comparison, face-to-face interventions provide larger and more enduring effects than computer-delivered interventions. Two SRs focused on college students who engaged in heavy or hazardous alcohol use. Brief, single-session interventions and the commercially available BASICS program were found to reduce alcohol use compared with no intervention. Among the brief behavioral interventions, MET/MI had the strongest effect. Two SRs focused on college students mandated to attend alcohol programs. On average, alcohol use decreased in the short- to medium-term regardless of intervention, but mostly did not persist. Four specific commercially available interventions were found to be more effective in the short term than others.

For opioid use disorder, longer courses (2 to 3 months) of buprenorphine are more effective than shorter courses (14 to 28 days) to reduce opioid use and achieve abstinence.

For alcohol and cannabis use disorder, evidence is insufficient regarding the effects of medications adding medications to treat substance use disorders. There is insufficient evidence relating to the effects of pharmacologic treatments used for the treatment of psychiatric comorbidities, depression, ADHD, and bipolar disorder in patients with concomitant substance use disorders.

When assigning strength of evidence (SoE) we considered various concepts including whether the conclusions are based on direct (head-to-head) or indirect comparisons (for which there were no head-to-head comparisons) and whether the reported outcomes are direct (true) measures of 
the outcome of interest. In the network meta-analyses we conducted related to nonbrief behavioral interventions, conclusions are based predominately on indirect evidence, with sparse direct evidence. Although there was some variability in the definitions of use days, abstinence and drug related problems, these were deemed to be sufficiently minor so as not to affect the overall directness. In contrast, for subpopulations and additional outcomes that were not meta-analyzed, conclusions were downgraded for being indirect if the outcomes were generally not well-defined and likely varied across studies.

The strength of evidence (SoE) for each conclusion, presented in Table 32, is based on a qualitative combination of the summary risk of bias across all relevant studies, the consistency of the studies, the precision of the available estimates, and the directness of the evidence. 
Table 32. Evidence profile for interventions for substance use disorders and problematic use in adolescents

\begin{tabular}{|c|c|c|c|c|c|c|c|c|c|c|}
\hline $\begin{array}{l}\text { Inter- } \\
\text { ventions }\end{array}$ & Topic & Outcome & Comparison & $\begin{array}{l}\text { No. } \\
\text { Studies } \\
\text { (Subjects) }\end{array}$ & $\begin{array}{l}\text { Risk of } \\
\text { Bias }\end{array}$ & Consistency & Precision & Directness & $\begin{array}{l}\text { Overall } \\
\text { SoE }\end{array}$ & $\begin{array}{l}\text { Conclusion } \\
\text { statements }\end{array}$ \\
\hline \multirow{7}{*}{$\begin{array}{l}\text { Brief } \\
\text { Behavioral } \\
\text { Inter- } \\
\text { ventions }\end{array}$} & \multirow[t]{3}{*}{$\begin{array}{l}\text { Alcohol } \\
\text { outcomes }\end{array}$} & $\begin{array}{l}\text { Heavy use } \\
\text { days }\end{array}$ & MI vs. TAU & $\begin{array}{l}7 \\
(1248)\end{array}$ & Moderate & Consistent & Imprecise & Direct & Low & $\begin{array}{l}\text { MI more } \\
\text { effective } \\
\text { than TAU }\end{array}$ \\
\hline & & Use days & MI vs. TAU & $\begin{array}{l}10 \\
(2153)\end{array}$ & Moderate & Consistent & Precise & $\begin{array}{l}\text { Direct \& } \\
\text { Indirect }\end{array}$ & Moderate & $\begin{array}{l}\text { MI more } \\
\text { effective } \\
\text { than TAU }\end{array}$ \\
\hline & & Abstinence & MI vs. TAU & $\begin{array}{l}7 \\
(2482)\end{array}$ & Moderate & Consistent & Imprecise & $\begin{array}{l}\text { Direct \& } \\
\text { Indirect }\end{array}$ & Insufficient & None \\
\hline & \multirow[t]{2}{*}{$\begin{array}{l}\text { Cannabis } \\
\text { outcomes }\end{array}$} & Use days & MI vs. TAU & $\begin{array}{l}13 \\
(2386)\end{array}$ & Moderate & Consistent & Precise & $\begin{array}{l}\text { Direct \& } \\
\text { Indirect }\end{array}$ & Moderate & $\begin{array}{l}\text { MI not } \\
\text { more } \\
\text { effective } \\
\text { than TAU }\end{array}$ \\
\hline & & Abstinence & MI vs. TAU & $\begin{array}{l}6 \\
(1119)\end{array}$ & Moderate & Consistent & Imprecise & Direct & Insufficient & None \\
\hline & \multirow[t]{2}{*}{$\begin{array}{l}\text { Substance } \\
\text { related } \\
\text { problem } \\
\text { scores }\end{array}$} & $\begin{array}{l}\text { Multiple } \\
\text { substance } \\
\text { use } \\
\text { problem } \\
\text { scales }\end{array}$ & MI vs. TAU & $\begin{array}{l}9 \\
(1854)\end{array}$ & Moderate & Consistent & Imprecise & $\begin{array}{l}\text { Direct \& } \\
\text { indirect }\end{array}$ & Low & $\begin{array}{l}\text { MI more } \\
\text { effective } \\
\text { than TAU }\end{array}$ \\
\hline & & & MI vs. Educ & $\begin{array}{l}3 \\
(646)\end{array}$ & Moderate & Inconsistent & Imprecise & Direct & Insufficient & None \\
\hline \multirow[t]{3}{*}{$\begin{array}{l}\text { Nonbrief } \\
\text { Behavioral } \\
\text { Inter- } \\
\text { ventions }\end{array}$} & $\begin{array}{l}\text { Alcohol } \\
\text { outcomes }\end{array}$ & Use days & $\begin{array}{l}\text { Network of } 11 \\
\text { interventions }\end{array}$ & $\begin{array}{l}11 \\
(2005)\end{array}$ & Moderate & Unclear & Precise & $\begin{array}{l}\text { Direct } \\
\text { (sparse) \& } \\
\text { indirect }\end{array}$ & Low & $\begin{array}{l}\text { Fam more } \\
\text { effective } \\
\text { than TAU, } \\
\text { ICM, CBT, } \\
\text { MI }\end{array}$ \\
\hline & $\begin{array}{l}\text { Cannabis } \\
\text { outcomes }\end{array}$ & Use days & $\begin{array}{l}\text { Network of } 13 \\
\text { Interventions }\end{array}$ & $\begin{array}{l}11 \\
(1643)\end{array}$ & Moderate & Unclear & Imprecise & $\begin{array}{l}\text { Direct } \\
\text { (sparse) \& } \\
\text { Indirect }\end{array}$ & Low & $\begin{array}{l}\text { CBT, } \\
\text { CBT+MI, } \\
\text { CBT+MI+ } \\
\text { CM, Educ } \\
\text { less } \\
\text { effective } \\
\text { than TAU }\end{array}$ \\
\hline & $\begin{array}{l}\text { Alcohol and } \\
\text { other drug } \\
\text { use }\end{array}$ & Use days & $\begin{array}{l}\text { Network of } 8 \\
\text { interventions }\end{array}$ & $\begin{array}{l}9 \\
(1170)\end{array}$ & Moderate & Unclear & Precise & $\begin{array}{l}\text { Direct } \\
\text { (sparse) \& } \\
\text { indirect }\end{array}$ & Low & $\begin{array}{l}\text { MI, CBT } \\
\text { more } \\
\text { effective } \\
\text { than TAU }\end{array}$ \\
\hline
\end{tabular}




\begin{tabular}{|c|c|c|c|c|c|c|c|c|c|c|}
\hline $\begin{array}{l}\text { Inter- } \\
\text { ventions }\end{array}$ & Topic & Outcome & Comparison & $\begin{array}{l}\text { No. } \\
\text { Studies } \\
\text { (Subjects) }\end{array}$ & $\begin{array}{l}\text { Risk of } \\
\text { Bias }\end{array}$ & Consistency & Precision & Directness & $\begin{array}{l}\text { Overall } \\
\text { SoE }\end{array}$ & $\begin{array}{l}\text { Conclusion } \\
\text { statements }\end{array}$ \\
\hline \multirow{11}{*}{$\begin{array}{l}\text { Nonbrief } \\
\text { Behavioral } \\
\text { Inter- } \\
\text { ventions } \\
\text { (contin- } \\
\text { ued) }\end{array}$} & $\begin{array}{l}\text { Illicit drug } \\
\text { use }\end{array}$ & Use days & $\begin{array}{l}\text { Network of } 7 \\
\text { interventions }\end{array}$ & $\begin{array}{l}5 \\
(1310)\end{array}$ & Moderate & Unclear & Precise & $\begin{array}{l}\text { Direct } \\
\text { (sparse) \& } \\
\text { indirect }\end{array}$ & Low & $\begin{array}{l}\text { CBT+MI } \\
\text { more } \\
\text { effective } \\
\text { than TAU }\end{array}$ \\
\hline & & Use days & & & Moderate & Unclear & Imprecise & $\begin{array}{l}\text { Direct } \\
\text { (sparse) \& } \\
\text { indirect }\end{array}$ & Insufficient & $\begin{array}{l}\text { None } \\
\text { (for } \\
\text { comparative } \\
\text { effects) }\end{array}$ \\
\hline & $\begin{array}{l}\text { Opioid use: } \\
\text { Medications, } \\
\text { plus } \\
\text { behavioral } \\
\text { therapies }\end{array}$ & $\begin{array}{l}\text { Use: } \\
\text { abstinence } \\
\text { or reported } \\
\text { use }\end{array}$ & $\begin{array}{l}\text { BUP (longer } \\
\text { vs. shorter } \\
\text { treatment } \\
\text { duration) }\end{array}$ & $\begin{array}{l}2 \\
(207)\end{array}$ & Moderate & Consistent & Imprecise & Direct & Low & $\begin{array}{l}\text { Longer } \\
\text { duration } \\
\text { buprenor- } \\
\text { phine more } \\
\text { effective }\end{array}$ \\
\hline & & & $\begin{array}{l}\text { BUP vs. } \\
\text { Clonidine }\end{array}$ & $\begin{array}{l}1 \\
(36)\end{array}$ & Low & NA & Imprecise & Direct & Insufficient & None \\
\hline & & & $\begin{array}{l}\text { BUP }( \pm 2 \\
\text { doses of } \\
\text { MEM }\end{array}$ & $\begin{array}{l}1 \\
(80)\end{array}$ & Low & NA & Imprecise & Direct & Insufficient & None \\
\hline & $\begin{array}{l}\text { Alcohol use: } \\
\text { Medications }\end{array}$ & $\begin{array}{l}\text { Use: } \\
\text { abstinence } \\
\text { and/or } \\
\text { reported } \\
\text { use }\end{array}$ & $\begin{array}{l}\text { Cyanamide } \\
\text { vs. } \\
\text { Placebo }\end{array}$ & $\begin{array}{l}1 \\
(26)\end{array}$ & Low & NA & Imprecise & Direct & Insufficient & None \\
\hline & & & $\begin{array}{l}\text { Disulfiram vs. } \\
\text { placebo }\end{array}$ & $\begin{array}{l}1 \\
(110)\end{array}$ & Low & NA & Imprecise & Direct & Insufficient & None \\
\hline & & & $\begin{array}{l}\text { Naltrexone } \\
\text { vs. } \\
\text { Placebot }\end{array}$ & $\begin{array}{l}3 \\
(188)\end{array}$ & Low & $\begin{array}{l}\text { Inconsistent } \\
\text { (effective in } 1 \\
\text { of } 3 \text { studies) }\end{array}$ & Imprecise & Direct & Insufficient & None \\
\hline & & & $\begin{array}{l}\text { Disulfiram+ } \\
\text { Educ(group) } \\
\text { vs. } \\
\text { Naltrexone+ } \\
\text { Educ(group) }\end{array}$ & $\begin{array}{l}2 \\
(110)\end{array}$ & Moderate & $\begin{array}{l}\text { Consistent } \\
\text { (single } \\
\text { center) }\end{array}$ & Imprecise & Direct & Insufficient & None \\
\hline & $\begin{array}{l}\text { Cannabis } \\
\text { use: } \\
\text { Medications }\end{array}$ & & $\begin{array}{l}\mathrm{NAC}+\mathrm{CM} \\
\text { vs. } \\
\text { Placebo+CM }\end{array}$ & $\begin{array}{l}1 \\
(116)\end{array}$ & & NA & Imprecise & Direct & Insufficient & None \\
\hline & & & $\begin{array}{l}\text { TOP+MI } \\
\text { vs. } \\
\text { Placebo+MI }\end{array}$ & $\begin{array}{l}1 \\
(66)\end{array}$ & & Inconsistent & Imprecise & Direct & Insufficient & None \\
\hline
\end{tabular}


Abbreviations: BUP = Buprenorphine/Buprenorphine-naloxone; $\mathrm{CBT}=$ cognitive behavioral therapy; $\mathrm{CM}=$ contingency management; Educ = psychoeducation; Fam = family therapy; ICM = intensive case management; $\mathrm{MEM}=$ Memantine; $\mathrm{MI}=$ motivational interviewing; $\mathrm{NAC}=\mathrm{N}$-acetylcysteine; SoE = strength of evidence; TAU = treatment as usual; TOP = Topiramate

†One study compared Naltrexone+MI with Placebo+MI. 


\section{Findings in Relationship to What Is Already Known}

\section{Brief Behavioral Interventions}

Our review of randomized controlled trials (RCTs) found that brief MI or motivation enhancement therapy (MET) reduces heavy alcohol use and overall alcohol use compared to TAU in adolescents with problematic alcohol use. Across brief behavioral interventions, we concluded that MI is more effective than TAU in reducing substance use associated problems. These findings are generally consistent with the recent systematic review with meta-analysis (SR/MA) of experimental and quasiexperimental studies by Tanner-Smith and Lipsey. ${ }^{266}$ Combining across multiple related outcomes and different interventions using a standardized effect size metric, they conclude that adolescents ages 11 to 18 who received brief alcohol interventions had lower levels of self-reported alcohol consumption and alcohol-related problems, and concluded that MI/MET strategies were most effective. ${ }^{266}$ In a subsequent paper, Tanner-Smith and Risser explore the variability of effects across different outcome measures. ${ }^{267}$

We found that MI does not reduce days of cannabis use compared to TAU. Recent reviews have reached mixed conclusions regarding effects on cannabis use. A SR/MA by Li et al. that identified 10 randomized trials evaluating MI interventions for illicit drug use in adolescents. ${ }^{268}$ They pooled use of multiple illicit substances, including cannabis (80\%), cocaine (30\%) and amphetamines/MDMA (20\%) and concluded that there was no statistically significant effect of MI on drug use behaviors. ${ }^{268}$ A SR/MA of adolescents and young adults, ${ }^{269}$ found that brief behavioral interventions targeting both alcohol and other illicit drugs effectively reduced use of "both of these substances." The review concludes that brief interventions that targeted only alcohol had no statistically significant secondary effects on untargeted illicit drug use. ${ }^{269}$ Our analyses of cannabis outcomes included all studies that reported a cannabis-specific outcome. As we discuss in the Limitations of the Evidence Base section, it was often unclear if specific substance use was targeted, and study participants often used multiple substances.

Given the apparent heterogeneity of treatment effects for alcohol and cannabis, it may be problematic to interpret effects for outcomes that combine multiple substances (e.g., alcohol, cannabis, and other drugs), as prior SRs have done.

\section{Nonbrief Behavioral Interventions}

Previous SRs have highlighted specific interventions and combinations of interventions as well established or showing particular promise for reducing substance use in adolescents. A 2018 SR and qualitative synthesis by Hogue et. al. identified several interventions as "well established."27 cognitive behavioral therapy (CBT, delivered both individually and in group format), Fam (delivered with an ecological orientation), MI + CBT, and MI + CBT + Fam (delivered with a behavioral orientation). Additional models were identified as "probably efficacious," including: MI, Fam (delivered with a behavioral orientation), and several multicomponent interventions the include contingency management. ${ }^{27}$

The SR/MA by Tanner-Smith et. al. of 61 experimental or quasi-experimental studies concluded that "most substance use treatment programs were beneficial in helping adolescents reduce their substance use when those treatment programs provide tailored treatment services beyond standard community services. Fam and CBT programs showed particular promise of effectiveness, and no program types showed evidence of harmful effects."270 
We performed separate network meta-analyses (NMA) for each substance specific outcome. This results in sparse networks and a reliance on primarily indirect evidence. Our NMA of the alcohol use days outcome suggests that intensive behavioral intervention with the entire family present (Fam) may be particularly effective. Furthermore, Fam may be more effective than intensive case management, CBT and MI.

We found little evidence to support the effectiveness of any intervention to reduce cannabis use days. Indeed, we found that CBT, $\mathrm{CBT}+\mathrm{MI}$ and $\mathrm{CBT}+\mathrm{MI}+\mathrm{CM}$ and Educ may result in relative increases in cannabis use compared to TAU. These conclusions are tentative, but if true, imply that there may be treatment effect heterogeneity by substance.

\section{Pharmacologic Interventions}

Only four studies evaluated treatment of opioid use disorder in adolescents or young adults. All addressed pharmacologic options for short-term opioid detoxification. There are currently three Food and Drug Administration (FDA)-approved medications for opioid use disorder in adults. For two of these (naltrexone and methadone) we found no studies in adolescents. None of the included studies evaluated long-term medication-assisted treatment.

\section{Pharmacologic Treatment of Psychiatric Comorbidities in Adolescents With Substance Use Disorders}

The narrative review by Brewer et. al. concludes that psychiatric and substance use disorder comorbidity is the rule rather than the exception. ${ }^{271}$ We reviewed outcomes from 10 RCTs of pharmacotherapies used for ADHD, bipolar disorder and depression in adolescents with concomitant substance use. No study demonstrated superior comparative effects on substance use outcomes in subjects given pharmacotherapy compared to placebo. However, most studies were small, with resultant imprecision (wide confidence intervals). Of note, a single RCT ${ }^{256,261}$ that evaluated fluoxetine with CBT+MI for comorbid depression found improvements in depression. Notably, none of the other 9 RCTs found demonstrated an improvement in the target psychiatric disorder.

\section{Applicability}

A number of factors may limit the applicability of our findings.

We aggregated brief behavioral interventions from studies done in various settings (e.g., emergency department (ED), inpatient, outpatient primary care, juvenile justice). Treatment effects may be moderated by context specific factors. For example, brief interventions in the ED after an alcohol-related presentation may represent a "teachable moment," or alternatively, the saliency of the event may prompt self-change. ${ }^{272}$

The diagnostic criteria for substance use disorders are well defined, albeit with variation by DSM version. However, our pragmatic inclusion criteria for problematic use necessarily defined a more heterogeneous group of substance users, likely with substantial variability in the degree of impairment.

Study inclusion criteria varied with regards to the primary substance of misuse, as detailed in Appendix D Some studies explicitly focused on adolescents who primarily misused a specific substance (i.e. alcohol or cannabis) and reported use-related outcomes for that substance only. However, other studies enrolled adolescents who misused a combination of substances, most commonly alcohol and cannabis, and reported some combination of cannabis and alcohol 
outcomes. Other studies reported outcomes for composite use of unspecified substances (e.g., "illicit drug use").

Despite this apparent heterogeneity of inclusion criteria, most studies enrolled alcohol and cannabis users (see Appendix Tables D-1 and D-2), with a minority using other drugs. Within studies, details of specific substances use were often incompletely reported (see Appendix Table D-3).

Behavioral interventions sometimes explicitly targeted multiple substances, e.g. alcohol and other drug use. Unlike some pharmacologic interventions, behavioral interventions are not inherently substance-specific. We therefore chose to aggregate our analysis by substance specific use outcomes, rather than attempting to disentangle effects for a specific use disorder or problematic use of alcohol or cannabis. This choice may limit the applicability of our conclusions to users of a single substance. Furthermore, this approach does not account for possible interaction effects between outcomes within a study, e.g. interventions that successfully target alcohol use might result an increase cannabis use due to substitution of cannabis for alcohol. $^{273}$

The specific outcomes reported in each substance-of-use category are detailed for each study in Appendix E-1 (Brief interventions) and E-2 (Nonbrief interventions). Table 33 below, summarizes the number of behavioral studies in each intervention category that reported an alcohol outcome only, a cannabis outcome only, or both an alcohol and a cannabis outcome.

Table 33. Number of meta-analyzed studies reporting outcomes for alcohol only, cannabis only, or both

\begin{tabular}{l|r|r|r}
\hline $\begin{array}{l}\text { Intervention } \\
\text { Type }\end{array}$ & $\begin{array}{r}\text { AlcoholOnly } \\
\text { Outcomes }\end{array}$ & $\begin{array}{r}\text { Cannabis-Only } \\
\text { Outcomes }\end{array}$ & $\begin{array}{r}\text { Alcohol \& } \\
\text { Cannabis }\end{array}$ \\
\hline Brief & 7 & 7 & 7 \\
\hline Intensive & 6 & 10 & 9 \\
\hline
\end{tabular}

Alcohol only outcomes: heavy-use days, use days, abstinence; Cannabis-only outcomes: use days, abstinence

A few studies explicitly focused on adolescents with SUD and a specific comorbid mental health diagnosis, whereas most studies focused on adolescents with SUD and often did not report on the frequency of comorbid psychiatric comorbidities.

Thus, even when samples were selected based on a specific substance or specific comorbid diagnosis, the populations identified were generally heterogeneous samples of polysubstance using adolescents, many of whom likely have psychiatric comorbidities.

Interventions identified across studies are multicomponent, complex interventions. Rather than simply comparing "brand name" therapy models, we attempted to identify a common set of core intervention components. Classification of active intervention components was dependent on the quality of reporting, and in many cases, specific intervention components were not well described. Determination of the list of classification components of interest and classification of the components for each study required judgements.

Comparator conditions - often generally described as treatment as usual (TAU) - were particularly challenging to classify, as the TAU category defines a heterogeneous collection of often poorly described active interventions. Any classification of interventions cannot perfectly 
capture the complexity of these interventions and relies on highly variable reporting in published reports.

\section{Implications for Clinical and Policy Decision Making}

As summarized in Table 32, there is evidence for the effectiveness of brief behavioral interventions for adolescents with problematic alcohol use. This supports the expansion of current initiatives to implement screening, brief intervention and referral to treatment (SBIRT) adolescents with alcohol use. ${ }^{274}$ However, based on the evidence, it should not be assumed that brief interventions have equal effects on alcohol and cannabis. In particular, brief motivational interviewing appears to be ineffective for adolescents with problematic cannabis use.

Limited evidence suggests that family focused behavioral interventions may be particularly effective in the treatment of alcohol use disorder. No intervention or combination of intensive interventions demonstrated a definite positive effect on increasing abstinence or decreasing overall cannabis use.

Evidence that longer courses of buprenorphine are more effective in the short-term management of opioid withdrawal supports recommendations for increased utilization of buprenorphine for longer-term maintenance therapy. ${ }^{17,275}$

\section{Limitations of the Systematic Review Process}

In our analyses, we used both direct and indirect information to inform comparisons between interventions. When interpreting the results, it is important to note that indirect comparisons rely on an assumption of consistency between indirect and direct evidence. For nonbrief behavioral interventions, the multiplicity of interventions and intervention components resulted in very sparse direct evidence.

We chose to rate an intervention component as present only if the intervention was wellspecified, as evidenced by use of a manual or fidelity monitoring. There were some multicomponent interventions in which the intervention was well-specified and monitored, but specific elements were much less so. Decisions about how to code these interventions were challenging and were directly influenced by the quality of intervention reporting. The use of a codebook and independent coding by three raters, one of which was a content expert and one of which was a methodological expert in multi-component interventions, helped to ensure that we used a consistent approach.

\section{Limitations of the Evidence Base}

For many topics, evidence was sparse and indeterminate — or absent entirely. The bulk of evidence relates to adolescents with problematic use or substance use disorders related to alcohol or cannabis.

Overall, we found very few studies in adolescents or young adults (ages 25 or less) that evaluated pharmacologic or combined pharmacologic and behavioral interventions. We identified 988 trials in adults that enrolled participants 18 years and older but found none that provided an extractable analysis of the adolescent/young adult subgroup.

The evidence regarding treatment of psychiatric comorbidities is quite limited. For the treatment of ADHD, we found no studies that evaluate agents that may have less potential for abuse (e.g., guanfacine and clonidine). 
Outcomes were measured and reported inconsistently. Most studies reported multiple outcomes. The most commonly reported outcome across studies was self-reported substance use days. However, use days were summarized over variable denominators (e.g., 7, 30, or 90 days), expressed as percent or log- or square root-transformed. Other studies quantified indicators of abstinence or substance-related problems use using a variety of scales. The lack of consistently reported outcomes across studies reduced the number of available comparisons.

The available evidence was too sparse to allow identification of key ingredients of successful interventions (moderators of treatment effect) or how intervention effects differ across demographic groups.

Studies took a wide variety of approaches to missing data in subjects who dropped out of treatment. When available, we preferred intention-to-treat analyses or model-based expected means. However, studies often reported only raw summary data based on number analyzed.

\section{Recommendations for Future Research}

There is a need to adopt a set of core outcome measures and standard approaches to reporting of these outcomes. For example, among studies to date, a wide range of outcomes have been used, including measures of frequency of use, abstinence, and substance-related problems. Even among studies that measure "frequency of use," the metrics used (e.g., count of days, proportion of days), specific substances of focus, and recall periods vary substantially. This required us to synthesize data over different recall periods. If all studies had consistently reported a core outcome set utilizing common measures, our summary findings would have been more reliable. Ideally, abstinence and use in studies would be modeled jointly, and may best be synthesized using a hurdle model utilizing individual patient data from individual studies. ${ }^{276,277}$

The observed variability of treatment effects by substance suggests it may difficult to interpret composite outcomes (i.e., alcohol and other drugs, illicit drugs and other drugs). If treatment effects vary by substance, estimates of composite effects will be determined by the relative proportion of alcohol, cannabis, and other drug use in individual studies. Furthermore, the increasing potency of cannabinoid products over time may impact response to therapy. ${ }^{278}$

A core outcome set would be especially valuable if it included standardized definitions of patient-centered outcomes, such as adolescent functioning in school, peer, and family domains. Some studies included secondary functional outcomes, but such outcomes were included inconsistently and varied across reports. More data on functional outcomes are needed to determine whether the reductions in days of use documented in this report translate into meaningful clinically improvements for adolescents.

To support evidence synthesis, studies need to more clearly describe intervention components received by study participants, including those assigned to TAU. For example, rather than simply stating that an intervention was designed to "build motivation" or "build skills," investigators should clearly explicate the underlying theoretical orientation of the intervention. In addition, each unique aspect of a multi-component intervention should be welldescribed, with references to a manual and supportive source materials. Data on fidelity monitoring should ideally be provided for each major component underlying the intervention.

Large "adult" trials that enroll older adolescents (age 18 to 20) should consider reporting this subgroup.

Future research should also seek to clarify whether specific subpopulations may benefit from specific intervention components. Specifically, studies should seek to clarify which interventions are most effective for a given primary substance of misuse, severity of use, and how 
effectiveness is moderated by the presence of co-occurring psychiatric disorders. Much of this research in the United States is funded by the National Institutes of Health via the National Institute on Drug Abuse (NIDA) and the National Institute on Alcohol Abuse and Alcoholism (NIAAA). Harmonized guidance from these agencies with respect to core outcomes might be particularly impactful.

\section{Conclusions}

Compared with TAU (e.g., brief advice and a handout), brief MI for adolescents with problematic substance use increases the likelihood of abstinence from alcohol and reduces both heavy alcohol use and overall days of use. However, brief MI did not decrease cannabis use. MI may decrease problems related to substance use, such as missed school or work or getting into trouble.

Among intensive interventions, Fam (with a focus on intervening in the entire family system) may be the most effective in reducing both days of heavy alcohol and overall alcohol use.

For opioid use disorder, buprenorphine and buprenorphine-naloxone are more effective in the short term management of opioid withdrawal if they are tapered over a longer period of time (i.e. 12 weeks versus 2 weeks, 56 days versus 28 days). Studies of long-term pharmacologic and/or behavioral treatment of opioid use disorder are urgently needed.

Further research is needed to identify: 1) brief and more intensive interventions for problematic cannabis use and cannabis use disorder, 2) effective combinations of behavioral treatments and medication to treat alcohol and cannabis use disorders, and 3) interventions that improve outcomes that are most meaningful to adolescents, such as better functioning in school and improved relationships with peers and parents. 


\section{References}

1. Lipari RN, Park-Lee E, Van Horn S. America's Need for and Receipt of Substance Use Treatment in 2015. The CBHSQ Report. Rockville (MD); 2016:1-7.

2. Dembo R, Belenko S, Childs K, et al. Drug use and sexually transmitted diseases among female and male arrested youths. J Behav Med. 2009 Apr;32(2):129-41. doi: 10.1007/s10865-0089183-2. PMID: 18979194.

3. Chapman SL, Wu LT. Substance Use among Adolescent Mothers: A Review. Child Youth Serv Rev. 2013 May 01;35(5):806-15. doi: 10.1016/j.childyouth.2013.02.004. PMID: 23641120.

4. Racz SJ, Saha S, Trent M, et al. Polysubstance Use among Minority Adolescent Males Incarcerated for Serious Offenses. Child Youth Care Forum. 2015 Apr 16;45(2):205-20. doi: 10.1007/s10566015-9334-x. PMID: 26997851.

5. Crosnoe R. The Connection Between Academic Failure and Adolescent Drinking in Secondary School. Sociol Educ. 2006;79(1):44-60. PMID: 20216913.

6. Volkow N. Comorbidity: Addiction and Other Mental Illnesses. National Institute on Drug Abuse. https://d14rmgtrwzf5a.cloudfront.net/sites/default /files/rrcomorbidity.pdf. Accessed on 21 January, 2017.

7. The National Center on Addiction and Substance Abuse (CASA). ADOLESCENT SUBSTANCE USE: AMERICA'S \#1 PUBLIC HEALTH PROBLEM. http://centeronaddiction.org/addictionresearch/reports/adolescent-substance-use. Accessed on 22 January, 2017.

8. Keyes KM, Brady JE, Li G. Effects of minimum legal drinking age on alcohol and marijuana use: evidence from toxicological testing data for fatally injured drivers aged 16 to 25 years. Inj Epidemiol. 2015 Jan;2. doi: 10.1186/s40621-0140032-1. PMID: 26301177.

9. Wong SS, Zhou B, Goebert D, et al. The risk of adolescent suicide across patterns of drug use: a nationally representative study of high school students in the United States from 1999 to 2009. Soc Psychiatry Psychiatr Epidemiol. 2013 Oct;48(10):1611-20. doi: 10.1007/s00127-0130721-z. PMID: 23744443.
10. Johnston LD, O’Malley PM, Miech RA, et al. Monitoring the Future national survey results on drug use, 1975-2015: Overview, key findings on adolescent drug use. Ann Arbor: Institute for Social Research, The University of Michigan; 2016.

http://www.monitoringthefuture.org/pubs/monog raphs/mtf-overview2015.pdf. Accessed on October 2, 2018.

11. Center for Behavioral Health Statistics and Quality (2016). Key substance use and mental health indicators in the United States: Results from the 2015 National Survey on Drug Use and Health (HHS Publication No. SMA 16-4984, NSDUH Series H-51).

https://www.samhsa.gov/data/sites/default/files/N SDUH-FFR1-2015/NSDUH-FFR12015/NSDUH-FFR1-2015.htm

12. Cerda M, Santaella J, Marshall BD, et al. Nonmedical Prescription Opioid Use in Childhood and Early Adolescence Predicts Transitions to Heroin Use in Young Adulthood: A National Study. The Journal of pediatrics. 2015 Sep;167(3):605-12 e1-2. doi: 10.1016/j.jpeds.2015.04.071. PMID: 26054942.

13. Curtin SC, Tejada-Vera B, Warner M. Drug Overdose Deaths among Adolescents Aged 1519 in the United States: 1999-2015. NCHS Data Brief. Number 282. National Center for Health Statistics. 2017.

14. Bukstein OG, Bernet W, Arnold V, et al. Practice parameter for the assessment and treatment of children and adolescents with substance use disorders. J Am Acad Child Adolesc Psychiatry. 2005 2005/6;44(6):609-21. doi: 10.1097/01.chi.0000159135.33706.37.

15. MacIntyre J, Pruitt D, Houston M, et al. Back to Project Future: plan for the coming decade. Washington, DC: American Academy of Child and Adolescent Psychiatry. 2014.

16. National Institute on Drug Abuse. Principles of Adolescent Substance Use Disorder Treatment: A Research-Based Guide. January 2014. https://d14rmgtrwzf5a.cloudfront.net/sites/default /files/podata_1_17_14.pdf

17. Committee On Substance Use and Prevention. Medication-Assisted Treatment of Adolescents With Opioid Use Disorders. Pediatrics. 2016 2016/9;138(3). doi: 10.1542/peds.2016-1893. 
18. Amini F, Zilberg NJ, Burke EL, et al. A controlled study of inpatient vs. outpatient treatment of delinquent drug abusing adolescents: one year results. Compr Psychiatry. 1982;23(5):436-44. PMID: CN-00182281.

19. American Psychiatric Association. Diagnostic and statistical manual of mental disorders: DSMIII-R. Washington, DC: American Psychiatric Association; 1987.

20. American Psychiatric Association. Diagnostic and statistical Manual of Mental Disorders: DSM-IV. Washington, DC: American Psychiatric Association. 1994.

21. American Psychiatric Association. Diagnostic and statistical manual of mental disorders (DSM5®): American Psychiatric Pub; 2013.

22. Bush K, Kivlahan DR, McDonell MB, et al. The AUDIT alcohol consumption questions (AUDITC): an effective brief screening test for problem drinking. Archives of Internal Medicine. 1998;158(16):1789-95. DOI: 10.1001/archinte.158.16.1789. PMID: 9738608

23. Hogue A, Henderson CE, Ozechowski TJ, et al. Evidence base on outpatient behavioral treatments for adolescent substance use: updates and recommendations 2007-2013. J Clin Child Adolesc Psychol. 2014 2014/6/13;43(5):695-720. doi: 10.1080/15374416.2014.915550.

24. Hogue A, Henderson CE, Schmidt AT. Multidimensional Predictors of Treatment Outcome in Usual Care for Adolescent Conduct Problems and Substance Use. Adm Policy Ment Health. 2017 May;44(3):380-94. doi: 10.1007/s10488-016-0724-7. PMID: 26884380.

25. Tanner-Smith EE, Wilson SJ, Lipsey MW. The comparative effectiveness of outpatient treatment for adolescent substance abuse: a meta-analysis. Journal of substance abuse treatment. 2013 Feb;44(2):145-58. doi: 10.1016/j.jsat.2012.05.006. PMID: 22763198.

26. Becker SJ, Curry JF. Outpatient interventions for adolescent substance abuse: a quality of evidence review. J Consult Clin Psychol. 2008 2008/8;76(4):531-43. doi: 10.1037/0022006X.76.4.531.
27. Hogue A, Henderson CE, Becker SJ, et al. Evidence Base on Outpatient Behavioral Treatments for Adolescent Substance Use, 20142017: Outcomes, Treatment Delivery, and Promising Horizons. J Clin Child Adolesc Psychol. 2018 Jul-Aug;47(4):499-526. doi: 10.1080/15374416.2018.1466307. PMID: 29893607.

28. Higgins JP, Altman DG, Gotzsche PC, et al. The Cochrane Collaboration's tool for assessing risk of bias in randomised trials. BMJ. 2011 Oct 18;343:d5928. doi: 10.1136/bmj.d5928. PMID: 22008217.

29. Wells GA, Shea B, O'Connell B, et al. The Newcastle-Ottawa Scale (NOS) for assessing the quality of nonrandomised studies in metaanalyses. http://www.ohri.ca/programs/clinical_epidemiolo gy/oxford.asp.

30. Shea BJ, Reeves BC, Wells G, et al. AMSTAR 2: a critical appraisal tool for systematic reviews that include randomised or non-randomised studies of healthcare interventions, or both. BMJ. 2017 Sep 21;358:j4008. doi: 10.1136/bmj.j4008. PMID: 28935701.

31. Marsden J, Stillwell G, Barlow H, et al. An evaluation of a brief motivational intervention among young ecstasy and cocaine users: no effect on substance and alcohol use outcomes. Addiction. 2006 Jul;101(7):1014-26. doi: 10.1111/j.1360-0443.2006.01290.x. PMID: 16771893.

32. Srisurapanont M, Sombatmai S, Boripuntakul T. Brief intervention for students with methamphetamine use disorders: a randomized controlled trial. Am J Addict. 2007 MarApr;16(2):111-6. doi: 10.1080/10550490601184431. PMID: 17453612.

33. Ogel K, Coskun S. Cognitive behavioral therapybased brief intervention for volatile substance misusers during adolescence: a follow-up study. Subst Use Misuse. 2011;46 Suppl 1:128-33. doi: 10.3109/10826084.2011.580233. PMID: 21609157.

34. R Core Team. R: A language and environment for statistical computing. 2013.

35. Viechtbauer W. Conducting Meta-Analyses in R with the metafor Package. Journal of Statistical Software. 2010;36(3). doi: 10.18637/jss.v036.i03. 
36. van Valkenhoef G, Kuiper J. gemtc: Network Meta-Analysis Using Bayesian Methods. 2016.

37. Dias S, Sutton AJ, Ades AE, et al. Evidence synthesis for decision making 2: a generalized linear modeling framework for pairwise and network meta-analysis of randomized controlled trials. Med Decis Making. 2012 26 October;33(5):607-17. doi: 10.1177/0272989X12458724.PMID: 23104435

38. Turner RM, Davey J, Clarke MJ, et al. Predicting the extent of heterogeneity in meta-analysis, using empirical data from the Cochrane Database of Systematic Reviews. Int J Epidemiol. 2012 Jun;41(3):818-27. doi: 10.1093/ije/dys041. PMID: 22461129.

39. Turner RM, Jackson D, Wei Y, et al. Predictive distributions for between-study heterogeneity and simple methods for their application in Bayesian meta-analysis. Stat Med. 2015 Mar 15;34(6):98498. doi: 10.1002/sim.6381. PMID: 25475839.

40. Rhodes KM, Turner RM, Higgins JP. Predictive distributions were developed for the extent of heterogeneity in meta-analyses of continuous outcome data. J Clin Epidemiol. 2015 Jan;68(1):52-60. doi: 10.1016/j.jclinepi.2014.08.012. PMID: 25304503.

41. Hornik K, Leisch F, Zeileis A. JAGS: A program for analysis of Bayesian graphical models using Gibbs sampling. Proceedings of the 3rd International Workshop on Distributed Statistical Computing (\{DSC\} 2003), March 20-22, Vienna, Austria.

42. van Valkenhoef G, Dias S, Ades AE, et al. Automated generation of node-splitting models for assessment of inconsistency in network metaanalysis. Res Synth Methods. 2016 March;7(1):80-93. DOI: 10.1002/jrsm.1167. PMID: 26461181.

43. Mbuagbaw L, Rochwerg B, Jaeschke R, et al. Approaches to interpreting and choosing the best treatments in network meta-analyses. Syst Rev. 2017 Apr 12;6(1):79. doi: 10.1186/s13643-0170473-z. PMID: 28403893.
44. Berkman ND, Lohr KN, Ansari M, et al. Grading the Strength of a Body of Evidence When Assessing Health Care Interventions for the Effective Health Care Program of the Agency for Healthcare Research and Quality: An Update.

Methods Guide for Effectiveness and Comparative Effectiveness Reviews. Rockville (MD): Agency for Healthcare Research and Quality (US); 2008.

45. Cuijpers P, Turner EH, Koole SL, et al. What is the threshold for a clinically relevant effect? The case of major depressive disorders. Depress Anxiety. 2014 May;31(5):374-8. doi: 10.1002/da.22249. PMID: 24677535.

46. Guyatt GH, Oxman AD, Vist GE, et al. GRADE: an emerging consensus on rating quality of evidence and strength of recommendations. Bmj. 2008 Apr 26;336(7650):924-6. doi: 10.1136/bmj.39489.470347.AD. PMID: 18436948.

47. Atkins D, Chang SM, Gartlehner G, et al. Assessing applicability when comparing medical interventions: AHRQ and the Effective Health Care Program. J Clin Epidemiol. 2011 Nov;64(11):1198-207. doi: 10.1016/j.jclinepi.2010.11.021. PMID: 21463926.

48. Arnaud N, Baldus C, Elgán TH, et al. Moderators of outcome in a web-based substance use intervention for adolescents. Sucht: Zeitschrift für Wissenschaft und Praxis. 2015;61(6):377-87. doi: 10.1024/0939-5911.a000397. PMID: 201603749-004.

49. Arnaud N, Baldus C, Elgan TH, et al. Effectiveness of a Web-Based Screening and Fully Automated Brief Motivational Intervention for Adolescent Substance Use: A Randomized Controlled Trial. J Med Internet Res. 2016 May 24;18(5):e103. doi: 10.2196/jmir.4643. PMID: 27220276.

50. Arnaud N, Diestelkamp S, Wartberg L, et al. Short- to Midterm Effectiveness of a Brief Motivational Intervention to Reduce Alcohol Use and Related Problems for Alcohol Intoxicated Children and Adolescents in Pediatric Emergency Departments: A Randomized Controlled Trial. Academic emergency medicine: official journal of the Society for Academic Emergency Medicine. 2017 Feb;24(2):186-200. doi: 10.1111/acem.13126. PMID: 27801991. 
51. Bernstein E, Edwards E, Dorfman D, et al. Screening and brief intervention to reduce marijuana use among youth and young adults in a pediatric emergency department. Academic emergency medicine: official journal of the Society for Academic Emergency Medicine. 2009 Nov;16(11):1174-85. doi: 10.1111/j.15532712.2009.00490.x. PMID: 20053238.

52. Bernstein J, Heeren T, Edward E, et al. A brief motivational interview in a pediatric emergency department, plus 10-day telephone follow-up, increases attempts to quit drinking among youth and young adults who screen positive for problematic drinking. Academic emergency medicine: official journal of the Society for Academic Emergency Medicine. 2010 Aug;17(8):890-902. doi: 10.1111/j.15532712.2010.00818.x. PMID: 20670329.

53. Braciszewski JM, Wernette GKT, Moore RS, et al. A pilot randomized controlled trial of a technology-based substance use intervention for youth exiting foster care. Children and Youth Services Review. 2018;94:466-76. doi: 10.1016/j.childyouth.2018.08.011. PMID: 132804409 .

54. Brown RA, Abrantes AM, Minami H, et al. Motivational Interviewing to Reduce Substance Use in Adolescents with Psychiatric Comorbidity. Journal of substance abuse treatment. 2015 Dec;59:20-9. doi: 10.1016/j.jsat.2015.06.016. PMID: 26362000.

55. Colby SM, Orchowski L, Magill M, et al. Brief Motivational Intervention for Underage Young Adult Drinkers: Results from a Randomized Clinical Trial. Alcohol Clin Exp Res. 2018 Jul;42(7):1342-51. doi: 10.1111/acer.13770. PMID: 29750362.

56. Magill M, Colby SM, Orchowski L, et al. How does brief motivational intervention change heavy drinking and harm among underage young adult drinkers? Journal of consulting and clinical psychology. 2017 May;85(5):447-58. doi: 10.1037/сcp0000200. PMID: 28287800.

57. Magill M, Janssen T, Mastroleo N, et al. Motivational interviewing technical process and moderated relational process with underage young adult heavy drinkers. Psychology of addictive behaviors: journal of the Society of Psychologists in Addictive Behaviors. 2019 Mar;33(2):128-38. doi: 10.1037/adb0000440. PMID: 30640505.
58. Cunningham RM, Chermack ST, Ehrlich PF, et al. Alcohol Interventions Among Underage Drinkers in the ED: A Randomized Controlled Trial. Pediatrics. 2015 Oct;136(4):e783-93. doi: 10.1542/peds.2015-1260. PMID: 26347440.

59. Ngo QM, Eisman AB, Walton MA, et al. Emergency Department Alcohol Intervention: Effects on Dating Violence and Depression. Pediatrics. 2018 Jul;142(1). doi: 10.1542/peds.2017-3525. PMID: 29871891.

60. Walton MA, Ngo QM, Chermack ST, et al. Understanding Mechanisms of Change for Brief Alcohol Interventions Among Youth: Examination of Within-Session Interactions. Journal of studies on alcohol and drugs. 2017 Sep;78(5):725-34. PMID: 28930060.

61. Ehrlich PF, Roche JS, Cunningham RM, et al. Underage drinking, brief interventions, and trauma patients: Are they really special? J Trauma Acute Care Surg. 2016 Jul;81(1):149-55. doi: 10.1097/ta.0000000000001093. PMID: 27120317.

62. Walton MA, Chermack ST, Blow FC, et al. Components of Brief Alcohol Interventions for Youth in the Emergency Department. Substance abuse. 2015;36(3):339-49. doi: 10.1080/08897077.2014.958607. PMID: 25222484.

63. D'Amico EJ, Miles JN, Stern SA, et al. Brief motivational interviewing for teens at risk of substance use consequences: a randomized pilot study in a primary care clinic. Journal of substance abuse treatment. $2008 \mathrm{Jul}$;35(1):53-61. doi: 10.1016/j.jsat.2007.08.008. PMID: 18037603.

64. D'Amico EJ, Parast L, Shadel WG, et al. Brief motivational interviewing intervention to reduce alcohol and marijuana use for at-risk adolescents in primary care. Journal of consulting and clinical psychology. 2018 Sep;86(9):775-86. doi: 10.1037/ccp0000332. PMID: 30138016.

65. de Gee EA, Verdurmen JE, Bransen E, et al. A randomized controlled trial of a brief motivational enhancement for non-treatmentseeking adolescent cannabis users. Journal of substance abuse treatment. 2014 Sep;47(3):1818. doi: 10.1016/j.jsat.2014.05.001. PMID: 24969735. 
66. Dembo R, Briones-Robinson R, Wareham J, et al. Impact of Brief Intervention Services on Drug Using Truant Youth Arrest Charges over Time. J Child Adolesc Subst Abuse. 2014;23(6):375-88. doi: 10.1080/1067828x.2012.741560. PMID: 25382960 .

67. Dembo R, Briones-Robinson R, Ungaro R, et al. Brief Intervention for Truant Youth Sexual Risk Behavior and Alcohol Use: A Parallel Process Growth Model Analysis. J Child Adolesc Subst Abuse. 2014;23(3):155-68. doi: 10.1080/1067828x.2013.786643. PMID: 25242878.

68. Dembo R, Briones-Robinson R, Wareham J, et al. Brief Intervention Impact on Truant Youth Attitudes to School and School Behavior Problems: A Longitudinal Study. J Educ Develop Psychol. 2014 Jan 1;4(1):163-93. PMID: 25247027.

69. Dembo R. Brief intervention impact on truant youths' marijuana use: Eighteen-month followup. Journal of child \& adolescent substance abuse. 2016;25(1):18 - EOA. doi: 10.1080/1067828X.2013.872068. PMID: 25642126.

70. Giles EL, McGeechan GJ, Coulton S, et al. Brief alcohol intervention for risky drinking in young people aged 14-15 years in secondary schools: the SIPS JR-HIGH RCT. Southampton (UK); 2019.

71. Martin G, Copeland J. The adolescent cannabis check-up: randomized trial of a brief intervention for young cannabis users. Journal of substance abuse treatment. 2008 Jun;34(4):407-14. doi: 10.1016/j.jsat.2007.07.004. PMID: 17869051.

72. Martínez Martínez KI, Cabrera FJP, De Los Ángeles Vicío Muro M, et al. Consejo breve para adolescentes escolares que abusan del alcohol. = School-based brief counseling for teenage drinkers. Revista Mexicana de Análisis de la Conducta. 2008;34(2):247-64. PMID: 200905582-007.

73. Mason M, Light J, Campbell L, et al. Peer Network Counseling with Urban Adolescents: A Randomized Controlled Trial with Moderate Substance Users. Journal of substance abuse treatment. 2015 Nov;58:16-24. doi: 10.1016/j.jsat.2015.06.013. PMID: 26234955.
74. Mason MJ, Zaharakis NM, Sabo R. Reducing social stress in urban adolescents with peer network counseling. Journal of Child and Family Studies. 2016;25(12):3488-96. doi: 10.1007/s10826-016-0515-5. PMID: 201640310-001.

75. Mason MJ, Sabo R, Zaharakis NM. Peer Network Counseling as Brief Treatment for Urban Adolescent Heavy Cannabis Users. Journal of studies on alcohol and drugs. 2017 Jan;78(1):152-7. PMID: 27936376.

76. McCambridge J, Strang J. The efficacy of singlesession motivational interviewing in reducing drug consumption and perceptions of drugrelated risk and harm among young people: results from a multi-site cluster randomized trial. Addiction. 2004 Jan;99(1):39-52. PMID: 14678061.

77. McCambridge J, Strang J. Deterioration over time in effect of Motivational Interviewing in reducing drug consumption and related risk among young people. Addiction. 2005 Apr;100(4):470-8. doi: 10.1111/j.13600443.2005.01013.x. PMID: 15784061.

78. McCambridge J, Slym RL, Strang J. Randomized controlled trial of motivational interviewing compared with drug information and advice for early intervention among young cannabis users. Addiction. 2008 Nov;103(11):1809-18. doi: 10.1111/j.1360-0443.2008.02331.x. PMID: 18778385.

79. McCambridge J, Day M, Thomas BA, et al. Fidelity to Motivational Interviewing and subsequent cannabis cessation among adolescents. Addictive behaviors. 2011 Jul;36(7):749-54. doi: 10.1016/j.addbeh.2011.03.002. PMID: 21440994.

80. Faulkner N, McCambridge J, Slym RL, et al. It ain't what you do, it's the way that you do it: a qualitative study of advice for young cannabis users. Drug Alcohol Rev. 2009 Mar;28(2):12934. doi: 10.1111/j.1465-3362.2008.00033.x. PMID: 19320697.

81. McCarty CA, Gersh E, Katzman K, et al. Screening and brief intervention with adolescents with risky alcohol use in school-based health centers: A randomized clinical trial of the Check Yourself tool. Substance abuse. 2019 Mar 18:1-9. doi: 10.1080/08897077.2019.1576090. PMID: 30883284. 
82. Monti PM, Colby SM, Barnett NP, et al. Brief intervention for harm reduction with alcoholpositive older adolescents in a hospital emergency department. Journal of consulting and clinical psychology. 1999 Dec;67(6):989-94. PMID: 10596521.

83. Peterson PL, Baer JS, Wells EA, et al. Short-term effects of a brief motivational intervention to reduce alcohol and drug risk among homeless adolescents. Psychology of addictive behaviors: journal of the Society of Psychologists in Addictive Behaviors. 2006 Sep;20(3):254-64. doi: 10.1037/0893-164x.20.3.254. PMID: 16938063.

84. Smith DC, Ureche DJ, Davis JP, et al. Motivational Interviewing With and Without Normative Feedback for Adolescents With Substance Use Problems: A Preliminary Study. Substance abuse. 2015;36(3):350-8. doi: 10.1080/08897077.2014.988838. PMID: 25551562.

85. Spijkerman R, Roek MA, Vermulst A, et al. Effectiveness of a web-based brief alcohol intervention and added value of normative feedback in reducing underage drinking: a randomized controlled trial. J Med Internet Res. 2010 Dec 19;12(5):e65. doi: 10.2196/jmir.1465. PMID: 21169172.

86. Spirito A, Monti PM, Barnett NP, et al. A randomized clinical trial of a brief motivational intervention for alcohol-positive adolescents treated in an emergency department. The Journal of pediatrics. 2004 Sep;145(3):396-402. doi: 10.1016/j.jpeds.2004.04.057. PMID: 15343198.

87. Spirito A, Sindelar-Manning H, Colby SM, et al. Individual and family motivational interventions for alcohol-positive adolescents treated in an emergency department: results of a randomized clinical trial. Archives of pediatrics \& adolescent medicine. 2011 Mar;165(3):269-74. doi: 10.1001/archpediatrics.2010.296. PMID: 21383276.

88. Becker SJ, Jones RN, Hernandez L, et al. Moderators of Brief Motivation-Enhancing Treatments for Alcohol-Positive Adolescents Presenting to the Emergency Department. Journal of substance abuse treatment. 2016 Oct;69:28-34. doi: 10.1016/j.jsat.2016.06.014. PMID: 27568507.
89. Spirito A, Hernandez L, Cancilliere MK, et al. Parent and Adolescent Motivational Enhancement Intervention for Substance-Using, Truant Adolescents: A Pilot Randomized Trial. J Clin Child Adolesc Psychol. 2017 Dec 18:1-13. doi: 10.1080/15374416.2017.1399402. PMID: 29252011.

90. Stein LA, Clair M, Lebeau R, et al. Motivational interviewing to reduce substance-related consequences: effects for incarcerated adolescents with depressed mood. Drug Alcohol Depend. 2011 Nov 1;118(2-3):475-8. doi: 10.1016/j.drugalcdep.2011.03.023. PMID: 21531089.

91. Stein LA, Lebeau R, Colby SM, et al. Motivational interviewing for incarcerated adolescents: effects of depressive symptoms on reducing alcohol and marijuana use after release. Journal of studies on alcohol and drugs. 2011 May;72(3):497-506. PMID: 21513687.

92. Clair M, Stein LA, Soenksen S, et al. Ethnicity as a moderator of motivational interviewing for incarcerated adolescents after release. Journal of substance abuse treatment. 2013 Oct;45(4):370-5. doi: 10.1016/j.jsat.2013.05.006. PMID: 23810265.

93. Stein LA, Colby SM, Barnett NP, et al. Effects of motivational interviewing for incarcerated adolescents on driving under the influence after release. Am J Addict. 2006;15 Suppl 1:50-7. doi: 10.1080/10550490601003680. PMID: 17182420.

94. Rosengard C, Stein LA, Barnett NP, et al. Randomized Clinical Trial of Motivational Enhancement of Substance Use Treatment Among Incarcerated Adolescents: Post-Release Condom Non-Use. J HIV AIDS Prev Child Youth. 2008 Feb 1;8(2):45-64. doi: 10.1300/J499v08n02_04. PMID: 19809580.

95. Tait RJ, Hulse GK, Robertson SI. Effectiveness of a brief-intervention and continuity of care in enhancing attendance for treatment by adolescent substance users. Drug Alcohol Depend. 2004 Jun 11;74(3):289-96. doi: 10.1016/j.drugalcdep.2004.01.003. PMID: 15194207.

96. Tait RJ, Hulse GK, Robertson SI, et al. Emergency department-based intervention with adolescent substance users: 12-month outcomes. Drug Alcohol Depend. 2005 Sep 1;79(3):359-63. doi: 10.1016/j.drugalcdep.2005.03.015. PMID: 16102378 . 
97. Tait RJ, Hulse GK. Adolescent substance use and hospital presentations: a record linkage assessment of 12-month outcomes. Drug Alcohol Depend. 2005 Sep 1;79(3):365-71. doi: 10.1016/j.drugalcdep.2005.03.016. PMID: 15896928.

98. Voogt CV, Kleinjan M, Poelen EA, et al. The effectiveness of a web-based brief alcohol intervention in reducing heavy drinking among adolescents aged 15-20 years with a low educational background: a two-arm parallel group cluster randomized controlled trial. BMC Public Health. 2013 Jul 30;13:694. doi: 10.1186/1471-2458-13-694. PMID: 23895403.

99. Walker DD, Roffman RA, Stephens RS, et al. Motivational enhancement therapy for adolescent marijuana users: a preliminary randomized controlled trial. Journal of consulting and clinical psychology. 2006 Jun;74(3):628-32. doi: 10.1037/0022-006x.74.3.628. PMID: 16822119.

100. Walker DD, Stephens R, Roffman R, et al. Randomized controlled trial of motivational enhancement therapy with nontreatment-seeking adolescent cannabis users: a further test of the teen marijuana check-up. Psychology of addictive behaviors: journal of the Society of Psychologists in Addictive Behaviors. 2011 Sep;25(3):474-84. doi: 10.1037/a0024076. PMID: 21688877.

101. Walker DD, Stephens RS, Blevins CE, et al. Augmenting brief interventions for adolescent marijuana users: The impact of motivational check-ins. Journal of consulting and clinical psychology. 2016 Nov;84(11):983-92. doi: 10.1037/сcp0000094. PMID: 27762569.

102. Blevins CE, Banes KE, Stephens RS, et al. A preliminary evaluation of synthetic cannabinoid use among adolescent cannabis users: Characteristics and treatment outcomes. Addictive behaviors. 2016 Dec;63:114-9. doi: 10.1016/j.addbeh.2016.07.005. PMID: 27454353.

103. Blevins CE, Banes KE, Stephens RS, et al. Change in motives among frequent cannabisusing adolescents: Predicting treatment outcomes. Drug Alcohol Depend. 2016 Oct 1;167:175-81. doi: 10.1016/j.drugalcdep.2016.08.018. PMID: 27577862.
104. Blevins CE, Walker DD, Stephens RS, et al. Changing social norms: The impact of normative feedback included in motivational enhancement therapy on cannabis outcomes among heavyusing adolescents. Addictive behaviors. 2018 Jan;76:270-4. doi: 10.1016/j.addbeh.2017.08.030. PMID: 28886575.

105. Winters KC, Leitten W. Brief intervention for drug-abusing adolescents in a school setting. Psychology of addictive behaviors: journal of the Society of Psychologists in Addictive Behaviors. 2007 Jun;21(2):249-54. doi: 10.1037/0893164x.21.2.249. PMID: 17563146.

106. Winters KC, Fahnhorst T, Botzet A, et al. Brief intervention for drug-abusing adolescents in a school setting: outcomes and mediating factors. Journal of substance abuse treatment. 2012 Apr;42(3):279-88. doi: 10.1016/j.jsat.2011.08.005. PMID: 22000326.

107. Winters KC, Lee S, Botzet A, et al. One-year outcomes and mediators of a brief intervention for drug abusing adolescents. Psychology of addictive behaviors: journal of the Society of Psychologists in Addictive Behaviors. 2014 Jun;28(2):464-74. doi: 10.1037/a0035041. PMID: 24955669.

108. Piehler TF, Winters KC. Parental involvement in brief interventions for adolescent marijuana use. Psychology of addictive behaviors: journal of the Society of Psychologists in Addictive Behaviors. 2015 Sep;29(3):512-21. doi: 10.1037/adb0000106. PMID: 26415058.

109. Piehler TF, Winters KC. Decision-making style and response to parental involvement in brief interventions for adolescent substance use. J Fam Psychol. 2017 Apr;31(3):336-46. doi: 10.1037/fam0000266. PMID: 27929312.

110. Braciszewski JM, Moore RS, Stout RL. Rationale for a New Direction in Foster Youth Substance Use Disorder Prevention. J Subst Use. 2014;19(1-2):108-11. doi: 10.3109/14659891.2012.750693. PMID: 26229518.

111. Arnaud N, Broning S, Drechsel M, et al. Webbased screening and brief intervention for polydrug use among teenagers: study protocol of a multicentre two-arm randomized controlled trial. BMC Public Health. 2012 Sep 26;12:826. doi: 10.1186/1471-2458-12-826. PMID: 23013141. 
112. Azrin NH, Donohue B, Besalel VA, et al. Youth drug abuse treatment: a controlled outcome study. Journal of child \& adolescent substance abuse. 1994;3:1-16. PMID: CN-00241903.

113. Azrin NH, Donohue B, Teichner GA, et al. A controlled evaluation and description of individual-cognitive problem solving and familybehavior therapies in dually-diagnosed conductdisordered and substance-dependent youth. Journal of Child \& Adolescent Substance Abuse. 2001;11(1):1-43. doi: 10.1300/J029v11n01_01. PMID: 2002-13926-001.

114. Baer JS, Garrett SB, Beadnell B, et al. Brief motivational intervention with homeless adolescents: evaluating effects on substance use and service utilization. Psychology of addictive behaviors: journal of the Society of Psychologists in Addictive Behaviors. 2007 Dec;21(4):582-6. doi: 10.1037/0893-164x.21.4.582. PMID: 18072842.

115. Burrow-Sanchez JJ, Wrona M. Comparing culturally accommodated versus standard group CBT for Latino adolescents with substance use disorders: a pilot study. Cultur Divers Ethnic Minor Psychol. 2012 Oct;18(4):373-83. doi: 10.1037/a0029439. PMID: 22866693.

116. Burrow-Sanchez JJ, Minami T, Hops H. Cultural accommodation of group substance abuse treatment for Latino adolescents: Results of an RCT. Cultur Divers Ethnic Minor Psychol. 2015 Oct;21(4):571-83. doi: 10.1037/cdp0000023. PMID: 25602465.

117. Burrow-Sanchez JJ, Hops H. A randomized trial of culturally accommodated versus standard group treatment for Latina/o adolescents with substance use disorders: Posttreatment through 12-month outcomes. Cultur Divers Ethnic Minor Psychol. 2018 Dec 3. doi: 10.1037/cdp0000249. PMID: 30507211.

118. D'Amico EJ, Hunter SB, Miles JN, et al. A randomized controlled trial of a group motivational interviewing intervention for adolescents with a first time alcohol or drug offense. Journal of substance abuse treatment. 2013 Nov-Dec;45(5):400-8. doi: 10.1016/j.jsat.2013.06.005. PMID: 23891459.

119. Dakof GA, Henderson CE, Rowe CL, et al. A randomized clinical trial of family therapy in juvenile drug court. J Fam Psychol. 2015 Apr;29(2):232-41. doi: 10.1037/fam0000053. PMID: 25621927.
120. Dennis M, Godley SH, Diamond G, et al. The Cannabis Youth Treatment (CYT) Study: main findings from two randomized trials. Journal of substance abuse treatment. 2004 Oct;27(3):197213. doi: 10.1016/j.jsat.2003.09.005. PMID: 15501373.

121. Esposito-Smythers C, Spirito A, Kahler CW, et al. Treatment of co-occurring substance abuse and suicidality among adolescents: a randomized trial. Journal of consulting and clinical psychology. 2011 Dec;79(6):728-39. doi: 10.1037/a0026074. PMID: 22004303.

122. Figurelli GA, Hartman BW, Kowalski FX, Jr. Assessment of change in scores on personal control orientation and use of drugs and alcohol of adolescents who participate in a cognitively oriented pretreatment intervention. Psychol Rep. 1994 Oct;75(2):939-44. doi: 10.2466/pr0.1994.75.2.939. PMID: 7862806.

123. Friedman AS. Family therapy vs. parent groups: Effects on adolescent drug abusers. Am J Family Therapy. 1989;17(4):335 - 47. PMID: CN00496580.

124. Godley MD, Godley SH, Dennis ML, et al. Preliminary outcomes from the assertive continuing care experiment for adolescents discharged from residential treatment. Journal of substance abuse treatment. 2002 Jul;23(1):21-32. PMID: 12127465.

125. Godley MD, Godley SH, Dennis ML, et al. The effect of assertive continuing care on continuing care linkage, adherence and abstinence following residential treatment for adolescents with substance use disorders. Addiction. 2007 Jan;102(1):81-93. doi: 10.1111/j.13600443.2006.01648.x. PMID: 17207126.

126. Garner BR, Godley MD, Funk RR, et al. The Washington Circle continuity of care performance measure: predictive validity with adolescents discharged from residential treatment. Journal of substance abuse treatment. 2010 Jan;38(1):3-11. doi: 10.1016/j.jsat.2009.05.008. PMID: 19553067.

127. Godley SH, Garner BR, Passetti LL, et al. Adolescent outpatient treatment and continuing care: main findings from a randomized clinical trial. Drug Alcohol Depend. 2010 Jul 1;110(12):44-54. doi: 10.1016/j.drugalcdep.2010.02.003. PMID: 20219293. 
128. Godley MD, Passetti LL, Hunter BD, et al. A randomized trial of Volunteer Recovery Support for Adolescents (VRSA) following residential treatment discharge. Journal of substance abuse treatment. 2019 Mar;98:15-25. doi: 10.1016/j.jsat.2018.11.014. PMID: 30665599.

129. Henderson CE, Wevodau AL, Henderson SE, et al. An independent replication of the AdolescentCommunity Reinforcement Approach with justice-involved youth. Am J Addict. 2016 Apr;25(3):233-40. doi: 10.1111/ajad.12366. PMID: 26992083.

130. Henggeler SW, Pickrel SG, Brondino MJ, et al. Eliminating (almost) treatment dropout of substance abusing or dependent delinquents through home-based multisystemic therapy. Am J Psychiatry. 1996 Mar;153(3):427-8. doi: 10.1176/ajp.153.3.427. PMID: 8610836.

131. Henggeler SW, Pickrel SG, Brondino MJ. Multisystemic treatment of substance-abusing and dependent delinquents: outcomes, treatment fidelity, and transportability. Ment Health Serv Res. 1999 Sep;1(3):171-84. PMID: 11258740.

132. Henggeler SW, Clingempeel WG, Brondino MJ, et al. Four-year follow-up of multisystemic therapy with substance-abusing and substancedependent juvenile offenders. J Am Acad Child Adolesc Psychiatry. 2002 Jul;41(7):868-74. doi: 10.1097/00004583-200207000-00021. PMID: 12108813.

133. Clingempeel WG, Henggeler SW, Pickrel SG, et al. Beyond treatment effects: predicting emerging adult alcohol and marijuana use among substance-abusing delinquents. Am J Orthopsychiatry. 2005 Oct;75(4):540-52. doi: 10.1037/0002-9432.75.4.540. PMID: 16262513.

134. Brown TI, Henggeler SW, Schoenwald SK, et al. Multisystemic treatment of substance abusing and dependent juvenile delinquents: effects on school attendance at posttreatment and 6-month follow-up. Children's services: social policy, research, and practice. 1999;2(2):81-93. PMID: CN-01415585.

135. Henggeler SW, Halliday-Boykins CA, Cunningham PB, et al. Juvenile drug court: enhancing outcomes by integrating evidencebased treatments. Journal of consulting and clinical psychology. 2006 Feb;74(1):42-54. doi: 10.1037/0022-006x.74.1.42. PMID: 16551142.
136. Henggeler SW, McCart MR, Cunningham PB, et al. Enhancing the effectiveness of juvenile drug courts by integrating evidence-based practices. Journal of consulting and clinical psychology. 2012 Apr;80(2):264-75. doi: 10.1037/a0027147. PMID: 22309470.

137. McCart MR, Henggeler SW, Chapman JE, et al. System-level effects of integrating a promising treatment into juvenile drug courts. Journal of substance abuse treatment. 2012 Sep;43(2):23143. doi: 10.1016/j.jsat.2011.10.030. PMID: 22154039.

138. Joanning HH, Thomas F, Quinn W, et al. Treating adolescent drug abuse: a comparison of family systems therapy, group therapy, and family drug education. Journal of martial \& family therapy. 1992;18(4):345-56. PMID: CN00631575.

139. Hogue A, Dauber S, Henderson CE, et al. Randomized Trial of Family Therapy Versus Nonfamily Treatment for Adolescent Behavior Problems in Usual Care. J Clin Child Adolesc Psychol. 2015;44(6):954-69. doi: 10.1080/15374416.2014.963857. PMID: 25496283.

140. Henderson CE, Hogue A, Dauber S. Family therapy techniques and one-year clinical outcomes among adolescents in usual care for behavior problems. Journal of consulting and clinical psychology. 2019 Mar;87(3):308-12. doi: 10.1037/ccp0000376. PMID: 30589350.

141. Kaminer Y, Burleson JA, Blitz C, et al. Psychotherapies for adolescent substance abusers: a pilot study. J Nerv Ment Dis. 1998 Nov;186(11):684-90. PMID: 9824170.

142. Kaminer Y, Burleson JA. Psychotherapies for adolescent substance abusers: 15-month followup of a pilot study. Am J Addict. 1999 Spring;8(2):114-9. PMID: 10365191.

143. Kaminer Y, Burleson JA, Goldberger R. Cognitive-behavioral coping skills and psychoeducation therapies for adolescent substance abuse. J Nerv Ment Dis. 2002 Nov;190(11):737-45. doi: 10.1097/01.Nmd.0000038168.51591.B6. PMID: 12436013.

144. Burleson JA, Kaminer Y. Self-efficacy as a predictor of treatment outcome in adolescent substance use disorders. Addictive behaviors. 2005 Oct;30(9):1751-64. doi: 10.1016/j.addbeh.2005.07.006. PMID: 16095844. 
145. Kaminer Y, Burleson JA. Does temperament moderate treatment response in adolescent substance use disorders? Substance abuse. 2008;29(2):89-95. doi: 10.1080/08897070802093387. PMID: 19042328.

146. Kaminer Y, Burleson JA, Burke RH. Efficacy of outpatient aftercare for adolescents with alcohol use disorders: a randomized controlled study. J Am Acad Child Adolesc Psychiatry. 2008 Dec;47(12):1405-12. doi: 10.1097/CHI.0b013e318189147c. PMID: 18978635.

147. Burleson JA, Kaminer Y, Burke RH. Twelvemonth follow-up of aftercare for adolescents with alcohol use disorders. Journal of substance abuse treatment. 2012 Jan;42(1):78-86. doi: 10.1016/j.jsat.2011.07.001. PMID: 21868186.

148. Kaminer Y, Burleson JA, Goldston DB, et al. Suicidal ideation among adolescents with alcohol use disorders during treatment and aftercare. Am J Addict. 2006;15 Suppl 1:43-9. doi: 10.1080/10550490601006154. PMID: 17182419.

149. Kelly JF, Kaminer Y, Kahler CW, et al. A pilot randomized clinical trial testing integrated 12Step facilitation (iTSF) treatment for adolescent substance use disorder. Addiction. 2017 Dec;112(12):2155-66. doi: 10.1111/add.13920. PMID: 28742932.

150. Killeen TK, McRae-Clark AL, Waldrop AE, et al. Contingency management in community programs treating adolescent substance abuse: a feasibility study. J Child Adolesc Psychiatr Nurs. 2012 Feb;25(1):33-41. doi: 10.1111/j.17446171.2011.00313.x. PMID: 22299805.

151. Latimer WW, Winters KC, D'Zurilla T, et al. Integrated family and cognitive-behavioral therapy for adolescent substance abusers: a stage I efficacy study. Drug Alcohol Depend. 2003 Sep 10;71(3):303-17. PMID: 12957348.

152. Letourneau EJ, McCart MR, Sheidow AJ, et al. First Evaluation of a Contingency Management Intervention Addressing Adolescent Substance Use and Sexual Risk Behaviors: Risk Reduction Therapy for Adolescents. Journal of substance abuse treatment. 2017 Jan;72:56-65. doi: 10.1016/j.jsat.2016.08.019. PMID: 27629581.

153. Liddle HA, Dakof GA, Parker K, et al. Multidimensional family therapy for adolescent drug abuse: results of a randomized clinical trial. Am J Drug Alcohol Abuse. 2001 Nov;27(4):65188. PMID: 11727882.
154. Liddle HA, Rowe CL, Dakof GA, et al. Early intervention for adolescent substance abuse: pretreatment to posttreatment outcomes of a randomized clinical trial comparing multidimensional family therapy and peer group treatment. J Psychoactive Drugs. 2004 Mar;36(1):49-63. doi: 10.1080/02791072.2004.10399723. PMID: 15152709.

155. Henderson CE, Rowe CL, Dakof GA, et al. Parenting practices as mediators of treatment effects in an early-intervention trial of multidimensional family therapy. Am J Drug Alcohol Abuse. 2009;35(4):220-6. doi: 10.1080/00952990903005890. PMID: 20180674.

156. Liddle HA, Rowe CL, Dakof GA, et al. Multidimensional family therapy for young adolescent substance abuse: twelve-month outcomes of a randomized controlled trial. Journal of consulting and clinical psychology. 2009 Feb;77(1):12-25. doi: 10.1037/a0014160. PMID: 19170450.

157. Liddle HA, Dakof GA, Turner RM, et al. Treating adolescent drug abuse: a randomized trial comparing multidimensional family therapy and cognitive behavior therapy. Addiction. 2008 Oct;103(10):1660-70. doi: 10.1111/j.13600443.2008.02274.x. PMID: 18705691.

158. Greenbaum PE, Wang W, Henderson CE, et al. Gender and ethnicity as moderators: Integrative data analysis of multidimensional family therapy randomized clinical trials. J Fam Psychol. 2015 2015/12;29(6):919-30. doi: 10.1037/fam0000127. PMID: 26213796.

159. Henderson CE, Dakof GA, Greenbaum PE, et al. Effectiveness of multidimensional family therapy with higher severity substance-abusing adolescents: report from two randomized controlled trials. Journal of consulting and clinical psychology. 2010 Dec;78(6):885-97. doi: 10.1037/a0020620. PMID: 20873891.

160. Hogue A, Henderson CE, Dauber S, et al. Treatment adherence, competence, and outcome in individual and family therapy for adolescent behavior problems. Journal of consulting and clinical psychology. 2008 Aug;76(4):544-55. doi: 10.1037/0022-006x.76.4.544. PMID: 18665684. 
161. Hogue A, Dauber S, Liddle HA, et al. Linking session focus to treatment outcome in evidencebased treatments for adolescent substance abuse. Psychotherapy (Chic). 2004 Summer;41(2):8396. doi: 10.1037/0033-3204.41.2.83. PMID: 20473370.

162. Liddle HA, Dakof GA, Rowe CL, et al. Multidimensional Family Therapy as a community-based alternative to residential treatment for adolescents with substance use and co-occurring mental health disorders. Journal of substance abuse treatment. $2018 \mathrm{Jul}$;90:47-56. doi: 10.1016/j.jsat.2018.04.011. PMID: 29866383.

163. Lowe J, Liang H, Riggs C, et al. Community partnership to affect substance abuse among Native American adolescents. Am J Drug Alcohol Abuse. 2012 Sep;38(5):450-5. doi: 10.3109/00952990.2012.694534. PMID: 22931079.

164. Najavits LM, Gallop RJ, Weiss RD. Seeking safety therapy for adolescent girls with PTSD and substance use disorder: a randomized controlled trial. J Behav Health Serv Res. 2006 Oct;33(4):453-63. doi: 10.1007/s11414-0069034-2. PMID: 16858633.

165. Rigter H, Henderson CE, Pelc I, et al. Multidimensional family therapy lowers the rate of cannabis dependence in adolescents: a randomised controlled trial in Western European outpatient settings. Drug Alcohol Depend. 2013 Jun 1;130(1-3):85-93. doi: 10.1016/j.drugalcdep.2012.10.013. PMID: 23140805.

166. Hendriks V, van der Schee E, Blanken P. Matching adolescents with a cannabis use disorder to multidimensional family therapy or cognitive behavioral therapy: treatment effect moderators in a randomized controlled trial. Drug Alcohol Depend. 2012 Sep 1;125(1-2):119-26. doi: 10.1016/j.drugalcdep.2012.03.023. PMID: 22560728.

167. Hendriks V, van der Schee E, Blanken P. Treatment of adolescents with a cannabis use disorder: main findings of a randomized controlled trial comparing multidimensional family therapy and cognitive behavioral therapy in The Netherlands. Drug Alcohol Depend. 2011 Dec 1;119(1-2):64-71. doi: 10.1016/j.drugalcdep.2011.05.021. PMID: 21684088.
168. Hendriks VM, van der Schee E, Blanken P. [Multidimensional family therapy and cognitive behavioral therapy in adolescents with a cannabis use disorder: a randomised controlled study]. Tijdschr Psychiatr. 2013;55(10):747-59. PMID: 24166335.

169. van der Pol TM, Hendriks V, Rigter H, et al. Multidimensional family therapy in adolescents with a cannabis use disorder: long-term effects on delinquency in a randomized controlled trial. Child Adolesc Psychiatry Ment Health. 2018;12:44. doi: 10.1186/s13034-018-0248-X. PMID: 30140308.

170. Schaub MP, Henderson CE, Pelc I, et al. Multidimensional family therapy decreases the rate of externalising behavioural disorder symptoms in cannabis abusing adolescents: outcomes of the INCANT trial. BMC Psychiatry. 2014 Jan 31;14:26. doi: 10.1186/1471-244x-1426. PMID: 24485347.

171. Lascaux M, Ionescu S, Phan O. Effectiveness of formalised therapy for adolescents with cannabis dependence: A randomised trial. Drugs: Education, Prevention \& Policy. 2016;23(5):4049. doi: 10.3109/09687637.2016.1153603. PMID: 2016-45389-007.

172. Tossmann P, Jonas B, Rigter H, et al. Multidimensionale Familientherapie (MDFT) bei cannabisbezogenen Störungen [Treating adolescents with cannabis use disorder with multidimensional family therapy (MDFT): Main results of a randomized controlled trial (RCT)]. Sucht. 2012;58(3):157-66. doi: 10.1024/09395911.a000180. PMID: 2012-18423-001.

173. Robbins MS, Szapocznik J, Dillon FR, et al. The efficacy of structural ecosystems therapy with drug-abusing/dependent African American and Hispanic American adolescents. J Fam Psychol. 2008 Feb;22(1):51-61. doi: 10.1037/08933200.22.1.51. PMID: 18266532.

174. Robbins MS, Feaster DJ, Horigian VE, et al. Brief strategic family therapy versus treatment as usual: results of a multisite randomized trial for substance using adolescents. Journal of consulting and clinical psychology. 2011 Dec;79(6):713-27. doi: 10.1037/a0025477. PMID: 21967492. 
175. Robbins MS, Szapocznik J, Horigian VE, et al. Brief strategic family therapy for adolescent drug abusers: a multi-site effectiveness study. Contemp Clin Trials. 2009 May;30(3):269-78. doi: 10.1016/j.cct.2009.01.004. PMID: 19470315.

176. Rynes KN, Rohrbaugh MJ, Lebensohn-Chialvo $F$, et al. Parallel demand-withdraw processes in family therapy for adolescent drug abuse. Psychology of addictive behaviors: journal of the Society of Psychologists in Addictive Behaviors. 2014 Jun;28(2):420-30. doi: 10.1037/a0031812. PMID: 23438248.

177. Feaster DJ, Robbins MS, Horigian V, et al. Statistical issues in multisite effectiveness trials: the case of brief strategic family therapy for adolescent drug abuse treatment. Clin Trials. 2004;1(5):428-39. doi: 10.1191/1740774504cn041oa. PMID: 16279281.

178. Feaster DJ, Robbins MS, Henderson C, et al. Equivalence of family functioning and externalizing behaviors in adolescent substance users of different race/ethnicity. Journal of substance abuse treatment. 2010 Jun;38 Suppl 1:S113-24. doi: 10.1016/j.jsat.2010.01.010. PMID: 20307791.

179. Horigian VE, Feaster DJ, Brincks A, et al. The effects of Brief Strategic Family Therapy (BSFT) on parent substance use and the association between parent and adolescent substance use. Addictive behaviors. 2015 Mar;42:44-50. doi: 10.1016/j.addbeh.2014.10.024. PMID: 25462653.

180. Horigian VE, Weems CF, Robbins MS, et al. Reductions in anxiety and depression symptoms in youth receiving substance use treatment. Am J Addict. 2013 Jul-Aug;22(4):329-37. doi: 10.1111/j.1521-0391.2013.12031.x. PMID: 23795871.

181. Horigian VE, Robbins MS, Dominguez R, et al. Principles for defining adverse events in behavioral intervention research: lessons from a family-focused adolescent drug abuse trial. Clin Trials. 2010 Feb;7(1):58-68. doi: 10.1177/1740774509356575. PMID: 20156957.

182. Rohde P, Waldron HB, Turner CW, et al. Sequenced versus coordinated treatment for adolescents with comorbid depressive and substance use disorders. Journal of consulting and clinical psychology. 2014 Apr;82(2):342-8. doi: 10.1037/a0035808. PMID: 24491069.
183. Rohde P, Turner CW, Waldron HB, et al. Depression Change Profiles in Adolescents Treated for Comorbid Depression/Substance Abuse and Profile Membership Predictors. J Clin Child Adolesc Psychol. 2018 Jul-Aug;47(4):595607. doi: 10.1080/15374416.2015.1118695. PMID: 26890999.

184. Rowe CL, Alberga L, Dakof GA, et al. FamilyBased HIV and Sexually Transmitted Infection Risk Reduction for Drug-Involved Young Offenders: 42-Month Outcomes. Family process. 2016 Jun;55(2):305-20. doi: 10.1111/famp.12206. PMID: 26879671.

185. Santisteban DA, Mena MP, McCabe BE. Preliminary results for an adaptive family treatment for drug abuse in Hispanic youth. J Fam Psychol. 2011 Aug;25(4):610-4. doi: 10.1037/a0024016. PMID: 21639636.

186. Santisteban DA, Mena MP, Muir J, et al. The efficacy of two adolescent substance abuse treatments and the impact of comorbid depression: results of a small randomized controlled trial. Psychiatr Rehabil J. 2015 Mar;38(1):55-64. doi: 10.1037/prj0000106. PMID: 25799306.

187. Schaeffer CM, Henggeler SW, Ford JD, et al. RCT of a promising vocational/employment program for high-risk juvenile offenders. Journal of substance abuse treatment. 2014 Feb;46(2):134-43. doi: 10.1016/j.jsat.2013.06.012. PMID: 23958035.

188. Slesnick N, Prestopnik JL. Ecologically based family therapy outcome with substance abusing runaway adolescents. J Adolesc. 2005 Apr;28(2):277-98. doi: 10.1016/j.adolescence.2005.02.008. PMID: 15878048.

189. Slesnick N, Prestopnik JL, Meyers RJ, et al. Treatment outcome for street-living, homeless youth. Addictive behaviors. 2007 Jun;32(6):1237-51. doi: 10.1016/j.addbeh.2006.08.010. PMID: 16989957.

190. Slesnick N, Prestopnik JL. Comparison of family therapy outcome with alcohol-abusing, runaway adolescents. J Marital Fam Ther. 2009 Jul;35(3):255-77. doi: 10.1111/j.17520606.2009.00121.x. PMID: 19522781. 
191. Slesnick N, Erdem G, Bartle-Haring S, et al. Intervention with substance-abusing runaway adolescents and their families: results of a randomized clinical trial. Journal of consulting and clinical psychology. 2013 Aug;81(4):600-14. doi: 10.1037/a0033463. PMID: 23895088.

192. Guo X, Slesnick N, Feng X. Changes in Family Relationships among Substance Abusing Runaway Adolescents: A Comparison between Family and Individual Therapies. J Marital Fam Ther. 2016 Apr;42(2):299-312. doi: 10.1111/jmft.12128. PMID: 25981755.

193. Slesnick N, Guo X, Feng X. Change in parentand child-reported internalizing and externalizing behaviors among substance abusing runaways: the effects of family and individual treatments. J Youth Adolesc. 2013 Jul;42(7):980-93. doi: 10.1007/s10964-012-9826-z. PMID: 23054350.

194. Slesnick N, Guo X, Brakenhoff B, et al. A comparison of three interventions for homeless youth evidencing substance use disorders: results of a randomized clinical trial. Journal of substance abuse treatment. 2015 Jul;54:1-13. doi: 10.1016/j.jsat.2015.02.001. PMID: 25736623.

195. Slesnick N. Evaluation of Treatments for Homeless Youth: CRA, MET and Case Management. 2013. https://clinicaltrials.gov/ct2/show/NCT01143792.

196. Smith DC, Hall JA, Williams JK, et al. Comparative efficacy of family and group treatment for adolescent substance abuse. Am J Addict. 2006;15 Suppl 1:131-6. doi: 10.1080/10550490601006253. PMID: 17182429.

197. Stanger C, Budney AJ, Kamon JL, et al. A randomized trial of contingency management for adolescent marijuana abuse and dependence. Drug Alcohol Depend. 2009 Dec 1;105(3):240-7. doi: 10.1016/j.drugalcdep.2009.07.009. PMID: 19717250.

198. Ryan SR, Stanger C, Thostenson J, et al. The impact of disruptive behavior disorder on substance use treatment outcome in adolescents. Journal of substance abuse treatment. 2013 MayJun;44(5):506-14. doi: 10.1016/j.jsat.2012.11.003. PMID: 23228436.

199. Brown PC, Budney AJ, Thostenson JD, et al. Initiation of abstinence in adolescents treated for marijuana use disorders. Journal of substance abuse treatment. 2013 Apr;44(4):384-90. doi: 10.1016/j.jsat.2012.08.223. PMID: 23085041.
200. Stanger C, Ryan SR, Scherer EA, et al. Clinicand home-based contingency management plus parent training for adolescent cannabis use disorders. J Am Acad Child Adolesc Psychiatry. 2015 Jun;54(6):445-53.e2. doi: 10.1016/j.jaac.2015.02.009. PMID: 26004659.

201. Stanger C, Scherer EA, Babbin SF, et al. Abstinence based incentives plus parent training for adolescent alcohol and other substance misuse. Psychology of addictive behaviors: journal of the Society of Psychologists in Addictive Behaviors. 2017 Jun;31(4):385-92. doi: 10.1037/adb0000279. PMID: 28414474.

202. Stanger C. Family Based Contingency Management for Adolescent Alcohol Abuse. 2018. https://clinicaltrials.gov/ct2/show/NCT00595478. Accessed on March 5, 2019.

203. Thush C, Wiers RW, Theunissen N, et al. A randomized clinical trial of a targeted intervention to moderate alcohol use and alcoholrelated problems in at-risk adolescents. Pharmacol Biochem Behav. 2007 Feb;86(2):36876. doi: 10.1016/j.pbb.2006.07.023. PMID: 16928395.

204. Tolou-Shams M, Dauria E, Conrad SM, et al. Outcomes of a family-based HIV prevention intervention for substance using juvenile offenders. Journal of substance abuse treatment. 2017 Jun;77:115-25. doi: 10.1016/j.jsat.2017.03.013. PMID: 28476263.

205. Trudeau KJ, Black RA, Kamon JL, et al. A randomized controlled trial of an online relapse prevention program for adolescents in substance abuse treatment. Child \& Youth Care Forum. 2017;46(3):437-54. doi: 10.1007/s10566-0169387-5. PMID: 2017-00657-001.

206. Wagner EF, Hospital MM, Graziano JN, et al. A randomized controlled trial of guided self-change with minority adolescents. Journal of consulting and clinical psychology. 2014 Dec;82(6):112839. doi: 10.1037/a0036939. PMID: 24841864.

207. Acosta SL, Hospital MM, Graziano JN, et al. Pathways to Drinking Among Hispanic/Latino Adolescents: Perceived Discrimination, Ethnic Identity, and Peer Affiliations. J Ethn Subst Abuse. 2015;14(3):270-86. doi: 10.1080/15332640.2014.993787. PMID: 26115004. 
208. Waldron HB, Slesnick N, Brody JL, et al. Treatment outcomes for adolescent substance abuse at 4- and 7-month assessments. Journal of consulting and clinical psychology. 2001 Oct;69(5):802-13. PMID: 11680557.

209. French MT, Zavala SK, McCollister KE, et al. Cost-effectiveness analysis of four interventions for adolescents with a substance use disorder. Journal of substance abuse treatment. 2008 Apr;34(3):272-81. doi: 10.1016/j.jsat.2007.04.008. PMID: 17600651.

210. Zhang J, Slesnick N. Substance use and social stability of homeless youth: A comparison of three interventions. Psychology of addictive behaviors: journal of the Society of Psychologists in Addictive Behaviors. 2018 Dec;32(8):873-84. doi: 10.1037/adb0000424. PMID: 30556713.

211. Carey KB, Scott-Sheldon LA, Carey MP, et al. Individual-level interventions to reduce college student drinking: a meta-analytic review. Addictive behaviors. 2007 Nov;32(11):2469-94. doi: 10.1016/j.addbeh.2007.05.004. PMID: 17590277.

212. Carey KB, Scott-Sheldon LA, Elliott JC, et al. Face-to-face versus computer-delivered alcohol interventions for college drinkers: a metaanalytic review, 1998 to 2010. Clinical psychology review. 2012 Dec;32(8):690-703. doi: 10.1016/j.cpr.2012.08.001. PMID: 23022767.

213. Barnett NP, Read JP. Mandatory alcohol intervention for alcohol-abusing college students: a systematic review. Journal of substance abuse treatment. 2005 Sep;29(2):147-58. doi: 10.1016/j.jsat.2005.05.007. PMID: 16135343.

214. Carey KB, Scott-Sheldon LA, Garey L, et al. Alcohol interventions for mandated college students: A meta-analytic review. Journal of consulting and clinical psychology. 2016 Jul;84(7):619-32. doi: 10.1037/a0040275. PMID: 27100126.

215. Fachini A, Aliane PP, Martinez EZ, et al. Efficacy of brief alcohol screening intervention for college students (BASICS): a meta-analysis of randomized controlled trials. Substance abuse treatment, prevention, and policy. 2012 Sep 12;7:40. doi: 10.1186/1747-597x-7-40. PMID: 22967716.
216. Samson JE, Tanner-Smith EE. Single-Session Alcohol Interventions for Heavy Drinking College Students: A Systematic Review and Meta-Analysis. Journal of studies on alcohol and drugs. 2015 Jul;76(4):530-43. PMID: 26098028.

217. Bogenschutz MP, Abbott PJ, Kushner R, et al. Effects of buprenorphine and hepatitis $\mathrm{C}$ on liver enzymes in adolescents and young adults. J Addict Med. 2010 Dec;4(4):211-6. doi: 10.1097/ADM.0b013e3181c4e27e. PMID: 21170166.

218. Chakrabarti A, Woody GE, Griffin ML, et al. Predictors of buprenorphine-naloxone dosing in a 12-week treatment trial for opioid-dependent youth: secondary analyses from a NIDA Clinical Trials Network study. Drug Alcohol Depend. 2010 Mar 1;107(2-3):253-6. doi: 10.1016/j.drugalcdep.2009.10.014. PMID: 19948382.

219. Gonzalez G, DiGirolamo G, Romero-Gonzalez $\mathrm{M}$, et al. Memantine improves buprenorphine/naloxone treatment for opioid dependent young adults. Drug Alcohol Depend. 2015 Nov 1;156:243-53. doi: 10.1016/j.drugalcdep.2015.09.020. PMID: 26454835.

220. Hill KP, Bennett HE, Griffin ML, et al. Association of cannabis use with opioid outcomes among opioid-dependent youth. Drug Alcohol Depend. 2013 Sep 1;132(1-2):342-5. doi: 10.1016/j.drugalcdep.2013.02.030. PMID: 23528523.

221. Marsch LA, Bickel WK, Badger GJ, et al. Comparison of pharmacological treatments for opioid-dependent adolescents: a randomized controlled trial. Arch Gen Psychiatry. 2005 Oct;62(10):1157-64. doi: 10.1001/archpsyc.62.10.1157. PMID: 16203961.

222. Marsch LA, Moore SK, Borodovsky JT, et al. A randomized controlled trial of buprenorphine taper duration among opioid-dependent adolescents and young adults. Addiction. 2016 Aug;111(8):1406-15. doi: 10.1111/add.13363. PMID: 26918564.

223. Meade CS, Weiss RD, Fitzmaurice GM, et al. HIV risk behavior in treatment-seeking opioiddependent youth: results from a NIDA clinical trials network multisite study. J Acquir Immune Defic Syndr. 2010 Sep;55(1):65-72. doi: 10.1097/QAI.0b013e3181d916db. PMID: 20393347. 
224. Moore SK, Marsch LA, Badger GJ, et al. Improvement in psychopathology among opioiddependent adolescents during behavioralpharmacological treatment. J Addict Med. 2011 Dec;5(4):264-71. doi: 10.1097/ADM.0b013e3182191099. PMID: 22107875.

225. Poole SA, Pecoraro A, Subramaniam G, et al. Presence or Absence of QTc Prolongation in Buprenorphine-Naloxone Among Youth With Opioid Dependence. J Addict Med. 2016 JanFeb;10(1):26-33. doi: 10.1097/adm.0000000000000176. PMID: 26690291.

226. Subramaniam GA, Warden D, Minhajuddin A, et al. Predictors of abstinence: National Institute of Drug Abuse multisite buprenorphine/naloxone treatment trial in opioid-dependent youth. J Am Acad Child Adolesc Psychiatry. 2011 Nov;50(11):1120-8. doi: 10.1016/j.jaac.2011.07.010. PMID: 22024000.

227. Wilcox CE, Bogenschutz MP, Nakazawa M, et al. Concordance between self-report and urine drug screen data in adolescent opioid dependent clinical trial participants. Addictive behaviors. 2013 Oct;38(10):2568-74. doi: 10.1016/j.addbeh.2013.05.015. PMID: 23811060 .

228. Woody GE, Poole SA, Subramaniam G, et al. Extended vs short-term buprenorphine-naloxone for treatment of opioid-addicted youth: a randomized trial. JAMA. 2008

2008/11/5;300(17):2003-11. doi: 10.1001/jama.2008.574. PMID: 18984887.

229. Gonzalez G. Memantine-enhanced Buprenorphine Treatment for Opioid-dependent Young Adults. 2015. https://clinicaltrials.gov/ct2/show/NCT01052662. Accessed on March 5, 2019.

230. De Sousa A. An open randomized trial comparing disulfiram and naltrexone in adolescents with alcohol dependence. Journal of substance use. 2008;13(6):382-8. doi: 10.1080/14659890802305861. PMID: CN00753784.

231. De Sousa A. A comparative study using Disulfiram and Naltrexone in alcohol-dependent adolescents. Journal of substance use. 2014;19(5):341-5. doi: 10.3109/14659891.2013.813084. PMID: CN01014147.
232.Miranda R, Ray L, Blanchard A, et al. Effects of naltrexone on adolescent alcohol cue reactivity and sensitivity: an initial randomized trial. Addict Biol. 2014 Sep;19(5):941-54. doi: 10.1111/adb.12050. PMID: 23489253.

233. Niederhofer H, Staffen W, Mair A. Comparison of cyanamide and placebo in the treatment of alcohol dependence of adolescents. Alcohol Alcohol. 2003 Jan-Feb;38(1):50-3. doi: 10.1093/alcalc/agg011. PMID: 12554608.

234. Niederhofer H, Staffen W, Mair A. Comparison of Naltrexone and Placebo in Treatment of Alcohol Dependence of Adolescents. Alcoholism Treatment Quarterly. 2003 2003/07/10;21(2):8795. doi: 10.1300/J020v21n02_06. PMID: CN00474316.

235. Niederhofer H, Staffen W. Comparison of disulfiram and placebo in treatment of alcohol dependence of adolescents. Drug Alcohol Rev. 2003 Sep;22(3):295-7. doi: 10.1080/0959523031000154436. PMID: 15385223.

236. O'Malley SS, Corbin WR, Leeman RF, et al. Reduction of alcohol drinking in young adults by naltrexone: a double-blind, placebo-controlled, randomized clinical trial of efficacy and safety. $\mathrm{J}$ Clin Psychiatry. 2015 2015/2;76(2):e207-13. doi: 10.4088/JCP.13m08934. PMID: 25742208.

237. Bold KW, Fucito LM, DeMartini KS, et al. Urgency traits moderate daily relations between affect and drinking to intoxication among young adults. Drug Alcohol Depend. 2017 Jan 1;170:59-65. doi: 10.1016/j.drugalcdep.2016.10.035. PMID: 27875802.

238. Bold KW, Fucito LM, Corbin WR, et al. Daily relations among affect, urge, targeted naltrexone, and alcohol use in young adults. Exp Clin Psychopharmacol. 2016 Oct;24(5):367-75. doi: 10.1037/pha0000090. PMID: 27690505.

239. DeMartini KS, Gueorguieva R, Leeman RF, et al. Longitudinal findings from a randomized clinical trial of naltrexone for young adult heavy drinkers. Journal of consulting and clinical psychology. 2016 Feb;84(2):185-90. doi: 10.1037/ccp0000053. PMID: 26654213.

240. Miranda R, Jr., Treloar H, Blanchard A, et al. Topiramate and motivational enhancement therapy for cannabis use among youth: a randomized placebo-controlled pilot study. Addict Biol. 2017 May;22(3):779-90. doi: 10.1111/adb.12350. PMID: 26752416. 
241. Bentzley JP, Tomko RL, Gray KM. Low Pretreatment Impulsivity and High Medication Adherence Increase the Odds of Abstinence in a Trial of N-Acetylcysteine in Adolescents with Cannabis Use Disorder. Journal of substance abuse treatment. 2016 Apr;63:72-7. doi: 10.1016/j.jsat.2015.12.003. PMID: 26827257.

242. Gray KM, Carpenter MJ, Baker NL, et al. A double-blind randomized controlled trial of Nacetylcysteine in cannabis-dependent adolescents. Am J Psychiatry. 2012 Aug;169(8):805-12. doi: 10.1176/appi.ajp.2012.12010055. PMID: 22706327.

243. Squeglia LM, Baker NL, McClure EA, et al. Alcohol use during a trial of $\mathrm{N}$-acetylcysteine for adolescent marijuana cessation. Addictive behaviors. 2016 Dec;63:172-7. doi: 10.1016/j.addbeh.2016.08.001. PMID: 27521979.

244. Tomko RL, Gilmore AK, Gray KM. The role of depressive symptoms in treatment of adolescent cannabis use disorder with N-Acetylcysteine. Addictive behaviors. 2018 Oct;85:26-30. doi: 10.1016/j.addbeh.2018.05.014. PMID: 29803870.

245. Gray JC, Treloar Padovano H, Wemm SE, et al. Predictors of Topiramate Tolerability in Heavy Cannabis-Using Adolescents and Young Adults: A Secondary Analysis of a Randomized, DoubleBlind, Placebo-Controlled Trial. J Clin Psychopharmacol. 2018 Apr;38(2):134-7. doi: 10.1097/jcp.0000000000000843. PMID: 29424802.

246. Cornelius JR, Bukstein OG, Douaihy AB, et al. Double-blind fluoxetine trial in comorbid MDDCUD youth and young adults. Drug Alcohol Depend. 2010 Nov 1;112(1-2):39-45. doi: 10.1016/j.drugalcdep.2010.05.010. PMID: 20576364.

247. Cornelius JR, Bukstein OG, Wood DS, et al. Double-blind placebo-controlled trial of fluoxetine in adolescents with comorbid major depression and an alcohol use disorder. Addictive behaviors. 2009 Oct;34(10):905-9. doi: 10.1016/j.addbeh.2009.03.008. PMID: 19321268.
248. Cornelius JR, Kirisci L. Assessing TLI as a predictor of treatment seeking for SUD among youth transitioning to young adulthood. In: Columbus AM, Columbus AM, eds. Advances in psychology research. Hauppauge, NY, US: Nova Science Publishers; 2013:85-94. PMID: 25379028.

249. Cornelius JR, Salloum IM, Ferrell R, et al. Treatment trial and long-term follow-up evaluation among comorbid youth with major depression and a cannabis use disorder. Int J Med Biol Front. 2012;18(6):399-411. PMID: 25328373.

250. Findling RL, Pagano ME, McNamara NK, et al. The short-term safety and efficacy of fluoxetine in depressed adolescents with alcohol and cannabis use disorders: a pilot randomized placebo-controlled trial. Child Adolesc Psychiatry Ment Health. 2009 Mar 19;3(1):11. doi: 10.1186/1753-2000-3-11. PMID: 19298659.

251. Geller B, Cooper TB, Sun K, et al. Double-blind and placebo-controlled study of lithium for adolescent bipolar disorders with secondary substance dependency. J Am Acad Child Adolesc Psychiatry. 1998 Feb;37(2):171-8. doi: 10.1097/00004583-199802000-00009. PMID: 9473913.

252. Gray KM, Riggs PD, Min SJ, et al. Cigarette and cannabis use trajectories among adolescents in treatment for attention-deficit/hyperactivity disorder and substance use disorders. Drug Alcohol Depend. 2011 Sep 1;117(2-3):242-7. doi: 10.1016/j.drugalcdep.2011.02.005. PMID: 21411243.

253. Delbelo M. Efficacy and Tolerability of Topiramate in Treatment of Bipolar Mania and Alcohol Use in Adolescents and Young Adults. 2017.

https://clinicaltrials.gov/ct2/show/NCT00550394. Accessed on March 5, 2019.

254. Delbelo M. Quetiapine Plus Topiramate for Reducing Cannabis Consumption and Bipolar Mania. 2017. https://clinicaltrials.gov/ct2/show/NCT00393978. March 5, 2019.

255. Mamey MR, Barbosa-Leiker C, McPherson S, et al. An application of analyzing the trajectories of two disorders: A parallel piecewise growth model of substance use and attentiondeficit/hyperactivity disorder. Exp Clin Psychopharmacol. 2015 Dec;23(6):422-7. doi: 10.1037/pha0000047. PMID: 26389639. 
256. Riggs PD, Mikulich-Gilbertson SK, Davies RD, et al. A randomized controlled trial of fluoxetine and cognitive behavioral therapy in adolescents with major depression, behavior problems, and substance use disorders. Archives of pediatrics \& adolescent medicine. 2007 Nov;161(11):1026-34. doi: 10.1001/archpedi.161.11.1026. PMID: 17984403.

257. Riggs PD, Winhusen T, Davies RD, et al. Randomized controlled trial of osmotic-release methylphenidate with cognitive-behavioral therapy in adolescents with attentiondeficit/hyperactivity disorder and substance use disorders. J Am Acad Child Adolesc Psychiatry. 2011 Sep;50(9):903-14. doi: 10.1016/j.jaac.2011.06.010. PMID: 21871372.

258. Tamm L, Adinoff B, Nakonezny PA, et al. Attention-deficit/hyperactivity disorder subtypes in adolescents with comorbid substance-use disorder. Am J Drug Alcohol Abuse. 2012 Jan;38(1):93-100. doi: 10.3109/00952990.2011.600395. PMID: 21834613.

259. Tamm L, Trello-Rishel K, Riggs P, et al. Predictors of treatment response in adolescents with comorbid substance use disorder and attention-deficit/hyperactivity disorder. Journal of substance abuse treatment. 2013 Feb;44(2):224-30. doi: 10.1016/j.jsat.2012.07.001. PMID: 22889694.

260. Thurstone C, Riggs PD, Salomonsen-Sautel S, et al. Randomized, controlled trial of atomoxetine for attention-deficit/hyperactivity disorder in adolescents with substance use disorder. J Am Acad Child Adolesc Psychiatry. 2010 Jun;49(6):573-82. doi: 10.1016/j.jaac.2010.02.013. PMID: 20494267.

261. Thurstone C, Salomensen-Sautel S, Riggs PD. How adolescents with substance use disorder spend research payments. Drug Alcohol Depend. 2010 Oct 1;111(3):262-4. doi: 10.1016/j.drugalcdep.2010.04.016. PMID: 20627618.

262. Warden D, Riggs PD, Min SJ, et al. Major depression and treatment response in adolescents with ADHD and substance use disorder. Drug Alcohol Depend. 2012 Jan 1;120(1-3):214-9. doi: 10.1016/j.drugalcdep.2011.08.001. PMID: 21885210 .
263. Winhusen TM, Lewis DF, Riggs PD, et al. Subjective effects, misuse, and adverse effects of osmotic-release methylphenidate treatment in adolescent substance abusers with attentiondeficit/hyperactivity disorder. J Child Adolesc Psychopharmacol. 2011 Oct;21(5):455-63. doi: 10.1089/cap.2011.0014. PMID: 22040190.

264. Riggs PD, Hall SK, Mikulich-Gilbertson SK, et al. A randomized controlled trial of pemoline for attention-deficit/hyperactivity disorder in substance-abusing adolescents. J Am Acad Child Adolesc Psychiatry. 2004 Apr;43(4):420-9. doi: 10.1097/00004583-200404000-00008. PMID: 15187802.

265. Cornelius JR. Fluoxetine for Major Depressive Disorder/Cannabis Disorder in Young People. 2013. https://clinicaltrials.gov/ct2/show/NCT00149643.

266. Tanner-Smith EE, Lipsey MW. Brief alcohol interventions for adolescents and young adults: a systematic review and meta-analysis. Journal of substance abuse treatment. 2015 Apr;51:1-18. doi: 10.1016/j.jsat.2014.09.001. PMID: 25300577.

267. Tanner-Smith EE, Risser MD. A meta-analysis of brief alcohol interventions for adolescents and young adults: variability in effects across alcohol measures. Am J Drug Alcohol Abuse. 2016 Mar;42(2):140-51. doi: 10.3109/00952990.2015.1136638. PMID: 26905387.

268. Li L, Zhu S, Tse N, et al. Effectiveness of motivational interviewing to reduce illicit drug use in adolescents: a systematic review and metaanalysis. Addiction. 2016 May;111(5):795-805. doi: 10.1111/add.13285. PMID: 26687544.

269. Tanner-Smith EE, Steinka-Fry KT, Hennessy EA, et al. Can brief alcohol interventions for youth also address concurrent illicit drug use? results from a meta-analysis. J Youth Adolesc. 2015 May;44(5):1011-23. doi: 10.1007/s10964015-0252-x. PMID: 25600491.

270. Tanner-Smith EE, Steinka-Fry KT, Kettrey HH, et al. Adolescent substance use treatment effectiveness: A systematic review and metaanalysis Peabody Research Institute, Vanderbilt University. 2016. https://www.ncjrs.gov/pdffiles1/ojjdp/grants/250 440.pdf 
271. Brewer S, Godley MD, Hulvershorn LA. Treating Mental Health and Substance Use Disorders in Adolescents: What Is on the Menu? Curr Psychiatry Rep. 2017 Jan;19(1):5. doi: 10.1007/s11920-017-0755-0. PMID: 28120255.

272. Field CA, Baird J, Saitz R, et al. The mixed evidence for brief intervention in emergency departments, trauma care centers, and inpatient hospital settings: what should we do? Alcohol Clin Exp Res. 2010 Dec;34(12):2004-10. doi: 10.1111/j.1530-0277.2010.01297.x. PMID: 20860610.

273. Subbaraman MS. Substitution and Complementarity of Alcohol and Cannabis: A Review of the Literature. Subst Use Misuse. 2016 Sep 18;51(11):1399-414. doi: 10.3109/10826084.2016.1170145. PMID: 27249324.

274. Committee on Substance Abuse, Levy SJ, Kokotailo PK. Substance use screening, brief intervention, and referral to treatment for pediatricians. Pediatrics. 2011

Nov;128(5):e1330-40. doi: 10.1542/peds.20111754. PMID: 22042818.
275. Saloner B, Feder KA, Krawczyk N. Closing the Medication-Assisted Treatment Gap for Youth With Opioid Use Disorder. JAMA Pediatr. 2017 Aug 1;171(8):729-31. doi: 10.1001/jamapediatrics.2017.1269. PMID: 28628699.

276. Huh D, Mun EY, Larimer ME, et al. Brief motivational interventions for college student drinking may not be as powerful as we think: an individual participant-level data meta-analysis. Alcohol Clin Exp Res. 2015 May;39(5):919-31. doi: 10.1111/acer.12714. PMID: 25872599.

277. Huh D, Mun EY, Walters ST, et al. A tutorial on individual participant data meta-analysis using Bayesian multilevel modeling to estimate alcohol intervention effects across heterogeneous studies. Addictive behaviors. 2019 Jul;94:162-70. doi: 10.1016/j.addbeh.2019.01.032. PMID: 30791977.

278. ElSohly MA, Mehmedic Z, Foster S, et al. Changes in Cannabis Potency Over the Last 2 Decades (1995-2014): Analysis of Current Data in the United States. Biol Psychiatry. 2016 Apr 1;79(7):613-9. doi: 10.1016/j.biopsych.2016.01.004. PMID: 26903403. 


\section{Abbreviations and Acronyms}

AACAP

AAP

ACC

ACRA

$\mathrm{ADE}$

ADHD

ADHD-RS

ADI

Ato

AUD

AUDIT

BAC

BASICS

BDI

CDRS-R

BSFT

CBT

CGAS

CI

$\mathrm{CM}$

CRAFT

CrI

DSM-III

DSM-IV

DSM-V

e-CHUG

EBFT

ED

Educ

Fam

FBT

FDA

FES

FFT

FSN

FST

GED
American Academy of Child and Adolescent Psychiatrists

American Academy of Pediatrics

Assertive continuing care

Adolescent community reinforcement approach

adverse event

attention deficit hyperactivity disorder

Attention Deficit Hyperactivity Disorder Rating Scale

Adolescent Diagnostic Interview

atomoxetine

alcohol use disorder

Alcohol Use Disorders Identification Test

blood alcohol concentration

Brief Alcohol Screening and Intervention for College Students

Beck's depression inventory

Children's Depression Rating Scale, Revised

Brief strategic family therapy

cognitive behavioral therapy

Children's Global Assessment Scale

confidence interval

contingency management

Community Restitution Apprentice-Focused Training

credible interval

Diagnostic and Statistical Manual of Mental Disorders, Third Edition

Diagnostic and Statistical Manual of Mental Disorders, Fourth Edition

Diagnostic and Statistical Manual of Mental Disorders, Fifth Edition

Electronic Check-Up To Go

Ecologically based family therapy

Emergency department

psychoeducation

family focused therapy

family behavioral therapy

U.S. Food and Drug Administration

Family Environment Scale

functional family therapy

family systems network

family systems therapy

General Equivalency Diploma 
ICM

$\mathrm{KQ}$

LSS-A

MDFT

MA

MET

MI

MST

NAC

NMA

NMD

NIDA

NOS

NRCS

OR

PeerGroup

PHYS

PP

PPQ

PU

RCT

RR

SE

SIQ-JR

SMD

SR

SRDR

STI

SUD

T-ASI

TAU

TEP

YHPS intensive case management

Key Question

Life Satisfaction Scale for Adolescents

Multidimensional family therapy

meta-analysis

motivation enhancement therapy

motivational interviewing

Multi-systemic therapy

$\mathrm{N}$-acetylcysteine

network meta-analysis

net mean difference

National Institute of Drug Abuse

not otherwise specified

nonrandomized comparative study

odds ratio

peer group therapy

Parent Happiness with Youth Scale

practice parameter

Parenting Practices Questionnaire

problematic use

randomized controlled trial

risk ratio

standard error

Suicidal Ideation Questionnaire for Adolescents

standardized mean difference

systematic review

Systematic Review Data Repository

sexually transmitted infection

substance use disorder

Teen Addiction Severity Index

treatment as usual

Technical Expert Panel

Youth Happiness with Parent Scale 


\title{
Appendix A. Search Strategies
}

\section{Primary Search for All Substances in Adolescents}

\author{
PubMed last run 10/31/2019
}

(((“Juvenile Delinquency/rehabilitation”[MeSH Terms] OR (juvenile AND (offender* or delinquency or prison)) OR ("Substance-Related Disorders"[Mesh] NOT ("Substance-Related Disorders/prevention and control"[Mesh] NOT "Substance-Related Disorders"[Mesh])) OR "drug offense” OR "Drug abuse” OR “drug misuse” OR "drug dependence” OR “drug addiction" OR "substance use" OR "substance abuse” OR "substance misuse” OR "substance dependence" OR "substance addiction” OR "prescription abuse” OR “Alcoholism”[Mesh] OR "cannabis use disorder" OR "alcohol use disorder" OR "stimulant use disorder" OR "hallucinogen use disorder" OR "opioid use disorder" OR "inhalant use disorder") OR ((Alcohol OR “Alcoholic Beverages”[Mesh] OR cannabis OR Marijuana OR “Cannabis”[Mesh] OR “Marijuana Abuse”[Mesh] OR “Marijuana Smoking”[Mesh] OR opioids OR "Narcotics”[Mesh] OR “Analgesics, Opioid”[Mesh] OR kratom OR hallucinogens OR “Psychotropic Drugs”[Mesh] OR inhalants OR toluene OR ((amyl OR butyl OR isobutyl) AND nitrites) OR stimulants OR “Central Nervous System Stimulants”[Mesh] OR “Amphetamines”[Mesh] OR Sedatives OR "Hypnotics and Sedatives”[Mesh] OR Benzodiazepines OR "Benzodiazepines”[Mesh] OR Anthramycin OR Bromazepam OR Clonazepam OR Devazepide OR Diazepam OR Flumazenil OR Flunitrazepam OR Flurazepam OR Fentanyl OR Alprazolam OR Clonidine OR Hashish Clonidine OR Lorazepam OR Nitrazepam OR Oxazepam OR Pirenzepine OR Prazepam OR Temazepam OR Chlordiazepoxide OR Clorazepate Dipotassium OR Estazolam OR Medazepam OR Midazolam OR Triazolam OR opioid* OR opiate* OR Heroin OR opium OR "Morphine Derivatives"[Mesh] OR Codeine OR Hydrocodone OR Oxycodone OR Dihydromorphine OR Ethylmorphine OR Heroin OR Hydromorphone OR Morphine OR Oxymorphone OR Thebaine OR Cocaine OR “Cocaine”[Mesh] OR Methamphetamine* OR "Methamphetamine”[Mesh] OR Benzphetamine OR anabolic steroids OR "Testosterone Congeners"[Mesh] OR antihistamines OR nitrous oxide OR betel nut OR kava OR Ecstasy OR phenylalkylamines OR mescaline OR 2,5-dimethoxy-4-methylamphetamine OR MDMA OR 3,4-methylenedioxymethamphetamine OR indoleamine* OR psilocybin OR psilocin OR dimethyltryptamine OR ergoline* OR lysergic acid diethylamide OR "morning glory seeds" OR "Salvia divinorum” OR jimsonweed OR anxiolytic OR benzodiazepine* OR zolpidem OR zaleplon OR carbamate* OR glutethimide OR meprobamate OR barbiturate* OR secobarbital OR barbiturate* OR glutethimide OR methaqualone OR amphetamine OR dextroamphetamine OR methamphetamine OR gabapentin OR baclofen OR diacetylmorphine OR kratom OR polydrug OR "poly-drug” OR polysubstance OR "poly-substance” OR "injection drug”) AND (addict* OR abus* OR misus* OR disorder* OR mis-use OR dependen*)))

AND 
(“Telemedicine/methods”[Mesh] OR "Active aftercare” OR "Adolescent Community Reinforcement Approach" OR "Alcoholics Anonymous” OR "Narcotics Anonymous” OR "12step” OR "assertive community treatment” OR "assertive continuing care” OR "Behavior Therapy"[Mesh] OR "brief intervention" OR "brief interventions” OR "Brief negotiated interview" OR "brief strategic family therapy" OR "Buprenorphine, Naloxone Drug Combination"[Mesh] OR "Buprenorphine”[Mesh] OR "Cognitive behavioral therapy" OR "Cognitive Therapy”[Mesh] OR "Cognitive-behavioral therapy” OR "Cognitive-behavioral treatment” OR “Combined Modality Therapy”[Mesh] OR “Contingency management” OR "Culturally Informed and Flexible Family-Based Treatment for Adolescents" OR "culturallybased intervention” OR “delinquency-treatment programs" OR “dopaminergic agent” OR “drug counseling” OR "Dual diagnosis therapy” OR "Dual diagnosis treatment” OR "Dual Recovery Therapy” OR “Family Therapy”[Mesh] OR "glutaminergic agent” OR "group therapy” OR "Harm reduction" OR "Interpersonal process groups" OR "Matrix-Model” OR "medication assisted treatment” OR “Methadone”[Mesh] OR “Motivational Enhancement Therapy” OR "Motivational incentives" OR "motivational interview" OR "motivational interviewing" OR "Motivational Interviewing”[Mesh] OR "Multidimensional Treatment Foster Care” OR "Multisystemic Therapy" OR "mutual help group" OR "Naloxone”[Mesh] OR "Narcotic Antagonists”[Mesh]) OR “Opioid replacement” OR “Opioid substitution” OR “oral THC” OR "Phoenix Academy” OR "Recovery Coach" OR "Recovery High School” OR "Reinforcementbased" OR "Seeking Safety" OR "Self-Help for Alcohol and Other Drug Use and Depression" OR "seven challenges" OR "Skills development groups" OR "Substance Abuse Program” OR "synthetic cannabinoids" OR "synthetic opioid" OR "systemic Therapy" OR "Teen Marijuana Check-Up” OR “Therapy, Computer-Assisted”[Mesh] OR ((family OR psychosocial OR Psychoeducational) and (therapy or therapies or treatment* or intervention* or counseling)) OR (behavior* AND (intervention* OR modification*)) OR A-CRA OR Acamprosate OR Acomplia OR amiodarone OR Buprenorphine OR Bupropion OR Cannabidiol OR CIFFTA OR citalopram OR dexamfetamine OR dexamphetamine OR Disulfiram OR duloxetine OR escitalopram OR fluoxetine OR gabapentin OR lisdexamfetamine OR Lithium OR Long-acting injectable OR meditation OR Methadone OR mindfulness OR Nabiximols OR Naltrexone OR paroxetine OR Peer-based OR "peer support" OR pemoline OR Pharmacological interventions OR prazosin OR pregabalin OR quetiapine OR Rimonabant OR Sativex OR SBIRT OR sertraline OR Topiramate OR varenicline OR venlafaxine OR Vilazodone )

\section{AND}

("Randomized Controlled Trial”[pt] OR "Cohort Studies"[Mesh] OR cohort OR "Clinical Trial" [Publication Type] OR longitudinal OR "Placebos"[Mesh] OR placebo* OR "Evaluation Studies" [Publication Type] OR "Comparative Study" [Publication Type] OR ((comparative or Intervention) AND study) OR pretest* OR preintervention OR posttest* OR prepost* OR "pre post" OR "before and after" OR interrupted time* OR time serie* OR ((quasi-experiment* OR quasiexperiment* OR quasi or experimental) and (method or study or trial or design*)) OR "Random Allocation"[Mesh] OR "Double-Blind Method"[Mesh] OR "Single-Blind Method"[Mesh] OR ((clinical OR controlled) and trial*) OR ((singl* or doubl* or trebl* or tripl*) and (blind* or mask*)) OR random*)

\section{AND}

(adolescent [MeSH] OR adolescen* OR teen* OR young people OR young person* OR young adult* OR youth* OR girl OR girls OR boy OR boys OR juvenile* OR "Young Adult"[Mesh])) 
NOT

(((“adult”[mesh] OR "Infant"[Mesh] OR “child”[mesh]) NOT “adolescent”[mesh]) OR neonat* OR infant* OR "addresses”[pt] or “autobiography”[pt] or "bibliography”[pt] or "biography”[pt] or "case reports"[pt] or "comment”[pt] or "congresses”[pt] or "dictionary”[pt] or "directory”[pt] or "festschrift"[pt] or "government publications"[pt] or "historical article"[pt] or "interview"[pt] or "lectures”[pt] or "legal cases”[pt] or "legislation”[pt] or "news”[pt] or "newspaper article”[pt] or "patient education handout”[pt] or "periodical index"[pt] or "comment on" or

("Animals"[Mesh] NOT "Humans"[Mesh]) OR rats[tw] or cow[tw] or cows[tw] or chicken*[tw] or horse[tw] or horses[tw] or mice[tw] or mouse[tw] or bovine[tw] or sheep or ovine or murinae or "animal model")

Cochrane last run on 10/31/19

ID Search Hits

\#1 MeSH descriptor: [Substance-Related Disorders] explode all trees

\#2 alcohol* or cannabis or marijuana or opioid* or narcotic* or hallucinogen* or

psychotropic or stimulant* or opiate* or steroid* or polydrug or polysubstance or drug* or substance* or prescription

\#3 addict* or abus* or misus* or mis-use or dependen*

\#4 \#2 NEAR \#3

\#5 \#1 OR \#4

\#6 treatment or therapy or intervention or counseling

\#7 \#5 AND \#6

\#8 adolescen* or teen* or "young people" or "young person" or "young adult" or "young adults" or youth* or girl or girls or boy or boys or juvenile*

\#9 \#7 and \#8

CINAHL/PsycINFO last run on 10/31/19

((alcohol* or cannabis or marijuana or opioid* or narcotic* or hallucinogen* or psychotropic or stimulant* or opiate* or steroid* or polydrug or polysubstance or drug* or substance* or prescription) N1 (addict* or abus* or misus* or mis-use or dependen*))

AND

(treatment or intervention or therapy or counseling)

AND

(adolescen* or teen* or "young people" or "young person" or "young adult" or "young adults" or youth* or girl or girls or boy or boys or juvenile*)

AND 
("Randomized Controlled Trial” OR "Cohort Studies" OR cohort OR "Clinical Trial" OR longitudinal OR "Placebos" OR placebo* OR "Evaluation Studies" OR "Comparative Study" OR ((comparative or Intervention) AND study) OR pretest* OR preintervention OR posttest* OR prepost* OR "pre post” OR "before and after" OR interrupted time* OR time serie* OR ((quasi-experiment* OR quasiexperiment* OR quasi or experimental) and (method or study or trial or design*)) OR "Random Allocation" OR "Double-Blind Method" OR "Single-Blind Method" OR ((clinical OR controlled) and trial*) OR ((singl* or doubl* or trebl* or tripl*) and (blind* or mask*)) OR random*)

Limit to journals, adolescent, young adult

Embase last run on 10/31/19

\#19

\#4 AND \#18 AND [embase]/lim AND ([article]/lim OR [article in press]/lim) AND

([adolescent]/lim OR [young adult]/lim) AND [humans]/lim

7289

\#18

\#5 OR \#6 OR \#7 OR \#8 OR \#9 OR \#10 OR \#11 OR \#12 OR \#13 OR \#14 OR \#15 OR \#16 OR $\# 17$

\#17

'cohort analysis'/exp OR 'cohort analysis'

\#16

'longitudinal study'/exp OR 'longitudinal study'

\#15

'clinical study'/exp OR 'clinical study'

\#14

'prospective study'/exp OR 'prospective study'

\#13

randomly AND allocated

\#12

'random allocation'

\#11

'placebo'/de

\#10

'crossover procedure'/de 
'double blind procedure'/de

\#8

'single blind procedure'/de

\#7

'randomization'/exp OR 'randomization'

\#6

'randomized controlled trial'/exp OR 'randomized controlled trial'

\#5

'clinical trial'/exp OR 'clinical trial'

\#4

\#1 AND \#2 AND \#3 AND ([article]/lim OR [article in press]/lim) AND [humans]/lim

\#3

adolescen* OR teen* OR 'young people' OR 'young person' OR 'young adult' OR 'young adults' OR youth* OR girl OR girls OR boy OR boys OR juvenile*

\#2

treatment $\mathrm{OR}$ therapy $\mathrm{OR}$ intervention $\mathrm{OR}$ counseling

\#1

'drug offense'/exp OR 'drug offense' OR 'drug abuse'/exp OR 'drug abuse' OR 'drug misuse'/exp OR 'drug misuse' OR 'drug dependence'/exp OR 'drug dependence' OR 'drug addiction'/exp OR 'drug addiction' OR 'substance use'/exp OR 'substance use' OR 'substance abuse'/exp OR 'substance abuse' OR 'substance misuse'/exp OR 'substance misuse' OR 'substance dependence'/exp OR 'substance dependence' OR 'substance addiction'/exp OR 'substance addiction' OR 'prescription abuse' OR 'alcoholism'/exp OR 'alcoholism' OR 'cannabis use disorder'/exp OR 'cannabis use disorder' OR 'alcohol use disorder'/exp OR 'alcohol use disorder' OR 'stimulant use disorder' OR 'hallucinogen use disorder' OR 'opioid use disorder'/exp OR 'opioid use disorder' OR 'inhalant use disorder'

CT.gov last run 10/31/19

Substance Use OR Substance Abuse OR Drug Abuse OR drug dependence OR drug addiction OR prescription abuse OR Alcoholism OR cannabis OR alcohol OR stimulant OR hallucinogen OR opioid OR inhalant

adolescent OR teen OR young people OR young person OR young adult OR youth OR juvenile 


\section{Search for Systematic Reviews of Alcohol in College Settings}

PubMed last run 10/31/19

(“Alcoholism”[Mesh] OR “alcohol use disorder” OR "Alcohol Drinking"[Mesh] OR pregam* OR ((Alcohol* OR “Alcoholic Beverages”[Mesh] OR drink*) AND (addict* OR disorder* OR abus* OR misus* OR mis-use OR dependen* OR binge OR heavy OR problematic OR highrisk)))

AND

(colleg*[tiab] OR undergraduate*[tiab] OR "Universities"[Mesh] OR University[tiab] OR universities[tiab])

AND

(systematic[sb] OR meta-analysis[pt] OR meta-analysis as topic[mh] OR meta-analysis[mh] OR meta analy* OR metanaly* OR metaanaly* OR met analy* OR (systematic AND (review* OR overview*)) OR "Review Literature as Topic"[Mesh] OR Cochrane[tiab] OR embase[tiab] OR (psychlit[tiab] or psyclit[tiab]) OR (psycINFO[tiab] or psycinfo[tiab])OR (cinahl[tiab] or cinhal[tiab]) OR science citation index[tiab] OR bids[tiab] OR cancerlit[tiab] OR reference list*[tiab] OR bibliograph*[tiab] OR hand-search*[tiab] OR relevant journals[tiab] OR manual search*[tiab] OR selection criteria[tiab] OR data extraction[tiab])

Cochrane/Epistemonikos last run 10/31/19

(((Alcohol* OR drink*) AND (addict* OR disorder* OR abus* OR misus* OR mis-use OR dependen* OR binge OR heavy OR problematic OR high-risk)) OR pregam*)

AND

(colleg* OR undergraduate* OR University OR universities) 


\section{Appendix B. Excluded Studies}

\begin{tabular}{|c|c|c|c|c|c|c|}
\hline No. & Author & Year & $\begin{array}{l}\text { PubMed or } \\
\text { (Other) ID }\end{array}$ & DOI & Title & Reason for Exclusion \\
\hline 1 & Abar & 2015 & 26402351 & & $\begin{array}{l}\text { Trajectories of Adolescent Alcohol Use in the Year Following a } \\
\text { Brief Alcohol Intervention }\end{array}$ & $\begin{array}{l}\text { No extractable or relevant } \\
\text { data for } \\
\text { interventions/outcomes of } \\
\text { interest }\end{array}$ \\
\hline 2 & Abdel-Salam & 2014 & $\begin{array}{l}\text { 2014-07010- } \\
005 \text { (psychinfo) }\end{array}$ & $10.3109 / 14659891.2012 .728670$ & $\begin{array}{l}\text { Examining the relationship between self-control and } \\
\text { adolescent TC treatment completion }\end{array}$ & $\begin{array}{l}\text { NRCS (nonpharm, } \\
\text { pharmacological } \\
\text { interventions } \mathrm{N}<100 \text { ) }\end{array}$ \\
\hline 3 & Agosti & 2007 & $\begin{array}{l}\text { L46871888 } \\
\text { (embase) }\end{array}$ & 10.1097/ADT.0b013e318059bb02 & $\begin{array}{l}\text { One-year posttreatment outcome of cannabis-dependent } \\
\text { adolescents }\end{array}$ & $\begin{array}{l}\text { NRCS (nonpharm, } \\
\text { pharmacological } \\
\text { interventions } N<100 \text { ) }\end{array}$ \\
\hline 4 & Akhtar & 2011 & $\begin{array}{l}\text { 2011-11112- } \\
002 \text { (psychinfo) }\end{array}$ & $10.1921 / 095182410 \times 576831$ & $\begin{array}{l}\text { Applying positive psychology to alcohol-misusing adolescents: } \\
\text { A group intervention }\end{array}$ & $\begin{array}{l}\text { NRCS (nonpharm, } \\
\text { pharmacological } \\
\text { interventions } N<100 \text { ) }\end{array}$ \\
\hline 5 & Albornoz & 2011 & $\begin{array}{l}\text { CN-00852012 } \\
\text { (cochrane) }\end{array}$ & & $\begin{array}{l}\text { The effects of group improvisational music therapy on } \\
\text { depression in adolescents and adults with substance abuse: a } \\
\text { randomized controlled trial }\end{array}$ & $\begin{array}{l}\text { No extractable or relevant } \\
\text { data for } \\
\text { interventions/outcomes of } \\
\text { interest }\end{array}$ \\
\hline 6 & Alderson & 2017 & 28536655 & 10.1186/s40814-017-0138-7 & $\begin{array}{l}\text { Supporting Looked After Children and Care Leavers In } \\
\text { Decreasing Drugs, and alcohol (SOLID): protocol for a pilot } \\
\text { feasibility randomised controlled trial of interventions to } \\
\text { decrease risky substance use (drugs and alcohol) and } \\
\text { improve mental health of looked after children and care } \\
\text { leavers aged 12-20 years }\end{array}$ & $\begin{array}{l}\text { No extractable or relevant } \\
\text { data for } \\
\text { interventions/outcomes of } \\
\text { interest }\end{array}$ \\
\hline 7 & Alizadehgoradel & 2019 & $\begin{array}{l}\text { L2002789837 } \\
\text { (Embase) }\end{array}$ & 10.1016/j.npbr.2019.08.002 & $\begin{array}{l}\text { Mindfulness-based substance abuse treatment (MBSAT) } \\
\text { improves executive functions in adolescents with substance } \\
\text { use disorders }\end{array}$ & $\begin{array}{l}\text { No extractable or relevant } \\
\text { data for } \\
\text { interventions/outcomes of } \\
\text { interest }\end{array}$ \\
\hline 8 & Andersson & 2017 & 28028732 & $10.1007 / s 12529-016-9625-0$ & $\begin{array}{l}\text { Interactive voice response with feedback intervention in } \\
\text { outpatient treatment of substance use problems in } \\
\text { adolescents and young adults: A randomized controlled trial }\end{array}$ & $\begin{array}{l}\text { Includes transition-aged } \\
\text { youth (non-pharmacological } \\
\text { interventions) }\end{array}$ \\
\hline 9 & Armitage & 2014 & 24491079 & 10.1037/a0035802 & $\begin{array}{l}\text { A brief psychological intervention that reduces adolescent } \\
\text { alcohol consumption }\end{array}$ & $\begin{array}{l}\text { Not all subjects with at least } \\
\text { problematic use }\end{array}$ \\
\hline
\end{tabular}




\begin{tabular}{|c|c|c|c|c|c|c|}
\hline No. & Author & Year & $\begin{array}{l}\text { PubMed or } \\
\text { (Other) ID }\end{array}$ & DOI & Title & Reason for Exclusion \\
\hline 10 & Arnaud & 2012 & 23013141 & 10.1186/1471-2458-12-826 & $\begin{array}{l}\text { Web-based screening and brief intervention for poly-drug use } \\
\text { among teenagers: study protocol of a multicentre two-arm } \\
\text { randomized controlled trial }\end{array}$ & $\begin{array}{l}\text { No extractable or relevant } \\
\text { data for } \\
\text { interventions/outcomes of } \\
\text { interest }\end{array}$ \\
\hline 11 & Arnaud & 2015 & 26135277 & $10.1055 / \mathrm{s}-0034-1387681$ & $\begin{array}{l}\text { Nachhaltiger Transfer des Gesundheitsnetz Alkohol im } \\
\text { Jugendalter: Eine Kooperation aus Forschung, Praxis und } \\
\text { Politik. = Sustainable transfer of the health network alcohol } \\
\text { use in adolescence: A cooperation of research, practice and } \\
\text { politics }\end{array}$ & $\begin{array}{l}\text { No extractable or relevant } \\
\text { data for } \\
\text { interventions/outcomes of } \\
\text { interest }\end{array}$ \\
\hline 12 & Asdigian & 2018 & 28032813 & 10.1080/00952990.2016.1265122 & $\begin{array}{l}\text { Effects of the 'Circle of Life' HIV-prevention program on } \\
\text { marijuana use among American Indian middle school youths: } \\
\text { a group randomized trial in a Northern Plains tribe }\end{array}$ & $\begin{array}{l}\text { Not all subjects with at least } \\
\text { problematic use }\end{array}$ \\
\hline 13 & Azrin & 1996 & 8561763 & & $\begin{array}{l}\text { Follow-up results of supportive versus behavioral therapy for } \\
\text { illicit drug use }\end{array}$ & $\mathrm{RCT}, \mathrm{N}<10$ per arm \\
\hline 14 & Babbin & 2016 & 27082747 & 10.1016/j.addbeh.2016.03.033 & $\begin{array}{l}\text { Identifying treatment response subgroups for adolescent } \\
\text { cannabis use }\end{array}$ & $\begin{array}{l}\text { NRCS (nonpharm, } \\
\text { pharmacological } \\
\text { interventions } N<100 \text { ) }\end{array}$ \\
\hline 15 & Bacio & 2017 & 28028740 & $10.1007 / s 11121-016-0741-5$ & $\begin{array}{l}\text { Impact of Ethnic Composition on Mechanisms of Change in } \\
\text { School-Based Substance Use Intervention Groups }\end{array}$ & $\begin{array}{l}\text { Not all subjects with at least } \\
\text { problematic use }\end{array}$ \\
\hline 16 & Baer & 2004 & $\begin{array}{l}2004-18304- \\
003 \text { (psychinfo) }\end{array}$ & $10.1080 / 1606635042000236475$ & $\begin{array}{l}\text { Rationale and design of a brief substance use intervention for } \\
\text { homeless adolescents }\end{array}$ & $\begin{array}{l}\text { No extractable or relevant } \\
\text { data for } \\
\text { interventions/outcomes of } \\
\text { interest }\end{array}$ \\
\hline 17 & Bailey & 2004 & 15370021 & $10.1080 / 09595230410001704136$ & $\begin{array}{l}\text { Pilot randomized controlled trial of a brief alcohol intervention } \\
\text { group for adolescents }\end{array}$ & $\begin{array}{l}\text { Not all subjects with at least } \\
\text { problematic use }\end{array}$ \\
\hline 18 & Baldus & 2011 & 21501479 & $10.1186 / 1472-6963-11-80$ & $\begin{array}{l}\text { 'CAN Stop'--implementation and evaluation of a secondary } \\
\text { group prevention for adolescent and young adult cannabis } \\
\text { users in various contexts--study protocol }\end{array}$ & $\begin{array}{l}\text { No extractable or relevant } \\
\text { data for } \\
\text { interventions/outcomes of } \\
\text { interest }\end{array}$ \\
\hline 19 & Bamberg & 2008 & 19004420 & $10.1080 / 02791072.2008 .10400643$ & $\begin{array}{l}\text { Including the siblings of youth substance abusers in a parent- } \\
\text { focused intervention: a pilot test of the Best Plus program }\end{array}$ & $\begin{array}{l}\text { NRCS (nonpharm, } \\
\text { pharmacological } \\
\text { interventions } \mathrm{N}<100 \text { ) }\end{array}$ \\
\hline 20 & Bantchevska & 2011 & $\begin{array}{l}\text { 2011-23331- } \\
009 \text { (psychinfo) }\end{array}$ & 10.1093/swr/35.1.58 & $\begin{array}{l}\text { Predictors of drop-in center attendance among substance- } \\
\text { abusing homeless adolescents }\end{array}$ & $\begin{array}{l}\text { No extractable or relevant } \\
\text { data for } \\
\text { interventions/outcomes of } \\
\text { interest }\end{array}$ \\
\hline
\end{tabular}




\begin{tabular}{|c|c|c|c|c|c|c|}
\hline No. & Author & Year & $\begin{array}{l}\text { PubMed or } \\
\text { (Other) ID }\end{array}$ & DOI & Title & Reason for Exclusion \\
\hline 21 & Barbosa & 2018 & 29885153 & & $\begin{array}{l}\text { Start-Up Costs of SBIRT Implementation for Adolescents in } \\
\text { Urban U.S. Federally Qualified Health Centers }\end{array}$ & $\begin{array}{l}\text { No extractable or relevant } \\
\text { data for } \\
\text { interventions/outcomes of } \\
\text { interest }\end{array}$ \\
\hline 22 & Barlow & 2013 & 23409290 & 10.1176/appi.ajp.2012.12010121 & $\begin{array}{l}\text { Effect of a paraprofessional home-visiting intervention on } \\
\text { American Indian teen mothers' and infants' behavioral risks: a } \\
\text { randomized controlled trial }\end{array}$ & $\begin{array}{l}\text { No extractable or relevant } \\
\text { data for } \\
\text { interventions/outcomes of } \\
\text { interest }\end{array}$ \\
\hline 23 & Barnett & 2002 & $\begin{array}{l}2002-01321- \\
003 \text { (psychinfo) }\end{array}$ & 10.1037/0893-164X.16.2.106 & $\begin{array}{l}\text { Predictors of motivation to change after medical treatment for } \\
\text { drinking-related events in adolescents }\end{array}$ & $\begin{array}{l}\text { No extractable or relevant } \\
\text { data for } \\
\text { interventions/outcomes of } \\
\text { interest }\end{array}$ \\
\hline 24 & Barnett & 2010 & 20402989 & 10.1111/j.1360-0443.2009.02814.x & $\begin{array}{l}\text { Moderators and mediators of two brief interventions for alcohol } \\
\text { in the emergency department }\end{array}$ & Includes adults ( $>25$ years) \\
\hline 25 & Bassett & 2016 & 27211991 & 10.1016/j.jsat.2016.02.011 & $\begin{array}{l}\text { Evaluating Measures of Fidelity for Substance Abuse Group } \\
\text { Treatment With Incarcerated Adolescents }\end{array}$ & $\begin{array}{l}\text { NRCS (nonpharm, } \\
\text { pharmacological } \\
\text { interventions } N<100 \text { ) }\end{array}$ \\
\hline 26 & Battjes & 2004 & 15450645 & 10.1016/j.jsat.2004.06.002 & $\begin{array}{l}\text { Evaluation of a group-based substance abuse treatment } \\
\text { program for adolescents }\end{array}$ & $\begin{array}{l}\text { Single arm (nonpharm, } \\
\text { pharmacological } \\
\text { interventions } N<200 \text { ) }\end{array}$ \\
\hline 27 & Beach & 2010 & 20954761 & $10.1037 / a 0020835$ & $\begin{array}{l}\text { Differential susceptibility to parenting among African American } \\
\text { youths: testing the DRD4 hypothesis }\end{array}$ & $\begin{array}{l}\text { Not all subjects with at least } \\
\text { problematic use }\end{array}$ \\
\hline 28 & Becan & 2015 & 25456094 & 10.1016/j.jsat.2014.10.002 & $\begin{array}{l}\text { Effectiveness of the Treatment Readiness and Induction } \\
\text { Program for increasing adolescent motivation for change }\end{array}$ & $\begin{array}{l}\text { NRCS (nonpharm, } \\
\text { pharmacological } \\
\text { interventions } N<100 \text { ) }\end{array}$ \\
\hline 29 & Becan & 2018 & 29654518 & $10.1186 / s 40352-018-0068-3$ & $\begin{array}{l}\text { A model for rigorously applying the Exploration, Preparation, } \\
\text { Implementation, Sustainment (EPIS) framework in the design } \\
\text { and measurement of a large scale collaborative multi-site } \\
\text { study }\end{array}$ & Review \\
\hline 30 & Becker & 2012 & 22560729 & 10.1016/j.drugalcdep.2012.03.021 & $\begin{array}{l}\text { Trajectories of adolescent alcohol use after brief treatment in } \\
\text { an Emergency Department }\end{array}$ & $\begin{array}{l}\text { No extractable or relevant } \\
\text { data for } \\
\text { interventions/outcomes of } \\
\text { interest }\end{array}$ \\
\hline 31 & Becker & 2017 & 28049542 & 10.1186/s13722-016-0067-4 & $\begin{array}{l}\text { Technology-assisted intervention for parents of adolescents in } \\
\text { residential substance use treatment: protocol of an open trial } \\
\text { and pilot randomized trial }\end{array}$ & $\begin{array}{l}\text { No extractable or relevant } \\
\text { data for } \\
\text { interventions/outcomes of } \\
\text { interest }\end{array}$ \\
\hline
\end{tabular}




\begin{tabular}{|c|c|c|c|c|c|c|}
\hline No. & Author & Year & $\begin{array}{l}\text { PubMed or } \\
\text { (Other) ID }\end{array}$ & DOI & Title & Reason for Exclusion \\
\hline 32 & Behar & 1996 & $\begin{array}{l}1996-02567- \\
010 \text { (psychinfo) }\end{array}$ & 10.1007/BF02518648 & $\begin{array}{l}\text { Policy implications of the evaluation of the Fort Bragg child } \\
\text { adolescent mental health demonstration project }\end{array}$ & $\begin{array}{l}\text { No extractable or relevant } \\
\text { data for } \\
\text { interventions/outcomes of } \\
\text { interest }\end{array}$ \\
\hline 33 & Belur & 2014 & $\begin{array}{l}\text { 2014-08701- } \\
002 \text { (psychinfo) }\end{array}$ & 10.1080/1754730X.2014.888223 & $\begin{array}{l}\text { Feasibility and impact of implementing motivational } \\
\text { enhancement therapy-cognitive behavioral therapy as a } \\
\text { substance use treatment intervention in school-based settings }\end{array}$ & $\begin{array}{l}\text { NRCS (nonpharm, } \\
\text { pharmacological } \\
\text { interventions } \mathrm{N}<100 \text { ) }\end{array}$ \\
\hline 34 & Benarous & 2016 & $\begin{array}{l}2016-59773- \\
001 \text { (psychinfo) }\end{array}$ & & $\begin{array}{l}\text { Ecological momentary assessment and smartphone } \\
\text { application intervention in adolescents with substance use and } \\
\text { comorbid severe psychiatric disorders: Study protocol }\end{array}$ & $\begin{array}{l}\text { No extractable or relevant } \\
\text { data for } \\
\text { interventions/outcomes of } \\
\text { interest }\end{array}$ \\
\hline 35 & Bergman & 2015 & 26116368 & 10.1016/j.drugalcdep.2015.05.017 & $\begin{array}{l}\text { The effects of continuing care on emerging adult outcomes } \\
\text { following residential addiction treatment }\end{array}$ & $\begin{array}{l}\text { Single arm (nonpharm, } \\
\text { pharmacological } \\
\text { interventions } \mathrm{N}<200 \text { ) }\end{array}$ \\
\hline 36 & Bernstein & 2017 & 26999582 & 10.1097/PEC.0000000000000662 & $\begin{array}{l}\text { Reaching adolescents for prevention: The role of pediatric } \\
\text { emergency department health promotion advocates }\end{array}$ & $\begin{array}{l}\text { No extractable or relevant } \\
\text { data for } \\
\text { interventions/outcomes of } \\
\text { interest }\end{array}$ \\
\hline 37 & Bertholet & 2012 & 22931392 & $10.1186 / 1471-2458-12-708$ & $\begin{array}{l}\text { Predictive value of readiness, importance, and confidence in } \\
\text { ability to change drinking and smoking }\end{array}$ & $\begin{array}{l}\text { Not all subjects with at least } \\
\text { problematic use }\end{array}$ \\
\hline 38 & Bertholet & 2016 & 27450907 & 10.1016/j.addbeh.2016.07.015 & $\begin{array}{l}\text { Are young men who overestimate drinking by others more } \\
\text { likely to respond to an electronic normative feedback brief } \\
\text { intervention for unhealthy alcohol use? }\end{array}$ & $\begin{array}{l}\text { No extractable or relevant } \\
\text { data for } \\
\text { interventions/outcomes of } \\
\text { interest }\end{array}$ \\
\hline 39 & Bertholet & 2018 & 29396897 & 10.1111/add.14179 & $\begin{array}{l}\text { Four-year follow-up of an internet-based brief intervention for } \\
\text { unhealthy alcohol use in young men }\end{array}$ & Includes adults ( $>25$ years) \\
\hline 40 & Bickman & 1996 & 8694389 & & A continuum of care. More is not always better & $\begin{array}{l}\text { No extractable or relevant } \\
\text { data for } \\
\text { interventions/outcomes of } \\
\text { interest }\end{array}$ \\
\hline 41 & Bohanna & 2014 & 25082422 & 10.1136/bmjopen-2014-005689 & $\begin{array}{l}\text { A service-level action research intervention to improve } \\
\text { identification and treatment of cannabis and related mental } \\
\text { health issues in young Indigenous Australians: A study } \\
\text { protocol }\end{array}$ & $\begin{array}{l}\text { No extractable or relevant } \\
\text { data for } \\
\text { interventions/outcomes of } \\
\text { interest }\end{array}$ \\
\hline 42 & Bond & 2004 & 15022372 & & $\begin{array}{l}\text { Long-term impact of the Gatehouse Project on cannabis use } \\
\text { of } 16 \text {-year-olds in Australia }\end{array}$ & $\begin{array}{l}\text { Not all subjects with at least } \\
\text { problematic use }\end{array}$ \\
\hline
\end{tabular}




\begin{tabular}{|c|c|c|c|c|c|c|}
\hline No. & Author & Year & $\begin{array}{l}\text { PubMed or } \\
\text { (Other) ID }\end{array}$ & DOI & Title & Reason for Exclusion \\
\hline 43 & Boyd & 2017 & 28583136 & $10.1186 / s 12954-017-0159-9$ & $\begin{array}{l}\text { Social-structural factors influencing periods of injection } \\
\text { cessation among marginalized youth who inject drugs in } \\
\text { Vancouver, Canada: an ethno-epidemiological study }\end{array}$ & Review \\
\hline 44 & Braciszewski & 2018 & 29367098 & 10.1016/j.chiabu.2018.01.013 & $\begin{array}{l}\text { Developing a tailored substance use intervention for youth } \\
\text { exiting foster care }\end{array}$ & $\begin{array}{l}\text { Single arm (nonpharm, } \\
\text { pharmacological } \\
\text { interventions } N<200 \text { ) }\end{array}$ \\
\hline 45 & Branson & & 22332855 & 10.1111/j.1521-0391.2011.00204.x & $\begin{array}{l}\text { A pilot study of low-cost contingency management to increase } \\
\text { attendance in an adolescent substance abuse program }\end{array}$ & $\begin{array}{l}\text { NRCS (nonpharm, } \\
\text { pharmacological } \\
\text { interventions } N<100 \text { ) }\end{array}$ \\
\hline 46 & Breda & 2004 & 15230075 & 10.1081/ADA-120037377 & $\begin{array}{l}\text { Predicting incentives to change among adolescents with } \\
\text { substance abuse disorder }\end{array}$ & $\begin{array}{l}\text { No extractable or relevant } \\
\text { data for } \\
\text { interventions/outcomes of } \\
\text { interest }\end{array}$ \\
\hline 47 & Brody & 2012 & 22157131 & 10.1542/peds.2011-0623 & $\begin{array}{l}\text { Family-centered program deters substance use, conduct } \\
\text { problems, and depressive symptoms in black adolescents }\end{array}$ & $\begin{array}{l}\text { Not all subjects with at least } \\
\text { problematic use }\end{array}$ \\
\hline 48 & Brody & 2012 & 22182263 & $10.1037 / a 0026592$ & $\begin{array}{l}\text { The Adults in the Making program: long-term protective } \\
\text { stabilizing effects on alcohol use and substance use problems } \\
\text { for rural African American emerging adults }\end{array}$ & $\begin{array}{l}\text { Not all subjects with at least } \\
\text { problematic use }\end{array}$ \\
\hline 49 & Broome & 2001 & $\begin{array}{l}2002-02226- \\
005 \text { (psychinfo) }\end{array}$ & $10.1177 / 0743558401166005$ & Engagement models for adolescents in DATOS-A & $\begin{array}{l}\text { NRCS (nonpharm, } \\
\text { pharmacological } \\
\text { interventions } N<100 \text { ) }\end{array}$ \\
\hline 50 & Bryan & 2009 & 19901006 & 10.1542/peds.2009-0679 & $\begin{array}{l}\text { HIV risk reduction among detained adolescents: a } \\
\text { randomized, controlled trial }\end{array}$ & Review \\
\hline 51 & Bryan & 2018 & 29435591 & 10.1001/jamapediatrics.2017.5621 & $\begin{array}{l}\text { Effect of Including Alcohol and Cannabis Content in a Sexual } \\
\text { Risk-Reduction Intervention on the Incidence of Sexually } \\
\text { Transmitted Infections in Adolescents: A Cluster Randomized } \\
\text { Clinical Trial }\end{array}$ & $\begin{array}{l}\text { Not all subjects with at least } \\
\text { problematic use }\end{array}$ \\
\hline 52 & Buchan & 2002 & $\begin{array}{l}\text { CN-00411895 } \\
\text { (cochrane) }\end{array}$ & & $\begin{array}{l}\text { Cannabis use: consistency and validity of self-report, on-site } \\
\text { urine testing and laboratory testing }\end{array}$ & $\begin{array}{l}\text { No extractable or relevant } \\
\text { data for } \\
\text { interventions/outcomes of } \\
\text { interest }\end{array}$ \\
\hline 53 & Burleson & 2006 & 17182415 & $10.1080 / 10550490601003656$ & $\begin{array}{l}\text { Absence of iatrogenic or contagion effects in adolescent group } \\
\text { therapy: findings from the Cannabis Youth Treatment (CYT) } \\
\text { study }\end{array}$ & $\begin{array}{l}\text { No extractable or relevant } \\
\text { data for } \\
\text { interventions/outcomes of } \\
\text { interest }\end{array}$ \\
\hline
\end{tabular}




\begin{tabular}{|c|c|c|c|c|c|c|}
\hline No. & Author & Year & $\begin{array}{l}\text { PubMed or } \\
\text { (Other) ID }\end{array}$ & DOI & Title & Reason for Exclusion \\
\hline 54 & Burton & 2007 & 16958129 & 10.1002/eat.20292 & $\begin{array}{l}\text { Experimental test of the affect-regulation theory of bulimic } \\
\text { symptoms and substance use: a randomized trial }\end{array}$ & $\begin{array}{l}\text { Not all subjects with at least } \\
\text { problematic use }\end{array}$ \\
\hline 55 & Callaghan & 2007 & 17618062 & 10.1016/j.addbeh.2007.06.007 & $\begin{array}{l}\text { A case-matched comparison of readmission patterns between } \\
\text { primary methamphetamine-using and primary cocaine-using } \\
\text { adolescents engaged in inpatient substance abuse treatment }\end{array}$ & Review \\
\hline 56 & Campos-Meledy & 2017 & 27929303 & $10.1037 / \mathrm{adb} 0000240$ & $\begin{array}{l}\text { 'The effect of therapists' adherence and competence in } \\
\text { delivering the adolescent community reinforcement approach } \\
\text { on client outcomes': Correction to Campos-Melady et al. } \\
\text { (2016) }\end{array}$ & $\begin{array}{l}\text { No extractable or relevant } \\
\text { data for } \\
\text { interventions/outcomes of } \\
\text { interest }\end{array}$ \\
\hline 57 & Carroll & 2006 & 17032099 & 10.1037/0022-006X.74.5.955 & $\begin{array}{l}\text { The use of contingency management and motivational/skills- } \\
\text { building therapy to treat young adults with marijuana } \\
\text { dependence }\end{array}$ & $\begin{array}{l}\text { Includes transition-aged } \\
\text { youth (non-pharmacological } \\
\text { interventions) }\end{array}$ \\
\hline 58 & Cassidy & 2019 & 31330464 & 10.1016/j.addbeh.2019.106044 & $\begin{array}{l}\text { Alcohol demand moderates brief motivational intervention } \\
\text { outcomes in underage young adult drinkers }\end{array}$ & $\begin{array}{l}\text { No extractable or relevant } \\
\text { data for } \\
\text { interventions/outcomes of } \\
\text { interest }\end{array}$ \\
\hline 59 & Caviness & 2013 & 23795877 & 10.1111/j.1521-0391.2013.12030.x & $\begin{array}{l}\text { Self-efficacy and motivation to quit marijuana use among } \\
\text { young women }\end{array}$ & Case control/cross sectional \\
\hline 60 & Chapman & 2013 & 23668668 & 10.1037/a0033021 & $\begin{array}{l}\text { Comparison of youth, caregiver, therapist, trained, and } \\
\text { treatment expert raters of therapist adherence to a substance } \\
\text { abuse treatment protocol }\end{array}$ & $\begin{array}{l}\text { No extractable or relevant } \\
\text { data for } \\
\text { interventions/outcomes of } \\
\text { interest }\end{array}$ \\
\hline 61 & Chassin & 2009 & 18657942 & 10.1016/j.jsat.2008.06.001 & $\begin{array}{l}\text { Substance use treatment outcomes in a sample of male } \\
\text { serious juvenile offenders }\end{array}$ & $\begin{array}{l}\text { NRCS (nonpharm, } \\
\text { pharmacological } \\
\text { interventions } N<100 \text { ) }\end{array}$ \\
\hline 62 & Cheung & 2013 & $\begin{array}{l}\text { 2013-01351- } \\
014 \text { (psychinfo) }\end{array}$ & 10.1016/j.childyouth.2012.11.006 & $\begin{array}{l}\text { Reducing youth's drug abuse through training social workers } \\
\text { for cognitive/havioral integrated treatment }\end{array}$ & $\begin{array}{l}\text { Not all subjects with at least } \\
\text { problematic use }\end{array}$ \\
\hline 63 & Chi & 2009 & 19344442 & 10.1111/j.1360-0443.2009.02524.x & $\begin{array}{l}\text { Twelve-Step affiliation and 3-year substance use outcomes } \\
\text { among adolescents: Social support and religious service } \\
\text { attendance as potential mediators }\end{array}$ & $\begin{array}{l}\text { Single arm (nonpharm, } \\
\text { pharmacological } \\
\text { interventions } \mathrm{N}<200 \text { ) }\end{array}$ \\
\hline 64 & Christoff Ade & 2015 & 25679364 & 10.1016/j.addbeh.2015.01.019 & $\begin{array}{l}\text { Reducing substance involvement in college students: a three- } \\
\text { arm parallel-group randomized controlled trial of a computer- } \\
\text { based intervention }\end{array}$ & $\begin{array}{l}\text { Includes transition-aged } \\
\text { youth (non-pharmacological } \\
\text { interventions) }\end{array}$ \\
\hline 65 & Chung & 2008 & 18412757 & 10.1111/j.1360-0443.2008.02158.x & $\begin{array}{l}\text { Cannabis withdrawal predicts severity of cannabis involvement } \\
\text { at 1-year follow-up among treated adolescents }\end{array}$ & $\begin{array}{l}\text { Single arm (nonpharm, } \\
\text { pharmacological } \\
\text { interventions } \mathrm{N}<200 \text { ) }\end{array}$ \\
\hline
\end{tabular}




\begin{tabular}{|c|c|c|c|c|c|c|}
\hline No. & Author & Year & $\begin{array}{l}\text { PubMed or } \\
\text { (Other) ID }\end{array}$ & DOI & Title & Reason for Exclusion \\
\hline 66 & Clark & 2005 & 16139960 & 10.1016/j.addbeh.2005.07.017 & $\begin{array}{l}\text { Supervisory neglect and adolescent alcohol use disorders: } \\
\text { effects on AUD onset and treatment outcome }\end{array}$ & $\begin{array}{l}\text { NRCS (nonpharm, } \\
\text { pharmacological } \\
\text { interventions } N<100 \text { ) }\end{array}$ \\
\hline 67 & Clark & 2010 & 19914003 & 10.1016/j.addbeh.2009.10.004 & $\begin{array}{l}\text { Project SUCCESS' effects on the substance use of alternative } \\
\text { high school students }\end{array}$ & $\begin{array}{l}\text { Not all subjects with at least } \\
\text { problematic use }\end{array}$ \\
\hline 68 & Clark & 2014 & 25358829 & 10.1111/jcap.12095 & $\begin{array}{l}\text { Facilitating access to effective and appropriate care for youth } \\
\text { with mild to moderate mental health concerns in New Zealand }\end{array}$ & $\begin{array}{l}\text { Not all subjects with at least } \\
\text { problematic use }\end{array}$ \\
\hline 69 & Clingempeel & 2008 & 18444724 & 10.1037/0002-9432.78.1.29 & $\begin{array}{l}\text { Beyond treatment effects: comorbid psychopathologies and } \\
\text { long-term outcomes among substance-abusing delinquents }\end{array}$ & $\begin{array}{l}\text { No extractable or relevant } \\
\text { data for } \\
\text { interventions/outcomes of } \\
\text { interest }\end{array}$ \\
\hline 70 & Coatsworth & 2001 & 11676271 & & $\begin{array}{l}\text { Brief Strategic Family Therapy versus community control: } \\
\text { engagement, retention, and an exploration of the moderating } \\
\text { role of adolescent symptom severity }\end{array}$ & $\begin{array}{l}\text { Not all subjects with at least } \\
\text { problematic use }\end{array}$ \\
\hline 71 & Collier & 2001 & 11696966 & 10.2190/GMC2-K3XX-XLHF-K2J0 & $\begin{array}{l}\text { The use of node-link mapping in the chemical dependency } \\
\text { treatment of adolescents }\end{array}$ & $\begin{array}{l}\text { No extractable or relevant } \\
\text { data for } \\
\text { interventions/outcomes of } \\
\text { interest }\end{array}$ \\
\hline 72 & Comulada & 2015 & 26114764 & $10.1080 / 15332640.2014 .986354$ & $\begin{array}{l}\text { Compliance to Cell Phone-Based EMA among Latino Youth in } \\
\text { Outpatient Treatment }\end{array}$ & $\begin{array}{l}\text { No extractable or relevant } \\
\text { data for } \\
\text { interventions/outcomes of } \\
\text { interest }\end{array}$ \\
\hline 73 & Conrod & 2006 & 17007600 & 10.1207/s15374424jccp3504_6 & $\begin{array}{l}\text { Efficacy of cognitive-behavioral interventions targeting } \\
\text { personality risk factors for youth alcohol misuse }\end{array}$ & $\begin{array}{l}\text { Not all subjects with at least } \\
\text { problematic use }\end{array}$ \\
\hline 74 & Conrod & 2011 & 21500886 & 10.1037/a0022997 & $\begin{array}{l}\text { Long-term effects of a personality-targeted intervention to } \\
\text { reduce alcohol use in adolescents }\end{array}$ & $\begin{array}{l}\text { Not all subjects with at least } \\
\text { problematic use }\end{array}$ \\
\hline 75 & Conrod & 2013 & 23344135 & 10.1001/jamapsychiatry.2013.651 & $\begin{array}{l}\text { Effectiveness of a selective, personality-targeted prevention } \\
\text { program for adolescent alcohol use and misuse: a cluster } \\
\text { randomized controlled trial }\end{array}$ & $\begin{array}{l}\text { Not all subjects with at least } \\
\text { problematic use }\end{array}$ \\
\hline 76 & Copeland & 2001 & $\begin{array}{l}\text { CN-00510570 } \\
\text { (cochrane) }\end{array}$ & & $\begin{array}{l}\text { A randomized controlled trial of brief interventions for cannabis } \\
\text { problems among young offenders }\end{array}$ & Review \\
\hline 77 & Corbin & 2013 & 23347236 & 10.1111/j.1530-0277.2012.01956.x & $\begin{array}{l}\text { Early subjective response and acquired tolerance as } \\
\text { predictors of alcohol use and related problems in a clinical } \\
\text { sample }\end{array}$ & $\begin{array}{l}\text { No extractable or relevant } \\
\text { data for } \\
\text { interventions/outcomes of } \\
\text { interest }\end{array}$ \\
\hline
\end{tabular}




\begin{tabular}{|c|c|c|c|c|c|c|}
\hline No. & Author & Year & $\begin{array}{l}\text { PubMed or } \\
\text { (Other) ID }\end{array}$ & DOI & Title & Reason for Exclusion \\
\hline 78 & Cornelius & 2005 & 15833583 & 10.1016/j.addbeh.2004.08.025 & $\begin{array}{l}\text { Fluoxetine in adolescents with comorbid major depression and } \\
\text { an alcohol use disorder: a 3-year follow-up study }\end{array}$ & $\begin{array}{l}\text { Single arm (nonpharm, } \\
\text { pharmacological } \\
\text { interventions } N<200 \text { ) }\end{array}$ \\
\hline 79 & Cornelius & 2006 & $\begin{array}{l}\text { CN-00714147 } \\
\text { (cochrane) }\end{array}$ & & $\begin{array}{l}\text { Alcohol use but not cannabis use reported to contribute to } \\
\text { depression in treatment trial of comorbid adolescents }\end{array}$ & Review \\
\hline 80 & Cornelius & 2008 & 18313860 & 10.1016/j.addbeh.2008.02.001 & $\begin{array}{l}\text { Cannabis withdrawal is common among treatment-seeking } \\
\text { adolescents with cannabis dependence and major depression, } \\
\text { and is associated with rapid relapse to dependence }\end{array}$ & $\begin{array}{l}\text { Single arm (nonpharm, } \\
\text { pharmacological } \\
\text { interventions } N<200 \text { ) }\end{array}$ \\
\hline 81 & Cornelius & 2011 & 21530092 & 10.1016/j.addbeh.2011.03.016 & $\begin{array}{l}\text { Evaluation of cognitive behavioral therapy/motivational } \\
\text { enhancement therapy (CBT/MET) in a treatment trial of } \\
\text { comorbid MDD/AUD adolescents }\end{array}$ & $\begin{array}{l}\text { NRCS (nonpharm, } \\
\text { pharmacological } \\
\text { interventions } \mathrm{N}<100 \text { ) }\end{array}$ \\
\hline 82 & Cornelius & 2013 & 25904826 & & $\begin{array}{l}\text { Paradoxical Decrease in Striatal Activation on an fMRI Reward } \\
\text { Task Following Treatment in Youth with Co-morbid Cannabis } \\
\text { Dependence/Major Depression }\end{array}$ & $\mathrm{RCT}, \mathrm{N}<10$ per arm \\
\hline 83 & Correia & 2005 & 15561446 & 10.1016/j.addbeh.2004.04.006 & $\begin{array}{l}\text { Decreased substance use following increases in alternative } \\
\text { behaviors: a preliminary investigation }\end{array}$ & $\begin{array}{l}\text { College setting (alcohol } \\
\text { interventions) }\end{array}$ \\
\hline 84 & Cosden & 2004 & $\begin{array}{l}\text { 2005-03363- } \\
011 \text { (psychinfo) }\end{array}$ & 10.1007/BF03340912 & $\begin{array}{l}\text { Strength-Based Assessment of Adolescents Who Abuse } \\
\text { Drugs: Implications for Helping High-Risk Youth }\end{array}$ & $\begin{array}{l}\text { Single arm (nonpharm, } \\
\text { pharmacological } \\
\text { interventions } \mathrm{N}<200 \text { ) }\end{array}$ \\
\hline 85 & Coulton & 2017 & 28284187 & 10.1186/s12889-017-4170-6 & $\begin{array}{l}\text { Pragmatic randomised controlled trial to evaluate the } \\
\text { effectiveness and cost effectiveness of a multi-component } \\
\text { intervention to reduce substance use and risk-taking } \\
\text { behaviour in adolescents involved in the criminal justice } \\
\text { system: A trial protocol (RISKIT-CJS) }\end{array}$ & $\begin{array}{l}\text { No extractable or relevant } \\
\text { data for } \\
\text { interventions/outcomes of } \\
\text { interest }\end{array}$ \\
\hline 86 & Cousins & 2016 & 26234389 & 10.1111/add.13087 & $\begin{array}{l}\text { Risk of mortality on and off methadone substitution treatment } \\
\text { in primary care: a national cohort study }\end{array}$ & $\begin{array}{l}\text { NRCS (nonpharm, } \\
\text { pharmacological } \\
\text { interventions } \mathrm{N}<100 \text { ) }\end{array}$ \\
\hline 87 & Cox & 2006 & 16470234 & 10.1188/06.ONF.51-60 & $\begin{array}{l}\text { Predicting and modifying substance use in childhood cancer } \\
\text { survivors: application of a conceptual model }\end{array}$ & $\begin{array}{l}\text { Not all subjects with at least } \\
\text { problematic use }\end{array}$ \\
\hline 88 & Cunningham & 1999 & $\begin{array}{l}2000-15331- \\
005 \text { (psychinfo) }\end{array}$ & 10.1023/A:1021951720298 & $\begin{array}{l}\text { Testing underlying assumptions of the family empowerment } \\
\text { perspective }\end{array}$ & $\begin{array}{l}\text { No extractable or relevant } \\
\text { data for } \\
\text { interventions/outcomes of } \\
\text { interest }\end{array}$ \\
\hline 89 & Cunningham & 2012 & 22614776 & 10.1542/peds.2011-3419 & $\begin{array}{l}\text { Brief motivational interviewing intervention for peer violence } \\
\text { and alcohol use in teens: one-year follow-up }\end{array}$ & $\begin{array}{l}\text { Not all subjects with at least } \\
\text { problematic use }\end{array}$ \\
\hline
\end{tabular}




\begin{tabular}{|c|c|c|c|c|c|c|}
\hline No. & Author & Year & $\begin{array}{l}\text { PubMed or } \\
\text { (Other) ID }\end{array}$ & DOI & Title & Reason for Exclusion \\
\hline 90 & Cunningham & 2012 & $\begin{array}{l}\text { CN-00845323 } \\
\text { (cochrane) }\end{array}$ & & $\begin{array}{l}\text { One-year peer violence outcomes following a brief } \\
\text { motivational interviewing intervention for violence and alcohol } \\
\text { among teens }\end{array}$ & Review \\
\hline 91 & Cunningham & 2013 & 23758302 & 10.1111/acem.12151 & $\begin{array}{l}\text { Dating violence: outcomes following a brief motivational } \\
\text { interviewing intervention among at-risk adolescents in an } \\
\text { urban emergency department }\end{array}$ & $\begin{array}{l}\text { Not all subjects with at least } \\
\text { problematic use }\end{array}$ \\
\hline 92 & Cunningham RM & 2009 & 20053240 & 10.1111/j.1553-2712.2009.00513.x & Three-Month Follow-up Of Brief Computerized And Therapist & $\begin{array}{l}\text { Not all subjects with at least } \\
\text { problematic use }\end{array}$ \\
\hline 93 & Curtis & 2015 & 25757693 & $10.1080 / 19371918.2014 .992713$ & $\begin{array}{l}\text { The East Tennessee assertive adolescent family treatment } \\
\text { program: a three-year evaluation }\end{array}$ & $\begin{array}{l}\text { Single arm (nonpharm, } \\
\text { pharmacological } \\
\text { interventions } \mathrm{N}<200 \text { ) }\end{array}$ \\
\hline 94 & D'Amico & 2007 & 18072844 & $10.1037 / 0893-164 X .21 .4 .592$ & $\begin{array}{l}\text { Pilot test of Project CHOICE: a voluntary afterschool } \\
\text { intervention for middle school youth }\end{array}$ & $\begin{array}{l}\text { NRCS (nonpharm, } \\
\text { pharmacological } \\
\text { interventions } N<100 \text { ) }\end{array}$ \\
\hline 95 & D'Amico & 2010 & 21113392 & $10.1080 / 07347324.2010 .511076$ & $\begin{array}{l}\text { Developing a group motivational interviewing intervention for } \\
\text { first-time adolescent offenders at-risk for an alcohol or drug } \\
\text { use disorder }\end{array}$ & $\begin{array}{l}\text { Not all subjects with at least } \\
\text { problematic use }\end{array}$ \\
\hline 96 & D'Amico & 2015 & 25365779 & $10.1037 / a 0038155$ & $\begin{array}{l}\text { Group motivational interviewing for adolescents: Change talk } \\
\text { and alcohol and marijuana outcomes }\end{array}$ & $\begin{array}{l}\text { Single arm (nonpharm, } \\
\text { pharmacological } \\
\text { interventions } \mathrm{N}<200 \text { ) }\end{array}$ \\
\hline 97 & D'Amico & 2017 & 28627914 & $10.1037 / a d b 0000288$ & $\begin{array}{l}\text { Group motivational interviewing for homeless young adults: } \\
\text { Associations of change talk with substance use and sexual } \\
\text { risk behavior }\end{array}$ & $\begin{array}{l}\text { Single arm (nonpharm, } \\
\text { pharmacological } \\
\text { interventions } N<200 \text { ) }\end{array}$ \\
\hline 98 & D'Amico & 2018 & 30138016 & $10.1037 /$ сср0000332 & $\begin{array}{l}\text { Brief motivational interviewing intervention to reduce alcohol } \\
\text { and marijuana use for at-risk adolescents in primary care. }\end{array}$ & $\begin{array}{l}\text { Not all subjects with at least } \\
\text { problematic use }\end{array}$ \\
\hline 99 & D'Amico & 2019 & $\begin{array}{l}\text { CN-01958806 } \\
\text { (Cochrane) }\end{array}$ & & $\begin{array}{l}\text { Engaging at-risk ethnically diverse teens in four primary care } \\
\text { settings in a clinical trial to reduce alcohol and marijuana use }\end{array}$ & $\begin{array}{l}\text { No extractable or relevant } \\
\text { data for } \\
\text { interventions/outcomes of } \\
\text { interest }\end{array}$ \\
\hline 100 & D'Amico & 2019 & 31296568 & 10.1542/peds.2018-3014 & $\begin{array}{l}\text { Understanding which teenagers benefit most from a brief } \\
\text { primary care substance use intervention }\end{array}$ & $\begin{array}{l}\text { Not all subjects with at least } \\
\text { problematic use }\end{array}$ \\
\hline 101 & D'Amico EJ & 2016 & 27940696 & 10.1542/peds.2016-1717 & $\begin{array}{l}\text { Screening in Primary Care: What Is the Best Way to Identify } \\
\text { At-Risk Youth for Substance Use? }\end{array}$ & Case control/cross sectional \\
\hline 102 & D'Onofrio & 2012 & 22459448 & 10.1016/j.annemergmed.2012.02.006 & $\begin{array}{l}\text { A brief intervention reduces hazardous and harmful drinking in } \\
\text { emergency department patients }\end{array}$ & $\begin{array}{l}\text { Includes transition-aged } \\
\text { youth (non-pharmacological } \\
\text { interventions) }\end{array}$ \\
\hline
\end{tabular}




\begin{tabular}{|c|c|c|c|c|c|c|}
\hline No. & Author & Year & $\begin{array}{l}\text { PubMed or } \\
\text { (Other) ID }\end{array}$ & DOI & Title & Reason for Exclusion \\
\hline 103 & Daeppen & 2011 & 20729010 & 10.1016/j.drugalcdep.2010.07.009 & $\begin{array}{l}\text { Efficacy of brief motivational intervention in reducing binge } \\
\text { drinking in young men: a randomized controlled trial }\end{array}$ & $\begin{array}{l}\text { Not all subjects with at least } \\
\text { problematic use }\end{array}$ \\
\hline 104 & Darnell & 2015 & 25767310 & 10.1016/j.childyouth.2015.01.013 & $\begin{array}{l}\text { Quasi-Experimental Study of Functional Family Therapy } \\
\text { Effectiveness for Juvenile Justice Aftercare in a Racially and } \\
\text { Ethnically Diverse Community Sample }\end{array}$ & $\begin{array}{l}\text { Not all subjects with at least } \\
\text { problematic use }\end{array}$ \\
\hline 105 & Dasinger & 2004 & 15152707 & 10.1080/02791072.2004.10399721 & $\begin{array}{l}\text { Assessing the Effectiveness of Community-Based Substance } \\
\text { Abuse Treatment for Adolescents }\end{array}$ & $\begin{array}{l}\text { NRCS (nonpharm, } \\
\text { pharmacological } \\
\text { interventions } N<100 \text { ) }\end{array}$ \\
\hline 106 & Davis & 2013 & 24006963 & $10.1186 / 1472-6882-13-215$ & $\begin{array}{l}\text { Pilot randomized trial on mindfulness training for smokers in } \\
\text { young adult binge drinkers }\end{array}$ & Includes adults ( $>25$ years) \\
\hline 107 & Davis & 2016 & 26710670 & 10.1016/j.jsat.2015.10.004 & $\begin{array}{l}\text { Brief Motivational Interviewing and Normative Feedback for } \\
\text { Adolescents: Change Language and Alcohol Use Outcomes }\end{array}$ & $\begin{array}{l}\text { No extractable or relevant } \\
\text { data for } \\
\text { interventions/outcomes of } \\
\text { interest }\end{array}$ \\
\hline 108 & Davis & 2016 & 27721646 & 10.1080/1067828X.2015.1056866 & $\begin{array}{l}\text { Informed assent recall among adolescents in substance use } \\
\text { disorder treatment research }\end{array}$ & $\begin{array}{l}\text { No extractable or relevant } \\
\text { data for } \\
\text { interventions/outcomes of } \\
\text { interest }\end{array}$ \\
\hline 109 & Davis & 2018 & 29758380 & 10.1016/j.drugalcdep.2018.03.044 & $\begin{array}{l}\text { Predictors of positive drinking outcomes among youth } \\
\text { receiving an alcohol brief intervention in the emergency } \\
\text { department }\end{array}$ & $\begin{array}{l}\text { No extractable or relevant } \\
\text { data for } \\
\text { interventions/outcomes of } \\
\text { interest }\end{array}$ \\
\hline 110 & Deady & 2014 & 24583824 & 10.2196/resprot.3192 & $\begin{array}{l}\text { Evaluating a brief, internet-based intervention for co-occurring } \\
\text { depression and problematic alcohol use in young people: } \\
\text { protocol for a randomized controlled trial }\end{array}$ & $\begin{array}{l}\text { No extractable or relevant } \\
\text { data for } \\
\text { interventions/outcomes of } \\
\text { interest }\end{array}$ \\
\hline 111 & Deady & 2016 & 27009465 & 10.2196/jmir.5178 & $\begin{array}{l}\text { An Online Intervention for Co-Occurring Depression and } \\
\text { Problematic Alcohol Use in Young People: Primary Outcomes } \\
\text { From a Randomized Controlled Trial }\end{array}$ & $\begin{array}{l}\text { Includes transition-aged } \\
\text { youth (non-pharmacological } \\
\text { interventions) }\end{array}$ \\
\hline 112 & Deluca & 2015 & 25886178 & 10.1186/s12889-015-1679-4 & $\begin{array}{l}\text { Linked randomised controlled trials of face-to-face and } \\
\text { electronic brief intervention methods to prevent alcohol related } \\
\text { harm in young people aged 14-17 years presenting to } \\
\text { Emergency Departments (SIPS junior) }\end{array}$ & $\begin{array}{l}\text { No extractable or relevant } \\
\text { data for } \\
\text { interventions/outcomes of } \\
\text { interest }\end{array}$ \\
\hline 113 & DeMartini KS & 2018 & 30138015 & $10.1037 / \operatorname{cсp} 0000323$ & $\begin{array}{l}\text { Drinking goals and attainment in a naltrexone trial of young } \\
\text { adult heavy drinkers }\end{array}$ & $\begin{array}{l}\text { No extractable or relevant } \\
\text { data for } \\
\text { interventions/outcomes of } \\
\text { interest }\end{array}$ \\
\hline
\end{tabular}




\begin{tabular}{|c|c|c|c|c|c|c|}
\hline No. & Author & Year & $\begin{array}{l}\text { PubMed or } \\
\text { (Other) ID }\end{array}$ & DOI & Title & Reason for Exclusion \\
\hline 114 & Dembo & 1999 & $\begin{array}{l}\text { 1999-05802- } \\
004 \text { (psychinfo) }\end{array}$ & 10.1016/S1359-1789(97)00028-1 & $\begin{array}{l}\text { Engaging high risk families in community based intervention } \\
\text { services }\end{array}$ & $\begin{array}{l}\text { Not all subjects with at least } \\
\text { problematic use }\end{array}$ \\
\hline 115 & Dembo & 2000 & $\begin{array}{l}\text { CN-00688968 } \\
\text { (cochrane) }\end{array}$ & & $\begin{array}{l}\text { A longitudinal study of the impact of a family empowerment } \\
\text { intervention on juvenile offender psychosocial functioning: an } \\
\text { expanded assessment }\end{array}$ & $\begin{array}{l}\text { Not all subjects with at least } \\
\text { problematic use }\end{array}$ \\
\hline 116 & Dembo & 2006 & $\begin{array}{l}\text { 2006-08089- } \\
001 \text { (psychinfo) }\end{array}$ & 10.1300/J029v15n04_01 & $\begin{array}{l}\text { The Correlates and Consequences of Drug Involvement } \\
\text { Among Youths Entering a Juvenile Justice Diversion Program }\end{array}$ & $\begin{array}{l}\text { No extractable or relevant } \\
\text { data for } \\
\text { interventions/outcomes of } \\
\text { interest }\end{array}$ \\
\hline 117 & Dembo & 2014 & 25400493 & 10.1080/1067828X.2014.928116 & $\begin{array}{l}\text { Brief Intervention for Truant Youth Sexual Risk Behavior and } \\
\text { Marijuana Use }\end{array}$ & $\begin{array}{l}\text { Not all subjects with at least } \\
\text { problematic use }\end{array}$ \\
\hline 118 & Dembo & & 27616873 & 10.1080/1067828X.2015.1103344 & $\begin{array}{l}\text { Impact of Brief Intervention Services on Drug-Using Truant } \\
\text { Youths' Self-Reported Delinquency and Arrest Charges: A } \\
\text { Longitudinal Study }\end{array}$ & $\begin{array}{l}\text { Single arm (nonpharm, } \\
\text { pharmacological } \\
\text { interventions } N<200 \text { ) }\end{array}$ \\
\hline 119 & Dennis & 2002 & 12460126 & & $\begin{array}{l}\text { The Cannabis Youth Treatment (CYT) experiment: rationale, } \\
\text { study design and analysis plans }\end{array}$ & $\begin{array}{l}\text { No extractable or relevant } \\
\text { data for } \\
\text { interventions/outcomes of } \\
\text { interest }\end{array}$ \\
\hline 120 & Diamond & 1999 & $\begin{array}{l}\text { 2000-05929- } \\
004 \text { (psychinfo) }\end{array}$ & 10.1037/h0087729 & $\begin{array}{l}\text { Alliance-building interventions with adolescents in family } \\
\text { therapy: A process study }\end{array}$ & $\begin{array}{l}\text { NRCS (nonpharm, } \\
\text { pharmacological } \\
\text { interventions } N<100 \text { ) }\end{array}$ \\
\hline 121 & Diamond & 2006 & 17182417 & $10.1080 / 10550490601003664$ & $\begin{array}{l}\text { Early therapeutic alliance as a predictor of treatment outcome } \\
\text { for adolescent cannabis users in outpatient treatment }\end{array}$ & $\begin{array}{l}\text { No extractable or relevant } \\
\text { data for } \\
\text { interventions/outcomes of } \\
\text { interest }\end{array}$ \\
\hline 122 & Diamond & 2006 & $\begin{array}{l}\text { 2006-08089- } \\
002 \text { (psychinfo) }\end{array}$ & 10.1300/J029v15n04_02 & $\begin{array}{l}\text { Psychiatric Syndromes in Adolescents with Marijuana Abuse } \\
\text { and Dependency in Outpatient Treatment }\end{array}$ & $\begin{array}{l}\text { No extractable or relevant } \\
\text { data for } \\
\text { interventions/outcomes of } \\
\text { interest }\end{array}$ \\
\hline 123 & Diaz & 2017 & 28704267 & 10.1097/MEJ.0000000000000488 & $\begin{array}{l}\text { Effect of a brief motivational intervention in reducing alcohol } \\
\text { consumption in the emergency department: a randomized } \\
\text { controlled trial }\end{array}$ & $\begin{array}{l}\text { Includes transition-aged } \\
\text { youth (non-pharmacologica } \\
\text { interventions) }\end{array}$ \\
\hline 124 & Dick & 2019 & 31500618 & 10.1186/s12889-019-7583-6 & $\begin{array}{l}\text { A systematic review of the effectiveness of digital interventions } \\
\text { for illicit substance misuse harm reduction in third-level } \\
\text { students }\end{array}$ & $\begin{array}{l}\text { No extractable or relevant } \\
\text { data for } \\
\text { interventions/outcomes of } \\
\text { interest }\end{array}$ \\
\hline
\end{tabular}

B-11 


\begin{tabular}{|c|c|c|c|c|c|c|}
\hline No. & Author & Year & $\begin{array}{l}\text { PubMed or } \\
\text { (Other) ID }\end{array}$ & DOI & Title & Reason for Exclusion \\
\hline 125 & Diestelkamp & 2014 & 24975110 & 10.1186/1471-227X-14-13 & $\begin{array}{l}\text { Brief motivational intervention for adolescents treated in } \\
\text { emergency departments for acute alcohol intoxication - a } \\
\text { randomized-controlled trial }\end{array}$ & $\begin{array}{l}\text { No extractable or relevant } \\
\text { data for } \\
\text { interventions/outcomes of } \\
\text { interest }\end{array}$ \\
\hline 126 & Diestelkamp & 2016 & 27595811 & 10.13109/prkk.2016.65.7.534 & $\begin{array}{l}\text { [Influence of Counsellor- and Intervention Variables on } \\
\text { Motivation to Change Following a Brief Motivational } \\
\text { Intervention to Reduce Risky Alcohol Use] }\end{array}$ & $\begin{array}{l}\text { No extractable or relevant } \\
\text { data for } \\
\text { interventions/outcomes of } \\
\text { interest }\end{array}$ \\
\hline 127 & Diestelkamp & 2019 & 30670102 & 10.1186/s13063-018-3160-z & $\begin{array}{l}\text { Effectiveness of a web-based screening and brief intervention } \\
\text { with weekly text-message-initiated individualised prompts for } \\
\text { reducing risky alcohol use among teenagers: Study protocol of } \\
\text { a randomised controlled trial within the ProHEAD consortium }\end{array}$ & $\begin{array}{l}\text { No extractable or relevant } \\
\text { data for } \\
\text { interventions/outcomes of } \\
\text { interest }\end{array}$ \\
\hline 128 & Donohue & 1998 & $\begin{array}{l}\text { 1999-10146- } \\
001 \text { (psychinfo) }\end{array}$ & 10.1300/J029v08n01_01 & $\begin{array}{l}\text { Improving initial session attendance of substance abusing and } \\
\text { conduct disordered adolescents: A controlled study }\end{array}$ & $\begin{array}{l}\text { NRCS (nonpharm, } \\
\text { pharmacological } \\
\text { interventions } N<100 \text { ) }\end{array}$ \\
\hline 129 & Doré-Gauthier & 2019 & 30731429 & 10.1016/j.psychres.2019.01.076 & $\begin{array}{l}\text { How to help homeless youth suffering from first episode } \\
\text { psychosis and substance use disorders? The creation of a } \\
\text { new intensive outreach intervention team }\end{array}$ & $\begin{array}{l}\text { Single arm (nonpharm, } \\
\text { pharmacological } \\
\text { interventions } \mathrm{N}<200 \text { ) }\end{array}$ \\
\hline 130 & Douglas-Siegel & 2013 & 23856594 & 10.1016/j.jsat.2013.05.010 & $\begin{array}{l}\text { The effect of recovery coaches for substance-involved } \\
\text { mothers in child welfare: impact on juvenile delinquency }\end{array}$ & $\begin{array}{l}\text { Not all subjects with at least } \\
\text { problematic use }\end{array}$ \\
\hline 131 & Doumas & 2008 & 17600650 & 10.1016/j.jsat.2007.04.006 & $\begin{array}{l}\text { Preventing high-risk drinking in youth in the workplace: a web- } \\
\text { based normative feedback program }\end{array}$ & $\begin{array}{l}\text { Not all subjects with at least } \\
\text { problematic use }\end{array}$ \\
\hline 132 & Doumas & 2014 & 24148137 & 10.1016/j.addbeh.2013.10.011 & $\begin{array}{l}\text { A test of the efficacy of a brief, web-based personalized } \\
\text { feedback intervention to reduce drinking among 9th grade } \\
\text { students }\end{array}$ & $\begin{array}{l}\text { Not all subjects with at least } \\
\text { problematic use }\end{array}$ \\
\hline 133 & Doumas & 2014 & 24666810 & 10.1016/j.jsat.2014.02.006 & $\begin{array}{l}\text { Reducing alcohol use among 9th grade students: } 6 \text { month } \\
\text { outcomes of a brief, Web-based intervention }\end{array}$ & $\begin{array}{l}\text { Not all subjects with at least } \\
\text { problematic use }\end{array}$ \\
\hline 134 & Doumas & 2015 & 25448614 & 10.1016/j.jsat.2014.09.005 & $\begin{array}{l}\text { Web-based personalized feedback: Is this an appropriate } \\
\text { approach for reducing drinking among high school students? }\end{array}$ & $\begin{array}{l}\text { No extractable or relevant } \\
\text { data for } \\
\text { interventions/outcomes of } \\
\text { interest }\end{array}$ \\
\hline 135 & Doumas & 2016 & $\begin{array}{l}\text { 2016-50240- } \\
012 \text { (psychinfo) }\end{array}$ & 10.1080/1067828X.2016.1171185 & $\begin{array}{l}\text { Age of drinking initiation as a moderator of the efficacy of a } \\
\text { brief, web-based personalized feedback alcohol intervention }\end{array}$ & $\begin{array}{l}\text { Not all subjects with at least } \\
\text { problematic use }\end{array}$ \\
\hline 136 & Doumas & 2017 & 28930058 & & $\begin{array}{l}\text { A Randomized Controlled Trial Testing the Efficacy of a Brief } \\
\text { Online Alcohol Intervention for High School Seniors }\end{array}$ & $\begin{array}{l}\text { Not all subjects with at least } \\
\text { problematic use }\end{array}$ \\
\hline
\end{tabular}

B-12 


\begin{tabular}{|c|c|c|c|c|c|c|}
\hline No. & Author & Year & $\begin{array}{l}\text { PubMed or } \\
\text { (Other) ID }\end{array}$ & DOI & Title & Reason for Exclusion \\
\hline 137 & Drost & 2016 & 27103154 & 10.2196/jmir.5223 & $\begin{array}{l}\text { A Web-Based Computer-Tailored Alcohol Prevention Program } \\
\text { for Adolescents: Cost-Effectiveness and Intersectoral Costs } \\
\text { and Benefits }\end{array}$ & $\begin{array}{l}\text { Not all subjects with at least } \\
\text { problematic use }\end{array}$ \\
\hline 138 & Dupont & 2015 & 25990860 & 10.1186/s12889-015-1826-y & $\begin{array}{l}\text { Developing the Moti- } 4 \text { intervention, assessing its feasibility } \\
\text { and pilot testing its effectiveness }\end{array}$ & $\begin{array}{l}\text { Single arm (nonpharm, } \\
\text { pharmacological } \\
\text { interventions } N<200 \text { ) }\end{array}$ \\
\hline 139 & Dupont & 2016 & 26780988 & 10.1016/j.jsat.2015.11.012 & $\begin{array}{l}\text { Assessing the Efficacy of MOTI-4 for Reducing the Use of } \\
\text { Cannabis Among Youth in the Netherlands: A Randomized } \\
\text { Controlled Trial }\end{array}$ & $\begin{array}{l}\text { Includes transition-aged } \\
\text { youth (non-pharmacological } \\
\text { interventions) }\end{array}$ \\
\hline 140 & Dupont & & 28548619 & 10.1080/02791072.2017.1325030 & $\begin{array}{l}\text { Stages of Change Model has Limited Value in Explaining the } \\
\text { Change in Use of Cannabis among Adolescent Participants in } \\
\text { an Efficacious Motivational Interviewing Intervention }\end{array}$ & $\begin{array}{l}\text { Includes transition-aged } \\
\text { youth (non-pharmacological } \\
\text { interventions) }\end{array}$ \\
\hline 141 & Dupouy & & 23337248 & 10.1016/j.jsat.2012.11.006 & $\begin{array}{l}\text { Effectiveness of drug tests in outpatients starting opioid } \\
\text { substitution therapy }\end{array}$ & $\begin{array}{l}\text { No extractable or relevant } \\
\text { data for } \\
\text { interventions/outcomes of } \\
\text { interest }\end{array}$ \\
\hline 142 & Easton & 2012 & 22242558 & 10.3109/00952990.2011.643989 & $\begin{array}{l}\text { Differences in treatment outcome among marijuana- } \\
\text { dependent young adults with and without antisocial personality } \\
\text { disorder }\end{array}$ & $\begin{array}{l}\text { Includes transition-aged } \\
\text { youth (non-pharmacological } \\
\text { interventions) }\end{array}$ \\
\hline 143 & Edelen & 2010 & 19819085 & 10.1016/j.drugalcdep.2009.09.008 & $\begin{array}{l}\text { Long-term effect of community-based treatment: evidence } \\
\text { from the Adolescent Outcomes Project }\end{array}$ & $\begin{array}{l}\text { Not all subjects with at least } \\
\text { problematic use }\end{array}$ \\
\hline 144 & Edwards & 2006 & 16836598 & 10.1111/j.1600-0447.2006.00783.x & $\begin{array}{l}\text { Randomized controlled trial of a cannabis-focused intervention } \\
\text { for young people with first-episode psychosis }\end{array}$ & Includes adults (> 25 years) \\
\hline 145 & Ellis & 1979 & 500887 & & Delinquent drug takers: a follow up & $\begin{array}{l}\text { NRCS (nonpharm, } \\
\text { pharmacological } \\
\text { interventions } N<100 \text { ) }\end{array}$ \\
\hline 146 & Engberg & 2006 & 17156173 & 10.1111/j.1360-0443.2006.01544.x & $\begin{array}{l}\text { Reducing substance use improves adolescents' school } \\
\text { attendance }\end{array}$ & $\begin{array}{l}\text { Single arm (nonpharm, } \\
\text { pharmacological } \\
\text { interventions } \mathrm{N}<200 \text { ) }\end{array}$ \\
\hline 147 & Ewing & 2014 & 24272742 & $10.1177 / 1078345813505445$ & $\begin{array}{l}\text { Continued detention involvement and adolescent marijuana } \\
\text { use trajectories }\end{array}$ & $\begin{array}{l}\text { NRCS (nonpharm, } \\
\text { pharmacological } \\
\text { interventions } \mathrm{N}<100 \text { ) }\end{array}$ \\
\hline 148 & Fagan & 2006 & $\begin{array}{l}\text { 2006-11654- } \\
002 \text { (psychinfo) }\end{array}$ & $10.1177 / 1066480706289651$ & $\begin{array}{l}\text { Counseling and Treating Adolescents With Alcohol and Other } \\
\text { Substance Use Problems and Their Families }\end{array}$ & Review \\
\hline
\end{tabular}




\begin{tabular}{|c|c|c|c|c|c|c|}
\hline No. & Author & Year & $\begin{array}{l}\text { PubMed or } \\
\text { (Other) ID }\end{array}$ & DOI & Title & Reason for Exclusion \\
\hline 149 & Farrow & 1999 & $\begin{array}{l}\text { CN-00159983 } \\
\text { (cochrane) }\end{array}$ & & $\begin{array}{l}\text { Pregnant adolescents in chemical dependency treatment. } \\
\text { Description and outcomes }\end{array}$ & $\begin{array}{l}\text { Single arm (nonpharm, } \\
\text { pharmacological } \\
\text { interventions } N<200 \text { ) }\end{array}$ \\
\hline 150 & Faw & 2004 & $\begin{array}{l}\text { CN-00476789 } \\
\text { (cochrane) }\end{array}$ & & $\begin{array}{l}\text { Multidimensional fidelity evaluation in a residential program for } \\
\text { adolescent substance abuse }\end{array}$ & Review \\
\hline 151 & Feigelman & & 3443889 & 10.1080/02791072.1987.10472421 & $\begin{array}{l}\text { Day-care treatment for multiple drug abusing adolescents: } \\
\text { Social factors linked with completing treatment }\end{array}$ & $\begin{array}{l}\text { Single arm (nonpharm, } \\
\text { pharmacological } \\
\text { interventions } \mathrm{N}<200 \text { ) }\end{array}$ \\
\hline 152 & Feldstein & 2009 & 19298319 & 10.1111/j.1369-1600.2009.00149.x & $\begin{array}{l}\text { Do genetic and individual risk factors moderate the efficacy of } \\
\text { motivational enhancement therapy? Drinking outcomes with } \\
\text { an emerging adult sample }\end{array}$ & $\begin{array}{l}\text { College setting (alcohol } \\
\text { interventions) }\end{array}$ \\
\hline 153 & $\begin{array}{l}\text { Fernández- } \\
\text { Artamendi }\end{array}$ & 2014 & $\begin{array}{l}\text { 2016-25085- } \\
003 \text { (psychinfo) }\end{array}$ & 10.1016/j.ijchp.2014.04.001 & $\begin{array}{l}\text { Evidence-based treatments for adolescents with cannabis use } \\
\text { disorders in the Spanish Public Health System }\end{array}$ & $\mathrm{RCT}, \mathrm{N}<10$ per arm \\
\hline 154 & Fernandes & 2010 & 20385444 & 10.1016/j.addbeh.2010.03.001 & $\begin{array}{l}\text { Brief Motivational Intervention and telemedicine: a new } \\
\text { perspective of treatment to marijuana users }\end{array}$ & $\begin{array}{l}\text { Includes transition-aged } \\
\text { youth (non-pharmacological } \\
\text { interventions) }\end{array}$ \\
\hline 155 & Fischer & 2012 & 22538183 & $10.1186 / 1747-597 X-7-15$ & $\begin{array}{l}\text { 12-month follow-up of an exploratory 'brief intervention' for } \\
\text { high-frequency cannabis users among Canadian university } \\
\text { students }\end{array}$ & Includes adults (> 25 years) \\
\hline 156 & Fischer & 2013 & 22520278 & 10.1016/j.jsat.2012.03.006 & $\begin{array}{l}\text { Feasibility and impact of brief interventions for frequent } \\
\text { cannabis users in Canada }\end{array}$ & Includes adults (> 25 years) \\
\hline 157 & Florsheim & 2008 & $\begin{array}{l}2008-15939- \\
005 \text { (psychinfo) }\end{array}$ & $10.1007 / s 10964-007-9232-0$ & $\begin{array}{l}\text { An experimental test of a craving management technique for } \\
\text { adolescents in substance-abuse treatment }\end{array}$ & $\begin{array}{l}\text { No extractable or relevant } \\
\text { data for } \\
\text { interventions/outcomes of } \\
\text { interest }\end{array}$ \\
\hline 158 & Forman & 1990 & $\begin{array}{l}\text { CN-00346459 } \\
\text { (cochrane) }\end{array}$ & & $\begin{array}{l}\text { Effects of coping skills training on adolescents at risk for } \\
\text { substance use }\end{array}$ & $\begin{array}{l}\text { Not all subjects with at least } \\
\text { problematic use }\end{array}$ \\
\hline 159 & Fors & 1995 & 8907403 & 10.2190/TU92-LX8W-G7FD-9LEM & $\begin{array}{l}\text { Evaluation of a peer-led drug abuse risk reduction project for } \\
\text { runaway/homeless youths }\end{array}$ & $\begin{array}{l}\text { NRCS (nonpharm, } \\
\text { pharmacological } \\
\text { interventions } \mathrm{N}<100 \text { ) }\end{array}$ \\
\hline 160 & Fox & 2011 & 21688873 & 10.1037/a0024331 & Motives for cannabis use in high-risk adolescent users & $\begin{array}{l}\text { No extractable or relevant } \\
\text { data for } \\
\text { interventions/outcomes of } \\
\text { interest }\end{array}$ \\
\hline
\end{tabular}




\begin{tabular}{|c|c|c|c|c|c|c|}
\hline No. & Author & Year & $\begin{array}{l}\text { PubMed or } \\
\text { (Other) ID }\end{array}$ & DOI & Title & Reason for Exclusion \\
\hline 161 & Freeborn & 1995 & 7558471 & & $\begin{array}{l}\text { Adolescent drug misuse treatment and use of medical care } \\
\text { services }\end{array}$ & $\begin{array}{l}\text { NRCS (nonpharm, } \\
\text { pharmacological } \\
\text { interventions } N<100 \text { ) }\end{array}$ \\
\hline 162 & Freedle & 2015 & $\begin{array}{l}2015-55090- \\
009 \text { (psychinfo) }\end{array}$ & & $\begin{array}{l}\text { The role of sandplay therapy in the treatment of adolescents } \\
\text { and young adults with co-occurring substance use disorders } \\
\text { and trauma }\end{array}$ & $\begin{array}{l}\text { NRCS (nonpharm, } \\
\text { pharmacological } \\
\text { interventions } N<100 \text { ) }\end{array}$ \\
\hline 163 & French & 2002 & $\begin{array}{l}\text { CN-00417055 } \\
\text { (cochrane) }\end{array}$ & & $\begin{array}{l}\text { The economic cost of outpatient marijuana treatment for } \\
\text { adolescents: findings from a multi-site field experiment }\end{array}$ & $\begin{array}{l}\text { No extractable or relevant } \\
\text { data for } \\
\text { interventions/outcomes of } \\
\text { interest }\end{array}$ \\
\hline 164 & Freudenberg & 2010 & 20970079 & 10.1016/j.jadohealth.2010.01.008 & $\begin{array}{l}\text { Reducing drug use, human immunodeficiency virus risk, and } \\
\text { recidivism among young men leaving jail: evaluation of the } \\
\text { REAL MEN re-entry program }\end{array}$ & $\begin{array}{l}\text { Not all subjects with at least } \\
\text { problematic use }\end{array}$ \\
\hline 165 & Friedman & 1986 & 3772356 & & $\begin{array}{l}\text { Program characteristics for successful treatment of adolescent } \\
\text { drug abuse }\end{array}$ & $\begin{array}{l}\text { No extractable or relevant } \\
\text { data for } \\
\text { interventions/outcomes of } \\
\text { interest }\end{array}$ \\
\hline 166 & Friedman & 2002 & $\begin{array}{l}\text { 2002-04532- } \\
003 \text { (psychinfo) }\end{array}$ & 10.1300/J029v11n04_03 & $\begin{array}{l}\text { Multimodel substance use intervention program for male } \\
\text { delinquents }\end{array}$ & $\begin{array}{l}\text { Not all subjects with at least } \\
\text { problematic use }\end{array}$ \\
\hline 167 & Fromme & & 8040918 & & $\begin{array}{l}\text { The Alcohol Skills Training Program: a group intervention for } \\
\text { young adult drinkers }\end{array}$ & $\begin{array}{l}\text { College setting (alcohol } \\
\text { interventions) }\end{array}$ \\
\hline 168 & Fulkerson & 2008 & 18607698 & $10.1007 / s 10900-008-9117-5$ & $\begin{array}{l}\text { Relationships between alcohol-related informal social control, } \\
\text { parental monitoring and adolescent problem behaviors among } \\
\text { racially diverse urban youth }\end{array}$ & $\begin{array}{l}\text { Not all subjects with at least } \\
\text { problematic use }\end{array}$ \\
\hline 169 & Galai & 2018 & 29966816 & 10.1016/j.socscimed.2018.06.013 & $\begin{array}{l}\text { A cluster randomized trial of community mobilization to reduce } \\
\text { methamphetamine use and HIV risk among youth in Thailand: } \\
\text { Design, implementation and results }\end{array}$ & $\begin{array}{l}\text { Not all subjects with at least } \\
\text { problematic use }\end{array}$ \\
\hline 170 & Galaif & 2001 & $\begin{array}{l}\text { 2002-02226- } \\
008 \text { (psychinfo) }\end{array}$ & $10.1177 / 0743558401166008$ & $\begin{array}{l}\text { Prospective risk factors and treatment outcomes among } \\
\text { adolescents in DATOS-A }\end{array}$ & $\begin{array}{l}\text { NRCS (nonpharm, } \\
\text { pharmacological } \\
\text { interventions } N<100 \text { ) }\end{array}$ \\
\hline 171 & Gantner & 2006 & 17058778 & & $\begin{array}{l}\text { [Multidimensional family therapy for adolescent clients with } \\
\text { cannabis use disorders--Results and experience from the } \\
\text { INCANT pilot study] }\end{array}$ & $\begin{array}{l}\text { No extractable or relevant } \\
\text { data for } \\
\text { interventions/outcomes of } \\
\text { interest }\end{array}$ \\
\hline
\end{tabular}




\begin{tabular}{|c|c|c|c|c|c|c|}
\hline No. & Author & Year & $\begin{array}{l}\text { PubMed or } \\
\text { (Other) ID }\end{array}$ & DOI & Title & Reason for Exclusion \\
\hline 172 & Gantner & 2006 & $\begin{array}{l}\text { 2006-21027- } \\
002 \text { (psychinfo) }\end{array}$ & & $\begin{array}{l}\text { Multidimensionale Familientherapie für cannabis-abhängige } \\
\text { Jugendliche--Ergebnisse und Erfahrungen aus der 'INCANT'- } \\
\text { Pilotstudie. = Multidimensional Family Therapy for adolescent } \\
\text { clients with cannabis use disorders--Results and experience } \\
\text { from the INCANT pilot study }\end{array}$ & $\begin{array}{l}\text { No extractable or relevant } \\
\text { data for } \\
\text { interventions/outcomes of } \\
\text { interest }\end{array}$ \\
\hline 173 & Gantner & & $\begin{array}{l}2010-11503- \\
006 \text { (psychinfo) }\end{array}$ & 10.1024/0939-5911/a000002 & $\begin{array}{l}\text { Multidimensionale familientherapie (MDFT) in der praxis: } \\
\text { Therapeutische erfahrungen mit jugendlichen cannabis- } \\
\text { abhängigen und ihren familien. = Multidimensional family } \\
\text { therapy in practice: Clinical experiences with adolescent } \\
\text { cannabis abusers and their families }\end{array}$ & $\begin{array}{l}\text { No extractable or relevant } \\
\text { data for } \\
\text { interventions/outcomes of } \\
\text { interest }\end{array}$ \\
\hline 174 & Garcia & 2019 & $\begin{array}{l}\text { CN-01958827 } \\
\text { (Cochrane) }\end{array}$ & & $\begin{array}{l}\text { Alcohol treatment response amonghispanic adolescents: a } \\
\text { randomized trial }\end{array}$ & $\begin{array}{l}\text { No extractable or relevant } \\
\text { data for } \\
\text { interventions/outcomes of } \\
\text { interest }\end{array}$ \\
\hline 175 & Gardner & 2016 & 27296978 & 10.1016/j.drugalcdep.2016.05.018 & $\begin{array}{l}\text { Faster entry into HIV care among HIV-infected drug users who } \\
\text { had been in drug-use treatment programs }\end{array}$ & $\begin{array}{l}\text { No extractable or relevant } \\
\text { data for } \\
\text { interventions/outcomes of } \\
\text { interest }\end{array}$ \\
\hline 176 & Garner & 2008 & 18472665 & $10.1080 / 02791072.2008 .10399761$ & $\begin{array}{l}\text { Predictors of early therapeutic alliance among adolescents in } \\
\text { Substance abuse treatment }\end{array}$ & $\begin{array}{l}\text { No extractable or relevant } \\
\text { data for } \\
\text { interventions/outcomes of } \\
\text { interest }\end{array}$ \\
\hline 177 & Garner & 2009 & 18715742 & 10.1016/j.jsat.2008.06.007 & $\begin{array}{l}\text { Exposure to Adolescent Community Reinforcement Approach } \\
\text { treatment procedures as a mediator of the relationship } \\
\text { between adolescent substance abuse treatment retention and } \\
\text { outcome }\end{array}$ & $\begin{array}{l}\text { No extractable or relevant } \\
\text { data for } \\
\text { interventions/outcomes of } \\
\text { interest }\end{array}$ \\
\hline 178 & Garner & 2010 & 20205824 & $10.1186 / 1748-5908-5-5$ & $\begin{array}{l}\text { The Reinforcing Therapist Performance (RTP) experiment: } \\
\text { study protocol for a cluster randomized trial }\end{array}$ & $\begin{array}{l}\text { No extractable or relevant } \\
\text { data for } \\
\text { interventions/outcomes of } \\
\text { interest }\end{array}$ \\
\hline 179 & Garner & 2012 & 22893231 & 10.1001/archpediatrics.2012.802 & $\begin{array}{l}\text { Using pay for performance to improve treatment } \\
\text { implementation for adolescent substance use disorders: } \\
\text { results from a cluster randomized trial }\end{array}$ & $\begin{array}{l}\text { No extractable or relevant } \\
\text { data for } \\
\text { interventions/outcomes of } \\
\text { interest }\end{array}$ \\
\hline 180 & Garner & 2014 & 25574502 & & $\begin{array}{l}\text { Recovery Support for Adolescents with Substance use } \\
\text { Disorders: The Impact of Recovery Support Telephone Calls } \\
\text { Provided by Pre-Professional Volunteers }\end{array}$ & $\begin{array}{l}\text { NRCS (nonpharm, } \\
\text { pharmacological } \\
\text { interventions } N<100 \text { ) }\end{array}$ \\
\hline
\end{tabular}




\begin{tabular}{|c|c|c|c|c|c|c|}
\hline No. & Author & Year & $\begin{array}{l}\text { PubMed or } \\
\text { (Other) ID }\end{array}$ & DOI & Title & Reason for Exclusion \\
\hline 181 & Garnick & 2012 & 22364777 & 10.1016/j.drugalcdep.2012.01.011 & $\begin{array}{l}\text { The Washington circle engagement performance measures' } \\
\text { association with adolescent treatment outcomes }\end{array}$ & $\begin{array}{l}\text { Single arm (nonpharm, } \\
\text { pharmacological } \\
\text { interventions } N<200 \text { ) }\end{array}$ \\
\hline 182 & Gattamorta & 2017 & 27849405 & 10.1080/10826084.2016.1229338 & $\begin{array}{l}\text { Psychiatric Symptoms, Parental Attachment, and Reasons for } \\
\text { Use as Correlates of Heavy Substance Use Among } \\
\text { Treatment-Seeking Hispanic Adolescents }\end{array}$ & $\begin{array}{l}\text { NRCS (nonpharm, } \\
\text { pharmacological } \\
\text { interventions } N<100 \text { ) }\end{array}$ \\
\hline 183 & Gattamorta & & 28661822 & $10.1080 / 15504263.2017 .1343965$ & $\begin{array}{l}\text { The Comorbidity of Psychiatric and Substance Use Disorders } \\
\text { Among Hispanic Adolescents }\end{array}$ & Case control/cross sectional \\
\hline 184 & Gau & 2012 & 22414236 & 10.1080/16506073.2011.649781 & $\begin{array}{l}\text { Negative life events and substance use moderate cognitive } \\
\text { behavioral adolescent depression prevention intervention }\end{array}$ & $\begin{array}{l}\text { Not all subjects with at least } \\
\text { problematic use }\end{array}$ \\
\hline 185 & Gaume & 2011 & 21777259 & 10.1111/j.1530-0277.2011.01526.x & $\begin{array}{l}\text { Is brief motivational intervention effective in reducing alcohol } \\
\text { use among young men voluntarily receiving it? A randomized } \\
\text { controlled trial }\end{array}$ & $\begin{array}{l}\text { Not all subjects with at least } \\
\text { problematic use }\end{array}$ \\
\hline 186 & Gaume & 2014 & 24961378 & 10.1111/acer.12469 & $\begin{array}{l}\text { Influence of counselor characteristics and behaviors on the } \\
\text { efficacy of a brief motivational intervention for heavy drinking } \\
\text { in young men-a randomized controlled trial }\end{array}$ & $\begin{array}{l}\text { Includes transition-aged } \\
\text { youth (non-pharmacological } \\
\text { interventions) }\end{array}$ \\
\hline 187 & Geller & 1992 & $\begin{array}{l}\text { CN-00185814 } \\
\text { (cochrane) }\end{array}$ & & $\begin{array}{l}\text { Early findings from a pharmacokinetically designed double- } \\
\text { blind and placebo-controlled study of lithium for adolescents } \\
\text { comorbid with biporal and substance dependency disorders }\end{array}$ & $\mathrm{RCT}, \mathrm{N}<10$ per arm \\
\hline 188 & Geller & 1992 & 1589586 & & $\begin{array}{l}\text { Early findings from a pharmacokinetically designed double- } \\
\text { blind and placebo-controlled study of lithium for adolescents } \\
\text { comorbid with bipolar and substance dependency disorders }\end{array}$ & $\mathrm{RCT}, \mathrm{N}<10$ per arm \\
\hline 189 & Gil & 2004 & 15488112 & 10.1111/j.1360-0443.2004.00861.x & $\begin{array}{l}\text { Culturally sensitive substance abuse intervention for Hispanic } \\
\text { and African American adolescents: empirical examples from } \\
\text { the Alcohol Treatment Targeting Adolescents in Need } \\
\text { (ATTAIN) Project }\end{array}$ & $\begin{array}{l}\text { No extractable or relevant } \\
\text { data for } \\
\text { interventions/outcomes of } \\
\text { interest }\end{array}$ \\
\hline 190 & Gilder & 2017 & 29021119 & 10.1016/j.jsat.2017.09.004 & $\begin{array}{l}\text { A pilot randomized trial of Motivational Interviewing compared } \\
\text { to Psycho-Education for reducing and preventing underage } \\
\text { drinking in American Indian adolescents }\end{array}$ & $\begin{array}{l}\text { Not all subjects with at least } \\
\text { problematic use }\end{array}$ \\
\hline 191 & Giles & 2016 & 28011807 & 10.1136/bmjopen-2016-012474 & $\begin{array}{l}\text { Multicentre individual randomised controlled trial of screening } \\
\text { and brief alcohol intervention to prevent risky drinking in young } \\
\text { people aged 14-15 in a high school setting (SIPS JR-HIGH): } \\
\text { study protocol }\end{array}$ & $\begin{array}{l}\text { No extractable or relevant } \\
\text { data for } \\
\text { interventions/outcomes of } \\
\text { interest }\end{array}$ \\
\hline
\end{tabular}




\begin{tabular}{|c|c|c|c|c|c|c|}
\hline No. & Author & Year & $\begin{array}{l}\text { PubMed or } \\
\text { (Other) ID }\end{array}$ & DOI & Title & Reason for Exclusion \\
\hline 192 & Gillespie & 2017 & 28340901 & 10.1016/j.jsat.2017.01.001 & $\begin{array}{l}\text { Predictive validity of an observer-rated adherence protocol for } \\
\text { multisystemic therapy with juvenile drug offenders }\end{array}$ & $\begin{array}{l}\text { No extractable or relevant } \\
\text { data for } \\
\text { interventions/outcomes of } \\
\text { interest }\end{array}$ \\
\hline 193 & Gmel & 2012 & 23089675 & $10.1007 / s 00038-012-0419-0$ & $\begin{array}{l}\text { A quasi-randomized group trial of a brief alcohol intervention } \\
\text { on risky single occasion drinking among secondary school } \\
\text { students }\end{array}$ & $\begin{array}{l}\text { NRCS (nonpharm, } \\
\text { pharmacological } \\
\text { interventions } N<100 \text { ) }\end{array}$ \\
\hline 194 & Gmel & 2013 & 22885010 & 10.1016/j.jsat.2012.07.005 & $\begin{array}{l}\text { Effectiveness of a brief integrative multiple substance use } \\
\text { intervention among young men with and without booster } \\
\text { sessions }\end{array}$ & $\begin{array}{l}\text { Not all subjects with at least } \\
\text { problematic use }\end{array}$ \\
\hline 195 & Godley & 2004 & 15488111 & 10.1111/j.1360-0443.2004.00860.x & $\begin{array}{l}\text { Thirty-month relapse trajectory cluster groups among } \\
\text { adolescents discharged from out-patient treatment }\end{array}$ & $\begin{array}{l}\text { No extractable or relevant } \\
\text { data for } \\
\text { interventions/outcomes of } \\
\text { interest }\end{array}$ \\
\hline 196 & Godley & 2004 & 15152708 & $10.1080 / 02791072.2004 .10399722$ & $\begin{array}{l}\text { Comparing Outcomes of Best-Practice and Research-Based } \\
\text { Outpatient Treatment Protocols for Adolescents }\end{array}$ & $\begin{array}{l}\text { NRCS (nonpharm, } \\
\text { pharmacological } \\
\text { interventions } N<100 \text { ) }\end{array}$ \\
\hline 197 & Godley & 2005 & 15783279 & 10.1037/0893-164X.19.1.62 & $\begin{array}{l}\text { The stability and impact of environmental factors on substance } \\
\text { use and problems after adolescent outpatient treatment for } \\
\text { cannabis abuse or dependence }\end{array}$ & $\begin{array}{l}\text { Single arm (nonpharm, } \\
\text { pharmacological } \\
\text { interventions } \mathrm{N}<200 \text { ) }\end{array}$ \\
\hline 198 & Godley & 2014 & 24294838 & 10.1037/a0035264 & $\begin{array}{l}\text { A randomized trial of assertive continuing care and } \\
\text { contingency management for adolescents with substance use } \\
\text { disorders }\end{array}$ & $\begin{array}{l}\text { NRCS (nonpharm, } \\
\text { pharmacological } \\
\text { interventions } N<100 \text { ) }\end{array}$ \\
\hline 199 & Godley & 2014 & 24462478 & 10.1016/j.jsat.2013.10.013 & $\begin{array}{l}\text { A comparison of treatment outcomes for adolescent } \\
\text { community reinforcement approach participants with and } \\
\text { without co-occurring problems }\end{array}$ & $\begin{array}{l}\text { NRCS (nonpharm, } \\
\text { pharmacological } \\
\text { interventions } N<100 \text { ) }\end{array}$ \\
\hline 200 & Godley & 2017 & 28282523 & 10.1016/j.drugalcdep.2016.12.029 & $\begin{array}{l}\text { Adolescent Community Reinforcement Approach } \\
\text { implementation and treatment outcomes for youth with opioid } \\
\text { problem use }\end{array}$ & $\begin{array}{l}\text { NRCS (nonpharm, } \\
\text { pharmacological } \\
\text { interventions } N<100 \text { ) }\end{array}$ \\
\hline 201 & Goldbach & 2011 & $\begin{array}{l}2011-12055- \\
003 \text { (psychinfo) }\end{array}$ & $10.1080 / 10538720.2011 .560135$ & $\begin{array}{l}\text { An examination of cultural adaptations performed by LGBT- } \\
\text { identified youths to a culturally grounded, evidence-based } \\
\text { substance abuse intervention }\end{array}$ & Review \\
\hline 202 & Goldstein & 2009 & 19858762 & 10.1097/CHI.0b013e3181bef6e8 & $\begin{array}{l}\text { Substance use and the treatment of resistant depression in } \\
\text { adolescents }\end{array}$ & $\begin{array}{l}\text { Not all subjects with at least } \\
\text { problematic use }\end{array}$ \\
\hline
\end{tabular}




\begin{tabular}{|c|c|c|c|c|c|c|}
\hline No. & Author & Year & $\begin{array}{l}\text { PubMed or } \\
\text { (Other) ID }\end{array}$ & DOI & Title & Reason for Exclusion \\
\hline 203 & Gonzales & 2008 & 19042326 & 10.1080/08897070802093312 & An emerging problem & $\begin{array}{l}\text { No extractable or relevant } \\
\text { data for } \\
\text { interventions/outcomes of } \\
\text { interest }\end{array}$ \\
\hline 204 & Gonzales & 2014 & 24629885 & 10.1016/j.jsat.2014.01.010 & $\begin{array}{l}\text { Substance use recovery outcomes among a cohort of youth } \\
\text { participating in a mobile-based texting aftercare pilot program }\end{array}$ & $\begin{array}{l}\text { Includes transition-aged } \\
\text { youth (non-pharmacologica } \\
\text { interventions) }\end{array}$ \\
\hline 205 & Gonzales & 2016 & 26689171 & 10.1111/ajad.12322 & $\begin{array}{l}\text { Youth recovery outcomes at } 6 \text { and } 9 \text { months following } \\
\text { participation in a mobile texting recovery support aftercare } \\
\text { pilot study }\end{array}$ & $\begin{array}{l}\text { Includes transition-aged } \\
\text { youth (non-pharmacologica } \\
\text { interventions) }\end{array}$ \\
\hline 206 & Goorden & 2016 & 27006273 & 10.1016/j.drugalcdep.2016.03.004 & $\begin{array}{l}\text { Cost-effectiveness of multidimensional family therapy } \\
\text { compared to cognitive behavioral therapy for adolescents with } \\
\text { a cannabis use disorder: Data from a randomized controlled } \\
\text { trial }\end{array}$ & $\begin{array}{l}\text { No extractable or relevant } \\
\text { data for } \\
\text { interventions/outcomes of } \\
\text { interest }\end{array}$ \\
\hline 207 & Goti & 2010 & 19779855 & $10.1007 / s 00787-009-0060-5$ & $\begin{array}{l}\text { Brief intervention in substance-use among adolescent } \\
\text { psychiatric patients: a randomized controlled trial }\end{array}$ & $\begin{array}{l}\text { Not all subjects with at least } \\
\text { problematic use }\end{array}$ \\
\hline 208 & Grafsky & 2011 & 21516226 & 10.1016/j.childyouth.2010.10.007 & $\begin{array}{l}\text { Comparison of treatment response among GLB and non-GLB } \\
\text { street-living youth }\end{array}$ & $\begin{array}{l}\text { No extractable or relevant } \\
\text { data for } \\
\text { interventions/outcomes of } \\
\text { interest }\end{array}$ \\
\hline 209 & Gray & & 16131498 & 10.1093/alcalc/agh199 & $\begin{array}{l}\text { The effectiveness of motivational interviewing delivered by } \\
\text { youth workers in reducing drinking, cigarette and cannabis } \\
\text { smoking among young people: quasi-experimental pilot study }\end{array}$ & $\begin{array}{l}\text { NRCS (nonpharm, } \\
\text { pharmacological } \\
\text { interventions } N<100 \text { ) }\end{array}$ \\
\hline 210 & Grazioli & 2015 & 25642586 & 10.1037/adb0000041 & $\begin{array}{l}\text { Protective behavioral strategies and future drinking behaviors: } \\
\text { Effect of drinking intentions }\end{array}$ & $\begin{array}{l}\text { No extractable or relevant } \\
\text { data for } \\
\text { interventions/outcomes of } \\
\text { interest }\end{array}$ \\
\hline 211 & Green & 2007 & 17218647 & $10.1177 / 1077559506296317$ & $\begin{array}{l}\text { How effective are family treatment drug courts? Outcomes } \\
\text { from a four-site national study }\end{array}$ & Includes adults ( $>25$ years) \\
\hline 212 & Gregor & 2003 & 12883517 & & $\begin{array}{l}\text { Feasibility of using an interactive laptop program in the } \\
\text { emergency department to prevent alcohol misuse among } \\
\text { adolescents }\end{array}$ & $\begin{array}{l}\text { No extractable or relevant } \\
\text { data for } \\
\text { interventions/outcomes of } \\
\text { interest }\end{array}$ \\
\hline 213 & Grella & 2001 & 11434639 & & $\begin{array}{l}\text { Drug treatment outcomes for adolescents with comorbid } \\
\text { mental and substance use disorders }\end{array}$ & $\begin{array}{l}\text { NRCS (nonpharm, } \\
\text { pharmacological } \\
\text { interventions } N<100 \text { ) }\end{array}$ \\
\hline
\end{tabular}




\begin{tabular}{|c|c|c|c|c|c|c|}
\hline No. & Author & Year & $\begin{array}{l}\text { PubMed or } \\
\text { (Other) ID }\end{array}$ & DOI & Title & Reason for Exclusion \\
\hline 214 & Grella & 2003 & 12568501 & $10.1177 / 1077559502239610$ & $\begin{array}{l}\text { Treatment processes and outcomes among adolescents with } \\
\text { a history of abuse who are in drug treatment }\end{array}$ & $\begin{array}{l}\text { No extractable or relevant } \\
\text { data for } \\
\text { interventions/outcomes of } \\
\text { interest }\end{array}$ \\
\hline 215 & Grenard & 2007 & 17259065 & 10.1016/j.jadohealth.2006.08.008 & $\begin{array}{l}\text { Brief intervention for substance use among at-risk } \\
\text { adolescents: a pilot study }\end{array}$ & $\begin{array}{l}\text { NRCS (nonpharm, } \\
\text { pharmacological } \\
\text { interventions } N<100 \text { ) }\end{array}$ \\
\hline 216 & Grenier & 1985 & 2991149 & & $\begin{array}{l}\text { Treatment effectiveness in an adolescent chemical } \\
\text { dependency treatment program: a quasi-experimental design }\end{array}$ & $\begin{array}{l}\text { NRCS (nonpharm, } \\
\text { pharmacological } \\
\text { interventions } N<100 \text { ) }\end{array}$ \\
\hline 217 & Griffin & 2012 & 22956890 & $10.1007 / s 10742-012-0089-7$ & $\begin{array}{l}\text { Assessing the Sensitivity of Treatment Effect Estimates to } \\
\text { Differential Follow-Up Rates: Implications for Translational } \\
\text { Research }\end{array}$ & $\begin{array}{l}\text { NRCS (nonpharm, } \\
\text { pharmacological } \\
\text { interventions } N<100 \text { ) }\end{array}$ \\
\hline 218 & Griffin & 2014 & 24440050 & 10.1016/j.drugalcdep.2013.12.017 & $\begin{array}{l}\text { Estimating the causal effects of cumulative treatment episodes } \\
\text { for adolescents using marginal structural models and inverse } \\
\text { probability of treatment weighting }\end{array}$ & $\begin{array}{l}\text { NRCS (nonpharm, } \\
\text { pharmacological } \\
\text { interventions } N<100 \text { ) }\end{array}$ \\
\hline 219 & Grossberg & 2004 & $\begin{array}{l}\text { CN-00505912 } \\
\text { (cochrane) }\end{array}$ & & $\begin{array}{l}\text { Brief physician advice for high-risk drinking among young } \\
\text { adults }\end{array}$ & Includes adults ( $>25$ years) \\
\hline 220 & Guo & 2014 & 24364361 & $10.1037 / a 0035380$ & $\begin{array}{l}\text { Reductions in depressive symptoms among substance- } \\
\text { abusing runaway adolescents and their primary caretakers: a } \\
\text { randomized clinical trial }\end{array}$ & $\begin{array}{l}\text { No extractable or relevant } \\
\text { data for } \\
\text { interventions/outcomes of } \\
\text { interest }\end{array}$ \\
\hline 221 & Guo & 2017 & 28426359 & $10.1080 / 10826084.2016 .1267219$ & $\begin{array}{l}\text { Reductions in Hard Drug Use Among Homeless Youth } \\
\text { Receiving a Strength-Based Outreach Intervention: } \\
\text { Comparing the Long-Term Effects of Shelter Linkage Versus } \\
\text { Drop-in Center Linkage }\end{array}$ & $\begin{array}{l}\text { Includes transition-aged } \\
\text { youth (non-pharmacological } \\
\text { interventions) }\end{array}$ \\
\hline 222 & Guyll & 2004 & 15222836 & 10.1037/0893-3200.18.2.293 & $\begin{array}{l}\text { Family-focused preventive interventions: evaluating parental } \\
\text { risk moderation of substance use trajectories }\end{array}$ & $\begin{array}{l}\text { Not all subjects with at least } \\
\text { problematic use }\end{array}$ \\
\hline 223 & Gwaltney & 2011 & 21126827 & 10.1016/j.addbeh.2010.10.010 & $\begin{array}{l}\text { Using daily drinking data to characterize the effects of a brief } \\
\text { alcohol intervention in an emergency room }\end{array}$ & $\begin{array}{l}\text { Includes transition-aged } \\
\text { youth (non-pharmacological } \\
\text { interventions) }\end{array}$ \\
\hline 224 & Hüsler & 2005 & 15974138 & $10.1081 / J A-200030560$ & $\begin{array}{l}\text { The Action Plan--A New Instrument to Collect Data on } \\
\text { Interventions in Secondary Prevention in Adolescents }\end{array}$ & $\begin{array}{l}\text { Not all subjects with at least } \\
\text { problematic use }\end{array}$ \\
\hline 225 & Haastrup & 1988 & 3348092 & & Eleven year follow-up of 300 young opioid addicts & $\begin{array}{l}\text { Not all subjects with at least } \\
\text { problematic use }\end{array}$ \\
\hline
\end{tabular}




\begin{tabular}{|c|c|c|c|c|c|c|}
\hline No. & Author & Year & $\begin{array}{l}\text { PubMed or } \\
\text { (Other) ID }\end{array}$ & DOI & Title & Reason for Exclusion \\
\hline 226 & Hadland SE & 2018 & 30208470 & 10.1001/jamapediatrics.2018.2143 & $\begin{array}{l}\text { Receipt of Timely Addiction Treatment and Association of } \\
\text { Early Medication Treatment With Retention in Care Among } \\
\text { Youths With Opioid Use Disorder }\end{array}$ & $\begin{array}{l}\text { No extractable or relevant } \\
\text { data for } \\
\text { interventions/outcomes of } \\
\text { interest }\end{array}$ \\
\hline 227 & Hall & 2014 & 24467198 & 10.1037/a0033845 & $\begin{array}{l}\text { Modeling motivation three ways: effects of MI metrics on } \\
\text { treatment outcomes among adolescents }\end{array}$ & $\begin{array}{l}\text { NRCS (nonpharm, } \\
\text { pharmacological } \\
\text { interventions } \mathrm{N}<100 \text { ) }\end{array}$ \\
\hline 228 & Haller & 2014 & 24616136 & 10.1503/cmaj.131301 & $\begin{array}{l}\text { Effectiveness of training family physicians to deliver a brief } \\
\text { intervention to address excessive substance use among } \\
\text { young patients: a cluster randomized controlled trial }\end{array}$ & $\begin{array}{l}\text { Not all subjects with at least } \\
\text { problematic use }\end{array}$ \\
\hline 229 & Hallfors & 2006 & 16809591 & 10.2105/AJPH.2005.067462 & $\begin{array}{l}\text { Efficacy vs effectiveness trial results of an indicated 'model' } \\
\text { substance abuse program: implications for public health }\end{array}$ & $\begin{array}{l}\text { Not all subjects with at least } \\
\text { problematic use }\end{array}$ \\
\hline 230 & Halliday-Boykins & 2010 & 20826076 & 10.1016/j.jsat.2010.07.011 & Predicting nonresponse to juvenile drug court interventions & $\begin{array}{l}\text { No extractable or relevant } \\
\text { data for } \\
\text { interventions/outcomes of } \\
\text { interest }\end{array}$ \\
\hline 231 & Hammond & 2019 & $\begin{array}{l}\text { CN-01958416 } \\
\text { (Cochrane) }\end{array}$ & & $\begin{array}{l}\text { Changes in psychiatric symptoms and opioid use during } \\
\text { buprenorphine/naloxone treatment in opioid-dependent youth }\end{array}$ & $\begin{array}{l}\text { No extractable or relevant } \\
\text { data for } \\
\text { interventions/outcomes of } \\
\text { interest }\end{array}$ \\
\hline 232 & Hammond & 2019 & $\begin{array}{l}\text { CN-01961002 } \\
\text { (Cochrane) }\end{array}$ & & $\begin{array}{l}\text { Association between opioid abstinence and anxious } \\
\text { depression in opioid-dependent youth receiving short-term and } \\
\text { extended buprenorphine/ naloxone-assisted treatment }\end{array}$ & $\begin{array}{l}\text { No extractable or relevant } \\
\text { data for } \\
\text { interventions/outcomes of } \\
\text { interest }\end{array}$ \\
\hline 233 & Harris & 2012 & 22566420 & 10.1542/peds.2011-1624 & $\begin{array}{l}\text { Computer-facilitated substance use screening and brief advice } \\
\text { for teens in primary care: an international trial }\end{array}$ & $\begin{array}{l}\text { Not all subjects with at least } \\
\text { problematic use }\end{array}$ \\
\hline 234 & Harrow & 2006 & 16864469 & $10.1080 / 00952990600753677$ & $\begin{array}{l}\text { The impact of publicly funded managed care on adolescent } \\
\text { substance abuse treatment outcomes }\end{array}$ & $\begin{array}{l}\text { Single arm (nonpharm, } \\
\text { pharmacological } \\
\text { interventions } N<200 \text { ) }\end{array}$ \\
\hline 235 & Hartzler & 2017 & 28797270 & 10.1186/s13012-017-0633-5 & $\begin{array}{l}\text { Implementing the teen marijuana check-up in schools-a study } \\
\text { protocol }\end{array}$ & $\begin{array}{l}\text { No extractable or relevant } \\
\text { data for } \\
\text { interventions/outcomes of } \\
\text { interest }\end{array}$ \\
\hline 236 & Haug & 2014 & 25099872 & 10.1186/1471-2458-14-809 & $\begin{array}{l}\text { Efficacy of a web-and text messaging-based intervention to } \\
\text { reduce problem drinking in young people: study protocol of a } \\
\text { cluster-randomised controlled trial }\end{array}$ & $\begin{array}{l}\text { Not all subjects with at least } \\
\text { problematic use }\end{array}$ \\
\hline
\end{tabular}




\begin{tabular}{|c|c|c|c|c|c|c|}
\hline No. & Author & Year & $\begin{array}{l}\text { PubMed or } \\
\text { (Other) ID }\end{array}$ & DOI & Title & Reason for Exclusion \\
\hline 237 & Haug & 2017 & 27606700 & $10.1037 /$ сср0000138 & $\begin{array}{l}\text { Efficacy of a Web-and Text Messaging-Based Intervention to } \\
\text { Reduce Problem Drinking in Adolescents: results of a Cluster- } \\
\text { Randomized Controlled Trial }\end{array}$ & $\begin{array}{l}\text { Not all subjects with at least } \\
\text { problematic use }\end{array}$ \\
\hline 238 & Haug & 2017 & 29021116 & 10.1016/j.jsat.2017.09.008 & $\begin{array}{l}\text { Efficacy of a technology-based, integrated smoking cessation } \\
\text { and alcohol intervention for smoking cessation in adolescents: } \\
\text { results of a cluster-randomised controlled trial }\end{array}$ & $\begin{array}{l}\text { Not all subjects with at least } \\
\text { problematic use }\end{array}$ \\
\hline 239 & Havard & 2012 & 22014309 & 10.1111/j.1530-0277.2011.01632.x & $\begin{array}{l}\text { Randomized controlled trial of mailed personalized feedback } \\
\text { for problem drinkers in the emergency department: The short- } \\
\text { term impact }\end{array}$ & Includes adults (> 25 years) \\
\hline 240 & Haynes & 2006 & 16676785 & & $\begin{array}{l}\text { Sleep and aggression in substance-abusing adolescents: } \\
\text { results from an integrative behavioral sleep-treatment pilot } \\
\text { program }\end{array}$ & $\begin{array}{l}\text { No extractable or relevant } \\
\text { data for } \\
\text { interventions/outcomes of } \\
\text { interest }\end{array}$ \\
\hline 241 & Helmer & 2016 & 26969585 & 10.1186/s12889-016-2898-z & $\begin{array}{l}\text { Development and evaluation of the efficacy of a web-based } \\
\text { 'social norms'-intervention for the prevention and reduction of } \\
\text { substance use in a cluster-controlled trial conducted at eight } \\
\text { German universities }\end{array}$ & $\begin{array}{l}\text { No extractable or relevant } \\
\text { data for } \\
\text { interventions/outcomes of } \\
\text { interest }\end{array}$ \\
\hline 242 & Henderson & 2017 & 28745011 & 10.1111/eip.12458 & $\begin{array}{l}\text { Enhancing prevention and intervention for youth concurrent } \\
\text { mental health and substance use disorders: The Research } \\
\text { and Action for Teens study }\end{array}$ & $\begin{array}{l}\text { NRCS (nonpharm, } \\
\text { pharmacological } \\
\text { interventions } N<100 \text { ) }\end{array}$ \\
\hline 243 & Henderson & 2017 & 28167747 & 10.1136/bmjopen-2016-014080 & $\begin{array}{l}\text { Integrated collaborative care teams to enhance service } \\
\text { delivery to youth with mental health and substance use } \\
\text { challenges: protocol for a pragmatic randomised controlled } \\
\text { trial }\end{array}$ & $\begin{array}{l}\text { No extractable or relevant } \\
\text { data for } \\
\text { interventions/outcomes of } \\
\text { interest }\end{array}$ \\
\hline 244 & Henggeler & 1991 & $\begin{array}{l}\text { CN-00241801 } \\
\text { (cochrane) }\end{array}$ & & $\begin{array}{l}\text { Effects of multisystemic therapy on drug use and abuse in } \\
\text { serious juvenile offenders: a progress report from two outcome } \\
\text { studies }\end{array}$ & $\begin{array}{l}\text { Not all subjects with at least } \\
\text { problematic use }\end{array}$ \\
\hline 245 & Herrington & 1981 & 7343183 & & $\begin{array}{l}\text { Alcohol and other drug dependence in adolescence: } \\
\text { characteristics of those who seek treatment, and outcome of } \\
\text { treatment }\end{array}$ & $\begin{array}{l}\text { NRCS (nonpharm, } \\
\text { pharmacological } \\
\text { interventions } N<100 \text { ) }\end{array}$ \\
\hline 246 & Hides & 2011 & 21806516 & & $\begin{array}{l}\text { Does the addition of integrated cognitive behaviour therapy } \\
\text { and motivational interviewing improve the outcomes of } \\
\text { standard care for young people with comorbid depression and } \\
\text { substance misuse? }\end{array}$ & $\begin{array}{l}\text { NRCS (nonpharm, } \\
\text { pharmacological } \\
\text { interventions } N<100 \text { ) }\end{array}$ \\
\hline 247 & Hides & 2013 & 23295899 & $10.1159 / 000341921$ & $\begin{array}{l}\text { Quik Fix: a randomized controlled trial of an enhanced brief } \\
\text { motivational interviewing intervention for alcohol/cannabis and } \\
\text { psychological distress in young people }\end{array}$ & $\begin{array}{l}\text { Includes transition-aged } \\
\text { youth (non-pharmacological } \\
\text { interventions) }\end{array}$ \\
\hline
\end{tabular}




\begin{tabular}{|c|c|c|c|c|c|c|}
\hline No. & Author & Year & $\begin{array}{l}\text { PubMed or } \\
\text { (Other) ID }\end{array}$ & DOI & Title & Reason for Exclusion \\
\hline 248 & Hides & 2014 & 25103779 & 10.1186/1471-227X-14-19 & $\begin{array}{l}\text { The Quik Fix study: a randomised controlled trial of brief } \\
\text { interventions for young people with alcohol-related injuries and } \\
\text { illnesses accessing emergency department and crisis support } \\
\text { care }\end{array}$ & $\begin{array}{l}\text { Includes transition-aged } \\
\text { youth (non-pharmacological } \\
\text { interventions) }\end{array}$ \\
\hline 249 & Hides & 2018 & 28992580 & 10.1016/j.addbeh.2017.09.020 & $\begin{array}{l}\text { Efficacy and outcomes of a mobile app targeting alcohol use in } \\
\text { young people }\end{array}$ & $\begin{array}{l}\text { Includes transition-aged } \\
\text { youth (non-pharmacological } \\
\text { interventions) }\end{array}$ \\
\hline 250 & Hides & & $\begin{array}{l}\text { 2007-01081- } \\
008 \text { (psychinfo) }\end{array}$ & & $\begin{array}{l}\text { Young people with co-existing mental health and drug and } \\
\text { alcohol problems }\end{array}$ & Review \\
\hline 251 & Himelstein & 2015 & $\begin{array}{l}\text { CN-01155528 } \\
\text { (cochrane) }\end{array}$ & & $\begin{array}{l}\text { Does mindfulness meditation increase effectiveness of } \\
\text { substance abuse treatment with incarcerated youth? A pilot } \\
\text { randomized controlled trial }\end{array}$ & $\begin{array}{l}\text { No extractable or relevant } \\
\text { data for } \\
\text { interventions/outcomes of } \\
\text { interest }\end{array}$ \\
\hline 252 & Hirschtritt & 2012 & 22116008 & 10.1016/j.jsat.2011.09.010 & $\begin{array}{l}\text { Moderators of fluoxetine treatment response for children and } \\
\text { adolescents with comorbid depression and substance use } \\
\text { disorders }\end{array}$ & $\begin{array}{l}\text { No extractable or relevant } \\
\text { data for } \\
\text { interventions/outcomes of } \\
\text { interest }\end{array}$ \\
\hline 253 & Hjorthø,j & 2013 & 23040144 & 10.1017/S0033291712002255 & $\begin{array}{l}\text { Specialized psychosocial treatment plus treatment as usual } \\
\text { (TAU) versus TAU for patients with cannabis use disorder and } \\
\text { psychosis: the CapOpus randomized trial }\end{array}$ & Includes adults ( $>25$ years) \\
\hline 254 & Hoch & 2012 & 21865014 & 10.1016/j.euroneuro.2011.07.014 & $\begin{array}{l}\text { Efficacy of a targeted cognitive-behavioral treatment program } \\
\text { for cannabis use disorders (CANDIS) }\end{array}$ & Includes adults ( $>25$ years) \\
\hline 255 & Hoeppner & 2014 & 25150401 & 10.1016/j.drugalcdep.2014.07.023 & $\begin{array}{l}\text { Do young people benefit from AA as much, and in the same } \\
\text { ways, as adult aged } 30+\text { ? A moderated multiple mediation } \\
\text { analysis }\end{array}$ & Includes adults ( $>25$ years) \\
\hline 256 & Hoffman & & 8699540 & & $\begin{array}{l}\text { Psychosocial treatments for cocaine abuse. 12-month } \\
\text { treatment outcomes }\end{array}$ & Includes adults (> 25 years) \\
\hline 257 & Hogue & 2013 & 23314000 & 10.1016/j.evalprogplan.2012.12.001 & $\begin{array}{l}\text { Assessing fidelity to evidence-based practices in usual care: } \\
\text { the example of family therapy for adolescent behavior } \\
\text { problems }\end{array}$ & $\begin{array}{l}\text { No extractable or relevant } \\
\text { data for } \\
\text { interventions/outcomes of } \\
\text { interest }\end{array}$ \\
\hline 258 & Hogue & 2015 & 24711046 & 10.1007/s10488-014-0548-2 & $\begin{array}{l}\text { Validity of therapist self-report ratings of fidelity to evidence- } \\
\text { based practices for adolescent behavior problems: } \\
\text { correspondence between therapists and observers }\end{array}$ & $\begin{array}{l}\text { No extractable or relevant } \\
\text { data for } \\
\text { interventions/outcomes of } \\
\text { interest }\end{array}$ \\
\hline
\end{tabular}




\begin{tabular}{|c|c|c|c|c|c|c|}
\hline No. & Author & Year & $\begin{array}{l}\text { PubMed or } \\
\text { (Other) ID }\end{array}$ & DOI & Title & Reason for Exclusion \\
\hline 259 & Hops & 2011 & 21833690 & $10.1007 / s 10461-011-0019-7$ & $\begin{array}{l}\text { Adolescent health-risk sexual behaviors: effects of a drug } \\
\text { abuse intervention }\end{array}$ & $\begin{array}{l}\text { No extractable or relevant } \\
\text { data for } \\
\text { interventions/outcomes of } \\
\text { interest }\end{array}$ \\
\hline 260 & Horigian & 2015 & 26359441 & 10.1111/ajad.12278 & $\begin{array}{l}\text { A cross-sectional assessment of the long term effects of brief } \\
\text { strategic family therapy for adolescent substance use }\end{array}$ & Case control/cross sectional \\
\hline 261 & Hser & 2001 & 11448377 & & An evaluation of drug treatments for adolescents in 4 US cities & $\begin{array}{l}\text { NRCS (nonpharm, } \\
\text { pharmacological } \\
\text { interventions } N<100 \text { ) }\end{array}$ \\
\hline 262 & Hser & 2003 & 12770530 & & $\begin{array}{l}\text { Drug-use initiation and conduct disorder among adolescents in } \\
\text { drug treatment }\end{array}$ & $\begin{array}{l}\text { NRCS (nonpharm, } \\
\text { pharmacological } \\
\text { interventions } N<100 \text { ) }\end{array}$ \\
\hline 263 & Huang & 2011 & 20735217 & $10.3109 / 10826084.2010 .501664$ & $\begin{array}{l}\text { Effects of motivational enhancement therapy on readiness to } \\
\text { change MDMA and methamphetamine use behaviors in } \\
\text { Taiwanese adolescents }\end{array}$ & $\begin{array}{l}\text { No extractable or relevant } \\
\text { data for } \\
\text { interventions/outcomes of } \\
\text { interest }\end{array}$ \\
\hline 264 & Huang & 2014 & 24611528 & 10.1111/famp.12068 & $\begin{array}{l}\text { An application of the Complier Average Causal Effect analysis } \\
\text { to examine the effects of a family intervention in reducing illicit } \\
\text { drug use among high-risk Hispanic adolescents }\end{array}$ & $\begin{array}{l}\text { Not all subjects with at least } \\
\text { problematic use }\end{array}$ \\
\hline 265 & Hunter & 2012 & 22209657 & 10.1016/j.jsat.2011.11.003 & $\begin{array}{l}\text { The effectiveness of community-based delivery of an } \\
\text { evidence-based treatment for adolescent substance use }\end{array}$ & $\begin{array}{l}\text { NRCS (nonpharm, } \\
\text { pharmacological } \\
\text { interventions } \mathrm{N}<100 \text { ) }\end{array}$ \\
\hline 266 & Hunter & 2014 & 24128291 & 10.1037/a0034199 & $\begin{array}{l}\text { Longitudinal change mechanisms for substance use and } \\
\text { illegal activity for adolescents in treatment }\end{array}$ & $\begin{array}{l}\text { Single arm (nonpharm, } \\
\text { pharmacological } \\
\text { interventions } N<200 \text { ) }\end{array}$ \\
\hline 267 & Hunter & 2014 & $\begin{array}{l}\text { 2014-08701- } \\
003 \text { (psychinfo) }\end{array}$ & 10.1080/1754730X.2014.888224 & $\begin{array}{l}\text { Feasibility of implementing the Adolescent Community } \\
\text { Reinforcement Approach in school settings for adolescents } \\
\text { with substance use disorders }\end{array}$ & $\begin{array}{l}\text { Single arm (nonpharm, } \\
\text { pharmacological } \\
\text { interventions } N<200 \text { ) }\end{array}$ \\
\hline 268 & Husted & 1995 & 8555351 & & Multi-dimensional adolescent treatment with American Indians & $\begin{array}{l}\text { Not all subjects with at least } \\
\text { problematic use }\end{array}$ \\
\hline 269 & Imel & 2011 & 21534654 & $10.1037 / a 0023284$ & $\begin{array}{l}\text { Racial/ethnic disparities in therapist effectiveness: A } \\
\text { conceptualization and initial study of cultural competence }\end{array}$ & $\begin{array}{l}\text { No extractable or relevant } \\
\text { data for } \\
\text { interventions/outcomes of } \\
\text { interest }\end{array}$ \\
\hline 270 & Ingels & 2013 & 23998376 & 10.1016/j.drugalcdep.2013.07.036 & $\begin{array}{l}\text { Cost-effectiveness of the strong African American families- } \\
\text { teen program: 1-year follow-up }\end{array}$ & $\begin{array}{l}\text { Not all subjects with at least } \\
\text { problematic use }\end{array}$ \\
\hline
\end{tabular}




\begin{tabular}{|c|c|c|c|c|c|c|}
\hline No. & Author & Year & $\begin{array}{l}\text { PubMed or } \\
\text { (Other) ID }\end{array}$ & DOI & Title & Reason for Exclusion \\
\hline 271 & Jacobus & 2018 & 29679914 & 10.1016/j.drugalcdep.2018.03.007 & $\begin{array}{l}\text { A multi-site proof-of-concept investigation of computerized } \\
\text { approach-avoidance training in adolescent cannabis users }\end{array}$ & $\begin{array}{l}\text { No extractable or relevant } \\
\text { data for } \\
\text { interventions/outcomes of } \\
\text { interest }\end{array}$ \\
\hline 272 & Jafari & 2012 & 24644477 & & $\begin{array}{l}\text { Comparing the effectiveness of Cognitive Behavioral Therapy } \\
\text { and Stages of Change Model on Improving Abstinence Self- } \\
\text { Efficacy in Iranian Substance Dependent Adolescents }\end{array}$ & $\begin{array}{l}\text { No extractable or relevant } \\
\text { data for } \\
\text { interventions/outcomes of } \\
\text { interest }\end{array}$ \\
\hline 273 & Jaffee & 2009 & 20180668 & $10.1080 / 00952990903150860$ & $\begin{array}{l}\text { Methods of recruiting adolescents with psychiatric and } \\
\text { substance use disorders for a clinical trial }\end{array}$ & $\begin{array}{l}\text { No extractable or relevant } \\
\text { data for } \\
\text { interventions/outcomes of } \\
\text { interest }\end{array}$ \\
\hline 274 & Jalling & & 26900316 & $10.1007 / s 10826-015-0263-y$ & $\begin{array}{l}\text { Parent Programs for Reducing Adolescent's Antisocial } \\
\text { Behavior and Substance Use: A Randomized Controlled Trial }\end{array}$ & $\begin{array}{l}\text { Not all subjects with at least } \\
\text { problematic use }\end{array}$ \\
\hline 275 & James & 2011 & $\begin{array}{l}\text { L361167650 } \\
\text { (embase) }\end{array}$ & 10.1080/07347324.2011.538305 & $\begin{array}{l}\text { Characteristics of treatment completers versus treatment } \\
\text { noncompleters in a targeted capacity expansion and HIVIAIDS } \\
\text { education program for adolescents with substance use } \\
\text { disorders }\end{array}$ & $\begin{array}{l}\text { Single arm (nonpharm, } \\
\text { pharmacological } \\
\text { interventions } N<200 \text { ) }\end{array}$ \\
\hline 276 & James-Burdumy & 2012 & 22265113 & 10.1016/j.jadohealth.2011.08.012 & $\begin{array}{l}\text { The effectiveness of mandatory-random student drug testing: } \\
\text { a cluster randomized trial }\end{array}$ & $\begin{array}{l}\text { Not all subjects with at least } \\
\text { problematic use }\end{array}$ \\
\hline 277 & Jander & 2014 & 25301695 & 10.1186/1471-2458-14-1054 & $\begin{array}{l}\text { A Web-based computer-tailored game to reduce binge } \\
\text { drinking among } 16 \text { to } 18 \text { year old Dutch adolescents: } \\
\text { development and study protocol }\end{array}$ & $\begin{array}{l}\text { No extractable or relevant } \\
\text { data for } \\
\text { interventions/outcomes of } \\
\text { interest }\end{array}$ \\
\hline 278 & Jander & 2016 & 26842694 & 10.2196/jmir.4708 & $\begin{array}{l}\text { Effects of a Web-Based Computer-Tailored Game to Reduce } \\
\text { Binge Drinking Among Dutch Adolescents: A Cluster } \\
\text { Randomized Controlled Trial }\end{array}$ & $\begin{array}{l}\text { No extractable or relevant } \\
\text { data for } \\
\text { interventions/outcomes of } \\
\text { interest }\end{array}$ \\
\hline 279 & Jaycox & 2003 & 12921478 & 10.1097/01.CHI.0000046846.56865.F9 & $\begin{array}{l}\text { Mental health and medical problems and service use among } \\
\text { adolescent substance users }\end{array}$ & $\begin{array}{l}\text { NRCS (nonpharm, } \\
\text { pharmacological } \\
\text { interventions } N<100 \text { ) }\end{array}$ \\
\hline 280 & Johnson & 2016 & 27770820 & $10.1186 / s 13063-016-1620-x$ & $\begin{array}{l}\text { A randomised controlled trial of the clinical and cost- } \\
\text { effectiveness of a contingency management intervention } \\
\text { compared to treatment as usual for reduction of cannabis use } \\
\text { and of relapse in early psychosis (CIRCLE): a study protocol } \\
\text { for a randomised controlled trial }\end{array}$ & Includes adults (> 25 years) \\
\hline
\end{tabular}




\begin{tabular}{|c|c|c|c|c|c|c|}
\hline No. & Author & Year & $\begin{array}{l}\text { PubMed or } \\
\text { (Other) ID }\end{array}$ & DOI & Title & Reason for Exclusion \\
\hline 281 & Kaminer & 2006 & 17182419 & $10.1080 / 10550490601006154$ & $\begin{array}{l}\text { Suicidal ideation among adolescents with alcohol use } \\
\text { disorders during treatment and aftercare }\end{array}$ & $\begin{array}{l}\text { No extractable or relevant } \\
\text { data for } \\
\text { interventions/outcomes of } \\
\text { interest }\end{array}$ \\
\hline 282 & Kaminer & 2014 & 25010430 & $10.1080 / 08897077.2014 .933724$ & $\begin{array}{l}\text { The efficacy of contingency management for adolescent } \\
\text { cannabis use disorder: a controlled study }\end{array}$ & $\begin{array}{l}\text { NRCS (nonpharm, } \\
\text { pharmacological } \\
\text { interventions } \mathrm{N}<100 \text { ) }\end{array}$ \\
\hline 283 & Kaminer & 2017 & 28232290 & 10.1016/j.addbeh.2017.02.013 & $\begin{array}{l}\text { Adolescents with cannabis use disorders: Adaptive treatment } \\
\text { for poor responders }\end{array}$ & $\begin{array}{l}\text { Single arm (nonpharm, } \\
\text { pharmacological } \\
\text { interventions } \mathrm{N}<200 \text { ) }\end{array}$ \\
\hline 284 & Kaminer & 2019 & 31403025 & $10.2174 / 2210676608666181102145040$ & $\begin{array}{l}\text { Retention and treatment outcome of youth with cannabis use } \\
\text { disorder referred by the legal system }\end{array}$ & $\begin{array}{l}\text { Single arm (nonpharm, } \\
\text { pharmacological } \\
\text { interventions } \mathrm{N}<200 \text { ) }\end{array}$ \\
\hline 285 & Kampman & 2004 & 15283944 & 10.1016/j.drugalcdep.2004.03.008 & $\begin{array}{l}\text { A pilot trial of topiramate for the treatment of cocaine } \\
\text { dependence }\end{array}$ & Includes adults ( $>25$ years) \\
\hline 286 & Kay-Lambkin & 2015 & 26444863 & 10.1186/s12889-015-2365-2 & $\begin{array}{l}\text { The iTreAD project: a study protocol for a randomised } \\
\text { controlled clinical trial of online treatment and social } \\
\text { networking for binge drinking and depression in young people }\end{array}$ & $\begin{array}{l}\text { No extractable or relevant } \\
\text { data for } \\
\text { interventions/outcomes of } \\
\text { interest }\end{array}$ \\
\hline 287 & Kellogg & 2006 & 16956865 & 10.1300/J069v25n03_03 & $\begin{array}{l}\text { Adolescent and young adult heroin patients: drug use and } \\
\text { success in methadone maintenance treatment }\end{array}$ & $\begin{array}{l}\text { NRCS (nonpharm, } \\
\text { pharmacological } \\
\text { interventions } \mathrm{N}<100 \text { ) }\end{array}$ \\
\hline 288 & Kelly & 2014 & 25294352 & 10.1093/alcalc/agu066 & $\begin{array}{l}\text { Do drug-dependent patients attending alcoholics anonymous } \\
\text { rather than narcotics anonymous do aswell? A prospective, } \\
\text { lagged, matching analysis }\end{array}$ & $\begin{array}{l}\text { No extractable or relevant } \\
\text { data for } \\
\text { interventions/outcomes of } \\
\text { interest }\end{array}$ \\
\hline 289 & Kelly & 2014 & 24945357 & 10.1371/journal.pone.0100121 & $\begin{array}{l}\text { Young adults, social networks, and addiction recovery: post } \\
\text { treatment changes in social ties and their role as a mediator of } \\
\text { 12-step participation }\end{array}$ & $\begin{array}{l}\text { No extractable or relevant } \\
\text { data for } \\
\text { interventions/outcomes of } \\
\text { interest }\end{array}$ \\
\hline 290 & Kemp & 2007 & 17852064 & $10.1080 / 10398560701439665$ & $\begin{array}{l}\text { Stop Using Stuff: trial of a drug and alcohol intervention for } \\
\text { young people with comorbid mental illness and drug and } \\
\text { alcohol problems }\end{array}$ & $\mathrm{RCT}, \mathrm{N}<10$ per arm \\
\hline 291 & Kempf & 1996 & 8703997 & 10.1300/J069v15n02_01 & $\begin{array}{l}\text { Impact of tobacco-free policy on recruitment and retention of } \\
\text { adolescents in residential substance abuse treatment }\end{array}$ & $\begin{array}{l}\text { Not all subjects with at least } \\
\text { problematic use }\end{array}$ \\
\hline
\end{tabular}




\begin{tabular}{|c|c|c|c|c|c|c|}
\hline No. & Author & Year & $\begin{array}{l}\text { PubMed or } \\
\text { (Other) ID }\end{array}$ & DOI & Title & Reason for Exclusion \\
\hline 292 & Kennedy & & 8411298 & & $\begin{array}{l}\text { The Beech Hill Hospital/Outward Bound Adolescent Chemical } \\
\text { Dependency Treatment Program }\end{array}$ & $\begin{array}{l}\text { Single arm (nonpharm, } \\
\text { pharmacological } \\
\text { interventions } N<200 \text { ) }\end{array}$ \\
\hline 293 & Kim & 2011 & 22004305 & 10.1037/a0025949 & $\begin{array}{l}\text { Substance use and delinquency among middle school girls in } \\
\text { foster care: a three-year follow-up of a randomized controlled } \\
\text { trial }\end{array}$ & $\begin{array}{l}\text { Not all subjects with at least } \\
\text { problematic use }\end{array}$ \\
\hline 294 & Kim & 2017 & 28523585 & $10.1007 / s 11121-017-0800-6$ & $\begin{array}{l}\text { Pathways to Preventing Substance Use Among Youth in } \\
\text { Foster Care }\end{array}$ & $\begin{array}{l}\text { Not all subjects with at least } \\
\text { problematic use }\end{array}$ \\
\hline 295 & Kirby & 1999 & 10462097 & & $\begin{array}{l}\text { Community reinforcement training for family and significant } \\
\text { others of drug abusers: a unilateral intervention to increase } \\
\text { treatment entry of drug users }\end{array}$ & Includes adults (> 25 years) \\
\hline 296 & Kirk & 1990 & $\begin{array}{l}\text { 1991-13660- } \\
001 \text { (psychinfo) }\end{array}$ & & $\begin{array}{l}\text { Documenting the effectiveness of adolescent substance abuse } \\
\text { treatment using public school archival records }\end{array}$ & $\begin{array}{l}\text { No extractable or relevant } \\
\text { data for } \\
\text { interventions/outcomes of } \\
\text { interest }\end{array}$ \\
\hline 297 & Knight & 2005 & 16026730 & 10.1016/j.jadohealth.2004.08.020 & $\begin{array}{l}\text { Motivational interviewing for adolescent substance use: a pilot } \\
\text { study }\end{array}$ & $\begin{array}{l}\text { Single arm (nonpharm, } \\
\text { pharmacological } \\
\text { interventions } \mathrm{N}<200 \text { ) }\end{array}$ \\
\hline 298 & Knight & 2015 & 24760288 & $10.1007 / s 10964-014-0127-6$ & $\begin{array}{l}\text { Effectiveness of a theoretically-based judgment and decision } \\
\text { making intervention for adolescents }\end{array}$ & $\begin{array}{l}\text { NRCS (nonpharm, } \\
\text { pharmacological } \\
\text { interventions } N<100 \text { ) }\end{array}$ \\
\hline 299 & Knight & 2016 & 27130175 & 10.1186/s13012-016-0423-5 & $\begin{array}{l}\text { Juvenile Justice-Translational Research on Interventions for } \\
\text { Adolescents in the Legal System (JJ-TRIALS): a cluster } \\
\text { randomized trial targeting system-wide improvement in } \\
\text { substance use services }\end{array}$ & $\begin{array}{l}\text { No extractable or relevant } \\
\text { data for } \\
\text { interventions/outcomes of } \\
\text { interest }\end{array}$ \\
\hline 300 & Knight & 2016 & 26742724 & 10.1016/j.jsat.2015.11.007 & $\begin{array}{l}\text { The Effectiveness of the Treatment Readiness and Induction } \\
\text { Program (TRIP) for Improving During-Treatment Outcomes }\end{array}$ & $\begin{array}{l}\text { NRCS (nonpharm, } \\
\text { pharmacological } \\
\text { interventions } \mathrm{N}<100 \text { ) }\end{array}$ \\
\hline 301 & Knight & 2018 & 29054734 & 10.1016/j.jadohealth.2017.08.013 & $\begin{array}{l}\text { Computer-Facilitated Screening and Brief Advice to Reduce } \\
\text { Adolescents' Heavy Episodic Drinking: A Study in Two } \\
\text { Countries }\end{array}$ & $\begin{array}{l}\text { Single arm (nonpharm, } \\
\text { pharmacological } \\
\text { interventions } N<200 \text { ) }\end{array}$ \\
\hline 302 & Knight & 2019 & 31225897 & 10.1001/jamanetworkopen.2019.6258 & $\begin{array}{l}\text { Effect of Computer-Based Substance Use Screening and Brief } \\
\text { Behavioral Counseling vs Usual Care for Youths in Pediatric } \\
\text { Primary Care: A Pilot Randomized Clinical Trial }\end{array}$ & $\begin{array}{l}\text { Not all subjects with at least } \\
\text { problematic use }\end{array}$ \\
\hline
\end{tabular}




\begin{tabular}{|c|c|c|c|c|c|c|}
\hline No. & Author & Year & $\begin{array}{l}\text { PubMed or } \\
\text { (Other) ID }\end{array}$ & DOI & Title & Reason for Exclusion \\
\hline 303 & Koning & 2011 & 21496753 & 10.1016/j.amepre.2010.12.030 & $\begin{array}{l}\text { Long-term effects of a parent and student intervention on } \\
\text { alcohol use in adolescents: a cluster randomized controlled } \\
\text { trial }\end{array}$ & $\begin{array}{l}\text { Not all subjects with at least } \\
\text { problematic use }\end{array}$ \\
\hline 304 & Koning & 2014 & 24462480 & 10.1016/j.jsat.2013.11.003 & $\begin{array}{l}\text { Differential effects of baseline drinking status: effects of an } \\
\text { alcohol prevention program targeting students and/or parents } \\
\text { (PAS) among weekly drinking students }\end{array}$ & $\begin{array}{l}\text { Not all subjects with at least } \\
\text { problematic use }\end{array}$ \\
\hline 305 & Kristiansen & 2001 & $\begin{array}{l}\text { 2002-02226- } \\
002 \text { (psychinfo) }\end{array}$ & $10.1177 / 0743558401166002$ & $\begin{array}{l}\text { Methodological overview and research design for adolescents } \\
\text { in the Drug Abuse Treatment Outcome Studies }\end{array}$ & Review \\
\hline 306 & Kulis & 2007 & 17096196 & $10.1007 / \mathrm{s} 11121-006-0052-3$ & $\begin{array}{l}\text { Promoting reduced and discontinued substance use among } \\
\text { adolescent substance users: effectiveness of a universal } \\
\text { prevention program }\end{array}$ & $\begin{array}{l}\text { Not all subjects with at least } \\
\text { problematic use }\end{array}$ \\
\hline 307 & LaBrie & 2015 & 25728042 & 10.1007/s11121-015-0549-8 & $\begin{array}{l}\text { The efficacy of a standalone protective behavioral strategies } \\
\text { intervention for students accessing mental health services }\end{array}$ & $\begin{array}{l}\text { College setting (alcohol } \\
\text { interventions) }\end{array}$ \\
\hline 308 & Lakshmana & 2016 & $\begin{array}{l}2016-52318- \\
002 \text { (psychinfo) }\end{array}$ & 10.1080/1533256X.2016.1235414 & $\begin{array}{l}\text { Efficacy of combination of motivational interviewing and } \\
\text { cognitive behavior intervention with substance abuse street } \\
\text { adolescents in India: A randomized control study }\end{array}$ & $\begin{array}{l}\text { No extractable or relevant } \\
\text { data for } \\
\text { interventions/outcomes of } \\
\text { interest }\end{array}$ \\
\hline 309 & Lammers & 2015 & 25892544 & 10.1111/add.12952 & $\begin{array}{l}\text { Effectiveness of a selective intervention program targeting } \\
\text { personality risk factors for alcohol misuse among young } \\
\text { adolescents: results of a cluster randomized controlled trial }\end{array}$ & $\begin{array}{l}\text { Not all subjects with at least } \\
\text { problematic use }\end{array}$ \\
\hline 310 & Lammers & 2017 & 28282524 & 10.1016/j.addbeh.2017.02.030 & $\begin{array}{l}\text { Effectiveness of a selective alcohol prevention program } \\
\text { targeting personality risk factors: Results of interaction } \\
\text { analyses }\end{array}$ & $\begin{array}{l}\text { Not all subjects with at least } \\
\text { problematic use }\end{array}$ \\
\hline 311 & Laporte & 2014 & 24479702 & $10.1186 / 1745-6215-15-40$ & $\begin{array}{l}\text { CANABIC: CANnabis and Adolescents: effect of a Brief } \\
\text { Intervention on their Consumption--study protocol for a } \\
\text { randomized controlled trial }\end{array}$ & $\begin{array}{l}\text { No extractable or relevant } \\
\text { data for } \\
\text { interventions/outcomes of } \\
\text { interest }\end{array}$ \\
\hline 312 & Laporte & 2017 & 28289112 & 10.1370/afm.2003 & $\begin{array}{l}\text { Cannabis and Young Users-A Brief Intervention to Reduce } \\
\text { Their Consumption (CANABIC): A Cluster Randomized } \\
\text { Controlled Trial in Primary Care }\end{array}$ & $\begin{array}{l}\text { Includes transition-aged } \\
\text { youth (non-pharmacological } \\
\text { interventions) }\end{array}$ \\
\hline 313 & Larm P & 2008 & 18375076 & 10.1016/j.drugalcdep.2008.01.026 & $\begin{array}{l}\text { Long-term outcomes of adolescents treated for substance } \\
\text { misuse }\end{array}$ & $\begin{array}{l}\text { Single arm (nonpharm, } \\
\text { pharmacological } \\
\text { interventions } \mathrm{N}<200 \text { ) }\end{array}$ \\
\hline
\end{tabular}




\begin{tabular}{|c|c|c|c|c|c|c|}
\hline No. & Author & Year & $\begin{array}{l}\text { PubMed or } \\
\text { (Other) ID }\end{array}$ & DOI & Title & Reason for Exclusion \\
\hline 314 & Lascaux & 2015 & 25526812 & 10.1016/j.encep.2014.10.013 & $\begin{array}{l}\text { [Comparison of European therapies for cannabis addiction } \\
\text { among adolescents] }\end{array}$ & $\begin{array}{l}\text { No extractable or relevant } \\
\text { data for } \\
\text { interventions/outcomes of } \\
\text { interest }\end{array}$ \\
\hline 315 & Latimer & 2000 & 10860115 & & $\begin{array}{l}\text { Demographic, individual, and interpersonal predictors of } \\
\text { adolescent alcohol and marijuana use following treatment }\end{array}$ & $\begin{array}{l}\text { NRCS (nonpharm, } \\
\text { pharmacological } \\
\text { interventions } N<100 \text { ) }\end{array}$ \\
\hline 316 & Lau-Barraco & 2018 & 29485676 & 10.1111/acer.13606 & $\begin{array}{l}\text { A Randomized Trial of a Personalized Feedback Intervention } \\
\text { for Nonstudent Emerging Adult At-Risk Drinkers }\end{array}$ & $\begin{array}{l}\text { Includes transition-aged } \\
\text { youth (non-pharmacological } \\
\text { interventions) }\end{array}$ \\
\hline 317 & Lecallier & 2012 & $\begin{array}{l}\text { CN-00845229 } \\
\text { (cochrane) }\end{array}$ & & $\begin{array}{l}\text { Screening, referring and counseling of adolescents for } \\
\text { substance abuse. A randomized controlled study on } 2120 \\
\text { students: reperer, orienter, conseiller les adolescents } \\
\text { consommateurs de substances psycho-actives (ROC-ADO). } \\
\text { etude prospective randomisee controlee aupres de } 2120 \\
\text { adolescents }\end{array}$ & $\begin{array}{l}\text { Not all subjects with at least } \\
\text { problematic use }\end{array}$ \\
\hline 318 & Lee & 2010 & 20565152 & 10.1037/a0018859 & $\begin{array}{l}\text { A brief, web-based personalized feedback selective } \\
\text { intervention for college student marijuana use: a randomized } \\
\text { clinical trial }\end{array}$ & $\begin{array}{l}\text { Not all subjects with at least } \\
\text { problematic use }\end{array}$ \\
\hline 319 & Lee & 2015 & 25643024 & 10.1037/a0038792 & $\begin{array}{l}\text { A comparison of delay discounting in adolescents and adults } \\
\text { in treatment for cannabis use disorders }\end{array}$ & $\begin{array}{l}\text { No extractable or relevant } \\
\text { data for } \\
\text { interventions/outcomes of } \\
\text { interest }\end{array}$ \\
\hline 320 & Lee MJ & 2013 & 23163605 & $10.1080 / 10810730.2012 .727949$ & $\begin{array}{l}\text { Underage drinkers' responses to negative-restrictive versus } \\
\text { proactive-nonrestrictive slogans in humorous anti-alcohol } \\
\text { abuse messages: are humorous responsible drinking } \\
\text { campaign messages effective? }\end{array}$ & $\begin{array}{l}\text { No extractable or relevant } \\
\text { data for } \\
\text { interventions/outcomes of } \\
\text { interest }\end{array}$ \\
\hline 321 & Lemma & 2017 & 28551714 & $10.1007 / s 00213-017-4639-0$ & $\begin{array}{l}\text { Cue avoidance training and inhibitory control training for the } \\
\text { reduction of alcohol consumption: a comparison of } \\
\text { effectiveness and investigation of their mechanisms of action }\end{array}$ & $\begin{array}{l}\text { College setting (alcohol } \\
\text { interventions) }\end{array}$ \\
\hline 322 & LeNoue & 2017 & 29064160 & 10.1111/ajad.12634 & $\begin{array}{l}\text { Marijuana commercialization and adolescent substance } \\
\text { treatment outcomes in Colorado }\end{array}$ & $\begin{array}{l}\text { NRCS (nonpharm, } \\
\text { pharmacological } \\
\text { interventions } N<100 \text { ) }\end{array}$ \\
\hline 323 & Leontieva & 2005 & 16253794 & 10.1016/j.jcrc.2005.05.009 & $\begin{array}{l}\text { Readiness to change problematic drinking assessed in the } \\
\text { emergency department as a predictor of change }\end{array}$ & $\begin{array}{l}\text { Single arm (nonpharm, } \\
\text { pharmacological } \\
\text { interventions } N<200 \text { ) }\end{array}$ \\
\hline
\end{tabular}




\begin{tabular}{|c|c|c|c|c|c|c|}
\hline No. & Author & Year & $\begin{array}{l}\text { PubMed or } \\
\text { (Other) ID }\end{array}$ & DOI & Title & Reason for Exclusion \\
\hline 324 & Letourneau & & 26413463 & $10.2174 / 22106766113036660002$ & $\begin{array}{l}\text { Caregiver Involvement in Sexual Risk Reduction with } \\
\text { Substance Using Juvenile Delinquents: Overview and } \\
\text { Preliminary Outcomes of a Randomized Trial }\end{array}$ & $\begin{array}{l}\text { No extractable or relevant } \\
\text { data for } \\
\text { interventions/outcomes of } \\
\text { interest }\end{array}$ \\
\hline 325 & Lewis & 2012 & 22988494 & $10.1155 / 2012 / 235646$ & $\begin{array}{l}\text { Consumer Feedback following Participation in a Family-Based } \\
\text { Intervention for Youth Mental Health }\end{array}$ & $\begin{array}{l}\text { No extractable or relevant } \\
\text { data for } \\
\text { interventions/outcomes of } \\
\text { interest }\end{array}$ \\
\hline 326 & Lewis & 2018 & 29511966 & 10.1007/s11121-018-0879-4 & $\begin{array}{l}\text { Evaluating Personalized Feedback Intervention Framing with a } \\
\text { Randomized Controlled Trial to Reduce Young Adult Alcohol- } \\
\text { Related Sexual Risk Taking }\end{array}$ & $\begin{array}{l}\text { Includes transition-aged } \\
\text { youth (non-pharmacological } \\
\text { interventions) }\end{array}$ \\
\hline 327 & Libby & 2005 & 16098679 & 10.1016/j.addbeh.2005.07.012 & $\begin{array}{l}\text { What came first, major depression or substance use disorder? } \\
\text { Clinical characteristics and substance use comparing teens in } \\
\text { a treatment cohort }\end{array}$ & $\begin{array}{l}\text { No extractable or relevant } \\
\text { data for } \\
\text { interventions/outcomes of } \\
\text { interest }\end{array}$ \\
\hline 328 & Liddle & 2002 & $\begin{array}{l}\text { CN-00384841 } \\
\text { (cochrane) }\end{array}$ & & $\begin{array}{l}\text { A randomized Controlled Trial of Intensive Outpatient, Family- } \\
\text { Based Therapy vs. Residential Drug Treatment for Co-Morbid } \\
\text { Adolescent Substance Abusers }\end{array}$ & Review \\
\hline 329 & Liddle & 2011 & 20427547 & $10.1177 / 0306624 X 10366960$ & $\begin{array}{l}\text { Implementation outcomes of Multidimensional Family } \\
\text { Therapy-Detention to Community: a reintegration program for } \\
\text { drug-using juvenile detainees }\end{array}$ & $\begin{array}{l}\text { NRCS (nonpharm, } \\
\text { pharmacological } \\
\text { interventions } N<100 \text { ) }\end{array}$ \\
\hline 330 & Liddle & & $\begin{array}{l}\text { CN-00642423 } \\
\text { (cochrane) }\end{array}$ & & $\begin{array}{l}\text { Multidimensional family therapy for severely impaired, dually } \\
\text { diagnosed youth: a randomized comparing outpatient and } \\
\text { residential treatment }\end{array}$ & Review \\
\hline 331 & Lifrak & 1997 & 9054806 & & Naltrexone for alcoholic adolescents & $\begin{array}{l}\text { Single arm (nonpharm, } \\
\text { pharmacological } \\
\text { interventions } \mathrm{N}<200 \text { ) }\end{array}$ \\
\hline 332 & Lin & 2016 & $\begin{array}{l}\text { 2016-48192- } \\
011 \text { (psychinfo) }\end{array}$ & $10.1080 / 16066359.2016 .1178244$ & $\begin{array}{l}\text { Trajectories of nonmedical use of prescription opioids among } \\
\text { adolescents in primary care }\end{array}$ & $\begin{array}{l}\text { No extractable or relevant } \\
\text { data for } \\
\text { interventions/outcomes of } \\
\text { interest }\end{array}$ \\
\hline 333 & Lindenberg & 2002 & 12173165 & & $\begin{array}{l}\text { Reducing substance use and risky sexual behavior among } \\
\text { young, low-income, Mexican-American women: comparison of } \\
\text { two interventions }\end{array}$ & $\begin{array}{l}\text { Not all subjects with at least } \\
\text { problematic use }\end{array}$ \\
\hline 334 & Lintz & 2019 & 31298564 & 10.1089/cap.2018.0178 & $\begin{array}{l}\text { Associations between School-Based Substance Use } \\
\text { Treatment and Academic Outcomes }\end{array}$ & $\begin{array}{l}\text { Single arm (nonpharm, } \\
\text { pharmacological } \\
\text { interventions } \mathrm{N}<200 \text { ) }\end{array}$ \\
\hline
\end{tabular}




\begin{tabular}{|c|c|c|c|c|c|c|}
\hline No. & Author & Year & $\begin{array}{l}\text { PubMed or } \\
\text { (Other) ID }\end{array}$ & DOI & Title & Reason for Exclusion \\
\hline 335 & Liu & 2009 & 19288196 & 10.1007/s11121-009-0125-1 & $\begin{array}{l}\text { Evaluating mediation in longitudinal multivariate data: } \\
\text { mediation effects for the Aban Aya Youth Project drug } \\
\text { prevention program }\end{array}$ & $\begin{array}{l}\text { Not all subjects with at least } \\
\text { problematic use }\end{array}$ \\
\hline 336 & Lloyd & 1974 & 4430521 & & $\begin{array}{l}\text { Evolution of a treatment approach for young heroin addicts. } \\
\text { Comparison of three treatment modalities }\end{array}$ & $\begin{array}{l}\text { NRCS (nonpharm, } \\
\text { pharmacological } \\
\text { interventions } N<100 \text { ) }\end{array}$ \\
\hline 337 & Lott & 2009 & 19250774 & 10.1016/j.drugalcdep.2009.01.010 & $\begin{array}{l}\text { Effectiveness of very low-cost contingency management in a } \\
\text { community adolescent treatment program }\end{array}$ & $\begin{array}{l}\text { Single arm (nonpharm, } \\
\text { pharmacological } \\
\text { interventions } N<200 \text { ) }\end{array}$ \\
\hline 338 & Louis-Jacques & 2014 & 24216313 & 10.1016/j.jadohealth.2013.09.012 & $\begin{array}{l}\text { Do risky friends change the efficacy of a primary care brief } \\
\text { intervention for adolescent alcohol use? }\end{array}$ & $\begin{array}{l}\text { NRCS (nonpharm, } \\
\text { pharmacological } \\
\text { interventions } N<100 \text { ) }\end{array}$ \\
\hline 339 & Luchansky & 2006 & 16597576 & 10.1300/J069v25n01_11 & $\begin{array}{l}\text { Treatment readmissions and criminal recidivism in youth } \\
\text { following participation in chemical dependency treatment }\end{array}$ & $\begin{array}{l}\text { No extractable or relevant } \\
\text { data for } \\
\text { interventions/outcomes of } \\
\text { interest }\end{array}$ \\
\hline 340 & Luchansky & 2007 & 17175402 & 10.1016/j.jsat.2006.06.007 & $\begin{array}{l}\text { Treatment response by primary drug of abuse: Does } \\
\text { methamphetamine make a difference? }\end{array}$ & $\begin{array}{l}\text { NRCS (nonpharm, } \\
\text { pharmacological } \\
\text { interventions } N<100 \text { ) }\end{array}$ \\
\hline 341 & Luehring-Jones & 2017 & 28992377 & 10.1111/acer.13520 & $\begin{array}{l}\text { A Single Session of Attentional Bias Modification Reduces } \\
\text { Alcohol Craving and Implicit Measures of Alcohol Bias in } \\
\text { Young Adult Drinkers }\end{array}$ & $\begin{array}{l}\text { No extractable or relevant } \\
\text { data for } \\
\text { interventions/outcomes of } \\
\text { interest }\end{array}$ \\
\hline 342 & Magill & 2009 & 19371492 & & $\begin{array}{l}\text { The role of marijuana use in brief motivational intervention with } \\
\text { young adult drinkers treated in an emergency department }\end{array}$ & $\begin{array}{l}\text { Includes transition-aged } \\
\text { youth (non-pharmacological } \\
\text { interventions) }\end{array}$ \\
\hline 343 & Mahu & 2015 & 26011508 & 10.1111/add.12991 & $\begin{array}{l}\text { Can cannabis use be prevented by targeting personality risk in } \\
\text { schools? Twenty-four-month outcome of the adventure trial on } \\
\text { cannabis use: a cluster-randomized controlled trial }\end{array}$ & $\begin{array}{l}\text { Not all subjects with at least } \\
\text { problematic use }\end{array}$ \\
\hline 344 & Maio & 2005 & 15795723 & 10.1016/j.annemergmed.2004.10.013 & $\begin{array}{l}\text { A randomized controlled trial of an emergency department- } \\
\text { based interactive computer program to prevent alcohol misuse } \\
\text { among injured adolescents }\end{array}$ & $\begin{array}{l}\text { Not all subjects with at least } \\
\text { problematic use }\end{array}$ \\
\hline 345 & March & 2009 & $\begin{array}{l}\text { CN-00726682 } \\
\text { (cochrane) }\end{array}$ & & $\begin{array}{l}\text { Predictors of outcome in Buprenorphine treatment for opioid- } \\
\text { dependent youth }\end{array}$ & Review \\
\hline 346 & Marlatt & 1993 & $\begin{array}{l}\text { 1994-15124- } \\
001 \text { (psychinfo) }\end{array}$ & $10.1016 / S 0005-7894(05) 80314-4$ & $\begin{array}{l}\text { Harm reduction for alcohol problems: Moving beyond the } \\
\text { controlled drinking controversy }\end{array}$ & Review \\
\hline
\end{tabular}




\begin{tabular}{|c|c|c|c|c|c|c|}
\hline No. & Author & Year & $\begin{array}{l}\text { PubMed or } \\
\text { (Other) ID }\end{array}$ & DOI & Title & Reason for Exclusion \\
\hline 347 & Marlowe & 2008 & 19192860 & & $\begin{array}{l}\text { An effectiveness trial of contingency management in a felony } \\
\text { preadjudication drug court }\end{array}$ & $\begin{array}{l}\text { Includes transition-aged } \\
\text { youth (non-pharmacological } \\
\text { interventions) }\end{array}$ \\
\hline 348 & Marsch & 2004 & $\begin{array}{l}\text { CN-00462191 } \\
\text { (cochrane) }\end{array}$ & & $\begin{array}{l}\text { Pharmacological and behavioral interventions for opioid- } \\
\text { dependent adolescents: a randomized, controlled trial }\end{array}$ & Review \\
\hline 349 & Marsiglia & 2015 & 25416154 & $10.1007 / s 10935-014-0380-1$ & $\begin{array}{l}\text { Long-term effects of the keepin' it REAL model program in } \\
\text { Mexico: substance use trajectories of Guadalajara middle } \\
\text { school students }\end{array}$ & $\begin{array}{l}\text { Not all subjects with at least } \\
\text { problematic use }\end{array}$ \\
\hline 350 & Martínez & 2008 & $\begin{array}{l}2008-07457- \\
006 \text { (psychinfo) }\end{array}$ & & $\begin{array}{l}\text { Resultados preliminares del programa de intervenci } \tilde{A}^{3} \mathrm{n} \text { breve } \\
\text { para adolescentes que inician el consumo de alcohol y otras } \\
\text { drogas. = Preliminary study of a brief intervention program for } \\
\text { adolescents who initiate alcohol and other drugs consumption }\end{array}$ & $\begin{array}{l}\text { Single arm (nonpharm, } \\
\text { pharmacological } \\
\text { interventions } N<200 \text { ) }\end{array}$ \\
\hline 351 & Marvel & 2009 & 19378646 & & $\begin{array}{l}\text { Multidimensional family therapy HIVISTD risk-reduction } \\
\text { intervention: an integrative family-based model for drug- } \\
\text { involved juvenile offenders }\end{array}$ & $\begin{array}{l}\text { No extractable or relevant } \\
\text { data for } \\
\text { interventions/outcomes of } \\
\text { interest }\end{array}$ \\
\hline 352 & Mason & 2009 & $\begin{array}{l}\text { 2009-04436- } \\
006 \text { (psychinfo) }\end{array}$ & $10.1080 / 10678280902724184$ & $\begin{array}{l}\text { Brief substance abuse treatment with urban adolescents: A } \\
\text { translational research study }\end{array}$ & $\begin{array}{l}\text { NRCS (nonpharm, } \\
\text { pharmacological } \\
\text { interventions } \mathrm{N}<100 \text { ) }\end{array}$ \\
\hline 353 & Mason & 2018 & 29706169 & 10.1016/j.jsat.2018.03.002 & $\begin{array}{l}\text { A pilot trial of text-delivered peer network counseling to treat } \\
\text { young adults with cannabis use disorder }\end{array}$ & $\begin{array}{l}\text { Includes transition-aged } \\
\text { youth (non-pharmacological } \\
\text { interventions) }\end{array}$ \\
\hline 354 & Mason MJ & 2018 & 30265057 & 10.1037/adb0000403 & $\begin{array}{l}\text { Who responds best to text-delivered cannabis use disorder } \\
\text { treatment? A randomized clinical trial with young adults }\end{array}$ & $\begin{array}{l}\text { Includes transition-aged } \\
\text { youth (non-pharmacological } \\
\text { interventions) }\end{array}$ \\
\hline 355 & Mathews & 2007 & 18351179 & 10.2190/DE.37.4.d & $\begin{array}{l}\text { An impact evaluation of two versions of a brief intervention } \\
\text { targeting alcohol use and physical activity among adolescents }\end{array}$ & $\begin{array}{l}\text { Not all subjects with at least } \\
\text { problematic use }\end{array}$ \\
\hline 356 & McCarthy & 2010 & 21121492 & & $\begin{array}{l}\text { Efficacy of a brief cognitive behavioral therapy program to } \\
\text { reduce excessive drinking behavior among new recruits } \\
\text { entering the Irish Navy: a pilot evaluation }\end{array}$ & $\begin{array}{l}\text { Not all subjects with at least } \\
\text { problematic use }\end{array}$ \\
\hline 357 & McClure & 2014 & 24720376 & $10.3109 / 00952990.2013 .878718$ & $\begin{array}{l}\text { Cigarette smoking during an } \mathrm{N} \text {-acetylcysteine-assisted } \\
\text { cannabis cessation trial in adolescents }\end{array}$ & $\begin{array}{l}\text { No extractable or relevant } \\
\text { data for } \\
\text { interventions/outcomes of } \\
\text { interest }\end{array}$ \\
\hline
\end{tabular}




\begin{tabular}{|c|c|c|c|c|c|c|}
\hline No. & Author & Year & $\begin{array}{l}\text { PubMed or } \\
\text { (Other) ID }\end{array}$ & DOI & Title & Reason for Exclusion \\
\hline 358 & McCollister & 2009 & 18172769 & 10.1007/s11414-007-9094-y & $\begin{array}{l}\text { Estimating the differential costs of criminal activity for juvenile } \\
\text { drug court participants: challenges and recommendations }\end{array}$ & $\begin{array}{l}\text { No extractable or relevant } \\
\text { data for } \\
\text { interventions/outcomes of } \\
\text { interest }\end{array}$ \\
\hline 359 & McGillicuddy & 2001 & 11239729 & & $\begin{array}{l}\text { Development of a skill training program for parents of } \\
\text { substance-abusing adolescents }\end{array}$ & $\mathrm{RCT}, \mathrm{N}<10$ per arm \\
\hline 360 & McKay & 2014 & 25134073 & 10.1016/j.adolescence.2014.07.014 & $\begin{array}{l}\text { The differential impact of a classroom-based, alcohol harm } \\
\text { reduction intervention, on adolescents with different alcohol } \\
\text { use experiences: a multi-level growth modelling analysis }\end{array}$ & $\begin{array}{l}\text { Not all subjects with at least } \\
\text { problematic use }\end{array}$ \\
\hline 361 & McMurran & 1990 & 2310865 & & $\begin{array}{l}\text { Evaluation of a self-help manual for young offenders who } \\
\text { drink: a pilot study }\end{array}$ & $\begin{array}{l}\text { NRCS (nonpharm, } \\
\text { pharmacological } \\
\text { interventions } N<100 \text { ) }\end{array}$ \\
\hline 362 & Mello & 2018 & 29471849 & 10.1186/s13012-018-0725-x & $\begin{array}{l}\text { Implementing Alcohol Misuse SBIRT in a National Cohort of } \\
\text { Pediatric Trauma Centers-a type III hybrid effectiveness- } \\
\text { implementation trial }\end{array}$ & $\begin{array}{l}\text { No extractable or relevant } \\
\text { data for } \\
\text { interventions/outcomes of } \\
\text { interest }\end{array}$ \\
\hline 363 & Melnick & 1997 & 9366969 & & $\begin{array}{l}\text { Motivation and readiness for therapeutic community treatment } \\
\text { among adolescents and adult substance abusers }\end{array}$ & $\begin{array}{l}\text { No extractable or relevant } \\
\text { data for } \\
\text { interventions/outcomes of } \\
\text { interest }\end{array}$ \\
\hline 364 & Meredith LS & 2018 & 29316897 & 10.1186/s12875-017-0689-y & $\begin{array}{l}\text { Influence of mental health and alcohol or other drug use risk } \\
\text { on adolescent reported care received in primary care settings. }\end{array}$ & Case control/cross sectional \\
\hline 365 & Mertens & & 24899076 & 10.1093/alcalc/agu030 & $\begin{array}{l}\text { Effectiveness of nurse-practitioner-delivered brief motivational } \\
\text { intervention for young adult alcohol and drug use in primary } \\
\text { care in South Africa: a randomized clinical trial }\end{array}$ & $\begin{array}{l}\text { Includes transition-aged } \\
\text { youth (non-pharmacological } \\
\text { interventions) }\end{array}$ \\
\hline 366 & Milburn & 2012 & 22443839 & 10.1016/j.jadohealth.2011.08.009 & $\begin{array}{l}\text { A family intervention to reduce sexual risk behavior, substance } \\
\text { use, and delinquency among newly homeless youth }\end{array}$ & $\begin{array}{l}\text { Not all subjects with at least } \\
\text { problematic use }\end{array}$ \\
\hline 367 & Millman & 1978 & 283716 & & Therapeutic detoxification of adolescent heroin addicts & $\begin{array}{l}\text { NRCS (nonpharm, } \\
\text { pharmacological } \\
\text { interventions } \mathrm{N}<100 \text { ) }\end{array}$ \\
\hline 368 & Mitchell & 2012 & 23786511 & 10.1111/j.1521-0391.2012.00299.x & $\begin{array}{l}\text { Screening, brief intervention, and referral to treatment (SBIRT) } \\
\text { for substance use in a school-based program: services and } \\
\text { outcomes }\end{array}$ & $\begin{array}{l}\text { NRCS (nonpharm, } \\
\text { pharmacological } \\
\text { interventions } N<100 \text { ) }\end{array}$ \\
\hline
\end{tabular}




\begin{tabular}{|c|c|c|c|c|c|c|}
\hline No. & Author & Year & $\begin{array}{l}\text { PubMed or } \\
\text { (Other) ID }\end{array}$ & DOI & Title & Reason for Exclusion \\
\hline 369 & Mitchell & 2016 & 26297321 & 10.1016/j.jsat.2015.06.011 & $\begin{array}{l}\text { SBIRT Implementation for Adolescents in Urban Federally } \\
\text { Qualified Health Centers }\end{array}$ & $\begin{array}{l}\text { No extractable or relevant } \\
\text { data for } \\
\text { interventions/outcomes of } \\
\text { interest }\end{array}$ \\
\hline 370 & Mohammadkhani & & $\begin{array}{l}\text { 2016-17978- } \\
003 \text { (psychinfo) }\end{array}$ & & $\begin{array}{l}\text { Effectiveness of guided adolescent problem solving on } \\
\text { craving, attitude toward drug abuse and coping strategies in } \\
\text { adolescents with substance abuse }\end{array}$ & $\begin{array}{l}\text { No extractable or relevant } \\
\text { data for } \\
\text { interventions/outcomes of } \\
\text { interest }\end{array}$ \\
\hline 371 & Moitra & 2016 & 26636547 & 10.1002/da.22460 & $\begin{array}{l}\text { Reductions in cannabis use are associated with mood } \\
\text { improvement in female emerging adults }\end{array}$ & $\begin{array}{l}\text { Includes transition-aged } \\
\text { youth (non-pharmacological } \\
\text { interventions) }\end{array}$ \\
\hline 372 & Molina & 2007 & 17667481 & 10.1097/chi.0b013e3180686d96 & $\begin{array}{l}\text { Delinquent behavior and emerging substance use in the MTA } \\
\text { at } 36 \text { months: prevalence, course, and treatment effects }\end{array}$ & $\begin{array}{l}\text { Not all subjects with at least } \\
\text { problematic use }\end{array}$ \\
\hline 373 & Molina & 2013 & 23452682 & 10.1016/j.jaac.2012.12.014 & $\begin{array}{l}\text { Adolescent substance use in the multimodal treatment study } \\
\text { of attention-deficit/hyperactivity disorder (ADHD) (MTA) as a } \\
\text { function of childhood ADHD, random assignment to childhood } \\
\text { treatments, and subsequent medication }\end{array}$ & $\begin{array}{l}\text { Not all subjects with at least } \\
\text { problematic use }\end{array}$ \\
\hline 374 & Montanaro & 2015 & 26510775 & 10.2196/jmir.4377 & $\begin{array}{l}\text { Using Videogame Apps to Assess Gains in Adolescents' } \\
\text { Substance Use Knowledge: New Opportunities for Evaluating } \\
\text { Intervention Exposure and Content Mastery }\end{array}$ & $\begin{array}{l}\text { Not all subjects with at least } \\
\text { problematic use }\end{array}$ \\
\hline 375 & Montgomery & 2012 & 22743160 & 10.1016/j.drugalcdep.2012.05.033 & $\begin{array}{l}\text { Moderating effects of race in clinical trial participation and } \\
\text { outcomes among marijuana-dependent young adults }\end{array}$ & $\begin{array}{l}\text { Includes transition-aged } \\
\text { youth (non-pharmacological } \\
\text { interventions) }\end{array}$ \\
\hline 376 & Monti & 2007 & 17565560 & 10.1111/j.1360-0443.2007.01878.x & $\begin{array}{l}\text { Motivational interviewing versus feedback only in emergency } \\
\text { care for young adult problem drinking }\end{array}$ & $\begin{array}{l}\text { Includes transition-aged } \\
\text { youth (non-pharmacological } \\
\text { interventions) }\end{array}$ \\
\hline 377 & Moore & 2009 & 19938941 & $10.1080 / 10826080802495229$ & $\begin{array}{l}\text { Efficacy of a brief alcohol consumption reintervention for } \\
\text { adolescents }\end{array}$ & $\begin{array}{l}\text { Not all subjects with at least } \\
\text { problematic use }\end{array}$ \\
\hline 378 & Moore & 2014 & 24041131 & $10.3109 / 10826084.2013 .832328$ & $\begin{array}{l}\text { 'This is not who I want to be:' experiences of opioid-dependent } \\
\text { youth before, and during, combined buprenorphine and } \\
\text { behavioral treatment }\end{array}$ & $\begin{array}{l}\text { No extractable or relevant } \\
\text { data for } \\
\text { interventions/outcomes of } \\
\text { interest }\end{array}$ \\
\hline 379 & Morehouse & 2000 & $\begin{array}{l}2000-07774- \\
001 \text { (psychinfo) }\end{array}$ & & $\begin{array}{l}\text { Preventing and reducing substance use among } \\
\text { institutionalized adolescents }\end{array}$ & $\begin{array}{l}\text { Not all subjects with at least } \\
\text { problematic use }\end{array}$ \\
\hline
\end{tabular}




\begin{tabular}{|c|c|c|c|c|c|c|}
\hline No. & Author & Year & $\begin{array}{l}\text { PubMed or } \\
\text { (Other) ID }\end{array}$ & DOI & Title & Reason for Exclusion \\
\hline 380 & Morgan-Lopez & 2019 & 30981034 & 10.1016/j.addbeh.2019.04.006 & $\begin{array}{l}\text { A quasi-experimental evaluation of partnerships for success's } \\
\text { impact on community-level ethanol and prescription drug } \\
\text { poisoning rates }\end{array}$ & $\begin{array}{l}\text { No extractable or relevant } \\
\text { data for } \\
\text { interventions/outcomes of } \\
\text { interest }\end{array}$ \\
\hline 381 & Morgenstern & 2009 & 19207348 & 10.1111/j.1360-0443.2008.02471.x & $\begin{array}{l}\text { School-based alcohol education: results of a cluster- } \\
\text { randomized controlled trial }\end{array}$ & $\begin{array}{l}\text { Not all subjects with at least } \\
\text { problematic use }\end{array}$ \\
\hline 382 & Morral & 2004 & 15482081 & 10.1037/0893-164X.18.3.257 & $\begin{array}{l}\text { Effectiveness of community-based treatment for substance- } \\
\text { abusing adolescents: 12-month outcomes of youths entering } \\
\text { phoenix academy or alternative probation dispositions }\end{array}$ & $\begin{array}{l}\text { NRCS (nonpharm, } \\
\text { pharmacological } \\
\text { interventions } N<100 \text { ) }\end{array}$ \\
\hline 383 & Motamed & 2008 & 21768987 & 10.1097/ADM.0b013e31816b2f84 & $\begin{array}{l}\text { Differences in Treatment Outcomes between Prescription } \\
\text { Opioid-Dependent and Heroin-Dependent Adolescents }\end{array}$ & $\begin{array}{l}\text { No extractable or relevant } \\
\text { data for } \\
\text { interventions/outcomes of } \\
\text { interest }\end{array}$ \\
\hline 384 & Mun & 2018 & 29229017 & 10.1017/S0954579417001742 & $\begin{array}{l}\text { Adolescence effortful control as a mediator between family } \\
\text { ecology and problematic substance use in early adulthood: A } \\
\text { 16-year prospective study }\end{array}$ & $\begin{array}{l}\text { Not all subjects with at least } \\
\text { problematic use }\end{array}$ \\
\hline 385 & Murphy & 2012 & 22191456 & 10.1089/apc.2011.0157 & $\begin{array}{l}\text { Alcohol and marijuana use outcomes in the Healthy Choices } \\
\text { motivational interviewing intervention for HIV-positive youth }\end{array}$ & $\begin{array}{l}\text { Includes transition-aged } \\
\text { youth (non-pharmacological } \\
\text { interventions) }\end{array}$ \\
\hline 386 & Mustafaoglu & 2019 & 31026384 & 10.1002/ppul.24330 & $\begin{array}{l}\text { Effects of core stabilization exercises on pulmonary function, } \\
\text { respiratory muscle strength, and functional capacity in } \\
\text { adolescents with substance use disorder: Randomized } \\
\text { controlled trial }\end{array}$ & $\begin{array}{l}\text { No extractable or relevant } \\
\text { data for } \\
\text { interventions/outcomes of } \\
\text { interest }\end{array}$ \\
\hline 387 & Myers & 2008 & 19042327 & $10.1080 / 08897070802093361$ & $\begin{array}{l}\text { Does smoking intervention influence adolescent substance } \\
\text { use disorder treatment outcomes? }\end{array}$ & $\begin{array}{l}\text { Not all subjects with at least } \\
\text { problematic use }\end{array}$ \\
\hline 388 & Naar-King & 2006 & 16539572 & 10.1521/aeap.2006.18.1.1 & $\begin{array}{l}\text { Healthy choices: motivational enhancement therapy for health } \\
\text { risk behaviors in HIV-positive youth }\end{array}$ & $\begin{array}{l}\text { Includes transition-aged } \\
\text { youth (non-pharmacological } \\
\text { interventions) }\end{array}$ \\
\hline 389 & Naar-King & 2009 & 19996045 & 10.1001/archpediatrics.2009.212 & $\begin{array}{l}\text { Improving health outcomes for youth living with the human } \\
\text { immunodeficiency virus: a multisite randomized trial of a } \\
\text { motivational intervention targeting multiple risk behaviors }\end{array}$ & $\begin{array}{l}\text { Not all subjects with at least } \\
\text { problematic use }\end{array}$ \\
\hline 390 & Needels & 2005 & 16014874 & 10.1093/jurban/jti092 & $\begin{array}{l}\text { Community case management for former jail inmates: its } \\
\text { impacts on rearrest, drug use, and HIV risk }\end{array}$ & $\begin{array}{l}\text { NRCS (nonpharm, } \\
\text { pharmacological } \\
\text { interventions } N<100 \text { ) }\end{array}$ \\
\hline
\end{tabular}




\begin{tabular}{|c|c|c|c|c|c|c|}
\hline No. & Author & Year & $\begin{array}{l}\text { PubMed or } \\
\text { (Other) ID }\end{array}$ & DOI & Title & Reason for Exclusion \\
\hline 391 & Neighbors & 2010 & 20409432 & & $\begin{array}{l}\text { Cost-effectiveness of a motivational intervention for alcohol- } \\
\text { involved youth in a hospital emergency department }\end{array}$ & $\begin{array}{l}\text { No extractable or relevant } \\
\text { data for } \\
\text { interventions/outcomes of } \\
\text { interest }\end{array}$ \\
\hline 392 & Newcomb & 2018 & 29332235 & $10.1007 / s 10461-018-2027-3$ & $\begin{array}{l}\text { Do Diary Studies Cause Behavior Change? An Examination of } \\
\text { Reactivity in Sexual Risk and Substance Use in Young Men } \\
\text { Who Have Sex with Men }\end{array}$ & Includes adults (> 25 years) \\
\hline 393 & Newton & 2017 & 28801399 & 10.1136/bmjopen-2016-015423 & $\begin{array}{l}\text { A randomised controlled pilot trial evaluating feasibility and } \\
\text { acceptability of a computer-based tool to identify and reduce } \\
\text { harmful and hazardous drinking among adolescents with } \\
\text { alcohol-related presentations in Canadian pediatric emergency } \\
\text { departments }\end{array}$ & $\begin{array}{l}\text { No extractable or relevant } \\
\text { data for } \\
\text { interventions/outcomes of } \\
\text { interest }\end{array}$ \\
\hline 394 & Newton & 2018 & 29783974 & 10.1186/s12889-018-5554-y & $\begin{array}{l}\text { Pathways to prevention: protocol for the CAP (Climate and } \\
\text { Preventure) study to evaluate the long-term effectiveness of } \\
\text { school-based universal, selective and combined alcohol } \\
\text { misuse prevention into early adulthood }\end{array}$ & $\begin{array}{l}\text { Not all subjects with at least } \\
\text { problematic use }\end{array}$ \\
\hline 395 & Niederhofer & 2003 & 12544017 & 10.1097/01.ALC.0000047305.32374.FE & $\begin{array}{l}\text { Tianeptine may be a useful adjunct in the treatment of alcohol } \\
\text { dependence of adolescents }\end{array}$ & Review \\
\hline 396 & Niederhofer & 2003 & 12768462 & $10.1007 / s 00787-003-0327-1$ & $\begin{array}{l}\text { Acamprosate and its efficacy in treating alcohol dependent } \\
\text { adolescents }\end{array}$ & Retracted article \\
\hline 397 & Nilsson & 2004 & $\begin{array}{l}\text { 2004-11429- } \\
004 \text { (psychinfo) }\end{array}$ & 10.1093/heapro/dah108 & $\begin{array}{l}\text { Evaluation of a health promotion programme to prevent the } \\
\text { misuse of androgenic anabolic steroids among Swedish } \\
\text { adolescents }\end{array}$ & $\begin{array}{l}\text { Not all subjects with at least } \\
\text { problematic use }\end{array}$ \\
\hline 398 & Nirenberg & 2013 & 23948537 & & $\begin{array}{l}\text { Treatment may influence self-report and jeopardize our } \\
\text { understanding of outcome }\end{array}$ & $\begin{array}{l}\text { Not all subjects with at least } \\
\text { problematic use }\end{array}$ \\
\hline 399 & Noel & 2006 & 16864466 & $10.1080 / 00952990500328646$ & $\begin{array}{l}\text { The impact of therapeutic case management on participation } \\
\text { in adolescent substance abuse treatment }\end{array}$ & $\begin{array}{l}\text { Not all subjects with at least } \\
\text { problematic use }\end{array}$ \\
\hline 400 & Novins & & 22880543 & $10.1080 / 02791072.2012 .684628$ & $\begin{array}{l}\text { Walking on: celebrating the journeys of Native American } \\
\text { adolescents with substance use problems on the winding road } \\
\text { to healing }\end{array}$ & Review \\
\hline 401 & O'Connor & 2016 & 27219498 & 10.1111/acer.13111 & $\begin{array}{l}\text { Alcohol Intervention for Adolescents with Fetal Alcohol } \\
\text { Spectrum Disorders: Project Step Up, a Treatment } \\
\text { Development Study }\end{array}$ & $\begin{array}{l}\text { NRCS (nonpharm, } \\
\text { pharmacological } \\
\text { interventions } N<100 \text { ) }\end{array}$ \\
\hline 402 & O'Leary-Barrett & 2010 & 20732631 & 10.1016/j.jaac.2010.04.011 & $\begin{array}{l}\text { Personality-targeted interventions delay uptake of drinking and } \\
\text { decrease risk of alcohol-related problems when delivered by } \\
\text { teachers }\end{array}$ & $\begin{array}{l}\text { Not all subjects with at least } \\
\text { problematic use }\end{array}$ \\
\hline
\end{tabular}




\begin{tabular}{|c|c|c|c|c|c|c|}
\hline No. & Author & Year & $\begin{array}{l}\text { PubMed or } \\
\text { (Other) ID }\end{array}$ & DOI & Title & Reason for Exclusion \\
\hline 403 & Ogborne & 1997 & 9143643 & & $\begin{array}{l}\text { Justice system clients of a Toronto youth addiction treatment } \\
\text { program }\end{array}$ & $\begin{array}{l}\text { No extractable or relevant } \\
\text { data for } \\
\text { interventions/outcomes of } \\
\text { interest }\end{array}$ \\
\hline 404 & Okulicz-Kozaryn & 2012 & 22551472 & $10.1186 / 1471-2458-12-319$ & $\begin{array}{l}\text { Effectiveness of the Strengthening Families Programme 10-14 } \\
\text { in Poland for the prevention of alcohol and drug misuse: } \\
\text { protocol for a randomized controlled trial }\end{array}$ & $\begin{array}{l}\text { Not all subjects with at least } \\
\text { problematic use }\end{array}$ \\
\hline 405 & Oliansky & 2009 & $\begin{array}{l}\text { CN-00198135 } \\
\text { (cochrane) }\end{array}$ & & $\begin{array}{l}\text { Effectiveness of brief interventions in reducing substance use } \\
\text { among at- risk primary care patients in three community-based } \\
\text { clinics }\end{array}$ & $\begin{array}{l}\text { Not all subjects with at least } \\
\text { problematic use }\end{array}$ \\
\hline 406 & Olmstead & 2007 & 17645430 & 10.1111/j.1360-0443.2007.01909.x & $\begin{array}{l}\text { The cost-effectiveness of four treatments for marijuana } \\
\text { dependence }\end{array}$ & $\begin{array}{l}\text { Includes transition-aged } \\
\text { youth (non-pharmacological } \\
\text { interventions) }\end{array}$ \\
\hline 407 & Orlando & 2003 & 12765210 & 10.1081/ADA-120020518 & $\begin{array}{l}\text { Retention of court-referred youths in residential treatment } \\
\text { programs: Client characteristics and treatment process effects }\end{array}$ & $\begin{array}{l}\text { No extractable or relevant } \\
\text { data for } \\
\text { interventions/outcomes of } \\
\text { interest }\end{array}$ \\
\hline 408 & Ozdemir & 2016 & 26381442 & 10.1111/add.13177 & $\begin{array}{l}\text { Does promoting parents' negative attitudes to underage } \\
\text { drinking reduce adolescents' drinking? The mediating process } \\
\text { and moderators of the effects of the Orebro Prevention } \\
\text { Programme }\end{array}$ & $\begin{array}{l}\text { Not all subjects with at least } \\
\text { problematic use }\end{array}$ \\
\hline 409 & Ozechowski & 2014 & 24512127 & 10.1037/a0035889 & $\begin{array}{l}\text { Empirical Bayes MCMC estimation for modeling treatment } \\
\text { processes, mechanisms of change, and clinical outcomes in } \\
\text { small samples }\end{array}$ & $\begin{array}{l}\text { No extractable or relevant } \\
\text { data for } \\
\text { interventions/outcomes of } \\
\text { interest }\end{array}$ \\
\hline 410 & Palfai & 2014 & 24845164 & 10.1016/j.addbeh.2014.04.025 & $\begin{array}{l}\text { Web-based screening and brief intervention for student } \\
\text { marijuana use in a university health center: pilot study to } \\
\text { examine the implementation of eCHECKUP TO GO in } \\
\text { different contexts }\end{array}$ & $\begin{array}{l}\text { Includes transition-aged } \\
\text { youth (non-pharmacological } \\
\text { interventions) }\end{array}$ \\
\hline 411 & Palm & 2016 & 27289105 & $10.1177 / 1403494816654047$ & $\begin{array}{l}\text { Motivational interviewing does not affect risk drinking among } \\
\text { young women: A randomised, controlled intervention study in } \\
\text { Swedish youth health centres }\end{array}$ & Review \\
\hline 412 & Pantin & 2009 & 19834053 & 10.1097/PSY.0b013e3181bb2913 & $\begin{array}{l}\text { A randomized controlled trial of Familias Unidas for Hispanic } \\
\text { adolescents with behavior problems }\end{array}$ & $\begin{array}{l}\text { Not all subjects with at least } \\
\text { problematic use }\end{array}$ \\
\hline 413 & Parsons & 2014 & 24364800 & $10.1037 / a 0035311$ & $\begin{array}{l}\text { A randomized controlled trial utilizing motivational interviewing } \\
\text { to reduce HIV risk and drug use in young gay and bisexual } \\
\text { men }\end{array}$ & $\begin{array}{l}\text { Includes transition-aged } \\
\text { youth (non-pharmacological } \\
\text { interventions) }\end{array}$ \\
\hline
\end{tabular}




\begin{tabular}{|c|c|c|c|c|c|c|}
\hline No. & Author & Year & $\begin{array}{l}\text { PubMed or } \\
\text { (Other) ID }\end{array}$ & DOI & Title & Reason for Exclusion \\
\hline 414 & Patel & 2018 & 29535906 & 10.7759/cureus.2033 & $\begin{array}{l}\text { Is Cannabis Use Associated With the Worst Inpatient } \\
\text { Outcomes in Attention Deficit Hyperactivity Disorder } \\
\text { Adolescents? }\end{array}$ & Case control/cross sectional \\
\hline 415 & Paz Castro & 2017 & 28371696 & 10.1016/j.addbeh.2017.03.013 & $\begin{array}{l}\text { Moderators of outcome in a technology-based intervention to } \\
\text { prevent and reduce problem drinking among adolescents }\end{array}$ & $\begin{array}{l}\text { Not all subjects with at least } \\
\text { problematic use }\end{array}$ \\
\hline 416 & Perrier-Menard & 2017 & 28734153 & 10.1016/j.addbeh.2017.07.015 & $\begin{array}{l}\text { The impact of youth internalising and externalising symptom } \\
\text { severity on the effectiveness of brief personality-targeted } \\
\text { interventions for substance misuse: A cluster randomised trial }\end{array}$ & $\begin{array}{l}\text { Not all subjects with at least } \\
\text { problematic use }\end{array}$ \\
\hline 417 & Peters & 2012 & 22189052 & 10.1016/j.addbeh.2011.11.036 & $\begin{array}{l}\text { Co-occurring marijuana use is associated with medication } \\
\text { nonadherence and nonplanning impulsivity in young adult } \\
\text { heavy drinkers }\end{array}$ & $\begin{array}{l}\text { Includes transition-aged } \\
\text { youth (non-pharmacological } \\
\text { interventions) }\end{array}$ \\
\hline 418 & Pfarrwaller & 2019 & 31330465 & 10.1016/j.addbeh.2019.106049 & $\begin{array}{l}\text { Excessive substance use screening to encourage behaviour } \\
\text { change among young people in primary care: Pilot study in } \\
\text { preparation for a randomized trial }\end{array}$ & $\begin{array}{l}\text { Not all subjects with at least } \\
\text { problematic use }\end{array}$ \\
\hline 419 & Phan & 2010 & $\begin{array}{l}\text { CN-00789450 } \\
\text { (cochrane) }\end{array}$ & 10.1016/j.amp.2009.12.013 & $\begin{array}{l}\text { A random clinical trial concerning the psychotherapy of } \\
\text { adolescents addicted to cannabis }\end{array}$ & $\begin{array}{l}\text { No extractable or relevant } \\
\text { data for } \\
\text { interventions/outcomes of } \\
\text { interest }\end{array}$ \\
\hline 420 & Phan & 2010 & $\begin{array}{l}2010-04145- \\
013 \text { (psychinfo) }\end{array}$ & 10.1016/j.amp.2009.12.013 & $\begin{array}{l}\text { Un essai clinique randomisé sur la psychothérapie des } \\
\text { adolescents dépendants au cannabis. = A random clinical trial } \\
\text { concerning the psychotherapy of adolescents addicted to } \\
\text { cannabis }\end{array}$ & $\begin{array}{l}\text { No extractable or relevant } \\
\text { data for } \\
\text { interventions/outcomes of } \\
\text { interest }\end{array}$ \\
\hline 421 & Phan & 2011 & 21749677 & 10.1186/1471-244X-11-110 & $\begin{array}{l}\text { European youth care sites serve different populations of } \\
\text { adolescents with cannabis use disorder. Baseline and referral } \\
\text { data from the INCANT trial }\end{array}$ & $\begin{array}{l}\text { No extractable or relevant } \\
\text { data for } \\
\text { interventions/outcomes of } \\
\text { interest }\end{array}$ \\
\hline 422 & Phan & & $\begin{array}{l}2011-08525- \\
005 \text { (psychinfo) }\end{array}$ & & $\begin{array}{l}\text { Aspect 'multidimensionnel' de la consommation problématique } \\
\text { de drogue chez les adolescents. = 'Multidimensional' aspect of } \\
\text { substance abuse in adolescents }\end{array}$ & Review \\
\hline 423 & Pirskanen & & 17456127 & 10.1111/j.1525-1446.2007.00632.x & $\begin{array}{l}\text { A formative evaluation to develop a school health nursing } \\
\text { early intervention model for adolescent substance use }\end{array}$ & $\begin{array}{l}\text { NRCS (nonpharm, } \\
\text { pharmacological } \\
\text { interventions } \mathrm{N}<100 \text { ) }\end{array}$ \\
\hline 424 & Planken & 2010 & $\begin{array}{l}\text { 2010-17904- } \\
004 \text { (psychinfo) }\end{array}$ & & $\begin{array}{l}\text { Effects of a 10-minutes peer education protocol to reduce } \\
\text { binge drinking among adolescents during holidays }\end{array}$ & $\begin{array}{l}\text { Not all subjects with at least } \\
\text { problematic use }\end{array}$ \\
\hline
\end{tabular}




\begin{tabular}{|c|c|c|c|c|c|c|}
\hline No. & Author & Year & $\begin{array}{l}\text { PubMed or } \\
\text { (Other) ID }\end{array}$ & DOI & Title & Reason for Exclusion \\
\hline 425 & Polsky & 2010 & 20626379 & 10.1111/j.1360-0443.2010.03001.x & $\begin{array}{l}\text { Cost-effectiveness of extended buprenorphine-naloxone } \\
\text { treatment for opioid-dependent youth: data from a randomized } \\
\text { trial }\end{array}$ & $\begin{array}{l}\text { No extractable or relevant } \\
\text { data for } \\
\text { interventions/outcomes of } \\
\text { interest }\end{array}$ \\
\hline 426 & Prado & 2012 & 22776441 & 10.1016/j.drugalcdep.2012.06.011 & $\begin{array}{l}\text { The efficacy of Familias Unidas on drug and alcohol outcomes } \\
\text { for Hispanic delinquent youth: main effects and interaction } \\
\text { effects by parental stress and social support }\end{array}$ & $\begin{array}{l}\text { Not all subjects with at least } \\
\text { problematic use }\end{array}$ \\
\hline 427 & Prado & 2013 & 23408280 & $10.1007 / s 11121-012-0326-x$ & $\begin{array}{l}\text { Ecodevelopmental and intrapersonal moderators of a family } \\
\text { based preventive intervention for Hispanic youth: a latent } \\
\text { profile analysis }\end{array}$ & $\begin{array}{l}\text { Not all subjects with at least } \\
\text { problematic use }\end{array}$ \\
\hline 428 & Prince & 2019 & 31144836 & 10.1037/pha0000301 & $\begin{array}{l}\text { A Preliminary Test of a Brief Intervention to Lessen Young } \\
\text { Adults' Cannabis Use: Episode-Level Smartphone Data } \\
\text { Highlights the Role of Protective Behavioral Strategies and } \\
\text { Exercise }\end{array}$ & $\begin{array}{l}\text { Includes transition-aged } \\
\text { youth (non-pharmacological } \\
\text { interventions) }\end{array}$ \\
\hline 429 & Rabbi M & 2018 & 30021714 & 10.2196/resprot.9850 & $\begin{array}{l}\text { Toward Increasing Engagement in Substance Use Data } \\
\text { Collection: Development of the Substance Abuse Research } \\
\text { Assistant App and Protocol for a Microrandomized Trial Using } \\
\text { Adolescents and Emerging Adults. }\end{array}$ & $\begin{array}{l}\text { No extractable or relevant } \\
\text { data for } \\
\text { interventions/outcomes of } \\
\text { interest }\end{array}$ \\
\hline 430 & Ramchand & 2011 & 21513674 & & $\begin{array}{l}\text { Using a cross-study design to assess the efficacy of } \\
\text { motivational enhancement therapy-cognitive behavioral } \\
\text { therapy } 5 \text { (MET/CBT5) in treating adolescents with cannabis- } \\
\text { related disorders }\end{array}$ & $\begin{array}{l}\text { No extractable or relevant } \\
\text { data for } \\
\text { interventions/outcomes of } \\
\text { interest }\end{array}$ \\
\hline 431 & Ramchand & 2015 & 25219932 & 10.1176/appi.ps.201300517 & $\begin{array}{l}\text { Provision of mental health services as a quality indicator for } \\
\text { adolescent substance abuse treatment facilities }\end{array}$ & $\begin{array}{l}\text { Single arm (nonpharm, } \\
\text { pharmacological } \\
\text { interventions } \mathrm{N}<200 \text { ) }\end{array}$ \\
\hline 432 & Ramo & 2018 & 29510223 & 10.1016/j.cct.2018.02.014 & $\begin{array}{l}\text { Using Facebook to address smoking and heavy drinking in } \\
\text { young adults: Protocol for a randomized, controlled trial }\end{array}$ & $\begin{array}{l}\text { No extractable or relevant } \\
\text { data for } \\
\text { interventions/outcomes of } \\
\text { interest }\end{array}$ \\
\hline 433 & Randall & 2011 & $\begin{array}{l}\text { CN-00605980 } \\
\text { (cochrane) }\end{array}$ & & $\begin{array}{l}\text { Adapting multisystemic therapy to treat adolescent substance } \\
\text { abuse more effectively }\end{array}$ & Review \\
\hline 434 & Rew & 2017 & 27411974 & 10.1177/0193945916658861 & $\begin{array}{l}\text { An Intervention to Enhance Psychological Capital and Health } \\
\text { Outcomes in Homeless Female Youths }\end{array}$ & $\begin{array}{l}\text { NRCS (nonpharm, } \\
\text { pharmacological } \\
\text { interventions } \mathrm{N}<100 \text { ) }\end{array}$ \\
\hline 435 & Rhoades & 2013 & 24003300 & 10.1080/1067828X.2013.788887 & $\begin{array}{l}\text { MTFC for High Risk Adolescent Girls: A Comparison of } \\
\text { Outcomes in England and the United States }\end{array}$ & $\begin{array}{l}\text { Not all subjects with at least } \\
\text { problematic use }\end{array}$ \\
\hline
\end{tabular}




\begin{tabular}{|c|c|c|c|c|c|c|}
\hline No. & Author & Year & $\begin{array}{l}\text { PubMed or } \\
\text { (Other) ID }\end{array}$ & DOI & Title & Reason for Exclusion \\
\hline 436 & Richard & & 8749724 & & Effectiveness of adjunct therapies in crack cocaine treatment & Includes adults (> 25 years) \\
\hline 437 & Richter & 2012 & 22722516 & 10.1097/JCP.0b013e31825e213e & $\begin{array}{l}\text { Efficacy and safety of levetiracetam for the prevention of } \\
\text { alcohol relapse in recently detoxified alcohol-dependent } \\
\text { patients: a randomized trial }\end{array}$ & Includes adults ( $>25$ years) \\
\hline 438 & Riggs & & $\begin{array}{l}\text { CN-00367159 } \\
\text { (cochrane) }\end{array}$ & & $\begin{array}{l}\text { Effects of pemoline on ADHD, antisocial behaviors and } \\
\text { substance use in adolescents with conduct disorder and } \\
\text { substance use disorder }\end{array}$ & Review \\
\hline 439 & Rigter & 2010 & 20380718 & $10.1186 / 1471-244 X-10-28$ & $\begin{array}{l}\text { INCANT: a transnational randomized trial of multidimensional } \\
\text { family therapy versus treatment as usual for adolescents with } \\
\text { cannabis use disorder }\end{array}$ & $\begin{array}{l}\text { No extractable or relevant } \\
\text { data for } \\
\text { interventions/outcomes of } \\
\text { interest }\end{array}$ \\
\hline 440 & Riley & 2008 & 18493858 & $10.1007 / s 11414-008-9111-9$ & Implementation of MET/CBT 5 for adolescents & $\begin{array}{l}\text { No extractable or relevant } \\
\text { data for } \\
\text { interventions/outcomes of } \\
\text { interest }\end{array}$ \\
\hline 441 & Robbins & 2010 & 22002455 & & $\begin{array}{l}\text { Transporting clinical research to community settings: } \\
\text { designing and conducting a multisite trial of brief strategic } \\
\text { family therapy }\end{array}$ & Review \\
\hline 442 & Robbins & 2011 & 21261433 & $10.1037 / a 0022146$ & $\begin{array}{l}\text { Therapist adherence in brief strategic family therapy for } \\
\text { adolescent drug abusers }\end{array}$ & $\begin{array}{l}\text { No extractable or relevant } \\
\text { data for } \\
\text { interventions/outcomes of } \\
\text { interest }\end{array}$ \\
\hline 443 & Rogers & 2004 & 15048860 & 10.1002/bsl.558 & $\begin{array}{l}\text { Predictors of Treatment Outcome in Dually-Diagnosed } \\
\text { Antisocial Youth: An Initial Study of Forensic Inpatients }\end{array}$ & $\begin{array}{l}\text { No extractable or relevant } \\
\text { data for } \\
\text { interventions/outcomes of } \\
\text { interest }\end{array}$ \\
\hline 444 & Rohde & 2001 & 11437018 & $10.1097 / 00004583-200107000-00014$ & $\begin{array}{l}\text { Impact of comorbidity on a cognitive-behavioral group } \\
\text { treatment for adolescent depression }\end{array}$ & $\begin{array}{l}\text { Not all subjects with at least } \\
\text { problematic use }\end{array}$ \\
\hline 445 & Rohde & 2012 & 22564206 & 10.1037/a0028269 & $\begin{array}{l}\text { Reduced substance use as a secondary benefit of an } \\
\text { indicated cognitive-behavioral adolescent depression } \\
\text { prevention program }\end{array}$ & $\begin{array}{l}\text { Not all subjects with at least } \\
\text { problematic use }\end{array}$ \\
\hline 446 & Rohrbach & & 20655946 & 10.1016/j.ypmed.2010.07.016 & $\begin{array}{l}\text { One-year follow-up evaluation of the Project Towards No Drug } \\
\text { Abuse (TND) dissemination trial }\end{array}$ & $\begin{array}{l}\text { Not all subjects with at least } \\
\text { problematic use }\end{array}$ \\
\hline 447 & Roll & 2006 & 16905197 & 10.1016/j.psychres.2005.12.003 & Contingency management: schedule effects & Includes adults ( $>25$ years) \\
\hline
\end{tabular}




\begin{tabular}{|c|c|c|c|c|c|c|}
\hline No. & Author & Year & $\begin{array}{l}\text { PubMed or } \\
\text { (Other) ID }\end{array}$ & $\mathrm{DOI}$ & Title & Reason for Exclusion \\
\hline 448 & Rosenberg & 1972 & 5067456 & & Methadone use in adolescent heroin addicts & $\begin{array}{l}\text { NRCS (nonpharm, } \\
\text { pharmacological } \\
\text { interventions } N<100 \text { ) }\end{array}$ \\
\hline 449 & Roten & 2013 & 23261493 & 10.1016/j.addbeh.2012.11.003 & $\begin{array}{l}\text { Marijuana craving trajectories in an adolescent marijuana } \\
\text { cessation pharmacotherapy trial }\end{array}$ & $\begin{array}{l}\text { No extractable or relevant } \\
\text { data for } \\
\text { interventions/outcomes of } \\
\text { interest }\end{array}$ \\
\hline 450 & Roten & 2015 & 25661990 & 10.1016/j.addbeh.2015.01.013 & $\begin{array}{l}\text { Cognitive performance in a placebo-controlled } \\
\text { pharmacotherapy trial for youth with marijuana dependence }\end{array}$ & $\begin{array}{l}\text { No extractable or relevant } \\
\text { data for } \\
\text { interventions/outcomes of } \\
\text { interest }\end{array}$ \\
\hline 451 & Rotheram-Borus & 2016 & 26837624 & $10.1007 / s 10461-015-1262-0$ & $\begin{array}{l}\text { Feasibility of Using Soccer and Job Training to Prevent Drug } \\
\text { Abuse and HIV }\end{array}$ & $\begin{array}{l}\text { Not all subjects with at least } \\
\text { problematic use }\end{array}$ \\
\hline 452 & Rounds-Bryant & 1999 & 10548436 & & $\begin{array}{l}\text { Drug abuse treatment outcome study of adolescents: a } \\
\text { comparison of client characteristics and pretreatment } \\
\text { behaviors in three treatment modalities }\end{array}$ & $\begin{array}{l}\text { No extractable or relevant } \\
\text { data for } \\
\text { interventions/outcomes of } \\
\text { interest }\end{array}$ \\
\hline 453 & Rowe & 2003 & $\begin{array}{l}\text { 2003-02519- } \\
005 \text { (psychinfo) }\end{array}$ & & Family therapy for early adolescent substance abuse & $\begin{array}{l}\text { No extractable or relevant } \\
\text { data for } \\
\text { interventions/outcomes of } \\
\text { interest }\end{array}$ \\
\hline 454 & Rowe & 2004 & 15050090 & $10.1016 / S 0740-5472(03) 00166-1$ & $\begin{array}{l}\text { Impact of psychiatric comorbidity on treatment of adolescent } \\
\text { drug abusers }\end{array}$ & $\begin{array}{l}\text { No extractable or relevant } \\
\text { data for } \\
\text { interventions/outcomes of } \\
\text { interest }\end{array}$ \\
\hline 455 & Rowe & 2013 & 23085040 & 10.1016/j.jsat.2012.08.225 & $\begin{array}{l}\text { Implementation fidelity of Multidimensional Family Therapy in } \\
\text { an international trial }\end{array}$ & $\begin{array}{l}\text { No extractable or relevant } \\
\text { data for } \\
\text { interventions/outcomes of } \\
\text { interest }\end{array}$ \\
\hline 456 & Rowland & 2008 & $\begin{array}{l}\text { 2014-27598- } \\
002 \text { (psychinfo) }\end{array}$ & $10.1080 / 15470650802071622$ & $\begin{array}{l}\text { Sibling outcomes from a randomized trial of evidence-based } \\
\text { treatments with substance abusing juvenile offenders }\end{array}$ & $\begin{array}{l}\text { Not all subjects with at least } \\
\text { problematic use }\end{array}$ \\
\hline 457 & Rupp & 2012 & $\begin{array}{l}\text { CN-00902300 } \\
\text { (cochrane) }\end{array}$ & & $\begin{array}{l}\text { Cognitive remediation therapy during treatment for alcohol } \\
\text { dependence }\end{array}$ & $\begin{array}{l}\text { No extractable or relevant } \\
\text { data for } \\
\text { interventions/outcomes of } \\
\text { interest }\end{array}$ \\
\hline 458 & Russell & 2018 & 28185103 & 10.1007/s11121-017-0751-y & $\begin{array}{l}\text { PROSPER Intervention Effects on Adolescents' Alcohol } \\
\text { Misuse Vary by GABRA2 Genotype and Age }\end{array}$ & $\begin{array}{l}\text { Not all subjects with at least } \\
\text { problematic use }\end{array}$ \\
\hline
\end{tabular}




\begin{tabular}{|c|c|c|c|c|c|c|}
\hline No. & Author & Year & $\begin{array}{l}\text { PubMed or } \\
\text { (Other) ID }\end{array}$ & $\mathrm{DOI}$ & Title & Reason for Exclusion \\
\hline 459 & Salazar Garcia & 2011 & $\begin{array}{l}\text { 2012-03248- } \\
012 \text { (psychinfo) }\end{array}$ & & $\begin{array}{l}\text { Intervenciones breves con adolescentes estudiantes rurales } \\
\text { que consumen alcohol en exceso. = Brief interventions with } \\
\text { adolescent rural students who drink alcohol in excess }\end{array}$ & $\begin{array}{l}\text { NRCS (nonpharm, } \\
\text { pharmacological } \\
\text { interventions } N<100 \text { ) }\end{array}$ \\
\hline 460 & Saloner & 2014 & 24613095 & 10.1016/j.jadohealth.2014.01.002 & $\begin{array}{l}\text { Explaining racial/ethnic differences in adolescent substance } \\
\text { abuse treatment completion in the United States: A } \\
\text { decomposition analysis }\end{array}$ & $\begin{array}{l}\text { No extractable or relevant } \\
\text { data for } \\
\text { interventions/outcomes of } \\
\text { interest }\end{array}$ \\
\hline 461 & Sambrano & 2005 & 16161731 & 10.1081/ADA-200068089 & $\begin{array}{l}\text { Understanding Prevention Effectiveness in Real-World } \\
\text { Settings: The National Cross-Site Evaluation of High Risk } \\
\text { Youth Programs }\end{array}$ & $\begin{array}{l}\text { Not all subjects with at least } \\
\text { problematic use }\end{array}$ \\
\hline 462 & Santisteban & 2003 & 12666468 & & $\begin{array}{l}\text { Efficacy of brief strategic family therapy in modifying Hispanic } \\
\text { adolescent behavior problems and substance use }\end{array}$ & $\begin{array}{l}\text { Not all subjects with at least } \\
\text { problematic use }\end{array}$ \\
\hline 463 & Saxon & 1996 & 8828247 & & $\begin{array}{l}\text { Pre-treatment characteristics, program philosophy and level of } \\
\text { ancillary services as predictors of methadone maintenance } \\
\text { treatment outcome }\end{array}$ & Includes adults ( $>25$ years) \\
\hline 464 & Schell & 2005 & 16033496 & 10.1111/j.1475-6773.2005.00399.x & $\begin{array}{l}\text { Dynamic Effects among Patients' Treatment Needs, Beliefs, } \\
\text { and Utilization: A Prospective Study of Adolescents in Drug } \\
\text { Treatment }\end{array}$ & $\begin{array}{l}\text { NRCS (nonpharm, } \\
\text { pharmacological } \\
\text { interventions } N<100 \text { ) }\end{array}$ \\
\hline 465 & $\begin{array}{l}\text { Schelleman- } \\
\text { Offermans }\end{array}$ & 2014 & 24210898 & 10.1016/j.jadohealth.2013.09.001 & $\begin{array}{l}\text { Preventing adolescent alcohol use: effects of a two-year } \\
\text { quasi-experimental community intervention intensifying formal } \\
\text { and informal control }\end{array}$ & $\begin{array}{l}\text { Not all subjects with at least } \\
\text { problematic use }\end{array}$ \\
\hline 466 & Schijven & 2015 & 26198744 & 10.1186/s12888-015-0563-1 & $\begin{array}{l}\text { Evaluating a selective prevention program for substance use } \\
\text { and comorbid behavioral problems in adolescents with mild to } \\
\text { borderline intellectual disabilities: Study protocol of a } \\
\text { randomized controlled trial }\end{array}$ & $\begin{array}{l}\text { No extractable or relevant } \\
\text { data for } \\
\text { interventions/outcomes of } \\
\text { interest }\end{array}$ \\
\hline 467 & Schinke & 2004 & 15376818 & & $\begin{array}{l}\text { Reducing the risks of alcohol use among urban youth: Three- } \\
\text { year effects of a computer-based intervention with and without } \\
\text { parent involvement }\end{array}$ & $\begin{array}{l}\text { Not all subjects with at least } \\
\text { problematic use }\end{array}$ \\
\hline 468 & Schmiege & 2009 & 19170452 & $10.1037 / a 0014513$ & $\begin{array}{l}\text { Randomized trial of group interventions to reduce HIVISTD } \\
\text { risk and change theoretical mediators among detained } \\
\text { adolescents }\end{array}$ & $\begin{array}{l}\text { No extractable or relevant } \\
\text { data for } \\
\text { interventions/outcomes of } \\
\text { interest }\end{array}$ \\
\hline 469 & Schoenwald & 1996 & $\begin{array}{l}\text { 1996-07046- } \\
004 \text { (psychinfo) }\end{array}$ & 10.1007/BF02233864 & $\begin{array}{l}\text { Multisystemic therapy treatment of substance abusing or } \\
\text { dependent adolescent offenders: Costs of reducing } \\
\text { incarceration, inpatient, and residential placement }\end{array}$ & $\begin{array}{l}\text { No extractable or relevant } \\
\text { data for } \\
\text { interventions/outcomes of } \\
\text { interest }\end{array}$ \\
\hline
\end{tabular}




\begin{tabular}{|c|c|c|c|c|c|c|}
\hline No. & Author & Year & $\begin{array}{l}\text { PubMed or } \\
\text { (Other) ID }\end{array}$ & DOI & Title & Reason for Exclusion \\
\hline 470 & Schuler & 2014 & 24650830 & & $\begin{array}{l}\text { Effectiveness of treatment for adolescent substance use: is } \\
\text { biological drug testing sufficient? }\end{array}$ & $\begin{array}{l}\text { NRCS (nonpharm, } \\
\text { pharmacological } \\
\text { interventions } N<100 \text { ) }\end{array}$ \\
\hline 471 & Schulte & 2010 & $\begin{array}{l}2010-23527- \\
002 \text { (psychinfo) }\end{array}$ & 10.1080/1067828X.2010.515877 & $\begin{array}{l}\text { Influencing adolescent social perceptions of alcohol use to } \\
\text { facilitate change through a school-based intervention }\end{array}$ & $\begin{array}{l}\text { Single arm (nonpharm, } \\
\text { pharmacological } \\
\text { interventions } \mathrm{N}<200 \text { ) }\end{array}$ \\
\hline 472 & Schuman-Olivier & 2014 & 24953168 & 10.1016/j.jsat.2014.04.006 & $\begin{array}{l}\text { Emerging adult age status predicts poor buprenorphine } \\
\text { treatment retention }\end{array}$ & Review \\
\hline 473 & Schwegler & & $\begin{array}{l}\text { CN-00309151 } \\
\text { (cochrane) }\end{array}$ & & $\begin{array}{l}\text { Clinical detoxification of juvenile drug addicts - drug therapy } \\
\text { with piracetam and doxepin }\end{array}$ & Review \\
\hline 474 & Schwinn & 2010 & 20553661 & 10.15288/jsad.2010.71.535 & $\begin{array}{l}\text { Preventing alcohol use among late adolescent urban youth: 6- } \\
\text { year results from a computer-based intervention }\end{array}$ & $\begin{array}{l}\text { Not all subjects with at least } \\
\text { problematic use }\end{array}$ \\
\hline 475 & Scott & 1988 & $\begin{array}{l}\text { CN-00058610 } \\
\text { (cochrane) }\end{array}$ & & $\begin{array}{l}\text { Impact of fitness training on native adolescents' self- } \\
\text { evaluations and substance use }\end{array}$ & $\begin{array}{l}\text { NRCS (nonpharm, } \\
\text { pharmacological } \\
\text { interventions } N<100 \text { ) }\end{array}$ \\
\hline 476 & Sealock & 1997 & $\begin{array}{l}\text { CN-00392595 } \\
\text { (cochrane) }\end{array}$ & & $\begin{array}{l}\text { Drug treatment for juvenile offenders: some good and bad } \\
\text { news }\end{array}$ & $\begin{array}{l}\text { NRCS (nonpharm, } \\
\text { pharmacological } \\
\text { interventions } N<100 \text { ) }\end{array}$ \\
\hline 477 & Segatto & 2011 & 21971774 & & $\begin{array}{l}\text { Brief motivational interview and educational brochure in } \\
\text { emergency room settings for adolescents and young adults } \\
\text { with alcohol-related problems: a randomized single-blind } \\
\text { clinical trial }\end{array}$ & $\begin{array}{l}\text { Includes transition-aged } \\
\text { youth (non-pharmacological } \\
\text { interventions) }\end{array}$ \\
\hline 478 & Segrott & 2014 & 24438460 & $10.1186 / 1471-2458-14-49$ & $\begin{array}{l}\text { Preventing substance misuse: study protocol for a randomised } \\
\text { controlled trial of the Strengthening Families Programme 10- } \\
14 \text { UK (SFP 10-14 UK) }\end{array}$ & $\begin{array}{l}\text { No extractable or relevant } \\
\text { data for } \\
\text { interventions/outcomes of } \\
\text { interest }\end{array}$ \\
\hline 479 & Selnow & 1985 & 3831285 & 10.2190/BBA3-FE34-M9UH-WNA3 & $\begin{array}{l}\text { Using a stratified approach in substance intervention and } \\
\text { prevention programs among adolescents: an empirical } \\
\text { analysis }\end{array}$ & $\begin{array}{l}\text { No extractable or relevant } \\
\text { data for } \\
\text { interventions/outcomes of } \\
\text { interest }\end{array}$ \\
\hline 480 & Serafini & 2018 & $\begin{array}{l}\text { 2018-11974- } \\
005 \text { (psychinfo) }\end{array}$ & $10.1080 / 16066359.2017 .1342819$ & $\begin{array}{l}\text { Perceived parental support and adolescent motivation for } \\
\text { substance use change: A preliminary investigation }\end{array}$ & $\begin{array}{l}\text { NRCS (nonpharm, } \\
\text { pharmacological } \\
\text { interventions } N<100 \text { ) }\end{array}$ \\
\hline 481 & Sevy & 2011 & 21636134 & 10.1016/j.psychres.2011.05.001 & $\begin{array}{l}\text { Olanzapine vs. risperidone in patients with first-episode } \\
\text { schizophrenia and a lifetime history of cannabis use disorders: } \\
\text { 16-week clinical and substance use outcomes }\end{array}$ & Includes adults (> 25 years) \\
\hline
\end{tabular}




\begin{tabular}{|c|c|c|c|c|c|c|}
\hline No. & Author & Year & $\begin{array}{l}\text { PubMed or } \\
\text { (Other) ID }\end{array}$ & DOI & Title & Reason for Exclusion \\
\hline 482 & Sexton & 2010 & 20545407 & 10.1037/a0019406 & $\begin{array}{l}\text { The effectiveness of functional family therapy for youth with } \\
\text { behavioral problems in a community practice setting }\end{array}$ & $\begin{array}{l}\text { Not all subjects with at least } \\
\text { problematic use }\end{array}$ \\
\hline 483 & Shakeshaft & 2014 & 24618831 & 10.1371/journal.pmed.1001617 & $\begin{array}{l}\text { The Effectiveness of Community Action in Reducing Risky } \\
\text { Alcohol Consumption and Harm: a Cluster Randomised } \\
\text { Controlled Trial }\end{array}$ & $\begin{array}{l}\text { Not all subjects with at least } \\
\text { problematic use }\end{array}$ \\
\hline 484 & Shane & 2006 & 17182418 & $10.1080 / 10550490601003714$ & $\begin{array}{l}\text { Impact of Victimization on Substance Abuse Treatment } \\
\text { Outcomes for Adolescents in Outpatient and Residential } \\
\text { Substance Abuse Treatment }\end{array}$ & $\begin{array}{l}\text { No extractable or relevant } \\
\text { data for } \\
\text { interventions/outcomes of } \\
\text { interest }\end{array}$ \\
\hline 485 & Sharp & & 9218237 & & $\begin{array}{l}\text { Facilitation of internal locus of control in adolescent alcoholics } \\
\text { through a brief biofeedback-assisted autogenic relaxation } \\
\text { training procedure }\end{array}$ & $\begin{array}{l}\text { No extractable or relevant } \\
\text { data for } \\
\text { interventions/outcomes of } \\
\text { interest }\end{array}$ \\
\hline 486 & Sheidow & 2012 & 22389577 & 10.1080/1067828X.2012.636701 & $\begin{array}{l}\text { Money Matters: Cost Effectiveness of Juvenile Drug Court with } \\
\text { and without Evidence-Based Treatments }\end{array}$ & $\begin{array}{l}\text { No extractable or relevant } \\
\text { data for } \\
\text { interventions/outcomes of } \\
\text { interest }\end{array}$ \\
\hline 487 & Sheidow & 2019 & 31393146 & $10.1037 / \mathrm{adb} 0000497$ & $\begin{array}{l}\text { Capacity of Juvenile Probation Officers in Low-Resourced, } \\
\text { Rural Settings to Deliver an Evidence-Based Substance Use } \\
\text { Intervention to Adolescents }\end{array}$ & $\begin{array}{l}\text { No extractable or relevant } \\
\text { data for } \\
\text { interventions/outcomes of } \\
\text { interest }\end{array}$ \\
\hline 488 & Sherman & 2009 & $\begin{array}{l}\text { CN-01601907 } \\
\text { (cochrane) }\end{array}$ & & $\begin{array}{l}\text { Evaluation of a peer network intervention trial among young } \\
\text { methamphetamine users in Chiang Mai, Thailand }\end{array}$ & $\begin{array}{l}\text { Includes transition-aged } \\
\text { youth (non-pharmacological } \\
\text { interventions) }\end{array}$ \\
\hline 489 & Shetgiri & 2011 & $\begin{array}{l}\text { CN-00845424 } \\
\text { (cochrane) }\end{array}$ & & $\begin{array}{l}\text { A randomized, controlled trial of a school-based intervention to } \\
\text { reduce violence and substance use in predominantly Latino } \\
\text { high school students }\end{array}$ & $\begin{array}{l}\text { Not all subjects with at least } \\
\text { problematic use }\end{array}$ \\
\hline 490 & Shift & 2001 & $\begin{array}{l}\text { CN-00367166 } \\
\text { (cochrane) }\end{array}$ & & Adolescent cannabis check-up and intervention trial & Review \\
\hline 491 & Sinha & & 14504024 & & $\begin{array}{l}\text { Engaging young probation-referred marijuana-abusing } \\
\text { individuals in treatment: a pilot trial }\end{array}$ & $\begin{array}{l}\text { Includes transition-aged } \\
\text { youth (non-pharmacological } \\
\text { interventions) }\end{array}$ \\
\hline 492 & Slesnick & 2004 & 18607515 & 10.1300/J020v22n02_02 & $\begin{array}{l}\text { Office versus home-based family therapy for runaway, alcohol } \\
\text { abusing adolescents: examination of factors associated with } \\
\text { treatment attendance }\end{array}$ & $\begin{array}{l}\text { No extractable or relevant } \\
\text { data for } \\
\text { interventions/outcomes of } \\
\text { interest }\end{array}$ \\
\hline
\end{tabular}




\begin{tabular}{|c|c|c|c|c|c|c|}
\hline No. & Author & Year & $\begin{array}{l}\text { PubMed or } \\
\text { (Other) ID }\end{array}$ & DOI & Title & Reason for Exclusion \\
\hline 493 & Slesnick & 2005 & $\begin{array}{l}\text { CN-00591039 } \\
\text { (cochrane) }\end{array}$ & & $\begin{array}{l}\text { Outcome of CRA with Homeless Adolescents: preliminary } \\
\text { findings }\end{array}$ & Review \\
\hline 494 & Slesnick & 2006 & 16933433 & & $\begin{array}{l}\text { Predictors of substance use and family therapy outcome } \\
\text { among physically and sexually abused runaway adolescents }\end{array}$ & $\begin{array}{l}\text { No extractable or relevant } \\
\text { data for } \\
\text { interventions/outcomes of } \\
\text { interest }\end{array}$ \\
\hline 495 & Slesnick & 2006 & 16564644 & 10.1016/j.addbeh.2006.02.006 & $\begin{array}{l}\text { Primary alcohol versus primary drug use among adolescents: } \\
\text { an examination of differences }\end{array}$ & $\begin{array}{l}\text { No extractable or relevant } \\
\text { data for } \\
\text { interventions/outcomes of } \\
\text { interest }\end{array}$ \\
\hline 496 & Slesnick & 2011 & $\begin{array}{l}\text { 2011-00923- } \\
005 \text { (psychinfo) }\end{array}$ & 10.1111/j.1467-6427.2010.00530.x & $\begin{array}{l}\text { Predictors of treatment attendance among adolescent } \\
\text { substance abusing runaways: A comparison of family and } \\
\text { individual therapy modalities }\end{array}$ & $\begin{array}{l}\text { No extractable or relevant } \\
\text { data for } \\
\text { interventions/outcomes of } \\
\text { interest }\end{array}$ \\
\hline 497 & Slesnick & 2013 & 24011094 & 10.1016/j.adolescence.2013.06.007 & $\begin{array}{l}\text { Two-year predictors of runaway and homeless episodes } \\
\text { following shelter services among substance abusing } \\
\text { adolescents }\end{array}$ & $\begin{array}{l}\text { No extractable or relevant } \\
\text { data for } \\
\text { interventions/outcomes of } \\
\text { interest }\end{array}$ \\
\hline 498 & Slice & 1998 & $\begin{array}{l}\text { L28294380 } \\
\text { (embase) }\end{array}$ & 10.1037/0893-164X.12.2.136 & $\begin{array}{l}\text { Relations of delinquency to adolescent substance use and } \\
\text { problem use: A prospective study }\end{array}$ & $\begin{array}{l}\text { Single arm (nonpharm, } \\
\text { pharmacological } \\
\text { interventions } \mathrm{N}<200 \text { ) }\end{array}$ \\
\hline 499 & Smeerdijk & 2014 & 24157087 & 10.1016/j.jsat.2013.09.006 & $\begin{array}{l}\text { Feasibility of teaching motivational interviewing to parents of } \\
\text { young adults with recent-onset schizophrenia and co-occurring } \\
\text { cannabis use }\end{array}$ & Includes adults (> 25 years) \\
\hline 500 & Smeerdijk & 2015 & 25959502 & 10.1017/S0033291715000793 & $\begin{array}{l}\text { Motivational interviewing and interaction skills training for } \\
\text { parents of young adults with recent-onset schizophrenia and } \\
\text { co-occurring cannabis use: } 15 \text {-month follow-up }\end{array}$ & Includes adults ( $>25$ years) \\
\hline 501 & Smith & 2010 & 20953309 & 10.1080/1067828X.2010.511986 & $\begin{array}{l}\text { Preliminary Support for Multidimensional Treatment Foster } \\
\text { Care in Reducing Substance Use in Delinquent Boys }\end{array}$ & $\begin{array}{l}\text { Not all subjects with at least } \\
\text { problematic use }\end{array}$ \\
\hline 502 & Smith & 2011 & 21831564 & 10.1016/j.jsat.2011.06.003 & $\begin{array}{l}\text { Adolescent Community Reinforcement Approach outcomes } \\
\text { differ among emerging adults and adolescents }\end{array}$ & $\begin{array}{l}\text { NRCS (nonpharm, } \\
\text { pharmacological } \\
\text { interventions } N<100 \text { ) }\end{array}$ \\
\hline 503 & Smith & 2014 & 23994049 & 10.1016/j.jsat.2013.07.004 & $\begin{array}{l}\text { Drug refusal skills training does not enhance outcomes of } \\
\text { African American adolescents with substance use problems }\end{array}$ & $\begin{array}{l}\text { NRCS (nonpharm, } \\
\text { pharmacological } \\
\text { interventions } N<100 \text { ) }\end{array}$ \\
\hline
\end{tabular}




\begin{tabular}{|c|c|c|c|c|c|c|}
\hline No. & Author & Year & $\begin{array}{l}\text { PubMed or } \\
\text { (Other) ID }\end{array}$ & DOI & Title & Reason for Exclusion \\
\hline 504 & Smith & 2015 & 26877622 & $10.1177 / 1049731514535851$ & $\begin{array}{l}\text { Normative Feedback and Adolescent Readiness to Change: A } \\
\text { Small Randomized Trial }\end{array}$ & $\begin{array}{l}\text { No extractable or relevant } \\
\text { data for } \\
\text { interventions/outcomes of } \\
\text { interest }\end{array}$ \\
\hline 505 & Smyth & 2018 & 26800851 & 10.1111/eip.12318 & $\begin{array}{l}\text { Changes in psychological well-being among heroin-dependent } \\
\text { adolescents during psychologically supported opiate } \\
\text { substitution treatment }\end{array}$ & $\begin{array}{l}\text { NRCS (nonpharm, } \\
\text { pharmacological } \\
\text { interventions } N<100 \text { ) }\end{array}$ \\
\hline 506 & Spaeth & 2010 & 20515209 & 10.1037/a0019550 & $\begin{array}{l}\text { Examining the differential effectiveness of a life skills program } \\
\text { (IPSY) on alcohol use trajectories in early adolescence }\end{array}$ & $\begin{array}{l}\text { Not all subjects with at least } \\
\text { problematic use }\end{array}$ \\
\hline 507 & Spirito & 2017 & 28259500 & 10.1016/j.jsat.2017.02.002 & $\begin{array}{l}\text { Effects of a brief, parent-focused intervention for substance } \\
\text { using adolescents and their sibling }\end{array}$ & $\begin{array}{l}\text { Not all subjects with at least } \\
\text { problematic use }\end{array}$ \\
\hline 508 & Stanczak & 1973 & 4808169 & & $\begin{array}{l}\text { Treatment of young suburban heroin addicts two and a half } \\
\text { years later }\end{array}$ & $\begin{array}{l}\text { Single arm (nonpharm, } \\
\text { pharmacological } \\
\text { interventions } \mathrm{N}<200 \text { ) }\end{array}$ \\
\hline 509 & Stanforth & 2016 & 27776675 & 10.1016/j.jsat.2016.08.005 & $\begin{array}{l}\text { Structure of Problem Recognition Questionnaire with } \\
\text { Hispanic/Latino Adolescents }\end{array}$ & $\begin{array}{l}\text { No extractable or relevant } \\
\text { data for } \\
\text { interventions/outcomes of } \\
\text { interest }\end{array}$ \\
\hline 510 & Stanger & 2012 & 22182419 & $10.1037 / a 0026543$ & $\begin{array}{l}\text { Delay discounting predicts adolescent substance abuse } \\
\text { treatment outcome }\end{array}$ & $\begin{array}{l}\text { No extractable or relevant } \\
\text { data for } \\
\text { interventions/outcomes of } \\
\text { interest }\end{array}$ \\
\hline 511 & Stanger & 2019 & 31246068 & 10.1037/adb0000480 & $\begin{array}{l}\text { Working Memory Training and High Magnitude Incentives for } \\
\text { Youth Cannabis Use: A SMART Pilot Trial }\end{array}$ & $\begin{array}{l}\text { Includes transition-aged } \\
\text { youth (non-pharmacological } \\
\text { interventions) }\end{array}$ \\
\hline 512 & Stanton & 2004 & 15466681 & 10.1001/archpedi.158.10.947 & $\begin{array}{l}\text { Randomized trial of a parent intervention: parents can make a } \\
\text { difference in long-term adolescent risk behaviors, perceptions, } \\
\text { and knowledge }\end{array}$ & $\begin{array}{l}\text { Not all subjects with at least } \\
\text { problematic use }\end{array}$ \\
\hline 513 & Stein & 2006 & 20617117 & & $\begin{array}{l}\text { Enhancing Substance Abuse Treatment Engagement in } \\
\text { Incarcerated Adolescents }\end{array}$ & $\begin{array}{l}\text { No extractable or relevant } \\
\text { data for } \\
\text { interventions/outcomes of } \\
\text { interest }\end{array}$ \\
\hline 514 & Stein & 2011 & 21185685 & 10.1016/j.jsat.2010.11.001 & $\begin{array}{l}\text { A brief marijuana intervention for non-treatment-seeking young } \\
\text { adult women }\end{array}$ & $\begin{array}{l}\text { Includes transition-aged } \\
\text { youth (non-pharmacological } \\
\text { interventions) }\end{array}$ \\
\hline
\end{tabular}




\begin{tabular}{|c|c|c|c|c|c|c|}
\hline No. & Author & Year & $\begin{array}{l}\text { PubMed or } \\
\text { (Other) ID }\end{array}$ & $\mathrm{DOI}$ & Title & Reason for Exclusion \\
\hline 515 & Stein & 2014 & 25127289 & $10.1080 / 08897077.2014 .949337$ & $\begin{array}{l}\text { Measuring behaviors of individual adolescents during group- } \\
\text { based substance abuse intervention }\end{array}$ & $\begin{array}{l}\text { No extractable or relevant } \\
\text { data for } \\
\text { interventions/outcomes of } \\
\text { interest }\end{array}$ \\
\hline 516 & Stein & 2018 & 28865169 & 10.1111/add.14026 & $\begin{array}{l}\text { A developmental-based motivational intervention to reduce } \\
\text { alcohol and marijuana use among non-treatment-seeking } \\
\text { young adults: a randomized controlled trial }\end{array}$ & $\begin{array}{l}\text { Includes transition-aged } \\
\text { youth (non-pharmacological } \\
\text { interventions) }\end{array}$ \\
\hline 517 & Stein MD & 2014 & 24439950 & 10.1016/j.whi.2013.10.005 & $\begin{array}{l}\text { Alcohol use potentiates marijuana problem severity in young } \\
\text { adult women. }\end{array}$ & $\begin{array}{l}\text { Single arm (nonpharm, } \\
\text { pharmacological } \\
\text { interventions } N<200 \text { ) }\end{array}$ \\
\hline 518 & Stephenson & 2018 & 29712625 & 10.2196/resprot.9414 & $\begin{array}{l}\text { Intervention to Increase HIV Testing Among Substance-Using } \\
\text { Young Men Who Have Sex With Men: Protocol for a } \\
\text { Randomized Controlled Trial }\end{array}$ & $\begin{array}{l}\text { No extractable or relevant } \\
\text { data for } \\
\text { interventions/outcomes of } \\
\text { interest }\end{array}$ \\
\hline 519 & Sterling & 2005 & $\begin{array}{l}\text { 2005-05432- } \\
015 \text { (psychinfo) }\end{array}$ & 10.1097/01.ALC.0000164373.89061.2C & $\begin{array}{l}\text { Chemical Dependency and Psychiatric Services for } \\
\text { Adolescents in Private Managed Care: Implications for } \\
\text { Outcomes }\end{array}$ & $\begin{array}{l}\text { Single arm (nonpharm, } \\
\text { pharmacological } \\
\text { interventions } N<200 \text { ) }\end{array}$ \\
\hline 520 & Sterling & 2009 & 19413644 & 10.1111/j.1530-0277.2009.00972.x & $\begin{array}{l}\text { Three-year chemical dependency and mental health treatment } \\
\text { outcomes among adolescents: the role of continuing care }\end{array}$ & $\begin{array}{l}\text { NRCS (nonpharm, } \\
\text { pharmacological } \\
\text { interventions } \mathrm{N}<100 \text { ) }\end{array}$ \\
\hline 521 & Sterling & 2015 & 26523821 & 10.1001/jamapediatrics.2015.3145 & $\begin{array}{l}\text { Implementation of Screening, Brief Intervention, and Referral } \\
\text { to Treatment for Adolescents in Pediatric Primary Care: A } \\
\text { Cluster Randomized Trial }\end{array}$ & $\begin{array}{l}\text { No extractable or relevant } \\
\text { data for } \\
\text { interventions/outcomes of } \\
\text { interest }\end{array}$ \\
\hline 522 & Sterling & 2017 & 29021115 & 10.1016/j.jsat.2017.09.005 & $\begin{array}{l}\text { Specialty addiction and psychiatry treatment initiation and } \\
\text { engagement: Results from an SBIRT randomized trial in } \\
\text { pediatrics }\end{array}$ & $\begin{array}{l}\text { No extractable or relevant } \\
\text { data for } \\
\text { interventions/outcomes of } \\
\text { interest }\end{array}$ \\
\hline 523 & Sterling & 2018 & 29396080 & 10.1016/j.jadohealth.2017.10.016 & $\begin{array}{l}\text { Pediatrician and Behavioral Clinician-Delivered Screening, } \\
\text { Brief Intervention and Referral to Treatment: Substance Use } \\
\text { and Depression Outcomes }\end{array}$ & $\begin{array}{l}\text { Not all subjects with at least } \\
\text { problematic use }\end{array}$ \\
\hline 524 & Sterling & 2019 & 31018988 & 10.1542/peds.2018-2803 & Health care use over 3 years after adolescent SBIRT & $\begin{array}{l}\text { Not all subjects with at least } \\
\text { problematic use }\end{array}$ \\
\hline 525 & Stevens & 2004 & 15152706 & 10.1080/02791072.2004.10399720 & $\begin{array}{l}\text { Gender Differences in Substance Use, Mental Health, and } \\
\text { Criminal Justice: Involvement of Adolescents at Treatment } \\
\text { Entry and at Three, Six, Twelve and Thirty Month Follow-Up }\end{array}$ & $\begin{array}{l}\text { No extractable or relevant } \\
\text { data for } \\
\text { interventions/outcomes of } \\
\text { interest }\end{array}$ \\
\hline
\end{tabular}




\begin{tabular}{|c|c|c|c|c|c|c|}
\hline No. & Author & Year & $\begin{array}{l}\text { PubMed or } \\
\text { (Other) ID }\end{array}$ & DOI & Title & Reason for Exclusion \\
\hline 526 & Stewart & 2015 & 26231697 & 10.1016/j.jsat.2015.06.002 & $\begin{array}{l}\text { Effectiveness of Motivational Incentives for Adolescent } \\
\text { Marijuana Users in a School-Based Intervention }\end{array}$ & $\begin{array}{l}\text { NRCS (nonpharm, } \\
\text { pharmacological } \\
\text { interventions } N<100 \text { ) }\end{array}$ \\
\hline 527 & Strang & 2004 & 15223098 & 10.1016/j.jsat.2004.05.003 & $\begin{array}{l}\text { Can the practitioner correctly predict outcome in motivational } \\
\text { interviewing? }\end{array}$ & $\begin{array}{l}\text { No extractable or relevant } \\
\text { data for } \\
\text { interventions/outcomes of } \\
\text { interest }\end{array}$ \\
\hline 528 & Suffoletto & 2012 & 22168137 & 10.1111/j.1530-0277.2011.01646.x & $\begin{array}{l}\text { Text-message-based drinking assessments and brief } \\
\text { interventions for young adults discharged from the emergency } \\
\text { department }\end{array}$ & $\begin{array}{l}\text { Includes transition-aged } \\
\text { youth (non-pharmacological } \\
\text { interventions) }\end{array}$ \\
\hline 529 & Suffoletto & 2013 & 23552023 & $10.1186 / 1745-6215-14-93$ & $\begin{array}{l}\text { Mobile phone text message intervention to reduce binge } \\
\text { drinking among young adults: study protocol for a randomized } \\
\text { controlled trial }\end{array}$ & $\begin{array}{l}\text { No extractable or relevant } \\
\text { data for } \\
\text { interventions/outcomes of } \\
\text { interest }\end{array}$ \\
\hline 530 & Suffoletto & 2014 & 25017822 & 10.1016/j.annemergmed.2014.06.010 & $\begin{array}{l}\text { A text message alcohol intervention for young adult } \\
\text { emergency department patients: a randomized clinical trial }\end{array}$ & $\begin{array}{l}\text { Includes transition-aged } \\
\text { youth (non-pharmacological } \\
\text { interventions) }\end{array}$ \\
\hline 531 & Suffoletto & 2015 & 26580802 & 10.1371/journal.pone.0142877 & $\begin{array}{l}\text { An Interactive Text Message Intervention to Reduce Binge } \\
\text { Drinking in Young Adults: a Randomized Controlled Trial with } \\
\text { 9-Month Outcomes }\end{array}$ & $\begin{array}{l}\text { Includes transition-aged } \\
\text { youth (non-pharmacological } \\
\text { interventions) }\end{array}$ \\
\hline 532 & Suffoletto & 2016 & $\begin{array}{l}\text { CN-01401609 } \\
\text { (cochrane) }\end{array}$ & & $\begin{array}{l}\text { Patterns of Change in Weekend Drinking Cognitions Among } \\
\text { Non-Treatment-Seeking Young Adults During Exposure to a } \\
\text { 12-Week Text Message Intervention }\end{array}$ & $\begin{array}{l}\text { Includes transition-aged } \\
\text { youth (non-pharmacological } \\
\text { interventions) }\end{array}$ \\
\hline 533 & Svikis & 1997 & $\begin{array}{l}\text { CN-00144272 } \\
\text { (cochrane) }\end{array}$ & & $\begin{array}{l}\text { Attendance incentives for outpatient treatment: effects in } \\
\text { methadone- and nonmethadone-maintained pregnant drug } \\
\text { dependent women }\end{array}$ & Includes adults (> 25 years) \\
\hline 534 & Szapocznik & 1986 & 3722570 & & $\begin{array}{l}\text { Conjoint versus one-person family therapy: further evidence } \\
\text { for the effectiveness of conducting family therapy through one } \\
\text { person with drug-abusing adolescents }\end{array}$ & $\begin{array}{l}\text { No extractable or relevant } \\
\text { data for } \\
\text { interventions/outcomes of } \\
\text { interest }\end{array}$ \\
\hline 535 & Szapocznik & 1988 & $\begin{array}{l}\text { 1989-06560- } \\
001 \text { (psychinfo) }\end{array}$ & $10.1037 / 0022-006 \times .56 .4 .552$ & $\begin{array}{l}\text { Engaging adolescent drug abusers and their families in } \\
\text { treatment: A strategic structural systems approach }\end{array}$ & $\begin{array}{l}\text { No extractable or relevant } \\
\text { data for } \\
\text { interventions/outcomes of } \\
\text { interest }\end{array}$ \\
\hline
\end{tabular}




\begin{tabular}{|c|c|c|c|c|c|c|}
\hline No. & Author & Year & $\begin{array}{l}\text { PubMed or } \\
\text { (Other) ID }\end{array}$ & DOI & Title & Reason for Exclusion \\
\hline 536 & Szobot & 2008 & $\begin{array}{l}\text { CN-00647808 } \\
\text { (cochrane) }\end{array}$ & & $\begin{array}{l}\text { A randomized crossover clinical study showing that } \\
\text { methylphenidate-SODAS improves attention- } \\
\text { deficit/hyperactivity disorder symptoms in adolescents with } \\
\text { substance use disorder }\end{array}$ & $\mathrm{RCT}, \mathrm{N}<10$ per arm \\
\hline 537 & Tait & 2016 & 27317044 & 10.1016/j.drugalcdep.2016.06.005 & $\begin{array}{l}\text { Emergency department based intervention with adolescent } \\
\text { substance users: 10year economic and health outcomes }\end{array}$ & $\begin{array}{l}\text { No extractable or relevant } \\
\text { data for } \\
\text { interventions/outcomes of } \\
\text { interest }\end{array}$ \\
\hline 538 & Tanner-Smith & 2018 & 29706171 & 10.1016/j.jsat.2018.03.003 & $\begin{array}{l}\text { Who attends recovery high schools after substance use } \\
\text { treatment? A descriptive analysis of school aged youth }\end{array}$ & Case control/cross sectional \\
\hline 539 & Tapert & 2003 & $\begin{array}{l}\text { 2003-09555- } \\
004 \text { (psychinfo) }\end{array}$ & 10.1300/J029v12n04_04 & Depressed mood, gender, and problem drinking in youth & $\begin{array}{l}\text { No extractable or relevant } \\
\text { data for } \\
\text { interventions/outcomes of } \\
\text { interest }\end{array}$ \\
\hline 540 & Tetzlaff & 2005 & 16011391 & 10.1037/0893-164X.19.2.199 & $\begin{array}{l}\text { Working alliance, treatment satisfaction, and patterns of } \\
\text { posttreatment use among adolescent substance users }\end{array}$ & $\begin{array}{l}\text { No extractable or relevant } \\
\text { data for } \\
\text { interventions/outcomes of } \\
\text { interest }\end{array}$ \\
\hline 541 & Thomasius & 2005 & 16097269 & 10.1024/1422-4917.33.3.217 & $\begin{array}{l}\text { Familientherapie als Frühintervention bei drogen-abhängigen } \\
\text { Jugendlichen, jungen Erwachsenen und deren Müttern-- } \\
\text { Effektstärken und individuelle Verbesserungsquoten bei den } \\
\text { Therapie-Beendern. = Early intervention family therapy in } \\
\text { drug-dependent adolescents, young adults, and their mothers- } \\
\text {-Effect sizes and intraindividual change indices in completers }\end{array}$ & $\begin{array}{l}\text { Single arm (nonpharm, } \\
\text { pharmacological } \\
\text { interventions } \mathrm{N}<200 \text { ) }\end{array}$ \\
\hline 542 & Thompson & 2017 & 28319159 & 10.1371/journal.pone.0173272 & $\begin{array}{l}\text { Drug therapy for alcohol dependence in primary care in the } \\
\text { UK: A Clinical Practice Research Datalink study }\end{array}$ & Includes adults ( $>25$ years) \\
\hline 543 & Thompson & 2017 & 28620272 & $10.1080 / 16066359.2016 .1193165$ & $\begin{array}{l}\text { Short-term effects of a brief intervention to reduce alcohol use } \\
\text { and sexual risk among homeless young adults: Results from a } \\
\text { randomized controlled trial }\end{array}$ & $\begin{array}{l}\text { Includes transition-aged } \\
\text { youth (non-pharmacological } \\
\text { interventions) }\end{array}$ \\
\hline 544 & Thush & 2009 & 19290699 & 10.1037/a0013789 & $\begin{array}{l}\text { Influence of motivational interviewing on explicit and implicit } \\
\text { alcohol-related cognition and alcohol use in at-risk } \\
\text { adolescents }\end{array}$ & $\begin{array}{l}\text { Not all subjects with at least } \\
\text { problematic use }\end{array}$ \\
\hline 545 & Timofeev & 1999 & 10467448 & 10.1142/S0192415X99000185 & $\begin{array}{l}\text { Effects of acupuncture and an agonist of opiate receptors on } \\
\text { heroin dependent patients }\end{array}$ & $\begin{array}{l}\text { No extractable or relevant } \\
\text { data for } \\
\text { interventions/outcomes of } \\
\text { interest }\end{array}$ \\
\hline
\end{tabular}




\begin{tabular}{|c|c|c|c|c|c|c|}
\hline No. & Author & Year & $\begin{array}{l}\text { PubMed or } \\
\text { (Other) ID }\end{array}$ & DOI & Title & Reason for Exclusion \\
\hline 546 & Tingey & 2016 & $\begin{array}{l}2017-07055- \\
013 \text { (psychinfo) }\end{array}$ & 10.5820/aian.2303.2016.248 & $\begin{array}{l}\text { Entrepreneurship education: A strength-based approach to } \\
\text { substance use and suicide prevention for American Indian } \\
\text { adolescents }\end{array}$ & $\begin{array}{l}\text { Not all subjects with at least } \\
\text { problematic use }\end{array}$ \\
\hline 547 & Tomko & 2019 & 30268706 & 10.1016/j.addbeh.2018.09.023 & $\begin{array}{l}\text { Corrigendum to 'The role of depressive symptoms in treatment } \\
\text { of adolescent cannabis use disorder with N-Acetylcysteine' }\end{array}$ & $\begin{array}{l}\text { No extractable or relevant } \\
\text { data for } \\
\text { interventions/outcomes of } \\
\text { interest }\end{array}$ \\
\hline 548 & Toumbourou & 2013 & 23968880 & 10.1016/j.jadohealth.2013.07.005 & $\begin{array}{l}\text { Reduction of adolescent alcohol use through familyâf"school } \\
\text { intervention: A randomized trial }\end{array}$ & $\begin{array}{l}\text { Not all subjects with at least } \\
\text { problematic use }\end{array}$ \\
\hline 549 & Treloar Padovano & 2018 & 29553345 & & $\begin{array}{l}\text { Using Ecological Momentary Assessment to Identify } \\
\text { Mechanisms of Change: An Application From a } \\
\text { Pharmacotherapy Trial With Adolescent Cannabis Users }\end{array}$ & $\begin{array}{l}\text { No extractable or relevant } \\
\text { data for } \\
\text { interventions/outcomes of } \\
\text { interest }\end{array}$ \\
\hline 550 & $\begin{array}{l}\text { Treloar Padovano } \\
\mathrm{H}\end{array}$ & 2018 & 29672090 & 10.1037/abn0000342 & $\begin{array}{l}\text { Subjective cannabis effects as part of a developing disorder in } \\
\text { adolescents and emerging adults. }\end{array}$ & $\begin{array}{l}\text { Single arm (nonpharm, } \\
\text { pharmacological } \\
\text { interventions } N<200 \text { ) }\end{array}$ \\
\hline 551 & Trupin & 2011 & $\begin{array}{l}2011-23745- \\
003 \text { (psychinfo) }\end{array}$ & 10.1080/1067828X.2011.614889 & $\begin{array}{l}\text { Family integrated transitions: A promising program for juvenile } \\
\text { offenders with co-occurring disorders }\end{array}$ & $\begin{array}{l}\text { NRCS (nonpharm, } \\
\text { pharmacological } \\
\text { interventions } N<100 \text { ) }\end{array}$ \\
\hline 552 & Tucker & 2017 & 28340904 & 10.1016/j.jsat.2017.02.008 & $\begin{array}{l}\text { A group-based motivational interviewing brief intervention to } \\
\text { reduce substance use and sexual risk behavior among } \\
\text { homeless young adults }\end{array}$ & $\begin{array}{l}\text { Not all subjects with at least } \\
\text { problematic use }\end{array}$ \\
\hline 553 & van der Pol & 2018 & 28076983 & $10.1177 / 0306624 X 16687536$ & $\begin{array}{l}\text { Multidimensional Family Therapy Reduces Self-Reported } \\
\text { Criminality Among Adolescents With a Cannabis Use Disorder }\end{array}$ & $\begin{array}{l}\text { No extractable or relevant } \\
\text { data for } \\
\text { interventions/outcomes of } \\
\text { interest }\end{array}$ \\
\hline 554 & Van Meter W & & 2324868 & 10.1080/02791072.1990.10472202 & $\begin{array}{l}\text { The case for shorter residential alcohol and other drug abuse } \\
\text { treatment adolescents }\end{array}$ & $\begin{array}{l}\text { Single arm (nonpharm, } \\
\text { pharmacological } \\
\text { interventions } \mathrm{N}<200 \text { ) }\end{array}$ \\
\hline 555 & Vargas-Martínez & 2019 & 31590139 & 10.1016/j.drugalcdep.2019.107597 & $\begin{array}{l}\text { Measuring the effects on quality of life and alcohol } \\
\text { consumption of a program to reduce binge drinking in Spanish } \\
\text { adolescents }\end{array}$ & $\begin{array}{l}\text { Not all subjects with at least } \\
\text { problematic use }\end{array}$ \\
\hline 556 & Voogt & 2012 & 22709609 & $10.1186 / 1745-6215-13-83$ & $\begin{array}{l}\text { The effectiveness of a web-based brief alcohol intervention in } \\
\text { reducing heavy drinking among adolescents aged } 15 \text { to } 20 \\
\text { years with a low educational background: study protocol for a } \\
\text { randomized controlled trial }\end{array}$ & $\begin{array}{l}\text { No extractable or relevant } \\
\text { data for } \\
\text { interventions/outcomes of } \\
\text { interest }\end{array}$ \\
\hline
\end{tabular}




\begin{tabular}{|c|c|c|c|c|c|c|}
\hline No. & Author & Year & $\begin{array}{l}\text { PubMed or } \\
\text { (Other) ID }\end{array}$ & DOI & Title & Reason for Exclusion \\
\hline 557 & Voogt & 2014 & 24613632 & 10.1016/j.drugalcdep.2014.02.009 & $\begin{array}{l}\text { The effect of the 'What Do You Drink' web-based brief alcohol } \\
\text { intervention on self-efficacy to better understand changes in } \\
\text { alcohol use over time: randomized controlled trial using } \\
\text { ecological momentary assessment }\end{array}$ & $\begin{array}{l}\text { College setting (alcohol } \\
\text { interventions) }\end{array}$ \\
\hline 558 & Waldron & 2005 & 16202539 & 10.1016/j.addbeh.2005.07.001 & $\begin{array}{l}\text { Profiles of drug use behavior change for adolescents in } \\
\text { treatment }\end{array}$ & $\begin{array}{l}\text { No extractable or relevant } \\
\text { data for } \\
\text { interventions/outcomes of } \\
\text { interest }\end{array}$ \\
\hline 559 & Walton & 2010 & 20682932 & 10.1001/jama.2010.1066 & $\begin{array}{l}\text { Effects of a brief intervention for reducing violence and alcohol } \\
\text { misuse among adolescents: a randomized controlled trial }\end{array}$ & $\begin{array}{l}\text { Not all subjects with at least } \\
\text { problematic use }\end{array}$ \\
\hline 560 & Walton & 2013 & 23711998 & 10.1016/j.drugalcdep.2013.04.020 & $\begin{array}{l}\text { Computer and therapist based brief interventions among } \\
\text { cannabis-using adolescents presenting to primary care: one } \\
\text { year outcomes }\end{array}$ & $\begin{array}{l}\text { Not all subjects with at least } \\
\text { problematic use }\end{array}$ \\
\hline 561 & Wang & 2016 & 27099500 & 10.2147/NDT.S105199 & $\begin{array}{l}\text { A family-oriented therapy program for youths with substance } \\
\text { abuse: long-term outcomes related to relapse and academic } \\
\text { or social status }\end{array}$ & $\begin{array}{l}\text { NRCS (nonpharm, } \\
\text { pharmacological } \\
\text { interventions } N<100 \text { ) }\end{array}$ \\
\hline 562 & Wang & & $\begin{array}{l}\text { 1997-41255- } \\
011 \text { (psychinfo) }\end{array}$ & 10.1016/S1001-0742(08)60025-X & An experimental study of drug-given-up in psychotherapy & Includes adults ( $>25$ years) \\
\hline 563 & Warden & 2012 & 22626890 & 10.1016/j.addbeh.2012.04.011 & $\begin{array}{l}\text { Predictors of attrition with buprenorphine/naloxone treatment } \\
\text { in opioid dependent youth }\end{array}$ & $\begin{array}{l}\text { No extractable or relevant } \\
\text { data for } \\
\text { interventions/outcomes of } \\
\text { interest }\end{array}$ \\
\hline 564 & Watson & 2015 & 27965788 & $10.1186 / s 40814-015-0004-4$ & $\begin{array}{l}\text { A randomised controlled feasibility trial of family and social } \\
\text { network intervention for young people who misuse alcohol and } \\
\text { drugs: study protocol (Y-SBNT) }\end{array}$ & $\begin{array}{l}\text { No extractable or relevant } \\
\text { data for } \\
\text { interventions/outcomes of } \\
\text { interest }\end{array}$ \\
\hline 565 & Watson & 2017 & 28399988 & 10.3310/hta21150 & $\begin{array}{l}\text { Youth social behaviour and network therapy (Y-SBNT): } \\
\text { adaptation of a family and social network intervention for } \\
\text { young people who misuse alcohol and drugs - a randomised } \\
\text { controlled feasibility trial }\end{array}$ & Includes adults ( $>25$ years) \\
\hline 566 & Watt & 2006 & $\begin{array}{l}\text { CN-00613379 } \\
\text { (cochrane) }\end{array}$ & $10.1080 / 09638230600998938$ & $\begin{array}{l}\text { Brief CBT for high anxiety sensitivity decreases drinking } \\
\text { problems, relief alcohol outcome expectancies, and conformity } \\
\text { drinking motives: evidence from a randomized controlled trial }\end{array}$ & $\begin{array}{l}\text { College setting (alcohol } \\
\text { interventions) }\end{array}$ \\
\hline 567 & Watterson & 2017 & 28464810 & 10.1186/s12889-017-4330-8 & $\begin{array}{l}\text { Measuring the effectiveness of in-hospital and on-base } \\
\text { Prevent Alcohol and Risk-related Trauma in Youth } \\
\text { (P.A.R.T.Y.) programs on reducing alcohol related harms in } \\
\text { naval trainees: P.A.R.T.Y. Defence study protocol }\end{array}$ & $\begin{array}{l}\text { No extractable or relevant } \\
\text { data for } \\
\text { interventions/outcomes of } \\
\text { interest }\end{array}$ \\
\hline
\end{tabular}




\begin{tabular}{|c|c|c|c|c|c|c|}
\hline No. & Author & Year & $\begin{array}{l}\text { PubMed or } \\
\text { (Other) ID }\end{array}$ & DOI & Title & Reason for Exclusion \\
\hline 568 & Wechsberg & 2017 & 28845096 & 10.1080/1067828X.2016.1260511 & $\begin{array}{l}\text { Efficacy of the Young Women's CoOp: An HIV Risk-Reduction } \\
\text { Intervention for Substance-Using African-American Female } \\
\text { Adolescents in the South }\end{array}$ & $\begin{array}{l}\text { No extractable or relevant } \\
\text { data for } \\
\text { interventions/outcomes of } \\
\text { interest }\end{array}$ \\
\hline 569 & Wechsberg & 2018 & 29996792 & 10.1186/s12889-018-5665-5 & $\begin{array}{l}\text { The Young Women's Health CoOp in Cape Town, South } \\
\text { Africa: Study protocol for a cluster-randomised trial for } \\
\text { adolescent women at risk for HIV }\end{array}$ & $\begin{array}{l}\text { No extractable or relevant } \\
\text { data for } \\
\text { interventions/outcomes of } \\
\text { interest }\end{array}$ \\
\hline 570 & Wegman & 2017 & 27964869 & 10.1016/S2214-109X(16)30303-5 & $\begin{array}{l}\text { Relapse to opioid use in opioid-dependent individuals released } \\
\text { from compulsory drug detention centres compared with those } \\
\text { from voluntary methadone treatment centres in Malaysia: a } \\
\text { two-arm, prospective observational study }\end{array}$ & $\begin{array}{l}\text { NRCS (nonpharm, } \\
\text { pharmacological } \\
\text { interventions } N<100 \text { ) }\end{array}$ \\
\hline 571 & Weidman & 1987 & 3612889 & & $\begin{array}{l}\text { Family therapy and reductions in treatment dropout in a } \\
\text { residential therapeutic community for chemically dependent } \\
\text { adolescents }\end{array}$ & $\begin{array}{l}\text { NRCS (nonpharm, } \\
\text { pharmacological } \\
\text { interventions } N<100 \text { ) }\end{array}$ \\
\hline 572 & Weiss & 2011 & 21463074 & $10.1037 / a 0023031$ & $\begin{array}{l}\text { Interaction effects of age and contingency management } \\
\text { treatments in cocaine-dependent outpatients }\end{array}$ & Includes adults (> 25 years) \\
\hline 573 & Weiss & 2014 & 24865619 & 10.1016/j.jsat.2014.04.003 & $\begin{array}{l}\text { Substance abuse treatment patients with early onset cocaine } \\
\text { use respond as well to contingency management interventions } \\
\text { as those with later onset cocaine use }\end{array}$ & $\begin{array}{l}\text { NRCS (nonpharm, } \\
\text { pharmacological } \\
\text { interventions } N<100 \text { ) }\end{array}$ \\
\hline 574 & Welsh & 2019 & 31088277 & 10.1080/02791072.2019.1613585 & $\begin{array}{l}\text { Treatment Retention and Outcomes with the Adolescent } \\
\text { Community Reinforcement Approach in Emerging Adults with } \\
\text { Opioid Use }\end{array}$ & $\begin{array}{l}\text { Includes transition-aged } \\
\text { youth (non-pharmacological } \\
\text { interventions) }\end{array}$ \\
\hline 575 & Wenzel & 2019 & $\begin{array}{l}\text { CN-01960977 } \\
\text { (Cochrane) }\end{array}$ & & $\begin{array}{l}\text { Youth opioid recovery support intervention: home delivery of } \\
\text { extended release naltrexone }\end{array}$ & $\begin{array}{l}\text { No extractable or relevant } \\
\text { data for } \\
\text { interventions/outcomes of } \\
\text { interest }\end{array}$ \\
\hline 576 & Werch & 2005 & 15957680 & & $\begin{array}{l}\text { A brief experimental alcohol beverage-tailored program for } \\
\text { adolescents }\end{array}$ & $\begin{array}{l}\text { Not all subjects with at least } \\
\text { problematic use }\end{array}$ \\
\hline 577 & Werch & 2010 & 20307126 & 10.1037/a0017997 & A brief image-based prevention intervention for adolescents & $\begin{array}{l}\text { Not all subjects with at least } \\
\text { problematic use }\end{array}$ \\
\hline 578 & Whicher & 2012 & $\begin{array}{l}2012-06310- \\
006 \text { (psychinfo) }\end{array}$ & 10.1097/ADT.0b013e3182387029 & $\begin{array}{l}\text { Pilot project to evaluate the effectiveness and acceptability of } \\
\text { single-session brief counseling for the prevention of substance } \\
\text { misuse in pregnant adolescents }\end{array}$ & $\begin{array}{l}\text { NRCS (nonpharm, } \\
\text { pharmacological } \\
\text { interventions } N<100 \text { ) }\end{array}$ \\
\hline 579 & White & 2015 & 25978822 & & $\begin{array}{l}\text { Are there secondary effects on marijuana use from brief } \\
\text { alcohol interventions for college students? }\end{array}$ & $\begin{array}{l}\text { College setting (alcohol } \\
\text { interventions) }\end{array}$ \\
\hline
\end{tabular}




\begin{tabular}{|c|c|c|c|c|c|c|}
\hline No. & Author & Year & $\begin{array}{l}\text { PubMed or } \\
\text { (Other) ID }\end{array}$ & DOI & Title & Reason for Exclusion \\
\hline 580 & Wilcox & 2012 & 21936751 & $10.3109 / 00952990.2011 .600393$ & $\begin{array}{l}\text { Compensation effects on clinical trial data collection in opioid- } \\
\text { dependent young adults }\end{array}$ & $\begin{array}{l}\text { No extractable or relevant } \\
\text { data for } \\
\text { interventions/outcomes of } \\
\text { interest }\end{array}$ \\
\hline 581 & Wildberger & 2019 & $\begin{array}{l}\text { CN-01961010 } \\
\text { (Cochrane) }\end{array}$ & & $\begin{array}{l}\text { Relationship between injectable naltrexone and IOP utilization } \\
\text { on opioid relapse in youth }\end{array}$ & $\begin{array}{l}\text { No extractable or relevant } \\
\text { data for } \\
\text { interventions/outcomes of } \\
\text { interest }\end{array}$ \\
\hline 582 & Wiljer & 2016 & 27815232 & 10.2196/resprot.6446 & $\begin{array}{l}\text { Enhancing Self-Efficacy for Help-Seeking Among Transition- } \\
\text { Aged Youth in Postsecondary Settings With Mental Health } \\
\text { and/or Substance Use Concerns, Using Crowd-Sourced } \\
\text { Online and Mobile Technologies: The Thought Spot Protocol }\end{array}$ & $\begin{array}{l}\text { Not all subjects with at least } \\
\text { problematic use }\end{array}$ \\
\hline 583 & Winn & 2019 & 31229188 & 10.1016/j.jsat.2019.05.009 & $\begin{array}{l}\text { Enhancing adolescent SBIRT with a peer-delivered } \\
\text { intervention: An implementation study }\end{array}$ & $\begin{array}{l}\text { Single arm (nonpharm, } \\
\text { pharmacological } \\
\text { interventions } N<200 \text { ) }\end{array}$ \\
\hline 584 & Winters & 2000 & 10829335 & & $\begin{array}{l}\text { The effectiveness of the Minnesota Model approach in the } \\
\text { treatment of adolescent drug abusers }\end{array}$ & $\begin{array}{l}\text { NRCS (nonpharm, } \\
\text { pharmacological } \\
\text { interventions } N<100 \text { ) }\end{array}$ \\
\hline 585 & Winters & 2007 & 17588490 & 10.1016/j.jsat.2006.12.003 & $\begin{array}{l}\text { Long-term outcome of substance-dependent youth following } \\
\text { 12-step treatment }\end{array}$ & $\begin{array}{l}\text { NRCS (nonpharm, } \\
\text { pharmacological } \\
\text { interventions } N<100 \text { ) }\end{array}$ \\
\hline 586 & Winters & & 25866459 & 10.1080/1067828X.2013.777377 & $\begin{array}{l}\text { Can Parents Provide Brief Intervention Services to Their Drug- } \\
\text { Abusing Teenager? }\end{array}$ & $\begin{array}{l}\text { No extractable or relevant } \\
\text { data for } \\
\text { interventions/outcomes of } \\
\text { interest }\end{array}$ \\
\hline 587 & Wintersteen & 2005 & $\begin{array}{l}\text { 2005-09654- } \\
008 \text { (psychinfo) }\end{array}$ & $10.1037 / 0735-7028.36 .4 .400$ & $\begin{array}{l}\text { Do Gender and Racial Differences Between Patient and } \\
\text { Therapist Affect Therapeutic Alliance and Treatment Retention } \\
\text { in Adolescents? }\end{array}$ & $\begin{array}{l}\text { Single arm (nonpharm, } \\
\text { pharmacological } \\
\text { interventions } \mathrm{N}<200 \text { ) }\end{array}$ \\
\hline 588 & Wodarski & 2010 & 20799128 & $10.1080 / 15433710903176112$ & $\begin{array}{l}\text { Prevention of adolescent reoccurring violence and alcohol } \\
\text { abuse: a multiple site evaluation }\end{array}$ & $\begin{array}{l}\text { No extractable or relevant } \\
\text { data for } \\
\text { interventions/outcomes of } \\
\text { interest }\end{array}$ \\
\hline 589 & Wright & 2017 & 28546136 & 10.2196/resprot.6760 & $\begin{array}{l}\text { An Ecological Momentary Intervention to Reduce Alcohol } \\
\text { Consumption in Young Adults Delivered During Drinking } \\
\text { Events: Protocol for a Pilot Randomized Controlled Trial }\end{array}$ & $\begin{array}{l}\text { No extractable or relevant } \\
\text { data for } \\
\text { interventions/outcomes of } \\
\text { interest }\end{array}$ \\
\hline
\end{tabular}




\begin{tabular}{|c|c|c|c|c|c|c|}
\hline No. & Author & Year & $\begin{array}{l}\text { PubMed or } \\
\text { (Other) ID }\end{array}$ & DOI & Title & Reason for Exclusion \\
\hline 590 & Yurasek & 2015 & 26191947 & 10.1037/pha0000025 & $\begin{array}{l}\text { A randomized controlled trial of a behavioral economic } \\
\text { intervention for alcohol and marijuana use }\end{array}$ & $\begin{array}{l}\text { College setting (alcohol } \\
\text { interventions) }\end{array}$ \\
\hline 591 & Zatzick & 2014 & 24733515 & 10.1001/jamapediatrics.2013.4784 & $\begin{array}{l}\text { Collaborative care intervention targeting violence risk } \\
\text { behaviors, substance use, and posttraumatic stress and } \\
\text { depressive symptoms in injured adolescents: a randomized } \\
\text { clinical trial }\end{array}$ & $\begin{array}{l}\text { Not all subjects with at least } \\
\text { problematic use }\end{array}$ \\
\hline 592 & Zhang & 2010 & 20802847 & 10.1016/j.jcrimjus.2010.04.012 & $\begin{array}{l}\text { Delinquency and alcohol-impaired driving among young } \\
\text { males: A longitudinal study }\end{array}$ & $\begin{array}{l}\text { Single arm (nonpharm, } \\
\text { pharmacological } \\
\text { interventions } N<200 \text { ) }\end{array}$ \\
\hline 593 & & 2015 & $\begin{array}{l}\text { NCT01632735 } \\
\text { (CT.gov) }\end{array}$ & & Mobile Continuing Care Approach for Youth & $\begin{array}{l}\text { Includes transition-aged } \\
\text { youth (non-pharmacological } \\
\text { interventions) }\end{array}$ \\
\hline 594 & & 2018 & 30484743 & 10.1089/tmj.2018.0201 & $\begin{array}{l}\text { Feasibility and Acceptability of an Electronic Parenting Skills } \\
\text { Intervention for Parents of Alcohol-Using Adolescent Trauma } \\
\text { Patients }\end{array}$ & $\begin{array}{l}\text { Not all subjects with at least } \\
\text { problematic use }\end{array}$ \\
\hline 595 & & 2018 & 30243410 & 10.1016/j.jsat.2018.08.013 & $\begin{array}{l}\text { Feasibility, acceptability, and preliminary effects of a brief } \\
\text { alcohol intervention for suicidal adolescents in inpatient } \\
\text { psychiatric treatment }\end{array}$ & $\begin{array}{l}\text { Not all subjects with at least } \\
\text { problematic use }\end{array}$ \\
\hline 596 & & 2018 & 29396897 & 10.1111/add.14179 & $\begin{array}{l}\text { Four-year follow-up of an internet-based brief intervention for } \\
\text { unhealthy alcohol use in young men }\end{array}$ & Includes adults (> 25 years) \\
\hline 597 & & 2018 & 30389649 & $10.2196 / 11298$ & $\begin{array}{l}\text { Efficacy of an Online Self-Help Treatment for Comorbid } \\
\text { Alcohol Misuse and Emotional Problems in Young Adults: } \\
\text { Protocol for a Randomized Controlled Trial }\end{array}$ & $\begin{array}{l}\text { No extractable or relevant } \\
\text { data for } \\
\text { interventions/outcomes of } \\
\text { interest }\end{array}$ \\
\hline 598 & & 2018 & 30587217 & 10.1186/s13063-018-3048-y & $\begin{array}{l}\text { Treatment effectiveness of a mindfulness-based inpatient } \\
\text { group psychotherapy in adolescent substance use disorder - } \\
\text { study protocol for a randomized controlled trial }\end{array}$ & $\begin{array}{l}\text { No extractable or relevant } \\
\text { data for } \\
\text { interventions/outcomes of } \\
\text { interest }\end{array}$ \\
\hline 599 & & 2018 & 29485676 & 10.1111/acer.13606 & $\begin{array}{l}\text { A Randomized Trial of a Personalized Feedback Intervention } \\
\text { for Nonstudent Emerging Adult At-Risk Drinkers }\end{array}$ & $\begin{array}{l}\text { Includes transition-aged } \\
\text { youth (non-pharmacological } \\
\text { interventions) }\end{array}$ \\
\hline 600 & & 2018 & 30148142 & 10.21037/mhealth.2018.07.04 & $\begin{array}{l}\text { Pilot randomized trial of MOMENT, a motivational counseling- } \\
\text { plus-ecological momentary intervention to reduce marijuana } \\
\text { use in youth }\end{array}$ & $\begin{array}{l}\text { Includes transition-aged } \\
\text { youth (non-pharmacological } \\
\text { interventions) }\end{array}$ \\
\hline
\end{tabular}




\begin{tabular}{|c|c|c|c|c|c|c|}
\hline No. & Author & Year & $\begin{array}{l}\text { PubMed or } \\
\text { (Other) ID }\end{array}$ & DOI & Title & Reason for Exclusion \\
\hline 601 & & 2018 & 28865169 & 10.1111/add.14026 & $\begin{array}{l}\text { A developmental-based motivational intervention to reduce } \\
\text { alcohol and marijuana use among non-treatment-seeking } \\
\text { young adults: a randomized controlled trial }\end{array}$ & $\begin{array}{l}\text { Includes transition-aged } \\
\text { youth (non-pharmacological } \\
\text { interventions) }\end{array}$ \\
\hline 602 & & 2018 & 29195590 & 10.1016/j.jsat.2017.10.012 & $\begin{array}{l}\text { Age differences in outcomes among patients in the 'Stimulant } \\
\text { Abuser Groups to Engage in 12-Step' (STAGE-12) } \\
\text { intervention }\end{array}$ & Includes adults ( $>25$ years) \\
\hline 603 & & 2018 & 29505456 & 10.1097/JAN.0000000000000207 & $\begin{array}{l}\text { Relational Health and Recovery: Adolescent Girls in Chemical } \\
\text { Dependency Treatment }\end{array}$ & $\begin{array}{l}\text { NRCS (nonpharm, } \\
\text { pharmacological } \\
\text { interventions } N<100 \text { ) }\end{array}$ \\
\hline 604 & & 2018 & 29960918 & 10.1016/j.drugalcdep.2018.05.020 & $\begin{array}{l}\text { Marijuana eCHECKUPTO GO: Effects of a personalized } \\
\text { feedback plus protective behavioral strategies intervention for } \\
\text { heavy marijuana-using college students }\end{array}$ & $\begin{array}{l}\text { Includes transition-aged } \\
\text { youth (non-pharmacological } \\
\text { interventions) }\end{array}$ \\
\hline 605 & & 2018 & 30021712 & $10.2196 / 11106$ & $\begin{array}{l}\text { The Family Check-Up Online Program for Parents of Middle } \\
\text { School Students: Protocol for a Randomized Controlled Trial }\end{array}$ & $\begin{array}{l}\text { Not all subjects with at least } \\
\text { problematic use }\end{array}$ \\
\hline 606 & & 2018 & 30030211 & 10.2196/mhealth.9324 & $\begin{array}{l}\text { Mobile Phone-Based Ecological Momentary Intervention to } \\
\text { Reduce Young Adults' Alcohol Use in the Event: A Three- } \\
\text { Armed Randomized Controlled Trial }\end{array}$ & Includes adults ( $>25$ years) \\
\hline 607 & & 2018 & 30126536 & 10.1016/j.jsat.2018.07.007 & $\begin{array}{l}\text { Young adults' perceptions of acceptability and effectiveness of } \\
\text { a text message-delivered treatment for cannabis use disorder }\end{array}$ & Includes adults (> 25 years) \\
\hline 608 & & 2018 & 30359047 & $10.1037 / \mathrm{adb} 0000413$ & $\begin{array}{l}\text { Feasibility of an interactive voice response system for daily } \\
\text { monitoring of illicit opioid use during buprenorphine treatment }\end{array}$ & Includes adults ( $>25$ years) \\
\hline 609 & & 2018 & 30422198 & 10.1001/jama.2018.12086 & $\begin{array}{l}\text { Screening and Behavioral Counseling Interventions to Reduce } \\
\text { Unhealthy Alcohol Use in Adolescents and Adults: Updated } \\
\text { Evidence Report and Systematic Review for the US } \\
\text { Preventive Services Task Force }\end{array}$ & Review \\
\hline 610 & & 2018 & 30092491 & 10.1016/j.evalprogplan.2018.07.001 & $\begin{array}{l}\text { Is culturally based prevention effective? Results from a 3-year } \\
\text { tribal substance use prevention program }\end{array}$ & $\begin{array}{l}\text { Not all subjects with at least } \\
\text { problematic use }\end{array}$ \\
\hline 611 & & 2019 & 30021470 & 10.1080/09540121.2018.1500008 & $\begin{array}{l}\text { Adolescent female school dropouts who use drugs and } \\
\text { engage in risky sex: effects of a brief pilot intervention in Cape } \\
\text { Town, South Africa }\end{array}$ & $\begin{array}{l}\text { No extractable or relevant } \\
\text { data for } \\
\text { interventions/outcomes of } \\
\text { interest }\end{array}$ \\
\hline 612 & & 2019 & 30670102 & 10.1186/s13063-018-3160-z & $\begin{array}{l}\text { Effectiveness of a web-based screening and brief intervention } \\
\text { with weekly text-message-initiated individualised prompts for } \\
\text { reducing risky alcohol use among teenagers: study protocol of } \\
\text { a randomised controlled trial within the ProHEAD consortium }\end{array}$ & $\begin{array}{l}\text { No extractable or relevant } \\
\text { data for } \\
\text { interventions/outcomes of } \\
\text { interest }\end{array}$ \\
\hline
\end{tabular}




\begin{tabular}{|c|c|c|c|c|c|c|}
\hline No. & Author & Year & $\begin{array}{l}\text { PubMed or } \\
\text { (Other) ID }\end{array}$ & DOI & Title & Reason for Exclusion \\
\hline 613 & & 2019 & 30866967 & 10.1186/s13012-019-0874-6 & $\begin{array}{l}\text { Measurement Training and Feedback System for } \\
\text { Implementation of family-based services for adolescent } \\
\text { substance use: protocol for a cluster randomized trial of two } \\
\text { implementation strategies }\end{array}$ & $\begin{array}{l}\text { No extractable or relevant } \\
\text { data for } \\
\text { interventions/outcomes of } \\
\text { interest }\end{array}$ \\
\hline 614 & & 2019 & 30782918 & 10.1136/bmjopen-2018-024776 & $\begin{array}{l}\text { A trauma-informed substance use and sexual risk reduction } \\
\text { intervention for young South African women: a mixed-methods } \\
\text { feasibility study }\end{array}$ & $\begin{array}{l}\text { Includes transition-aged } \\
\text { youth (non-pharmacological } \\
\text { interventions) }\end{array}$ \\
\hline 615 & & 2019 & 30940206 & 10.1186/s13722-019-0141-9 & $\begin{array}{l}\text { Development of a social media-based intervention targeting } \\
\text { tobacco use and heavy episodic drinking in young adults }\end{array}$ & $\begin{array}{l}\text { Includes transition-aged } \\
\text { youth (non-pharmacological } \\
\text { interventions) }\end{array}$ \\
\hline 616 & & 2019 & 30577903 & 10.1016/j.jsat.2018.11.012 & $\begin{array}{l}\text { Randomized effectiveness trial of a parent and youth } \\
\text { combined intervention on the substance use norms of Latino } \\
\text { middle school students }\end{array}$ & $\begin{array}{l}\text { Not all subjects with at least } \\
\text { problematic use }\end{array}$ \\
\hline 617 & & 2019 & 30640148 & 10.1016/j.addbeh.2019.01.006 & $\begin{array}{l}\text { Which behavior change techniques help young adults reduce } \\
\text { binge drinking? A pilot randomized clinical trial of } 5 \text { text } \\
\text { message interventions }\end{array}$ & Includes adults (> 25 years) \\
\hline 618 & & 2019 & $\begin{array}{l}135476645 \\
\text { (embase) }\end{array}$ & 10.1080/1067828X.2018.1529645 & $\begin{array}{l}\text { Brief alcohol interventions for youth in the emergency } \\
\text { department: Exploring proximal and distal outcomes }\end{array}$ & $\begin{array}{l}\text { No extractable or relevant } \\
\text { data for } \\
\text { interventions/outcomes of } \\
\text { interest }\end{array}$ \\
\hline 619 & & & $\begin{array}{l}127619233 \\
\text { (embase) }\end{array}$ & & $\begin{array}{l}23 \text { - Practical Tools to Support Adolescent Substance Abuse } \\
\text { Prevention in Primary Care: A Multi-Site Randomized } \\
\text { Controlled Trial of Computer-Facilitated Screening and } \\
\text { Provider Brief Advice in the Medical Office }\end{array}$ & Review \\
\hline 620 & & & $\begin{array}{l}\text { CN-01613001 } \\
\text { (cochrane) }\end{array}$ & & $\begin{array}{l}\text { Effects of topiramate on cannabis use among adolescents and } \\
\text { young adults in a randomized controlled clinical trial targeting } \\
\text { alcohol misuse }\end{array}$ & Review \\
\hline 621 & & & $\begin{array}{l}\text { CN-01907359 } \\
\text { (cochrane) }\end{array}$ & & $\begin{array}{l}\text { Implementation and effectiveness of an early intervention } \\
\text { program (QuikFix) for young people experiencing alcohol and } \\
\text { other drug-related harm }\end{array}$ & $\begin{array}{l}\text { No extractable or relevant } \\
\text { data for } \\
\text { interventions/outcomes of } \\
\text { interest }\end{array}$ \\
\hline 622 & & & $\begin{array}{l}\text { CN-01899196 } \\
\text { (cochrane) }\end{array}$ & & $\begin{array}{l}\text { Treatment of mindfulness-based psychotherapy in adolescent } \\
\text { inpatients with substance use disorders }\end{array}$ & $\begin{array}{l}\text { No extractable or relevant } \\
\text { data for } \\
\text { interventions/outcomes of } \\
\text { interest }\end{array}$ \\
\hline
\end{tabular}




\begin{tabular}{|l|l|l|l|l|l|l|}
\hline No. & Author & Year & $\begin{array}{l}\text { PubMed or } \\
\text { (Other) ID }\end{array}$ & DoI & Title & Reason for Exclusion \\
\hline 623 & & $\begin{array}{l}\text { CN-01899372 } \\
\text { (cochrane) }\end{array}$ & & $\begin{array}{l}\text { Promoting Help-seeking using E-technology for Adolescents } \\
\text { (Pro-HEAD). Sub-project 3: web-Based Screening and Brief } \\
\text { Intervention for Alcohol Use among Teenagers: added Effects } \\
\text { through Extended User Engagement? }\end{array}$ & $\begin{array}{l}\text { No extractable or relevant } \\
\text { data for } \\
\text { interventions/outcomes of } \\
\text { interest }\end{array}$ \\
\hline 624 & & $\begin{array}{l}\text { CN-01906948 } \\
\text { (cochrane) }\end{array}$ & $\begin{array}{l}\text { Prevention of Substance Abuse and Mental Disorders in } \\
\text { Children using the Mindfulness- Augmented "Trampoline" } \\
\text { Program }\end{array}$ & $\begin{array}{l}\text { No extractable or relevant } \\
\text { data for } \\
\text { interventions/outcomes of } \\
\text { interest }\end{array}$ \\
\hline 625 & & & $\begin{array}{l}\text { NCT02646449 } \\
\text { (CT.gov) }\end{array}$ & & $\begin{array}{l}\text { Treatment of Young Adults With Comorbid AUD/MDD: A Pilot } \\
\text { Medication Trial }\end{array}$ & RCT, N < 10 per arm \\
\hline
\end{tabular}

Abbreviations: NRCS = non-randomised controlled study; RCT = randomized controlled trial; nonpharm = study did not evaluate a pharmacological agent 


\section{Appendix C. Intervention Coding Manual}

\section{Determinations for Each Study}

1. Intervention type? (category: Drug; Behav; Drug \& Behav)

2. Drugs used (text=drug(s) used in each arm, if applicable)

2a. Drug note (text=details reported about drug dose, delivery, frequency, etc.)

3. Source (text=additional sources other than primary extracted PMID [e.g., included co-pubs, protocols, cited manuals] used to code intervention content)

\section{Table C-2. Determinations for Each Arm}

\section{LABELS}

4. SRDR arm name (text=comprised of content labels in order of appearance in extraction sheet)

5. Article arm name (text=specific label for arm as written in article)

6. Is the intervention (as a whole, if includes multiple content components below) well specified? (binary: $0=$ no; 1 =yes)

\section{Code YES if:}

Intervention has a specific name that describes the approach

- Primary intervention of interest is guided by a manual or comparable guide to ensure that others could replicate the approach

- The study references treatment fidelity or adherence

\section{Code NO if:}

- Intervention is generally not well specified

7. Is the intervention well specified note? (text=specific text describing why intervention is/is not well specified). Consider TIDieR checklist.

INTERVENTION CONTENT

8. Content: Cognitive behavioral (binary: $0=$ no; $1=$ yes)

\section{Code YES if:}

- Intervention described as focusing on changing the adolescent's thoughts and/or behaviors

- Common brand names may include:

o Adolescent Community Reinforcement Approach (ACRA)

o Dialectical Behavior Therapy (DBT)

o Cognitive Behavioral Therapy (CBT)

o Cognitive Therapy (CT)

\section{Code NO if:}

- No mention of cognitive behavioral therapy

9. Content: Cognitive behavioral note (text=specific labels and/or text to support content label)

10. Content: Motivation building (binary: $0=$ no; $1=$ yes)

\section{Code YES if:}

- Intervention described as focusing on increasing the adolescent's motivation to change

- Common brand names may include:

o Motivational Interviewing (MI)

o Brief Motivational Intervention (BMI)

o Motivation-Enhancement Therapy (MET)

\section{Code NO if:}


- No mention of motivation-building therapy

11. Content: Motivation building note (text=specific labels and/or text to support content label)

12. Content: Educational (binary: $0=$ no; $1=$ yes)

\section{Code YES if:}

- The intervention is described as psychoeducation, education, or general education

- Common brand names may include:

o Psychoeducation(al) (PE)

o Psychoeducation therapy or treatment (PET)

o Education (ED or EDUC)

\section{Code NO if:}

- No mention of psychoeducation or education

13. Content: Educational note (text=specific labels and/or text to support content label)

14. Content: Family focused (binary: $0=$ no; $1=y e s$ )

\section{Code YES if:}

- Common descriptions may include:

o Family therapy

o Family-based therapy

o Family strategic therapy

- Common brand names may include:

o Brief Strategic Family Therapy (BSFT)

o Ecological Family Therapy

o Ecologically Based Family Therapy (EBFT)

o Educational Family Therapy

o Family Behavioral Therapy (FBT)

o Family Functional Therapy/Functional Family Therapy (FFT)

o Family Systems Therapy (FST)

o Family Systems Network (FSN)

o Multidimensional Family Therapy (MDFT)

o Multi-systemic Therapy (MST)

- The intervention will include BOTH teen and a parent or legal guardian. Do NOT select this category if the intervention targets sibling only or teen + sibling or parents only

\section{Code NO if:}

- No mention of family therapy

15. Content: Family focused note (text=specific labels and/or text to support content label)

16. Content: Contingency management (binary: $0=$ no; $1=$ yes)

\section{Code YES if:}

- Intervention described as using contingency management, motivational incentives, vouchers, or prize draws

- Common brand names may include:

0 Contingency management (CM)

o Motivational Incentives

o Voucher-Based Therapy

\section{Code NO if:}

- No mention of contingency management

17. Content: Contingency management note (text=specific labels and/or text to support content label) 
18. Content: Peer group therapy (binary: $0=$ no; $1=$ yes)

\section{Code YES if:}

- Intervention described as providing peer group therapy (e.g. nondirective therapy interventions) delivered to

adolescents in a group format

\section{Code NO if:}

- No mention of peer group therapy

19. Content: Peer group therapy note (text=specific labels and/or text to support content label)

20. Content: Intensive case management (binary: $0=$ no; $1=y e s$ )

\section{Code YES if:}

- Intervention described as providing support to link adolescents to supportive services (e.g., continuity of care, etc.)

\section{Code NO if:}

- No mention of peer group therapy

21. Content: Intensive case management note (text=specific labels and/or text to support content label)

\section{EFFECT MODIFIERS}

\section{Brief duration (binary $0=$ no; $1=y e s$ )}

\section{Code YES if:}

- The intervention content is delivered in $<2$ sessions

\section{Code NO if:}

- The intervention context is delivered $>2$ sessions

23. Brief duration note (text=specific description of the duration and frequency of the intervention content above)

24. Delivery group (binary: $0=$ no; $1=$ yes)

The goal of the intervention is to address each individual adolescent's substance use, but the delivery mechanism is simultaneous treatment of a group of adolescents

\section{Code YES if:}

- The intervention content is described as being delivered in a group format (i.e., delivered to multiple adolescents at the same time

- Any intervention model is fine. Focus on whether the intervention is described as delivered in groups of adolescents

\section{Code NO if:}

- No mention of group therapy

25. Delivery group note (text=specific description of the group format)

26. Culturally accommodated intervention (binary: 0=no; $1=y e s$ )

\section{Code YES if:}

- The intervention content is described as being culturally sensitive/culturally adapted/specific/tailored

\section{Code NO if:}

- No mention of cultural adaptation

27. Culturally accommodated intervention note (text=brief description of how study was culturally sensitive; consider PROGRESS framework)

28. Integrated intervention (binary: $0=$ no; $1=y e s$ )

\section{Code YES if:}


- The intervention content is described targeting substance abuse and a co-occurring disorder

\section{Code NO if:}

- No mention of integrated intervention

29. Integrated intervention note (text= specific description of the integrated intervention)

30. General note (text=other information relevant to the intervention not already captured. Note, that this field should not be used to flag studies to be excluded but may be used to flag potential co-publications if not already linked. Also a place to note ordering (e.g., if a study compares MET + CBT vs. CBT + MET) 


\section{Appendix D. Baselines}

Table D-1. Brief behavioral interventions - baseline data and interventions

\begin{tabular}{|c|c|c|c|c|c|c|c|c|}
\hline $\begin{array}{l}\text { Author, Year } \\
\text { PMID* }\end{array}$ & $\mathrm{N}$ & $\begin{array}{l}\text { Substances } \\
\text { Used }\end{array}$ & Severity & $\begin{array}{l}\text { Ages } \\
\text { [eligible] } \\
\text { Mean } \\
\text { (sd) }\end{array}$ & $\begin{array}{r}\text { Male } \\
\%\end{array}$ & Setting & $\begin{array}{l}\text { Intervention } \\
\text { Delivery }\end{array}$ & Arm Names \\
\hline $\begin{array}{l}\text { Arnaud, } 2015 \\
2016-03749- \\
004 \\
\text { (psychinfo) }\end{array}$ & 1449 & alcohol & PU & $\begin{array}{l}{[16,18]} \\
16.9 \\
(0.7)\end{array}$ & 47 & $\begin{array}{l}\text { outpatient } \\
\text { online }\end{array}$ & online & $\begin{array}{l}\text { 1. TAU: "Control" } \\
\text { 2. MI: } \\
\text { "WISEteens" }\end{array}$ \\
\hline $\begin{array}{l}\text { Arnaud, } 2017 \\
27801991\end{array}$ & 320 & alcohol & PU & $\begin{array}{l}{[\mathrm{nr}, 18]} \\
15.7 \\
(1.2)\end{array}$ & 49 & $\begin{array}{l}\text { hospital } \\
\text { ED }\end{array}$ & hospital staff & $\begin{array}{l}\text { 1. TAU: } \\
\text { "Treatment as } \\
\text { usual" } \\
\text { 2. MI (parent): } \\
\text { "Brief Ml" }\end{array}$ \\
\hline $\begin{array}{l}\text { Bernstein, } \\
2009 \\
20053238\end{array}$ & 210 & cannabis & PU & $\begin{array}{l}{[14,21]} \\
\mathrm{nr}\end{array}$ & 63 & $\begin{array}{l}\text { hospital } \\
\text { ED }\end{array}$ & $\begin{array}{l}\text { peer } \\
\text { educators }\end{array}$ & $\begin{array}{l}\text { 1. TAU: } \\
\text { "Assessed control" } \\
\text { 2. Ml: } \\
\text { "Intervention" }\end{array}$ \\
\hline $\begin{array}{l}\text { Bernstein, } \\
2010 \\
20670329\end{array}$ & 853 & $\begin{array}{l}\text { alcohol } \\
\text { cannabis }\end{array}$ & PU & $\begin{array}{l}{[14,21]} \\
\mathrm{nr}\end{array}$ & 53 & $\begin{array}{l}\text { hospital } \\
\text { ED }\end{array}$ & $\begin{array}{l}\text { peer } \\
\text { educators }\end{array}$ & $\begin{array}{l}\text { 1. TAU: "Standard } \\
\text { assessed control" } \\
\text { 2. MI: } \\
\text { "Intervention" }\end{array}$ \\
\hline $\begin{array}{l}\text { Braciszewski, } \\
2018 \\
132804409 \\
\text { (embase) }\end{array}$ & 33 & $\begin{array}{l}\text { alcohol } \\
\text { cannabis } \\
\text { other drugs }\end{array}$ & PU & $\begin{array}{l}{[18,19]} \\
18.9 \\
(0.5)\end{array}$ & 52 & $\begin{array}{l}\text { exiting } \\
\text { foster care }\end{array}$ & computerized & $\begin{array}{l}\text { 1. TAU: "Control" } \\
\text { 2. MI: "iHeLP" }\end{array}$ \\
\hline $\begin{array}{l}\text { Brown, } 2015 \\
26362000\end{array}$ & 151 & $\begin{array}{l}\text { cannabis } \\
\text { alcohol }\end{array}$ & SUD & $\begin{array}{l}{[13,17]} \\
15.8(1)\end{array}$ & 35 & $\begin{array}{l}\text { inpatient } \\
\text { psychiatric } \\
\text { hospital }\end{array}$ & $\begin{array}{l}\text { research staff } \\
\text { (Ph.D. } \\
\text { psychologists, } \\
\text { postdoctoral } \\
\text { fellows) }\end{array}$ & $\begin{array}{l}\text { 1. TAU: } \\
\text { "Treatment as } \\
\text { usual" } \\
\text { 2. Ml: } \\
\text { "Motivational } \\
\text { interviewing" }\end{array}$ \\
\hline $\begin{array}{l}\text { Colby, } 2018 \\
29750362\end{array}$ & 167 & alcohol & PU & $\begin{array}{l}{[17,20]} \\
\mathrm{nr}\end{array}$ & 59 & $\begin{array}{l}\text { community } \\
\text { outpatienr }\end{array}$ & $\begin{array}{l}\text { research staff } \\
\text { (PhD } \\
\text { psychologist, } \\
\text { postdoctoral } \\
\text { fellow, social } \\
\text { worker) }\end{array}$ & $\begin{array}{l}\text { 1. TAU: "Attention } \\
\text { control" } \\
\text { 2. MI: "Brief } \\
\text { motivational } \\
\text { intervention" }\end{array}$ \\
\hline $\begin{array}{l}\text { Cunningham, } \\
2015 \\
26347440\end{array}$ & 836 & $\begin{array}{l}\text { alcohol } \\
\text { cannabis } \\
\text { other drugs }\end{array}$ & PU & $\begin{array}{l}{[14,20]} \\
18.6 \\
(1.4)\end{array}$ & 52 & $\begin{array}{l}\text { hospital } \\
\text { ED }\end{array}$ & $\begin{array}{l}\text { online, } \\
\text { therapist (no } \\
\text { detail) }\end{array}$ & $\begin{array}{l}\text { 1. TAU: "Control" } \\
\text { 2. MI: "Computer } \\
\text { brief intervention" } \\
\text { 3. MI: "Therapist } \\
\text { brief intervention" }\end{array}$ \\
\hline $\begin{array}{l}\text { D'Amico, } 2008 \\
18037603\end{array}$ & 64 & $\begin{array}{l}\text { alcohol } \\
\text { cannabis }\end{array}$ & PU & $\begin{array}{l}{[12,18]} \\
16(1.8)\end{array}$ & 48 & $\begin{array}{l}\text { primary } \\
\text { care }\end{array}$ & $\begin{array}{l}\text { therapists (no } \\
\text { detail) }\end{array}$ & $\begin{array}{l}\text { 1. TAU: "Usual } \\
\text { care" } \\
\text { 2. MI: "Project } \\
\text { CHAT" }\end{array}$ \\
\hline
\end{tabular}




\begin{tabular}{|c|c|c|c|c|c|c|c|c|}
\hline $\begin{array}{l}\text { Author, Year } \\
\text { PMID* }\end{array}$ & $\mathrm{N}$ & $\begin{array}{l}\text { Substances } \\
\text { Used }\end{array}$ & Severity & $\begin{array}{l}\text { Ages } \\
\text { [eligible] } \\
\text { Mean } \\
\text { (sd) }\end{array}$ & $\begin{array}{r}\text { Male } \\
\%\end{array}$ & Setting & $\begin{array}{l}\text { Intervention } \\
\text { Delivery }\end{array}$ & Arm Names \\
\hline $\begin{array}{l}\text { D'Amico, } 2018 \\
30138016\end{array}$ & 294 & $\begin{array}{l}\text { cannabis } \\
\text { alcohol }\end{array}$ & PU & $\begin{array}{l}{[12,18]} \\
15.9 \\
(1.6)\end{array}$ & 45 & $\begin{array}{l}\text { outpatient } \\
\text { primary } \\
\text { care clinic }\end{array}$ & facilitators & $\begin{array}{l}\text { 1. TAU: "Usual } \\
\text { care" } \\
\text { 2. MI: "CHAT } \\
\text { intervention" }\end{array}$ \\
\hline $\begin{array}{l}\text { de Gee, } 2014 \\
24969735\end{array}$ & 171 & cannabis & PU & $\begin{array}{l}{[14,21]} \\
17.9 \\
(1.8)\end{array}$ & 76 & $\begin{array}{l}\text { outpatient } \\
\text { community }\end{array}$ & $\begin{array}{l}\text { community } \\
\text { clinicians } \\
\text { (prevention } \\
\text { workers) }\end{array}$ & $\begin{array}{l}\text { 1. Ml: "'Weed } \\
\text { Check" in Dutch" } \\
\text { 2. Educ: } \\
\text { "Information } \\
\text { session" }\end{array}$ \\
\hline $\begin{array}{l}\text { Dembo, 2014 } \\
\text { 2014-42452- } \\
005 \\
\text { (psychinfo) }\end{array}$ & 180 & $\begin{array}{l}\text { alcohol } \\
\text { cannabis } \\
\text { other drugs }\end{array}$ & PU & $\begin{array}{l}{[11,17]} \\
14.8 \\
(1.3)\end{array}$ & 65 & $\begin{array}{l}\text { outpatient } \\
\text { community } \\
\text { (juvenile } \\
\text { justice) }\end{array}$ & $\begin{array}{l}\text { therapists (no } \\
\text { detail) }\end{array}$ & $\begin{array}{l}\text { 1. TAU: "Standard } \\
\text { truancy services" } \\
\text { 2. MI (parent): "BI- } \\
\text { Youth and Parent" } \\
\text { 3. MI: "BI-Youth" }\end{array}$ \\
\hline $\begin{array}{l}\text { Giles, } 2019 \\
\text { CN-01953820 } \\
\text { (cochrane) }\end{array}$ & 443 & alcohol & PU & $\begin{array}{l}{[14,15]} \\
\mathrm{nr}\end{array}$ & 51 & $\begin{array}{l}\text { outpatient } \\
\text { school }\end{array}$ & $\begin{array}{l}\text { school staff } \\
\text { (learning } \\
\text { mentors) }\end{array}$ & $\begin{array}{l}\text { 1. TAU: "Control" } \\
\text { 2. MI: "Brief } \\
\text { alcohol } \\
\text { intervention" }\end{array}$ \\
\hline $\begin{array}{l}\text { Marsden, } \\
2006 \\
16771893\end{array}$ & 342 & $\begin{array}{l}\text { cannabis } \\
\text { alcohol } \\
\text { MDMA } \\
\text { cocaine }\end{array}$ & PU & $\begin{array}{l}{[16,22]} \\
18.3(2)\end{array}$ & 67 & $\begin{array}{l}\text { community } \\
\text { outpatiet }\end{array}$ & $\begin{array}{l}\text { community } \\
\text { clinicians, } \\
\text { research staff } \\
\text { (no detail) }\end{array}$ & $\begin{array}{l}\text { 1. TAU: "Control" } \\
\text { 2. MI: } \\
\text { "Intervention" }\end{array}$ \\
\hline $\begin{array}{l}\text { Martin, } 2008 \\
17869051\end{array}$ & 40 & $\begin{array}{l}\text { cannabis } \\
\text { alcohol } \\
\text { other drugs }\end{array}$ & PU & $\begin{array}{l}{[14,19]} \\
16.5 \\
(1.3)\end{array}$ & 67 & $\begin{array}{l}\text { outpatient } \\
\text { community }\end{array}$ & $\begin{array}{l}\text { therapists (no } \\
\text { detail) }\end{array}$ & $\begin{array}{l}\text { 1. TAU: "Delayed } \\
\text { treatment } \\
\text { condition" } \\
\text { 2. MI: "Adolescent } \\
\text { Cannabis Check- } \\
\text { Up" }\end{array}$ \\
\hline $\begin{array}{l}\text { Martínez } \\
\text { Martínez, } \\
2008 \\
2009-05582- \\
007 \\
\text { (psychinfo) }\end{array}$ & 52 & alcohol & PU & $\begin{array}{l}{[14,18]} \\
16(1.5)\end{array}$ & 65 & $\begin{array}{l}\text { outpatient } \\
\text { school }\end{array}$ & $\begin{array}{l}\text { therapists (no } \\
\text { detail) }\end{array}$ & $\begin{array}{l}\text { 1. TAU: "Control } \\
\text { waitlist" } \\
\text { 2. Ml: } \\
\text { "Experimental" }\end{array}$ \\
\hline $\begin{array}{l}\text { Mason, } 2015 \\
26234955\end{array}$ & 119 & $\begin{array}{l}\text { cannabis } \\
\text { alcohol }\end{array}$ & PU & $\begin{array}{l}{[14,18]} \\
16.4 \\
(1.2)\end{array}$ & 29 & $\begin{array}{l}\text { outpatient } \\
\text { research } \\
\text { clinic }\end{array}$ & $\begin{array}{l}\text { therapists (no } \\
\text { detail) }\end{array}$ & $\begin{array}{l}\text { 1. TAU: "Attention } \\
\text { control condition" } \\
\text { 2. MI: "Peer } \\
\text { network } \\
\text { counselling" }\end{array}$ \\
\hline $\begin{array}{l}\text { McCambridge, } \\
2004 \\
14678061\end{array}$ & 179 & $\begin{array}{l}\text { cannabis } \\
\text { alcohol } \\
\text { other drugs }\end{array}$ & PU & $\begin{array}{l}{[16,20]} \\
17.6 \\
(1.1)\end{array}$ & 55 & $\begin{array}{l}\text { outpatient } \\
\text { school }\end{array}$ & $\begin{array}{l}\text { research staff } \\
\text { (PhD } \\
\text { psychologist) }\end{array}$ & $\begin{array}{l}\text { 1. TAU: } \\
\text { "Education-as- } \\
\text { usual" } \\
\text { 2. Ml: } \\
\text { "Motivational } \\
\text { interviewing" }\end{array}$ \\
\hline
\end{tabular}




\begin{tabular}{|c|c|c|c|c|c|c|c|c|}
\hline $\begin{array}{l}\text { Author, Year } \\
\text { PMID* }\end{array}$ & $\mathrm{N}$ & $\begin{array}{l}\text { Substances } \\
\text { Used }\end{array}$ & Severity & $\begin{array}{l}\text { Ages } \\
\text { [eligible] } \\
\text { Mean } \\
\text { (sd) }\end{array}$ & $\begin{array}{r}\text { Male } \\
\%\end{array}$ & Setting & $\begin{array}{l}\text { Intervention } \\
\text { Delivery }\end{array}$ & Arm Names \\
\hline $\begin{array}{l}\text { McCambridge, } \\
2008 \\
18778385\end{array}$ & 326 & $\begin{array}{l}\text { cannabis } \\
\text { alcohol } \\
\text { other drugs }\end{array}$ & PU & $\begin{array}{l}{[16,19]} \\
18(1.7)\end{array}$ & 69 & $\begin{array}{l}\text { outpatient } \\
\text { school }\end{array}$ & $\begin{array}{l}\text { research staff } \\
\text { (PhD } \\
\text { psychologist, } \\
\text { psychology } \\
\text { graduates) }\end{array}$ & $\begin{array}{l}\text { 1. Ml: } \\
\text { "Motivational } \\
\text { interviewing" } \\
\text { 2. Educ: "Drug } \\
\text { information and } \\
\text { advice-giving" }\end{array}$ \\
\hline $\begin{array}{l}\text { McCarty, } 2019 \\
30883284\end{array}$ & 148 & $\begin{array}{l}\text { alcohol } \\
\text { cannabis }\end{array}$ & PU & $\begin{array}{l}{[13,18]} \\
\mathrm{nr}\end{array}$ & 20 & outpatient & $\begin{array}{l}\text { school } \\
\text { providers }\end{array}$ & $\begin{array}{l}\text { 1. TAU: "School- } \\
\text { based health clinic } \\
\text { visit" } \\
\text { 2. MI: "Check } \\
\text { Yourself feedback } \\
\text { + School-based } \\
\text { health visit" }\end{array}$ \\
\hline $\begin{array}{l}\text { Monti, } 1999 \\
10596521\end{array}$ & 94 & alcohol & PU & $\begin{array}{l}{[18,19]} \\
18.4 \\
(0.5)\end{array}$ & 65 & $\begin{array}{l}\text { hospital } \\
\text { ED }\end{array}$ & $\begin{array}{l}\text { research staff } \\
\text { (no detail) }\end{array}$ & $\begin{array}{l}\text { 1. TAU: "Standard } \\
\text { card" } \\
\text { 2. MI: "Brief } \\
\text { motivational } \\
\text { interviewing" }\end{array}$ \\
\hline $\begin{array}{l}\text { Peterson, } \\
2006 \\
16938063\end{array}$ & 285 & $\begin{array}{l}\text { alcohol } \\
\text { cannabis } \\
\text { amphetamines } \\
\text { cocaine } \\
\text { heroin }\end{array}$ & PU & $\begin{array}{l}{[13,19]} \\
17.4 \\
(1.5)\end{array}$ & 55 & $\begin{array}{l}\text { outpatient } \\
\text { community }\end{array}$ & $\begin{array}{l}\text { research staff } \\
\text { (Master's } \\
\text { level } \\
\text { therapists) }\end{array}$ & $\begin{array}{l}\text { 1. TAU: } \\
\text { "Assessment only" } \\
\text { 2. TAU: } \\
\text { "Assessment at } \\
\text { follow-up only" } \\
\text { 3. MI: "Brief } \\
\text { motivational } \\
\text { enhancement" }\end{array}$ \\
\hline $\begin{array}{l}\text { Smith, } 2015 \\
25551562\end{array}$ & 48 & unspecified & PU & $\begin{array}{l}{[13,19]} \\
16.3 \\
(1.4)\end{array}$ & 77 & $\begin{array}{l}\text { outpatient } \\
\text { community }\end{array}$ & $\begin{array}{l}\text { therapists (no } \\
\text { detail) }\end{array}$ & $\begin{array}{l}\text { 1. Ml: "Ml" } \\
\text { 2. Ml: "MI + } \\
\text { normative } \\
\text { feedback" }\end{array}$ \\
\hline $\begin{array}{l}\text { Spijkerman, } \\
2010 \\
21169172\end{array}$ & 575 & alcohol & PU & $\begin{array}{l}{[15,20]} \\
18.2 \\
(1.6)\end{array}$ & 38 & $\begin{array}{l}\text { outpatient } \\
\text { online }\end{array}$ & online & $\begin{array}{l}\text { 1. TAU: "Control" } \\
\text { 2. MI: "Brief } \\
\text { intervention } \\
\text { without normative } \\
\text { feedback" } \\
\text { 3. MI: "Brief } \\
\text { intervention with } \\
\text { normative } \\
\text { feedback" }\end{array}$ \\
\hline $\begin{array}{l}\text { Spirito, } 2004 \\
15343198\end{array}$ & 152 & alcohol & PU & $\begin{array}{l}{[13,17]} \\
15.6 \\
(1.2)\end{array}$ & 64 & $\begin{array}{l}\text { hospital } \\
\text { ED }\end{array}$ & $\begin{array}{l}\text { research staff } \\
\text { (Bachelor's } \\
\text { level } \\
\text { therapists and } \\
\text { Master's level } \\
\text { therapists) }\end{array}$ & $\begin{array}{l}\text { 1. TAU: "Standard } \\
\text { care" } \\
\text { 2. MI: } \\
\text { "Motivational } \\
\text { interview" }\end{array}$ \\
\hline
\end{tabular}




\begin{tabular}{|c|c|c|c|c|c|c|c|c|}
\hline $\begin{array}{l}\text { Author, Year } \\
\text { PMID* }\end{array}$ & $\mathrm{N}$ & $\begin{array}{l}\text { Substances } \\
\text { Used }\end{array}$ & Severity & $\begin{array}{l}\text { Ages } \\
\text { [eligible] } \\
\text { Mean } \\
\text { (sd) }\end{array}$ & $\begin{array}{r}\text { Male } \\
\%\end{array}$ & Setting & $\begin{array}{l}\text { Intervention } \\
\text { Delivery }\end{array}$ & Arm Names \\
\hline $\begin{array}{l}\text { Spirito, } 2011 \\
21383276\end{array}$ & 125 & alcohol & PU & $\begin{array}{l}{[13,17]} \\
15.5 \\
(1.2)\end{array}$ & 48 & $\begin{array}{l}\text { hospital } \\
\text { ED }\end{array}$ & $\begin{array}{l}\text { research staff } \\
\text { (Master's } \\
\text { level } \\
\text { therapists) }\end{array}$ & $\begin{array}{l}\text { 1. MI (parent): } \\
\text { "Individual } \\
\text { Motivational } \\
\text { Interview + Family } \\
\text { Motivational } \\
\text { Interview (Family } \\
\text { Check Up)" } \\
\text { 2. MI: } \\
\text { "Motivational } \\
\text { interview" }\end{array}$ \\
\hline $\begin{array}{l}\text { Spirito, } 2017 \\
29252011\end{array}$ & 69 & $\begin{array}{l}\text { cannabis } \\
\text { alcohol } \\
\text { other drugs }\end{array}$ & PU & $\begin{array}{l}{[13,18]} \\
15.8 \\
(1.4)\end{array}$ & 59 & $\begin{array}{l}\text { outpatient } \\
\text { research } \\
\text { clinic } \\
\text { (juvenile } \\
\text { justice } \\
\text { truant } \\
\text { court) }\end{array}$ & $\begin{array}{l}\text { research staff } \\
\text { (graduate } \\
\text { students) }\end{array}$ & $\begin{array}{l}\text { 1. MI (parent): } \\
\text { "Motivational } \\
\text { enhancement } \\
\text { therapy + Family } \\
\text { Check Up" } \\
\text { 2. Educ: } \\
\text { "Psychoeducation" }\end{array}$ \\
\hline $\begin{array}{l}\text { Srisurapanont, } \\
2007 \\
17453612\end{array}$ & 48 & methamphetamine & SUD & $\begin{array}{l}{[14,19]} \\
16.9 \\
(1.4)\end{array}$ & 88 & $\begin{array}{l}\text { outpatient } \\
\text { research } \\
\text { clinic }\end{array}$ & $\begin{array}{l}\text { therapists (no } \\
\text { detail) }\end{array}$ & $\begin{array}{l}\text { 1. Ml: "Brief } \\
\text { intervention" } \\
\text { 2. Educ: } \\
\text { "Psychoeducation" }\end{array}$ \\
\hline $\begin{array}{l}\text { Stein, } 2011 \\
21531089\end{array}$ & 189 & $\begin{array}{l}\text { cannabis } \\
\text { alcohol }\end{array}$ & PU & $\begin{array}{l}{[14,19]} \\
\mathrm{nr}\end{array}$ & $\mathrm{nr}$ & $\begin{array}{l}\text { residential } \\
\text { (juvenile } \\
\text { justice) }\end{array}$ & $\begin{array}{l}\text { research staff } \\
\text { (no detail) }\end{array}$ & $\begin{array}{l}\text { 1. TAU: } \\
\text { "Relaxation } \\
\text { training" } \\
\text { 2. MI: } \\
\text { "Motivational } \\
\text { interviewing" }\end{array}$ \\
\hline $\begin{array}{l}\text { Tait, } 2004 \\
15194207\end{array}$ & 249 & $\begin{array}{l}\text { alcohol } \\
\text { cannabis } \\
\text { other drugs }\end{array}$ & PU & $\begin{array}{l}{[12,19]} \\
16.7 \\
(1.7)\end{array}$ & 48 & $\begin{array}{l}\text { hospital } \\
\text { ED }\end{array}$ & $\begin{array}{l}\text { research staff } \\
\text { (no detail) }\end{array}$ & $\begin{array}{l}\text { 1. TAU: "Standard } \\
\text { hospital care" } \\
\text { 2. ICM: "Usual } \\
\text { hospital care + BI } \\
\text { focused on } \\
\text { engagement" }\end{array}$ \\
\hline $\begin{array}{l}\text { Voogt, } 2013 \\
\text { CN-01122318 } \\
\text { (cochrane) }\end{array}$ & 609 & alcohol & PU & $\begin{array}{l}{[15,20]} \\
17.3 \\
(1.3)\end{array}$ & 60 & $\begin{array}{l}\text { outpatient } \\
\text { school }\end{array}$ & online & $\begin{array}{l}\text { 1. TAU: "Control" } \\
\text { 2. MI: } \\
\text { "Experimental" }\end{array}$ \\
\hline $\begin{array}{l}\text { Walker, } 2006 \\
16822119\end{array}$ & 97 & $\begin{array}{l}\text { cannabis } \\
\text { alcohol } \\
\text { other drugs }\end{array}$ & PU & $\begin{array}{l}{[14,19]} \\
15.8 \\
(1.2)\end{array}$ & 48 & $\begin{array}{l}\text { outpatient } \\
\text { school }\end{array}$ & $\begin{array}{l}\text { research staff } \\
\text { (health } \\
\text { educators) }\end{array}$ & $\begin{array}{l}\text { 1. TAU: "Waitlist } \\
\text { control" } \\
\text { 2. MI: } \\
\text { "Motivational } \\
\text { enchancement } \\
\text { therapy" }\end{array}$ \\
\hline
\end{tabular}




\begin{tabular}{|c|c|c|c|c|c|c|c|c|}
\hline $\begin{array}{l}\text { Author, Year } \\
\text { PMID* }\end{array}$ & $\mathrm{N}$ & $\begin{array}{l}\text { Substances } \\
\text { Used }\end{array}$ & Severity & $\begin{array}{l}\text { Ages } \\
\text { [eligible] } \\
\text { Mean } \\
\text { (sd) }\end{array}$ & $\begin{array}{r}\text { Male } \\
\%\end{array}$ & Setting & $\begin{array}{l}\text { Intervention } \\
\text { Delivery }\end{array}$ & Arm Names \\
\hline $\begin{array}{l}\text { Walker, } 2011 \\
21688877\end{array}$ & 310 & $\begin{array}{l}\text { cannabis } \\
\text { alcohol } \\
\text { other drugs }\end{array}$ & PU & $\begin{array}{l}{[14,19]} \\
16(1.2)\end{array}$ & 61 & $\begin{array}{l}\text { outpatient } \\
\text { school }\end{array}$ & $\begin{array}{l}\text { health } \\
\text { educators } \\
\text { (Bachelor's } \\
\text { and Master's } \\
\text { level } \\
\text { therapists) }\end{array}$ & $\begin{array}{l}\text { 1. TAU: "Delayed } \\
\text { feedback control" } \\
\text { 2. Ml: } \\
\text { "Motivational } \\
\text { enhancement } \\
\text { therapy" } \\
\text { 3. Educ: } \\
\text { "Educational } \\
\text { feedback control" }\end{array}$ \\
\hline $\begin{array}{l}\text { Walker, } 2016 \\
27762569\end{array}$ & 252 & $\begin{array}{l}\text { cannabis } \\
\text { alcohol } \\
\text { other drugs }\end{array}$ & PU & $\begin{array}{l}{[\mathrm{nr}, \mathrm{nr}]} \\
15.8(1)\end{array}$ & 68 & $\begin{array}{l}\text { outpatient } \\
\text { school }\end{array}$ & $\begin{array}{l}\text { research staff } \\
\text { (Bachelor's } \\
\text { level } \\
\text { therapists and } \\
\text { Master's level } \\
\text { therapists) }\end{array}$ & $\begin{array}{l}\text { 1. CBT+MI: } \\
\text { "Assessment only } \\
\text { check-in" } \\
\text { 2. CBT+MI: } \\
\text { "Motivational } \\
\text { check-in" }\end{array}$ \\
\hline $\begin{array}{l}\text { Winters, } 2007 \\
17563146\end{array}$ & 79 & $\begin{array}{l}\text { alcohol } \\
\text { cannabis }\end{array}$ & SUD & $\begin{array}{l}{[13,17]} \\
15.6(n r)\end{array}$ & 62 & $\begin{array}{l}\text { outpatient } \\
\text { school }\end{array}$ & $\begin{array}{l}\text { therapists (no } \\
\text { detail) }\end{array}$ & $\begin{array}{l}\text { 1. TAU: "Control" } \\
\text { 2. MI: "BI-A" } \\
\text { 3. MI: "BI-AP" }\end{array}$ \\
\hline $\begin{array}{l}\text { Winters, } 2012 \\
22000326\end{array}$ & 315 & $\begin{array}{l}\text { cannabis } \\
\text { alcohol } \\
\text { other drugs }\end{array}$ & PU & $\begin{array}{l}{[12,18]} \\
\mathrm{nr}\end{array}$ & $\mathrm{nr}$ & $\begin{array}{l}\text { outpatient } \\
\text { school }\end{array}$ & $\begin{array}{l}\text { therapists (no } \\
\text { detail) }\end{array}$ & $\begin{array}{l}\text { 1. TAU: } \\
\text { "Assessment only } \\
\text { control" } \\
\text { 2. MI (parent): } \\
\text { "Brief Intervention } \\
\text { - Adolescent Plus } \\
\text { Parent Session" } \\
\text { 3. MI: "Brief } \\
\text { Intervention - } \\
\text { Adolescent Only" }\end{array}$ \\
\hline
\end{tabular}

. PMID* = Pubmed identifier if available, otherwise (database name). Abbreviations: $\mathrm{N}=$ number randomized; PU = problematic use; $\mathrm{SD}$ = standard deviation; $\mathrm{ED}=$ emergency department; $\mathrm{nr}=$ not reported; $\mathrm{CBT}=$ cognitive behavioral therapy; $\mathrm{CM}=$ contingency management; Edu = education; Fam = family therapy; ICM = intensive case management; $\mathrm{MI}=$ motivational interviewing; TAU = treatment as usual; parent = at least one component of the intervention included parent involvement/was targeted towards parents 
Table D-2. Nonbrief behavioral interventions - baseline data and interventions

\begin{tabular}{|c|c|c|c|c|c|c|c|c|}
\hline $\begin{array}{l}\text { Author, Year } \\
\text { PMID* }\end{array}$ & $\mathrm{N}$ & $\begin{array}{l}\text { Substances } \\
\text { Used }\end{array}$ & Severity & $\begin{array}{l}\text { Ages } \\
\text { [eligible] } \\
\text { Mean } \\
\text { (sd) }\end{array}$ & $\begin{array}{r}\text { Male } \\
\%\end{array}$ & Setting & $\begin{array}{l}\text { Intervention } \\
\text { Delivery }\end{array}$ & Arm Names \\
\hline $\begin{array}{l}\text { Amini, } 1982 \\
\text { CN-00182281 } \\
\text { (cochrane) }\end{array}$ & 87 & unspecified & PU & $\begin{array}{l}{[\mathrm{nr}, \mathrm{nr}]} \\
16.1(1)\end{array}$ & 69 & $\begin{array}{l}\text { inpatient } \\
\text { (experimental) } \\
\text { outpatient } \\
\text { community } \\
\text { (control) }\end{array}$ & $\begin{array}{l}\text { research staff } \\
\text { (psychiatry } \\
\text { residents, } \\
\text { psychology } \\
\text { interns) }\end{array}$ & $\begin{array}{l}\text { 1. TAU (group): "Inpatient" } \\
\text { 2. TAU: "Outpatient" }\end{array}$ \\
\hline $\begin{array}{l}\text { Azrin, } 1994 \\
\text { CN-00241903 } \\
\text { (cochrane) }\end{array}$ & 26 & $\begin{array}{l}\text { cannabis } \\
\text { cocaine } \\
\text { hallucinogens } \\
\text { methamphetamine } \\
\text { benzodiazepines }\end{array}$ & PU & $\begin{array}{l}{[\mathrm{nr}, 18]} \\
16(\mathrm{nr})\end{array}$ & 77 & $\begin{array}{l}\text { outpatient } \\
\text { research clinic }\end{array}$ & $\begin{array}{l}\text { research staff } \\
\text { (college } \\
\text { graduates, } \\
\text { graduate } \\
\text { students) }\end{array}$ & $\begin{array}{l}\text { 1. PeerGroup (group): } \\
\text { "Supportive counseling" } \\
\text { 2. CBT (parent): "Behavioral } \\
\text { program" }\end{array}$ \\
\hline $\begin{array}{l}\text { Azrin, } 2001 \\
2002-13926- \\
001 \\
\text { (psychinfo) }\end{array}$ & 56 & $\begin{array}{l}\text { alcohol } \\
\text { cannabis } \\
\text { "hard drugs" }\end{array}$ & SUD & $\begin{array}{l}{[12,17]} \\
15.4 \\
(1.3)\end{array}$ & 82 & $\begin{array}{l}\text { outpatient } \\
\text { research clinic }\end{array}$ & $\begin{array}{l}\text { research staff } \\
\text { (graduate } \\
\text { students) }\end{array}$ & $\begin{array}{l}\text { 1. Fam[behavioral] } \\
\text { (integrated): "Family- } \\
\text { behavioral therapy" } \\
\text { 2. CBT (integrated): } \\
\text { "Individual-cognitive therapy" }\end{array}$ \\
\hline $\begin{array}{l}\text { Baer, } 2007 \\
18072842\end{array}$ & 117 & $\begin{array}{l}\text { cannabis } \\
\text { alcohol } \\
\text { other drug }\end{array}$ & PU & $\begin{array}{l}{[13,19]} \\
17.9 \\
(1.2)\end{array}$ & 56 & $\begin{array}{l}\text { outpatient } \\
\text { community } \\
\text { (drop in } \\
\text { center) }\end{array}$ & $\begin{array}{l}\text { therapists (no } \\
\text { detail) }\end{array}$ & $\begin{array}{l}\text { 1. TAU: "Treatment as usual" } \\
\text { 2. MI: "Brief motivational } \\
\text { interview" }\end{array}$ \\
\hline $\begin{array}{l}\text { Burrow- } \\
\text { Sanchez, } \\
2012 \\
22866693\end{array}$ & 35 & unspecified & SUD & $\begin{array}{l}{[13,18]} \\
15.5 \\
(1.3)\end{array}$ & 94 & $\begin{array}{l}\text { outpatient } \\
\text { reseach clinic } \\
\text { (juvenile } \\
\text { justice) }\end{array}$ & $\begin{array}{l}\text { research staff } \\
\text { (graduate } \\
\text { students) }\end{array}$ & $\begin{array}{l}\text { 1. CBT (group, cultural): } \\
\text { "Culturally accomodated CBT" } \\
\text { 2. CBT (group): "Standard } \\
\text { CBT" }\end{array}$ \\
\hline $\begin{array}{l}\text { Burrow- } \\
\text { Sanchez, } \\
2015 \\
25602465\end{array}$ & 70 & $\begin{array}{l}\text { alcohol } \\
\text { marijuana } \\
\text { other drugs }\end{array}$ & SUD & $\begin{array}{l}{[13,18]} \\
15.2 \\
(1.2)\end{array}$ & 90 & $\begin{array}{l}\text { outpatient } \\
\text { reseach clinic } \\
\text { (juvenile } \\
\text { justice) }\end{array}$ & $\begin{array}{l}\text { research staff } \\
\text { (graduate } \\
\text { students) }\end{array}$ & $\begin{array}{l}\text { 1. CBT (group, cultural): } \\
\text { "Culturally accomodated CBT" } \\
\text { 2. CBT (group): "Standard } \\
\text { CBT" }\end{array}$ \\
\hline $\begin{array}{l}\text { D'Amico, } 2013 \\
\text { CN-00917707 } \\
\text { (cochrane) }\end{array}$ & 193 & $\begin{array}{l}\text { cannabis } \\
\text { alcohol }\end{array}$ & PU & $\begin{array}{l}{[14,18]} \\
16.6 \\
(1.1)\end{array}$ & 67 & $\begin{array}{l}\text { outpatient } \\
\text { community }\end{array}$ & $\begin{array}{l}\text { community } \\
\text { clinicians } \\
\text { (control) } \\
\text { research staff } \\
\text { (graduate } \\
\text { students; } \\
\text { experimental) }\end{array}$ & $\begin{array}{l}\text { 1. PeerGroup (group): "Usual } \\
\text { care" } \\
\text { 2. MI (group): "Group } \\
\text { motivational interviewing } \\
\text { (FreeTalk)" }\end{array}$ \\
\hline $\begin{array}{l}\text { Dakof, } 2015 \\
25621927\end{array}$ & 112 & $\begin{array}{l}\text { cannabis } \\
\text { alcohol } \\
\text { other drug }\end{array}$ & SUD & $\begin{array}{l}{[13,18]} \\
16(1.1)\end{array}$ & 89 & $\begin{array}{l}\text { outpatient } \\
\text { community } \\
\text { (juvenile } \\
\text { justice) }\end{array}$ & $\begin{array}{l}\text { community } \\
\text { clinicians }\end{array}$ & $\begin{array}{l}\text { 1. Fam[ecological]: } \\
\text { "Multidimensional family } \\
\text { therapy" } \\
\text { 2. CBT+MI (group): } \\
\text { "Adolescent group therapy" }\end{array}$ \\
\hline
\end{tabular}




\begin{tabular}{|c|c|c|c|c|c|c|c|c|}
\hline $\begin{array}{l}\text { Author, Year } \\
\text { PMID* }\end{array}$ & $N$ & $\begin{array}{l}\text { Substances } \\
\text { Used }\end{array}$ & Severity & $\begin{array}{l}\text { Ages } \\
\text { [eligible] } \\
\text { Mean } \\
\text { (sd) }\end{array}$ & $\begin{array}{r}\text { Male } \\
\%\end{array}$ & Setting & $\begin{array}{l}\text { Intervention } \\
\text { Delivery }\end{array}$ & Arm Names \\
\hline $\begin{array}{l}\text { Dennis, } 2004 \\
15501373 b\end{array}$ & 300 & $\begin{array}{l}\text { cannabis } \\
\text { alcohol } \\
\text { other drugs }\end{array}$ & PU & $\begin{array}{l}{[12,18]} \\
\mathrm{nr}\end{array}$ & 85 & $\begin{array}{l}\text { outpatient } \\
\text { community }\end{array}$ & $\begin{array}{l}\text { community } \\
\text { clinicians }\end{array}$ & $\begin{array}{l}\text { 1. Fam[ecological] (cultural): } \\
\text { "Multidimensional Family } \\
\text { Therapy" } \\
\text { 2. CBT+MI (group): } \\
\text { "Motivational Enhancement } \\
\text { Therapy plus Cognitive } \\
\text { Behavioral Therapy } \\
\text { (MET/CBT) - } 5 \text { sessions" } \\
\text { 3. CBT (parent): "Adolescent } \\
\text { Community Reinforcement } \\
\text { Approach" }\end{array}$ \\
\hline $\begin{array}{l}\text { Dennis, } 2004 \\
15501373\end{array}$ & 213 & $\begin{array}{l}\text { cannabis } \\
\text { alcohol } \\
\text { other drugs }\end{array}$ & PU & $\begin{array}{l}{[12,18]} \\
\mathrm{nr}\end{array}$ & 84 & $\begin{array}{l}\text { outpatient } \\
\text { community }\end{array}$ & $\begin{array}{l}\text { community } \\
\text { clinicians }\end{array}$ & $\begin{array}{l}\text { 1. CBT+MI+Educ+ICM (parent, } \\
\text { group): "Family education and } \\
\text { therapy components (Family } \\
\text { Support Network)" } \\
\text { 2. CBT+MI (group): } \\
\text { "Motivational Enhancement } \\
\text { Therapy plus Cognitive } \\
\text { Behavioral Therapy } \\
\text { (MET/CBT) - } 5 \text { sessions" } \\
\text { 3. CBT+MI (group): } \\
\text { "Motivational Enhancement } \\
\text { Therapy plus Cognitive } \\
\text { Behavioral Therapy } \\
\text { (MET/CBT) - } 12 \text { sessions" }\end{array}$ \\
\hline $\begin{array}{l}\text { Esposito- } \\
\text { Smythers, } \\
2011 \\
22004303\end{array}$ & 80 & $\begin{array}{l}\text { cannabis } \\
\text { alcohol }\end{array}$ & SUD & $\begin{array}{l}{[13,17]} \\
15.7 \\
(1.2)\end{array}$ & 33 & $\begin{array}{l}\text { outpatient } \\
\text { research clinic }\end{array}$ & $\begin{array}{l}\text { research staff } \\
\text { (PhD } \\
\text { psychologist, } \\
\text { postdoctoral } \\
\text { fellow; } \\
\text { experimental) } \\
\text { community } \\
\text { clinicians } \\
\text { (control) }\end{array}$ & $\begin{array}{l}\text { 1. TAU (integrated): } \\
\text { "Enhanced TAU" } \\
\text { 2. CBT+MI (parent, integrated): } \\
\text { "Integrated CBT" }\end{array}$ \\
\hline $\begin{array}{l}\text { Figurelli, } 1994 \\
7862806\end{array}$ & 48 & $\begin{array}{l}\text { alcohol } \\
\text { other drugs }\end{array}$ & PU & $\begin{array}{l}{[13,19]} \\
\mathrm{nr}\end{array}$ & 62 & $\begin{array}{l}\text { outpatient } \\
\text { community }\end{array}$ & $\begin{array}{l}\text { therapists } \\
\text { (substance } \\
\text { abuse } \\
\text { counselors) }\end{array}$ & $\begin{array}{l}\text { 1. TAU: "TAU" } \\
\text { 2. CBT: "Cognitively-oriented } \\
\text { pre-intervention" }\end{array}$ \\
\hline $\begin{array}{l}\text { Friedman, } \\
1989 \\
\text { CN-00496580 } \\
\text { (cochrane) }\end{array}$ & 169 & $\begin{array}{l}\text { alcohol } \\
\text { cannabis } \\
\text { other drugs }\end{array}$ & PU & $\begin{array}{l}{[14,21]} \\
17.9 \\
(1.8)\end{array}$ & 61 & $\begin{array}{l}\text { outpatient } \\
\text { community }\end{array}$ & $\begin{array}{l}\text { therapists (no } \\
\text { detail) }\end{array}$ & $\begin{array}{l}\text { 1. Fam[functional]: "Family } \\
\text { therapy" } \\
\text { 2. CBT (parent, group): "Parent } \\
\text { group" }\end{array}$ \\
\hline $\begin{array}{l}\text { Godley, } 2002 \\
12127465\end{array}$ & 114 & $\begin{array}{l}\text { alcohol } \\
\text { cannabis }\end{array}$ & SUD & $\begin{array}{l}{[12,17]} \\
\mathrm{nr}\end{array}$ & 80 & $\begin{array}{l}\text { outpatient } \\
\text { community }\end{array}$ & $\begin{array}{l}\text { case } \\
\text { managers }\end{array}$ & $\begin{array}{l}\text { 1. TAU: "Usual continuing } \\
\text { care" } \\
\text { 2. CBT+ICM: "UCC plus an } \\
\text { assertive continuing care } \\
\text { protocol" }\end{array}$ \\
\hline
\end{tabular}




\begin{tabular}{|c|c|c|c|c|c|c|c|c|}
\hline $\begin{array}{l}\text { Author, Year } \\
\text { PMID* }\end{array}$ & $\mathrm{N}$ & $\begin{array}{l}\text { Substances } \\
\text { Used }\end{array}$ & Severity & $\begin{array}{l}\text { Ages } \\
\text { [eligible] } \\
\text { Mean } \\
\text { (sd) }\end{array}$ & $\begin{array}{r}\text { Male } \\
\%\end{array}$ & Setting & $\begin{array}{l}\text { Intervention } \\
\text { Delivery }\end{array}$ & Arm Names \\
\hline $\begin{array}{l}\text { Godley, } 2010 \\
20219293\end{array}$ & 320 & $\begin{array}{l}\text { cannabis } \\
\text { alcohol } \\
\text { other drugs }\end{array}$ & SUD & $\begin{array}{l}{[12,18]} \\
\mathrm{nr}\end{array}$ & 76 & $\begin{array}{l}\text { outpatient } \\
\text { community }\end{array}$ & $\begin{array}{l}\text { community } \\
\text { clinicians }\end{array}$ & $\begin{array}{l}\text { 1. TAU (group): "Chestnut's } \\
\text { Bloomington Outpatient } \\
\text { Treatment" } \\
\text { 2. CBT+MI+ICM (group): } \\
\text { "Motivational Enhancement } \\
\text { Therapy/Cognitive Behavior } \\
\text { Therapy-7 session model + } \\
\text { Assertive Continuing Care" } \\
\text { 3. CBT+MI (group): } \\
\text { "Motivational Enhancement } \\
\text { Therapy/Cognitive Behavior } \\
\text { Therapy-7 session model" } \\
\text { 4. CBT+ICM (group): } \\
\text { "Chestnut's Bloomington } \\
\text { Outpatient Treatment + } \\
\text { Assertive Continuing Care" }\end{array}$ \\
\hline $\begin{array}{l}\text { Godley, } 2019 \\
\text { CN-01745749 } \\
\text { (cochrane) }\end{array}$ & 402 & $\begin{array}{l}\text { cannabis } \\
\text { alcohol } \\
\text { other drugs }\end{array}$ & SUD & $\begin{array}{l}{[12,18]} \\
\mathrm{nr}\end{array}$ & 84 & $\begin{array}{l}\text { residential } \\
\text { treatment }\end{array}$ & volunteers & $\begin{array}{l}\text { 1. TAU: "Continuing care } \\
\text { services as usual" } \\
\text { 2. CBT+ICM: "Volunteer } \\
\text { recovery support for } \\
\text { adolescents" }\end{array}$ \\
\hline $\begin{array}{l}\text { Henderson, } \\
2016 \\
26992083\end{array}$ & 126 & $\begin{array}{l}\text { alcohol } \\
\text { other drugs }\end{array}$ & PU & $\begin{array}{l}{[12,17]} \\
15.2 \\
(1.1)\end{array}$ & 74 & $\begin{array}{l}\text { outpatient } \\
\text { community } \\
\text { (juvenile } \\
\text { justice) }\end{array}$ & $\begin{array}{l}\text { therapists (no } \\
\text { detail) }\end{array}$ & $\begin{array}{l}\text { 1. TAU: "Services as usual } \\
\text { (SAU)" } \\
\text { 2. CBT+ICM: "Adolescent- } \\
\text { community reinforcement } \\
\text { approach + assertive } \\
\text { continuing care" }\end{array}$ \\
\hline $\begin{array}{l}\text { Henggeler, } \\
1996 \\
8610836\end{array}$ & 118 & $\begin{array}{l}\text { alcohol } \\
\text { cannabis } \\
\text { other drugs }\end{array}$ & SUD & $\begin{array}{l}{[12,17]} \\
15.7(1)\end{array}$ & 79 & $\begin{array}{l}\text { outpatient } \\
\text { community }\end{array}$ & $\begin{array}{l}\text { therapists (no } \\
\text { detail; } \\
\text { experimental); } \\
\text { community } \\
\text { clinicians } \\
\text { (control) }\end{array}$ & $\begin{array}{l}\text { 1. TAU (group): "Usual } \\
\text { community services" } \\
\text { 2. Fam[ecological]: "Home- } \\
\text { based multisystemic therapy" }\end{array}$ \\
\hline $\begin{array}{l}\text { Henggeler, } \\
2006 \\
16551142\end{array}$ & 161 & $\begin{array}{l}\text { cannabis } \\
\text { alcohol } \\
\text { cocaine }\end{array}$ & SUD & $\begin{array}{l}{[12,17]} \\
15.2 \\
(1.1)\end{array}$ & 83 & $\begin{array}{l}\text { outpatient } \\
\text { community } \\
\text { (juvenile } \\
\text { justice) }\end{array}$ & $\begin{array}{l}\text { community } \\
\text { clinicians }\end{array}$ & $\begin{array}{l}\text { 1. PeerGroup (group): "Drug } \\
\text { court with community services" } \\
\text { 2. PeerGroup (group): "Family } \\
\text { court with community services" } \\
\text { 3. Fam[ecological]+PeerGroup } \\
\text { (group): "Drug court + } \\
\text { multisystemic therapy" } \\
\text { 4. } \\
\text { Fam[ecological]+CM+PeerGro } \\
\text { up (group): "Drug court + } \\
\text { multisystemic therapy + } \\
\text { contingency managment" }\end{array}$ \\
\hline
\end{tabular}




\begin{tabular}{|c|c|c|c|c|c|c|c|c|}
\hline $\begin{array}{l}\text { Author, Year } \\
\text { PMID* }\end{array}$ & $\mathrm{N}$ & $\begin{array}{l}\text { Substances } \\
\text { Used }\end{array}$ & Severity & $\begin{array}{l}\text { Ages } \\
\text { [eligible] } \\
\text { Mean } \\
\text { (sd) }\end{array}$ & $\begin{array}{r}\text { Male } \\
\%\end{array}$ & Setting & $\begin{array}{l}\text { Intervention } \\
\text { Delivery }\end{array}$ & Arm Names \\
\hline $\begin{array}{l}\text { Henggeler, } \\
2012 \\
22309470\end{array}$ & 115 & $\begin{array}{l}\text { cannabis } \\
\text { alcohol } \\
\text { other drugs }\end{array}$ & PU & $\begin{array}{l}{[12,17]} \\
15.4(1)\end{array}$ & 83 & $\begin{array}{l}\text { outpatient } \\
\text { community } \\
\text { (juvenile } \\
\text { justice) }\end{array}$ & $\begin{array}{l}\text { community } \\
\text { clinicians }\end{array}$ & $\begin{array}{l}\text { 1. PeerGroup (group): "Usual } \\
\text { services" } \\
\text { 2. Fam[ecological]+CM: } \\
\text { "Contingency management } \\
\text { and family engagement } \\
\text { strategies" }\end{array}$ \\
\hline $\begin{array}{l}\text { Hogue, } 2015 \\
25496283\end{array}$ & 297 & $\begin{array}{l}\text { alcohol and other } \\
\text { drugs }\end{array}$ & PU & $\begin{array}{l}{[12,18]} \\
15.7 \\
(1.5)\end{array}$ & 52 & $\begin{array}{l}\text { outpatient } \\
\text { community }\end{array}$ & $\begin{array}{l}\text { community } \\
\text { clinicians }\end{array}$ & $\begin{array}{l}\text { 1. TAU: "Usual care other" } \\
\text { 2. Fam[systems/structural]: } \\
\text { "Usual care family therapy" }\end{array}$ \\
\hline $\begin{array}{l}\text { Joanning, } \\
1992 \\
\text { CN-00631575 } \\
\text { (cochrane) }\end{array}$ & 134 & $\begin{array}{l}\text { alcohol } \\
\text { cannabis } \\
\text { amphetamines } \\
\text { barbiturates } \\
\text { or hallucinogens }\end{array}$ & PU & $\begin{array}{l}{[11,20]} \\
15.4 \\
(1.9)\end{array}$ & $\mathrm{nr}$ & $\begin{array}{l}\text { outpatient } \\
\text { research clinic }\end{array}$ & $\begin{array}{l}\text { research staff } \\
\text { (graduate } \\
\text { students) }\end{array}$ & $\begin{array}{l}\text { 1. PeerGroup (group): } \\
\text { "Adolescent group therapy" } \\
\text { 2. Fam[systems/structural]: } \\
\text { "Family systems therapy" } \\
\text { 3. Fam[education] (group): } \\
\text { "Family drug education" }\end{array}$ \\
\hline $\begin{array}{l}\text { Kaminer, } 1998 \\
9824170\end{array}$ & 32 & unspecified & SUD & $\begin{array}{l}{[13,18]} \\
15.4 \\
(1.5)\end{array}$ & 62 & $\begin{array}{l}\text { outpatient } \\
\text { research clinic }\end{array}$ & & $\begin{array}{l}\text { 1. PeerGroup (group): } \\
\text { "Interactional group treatment" } \\
\text { 2. CBT (group): "Cognitive- } \\
\text { behavioral group treatment" }\end{array}$ \\
\hline $\begin{array}{l}\text { Kaminer, } 2002 \\
12436013\end{array}$ & 88 & $\begin{array}{l}\text { cannabis } \\
\text { alcohol }\end{array}$ & SUD & $\begin{array}{l}{[13,18]} \\
15.4 \\
(1.3)\end{array}$ & 70 & $\begin{array}{l}\text { outpatient } \\
\text { research clinic }\end{array}$ & $\begin{array}{l}\text { therapist (no } \\
\text { detail) }\end{array}$ & $\begin{array}{l}\text { 1. Educ (group): } \\
\text { "Psychoeducational therapy" } \\
\text { 2. CBT (group): "Cognitive } \\
\text { behavioral therapy" }\end{array}$ \\
\hline $\begin{array}{l}\text { Kaminer, } 2008 \\
18978635\end{array}$ & 144 & $\begin{array}{l}\text { cannabis } \\
\text { alcohol }\end{array}$ & SUD & $\begin{array}{l}{[13,18]} \\
15.9 \\
(1.2)\end{array}$ & 67 & $\begin{array}{l}\text { outpatient } \\
\text { research clinic }\end{array}$ & $\begin{array}{l}\text { therapists (no } \\
\text { detail) }\end{array}$ & $\begin{array}{l}\text { 1. TAU: "No-active aftercare" } \\
\text { 2. CBT+MI: "Brief telephone } \\
\text { MI" } \\
\text { 3. CBT+MI: "In-person Ml" }\end{array}$ \\
\hline $\begin{array}{l}\text { Kelly, } 2017 \\
28742932\end{array}$ & 59 & $\begin{array}{l}\text { alcohol and other } \\
\text { drugs }\end{array}$ & SUD & $\begin{array}{l}{[14,21]} \\
16.9(2)\end{array}$ & 73 & $\begin{array}{l}\text { outpatient } \\
\text { community }\end{array}$ & $\begin{array}{l}\text { therapists (no } \\
\text { detail) }\end{array}$ & $\begin{array}{l}\text { 1. PeerGroup (group): } \\
\text { "Integrated 12-step" } \\
\text { 2. CBT+MI (group): } \\
\text { "MET/CBT" }\end{array}$ \\
\hline $\begin{array}{l}\text { Killeen, } 2012 \\
22299805\end{array}$ & 31 & $\begin{array}{l}\text { cannabis } \\
\text { other drugs }\end{array}$ & SUD & $\begin{array}{l}{[14,18]} \\
15.5 \\
(1.2)\end{array}$ & 84 & $\begin{array}{l}\text { outpatient } \\
\text { community }\end{array}$ & $\begin{array}{l}\text { therapists (no } \\
\text { detail) }\end{array}$ & $\begin{array}{l}\text { 1. TAU: "Control + community } \\
\text { treatment" } \\
\text { 2. CM: "Contingency } \\
\text { management + community } \\
\text { treatment" }\end{array}$ \\
\hline $\begin{array}{l}\text { Latimer, } 2003 \\
12957348\end{array}$ & 43 & $\begin{array}{l}\text { cannabis } \\
\text { alcohol } \\
\text { other drugs }\end{array}$ & SUD & $\begin{array}{l}{[14,17]} \\
\mathrm{nr}\end{array}$ & 76 & $\begin{array}{l}\text { outpatient } \\
\text { community }\end{array}$ & $\begin{array}{l}\text { therapists (no } \\
\text { detail) }\end{array}$ & $\begin{array}{l}\text { 1. Educ (group): "Drugs harm } \\
\text { psychoeducation curriculum" } \\
\text { 2. CBT+Fam[behavioral] } \\
\text { (group): "Integrated family and } \\
\text { cognitive-behavioral therapy" }\end{array}$ \\
\hline
\end{tabular}




\begin{tabular}{|c|c|c|c|c|c|c|c|c|}
\hline $\begin{array}{l}\text { Author, Year } \\
\text { PMID* }\end{array}$ & $\mathrm{N}$ & $\begin{array}{l}\text { Substances } \\
\text { Used }\end{array}$ & Severity & $\begin{array}{l}\text { Ages } \\
\text { [eligible] } \\
\text { Mean } \\
\text { (sd) }\end{array}$ & $\begin{array}{r}\text { Male } \\
\%\end{array}$ & Setting & $\begin{array}{l}\text { Intervention } \\
\text { Delivery }\end{array}$ & Arm Names \\
\hline $\begin{array}{l}\text { Letourneau, } \\
2017 \\
27629581\end{array}$ & 107 & $\begin{array}{l}\text { cannabis } \\
\text { alcohol } \\
\text { cocaine }\end{array}$ & PU & $\begin{array}{l}{[11,17]} \\
14.9 \\
(0.1)\end{array}$ & 84 & $\begin{array}{l}\text { outpatient } \\
\text { research clinic } \\
\text { (juvenile } \\
\text { justice) }\end{array}$ & $\begin{array}{l}\text { research staff } \\
\text { (Master's level } \\
\text { therapists) }\end{array}$ & $\begin{array}{l}\text { 1. TAU (group): "Usual } \\
\text { services" } \\
\text { 2. CBT+Fam[behavioral]+CM: } \\
\text { "Risk reduction therapy for } \\
\text { adolescents" }\end{array}$ \\
\hline $\begin{array}{l}\text { Liddle, } 2001 \\
11727882\end{array}$ & 182 & $\begin{array}{l}\text { cannabis } \\
\text { alcohol } \\
\text { and other drugs }\end{array}$ & PU & $\begin{array}{l}{[13,18]} \\
15.9 \\
(1.2)\end{array}$ & $\mathrm{nr}$ & $\begin{array}{l}\text { outpatient } \\
\text { community }\end{array}$ & $\begin{array}{l}\text { community } \\
\text { clinicians }\end{array}$ & $\begin{array}{l}\text { 1. PeerGroup (group): } \\
\text { "Adolescent group therapy" } \\
\text { 2. Fam[education] (group): } \\
\text { "Multifamily educational } \\
\text { intervention" } \\
\text { 3. Fam[ecological]: } \\
\text { "Multidimensional family } \\
\text { therapy" }\end{array}$ \\
\hline $\begin{array}{l}\text { Liddle, } 2004 \\
15152709\end{array}$ & 80 & unspecified & PU & $\begin{array}{l}{[11,15]} \\
13.7 \\
(1.1)\end{array}$ & 72 & $\begin{array}{l}\text { outpatient } \\
\text { community }\end{array}$ & $\begin{array}{l}\text { community } \\
\text { clinicians }\end{array}$ & $\begin{array}{l}\text { 1. Fam[ecological]: } \\
\text { "Multidimensional family } \\
\text { therapy" } \\
\text { 2. CBT (group): "Peer group" }\end{array}$ \\
\hline $\begin{array}{l}\text { Liddle, } 2008 \\
18705691\end{array}$ & 224 & $\begin{array}{l}\text { cannabis } \\
\text { alcohol } \\
\text { other drugs }\end{array}$ & PU & $\begin{array}{l}{[12} \\
17.5] \\
15.3 \\
(1.2)\end{array}$ & 18 & $\begin{array}{l}\text { outpatient } \\
\text { community }\end{array}$ & $\begin{array}{l}\text { therapists } \\
\text { (Ph.D. } \\
\text { psychologists } \\
\text { and Master's } \\
\text { level } \\
\text { therapists) }\end{array}$ & $\begin{array}{l}\text { 1. Fam[ecological]: } \\
\text { "Multidimensional family } \\
\text { therapy" } \\
\text { 2. CBT: "Cognitive behavioral } \\
\text { therapy" }\end{array}$ \\
\hline $\begin{array}{l}\text { Liddle, } 2018 \\
29866383\end{array}$ & 113 & $\begin{array}{l}\text { cannabis } \\
\text { alcohol } \\
\text { stimulants } \\
\text { opioids }\end{array}$ & SUD & $\begin{array}{l}{[13,18]} \\
15.4 \\
(1.1)\end{array}$ & 75 & $\begin{array}{l}\text { outpatient } \\
\text { community }\end{array}$ & $\begin{array}{l}\text { community } \\
\text { clinicians }\end{array}$ & $\begin{array}{l}\text { 1. TAU (group): "Residential } \\
\text { treatment" } \\
\text { 2. Fam[ecological]: } \\
\text { "Multidimensional family } \\
\text { therapy" }\end{array}$ \\
\hline $\begin{array}{l}\text { Lowe, } 2012 \\
22931079\end{array}$ & 187 & unspecified & PU & $\begin{array}{l}{[13,18]} \\
16.4 \\
(1.3)\end{array}$ & 58 & $\begin{array}{l}\text { outpatient } \\
\text { community } \\
\text { (Native } \\
\text { American } \\
\text { tribal area) }\end{array}$ & $\begin{array}{l}\text { therapist and } \\
\text { cultural expert } \\
\text { (no detail) }\end{array}$ & $\begin{array}{l}\text { 1. PeerGroup (group, cultural): } \\
\text { "Cherokee Talking Circle" } \\
\text { 2. Educ (group): "Standard } \\
\text { Substance Abuse Education" }\end{array}$ \\
\hline $\begin{array}{l}\text { Najavits, } 2006 \\
16858633\end{array}$ & 33 & $\begin{array}{l}\text { cannabis } \\
\text { alcohol } \\
\text { hallucinogens } \\
\text { amphetamines } \\
\text { cocaine } \\
\text { opioids } \\
\text { inhalants } \\
\text { barbiturates }\end{array}$ & SUD & $\begin{array}{l}{[\mathrm{nr}, \mathrm{nr}]} \\
16.1 \\
(1.2)\end{array}$ & 0 & $\begin{array}{l}\text { outpatient } \\
\text { research clinic }\end{array}$ & $\begin{array}{l}\text { research staff } \\
\text { (PhD } \\
\text { psychologists, } \\
\text { postdoctoral } \\
\text { fellows) }\end{array}$ & $\begin{array}{l}\text { 1. TAU (integrated): } \\
\text { "Treatment as usual" } \\
\text { 2. CBT (integrated): "Seeking } \\
\text { Safety psychotherapy" }\end{array}$ \\
\hline $\begin{array}{l}\text { Ogel, } 2011 \\
21609157\end{array}$ & 62 & $\begin{array}{l}\text { inhalant } \\
\text { cannabis } \\
\text { other drugs }\end{array}$ & SUD & $\begin{array}{l}{[13,18]} \\
15.3 \\
(1.4)\end{array}$ & $\mathrm{nr}$ & inpatient & $\begin{array}{l}\text { therapists (no } \\
\text { detail) }\end{array}$ & $\begin{array}{l}\text { 1. Educ (group): "Control" } \\
\text { 2. CBT+Educ (group): } \\
\text { "Experimental" }\end{array}$ \\
\hline
\end{tabular}




\begin{tabular}{|c|c|c|c|c|c|c|c|c|}
\hline $\begin{array}{l}\text { Author, Year } \\
\text { PMID* }\end{array}$ & $N$ & $\begin{array}{l}\text { Substances } \\
\text { Used }\end{array}$ & Severity & $\begin{array}{l}\text { Ages } \\
\text { [eligible] } \\
\text { Mean } \\
\text { (sd) }\end{array}$ & $\begin{array}{r}\text { Male } \\
\%\end{array}$ & Setting & $\begin{array}{l}\text { Intervention } \\
\text { Delivery }\end{array}$ & Arm Names \\
\hline $\begin{array}{l}\text { Rigter, } 2013 \\
23140805\end{array}$ & 450 & $\begin{array}{l}\text { cannabis } \\
\text { alcohol }\end{array}$ & SUD & $\begin{array}{l}{[13,18]} \\
16.3(\mathrm{nr})\end{array}$ & 85 & $\begin{array}{l}\text { outpatient } \\
\text { community } \\
\text { (forensic } \\
\text { centers) }\end{array}$ & $\begin{array}{l}\text { community } \\
\text { clinicians }\end{array}$ & $\begin{array}{l}\text { 1. TAU: "Individual } \\
\text { psychotherapy" } \\
\text { 2. Fam[ecological]: } \\
\text { "Multidimensional family } \\
\text { therapy" }\end{array}$ \\
\hline $\begin{array}{l}\text { Robbins, } 2008 \\
18266532\end{array}$ & 190 & $\begin{array}{l}\text { cannabis } \\
\text { cocaine } \\
\text { other drugs }\end{array}$ & SUD & $\begin{array}{l}{[12,17]} \\
15.6 \\
(1.1)\end{array}$ & 78 & $\begin{array}{l}\text { outpatient } \\
\text { community }\end{array}$ & $\begin{array}{l}\text { research staff } \\
\text { (postdoctoral } \\
\text { fellow, } \\
\text { predoctoral } \\
\text { intern, } \\
\text { Master's level } \\
\text { therapists) }\end{array}$ & $\begin{array}{l}\text { 1. TAU: "Community services } \\
\text { control" } \\
\text { 2. Fam[systems/structural]: } \\
\text { "Family process only" } \\
\text { 3. Fam[ecological]: "Fully } \\
\text { integrated ecosystemic family } \\
\text { approach" }\end{array}$ \\
\hline $\begin{array}{l}\text { Robbins, } 2011 \\
21967492\end{array}$ & 481 & $\begin{array}{l}\text { cannabis } \\
\text { alcohol } \\
\text { other drugs }\end{array}$ & PU & $\begin{array}{l}{[13,17]} \\
15.2 \\
(1.2)\end{array}$ & 78 & $\begin{array}{l}\text { outpatient } \\
\text { community }\end{array}$ & $\begin{array}{l}\text { community } \\
\text { clinicians }\end{array}$ & $\begin{array}{l}\text { 1. TAU: "Treatment as usual" } \\
\text { 2. Fam[systems/structural]: } \\
\text { "Brief strategic family therapy" }\end{array}$ \\
\hline $\begin{array}{l}\text { Rohde, } 2014 \\
24491069\end{array}$ & 170 & $\begin{array}{l}\text { cannabis } \\
\text { alcohol }\end{array}$ & SUD & $\begin{array}{l}{[13,18]} \\
16.2 \\
(1.4)\end{array}$ & 74 & $\begin{array}{l}\text { outpatient } \\
\text { research clinic }\end{array}$ & $\begin{array}{l}\text { therapists } \\
\text { (Master's level } \\
\text { therapists; no } \\
\text { detail) }\end{array}$ & $\begin{array}{l}\text { 1. CBT+Fam[functional] } \\
\text { (group, integrated): "FFT } \\
\text { followed by CWD" } \\
\text { 2. CBT+Fam[functional] } \\
\text { (group, integrated): "CWD } \\
\text { followed by FFT" } \\
\text { 3. CBT+Fam[functional] } \\
\text { (group, integrated): } \\
\text { "Combining FFT and CWD } \\
\text { (Coordinated treatment)" }\end{array}$ \\
\hline $\begin{array}{l}\text { Rowe, } 2016 \\
26879671\end{array}$ & 154 & $\begin{array}{l}\text { cannabis } \\
\text { alcohol } \\
\text { other drug }\end{array}$ & PU & $\begin{array}{l}{[13,17]} \\
15.5 \\
(1.3)\end{array}$ & 83 & $\begin{array}{l}\text { Residential } \\
\text { (juvenile } \\
\text { justice), } \\
\text { Outpatient } \\
\text { community } \\
\text { (juvenile } \\
\text { justice) }\end{array}$ & $\begin{array}{l}\text { juvenile } \\
\text { detention staff }\end{array}$ & $\begin{array}{l}\text { 1. TAU (group, integrated): } \\
\text { "Enhanced services as usual" } \\
\text { 2. Fam[ecological]: } \\
\text { "Multidimensional family } \\
\text { therapy" }\end{array}$ \\
\hline $\begin{array}{l}\text { Santisteban, } \\
2011 \\
21639636\end{array}$ & 28 & $\begin{array}{l}\text { cannabis } \\
\text { cocaine } \\
\text { other drugs }\end{array}$ & SUD & $\begin{array}{l}{[14,17]} \\
\mathrm{nr}\end{array}$ & $\mathrm{nr}$ & $\begin{array}{l}\text { outpatient } \\
\text { community }\end{array}$ & $\begin{array}{l}\text { therapists (no } \\
\text { detail) }\end{array}$ & $\begin{array}{l}1 . \\
\text { Ml+Educ+Fam[systems/structu } \\
\text { ral] (cultural): "Culturally } \\
\text { informed and flexible family- } \\
\text { based treatment for } \\
\text { adolescents (CIFFTA)" } \\
\text { 2. Fam[systems/structural]: } \\
\text { "Traditional Family Therapy" }\end{array}$ \\
\hline
\end{tabular}




\begin{tabular}{|c|c|c|c|c|c|c|c|c|}
\hline $\begin{array}{l}\text { Author, Year } \\
\text { PMID* }\end{array}$ & $\mathrm{N}$ & $\begin{array}{l}\text { Substances } \\
\text { Used }\end{array}$ & Severity & $\begin{array}{l}\text { Ages } \\
\text { [eligible] } \\
\text { Mean } \\
\text { (sd) }\end{array}$ & $\begin{array}{r}\text { Male } \\
\%\end{array}$ & Setting & $\begin{array}{l}\text { Intervention } \\
\text { Delivery }\end{array}$ & Arm Names \\
\hline $\begin{array}{l}\text { Santisteban, } \\
2015 \\
25799306\end{array}$ & 40 & $\begin{array}{l}\text { cannabis } \\
\text { alcohol } \\
\text { cocaine }\end{array}$ & SUD & $\begin{array}{l}{[14,17]} \\
\mathrm{nr}(16)\end{array}$ & 1 & $\begin{array}{l}\text { outpatient } \\
\text { community } \\
\text { (juvenile } \\
\text { justice) }\end{array}$ & $\begin{array}{l}\text { research staff } \\
\text { (Ph.D. } \\
\text { psychologists; } \\
\text { Master's level } \\
\text { therapists) }\end{array}$ & $\begin{array}{l}\text { 1. TAU: "Individual drug } \\
\text { counseling" } \\
\text { 2. } \\
\text { CBT+Fam[systems/structural] } \\
\text { (integrated): "Personality } \\
\text { Disorder-Oriented Adolescent } \\
\text { Family Therapy (I-BAFT)" }\end{array}$ \\
\hline $\begin{array}{l}\text { Schaeffer, } \\
2014 \\
23958035\end{array}$ & 97 & $\begin{array}{l}\text { cannabis } \\
\text { alcohol } \\
\text { other drugs }\end{array}$ & PU & $\begin{array}{l}{[15,18]} \\
15.8 \\
(0.9)\end{array}$ & 83 & $\begin{array}{l}\text { outpatient } \\
\text { community } \\
\text { (juvenile } \\
\text { justice) }\end{array}$ & $\begin{array}{l}\text { community } \\
\text { clinicians }\end{array}$ & $\begin{array}{l}\text { 1. TAU: "Community restitution } \\
\text { apprentice-focused training" } \\
\text { 2. TAU: "Education as usual" }\end{array}$ \\
\hline $\begin{array}{l}\text { Slesnick, } 2005 \\
15878048\end{array}$ & 124 & $\begin{array}{l}\text { cannabis } \\
\text { alcohol } \\
\text { cocaine } \\
\text { opiates }\end{array}$ & PU & $\begin{array}{l}{[12,17]} \\
14.9 \\
(1.4)\end{array}$ & 41 & $\begin{array}{l}\text { outpatient } \\
\text { community }\end{array}$ & $\begin{array}{l}\text { therapists (no } \\
\text { detail) }\end{array}$ & $\begin{array}{l}\text { 1. TAU: "Service as usual" } \\
\text { 2. Fam[ecological]: } \\
\text { "Ecologically based family } \\
\text { therapy" }\end{array}$ \\
\hline $\begin{array}{l}\text { Slesnick, } 2007 \\
16989957\end{array}$ & 180 & $\begin{array}{l}\text { cannabis } \\
\text { alcohol } \\
\text { other drugs }\end{array}$ & SUD & $\begin{array}{l}{[14,22]} \\
19.2 \\
(2.1)\end{array}$ & 66 & $\begin{array}{l}\text { outpatient } \\
\text { community } \\
\text { (drop in } \\
\text { center) }\end{array}$ & $\begin{array}{l}\text { therapists (no } \\
\text { detail) }\end{array}$ & $\begin{array}{l}\text { 1. TAU: "TAU through the drop } \\
\text { in center" } \\
\text { 2. CBT: "Community } \\
\text { reinforcement approach" }\end{array}$ \\
\hline $\begin{array}{l}\text { Slesnick, } 2009 \\
19522781\end{array}$ & 119 & $\begin{array}{l}\text { alcohol } \\
\text { cannabis } \\
\text { other drugs }\end{array}$ & PU & $\begin{array}{l}{[12,17]} \\
15.1 \\
(1.4)\end{array}$ & 45 & $\begin{array}{l}\text { outpatient } \\
\text { community }\end{array}$ & $\begin{array}{l}\text { therapists (no } \\
\text { detail) }\end{array}$ & $\begin{array}{l}\text { 1. TAU: "Service as usual" } \\
\text { 2. Fam[functional]: "Office- } \\
\text { based functional family } \\
\text { therapy" } \\
\text { 3. Fam[ecological]: "Home- } \\
\text { based ecologically based } \\
\text { family therapy" }\end{array}$ \\
\hline $\begin{array}{l}\text { Slesnick, } 2013 \\
23895088\end{array}$ & 179 & unspecified & SUD & $\begin{array}{l}{[12,17]} \\
15.4 \\
(1.2)\end{array}$ & 48 & $\begin{array}{l}\text { outpatient } \\
\text { community } \\
\text { (shelter) }\end{array}$ & $\begin{array}{l}\text { community } \\
\text { clinicians, } \\
\text { research staff } \\
\text { (graduate } \\
\text { students) }\end{array}$ & $\begin{array}{l}\text { 1. MI: "Motivational } \\
\text { Interviewing" } \\
\text { 2. Fam[ecological]: } \\
\text { "Ecologically-Based Family } \\
\text { Therapy" } \\
\text { 3. CBT: "Community } \\
\text { Reinforcement Approach" }\end{array}$ \\
\hline $\begin{array}{l}\text { Slesnick, } 2015 \\
25736623\end{array}$ & 270 & $\begin{array}{l}\text { cannabis } \\
\text { alcohol } \\
\text { other drugs }\end{array}$ & SUD & $\begin{array}{l}{[14,20]} \\
18.7 \\
(1.3)\end{array}$ & 53 & $\begin{array}{l}\text { outpatient } \\
\text { community } \\
\text { (shelter) }\end{array}$ & $\begin{array}{l}\text { therapists (no } \\
\text { detail) }\end{array}$ & $\begin{array}{l}\text { 1. MI: "Motivational } \\
\text { enhancement therapy" } \\
\text { 2. ICM: "Case management" } \\
\text { 3. CBT: "Community } \\
\text { reinforcement approach" }\end{array}$ \\
\hline $\begin{array}{l}\text { Smith, } 2006 \\
17182429\end{array}$ & 98 & $\begin{array}{l}\text { cannabis } \\
\text { alcohol } \\
\text { other drugs }\end{array}$ & PU & $\begin{array}{l}{[12,18]} \\
15.8(\mathrm{nr})\end{array}$ & 71 & $\begin{array}{l}\text { outpatient } \\
\text { community }\end{array}$ & $\begin{array}{l}\text { therapists (no } \\
\text { detail) }\end{array}$ & $\begin{array}{l}\text { 1. Fam[behavioral] (group): } \\
\text { "Strengths oriented family } \\
\text { therapy" } \\
\text { 2. CBT+Ml+PeerGroup } \\
\text { (group): "The Seven } \\
\text { Challenges" }\end{array}$ \\
\hline
\end{tabular}




\begin{tabular}{|c|c|c|c|c|c|c|c|c|}
\hline $\begin{array}{l}\text { Author, Year } \\
\text { PMID* }\end{array}$ & $\mathrm{N}$ & $\begin{array}{l}\text { Substances } \\
\text { Used }\end{array}$ & Severity & $\begin{array}{l}\text { Ages } \\
\text { [eligible] } \\
\text { Mean } \\
\text { (sd) }\end{array}$ & $\begin{array}{r}\text { Male } \\
\%\end{array}$ & Setting & $\begin{array}{l}\text { Intervention } \\
\text { Delivery }\end{array}$ & Arm Names \\
\hline $\begin{array}{l}\text { Stanger, } 2009 \\
19717250\end{array}$ & 69 & $\begin{array}{l}\text { cannabis } \\
\text { alcohol }\end{array}$ & PU & $\begin{array}{l}{[12,18]} \\
15.9(1)\end{array}$ & 83 & $\begin{array}{l}\text { outpatient } \\
\text { research clinic }\end{array}$ & $\begin{array}{l}\text { research staff } \\
\text { (postdoctoral } \\
\text { fellows, } \\
\text { Master's level } \\
\text { therapists) }\end{array}$ & $\begin{array}{l}\text { 1. } \\
\text { CBT+MI+Fam[behavioral]+CM: } \\
\text { "MET/CBT + abstinence CM + } \\
\text { family management" } \\
\text { 2. CBT+MI+Educ+CM (parent): } \\
\text { "MET/CBT + attendance CM + } \\
\text { parent psychoeducation" }\end{array}$ \\
\hline $\begin{array}{l}\text { Stanger, } 2015 \\
26004659\end{array}$ & 153 & $\begin{array}{l}\text { cannabis } \\
\text { alcohol }\end{array}$ & SUD & $\begin{array}{l}{[12,18]} \\
15.8 \\
(1.4)\end{array}$ & 89 & $\begin{array}{l}\text { outpatient } \\
\text { community }\end{array}$ & $\begin{array}{l}\text { research staff } \\
\text { (Master's level } \\
\text { therapists) }\end{array}$ & $\begin{array}{l}\text { 1. CBT+MI+CM (parent): } \\
\text { "MET/CBT+CM+Parent } \\
\text { Training" } \\
\text { 2. CBT+MI+CM: } \\
\text { "MET/CBT+abstinence-based } \\
\text { contingency management } \\
\text { (CM)" } \\
\text { 3. CBT+MI: "Motivational } \\
\text { enhancement } \\
\text { therapy/cognitive-behavioral } \\
\text { therapy (MET/CBT)" }\end{array}$ \\
\hline $\begin{array}{l}\text { Stanger, } 2017 \\
28414474\end{array}$ & 75 & $\begin{array}{l}\text { cannabis } \\
\text { alcohol }\end{array}$ & PU & $\begin{array}{l}{[12,18]} \\
16.2 \\
(1.2)\end{array}$ & 75 & $\begin{array}{l}\text { outpatient } \\
\text { research clinic }\end{array}$ & $\begin{array}{l}\text { research staff } \\
\text { (postdoctoral } \\
\text { fellows) }\end{array}$ & $\begin{array}{l}\text { 1. } \\
\text { CBT+MI+Fam[behavioral]+CM } \\
\text { +ICM: "Experimental" } \\
\text { 2. CBT+MI+CM+ICM: } \\
\text { "Contriol" }\end{array}$ \\
\hline $\begin{array}{l}\text { Thush, } 2007 \\
16928395\end{array}$ & 107 & alcohol & PU & $\begin{array}{l}{[14,18]} \\
15.5(1)\end{array}$ & 57 & $\begin{array}{l}\text { outpatient } \\
\text { research clinic }\end{array}$ & $\begin{array}{l}\text { research staff } \\
\text { (no detail) }\end{array}$ & $\begin{array}{l}\text { 1. TAU: "Information-only } \\
\text { control" } \\
\text { 2. CBT+MI (group): "Learning } \\
\text { to drink" }\end{array}$ \\
\hline $\begin{array}{l}\text { Tolou-Shams, } \\
2017 \\
\text { CN-01365355 } \\
\text { (cochrane) }\end{array}$ & 60 & $\begin{array}{l}\text { cannabis } \\
\text { alcohol } \\
\text { other drugs }\end{array}$ & PU & $\begin{array}{l}{[12,18]} \\
15.6 \\
(1.3)\end{array}$ & 70 & $\begin{array}{l}\text { outpatient } \\
\text { community } \\
\text { (juvenile } \\
\text { justice } \\
\text { family court) }\end{array}$ & $\begin{array}{l}\text { therapists (no } \\
\text { detail) }\end{array}$ & $\begin{array}{l}\text { 1. Educ: "Adolescent-only } \\
\text { health promotion intervention" } \\
\text { 2. CBT+Fam[behavioral]: } \\
\text { "Family-based affect } \\
\text { management" }\end{array}$ \\
\hline $\begin{array}{l}\text { Trudeau, 2017 } \\
2017-00657- \\
001 \\
\text { (psychinfo) }\end{array}$ & 160 & $\begin{array}{l}\text { alcohol and other } \\
\text { drugs }\end{array}$ & PU & $\begin{array}{l}{[13,21]} \\
17.6 \\
(2.1)\end{array}$ & 43 & $\begin{array}{l}\text { outpatient } \\
\text { onlline }\end{array}$ & $\begin{array}{l}\text { community } \\
\text { clinicians }\end{array}$ & $\begin{array}{l}\text { 1. TAU: "Attention control" } \\
\text { 2. CBT: "Navigating my } \\
\text { Journey" }\end{array}$ \\
\hline $\begin{array}{l}\text { Wagner, } 2014 \\
24841864\end{array}$ & 514 & $\begin{array}{l}\text { cannabis } \\
\text { alcohol } \\
\text { other drugs }\end{array}$ & PU & $\begin{array}{l}{[14,18]} \\
16.2 \\
(1.2)\end{array}$ & 59 & $\begin{array}{l}\text { outpatient } \\
\text { school }\end{array}$ & $\begin{array}{l}\text { therapists (no } \\
\text { detail) }\end{array}$ & $\begin{array}{l}\text { 1. TAU: "Standard care" } \\
\text { 2. CBT+MI: "Guided self- } \\
\text { change" }\end{array}$ \\
\hline $\begin{array}{l}\text { Waldron, } 2001 \\
11680557\end{array}$ & 120 & $\begin{array}{l}\text { cannabis } \\
\text { other drugs }\end{array}$ & SUD & $\begin{array}{l}{[13,17]} \\
15.4(1)\end{array}$ & 80 & $\begin{array}{l}\text { outpatient } \\
\text { research clinic }\end{array}$ & $\begin{array}{l}\text { research staff } \\
\text { (PhD } \\
\text { psychologists, } \\
\text { graduate } \\
\text { students) }\end{array}$ & $\begin{array}{l}\text { 1. Fam[functional]: "FFT" } \\
\text { 2. Educ (group): "Group" } \\
\text { 3. CBT+Ml+Fam[functional]: } \\
\text { "Joint" } \\
\text { 4. CBT+MI: "CBT" }\end{array}$ \\
\hline
\end{tabular}




\begin{tabular}{|c|c|c|c|c|c|c|c|c|}
\hline $\begin{array}{l}\text { Author, Year } \\
\text { PMID* }\end{array}$ & $N$ & $\begin{array}{l}\text { Substances } \\
\text { Used }\end{array}$ & Severity & $\begin{array}{l}\text { Ages } \\
\text { [eligible] } \\
\text { Mean } \\
\text { (sd) }\end{array}$ & $\begin{array}{r}\text { Male } \\
\%\end{array}$ & Setting & $\begin{array}{l}\text { Intervention } \\
\text { Delivery }\end{array}$ & Arm Names \\
\hline $\begin{array}{l}\text { Zhang, } 2018 \\
30556713\end{array}$ & 270 & $\begin{array}{l}\text { alcohol or other } \\
\text { drugs }\end{array}$ & SUD & $\begin{array}{l}{[14,20]} \\
18.7 \\
(1.3)\end{array}$ & 53 & $\begin{array}{l}\text { Outpatient } \\
\text { (drop-in center } \\
\text { for homeless } \\
\text { youth) }\end{array}$ & $\begin{array}{l}\text { Master's level } \\
\text { (counelors, } \\
\text { marriage and } \\
\text { family } \\
\text { therapists or } \\
\text { social } \\
\text { workers) }\end{array}$ & $\begin{array}{l}\text { 1. MI: "Motivational } \\
\text { enhancement therapy" } \\
\text { 2. ICM: "Case management" } \\
\text { 3. CBT: "Community } \\
\text { reinforcement approach" }\end{array}$ \\
\hline
\end{tabular}

PMID* = Pubmed identifier if available, otherwise (database name). Arm names = Intervention codes, (intervention modifiers) and [family subclassification]: "study arm name". Abbreviations: N=number randomized; SD = standard deviation; ED = emergency department; $\mathrm{nr}=$ not reported; $\mathrm{CBT}=$ cognitive behavioral therapy; $\mathrm{CM}=$ contingency management; Edu = education; Fam = family therapy; ICM = intensive case management; $\mathrm{MI}=$ motivational interviewing; TAU = treatment as usual; parent $=$ at least one component of the intervention included parent involvement/was targeted towards parents; Fam = Family therapy; ICM= intensive case management; group = at least one component of the intervention was delivered in a group setting; parent $=$ at least one component of the intervention included parent involvement/was targeted towards parents; integrated = intervention as a whole was designed to treat substance use disorder/problematic use and at least one other diagnoses (e.g., mental health); cultural = intervention designed to meet the unique characteristics of the population in which it was delivered. 


\begin{tabular}{|c|c|c|c|c|c|c|c|c|c|c|c|c|c|c|}
\hline $\begin{array}{l}\text { Author, Year } \\
\text { PMID }\end{array}$ & Country & Site(s) & Funding Source & Design & $\begin{array}{r}\# \\
\text { Arms }\end{array}$ & $\begin{array}{l}\text { Percent } \\
\text { WhitelBlacklOther(details) }\end{array}$ & $\frac{\frac{9}{0}}{\frac{0}{0}}$ & 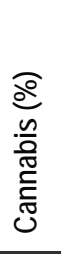 & $\begin{array}{l}\frac{\overline{9}}{0} \\
\frac{0}{0} \\
\frac{0}{0} \\
0\end{array}$ & $\frac{\sqrt{9}}{00}$ & 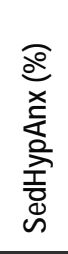 & 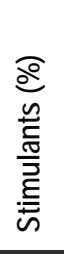 & 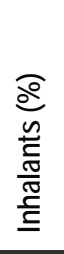 & 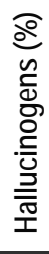 \\
\hline $\begin{array}{l}\text { Amini, } 1982 \\
\text { CN-00182281 } \\
\text { (cochrane) }\end{array}$ & United States & single center & $\mathrm{nr}$ & RCT & 2 & $52 / 16 / n r$ & $\mathrm{nr}$ & $\mathrm{nr}$ & $\mathrm{nr}$ & $\mathrm{nr}$ & $\mathrm{nr}$ & $\mathrm{nr}$ & $\mathrm{nr}$ & $\mathrm{nr}$ \\
\hline $\begin{array}{l}\text { Arnaud, } 2015 \\
2016-03749-004 \\
\text { (psychinfo) }\end{array}$ & $\begin{array}{l}\text { Sweden, } \\
\text { Belgium, Czech } \\
\text { Republic, } \\
\text { Germany }\end{array}$ & multi-center & $\begin{array}{l}\text { Drug Prevention and } \\
\text { Information Programme of } \\
\text { the European Union }\end{array}$ & RCT & 2 & $\mathrm{nr} / \mathrm{nr} / \mathrm{nr}$ & $\mathrm{nr}$ & $\mathrm{nr}$ & $\mathrm{nr}$ & $\mathrm{nr}$ & $\mathrm{nr}$ & $\mathrm{nr}$ & $\mathrm{nr}$ & $\mathrm{nr}$ \\
\hline $\begin{array}{l}\text { Arnaud, } 2017 \\
27801991\end{array}$ & Germany & multi-center & $\begin{array}{l}\text { German Federal Ministry } \\
\text { for Education and } \\
\text { Research }\end{array}$ & $\begin{array}{l}\text { cluster } \\
\text { RCT }\end{array}$ & 2 & $\mathrm{nr} / \mathrm{nr} / \mathrm{nr}$ & $\mathrm{nr}$ & $\mathrm{nr}$ & $\mathrm{nr}$ & $\mathrm{nr}$ & $\mathrm{nr}$ & $\mathrm{nr}$ & $\mathrm{nr}$ & $\mathrm{nr}$ \\
\hline $\begin{array}{l}\text { Azrin, } 1994 \\
\text { CN-00241903 } \\
\text { (cochrane) }\end{array}$ & United States & single center & NIDA & $\mathrm{RCT}$ & 2 & nr//nr/19 (Minority) & $\mathrm{nr}$ & 96 & $\mathrm{nr}$ & 4 & $\mathrm{nr}$ & 35 & $\mathrm{nr}$ & 8 \\
\hline $\begin{array}{l}\text { Azrin, 2001 } \\
\text { 2002-13926-001 } \\
\text { (psychinfo) }\end{array}$ & United States & single center & $\mathrm{NIMH}$ & RCT & 2 & $79 / 2 / \mathrm{nr}$ & $\mathrm{nr}$ & $\mathrm{nr}$ & $\mathrm{nr}$ & $\mathrm{nr}$ & $\mathrm{nr}$ & $\mathrm{nr}$ & $\mathrm{nr}$ & $\mathrm{nr}$ \\
\hline $\begin{array}{l}\text { Baer, } 2007 \\
18072842 \\
\end{array}$ & United States & single center & NIDA & RCT & 2 & 58/8/19 (Multiracial) & $\mathrm{nr}$ & $\mathrm{nr}$ & $\mathrm{nr}$ & $\mathrm{nr}$ & $\mathrm{nr}$ & $\mathrm{nr}$ & $\mathrm{nr}$ & $\mathrm{nr}$ \\
\hline $\begin{array}{l}\text { Bernstein, } 2009 \\
20053238\end{array}$ & United States & single center & $\mathrm{NIH}, \mathrm{NIDA}$ & RCT & 3 & 4/84/2 (Other) & $\mathrm{nr}$ & 62 & $\mathrm{nr}$ & $\mathrm{nr}$ & $\mathrm{nr}$ & $\mathrm{nr}$ & $\mathrm{nr}$ & $\mathrm{nr}$ \\
\hline $\begin{array}{l}\text { Bernstein, } 2010 \\
20670329\end{array}$ & United States & single center & NIAA & RCT & 3 & 26/51/2 (Asian) & $\mathrm{nr}$ & $\mathrm{nr}$ & $\mathrm{nr}$ & $\mathrm{nr}$ & $\mathrm{nr}$ & $\mathrm{nr}$ & $\mathrm{nr}$ & $\mathrm{nr}$ \\
\hline $\begin{array}{l}\text { Braciszewski, } \\
2018 \\
132804409 \\
\text { (embase) }\end{array}$ & United States & single center & NIDA & $\mathrm{RCT}$ & 2 & 52/27/21 (Mixed/other) & 4 & 94 & $\mathrm{nr}$ & $\mathrm{nr}$ & $\mathrm{nr}$ & $\mathrm{nr}$ & $\mathrm{nr}$ & $\mathrm{nr}$ \\
\hline $\begin{array}{l}\text { Brown, } 2015 \\
26362000\end{array}$ & United States & multicenter & NIDA & RCT & 2 & $\begin{array}{l}88 / 6 / 5 \text { (Other races or more than } \\
\text { one race) }\end{array}$ & 73 & 95 & 100 & 18 & 14 & 19 & 6 & 8 \\
\hline
\end{tabular}




\begin{tabular}{|c|c|c|c|c|c|c|c|c|c|c|c|c|c|c|}
\hline $\begin{array}{l}\text { Author, Year } \\
\text { PMID }\end{array}$ & Country & Site(s) & Funding Source & Design & $\begin{array}{r}\# \\
\text { Arms }\end{array}$ & $\begin{array}{l}\text { Percent } \\
\text { WhitelBlacklOther(details) }\end{array}$ & $\begin{array}{l}\bar{\Xi} \\
\frac{0}{0} \\
\frac{0}{0} \\
\frac{0}{\pi}\end{array}$ & 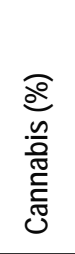 & $\frac{\bar{a}}{\frac{0}{0}}$ & $\begin{array}{l}\text { @ } \\
\frac{0}{20} \\
\frac{1}{2}\end{array}$ & 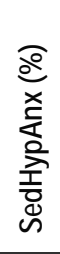 & 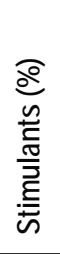 & 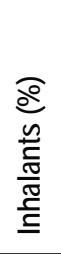 & 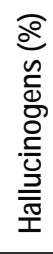 \\
\hline $\begin{array}{l}\text { Burrow-Sanchez, } \\
2012 \\
22866693\end{array}$ & United States & single center & NIDA & $\mathrm{RCT}$ & 2 & $\mathrm{nr} / \mathrm{nr} / \mathrm{nr}$ & $\mathrm{nr}$ & $\mathrm{nr}$ & 100 & $\mathrm{nr}$ & $\mathrm{nr}$ & $\mathrm{nr}$ & $\mathrm{nr}$ & $\mathrm{nr}$ \\
\hline $\begin{array}{l}\text { Burrow-Sanchez, } \\
2015 \\
25602465\end{array}$ & United States & single center & NIDA & RCT & 2 & $n r / n r / n r$ & 6 & 67 & 100 & $\mathrm{nr}$ & $\mathrm{nr}$ & $\mathrm{nr}$ & $\mathrm{nr}$ & $\mathrm{nr}$ \\
\hline $\begin{array}{l}\text { Colby, } 2018 \\
29750362\end{array}$ & United States & single center & NIAA & $\mathrm{RCT}$ & 2 & 58/0/1 (Native American) & $\mathrm{nr}$ & $\mathrm{nr}$ & $\mathrm{nr}$ & $\mathrm{nr}$ & $\mathrm{nr}$ & $\mathrm{nr}$ & $\mathrm{nr}$ & $\mathrm{nr}$ \\
\hline $\begin{array}{l}\text { Cornelius, } 2009 \\
19321268\end{array}$ & United States & single center & NIDA, NIAAA & RCT & 2 & 86/8/6 (Mixed race) & 100 & $\mathrm{nr}$ & $\mathrm{nr}$ & 0 & 0 & 0 & 0 & 0 \\
\hline $\begin{array}{l}\text { Cornelius, } 2010 \\
20576364\end{array}$ & United States & single center & NIDA, NIAAA, VA & RCT & 2 & $57 / n r / n r$ & $\mathrm{nr}$ & 100 & $\mathrm{nr}$ & 0 & 0 & 0 & 0 & 0 \\
\hline $\begin{array}{l}\text { Cunningham, } \\
2015 \\
26347440\end{array}$ & United States & single center & $\mathrm{NIH}$ & RCT & 3 & 79/10/11 (Other) & 100 & $\mathrm{nr}$ & $\mathrm{nr}$ & $\mathrm{nr}$ & $\mathrm{nr}$ & $\mathrm{nr}$ & $\mathrm{nr}$ & $\mathrm{nr}$ \\
\hline $\begin{array}{l}\text { D'Amico, } 2013 \\
\text { CN-00917707 } \\
\text { (cochrane) }\end{array}$ & United States & single center & NIDA & RCT & 2 & 45/nr/10 (Mixed and other) & $\mathrm{nr}$ & $\mathrm{nr}$ & $\mathrm{nr}$ & $\mathrm{nr}$ & $\mathrm{nr}$ & $\mathrm{nr}$ & $\mathrm{nr}$ & $\mathrm{nr}$ \\
\hline $\begin{array}{l}\text { D'Amico, } 2008 \\
18037603\end{array}$ & United States & single center & NIDA & RCT & 2 & $5 / 10 / n r$ & $\mathrm{nr}$ & $\mathrm{nr}$ & $\mathrm{nr}$ & $\mathrm{nr}$ & $\mathrm{nr}$ & $\mathrm{nr}$ & $\mathrm{nr}$ & $\mathrm{nr}$ \\
\hline $\begin{array}{l}\text { Dakof, } 2015 \\
25621927\end{array}$ & United States & single center & NIDA & RCT & 2 & $\mathrm{nr} / 33 / 5$ (Other) & 26 & 91 & $\mathrm{nr}$ & $\mathrm{nr}$ & $\mathrm{nr}$ & $\mathrm{nr}$ & $\mathrm{nr}$ & $\mathrm{nr}$ \\
\hline $\begin{array}{l}\text { D'Amico, } 2018 \\
30138016\end{array}$ & United States & multi-center & NIAAA & RCT & 2 & 11/13/8 (Other/multiracial) & 94 & 82 & $\mathrm{nr}$ & $\mathrm{nr}$ & $\mathrm{nr}$ & $\mathrm{nr}$ & $\mathrm{nr}$ & $\mathrm{nr}$ \\
\hline $\begin{array}{l}\text { de Gee, } 2014 \\
24969735\end{array}$ & Netherland & single center & ZonMW & $\mathrm{RCT}$ & 2 & $\mathrm{nr} / \mathrm{nr} / \mathrm{nr}$ & $\mathrm{nr}$ & $\mathrm{nr}$ & $\mathrm{nr}$ & $\mathrm{nr}$ & $\mathrm{nr}$ & $\mathrm{nr}$ & $\mathrm{nr}$ & $\mathrm{nr}$ \\
\hline $\begin{array}{l}\text { De Sousa, } 2008 \\
\text { CN-00753784 } \\
\text { (cochrane) }\end{array}$ & India & single center & unclear & $\mathrm{RCT}$ & 2 & $\mathrm{nr} / \mathrm{nr} / \mathrm{nr}$ & 100 & $\mathrm{nr}$ & $\mathrm{nr}$ & $\mathrm{nr}$ & $\mathrm{nr}$ & $\mathrm{nr}$ & $\mathrm{nr}$ & $\mathrm{nr}$ \\
\hline
\end{tabular}




\begin{tabular}{|c|c|c|c|c|c|c|c|c|c|c|c|c|c|c|}
\hline $\begin{array}{l}\text { Author, Year } \\
\text { PMID }\end{array}$ & Country & Site(s) & Funding Source & Design & $\begin{array}{r}\# \\
\text { Arms }\end{array}$ & $\begin{array}{l}\text { Percent } \\
\text { WhitelBlacklOther(details) }\end{array}$ & $\begin{array}{l}\overline{\mathrm{a}} \\
\overline{0} \\
\frac{0}{0} \\
\frac{0}{4}\end{array}$ & 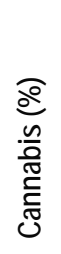 & 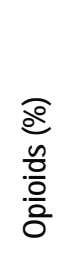 & $\begin{array}{l}\text { a } \\
\text { no } \\
\text { 20 }\end{array}$ & 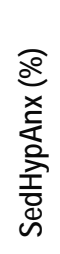 & 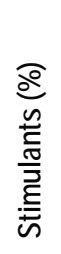 & 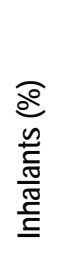 & 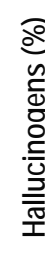 \\
\hline $\begin{array}{l}\text { Dembo, } 2014 \\
\text { 2014-42452-005 } \\
\text { (psychinfo) }\end{array}$ & United States & single center & NIAA & RCT & 3 & 39/23/8 (Other) & $\mathrm{nr}$ & $\mathrm{nr}$ & $\mathrm{nr}$ & $\mathrm{nr}$ & $\mathrm{nr}$ & $\mathrm{nr}$ & $\mathrm{nr}$ & $\mathrm{nr}$ \\
\hline $\begin{array}{l}\text { Dennis, } 2004 \\
15501373\end{array}$ & United States & multicenter & SAMHSA & RCT & 3 & 70/15/9 (Other/mixed) & 11 & 76 & 1 & $\mathrm{nr}$ & $\mathrm{nr}$ & $\mathrm{nr}$ & $\mathrm{nr}$ & $\mathrm{nr}$ \\
\hline $\begin{array}{l}\text { Dennis, } 2004 \\
15501373 b\end{array}$ & United States & multicenter & SAMHSA & RCT & 3 & 47/47/5 (Other/mixed) & 18 & 74 & 1 & $\mathrm{nr}$ & $\mathrm{nr}$ & $\mathrm{nr}$ & $\mathrm{nr}$ & $\mathrm{nr}$ \\
\hline $\begin{array}{l}\text { Esposito- } \\
\text { Smythers, } 2011 \\
22004303\end{array}$ & United States & single center & NIAAA & RCT & 2 & $89 / 3 / n r$ & 64 & 83 & $\mathrm{nr}$ & $\mathrm{nr}$ & $\mathrm{nr}$ & $\mathrm{nr}$ & $\mathrm{nr}$ & $\mathrm{nr}$ \\
\hline $\begin{array}{l}\text { Figurelli, } 1994 \\
7862806\end{array}$ & United States & single center & $\mathrm{nr}$ & $\mathrm{RCT}$ & 2 & $81 / 11 / n r$ & $\mathrm{nr}$ & $\mathrm{nr}$ & 100 & $\mathrm{nr}$ & $\mathrm{nr}$ & $\mathrm{nr}$ & $\mathrm{nr}$ & $\mathrm{nr}$ \\
\hline $\begin{array}{l}\text { Findling, } 2009 \\
19298659\end{array}$ & United States & single center & $\begin{array}{l}\text { American Foundation for } \\
\text { Suicide Prevention, the St. } \\
\text { Luke's Foundation of } \\
\text { Cleveland, Ohio, and by a } \\
\text { clinical research grant } \\
\text { from Lilly. }\end{array}$ & RCT & 2 & 73/18/9 (Other) & 37 & 88 & $\mathrm{nr}$ & $\mathrm{nr}$ & $\mathrm{nr}$ & $\mathrm{nr}$ & $\mathrm{nr}$ & $\mathrm{nr}$ \\
\hline $\begin{array}{l}\text { Friedman, } 1989 \\
\text { CN-00496580 } \\
\text { (cochrane) }\end{array}$ & United States & single center & NIDA & RCT & 2 & 90/nr/10 (Not white) & 88 & 87 & $\mathrm{nr}$ & 28 & 14 & $\mathrm{nr}$ & 7 & 22 \\
\hline $\begin{array}{l}\text { Geller, } 1998 \\
9473913\end{array}$ & United States & single center & NIDA & $\mathrm{RCT}$ & 2 & $\mathrm{nr} / \mathrm{nr} / \mathrm{nr}$ & 7 & 2 & 100 & $\mathrm{nr}$ & $\mathrm{nr}$ & $\mathrm{nr}$ & 1 & $\mathrm{nr}$ \\
\hline $\begin{array}{l}\text { Giles, } 2019 \\
\text { CN-01953820 } \\
\text { (cochrane) }\end{array}$ & $\begin{array}{l}\text { United } \\
\text { Kingdom }\end{array}$ & multi-center & NIHR & RCT & 2 & 91/2/6 (Asian/other) & 100 & $\mathrm{nr}$ & $\mathrm{nr}$ & $\mathrm{nr}$ & $\mathrm{nr}$ & $\mathrm{nr}$ & $\mathrm{nr}$ & $\mathrm{nr}$ \\
\hline $\begin{array}{l}\text { Godley, } 2002 \\
12127465\end{array}$ & United States & single center & NIAA & RCT & 2 & 75/18/6 (Other) & 59 & 90 & $\mathrm{nr}$ & $\mathrm{nr}$ & $\mathrm{nr}$ & 22 & $\mathrm{nr}$ & $\mathrm{nr}$ \\
\hline $\begin{array}{l}\text { Godley, } 2010 \\
20219293\end{array}$ & United States & single center & NIDA & RCT & 4 & $75 / 13 / \mathrm{nr}$ & 49 & 76 & 100 & $\mathrm{nr}$ & $\mathrm{nr}$ & $\mathrm{nr}$ & $\mathrm{nr}$ & $\mathrm{nr}$ \\
\hline
\end{tabular}




\begin{tabular}{|c|c|c|c|c|c|c|c|c|c|c|c|c|c|c|}
\hline $\begin{array}{l}\text { Author, Year } \\
\text { PMID }\end{array}$ & Country & Site(s) & Funding Source & Design & $\begin{array}{r}\# \\
\text { Arms } \\
\end{array}$ & $\begin{array}{l}\text { Percent } \\
\text { WhitelBlacklOther(details) }\end{array}$ & $\begin{array}{l}\frac{\bar{c}}{\overline{0}} \\
\frac{0}{\bar{c}}\end{array}$ & 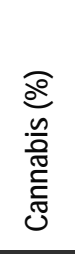 & $\begin{array}{l}\bar{a} \\
\frac{0}{0} \\
\frac{0}{0} \\
\overline{0}\end{array}$ & $\begin{array}{l}\frac{\bar{a}}{2} \\
\frac{0}{2}\end{array}$ & 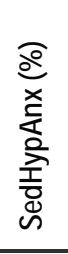 & 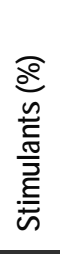 & 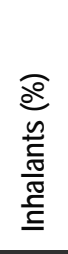 & 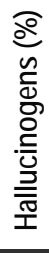 \\
\hline $\begin{array}{l}\text { Godley, } 2019 \\
\text { CN-01745749 } \\
\text { (cochrane) }\end{array}$ & United States & $\mathrm{nr}$ & NIAAA & $\mathrm{RCT}$ & 2 & 49/18/19 (Other/mixed) & $\mathrm{nr}$ & $\mathrm{nr}$ & 95 & $\mathrm{nr}$ & $\mathrm{nr}$ & $\mathrm{nr}$ & $\mathrm{nr}$ & $\mathrm{nr}$ \\
\hline $\begin{array}{l}\text { Gonzalez, } 2015 \\
26454835\end{array}$ & United States & single center & NIDA & $\mathrm{RCT}$ & 3 & $73 / n r / n r$ & 0 & 51 & $\mathrm{nr}$ & 100 & $\mathrm{nr}$ & $\mathrm{nr}$ & $\mathrm{nr}$ & $\mathrm{nr}$ \\
\hline $\begin{array}{l}\text { Gray, } 2012 \\
22706327 \\
\end{array}$ & United States & single center & NIDA & RCT & 2 & $83 / n r / n r$ & $\mathrm{nr}$ & 100 & $\mathrm{nr}$ & $\mathrm{nr}$ & $\mathrm{nr}$ & $\mathrm{nr}$ & $\mathrm{nr}$ & $\mathrm{nr}$ \\
\hline $\begin{array}{l}\text { Henderson, } 2016 \\
26992083\end{array}$ & United States & single center & CSA/NIDA & RCT & 2 & 79/6/1 (Asian) & $\mathrm{nr}$ & $\mathrm{nr}$ & 100 & $\mathrm{nr}$ & $\mathrm{nr}$ & $\mathrm{nr}$ & $\mathrm{nr}$ & $\mathrm{nr}$ \\
\hline $\begin{array}{l}\text { Henggeler, } 1996 \\
8610836\end{array}$ & United States & single center & NIDA & RCT & 2 & $\mathrm{nr} / \mathrm{nr} / \mathrm{nr}$ & $\mathrm{nr}$ & $\mathrm{nr}$ & 100 & $\mathrm{nr}$ & $\mathrm{nr}$ & $\mathrm{nr}$ & $\mathrm{nr}$ & $\mathrm{nr}$ \\
\hline $\begin{array}{l}\text { Henggeler, } 2006 \\
16551142\end{array}$ & United States & single center & NIAA & RCT & 4 & 31/67/2 (Biracial) & $\mathrm{nr}$ & $\mathrm{nr}$ & 100 & $\mathrm{nr}$ & $\mathrm{nr}$ & $\mathrm{nr}$ & $\mathrm{nr}$ & $\mathrm{nr}$ \\
\hline $\begin{array}{l}\text { Henggeler, } 2012 \\
22309470\end{array}$ & United States & multicenter & NIDA & $\mathrm{RCT}$ & 2 & 57/40/3 (Biracial) & $\mathrm{nr}$ & $\mathrm{nr}$ & $\mathrm{nr}$ & $\mathrm{nr}$ & $\mathrm{nr}$ & $\mathrm{nr}$ & $\mathrm{nr}$ & $\mathrm{nr}$ \\
\hline $\begin{array}{l}\text { Hogue, } 2015 \\
25496283\end{array}$ & United States & single center & NIDA & RCT & 2 & nr/21/6 (Other/mixed) & $\mathrm{nr}$ & $\mathrm{nr}$ & $\mathrm{nr}$ & $\mathrm{nr}$ & $\mathrm{nr}$ & $\mathrm{nr}$ & $\mathrm{nr}$ & $\mathrm{nr}$ \\
\hline $\begin{array}{l}\text { Delbelo, } 2017 \\
\text { NCT00393978 } \\
\text { (ctg) }\end{array}$ & United States & single center & NIDA & RCT & 2 & $\mathrm{nr} / \mathrm{nr} / \mathrm{nr}$ & $\mathrm{nr}$ & 100 & $\mathrm{nr}$ & $\mathrm{nr}$ & $\mathrm{nr}$ & $\mathrm{nr}$ & $\mathrm{nr}$ & $\mathrm{nr}$ \\
\hline $\begin{array}{l}\text { Delbelo, } 2017 \\
\text { NCT00550394 } \\
\text { (ctg) }\end{array}$ & United States & single center & NIAAA & RCT & 2 & $\mathrm{nr} / \mathrm{nr} / \mathrm{nr}$ & $\mathrm{nr}$ & $\mathrm{nr}$ & $\mathrm{nr}$ & $\mathrm{nr}$ & $\mathrm{nr}$ & $\mathrm{nr}$ & $\mathrm{nr}$ & $\mathrm{nr}$ \\
\hline $\begin{array}{l}\text { HJoanning, } 1992 \\
\text { CN-00631575 } \\
\text { (cochrane) }\end{array}$ & United States & single center & NIDA & $\mathrm{RCT}$ & 3 & $68 / 2 / \mathrm{nr}$ & $\mathrm{nr}$ & $\mathrm{nr}$ & $\mathrm{nr}$ & $\mathrm{nr}$ & $\mathrm{nr}$ & $\mathrm{nr}$ & $\mathrm{nr}$ & $\mathrm{nr}$ \\
\hline $\begin{array}{l}\text { Kaminer, } 1998 \\
9824170\end{array}$ & United States & single center & $\mathrm{nr}$ & $\mathrm{RCT}$ & 2 & $\mathrm{nr} / \mathrm{nr} / \mathrm{nr}$ & $\mathrm{nr}$ & $\mathrm{nr}$ & 100 & $\mathrm{nr}$ & $\mathrm{nr}$ & $\mathrm{nr}$ & $\mathrm{nr}$ & $\mathrm{nr}$ \\
\hline $\begin{array}{l}\text { Kaminer, } 2002 \\
12436013\end{array}$ & United States & single center & NIDA & RCT & 2 & 90/nr/10 (Non white) & $\mathrm{nr}$ & $\mathrm{nr}$ & 100 & $\mathrm{nr}$ & $\mathrm{nr}$ & $\mathrm{nr}$ & $\mathrm{nr}$ & $\mathrm{nr}$ \\
\hline
\end{tabular}




\begin{tabular}{|c|c|c|c|c|c|c|c|c|c|c|c|c|c|c|}
\hline $\begin{array}{l}\text { Author, Year } \\
\text { PMID }\end{array}$ & Country & Site(s) & Funding Source & Design & $\begin{array}{r}\# \\
\text { Arms }\end{array}$ & $\begin{array}{l}\text { Percent } \\
\text { WhitelBlacklOther(details) }\end{array}$ & 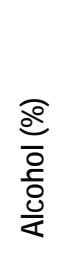 & 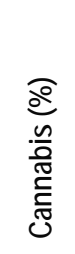 & $\begin{array}{l}\frac{0}{0} \\
\frac{0}{0} \\
\frac{.0}{0} \\
\frac{0}{0}\end{array}$ & $\begin{array}{l}\bar{\Xi} \\
\text { o } \\
\text { 20 }\end{array}$ & 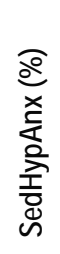 & 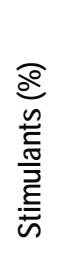 & 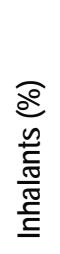 & 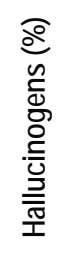 \\
\hline $\begin{array}{l}\text { Kaminer, } 2008 \\
18978635\end{array}$ & United States & single center & NIAA & RCT & 3 & 82/4/4 (Biracial//other) & $\mathrm{nr}$ & 100 & $\mathrm{nr}$ & $\mathrm{nr}$ & $\mathrm{nr}$ & $\mathrm{nr}$ & $\mathrm{nr}$ & $\mathrm{nr}$ \\
\hline $\begin{array}{l}\text { Kelly, } 2017 \\
28742932\end{array}$ & United States & single center & NIAAA & RCT & 2 & 68/11/14 (Mixed) & $\mathrm{nr}$ & $\mathrm{nr}$ & 100 & $\mathrm{nr}$ & $\mathrm{nr}$ & $\mathrm{nr}$ & $\mathrm{nr}$ & $\mathrm{nr}$ \\
\hline $\begin{array}{l}\text { Killeen, } 2012 \\
22299805\end{array}$ & United States & multi-center & NIDA & $\mathrm{RCT}$ & 2 & $19 / 77 / 3$ & $\mathrm{nr}$ & 100 & 32 & $\mathrm{nr}$ & $\mathrm{nr}$ & $\mathrm{nr}$ & $\mathrm{nr}$ & $\mathrm{nr}$ \\
\hline $\begin{array}{l}\text { Latimer, } 2003 \\
12957348\end{array}$ & United States & single center & NIDA & RCT & 2 & 81/0/0 (Asian) & $\mathrm{nr}$ & $\mathrm{nr}$ & 100 & $\mathrm{nr}$ & $\mathrm{nr}$ & $\mathrm{nr}$ & $\mathrm{nr}$ & $\mathrm{nr}$ \\
\hline $\begin{array}{l}\text { Letourneau, } 2017 \\
27629581\end{array}$ & United States & multicenter & $\mathrm{NIDA}, \mathrm{NIH}$ & RCT & 2 & $33 / 30 / n r$ & 40 & 87 & 23 & 0 & $\mathrm{nr}$ & 1 & $\mathrm{nr}$ & $\mathrm{nr}$ \\
\hline $\begin{array}{l}\text { Liddle, } 2001 \\
11727882\end{array}$ & United States & single center & NIDA & RCT & 3 & 51/15/16 (Asian/other) & $\mathrm{nr}$ & $\mathrm{nr}$ & 51 & $\mathrm{nr}$ & $\mathrm{nr}$ & $\mathrm{nr}$ & $\mathrm{nr}$ & $\mathrm{nr}$ \\
\hline $\begin{array}{l}\text { Liddle, } 2004 \\
15152709\end{array}$ & United States & single center & $\begin{array}{l}\text { Substance Abuse and } \\
\text { Mental Health Services } \\
\text { Administration/Center for } \\
\text { Substance Abuse } \\
\text { Treatment }\end{array}$ & RCT & 2 & 3/38/4 (Other) & $\mathrm{nr}$ & $\mathrm{nr}$ & $\mathrm{nr}$ & $\mathrm{nr}$ & $\mathrm{nr}$ & $\mathrm{nr}$ & $\mathrm{nr}$ & $\mathrm{nr}$ \\
\hline $\begin{array}{l}\text { Liddle, } 2008 \\
18705691\end{array}$ & United States & single center & NIDA & RCT & 2 & $21 / 71 / n r$ & 26 & 85 & 100 & $\mathrm{nr}$ & $\mathrm{nr}$ & $\mathrm{nr}$ & $\mathrm{nr}$ & $\mathrm{nr}$ \\
\hline $\begin{array}{l}\text { Liddle, } 2018 \\
29866383\end{array}$ & United States & single-center & NIDA & RCT & 2 & $13 / 18 / n r$ & 71 & 100 & 33 & $\mathrm{nr}$ & $\mathrm{nr}$ & $\mathrm{nr}$ & $\mathrm{nr}$ & $\mathrm{nr}$ \\
\hline $\begin{array}{l}\text { Lowe, } 2012 \\
22931079\end{array}$ & United States & multi-center & NIDA & RCT & 2 & 0/0/100 (Native American) & $\mathrm{nr}$ & $\mathrm{nr}$ & $\mathrm{nr}$ & $\mathrm{nr}$ & $\mathrm{nr}$ & $\mathrm{nr}$ & $\mathrm{nr}$ & $\mathrm{nr}$ \\
\hline $\begin{array}{l}\text { Marsch, } 2005 \\
16203961\end{array}$ & United States & single center & NIDA & RCT & 2 & $100 / 0 / 0$ & $\mathrm{nr}$ & 22 & 100 & 100 & $\mathrm{nr}$ & 22 & $\mathrm{nr}$ & $\mathrm{nr}$ \\
\hline $\begin{array}{l}\text { Marsch, } 2016 \\
26918564\end{array}$ & United States & multicenter & NIDA & RCT & 2 & $81 / n r / n r$ & 25 & 21 & $\mathrm{nr}$ & 100 & $\mathrm{nr}$ & 32 & $\mathrm{nr}$ & $\mathrm{nr}$ \\
\hline $\begin{array}{l}\text { Marsden, } 2006 \\
16771893\end{array}$ & UK & multi-center & $\begin{array}{l}\text { Department of Health for } \\
\text { England and Wales }\end{array}$ & RCT & 2 & 75/13/14 (Other) & 80 & 90 & $\mathrm{nr}$ & $\mathrm{nr}$ & $\mathrm{nr}$ & 78 & $\mathrm{nr}$ & $\mathrm{nr}$ \\
\hline
\end{tabular}




\begin{tabular}{|c|c|c|c|c|c|c|c|c|c|c|c|c|c|c|}
\hline $\begin{array}{l}\text { Author, Year } \\
\text { PMID }\end{array}$ & Country & Site(s) & Funding Source & Design & $\begin{array}{r}\# \\
\text { Arms }\end{array}$ & $\begin{array}{l}\text { Percent } \\
\text { WhitelBlacklOther(details) }\end{array}$ & $\begin{array}{l}\overline{9} \\
\frac{0}{0} \\
\frac{0}{4}\end{array}$ & 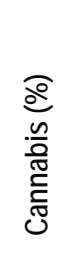 & $\begin{array}{l}\overline{\frac{9}{0}} \\
\frac{0}{0} \\
\frac{0}{0} \\
0\end{array}$ & $\begin{array}{l}\frac{\Im}{2} \\
\text { o } \\
2\end{array}$ & 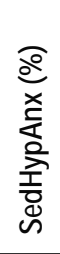 & 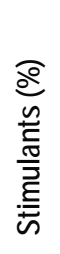 & 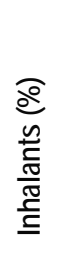 & 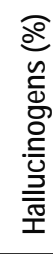 \\
\hline $\begin{array}{l}\text { Martin, } 2008 \\
17869051\end{array}$ & United States & single center & $\begin{array}{l}\text { National Health and } \\
\text { Medical Research } \\
\text { Council. }\end{array}$ & RCT & 2 & $\mathrm{nr} / \mathrm{nr} / \mathrm{nr}$ & $\mathrm{nr}$ & 85 & $\mathrm{nr}$ & $\mathrm{nr}$ & $\mathrm{nr}$ & $\mathrm{nr}$ & $\mathrm{nr}$ & $\mathrm{nr}$ \\
\hline $\begin{array}{l}\text { Martínez } \\
\text { Martínez, } 2008 \\
\text { 2009-05582-007 } \\
\text { (psychinfo) }\end{array}$ & Mexico & single center & PROMEP (Govt) & RCT & 2 & $\mathrm{nr} / \mathrm{nr} / \mathrm{nr}$ & 100 & $\mathrm{nr}$ & $\mathrm{nr}$ & $\mathrm{nr}$ & $\mathrm{nr}$ & $\mathrm{nr}$ & $\mathrm{nr}$ & $\mathrm{nr}$ \\
\hline $\begin{array}{l}\text { Mason, } 2015 \\
26234955\end{array}$ & United States & single center & NIDA & $\mathrm{RCT}$ & 2 & $\begin{array}{l}\text { 84/nr/16 (White, mixed race, } \\
\text { hispanic) }\end{array}$ & $\mathrm{nr}$ & $\mathrm{nr}$ & $\mathrm{nr}$ & $\mathrm{nr}$ & $\mathrm{nr}$ & $\mathrm{nr}$ & $\mathrm{nr}$ & $\mathrm{nr}$ \\
\hline $\begin{array}{l}\text { McCambridge, } \\
2004 \\
14678061\end{array}$ & UK & multi-center & NHS & $\begin{array}{l}\text { Cluster } \\
\text { RCT }\end{array}$ & 2 & 46/37/20 (Asian/other) & 48 & 76 & 21 & 0 & $\mathrm{nr}$ & 23 & $\mathrm{nr}$ & $\mathrm{nr}$ \\
\hline $\begin{array}{l}\text { McCambridge, } \\
2008 \\
18778385\end{array}$ & UK & multi-center & Wellcome Trust & RCT & 2 & 11/52/19 (Asian) & 78 & 100 & $\mathrm{nr}$ & 4 & $\mathrm{nr}$ & 3 & $\mathrm{nr}$ & 8 \\
\hline $\begin{array}{l}\text { McCarty, } 2019 \\
30883284\end{array}$ & United States & single center & NIAAA & RCT & 2 & $\begin{array}{l}\text { 48/9/43 (Asian, hispanic, native } \\
\text { American) }\end{array}$ & 100 & $\mathrm{nr}$ & $\mathrm{nr}$ & $\mathrm{nr}$ & $\mathrm{nr}$ & $\mathrm{nr}$ & $\mathrm{nr}$ & $\mathrm{nr}$ \\
\hline $\begin{array}{l}\text { Miranda, } 2014 \\
23489253\end{array}$ & United States & single center & NIAA & $\mathrm{RCT}$ & 2 & 70/0/10 (Native American) & 70 & 50 & $\mathrm{nr}$ & $\mathrm{nr}$ & $\mathrm{nr}$ & $\mathrm{nr}$ & $\mathrm{nr}$ & $\mathrm{nr}$ \\
\hline $\begin{array}{l}\text { Miranda, } 2017 \\
26752416\end{array}$ & United States & single center & NIDA & $\mathrm{RCT}$ & 2 & 50/nr/50 (Minority) & $\mathrm{nr}$ & 100 & $\mathrm{nr}$ & $\mathrm{nr}$ & $\mathrm{nr}$ & $\mathrm{nr}$ & $\mathrm{nr}$ & $\mathrm{nr}$ \\
\hline $\begin{array}{l}\text { Monti, } 1999 \\
10596521\end{array}$ & United States & single center & NIAA & RCT & 2 & 79/10/8 (Asian/east indian) & 100 & $\mathrm{nr}$ & $\mathrm{nr}$ & $\mathrm{nr}$ & $\mathrm{nr}$ & $\mathrm{nr}$ & $\mathrm{nr}$ & $\mathrm{nr}$ \\
\hline $\begin{array}{l}\text { Najavits, } 2006 \\
16858633\end{array}$ & United States & single center & NIAAA & $\mathrm{RCT}$ & 2 & $\begin{array}{l}\text { 79/3/15 (Asian/pacific } \\
\text { islander/multiethnic) }\end{array}$ & 67 & 79 & 6 & 9 & 6 & 24 & 9 & 24 \\
\hline $\begin{array}{l}\text { Niederhofer, } 2003 \\
12554608\end{array}$ & Austria & single center & unclear & $\mathrm{RCT}$ & 2 & $n r / n r / n r$ & 100 & $\mathrm{nr}$ & $\mathrm{nr}$ & $\mathrm{nr}$ & $\mathrm{nr}$ & $\mathrm{nr}$ & $\mathrm{nr}$ & $\mathrm{nr}$ \\
\hline $\begin{array}{l}\text { Niederhofer, } 2003 \\
15385223\end{array}$ & Austria & single center & $\mathrm{nr}$ & $\mathrm{RCT}$ & 2 & $\mathrm{nr} / \mathrm{nr} / \mathrm{nr}$ & 100 & 0 & 100 & 0 & 0 & 0 & 0 & 0 \\
\hline
\end{tabular}




\begin{tabular}{|c|c|c|c|c|c|c|c|c|c|c|c|c|c|c|}
\hline $\begin{array}{l}\text { Author, Year } \\
\text { PMID }\end{array}$ & Country & Site(s) & Funding Source & Design & $\begin{array}{r}\# \\
\text { Arms } \\
\end{array}$ & $\begin{array}{l}\text { Percent } \\
\text { WhitelBlacklOther(details) }\end{array}$ & $\begin{array}{l}\frac{\overline{9}}{\overline{0}} \\
\frac{0}{0} \\
\frac{0}{4}\end{array}$ & 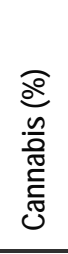 & $\begin{array}{l}\bar{\Xi} \\
\frac{0}{0} \\
\frac{0}{0} \\
0\end{array}$ & $\begin{array}{l}\frac{\Im}{9} \\
\frac{0}{2} \\
\frac{0}{2}\end{array}$ & 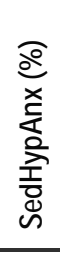 & 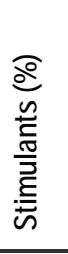 & 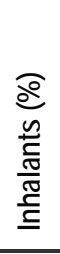 & 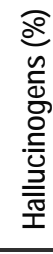 \\
\hline $\begin{array}{l}\text { Niederhofer, } 2003 \\
\text { CN-00474316 } \\
\text { (cochrane) }\end{array}$ & Austria & single center & unclear & $\mathrm{RCT}$ & 2 & $\mathrm{nr} / \mathrm{nr} / \mathrm{nr}$ & 100 & $\mathrm{nr}$ & $\mathrm{nr}$ & $\mathrm{nr}$ & $\mathrm{nr}$ & $\mathrm{nr}$ & $\mathrm{nr}$ & $\mathrm{nr}$ \\
\hline $\begin{array}{l}\text { O'Malley, } 2015 \\
25742208\end{array}$ & Unted States & multi-center & $\mathrm{NIH}$ & $\mathrm{RCT}$ & 2 & $\begin{array}{l}\text { 77/8/15 (Various - native } \\
\text { American, asian, multiple, and } \\
\text { other/refused/unknown.) }\end{array}$ & 100 & 33 & 79 & 0 & $\mathrm{nr}$ & $\mathrm{nr}$ & $\mathrm{nr}$ & $\mathrm{nr}$ \\
\hline $\begin{array}{l}\text { Ogel, } 2011 \\
21609157\end{array}$ & Turkey & single center & $\mathrm{nr}$ & $\mathrm{RCT}$ & 2 & $\mathrm{nr} / \mathrm{nr} / \mathrm{nr}$ & $\mathrm{nr}$ & 64 & $\mathrm{nr}$ & $\mathrm{nr}$ & $\mathrm{nr}$ & $\mathrm{nr}$ & $\mathrm{nr}$ & $\mathrm{nr}$ \\
\hline $\begin{array}{l}\text { Peterson, } 2006 \\
16938063\end{array}$ & United States & single center & NIAAA & RCT & 3 & $\begin{array}{l}72 / 3 / 20 \text { (Native American, asian, } \\
\text { other) }\end{array}$ & 87 & 94 & $\mathrm{nr}$ & 27 & 10 & 53 & 7 & 36 \\
\hline $\begin{array}{l}\text { Riggs, } 2004 \\
15187802 \\
\end{array}$ & United States & single center & NIDA & RCT & 2 & $71 / 3 / 0(\mathrm{Na})$ & 47 & 69 & 100 & $\mathrm{nr}$ & $\mathrm{nr}$ & $\mathrm{nr}$ & $\mathrm{nr}$ & $\mathrm{nr}$ \\
\hline $\begin{array}{l}\text { Riggs, } 2007 \\
17984403 \\
\end{array}$ & United States & single center & NIDA & $\mathrm{RCT}$ & 2 & $48 / 14 / 27$ & 75 & 88 & 100 & 10 & 8 & 19 & 2 & 18 \\
\hline $\begin{array}{l}\text { Riggs, } 2011 \\
21871372 \\
\end{array}$ & United States & multi-center & NIDA & $\mathrm{RCT}$ & 2 & $62 / 23 / n r$ & 30 & 67 & 0 & 0 & 3 & 9 & 0 & 5 \\
\hline $\begin{array}{l}\text { Rigter, } 2013 \\
23140805\end{array}$ & $\begin{array}{l}\text { Belgium, } \\
\text { France, } \\
\text { Germany, The } \\
\text { Netherlands, } \\
\text { Switzerland }\end{array}$ & multi-center & $\begin{array}{l}\text { (federal) Ministries of } \\
\text { Health of Belgium, } \\
\text { Germany, The } \\
\text { Netherlands, Switzerland, } \\
\text { and by MILDT:the Mission } \\
\text { Interministerielle de Lutte } \\
\text { Contre la Drogue et de } \\
\text { Toximanie, France }\end{array}$ & RCT & 2 & nr/nr/40 (Foreign descent) & 40 & 100 & $\mathrm{nr}$ & $\mathrm{nr}$ & $\mathrm{nr}$ & $\mathrm{nr}$ & $\mathrm{nr}$ & $\mathrm{nr}$ \\
\hline $\begin{array}{l}\text { Robbins, } 2008 \\
18266532\end{array}$ & United States & single center & NIDA & $\mathrm{RCT}$ & 3 & 0/40/0 (Na) & $\mathrm{nr}$ & $\mathrm{nr}$ & 100 & $\mathrm{nr}$ & $\mathrm{nr}$ & $\mathrm{nr}$ & $\mathrm{nr}$ & $\mathrm{nr}$ \\
\hline $\begin{array}{l}\text { Robbins, } 2011 \\
21967492 \\
\end{array}$ & United States & multi-center & NIDA & $\mathrm{RCT}$ & 2 & 31/22/2 (Other) & 26 & 41 & 20 & $\mathrm{nr}$ & $\mathrm{nr}$ & $\mathrm{nr}$ & $\mathrm{nr}$ & $\mathrm{nr}$ \\
\hline $\begin{array}{l}\text { Rohde, } 2014 \\
24491069\end{array}$ & United States & single center & NIDA & $\mathrm{RCT}$ & 3 & $61 / n r / n r$ & $\mathrm{nr}$ & $\mathrm{nr}$ & 100 & $\mathrm{nr}$ & $\mathrm{nr}$ & $\mathrm{nr}$ & $\mathrm{nr}$ & $\mathrm{nr}$ \\
\hline $\begin{array}{l}\text { Rowe, } 2016 \\
26879671\end{array}$ & United States & multicenter & $\begin{array}{l}\text { NIDA, SAMHSA, CDC, } \\
\text { NIAAA, DOJ }\end{array}$ & RCT & 2 & $\mathrm{nr} / 61 / \mathrm{nr}$ & 20 & 60 & 8 & $\mathrm{nr}$ & $\mathrm{nr}$ & $\mathrm{nr}$ & $\mathrm{nr}$ & $\mathrm{nr}$ \\
\hline
\end{tabular}




\begin{tabular}{|c|c|c|c|c|c|c|c|c|c|c|c|c|c|c|}
\hline $\begin{array}{l}\text { Author, Year } \\
\text { PMID }\end{array}$ & Country & Site(s) & Funding Source & Design & $\begin{array}{r}\# \\
\text { Arms }\end{array}$ & $\begin{array}{l}\text { Percent } \\
\text { WhitelBlacklOther(details) }\end{array}$ & $\begin{array}{l}\bar{\Xi} \\
\frac{0}{0} \\
\frac{0}{0} \\
\frac{0}{\pi}\end{array}$ & 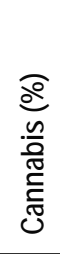 & $\begin{array}{l}\overline{\frac{0}{0}} \\
\frac{0}{0} \\
\frac{0}{0} \\
\frac{0}{0}\end{array}$ & 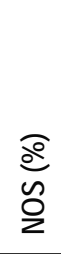 & 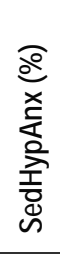 & 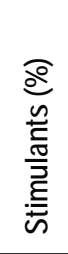 & 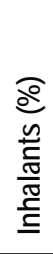 & 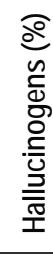 \\
\hline $\begin{array}{l}\text { Santisteban, } 2011 \\
21639636\end{array}$ & United States & single center & $\begin{array}{l}\text { NIDA, Center for Minority } \\
\text { Health and Health } \\
\text { Disparities }\end{array}$ & RCT & 2 & $\mathrm{nr} / \mathrm{nr} / \mathrm{nr}$ & $\mathrm{nr}$ & $\mathrm{nr}$ & 100 & $\mathrm{nr}$ & $\mathrm{nr}$ & $\mathrm{nr}$ & $\mathrm{nr}$ & $\mathrm{nr}$ \\
\hline $\begin{array}{l}\text { Santisteban, } 2015 \\
25799306\end{array}$ & United States & single center & NIDA & $\mathrm{RCT}$ & 2 & $\mathrm{nr} / \mathrm{nr} / \mathrm{nr}$ & 59 & 79 & 100 & $\mathrm{nr}$ & $\mathrm{nr}$ & $\mathrm{nr}$ & $\mathrm{nr}$ & $\mathrm{nr}$ \\
\hline $\begin{array}{l}\text { Schaeffer, } 2014 \\
23958035\end{array}$ & United States & single center & NIDA & $\mathrm{RCT}$ & 2 & 18/26/4 (Mixed) & $\mathrm{nr}$ & $\mathrm{nr}$ & $\mathrm{nr}$ & $\mathrm{nr}$ & $\mathrm{nr}$ & $\mathrm{nr}$ & $\mathrm{nr}$ & $\mathrm{nr}$ \\
\hline $\begin{array}{l}\text { Slesnick, } 2005 \\
15878048\end{array}$ & United States & single center & NIDA & $\mathrm{RCT}$ & 2 & $\begin{array}{l}37 / 7 / 14 \text { (Native American or } \\
\text { other) }\end{array}$ & 11 & 36 & 34 & 10 & $\mathrm{nr}$ & $\mathrm{nr}$ & $\mathrm{nr}$ & $\mathrm{nr}$ \\
\hline $\begin{array}{l}\text { Slesnick, } 2007 \\
16989957\end{array}$ & United States & single center & $\mathrm{nr}$ & RCT & 2 & 41/3/13 (Native American) & 70 & 85 & 49 & $\mathrm{nr}$ & $\mathrm{nr}$ & $\mathrm{nr}$ & $\mathrm{nr}$ & $\mathrm{nr}$ \\
\hline $\begin{array}{l}\text { Slesnick, } 2009 \\
19522781\end{array}$ & United States & single center & NIAAA/CSAT & $\mathrm{RCT}$ & 3 & $\begin{array}{l}\text { 29/5/22 (Native American or } \\
\text { other) }\end{array}$ & 89 & 29 & 17 & $\mathrm{nr}$ & $\mathrm{nr}$ & $\mathrm{nr}$ & $\mathrm{nr}$ & $\mathrm{nr}$ \\
\hline $\begin{array}{l}\text { Slesnick, } 2013 \\
23895088\end{array}$ & United States & single center & NIDA & RCT & 3 & $\begin{array}{l}\text { 26/66/7 (Native American, asian, } \\
\text { other) }\end{array}$ & $\mathrm{nr}$ & $\mathrm{nr}$ & 100 & $\mathrm{nr}$ & $\mathrm{nr}$ & $\mathrm{nr}$ & $\mathrm{nr}$ & $\mathrm{nr}$ \\
\hline $\begin{array}{l}\text { Slesnick, } 2015 \\
25736623\end{array}$ & United States & single center & NIDA & RCT & 3 & $\begin{array}{l}\text { 20/66/13 (Native American, asian, } \\
\text { or other) }\end{array}$ & $\mathrm{nr}$ & 78 & $\mathrm{nr}$ & 7 & $\mathrm{nr}$ & 2 & $\mathrm{nr}$ & $\mathrm{nr}$ \\
\hline $\begin{array}{l}\text { Smith, } 2006 \\
17182429\end{array}$ & United States & multicenter & SAMHSA & $\mathrm{RCT}$ & 2 & $n r / n r / n r$ & 23 & 49 & 50 & $\mathrm{nr}$ & $\mathrm{nr}$ & $\mathrm{nr}$ & $\mathrm{nr}$ & $\mathrm{nr}$ \\
\hline $\begin{array}{l}\text { Smith, } 2015 \\
25551562\end{array}$ & United States & multicenter & NIAA & $\mathrm{RCT}$ & 2 & 23/36/36 (Multiracial) & $\mathrm{nr}$ & $\mathrm{nr}$ & $\mathrm{nr}$ & $\mathrm{nr}$ & $\mathrm{nr}$ & $\mathrm{nr}$ & $\mathrm{nr}$ & $\mathrm{nr}$ \\
\hline $\begin{array}{l}\text { De Sousa, } 2014 \\
\text { CN-01014147 } \\
\text { (cochrane) }\end{array}$ & India & single center & $\mathrm{nr}$ & $\mathrm{RCT}$ & 2 & nr/nr/100 (Indian) & 100 & $\mathrm{nr}$ & $\mathrm{nr}$ & $\mathrm{nr}$ & $\mathrm{nr}$ & $\mathrm{nr}$ & $\mathrm{nr}$ & $\mathrm{nr}$ \\
\hline $\begin{array}{l}\text { Spijkerman, } 2010 \\
21169172\end{array}$ & Netherlands & single center & ZonMw & RCT & 3 & $\mathrm{nr} / \mathrm{nr} / \mathrm{nr}$ & 55 & $\mathrm{nr}$ & $\mathrm{nr}$ & $\mathrm{nr}$ & $\mathrm{nr}$ & $\mathrm{nr}$ & $\mathrm{nr}$ & $\mathrm{nr}$ \\
\hline $\begin{array}{l}\text { Spirito, } 2004 \\
15343198\end{array}$ & United States & single center & $\mathrm{nr}$ & RCT & 2 & 72/7/1 (Asian/east indian) & 100 & $\mathrm{nr}$ & $\mathrm{nr}$ & $\mathrm{nr}$ & $\mathrm{nr}$ & $\mathrm{nr}$ & $\mathrm{nr}$ & $\mathrm{nr}$ \\
\hline $\begin{array}{l}\text { Spirito, } 2011 \\
21383276\end{array}$ & United States & single center & $\mathrm{NHH}$ & RCT & 2 & $\begin{array}{l}\text { 71/2/0 (Asian American/ east } \\
\text { indian) }\end{array}$ & 100 & $\mathrm{nr}$ & $\mathrm{nr}$ & $\mathrm{nr}$ & $\mathrm{nr}$ & $\mathrm{nr}$ & $\mathrm{nr}$ & $\mathrm{nr}$ \\
\hline
\end{tabular}




\begin{tabular}{|c|c|c|c|c|c|c|c|c|c|c|c|c|c|c|}
\hline $\begin{array}{l}\text { Author, Year } \\
\text { PMID }\end{array}$ & Country & Site(s) & Funding Source & Design & $\begin{array}{r}\# \\
\text { Arms }\end{array}$ & $\begin{array}{l}\text { Percent } \\
\text { WhitelBlacklOther(details) }\end{array}$ & $\begin{array}{l}\frac{\overline{9}}{\overline{0}} \\
\frac{0}{\frac{0}{4}}\end{array}$ & 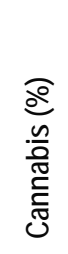 & $\begin{array}{l}\frac{9}{0} \\
\frac{0}{0} \\
\frac{0}{0}\end{array}$ & 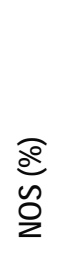 & 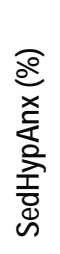 & 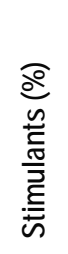 & 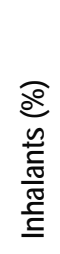 & 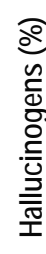 \\
\hline $\begin{array}{l}\text { Spirito, } 2017 \\
29252011 \\
\end{array}$ & United States & single center & NIDA & $\mathrm{RCT}$ & 2 & 67/19/14 (Multiracial) & $\mathrm{nr}$ & $\mathrm{nr}$ & $\mathrm{nr}$ & $\mathrm{nr}$ & $\mathrm{nr}$ & $\mathrm{nr}$ & $\mathrm{nr}$ & $\mathrm{nr}$ \\
\hline $\begin{array}{l}\text { Srisurapanont, } \\
2007 \\
17453612\end{array}$ & Thailand & single center & $\begin{array}{l}\text { The Office of Narcotics } \\
\text { Control Board, Thailand }\end{array}$ & RCT & 2 & $\mathrm{nr} / \mathrm{nr} / \mathrm{nr}$ & $\mathrm{nr}$ & $\mathrm{nr}$ & $\mathrm{nr}$ & $\mathrm{nr}$ & $\mathrm{nr}$ & 75 & $\mathrm{nr}$ & $\mathrm{nr}$ \\
\hline $\begin{array}{l}\text { Stanger, } 2009 \\
19717250\end{array}$ & United States & single center & $\begin{array}{l}\text { NIDA, NIAAA, Arkansas } \\
\text { Tobacco Settlement fund }\end{array}$ & RCT & 2 & $91 / 6 / 0$ & $\mathrm{nr}$ & $\mathrm{nr}$ & $\mathrm{nr}$ & $\mathrm{nr}$ & $\mathrm{nr}$ & $\mathrm{nr}$ & $\mathrm{nr}$ & $\mathrm{nr}$ \\
\hline $\begin{array}{l}\text { Stanger, } 2015 \\
26004659\end{array}$ & United States & single center & $\mathrm{NIH}$ & $\mathrm{RCT}$ & 3 & $\begin{array}{l}\text { 35/62/3 (Native American, multi- } \\
\text { racial) }\end{array}$ & $\mathrm{nr}$ & 100 & $\mathrm{nr}$ & 0 & 0 & 0 & 0 & 0 \\
\hline $\begin{array}{l}\text { Stanger, } 2017 \\
28414474\end{array}$ & United States & single center & $\mathrm{NIH}$ & $\mathrm{RCT}$ & 2 & 81/nr/19 (Minority) & 100 & 35 & $\mathrm{nr}$ & $\mathrm{nr}$ & $\mathrm{nr}$ & $\mathrm{nr}$ & $\mathrm{nr}$ & $\mathrm{nr}$ \\
\hline $\begin{array}{l}\text { Stein, } 2011 \\
21531089\end{array}$ & United States & single center & NIDA & RCT & 2 & $\mathrm{nr} / \mathrm{nr} / \mathrm{nr}$ & $\mathrm{nr}$ & $\mathrm{nr}$ & $\mathrm{nr}$ & $\mathrm{nr}$ & $\mathrm{nr}$ & $\mathrm{nr}$ & $\mathrm{nr}$ & $\mathrm{nr}$ \\
\hline $\begin{array}{l}\text { Tait, } 2004 \\
15194207\end{array}$ & Australia & multicenter & $\begin{array}{l}\text { Healthway, the West } \\
\text { Australian Health } \\
\text { Promotion Foundation. }\end{array}$ & $\mathrm{RCT}$ & 3 & $\mathrm{nr} / \mathrm{nr} / \mathrm{nr}$ & 83 & $\mathrm{nr}$ & $\mathrm{nr}$ & $\mathrm{nr}$ & $\mathrm{nr}$ & $\mathrm{nr}$ & $\mathrm{nr}$ & $\mathrm{nr}$ \\
\hline $\begin{array}{l}\text { Thurstone, } 2010 \\
20494267\end{array}$ & United States & single center & $\begin{array}{l}\text { NIDA, AACAP (Rx from Eli } \\
\text { Lilly) }\end{array}$ & RCT & 2 & 19/9/59 (Other) & $\mathrm{nr}$ & $\mathrm{nr}$ & $\mathrm{nr}$ & $\mathrm{nr}$ & $\mathrm{nr}$ & $\mathrm{nr}$ & $\mathrm{nr}$ & $\mathrm{nr}$ \\
\hline $\begin{array}{l}\text { Thush, } 2007 \\
16928395\end{array}$ & Netherlands & single center & $\begin{array}{l}\text { Dutch Health Care } \\
\text { Research Organization }\end{array}$ & RCT & 2 & $\mathrm{nr} / \mathrm{nr} / \mathrm{nr}$ & 100 & $\mathrm{nr}$ & $\mathrm{nr}$ & $\mathrm{nr}$ & $\mathrm{nr}$ & $\mathrm{nr}$ & $\mathrm{nr}$ & $\mathrm{nr}$ \\
\hline $\begin{array}{l}\text { Tolou-Shams, } \\
2017 \\
\text { CN-01365355 } \\
\text { (cochrane) }\end{array}$ & United States & single center & NIDA & RCT & 2 & 69/10/22 (Asian/other) & 78 & 90 & 28 & $\mathrm{nr}$ & $\mathrm{nr}$ & $\mathrm{nr}$ & $\mathrm{nr}$ & $\mathrm{nr}$ \\
\hline $\begin{array}{l}\text { Trudeau, 2017 } \\
\text { 2017-00657-001 } \\
\text { (psychinfo) }\end{array}$ & United States & single center & NIDA & $\mathrm{RCT}$ & 2 & 9/nr/91 (Non-caucasian) & 76 & $\mathrm{nr}$ & 74 & $\mathrm{nr}$ & $\mathrm{nr}$ & $\mathrm{nr}$ & $\mathrm{nr}$ & $\mathrm{nr}$ \\
\hline $\begin{array}{l}\text { Voogt, } 2013 \\
\text { CN-01122318 } \\
\text { (cochrane) }\end{array}$ & Netherlands & multicenter & $\begin{array}{l}\text { The Netherlands } \\
\text { Organization for Health } \\
\text { Research and } \\
\text { Development }\end{array}$ & $\begin{array}{l}\text { cluster } \\
\text { RCT }\end{array}$ & 2 & $\mathrm{nr} / \mathrm{nr} / \mathrm{nr}$ & 100 & $\mathrm{nr}$ & $\mathrm{nr}$ & $\mathrm{nr}$ & $\mathrm{nr}$ & $\mathrm{nr}$ & $\mathrm{nr}$ & $\mathrm{nr}$ \\
\hline
\end{tabular}




\begin{tabular}{|c|c|c|c|c|c|c|c|c|c|c|c|c|c|c|}
\hline $\begin{array}{l}\text { Author, Year } \\
\text { PMID }\end{array}$ & Country & Site(s) & Funding Source & Design & $\begin{array}{r}\# \\
\text { Arms } \\
\end{array}$ & $\begin{array}{l}\text { Percent } \\
\text { WhitelBlacklOther(details) }\end{array}$ & $\begin{array}{l}\overline{\frac{a}{0}} \\
\frac{0}{0} \\
\frac{0}{\alpha}\end{array}$ & 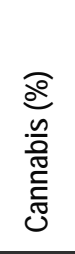 & $\begin{array}{l}\overline{0} \\
\frac{0}{0} \\
\frac{0}{0} \\
\frac{0}{0}\end{array}$ & $\begin{array}{l}\bar{\Xi} \\
\text { o } \\
\text { 20 }\end{array}$ & 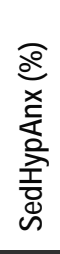 & 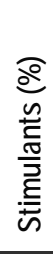 & 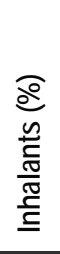 & 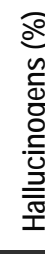 \\
\hline $\begin{array}{l}\text { Wagner, } 2014 \\
24841864\end{array}$ & United States & multicenter & NIAAA & RCT & 2 & 6/23/14 (Other) & 92 & $\mathrm{nr}$ & 90 & $\mathrm{nr}$ & $\mathrm{nr}$ & $\mathrm{nr}$ & $\mathrm{nr}$ & $\mathrm{nr}$ \\
\hline $\begin{array}{l}\text { Waldron, } 2001 \\
11680557\end{array}$ & United States & single center & NIDA & RCT & 4 & 38/nr/8 (Native American) & $\mathrm{nr}$ & $\mathrm{nr}$ & $\mathrm{nr}$ & $\mathrm{nr}$ & $\mathrm{nr}$ & $\mathrm{nr}$ & $\mathrm{nr}$ & $\mathrm{nr}$ \\
\hline $\begin{array}{l}\text { Walker, } 2006 \\
16822119\end{array}$ & United States & multicenter & NIDA & $\mathrm{RCT}$ & 2 & $\begin{array}{l}\text { 53/17/25 (Asian/pacific } \\
\text { islander/other) }\end{array}$ & $\mathrm{nr}$ & 100 & $\mathrm{nr}$ & $\mathrm{nr}$ & $\mathrm{nr}$ & $\mathrm{nr}$ & $\mathrm{nr}$ & $\mathrm{nr}$ \\
\hline $\begin{array}{l}\text { Walker, } 2011 \\
21688877\end{array}$ & United States & single center & NIDA & $\mathrm{RCT}$ & 2 & 66/10/13 (Multiracial) & $\mathrm{nr}$ & 100 & $\mathrm{nr}$ & $\mathrm{nr}$ & $\mathrm{nr}$ & $\mathrm{nr}$ & $\mathrm{nr}$ & $\mathrm{nr}$ \\
\hline $\begin{array}{l}\text { Walker, } 2016 \\
27762569\end{array}$ & United States & single center & NIDA & $\mathrm{RCT}$ & 2 & $\begin{array}{l}59 / 6 / 35 \text { (Multiracial or asian or } \\
\text { other) }\end{array}$ & $\mathrm{nr}$ & 100 & $\mathrm{nr}$ & $\mathrm{nr}$ & $\mathrm{nr}$ & $\mathrm{nr}$ & $\mathrm{nr}$ & $\mathrm{nr}$ \\
\hline $\begin{array}{l}\text { Winters, } 2007 \\
17563146\end{array}$ & United States & single center & NIDA & $\mathrm{RCT}$ & 2 & 81/nr/19 (Nonwhite) & $\mathrm{nr}$ & $\mathrm{nr}$ & 100 & $\mathrm{nr}$ & $\mathrm{nr}$ & $\mathrm{nr}$ & $\mathrm{nr}$ & $\mathrm{nr}$ \\
\hline $\begin{array}{l}\text { Winters, } 2012 \\
22000326\end{array}$ & United States & single center & $\mathrm{NIH}$ & $\mathrm{RCT}$ & 3 & $66 / n r / 34$ & $\mathrm{nr}$ & $\mathrm{nr}$ & $\mathrm{nr}$ & $\mathrm{nr}$ & $\mathrm{nr}$ & $\mathrm{nr}$ & $\mathrm{nr}$ & $\mathrm{nr}$ \\
\hline $\begin{array}{l}\text { Woody, } 2008 \\
18984887\end{array}$ & United States & multi-center & NIDA & $\mathrm{RCT}$ & 2 & $n r / n r / n r$ & $\mathrm{nr}$ & $\mathrm{nr}$ & $\mathrm{nr}$ & $\mathrm{nr}$ & $\mathrm{nr}$ & $\mathrm{nr}$ & $\mathrm{nr}$ & $\mathrm{nr}$ \\
\hline $\begin{array}{l}\text { Zhang, } 2018 \\
30556713\end{array}$ & United States & $\mathrm{nr}$ & NIDA & $\mathrm{RCT}$ & 3 & $17 / 68 / 15$ & $\mathrm{nr}$ & $\mathrm{nr}$ & $\mathrm{nr}$ & $\mathrm{nr}$ & $\mathrm{nr}$ & $\mathrm{nr}$ & $\mathrm{nr}$ & $\mathrm{nr}$ \\
\hline
\end{tabular}

Abbreviations: PMID* = Pubmed identifier if available, otherwise (database name); NOS = drug of abuse tot otherwise specified; SedHypAnx = sedatative, hypnotic or anxiolytic; BMBF = German Federal Ministry for Education and Research; $\mathrm{CSA}=; \mathrm{CSAT}=; \mathrm{CMHD}=$ Center for Minority Health and Health Disparities; DHCO = Dutch Health Care Research Organization; DPIP=Drug Prevention and Information Programme of the European Union; NHMRC = National Health and Medical Research Council; NIAA (or NIAAA) = National Institute on Alcohol Abuse and Alcoholism ; NIDA = National Institute on Drug Abuse; NIH = National Institutes of Health ; NIMH = National Institutes of Mental Health; ONCB (Thailand) = The Office of Narcotics Control Board, Thailand ; SAMHSA = Substance Abuse and Mental Health Services Administration; VA = Department of Veteran Affairs ; ZonMw = The Netherlands Organization for Health Research and Developmen 


\section{Appendix E. Outcomes Extracted by Study}

Table E-1. Brief interventions

Study, Year

PubMed (other database) ID

Arnaud, 2015

2016-03749-004 (psycINFO)

Arnaud, 2017

27801991

Bernstein, 2009

20053238

Bernstein, 2010

20670329

Braciszewski, 2018

132804409 (Embase)

Brown, 2015

26362000

Colby, 2018

29750362

D'Amico, 2018

30138016

de Gee, 2014

24969735

Martin, 2008

17869051

Giles, 2019

CN-01953820 (cochrane)

Martínez Martínez, 2008

2009-05582-007 (psycINFO)

McCambridge, 2004

14678061

McCambridge, 2008

18778385

\begin{tabular}{|c|c|c|c|c|c|c|c|c|c|c|}
\hline 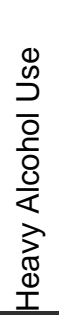 & 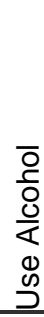 & 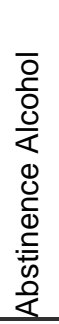 & 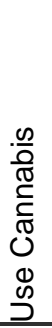 & 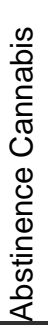 & $\begin{array}{l}\frac{\omega}{\pi} \\
\tilde{d} \\
\frac{1}{0} \\
\frac{0}{0} \\
\frac{0}{0} \\
0 \\
0\end{array}$ & 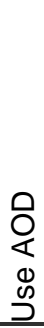 & 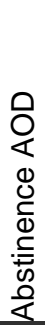 & 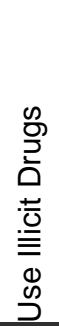 & 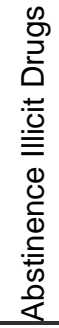 & 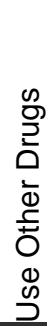 \\
\hline$\checkmark$ & $\checkmark$ & $\checkmark$ & $\circ$ & $\circ$ & $\circ$ & $\checkmark$ & $\checkmark$ & $\circ$ & $\circ$ & ○ \\
\hline$\checkmark$ & ○ & $\circ$ & $\circ$ & ० & $\checkmark$ & ० & ○ & $\circ$ & ० & ○ \\
\hline$\circ$ & $\circ$ & $\circ$ & $\checkmark$ & $\checkmark$ & o & $\circ$ & o & $\circ$ & ० & ○ \\
\hline$\checkmark$ & $\checkmark$ & $\circ$ & 0 & $\circ$ & $\circ$ & $\circ$ & $\circ$ & $\circ$ & $\circ$ & ○ \\
\hline$\circ$ & ○ & $\circ$ & $\checkmark$ & $\circ$ & o & ० & ० & $\circ$ & ○ & 0 \\
\hline$\circ$ & $\checkmark$ & $\checkmark$ & $\checkmark$ & $\checkmark$ & o & $\checkmark$ & $\checkmark$ & $\circ$ & o & ० \\
\hline$\checkmark$ & $\checkmark$ & $\circ$ & o & ० & $\checkmark$ & ० & ○ & $\circ$ & ० & ० \\
\hline$\checkmark$ & $\checkmark$ & $\circ$ & $\checkmark$ & $\circ$ & $\checkmark$ & ० & ○ & $\circ$ & o & o \\
\hline$\circ$ & ○ & $\circ$ & $\checkmark$ & $\circ$ & $\checkmark$ & ० & ○ & $\circ$ & o & o \\
\hline$\circ$ & ○ & $\circ$ & $\checkmark$ & ० & $\checkmark$ & ० & ० & $\circ$ & ○ & 0 \\
\hline$\checkmark$ & $\checkmark$ & $\circ$ & $\circ$ & 0 & $\checkmark$ & ० & ० & ○ & ○ & $\circ$ \\
\hline ० & $\circ$ & $\checkmark$ & ○ & $\circ$ & $\circ$ & $\circ$ & $\circ$ & ○ & ० & $\circ$ \\
\hline$\circ$ & $\circ$ & $\checkmark$ & $\circ$ & $\checkmark$ & $\circ$ & ० & ० & $\circ$ & o & $\circ$ \\
\hline$\circ$ & $\checkmark$ & $\checkmark$ & $\checkmark$ & $\checkmark$ & $\checkmark$ & $\circ$ & $\circ$ & $\circ$ & $\circ$ & $\circ$ \\
\hline
\end{tabular}


Study, Year

PubMed (other database) ID
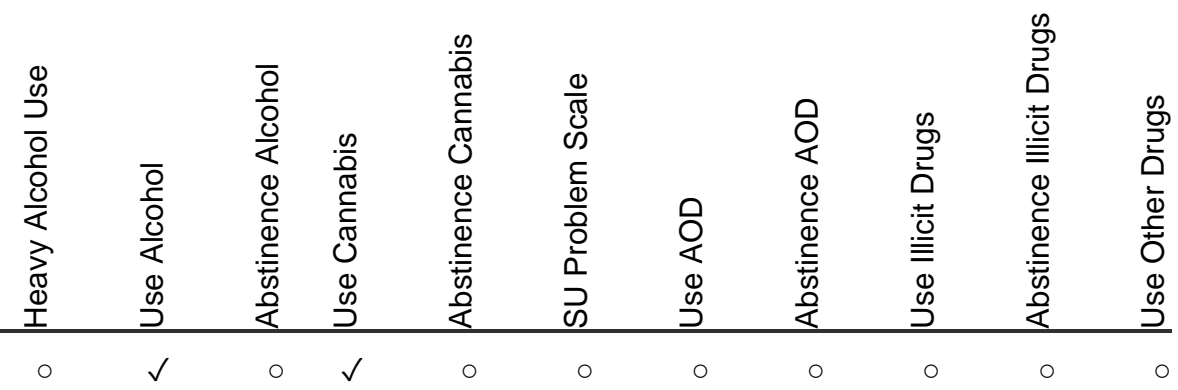

McCarty, 2019

Peterson, 2006

16938063

Spirito, 2004

15343198

Spirito, 2017

29252011

Stein, 2011

21531089

Walker, 2006

16822119

Walker, 2011

21688877

Winters, 2007

17563146

Winters, 2012

22000326

Abbreviations: $\checkmark$ = outcom e reported ; $\mathrm{O}=$ outcom e not reported ; $\mathrm{A} O \mathrm{D}=$ alcoho l and other drugs

\section{Table E-2. Nonbrief interventions}

Study, Year

PubMed (other database) ID

Azrin, 1994

CN-00241903 (Cochrane)

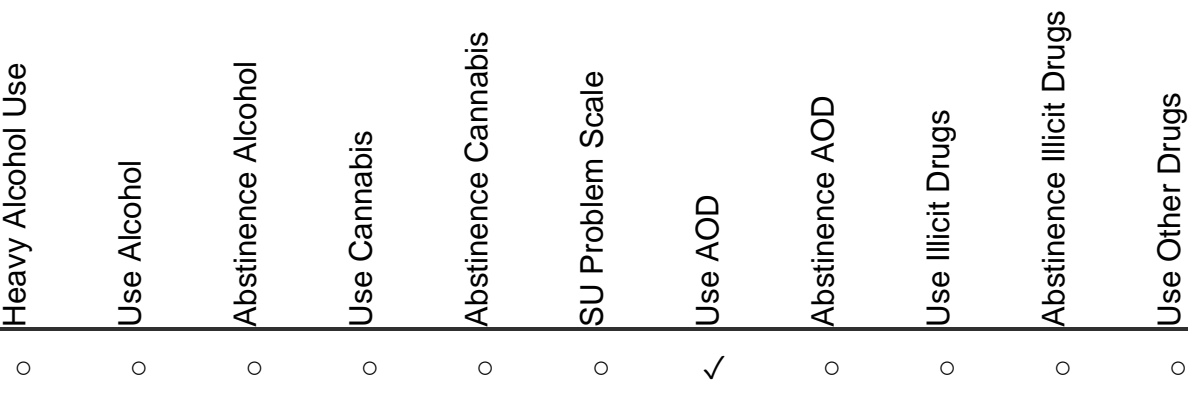


Study, Year

PubMed (other database) ID

Azrin, 2001

2002-13926-001 (psycINFO)

Baer, 2007

18072842

D'Amico, 2013

CN-00917707 (Cochrane)

Dakof, 2015

25621927

Dennis, 2004

15501373

Figurelli, 1994

7862806

Godley, 2002

12127465

Godley, 2010

20219293

Henderson, 2016

26992083

Henggeler, 1996

8610836

Henggeler, 2006

16551142

Henggeler, 2012

22309470

HJoanning, 1992

CN-00631575 (Cochrane)

Hogue, 2015

25496283

Kaminer, 1998

9824170

Kaminer, 2002

12436013

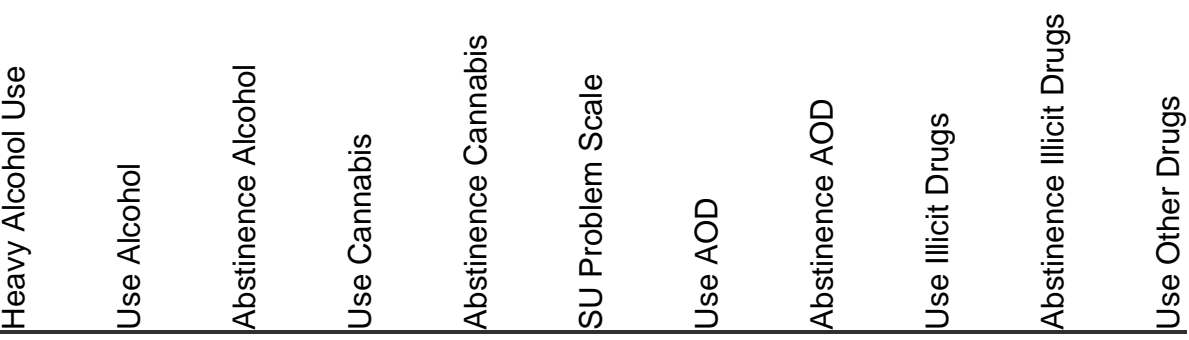

$\begin{array}{lllllllllll}\checkmark & 0 & \checkmark & 0 & 0 & 0 & 0 & 0 & 0 & \checkmark\end{array}$

\begin{tabular}{lllllllllll}
$\checkmark$ & $\checkmark$ & 0 & $\checkmark$ & 0 & $\checkmark$ & 0 & 0 & 0 & 0 & 0 \\
\hline
\end{tabular}


Study, Year

PubMed (other database) ID

Kaminer, 2008

18978635

Kelly, 2017

28742932

Latimer, 2003

12957348

Letourneau, 2017

27629581

Liddle, 2001

11727882

Liddle, 2004

15152709

Liddle, 2008

18705691

Liddle, 2018

29866383

Lowe, 2012

22931079

Najavits, 2006

16858633

Rigter, 2013

23140805

Robbins, 2011

21967492

Santisteban, 2011

21639636

Slesnick, 2007

16989957

Slesnick, 2009

19522781

Slesnick, 2015

25736623

$\begin{array}{lllll}0 & \checkmark & 0 & \checkmark & 0 \\ 0 & 0 & 0 & 0 & 0\end{array}$

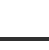


Study, Year

PubMed (other database) ID

Smith, 2006

17182429

Stanger, 2009

19717250

Stanger, 2015

26004659

Stanger, 2017

28414474

Tolou-Shams, 2017

CN-01365355 (Cochrane)

Trudeau, 2017

2017-00657-001 (psycINFO)

Wagner, 2014

24841864

Waldron, 2001

11680557

Zhang, 2018

30556713

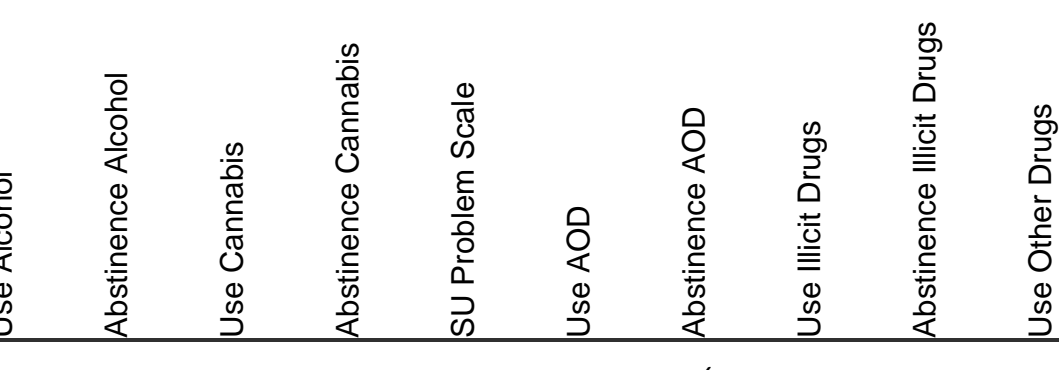

Abbreviations: $\checkmark=$ outcom e reported; $\mathrm{O}=$ outcom e not reported; $\mathrm{A}$ O D = alcohol and other drugs 


\section{Appendix F. Brief interventions: Detailed Results}

Table F-1. Brief interventions, heavy alcohol use days

\begin{tabular}{|c|c|c|c|c|c|c|c|c|c|c|}
\hline PMID & Citation & Intervention & $\begin{array}{r}\mathrm{N} \\
\text { (endtime) }\end{array}$ & Scale & $\begin{array}{r}\text { Baseline } \\
\text { Mean } \\
\text { (SD) }\end{array}$ & $\begin{array}{l}\text { End Mean } \\
\text { (SD) }\end{array}$ & $\begin{array}{r}\text { MD } \\
(95 \% \mathrm{Cl})\end{array}$ & $\begin{array}{r}\text { NMD } \\
(95 \% \mathrm{Cl})\end{array}$ & $\begin{array}{r}\text { SMD } \\
(95 \% \mathrm{Cl})\end{array}$ & $\begin{array}{r}\text { SNMD } \\
(95 \% \mathrm{Cl})\end{array}$ \\
\hline 27801991 & $\begin{array}{l}\text { Arnaud, } \\
2017\end{array}$ & Ml & $\begin{array}{r}141 \\
(3 \mathrm{mo})\end{array}$ & No & $\begin{array}{r}2.69 \\
(2.82)\end{array}$ & $\begin{array}{r}1.02 \\
(1.51)\end{array}$ & $\begin{array}{r}-1.67 \\
(-2.1,-1.2)\end{array}$ & $\begin{array}{r}-0.64 \\
(-1.2,-0.1)\end{array}$ & $\begin{array}{r}-0.65 \\
(-0.8,-0.5)\end{array}$ & $\begin{array}{r}-0.25 \\
(-0.5,0)\end{array}$ \\
\hline 27801991 & $\begin{array}{l}\text { Arnaud, } \\
2017\end{array}$ & TAU & $\begin{array}{r}175 \\
(3 \mathrm{mo})\end{array}$ & No & $\begin{array}{r}2.1 \\
(1.96)\end{array}$ & $\begin{array}{r}1.07 \\
(1.82)\end{array}$ & $\begin{array}{r}-1.03 \\
(-1.4,-0.7)\end{array}$ & & $\begin{array}{r}-0.4 \\
(-0.5,-0.3)\end{array}$ & \\
\hline 20670329 & $\begin{array}{l}\text { Bernstein, } \\
2010\end{array}$ & MI & $\begin{array}{r}202 \\
(3 \mathrm{mo})\end{array}$ & No & $\begin{array}{l}3.1 \\
(5)\end{array}$ & $\begin{array}{r}3.9 \\
(3.6)\end{array}$ & $\begin{array}{r}0.8 \\
(0.1,1.5)\end{array}$ & $\begin{array}{r}-1 \\
(-1.9,-0.1)\end{array}$ & $\begin{array}{r}0.18 \\
(0,0.3)\end{array}$ & $\begin{array}{r}-0.22 \\
(-0.4,0)\end{array}$ \\
\hline 20670329 & $\begin{array}{l}\text { Bernstein, } \\
2010\end{array}$ & TAU & $\begin{array}{r}197 \\
(3 \mathrm{mo})\end{array}$ & No & $\begin{array}{l}2.7 \\
(4)\end{array}$ & $\begin{array}{r}4.5 \\
(3.6)\end{array}$ & $\begin{array}{r}1.8 \\
(1.2,2.4)\end{array}$ & & $\begin{array}{r}0.4 \\
(0.3,0.5)\end{array}$ & \\
\hline 29750362 & $\begin{array}{l}\text { Colby, } \\
2018\end{array}$ & Ml & $\begin{array}{r}80 \\
(3 \mathrm{mo}) \\
\end{array}$ & No & $\begin{array}{r}5.63 \\
(4.34) \\
\end{array}$ & $\begin{array}{r}2.41 \\
(2.99) \\
\end{array}$ & $\begin{array}{r}-3.22 \\
(-4.2,-2.2) \\
\end{array}$ & $\begin{array}{r}-1.96 \\
(-3.4,-0.5) \\
\end{array}$ & $\begin{array}{r}-0.67 \\
(-0.9,-0.5) \\
\end{array}$ & $\begin{array}{r}-0.41 \\
(-0.7,-0.1) \\
\end{array}$ \\
\hline 29750362 & $\begin{array}{l}\text { Colby, } \\
2018\end{array}$ & TAU & $\begin{array}{r}81 \\
(3 \mathrm{mo})\end{array}$ & No & $\begin{array}{r}5.19 \\
(4.18)\end{array}$ & $\begin{array}{r}3.93 \\
(4.18)\end{array}$ & $\begin{array}{r}-1.26 \\
(-2.4,-0.2)\end{array}$ & & $\begin{array}{r}-0.26 \\
(-0.5,0)\end{array}$ & \\
\hline 30138016 & $\begin{array}{l}\text { D'Amico, } \\
2018\end{array}$ & $\mathrm{Ml}$ & $\begin{array}{r}153 \\
(3 \mathrm{mo})\end{array}$ & No & $\begin{array}{r}0.43 \\
(0.51)\end{array}$ & $\begin{array}{r}0.92 \\
(1.52)\end{array}$ & $\begin{array}{r}0.49 \\
(0.3,0.7)\end{array}$ & $\begin{array}{r}-0.07 \\
(-0.4,0.3)\end{array}$ & $\begin{array}{r}0.32 \\
(0.2,0.5)\end{array}$ & $\begin{array}{r}-0.05 \\
(-0.3,0.2)\end{array}$ \\
\hline 30138016 & $\begin{array}{l}\text { D'Amico, } \\
2018\end{array}$ & TAU & $\begin{array}{r}141 \\
(3 \mathrm{mo})\end{array}$ & No & $\begin{array}{r}0.45 \\
(0.59)\end{array}$ & $\begin{array}{l}1.01 \\
(1.6)\end{array}$ & $\begin{array}{r}0.56 \\
(0.3,0.8) \\
\end{array}$ & & $\begin{array}{r}0.37 \\
(0.2,0.5) \\
\end{array}$ & \\
\hline 17563146 & $\begin{array}{l}\text { Winters, } \\
2007\end{array}$ & MI & $\begin{array}{r}52 \\
(6 \mathrm{mo}) \\
\end{array}$ & No & $\begin{array}{r}1.89 \\
(0.75) \\
\end{array}$ & $\begin{array}{r}1.07 \\
(0.68) \\
\end{array}$ & $\begin{array}{r}-0.82 \\
(-1.1,-0.6) \\
\end{array}$ & $\begin{array}{r}-0.75 \\
(-1.3,-0.2) \\
\end{array}$ & $\begin{array}{r}-0.84 \\
(-1.1,-0.6) \\
\end{array}$ & $\begin{array}{r}-0.77 \\
(-1.3,-0.2) \\
\end{array}$ \\
\hline $\begin{array}{r}\text { CN- } \\
01953820 \\
\text { (cochrane) } \\
\end{array}$ & $\begin{array}{l}\text { Giles, } \\
2019\end{array}$ & MI & $\begin{array}{r}178 \\
(12 \mathrm{mo})\end{array}$ & No & $\mathrm{nr}$ & $\begin{array}{r}1.5 \\
(1.7)\end{array}$ & $\begin{array}{r}0.3 \\
(-0.1,0.7)\end{array}$ & & & \\
\hline $\begin{array}{r}\text { CN- } \\
01953820 \\
\text { (cochrane) } \\
\end{array}$ & $\begin{array}{l}\text { Giles, } \\
2019\end{array}$ & TAU & $\begin{array}{r}196 \\
(12 \mathrm{mo})\end{array}$ & No & $\mathrm{nr}$ & $\begin{array}{r}1.8 \\
(2.2)\end{array}$ & & & & \\
\hline 17563146 & $\begin{array}{l}\text { Winters, } \\
2007\end{array}$ & TAU & $\begin{array}{r}26 \\
(6 \mathrm{mo}) \\
\end{array}$ & No & $\begin{array}{r}1.79 \\
(0.93)\end{array}$ & $\begin{array}{r}1.71 \\
(1)\end{array}$ & $\begin{array}{r}-0.07 \\
(-0.5,0.4) \\
\end{array}$ & & $\begin{array}{r}-0.07 \\
(-0.5,0.4) \\
\end{array}$ & \\
\hline $\begin{array}{r}2016-03749- \\
004 \\
\text { (psycINFO) }\end{array}$ & $\begin{array}{l}\text { Arnaud, } \\
2015\end{array}$ & $\mathrm{Ml}$ & $\begin{array}{r}715 \\
(3 \mathrm{mo})\end{array}$ & Yes & $\begin{array}{r}1.54 \\
(0.99)\end{array}$ & $\begin{array}{l}1.39 \\
(0.5)\end{array}$ & & & $\begin{array}{r}-0.15 \\
(-0.2,-0.1)\end{array}$ & $\begin{array}{r}0.01 \\
(-0.1,0.1)\end{array}$ \\
\hline
\end{tabular}




\begin{tabular}{|c|c|c|c|c|c|c|c|c|c|c|}
\hline PMID & Citation & Intervention & $\begin{array}{r}\mathrm{N} \\
\text { (endtime) }\end{array}$ & Scale & $\begin{array}{r}\text { Baseline } \\
\text { Mean } \\
(\mathrm{SD})\end{array}$ & $\begin{array}{r}\text { End Mean } \\
\text { (SD) }\end{array}$ & $\begin{array}{r}\text { MD } \\
(95 \% \mathrm{Cl})\end{array}$ & $\begin{array}{r}\text { NMD } \\
(95 \% \mathrm{Cl})\end{array}$ & $\begin{array}{r}\text { SMD } \\
(95 \% \mathrm{Cl})\end{array}$ & $\begin{array}{r}\text { SNMD } \\
(95 \% \mathrm{Cl})\end{array}$ \\
\hline $\begin{array}{r}2016-03749- \\
004 \\
\text { (psycINFO) }\end{array}$ & $\begin{array}{l}\text { Arnaud, } \\
2015\end{array}$ & TAU & $\begin{array}{r}734 \\
(3 \mathrm{mo})\end{array}$ & Yes & $\begin{array}{r}1.58 \\
(1.02)\end{array}$ & $\begin{array}{r}1.42 \\
(0.47)\end{array}$ & & & $\begin{array}{r}-0.16 \\
(-0.2,-0.1)\end{array}$ & \\
\hline 15343198 & $\begin{array}{l}\text { Spirito, } \\
2004\end{array}$ & $\mathrm{Ml}$ & $\begin{array}{r}64 \\
(3 \mathrm{mo})\end{array}$ & Yes & $\begin{array}{r}1.82 \\
(3.46)\end{array}$ & $\begin{array}{r}1 \\
(2.08)\end{array}$ & & & $\begin{array}{r}-0.2 \\
(-0.4,0)\end{array}$ & $\begin{array}{r}-0.07 \\
(-0.4,0.3)\end{array}$ \\
\hline 15343198 & $\begin{array}{l}\text { Spirito, } \\
2004\end{array}$ & TAU & $\begin{array}{r}60 \\
(3 \mathrm{mo})\end{array}$ & Yes & $\begin{array}{r}2.59 \\
(4.01)\end{array}$ & $\begin{array}{r}2.06 \\
(3.75)\end{array}$ & & & $\begin{array}{r}-0.13 \\
(-0.4,0.2)\end{array}$ & \\
\hline
\end{tabular}

Abbreviations: PMID = PubMed ID (or other ID), N=number of subjects;SD = standard deviation;End mean = mean at End;MD = mean difference;NMD = net mean difference;SMD = standardized mean difference; SNMD = standardized net mean difference; $\mathrm{MI}=$ motivational interviewing; Educ = psychoeducation; TAU = treatment as usual 
Table F-2. Brief interventions, alcohol use days

\begin{tabular}{|c|c|c|c|c|c|c|c|c|c|c|}
\hline PMID & Citation & Intervention & $\begin{array}{r}\mathrm{N} \\
\text { (endtime) }\end{array}$ & Scale & $\begin{array}{r}\text { Baseline } \\
\text { Mean } \\
(\mathrm{SD})\end{array}$ & $\begin{array}{r}\text { End Mean } \\
\text { (SD) }\end{array}$ & $\begin{array}{r}M D \\
(95 \% \mathrm{Cl})\end{array}$ & $\begin{array}{r}\text { NMD } \\
(95 \% \mathrm{Cl})\end{array}$ & $\begin{array}{r}\text { SMD } \\
(95 \% \mathrm{Cl})\end{array}$ & $\begin{array}{r}\text { SNMD } \\
(95 \% \mathrm{Cl})\end{array}$ \\
\hline 20670329 & $\begin{array}{l}\text { Bernstein, } \\
2010\end{array}$ & $\mathrm{Ml}$ & $\begin{array}{r}202 \\
(3 \mathrm{mo}) \\
\end{array}$ & No & $\begin{array}{r}6.7 \\
(6.6) \\
\end{array}$ & $\begin{array}{r}5.5 \\
(4.5) \\
\end{array}$ & $\begin{array}{r}-1.2 \\
(-2.1,-0.3) \\
\end{array}$ & $\begin{array}{r}-0.8 \\
(-2,0.4) \\
\end{array}$ & $\begin{array}{r}-0.2 \\
(-0.3,-0.1) \\
\end{array}$ & $\begin{array}{r}-0.13 \\
(-0.3,0.1) \\
\end{array}$ \\
\hline 20670329 & $\begin{array}{l}\text { Bernstein, } \\
2010\end{array}$ & TAU & $\begin{array}{r}197 \\
(3 \mathrm{mo})\end{array}$ & No & $\begin{array}{l}6.1 \\
(6)\end{array}$ & $\begin{array}{r}5.7 \\
(4.5)\end{array}$ & $\begin{array}{r}-0.4 \\
(-1.2,0.4)\end{array}$ & & $\begin{array}{r}-0.07 \\
(-0.2,0.1)\end{array}$ & \\
\hline 26362000 & $\begin{array}{l}\text { Brown, } \\
2015\end{array}$ & $\mathrm{Ml}$ & $\begin{array}{r}79 \\
(3 \mathrm{mo}) \\
\end{array}$ & No & $\begin{array}{r}3.7 \\
(5.55) \\
\end{array}$ & $\begin{array}{r}1.1 \\
(1.72)\end{array}$ & $\begin{array}{r}-2.6 \\
(-3.8,-1.4) \\
\end{array}$ & $\begin{array}{r}-2.15 \\
(-3.6,-0.7) \\
\end{array}$ & $\begin{array}{r}-0.56 \\
(-0.8,-0.3) \\
\end{array}$ & $\begin{array}{r}-0.46 \\
(-0.8,-0.1) \\
\end{array}$ \\
\hline 26362000 & $\begin{array}{l}\text { Brown, } \\
2015\end{array}$ & TAU & $\begin{array}{r}72 \\
(3 \mathrm{mo})\end{array}$ & No & $\begin{array}{r}2.35 \\
(3.49)\end{array}$ & $\begin{array}{r}1.9 \\
(2.71)\end{array}$ & $\begin{array}{r}-0.45 \\
(-1.3,0.4)\end{array}$ & & $\begin{array}{r}-0.1 \\
(-0.3,0.1)\end{array}$ & \\
\hline 29750362 & $\begin{array}{l}\text { Colby, } \\
2018\end{array}$ & $\mathrm{Ml}$ & $\begin{array}{r}82 \\
(3 \mathrm{mo})\end{array}$ & No & $\begin{array}{r}9.1 \\
(4.32)\end{array}$ & $\begin{array}{r}4.3 \\
(3.67)\end{array}$ & $\begin{array}{r}-4.81 \\
(-5.9,-3.7)\end{array}$ & $\begin{array}{r}-2.64 \\
(-4.4,-0.8)\end{array}$ & $\begin{array}{r}-0.82 \\
(-1,-0.6)\end{array}$ & $\begin{array}{r}-0.45 \\
(-0.8,-0.1)\end{array}$ \\
\hline 29750362 & $\begin{array}{l}\text { Colby, } \\
2018\end{array}$ & TAU & $\begin{array}{r}84 \\
(3 \mathrm{mo})\end{array}$ & No & $\begin{array}{r}8.02 \\
(5.67)\end{array}$ & $\begin{array}{r}5.86 \\
(5.38)\end{array}$ & $\begin{array}{r}-2.17 \\
(-3.6,-0.7)\end{array}$ & & $\begin{array}{r}-0.37 \\
(-0.6,-0.1)\end{array}$ & \\
\hline 30138016 & $\begin{array}{l}\text { D'Amico, } \\
2018\end{array}$ & $\mathrm{Ml}$ & $\begin{array}{r}153 \\
(3 \mathrm{mo})\end{array}$ & No & $\begin{array}{r}0.86 \\
(0.65)\end{array}$ & $\begin{array}{r}1.73 \\
(1.86)\end{array}$ & $\begin{array}{r}0.87 \\
(0.6,1.2)\end{array}$ & $\begin{array}{r}-0.23 \\
(-0.7,0.2)\end{array}$ & $\begin{array}{r}0.47 \\
(0.3,0.6)\end{array}$ & $\begin{array}{r}-0.13 \\
(-0.4,0.1)\end{array}$ \\
\hline 30138016 & $\begin{array}{l}\text { D'Amico, } \\
2018\end{array}$ & TAU & $\begin{array}{r}141 \\
(3 \mathrm{mo})\end{array}$ & No & $\begin{array}{r}0.78 \\
(0.64)\end{array}$ & $\begin{array}{r}1.88 \\
(1.95)\end{array}$ & $\begin{array}{r}1.1 \\
(0.8,1.4) \\
\end{array}$ & & $\begin{array}{r}0.6 \\
(0.4,0.8) \\
\end{array}$ & \\
\hline 18778385 & $\begin{array}{l}\text { McCambridge, } \\
2008\end{array}$ & $\mathrm{Ml}$ & $\begin{array}{r}164 \\
(3 \mathrm{mo}) \\
\end{array}$ & No & $\begin{array}{r}4.4 \\
(5.8) \\
\end{array}$ & $\begin{array}{r}4 \\
(5.5)\end{array}$ & $\begin{array}{r}-0.4 \\
(-1.5,0.7) \\
\end{array}$ & $\begin{array}{r}0.3 \\
(-1.27,1.87) \\
\end{array}$ & $\begin{array}{r}-0.06 \\
(-0.2,0.1) \\
\end{array}$ & $\begin{array}{r}0.04 \\
(-0.18,0.26) \\
\end{array}$ \\
\hline $\begin{array}{r}\text { CN- } \\
01953820 \\
\text { (cochrane) }\end{array}$ & Giles, 2019 & MI & $\begin{array}{r}178 \\
(12 \mathrm{mo})\end{array}$ & No & $\mathrm{nr}$ & & $\begin{array}{r}0.27 \\
(-1.4,1.9)\end{array}$ & NA & NA & NA \\
\hline $\begin{array}{r}\text { CN- } \\
01953820 \\
\text { (cochrane) }\end{array}$ & Giles, 2019 & TAU & $\begin{array}{r}196 \\
(12 \mathrm{mo})\end{array}$ & No & $\mathrm{nr}$ & & & & & \\
\hline 18778385 & $\begin{array}{l}\text { McCambridge, } \\
2008\end{array}$ & Educ & $\begin{array}{r}162 \\
(3 \mathrm{mo})\end{array}$ & No & $\begin{array}{r}4.4 \\
(6.5)\end{array}$ & $\begin{array}{r}3.7 \\
(5.7)\end{array}$ & $\begin{array}{r}-0.7 \\
(-1.9,0.5)\end{array}$ & & $\begin{array}{r}-0.1 \\
(-0.3,0.1)\end{array}$ & \\
\hline 30883284 & $\begin{array}{l}\text { McCarty, } \\
2019\end{array}$ & $\mathrm{Ml}$ & $\begin{array}{r}214 \\
(2 \mathrm{mo})\end{array}$ & No & $\begin{array}{r}2.84 \\
(3.88) \\
\end{array}$ & $\begin{array}{r}2.66 \\
(4.72) \\
\end{array}$ & $\begin{array}{r}-0.18 \\
(-0.9,0.5)\end{array}$ & $\begin{array}{r}0.18 \\
(-0.6,1)\end{array}$ & $\begin{array}{r}-0.04 \\
(-0.2,0.1)\end{array}$ & $\begin{array}{r}0.04 \\
(-0.1,0.2)\end{array}$ \\
\hline 30883284 & $\begin{array}{l}\text { McCarty, } \\
2019\end{array}$ & TAU & $\begin{array}{r}214 \\
(2 \mathrm{mo})\end{array}$ & No & $\begin{array}{r}2.5 \\
(2.78)\end{array}$ & $\begin{array}{r}2.14 \\
(2.32)\end{array}$ & $\begin{array}{r}-0.36 \\
(-0.8,0.1)\end{array}$ & & $\begin{array}{r}-0.08 \\
(-0.2,0)\end{array}$ & \\
\hline 22000326 & $\begin{array}{l}\text { Winters, } \\
2012\end{array}$ & $\mathrm{Ml}$ & $\begin{array}{r}256 \\
(6 \mathrm{mo})\end{array}$ & No & $\begin{array}{r}1.51 \\
(2.25)\end{array}$ & $\begin{array}{r}1.13 \\
(1.73)\end{array}$ & $\begin{array}{r}-0.38 \\
(-0.7,-0.1)\end{array}$ & $\begin{array}{r}-2.38 \\
(-3.5,-1.3)\end{array}$ & $\begin{array}{r}-0.14 \\
(-0.2,0)\end{array}$ & $\begin{array}{r}-0.86 \\
(-1.3,-0.5)\end{array}$ \\
\hline
\end{tabular}




\begin{tabular}{|c|c|c|c|c|c|c|c|c|c|c|}
\hline PMID & Citation & Intervention & $\begin{array}{r}\mathrm{N} \\
\text { (endtime) }\end{array}$ & Scale & $\begin{array}{r}\text { Baseline } \\
\text { Mean } \\
(\mathrm{SD})\end{array}$ & $\begin{array}{r}\text { End Mean } \\
\text { (SD) }\end{array}$ & $\begin{array}{r}\text { MD } \\
(95 \% \mathrm{Cl})\end{array}$ & $\begin{array}{r}\text { NMD } \\
(95 \% \mathrm{Cl})\end{array}$ & $\begin{array}{r}\text { SMD } \\
(95 \% \mathrm{Cl})\end{array}$ & $\begin{array}{r}\text { SNMD } \\
(95 \% \mathrm{Cl})\end{array}$ \\
\hline 22000326 & $\begin{array}{l}\text { Winters, } \\
2012\end{array}$ & TAU & $\begin{array}{r}55 \\
(6 \mathrm{mo})\end{array}$ & No & $\begin{array}{r}1.5 \\
(2.23)\end{array}$ & $\begin{array}{r}3.5 \\
(3.93)\end{array}$ & $\begin{array}{r}2 \\
(0.9,3.1)\end{array}$ & & $\begin{array}{r}0.72 \\
(0.3,1.1)\end{array}$ & \\
\hline 17563146 & $\begin{array}{l}\text { Winters, } \\
2007\end{array}$ & MI & $\begin{array}{r}52 \\
(6 \mathrm{mo})\end{array}$ & No & $\begin{array}{r}4.46 \\
(0.76)\end{array}$ & $\begin{array}{r}2.96 \\
(0.76)\end{array}$ & $\begin{array}{r}-1.5 \\
(-1.8,-1.2)\end{array}$ & $\begin{array}{r}-1.21 \\
(-1.7,-0.7)\end{array}$ & $\begin{array}{r}-1.56 \\
(-1.8,-1.3)\end{array}$ & $\begin{array}{r}-1.27 \\
(-1.8,-0.8)\end{array}$ \\
\hline 17563146 & $\begin{array}{l}\text { Winters, } \\
2007\end{array}$ & TAU & $\begin{array}{r}26 \\
(6 \mathrm{mo})\end{array}$ & No & $\begin{array}{r}4.36 \\
(0.93)\end{array}$ & $\begin{array}{r}4.07 \\
(0.79)\end{array}$ & $\begin{array}{r}-0.29 \\
(-0.7,0.1)\end{array}$ & & $\begin{array}{r}-0.3 \\
(-0.7,0.1)\end{array}$ & \\
\hline $\begin{array}{r}\text { 2016-03749- } \\
004 \\
\text { (psycINFO) }\end{array}$ & $\begin{array}{l}\text { Arnaud, } \\
2015\end{array}$ & MI & $\begin{array}{r}715 \\
(3 \mathrm{mo})\end{array}$ & Yes & $\begin{array}{r}1.98 \\
(0.81)\end{array}$ & $\begin{array}{r}1.75 \\
(0.47)\end{array}$ & & & $\begin{array}{r}-0.24 \\
(-0.3,-0.2)\end{array}$ & $\begin{array}{r}-0.24 \\
(-0.3,-0.1)\end{array}$ \\
\hline $\begin{array}{r}2016-03749- \\
004 \\
\text { (psycINFO) }\end{array}$ & $\begin{array}{l}\text { Arnaud, } \\
2015\end{array}$ & TAU & $\begin{array}{r}734 \\
(3 \mathrm{mo})\end{array}$ & Yes & $\begin{array}{l}1.93 \\
(0.9)\end{array}$ & $\begin{array}{l}1.93 \\
(0.9)\end{array}$ & & & $\begin{array}{r}0 \\
(-0.1,0.1)\end{array}$ & \\
\hline 15343198 & $\begin{array}{l}\text { Spirito, } \\
2004\end{array}$ & MI & $\begin{array}{r}64 \\
(3 \mathrm{mo})\end{array}$ & Yes & $\begin{array}{r}3.53 \\
(4.67)\end{array}$ & $\begin{array}{r}2.55 \\
(4.06)\end{array}$ & & & $\begin{array}{r}-0.17 \\
(-0.4,0.1)\end{array}$ & $\begin{array}{r}-0.06 \\
(-0.4,0.3)\end{array}$ \\
\hline 15343198 & $\begin{array}{l}\text { Spirito, } \\
2004\end{array}$ & TAU & $\begin{array}{r}60 \\
(3 \mathrm{mo})\end{array}$ & Yes & $\begin{array}{r}4.18 \\
(4.97)\end{array}$ & $\begin{array}{r}3.54 \\
(5.39)\end{array}$ & & & $\begin{array}{r}-0.11 \\
(-0.4,0.2)\end{array}$ & \\
\hline
\end{tabular}

Abbreviations: PMID = PubMed ID (or other ID), N=number subjects;SD = standard deviation;End mean = mean at End;MD = mean difference;NMD = net mean difference;SMD = standardized mean difference; SNMD = standardized net mean difference; $\mathrm{MI}$ = motivational interviewing; Educ = psychoeducation; TAU = treatment as usual; $\mathrm{NA}$ = not applicable

Table F-3. Brief interventions, alcohol abstinence

\begin{tabular}{|c|c|c|c|c|c|c|}
\hline PMID & Citation & Intervention & $x$ & $N$ & $\begin{array}{l}\log \text { OR } \\
(95 \% \mathrm{Cl})\end{array}$ & $\begin{array}{l}\text { OR } \\
\text { (95\% Cl) }\end{array}$ \\
\hline $\begin{array}{r}2016-03749-004 \\
\text { (psycINFO) }\end{array}$ & $\begin{array}{r}\text { Arnaud, } \\
2015\end{array}$ & Ml & 491 & 715 & $\begin{array}{l}0.24 \\
(95 \% \mathrm{Cl}: 0.03,0.46)\end{array}$ & $\begin{array}{l}1.28 \\
(95 \% \mathrm{Cl}: 1.03,1.59)\end{array}$ \\
\hline $\begin{array}{r}2016-03749-004 \\
\text { (psycINFO) }\end{array}$ & $\begin{array}{r}\text { Arnaud, } \\
2015\end{array}$ & TAU & 464 & 734 & & \\
\hline 26362000 & $\begin{array}{r}\text { Brown, } \\
2015\end{array}$ & Ml & 23 & 69 & $\begin{array}{l}-0.52 \\
(95 \% \mathrm{Cl}:-1.21,0.17)\end{array}$ & $\begin{array}{l}0.59 \\
(95 \% \mathrm{Cl}: 0.3,1.18)\end{array}$ \\
\hline 26362000 & $\begin{array}{r}\text { Brown, } \\
2015\end{array}$ & TAU & 32 & 70 & & \\
\hline $\begin{array}{r}2009-05582-007 \\
\text { (psyclNFO) }\end{array}$ & $\begin{array}{r}\text { Martínez Martínez, } \\
2008\end{array}$ & $\mathrm{Ml}$ & 8 & 23 & $\begin{array}{l}2.95 \\
(95 \% \text { Cl: } 0.02,5.89)\end{array}$ & $\begin{array}{l}19.19 \\
(95 \% \text { Cl: } 1.02,360.51)\end{array}$ \\
\hline
\end{tabular}




\begin{tabular}{|c|c|c|c|c|c|c|c|}
\hline & PMID & Citation & Intervention & $x$ & $\mathrm{~N}$ & $\begin{array}{l}\log \text { OR } \\
(95 \% \mathrm{Cl})\end{array}$ & $\begin{array}{l}\text { OR } \\
(95 \% \mathrm{Cl})\end{array}$ \\
\hline & $\begin{array}{r}2009-05582-007 \\
\text { (psycINFO) }\end{array}$ & $\begin{array}{r}\text { Martínez Martínez, } \\
2008\end{array}$ & TAU & 0 & 17 & & \\
\hline & 14678061 & $\begin{array}{r}\text { McCambridge, } \\
2004\end{array}$ & Ml & 7 & 86 & $\begin{array}{l}1.74 \\
(95 \% \mathrm{Cl}:-0.39,3.86)\end{array}$ & $\begin{array}{l}5.67 \\
(95 \% \mathrm{Cl}: 0.68,47.29)\end{array}$ \\
\hline & 14678061 & $\begin{array}{r}\text { McCambridge, } \\
2004\end{array}$ & TAU & 1 & 65 & & \\
\hline & 18778385 & $\begin{array}{r}\text { McCambridge, } \\
2008\end{array}$ & Ml & 71 & 164 & $\begin{array}{l}0.26 \\
(95 \% \mathrm{Cl}:-0.18,0.7)\end{array}$ & $\begin{array}{l}1.3 \\
\text { (95\% Cl: } 0.83,2.02)\end{array}$ \\
\hline & 18778385 & $\begin{array}{r}\text { McCambridge, } \\
2008\end{array}$ & Educ & 60 & 162 & & \\
\hline & 29252011 & $\begin{array}{r}\text { Spirito, } \\
2017\end{array}$ & Ml & 11 & 32 & $\begin{array}{l}0.19 \\
(95 \% \mathrm{Cl}:-0.85,1.23)\end{array}$ & $\begin{array}{l}1.2 \\
(95 \% \mathrm{Cl}: 0.43,3.41)\end{array}$ \\
\hline & 29252011 & $\begin{array}{r}\text { Spirito, } \\
2017 \\
\end{array}$ & Educ & 10 & 33 & & \\
\hline & 22000326 & $\begin{array}{r}\text { Winters, } \\
2012\end{array}$ & Ml & 130 & 256 & $\begin{array}{l}1.04 \\
(95 \% \mathrm{Cl}: 0.4,1.68)\end{array}$ & $\begin{array}{l}2.82 \\
(95 \% \text { Cl: } 1.49,5.35)\end{array}$ \\
\hline & 22000326 & $\begin{array}{r}\text { Winters, } \\
2012\end{array}$ & TAU & 15 & 56 & & \\
\hline 22000326 & $\begin{array}{r}\text { Winters, } \\
2012\end{array}$ & TAU & 15 & & & & \\
\hline
\end{tabular}

Abbreviations: PMID = PubMed ID (or other ID), N=number of subjects; $x=$ number abstinent; $\mathrm{N}=$ number of subjects; Log OR=log(odds ratio); OR=odds ratio; 95\%CI=95\% confidence interval ; MI = motivational interviewing; Educ = psychoeducation; TAU = treatment as usual

Table F-4. Brief interventions, cannabis use days

\begin{tabular}{|c|c|c|c|c|c|c|c|c|c|c|}
\hline PMID & Citation & Intervention & $\begin{array}{r}\mathrm{N} \\
\text { (endtime) }\end{array}$ & Scale & $\begin{array}{r}\text { Baseline } \\
\text { Mean } \\
\text { (SD) }\end{array}$ & $\begin{array}{r}\text { End Mean } \\
\text { (SD) }\end{array}$ & $\begin{array}{r}\text { MD } \\
(95 \% \mathrm{Cl})\end{array}$ & $\begin{array}{r}\text { NMD } \\
(95 \% \mathrm{Cl})\end{array}$ & $\begin{array}{r}\text { SMD } \\
(95 \% \mathrm{Cl})\end{array}$ & $\begin{array}{r}\text { SNMD } \\
(95 \% \mathrm{Cl})\end{array}$ \\
\hline 20053238 & $\begin{array}{l}\text { Bernstein, } \\
2009\end{array}$ & $\mathrm{Ml}$ & $\begin{array}{r}41 \\
(3 \mathrm{mo})\end{array}$ & No & $\begin{array}{r}19 \\
(10.9)\end{array}$ & $\begin{array}{r}14.2 \\
(10.8)\end{array}$ & $\begin{array}{r}-4.8 \\
(-8.5,-1.1)\end{array}$ & $\begin{array}{r}-3.2 \\
(-8.1,1.7)\end{array}$ & $\begin{array}{r}-0.4 \\
(-0.7,-0.1)\end{array}$ & $\begin{array}{r}-0.27 \\
(-0.7,0.1)\end{array}$ \\
\hline 20053238 & $\begin{array}{l}\text { Bernstein, } \\
2009\end{array}$ & TAU & $\begin{array}{r}54 \\
(3 \mathrm{mo})\end{array}$ & No & $\begin{array}{r}15.3 \\
(10.1)\end{array}$ & $\begin{array}{r}13.7 \\
(11.1)\end{array}$ & $\begin{array}{r}-1.6 \\
(-4.9,1.7)\end{array}$ & & $\begin{array}{r}-0.13 \\
(-0.4,0.1)\end{array}$ & \\
\hline $\begin{array}{r}132804409 \\
\text { (embase) }\end{array}$ & $\begin{array}{l}\text { Braciszewski, } \\
2018\end{array}$ & $\mathrm{Ml}$ & $\begin{array}{r}12 \\
(3 \mathrm{mo})\end{array}$ & No & $\begin{array}{r}19.79 \\
(12)\end{array}$ & $\begin{array}{r}14.09 \\
(11.83)\end{array}$ & $\begin{array}{r}-5.69 \\
(-13.6,2.3)\end{array}$ & $\begin{array}{r}-2.22 \\
(-12.3,7.9)\end{array}$ & $\begin{array}{r}-0.43 \\
(-1,0.2)\end{array}$ & $\begin{array}{r}-0.17 \\
(-0.9,0.6)\end{array}$ \\
\hline $\begin{array}{r}132804409 \\
\text { (embase) }\end{array}$ & $\begin{array}{l}\text { Braciszewski, } \\
2018\end{array}$ & TAU & $\begin{array}{r}18 \\
(3 \mathrm{mo})\end{array}$ & No & $\begin{array}{r}22.1 \\
(10.61)\end{array}$ & $\begin{array}{r}18.63 \\
(11.78)\end{array}$ & $\begin{array}{r}-3.47 \\
(-9.8,2.8)\end{array}$ & & $\begin{array}{r}-0.26 \\
(-0.7,0.2)\end{array}$ & \\
\hline
\end{tabular}




\begin{tabular}{|c|c|c|c|c|c|c|c|c|c|c|}
\hline PMID & Citation & Intervention & $\begin{array}{r}\mathrm{N} \\
\text { (endtime) }\end{array}$ & Scale & $\begin{array}{r}\text { Baseline } \\
\text { Mean } \\
\text { (SD) }\end{array}$ & $\begin{array}{r}\text { End Mean } \\
\text { (SD) }\end{array}$ & $\begin{array}{r}M D \\
(95 \% \mathrm{Cl})\end{array}$ & $\begin{array}{r}\text { NMD } \\
(95 \% \mathrm{Cl})\end{array}$ & $\begin{array}{r}\text { SMD } \\
(95 \% \mathrm{Cl})\end{array}$ & $\begin{array}{r}\text { SNMD } \\
(95 \% \mathrm{Cl})\end{array}$ \\
\hline 26362000 & $\begin{array}{l}\text { Brown, } \\
2015\end{array}$ & $\mathrm{Ml}$ & $\begin{array}{r}79 \\
(3 \mathrm{mo})\end{array}$ & No & $\begin{array}{r}14.9 \\
(10.1)\end{array}$ & $\begin{array}{r}9 \\
(8.91)\end{array}$ & $\begin{array}{r}-5.9 \\
(-8.5,-3.3) \\
\end{array}$ & $\begin{array}{r}-0.5 \\
(-4.3,3.3) \\
\end{array}$ & $\begin{array}{r}-0.5 \\
(-0.7,-0.3) \\
\end{array}$ & $\begin{array}{r}-0.04 \\
(-0.4,0.3)\end{array}$ \\
\hline 26362000 & $\begin{array}{l}\text { Brown, } \\
2015\end{array}$ & TAU & $\begin{array}{r}72 \\
(3 \mathrm{mo})\end{array}$ & No & $\begin{array}{r}14.6 \\
(10.8)\end{array}$ & $\begin{array}{r}9.2 \\
(9.05)\end{array}$ & $\begin{array}{r}-5.4 \\
(-8.2,-2.6)\end{array}$ & & $\begin{array}{r}-0.46 \\
(-0.7,-0.2)\end{array}$ & \\
\hline 30138016 & $\begin{array}{l}\text { D'Amico, } \\
2018\end{array}$ & $\mathrm{Ml}$ & $\begin{array}{r}153 \\
(3 \mathrm{mo})\end{array}$ & No & $\begin{array}{l}0.82 \\
(0.7)\end{array}$ & $\begin{array}{r}2.13 \\
(2.68)\end{array}$ & $\begin{array}{r}1.3 \\
(0.9,1.7)\end{array}$ & $\begin{array}{r}0.1 \\
(-0.5,0.7)\end{array}$ & $\begin{array}{r}0.52 \\
(0.4,0.7)\end{array}$ & $\begin{array}{r}0.04 \\
(-0.2,0.3)\end{array}$ \\
\hline 30138016 & $\begin{array}{l}\text { D'Amico, } \\
2018\end{array}$ & TAU & $\begin{array}{r}141 \\
(3 \mathrm{mo})\end{array}$ & No & $\begin{array}{r}0.78 \\
(0.68)\end{array}$ & $\begin{array}{r}1.98 \\
(2.53)\end{array}$ & $\begin{array}{r}1.2 \\
(0.8,1.6)\end{array}$ & & $\begin{array}{r}0.48 \\
(0.3,0.6)\end{array}$ & \\
\hline 24969735 & $\begin{array}{l}\text { de Gee, } \\
2014\end{array}$ & Ml & $\begin{array}{r}45 \\
(3 \mathrm{mo})\end{array}$ & No & $\begin{array}{l}19.71 \\
(9.43)\end{array}$ & $\begin{array}{l}18.86 \\
(9.86)\end{array}$ & $\begin{array}{r}-0.86 \\
(-4.1,2.4)\end{array}$ & $\begin{array}{r}0 \\
(-10.8,10.8) \\
\end{array}$ & $\begin{array}{r}-0.07 \\
(-0.4,0.2)\end{array}$ & $\begin{array}{r}0 \\
(-0.1,0.1\end{array}$ \\
\hline 24969735 & $\begin{array}{l}\text { de Gee, } \\
2014\end{array}$ & Educ & $\begin{array}{r}53 \\
(3 \mathrm{mo})\end{array}$ & No & $\begin{array}{l}18.43 \\
(9.43)\end{array}$ & $\begin{array}{r}17.57 \\
(10.71)\end{array}$ & $\begin{array}{r}-0.86 \\
(-4.1,2.4)\end{array}$ & & $\begin{array}{r}-0.07 \\
(-0.4,0.2)\end{array}$ & \\
\hline 17869051 & $\begin{array}{l}\text { Martin, } \\
2008\end{array}$ & $\mathrm{Ml}$ & $\begin{array}{r}20 \\
(3 \mathrm{mo})\end{array}$ & No & $\begin{array}{l}24.7 \\
(8.2)\end{array}$ & $\begin{array}{r}18.1 \\
(12.03)\end{array}$ & $\begin{array}{r}-6.6 \\
(-12.2,-1)\end{array}$ & $\begin{array}{r}-6.3 \\
(-14.2,1.6)\end{array}$ & $\begin{array}{r}-0.53 \\
(-1,-0.1)\end{array}$ & $\begin{array}{r}-0.5 \\
(-1.1,0.1)\end{array}$ \\
\hline 17869051 & $\begin{array}{l}\text { Martin, } \\
2008\end{array}$ & TAU & $\begin{array}{r}20 \\
(3 \mathrm{mo})\end{array}$ & No & $\begin{array}{r}18.47 \\
(10.47)\end{array}$ & $\begin{array}{r}18.17 \\
(10.53)\end{array}$ & $\begin{array}{r}-0.3 \\
(-5.9,5.3)\end{array}$ & & $\begin{array}{r}-0.02 \\
(-0.5,0.4)\end{array}$ & \\
\hline 18778385 & $\begin{array}{l}\text { McCambridge, } \\
2008\end{array}$ & $\mathrm{Ml}$ & $\begin{array}{r}164 \\
(3 \mathrm{mo})\end{array}$ & No & $\begin{array}{l}17.3 \\
(9.8)\end{array}$ & $\begin{array}{r}14.6 \\
(11.7)\end{array}$ & $\begin{array}{r}-2.7 \\
(-4.7,-0.7)\end{array}$ & $\begin{array}{r}-0.3 \\
(-4.6,4) \\
\end{array}$ & $\begin{array}{r}-0.2 \\
(-0.4,-0.1)\end{array}$ & $\begin{array}{r}-0.02 \\
(0,0))\end{array}$ \\
\hline 18778385 & $\begin{array}{l}\text { McCambridge, } \\
2008\end{array}$ & Educ & $\begin{array}{r}162 \\
(3 \mathrm{mo})\end{array}$ & No & $\begin{array}{r}18.3 \\
(10.4)\end{array}$ & $\begin{array}{r}15.9 \\
(11.6)\end{array}$ & $\begin{array}{r}-2.4 \\
(-4.5,-0.3)\end{array}$ & & $\begin{array}{r}-0.18 \\
(-0.3,0)\end{array}$ & \\
\hline 30883284 & $\begin{array}{l}\text { McCarty, } \\
2019\end{array}$ & $\mathrm{Ml}$ & $\begin{array}{r}214 \\
(2 \mathrm{mo}) \\
\end{array}$ & No & $\begin{array}{r}4.78 \\
(8.12) \\
\end{array}$ & $\begin{array}{r}3.92 \\
(7.54) \\
\end{array}$ & $\begin{array}{r}-0.86 \\
(-2.1,0.4) \\
\end{array}$ & $\begin{array}{r}0.81 \\
(-1,2.7) \\
\end{array}$ & $\begin{array}{r}-0.09 \\
(-0.2,0) \\
\end{array}$ & $\begin{array}{r}0.08 \\
(-0.1,0.3) \\
\end{array}$ \\
\hline 30883284 & $\begin{array}{l}\text { McCarty, } \\
2019\end{array}$ & TAU & $\begin{array}{r}214 \\
(2 \mathrm{mo})\end{array}$ & No & $\begin{array}{r}5.47 \\
(9.15)\end{array}$ & $\begin{array}{r}3.8 \\
(6.72)\end{array}$ & $\begin{array}{r}-1.67 \\
(-3,-0.3)\end{array}$ & & $\begin{array}{r}-0.17 \\
(-0.3,0)\end{array}$ & \\
\hline 16938063 & $\begin{array}{l}\text { Peterson, } \\
2006\end{array}$ & $\mathrm{Ml}$ & $\begin{array}{r}69 \\
(3 \mathrm{mo})\end{array}$ & No & $\begin{array}{r}15.77 \\
(11.05)\end{array}$ & $\begin{array}{r}11.83 \\
(11.74)\end{array}$ & $\begin{array}{r}-3.94 \\
(-7.2,-0.6)\end{array}$ & $\begin{array}{r}0.5 \\
(-4.1,5.1)\end{array}$ & $\begin{array}{r}-0.28 \\
(-0.5,0)\end{array}$ & $\begin{array}{r}0.04 \\
(-0.3,0.4)\end{array}$ \\
\hline 16938063 & $\begin{array}{l}\text { Peterson, } \\
2006\end{array}$ & TAU & $\begin{array}{r}77 \\
(3 \mathrm{mo})\end{array}$ & No & $\begin{array}{r}16.58 \\
(11.83)\end{array}$ & $\begin{array}{r}12.14 \\
(12.08)\end{array}$ & $\begin{array}{r}-4.44 \\
(-7.7,-1.2)\end{array}$ & & $\begin{array}{r}-0.31 \\
(-0.5,-0.1)\end{array}$ & \\
\hline 29252011 & $\begin{array}{l}\text { Spirito, } \\
2017\end{array}$ & $\mathrm{Ml}$ & $\begin{array}{r}32 \\
(3 \mathrm{mo})\end{array}$ & No & $\begin{array}{l}11.67 \\
(9.67)\end{array}$ & $\begin{array}{r}9 \\
(11.67)\end{array}$ & $\begin{array}{r}-2.67 \\
(-7.2,1.8)\end{array}$ & $\begin{array}{r}0 \\
(-22.5,22.5))\end{array}$ & $\begin{array}{r}-0.2 \\
(-0.5,0.1)\end{array}$ & $\begin{array}{r}0 \\
(-0.1,0.1) \\
\end{array}$ \\
\hline 29252011 & $\begin{array}{l}\text { Spirito, } \\
2017\end{array}$ & Educ & $\begin{array}{r}33 \\
(3 \mathrm{mo})\end{array}$ & No & $\begin{array}{r}16.33 \\
(11.67)\end{array}$ & $\begin{array}{r}13.67 \\
(12)\end{array}$ & $\begin{array}{r}-2.67 \\
(-7.5,2.2)\end{array}$ & & $\begin{array}{r}-0.2 \\
(-0.6,0.2)\end{array}$ & \\
\hline
\end{tabular}




\begin{tabular}{|c|c|c|c|c|c|c|c|c|c|c|}
\hline PMID & Citation & Intervention & $\begin{array}{r}\mathrm{N} \\
\text { (endtime) }\end{array}$ & Scale & $\begin{array}{r}\text { Baseline } \\
\text { Mean } \\
\text { (SD) }\end{array}$ & $\begin{array}{r}\text { End Mean } \\
\text { (SD) }\end{array}$ & $\begin{array}{r}\mathrm{MD} \\
(95 \% \mathrm{Cl})\end{array}$ & $\begin{array}{r}\text { NMD } \\
(95 \% \mathrm{Cl})\end{array}$ & $\begin{array}{r}\text { SMD } \\
(95 \% \mathrm{Cl})\end{array}$ & $\begin{array}{r}\text { SNMD } \\
(95 \% \mathrm{Cl})\end{array}$ \\
\hline 21688877 & $\begin{array}{l}\text { Walker, } \\
2011\end{array}$ & $\mathrm{Ml}$ & $\begin{array}{r}101 \\
(3 \mathrm{mo})\end{array}$ & No & $\begin{array}{l}19.74 \\
(6.71)\end{array}$ & $\begin{array}{r}15.9 \\
(9.84)\end{array}$ & $\begin{array}{r}-3.84 \\
(-5.9,-1.8)\end{array}$ & $\begin{array}{r}-3.48 \\
(-6.3,-0.6)\end{array}$ & $\begin{array}{r}-0.37 \\
(-0.6,-0.2)\end{array}$ & $\begin{array}{r}-0.33 \\
(-0.6,-0.1)\end{array}$ \\
\hline 21688877 & $\begin{array}{l}\text { Walker, } \\
2011\end{array}$ & Educ & $\begin{array}{r}100 \\
(3 \mathrm{mo})\end{array}$ & No & $\begin{array}{l}19.74 \\
(7.22)\end{array}$ & $\begin{array}{l}17.27 \\
(9.89)\end{array}$ & $\begin{array}{r}-2.47 \\
(-4.6,-0.4)\end{array}$ & $-1.37(-5.7,3)$ & $\begin{array}{r}-0.24 \\
(-0.4,0)\end{array}$ & $\begin{array}{r}-0.13 \\
(-0.2,-0.1)\end{array}$ \\
\hline 21688877 & $\begin{array}{l}\text { Walker, } \\
2011\end{array}$ & TAU & $\begin{array}{r}104 \\
(3 \mathrm{mo})\end{array}$ & No & $\begin{array}{l}19.09 \\
(7.28)\end{array}$ & $\begin{array}{l}18.73 \\
(9.49)\end{array}$ & $\begin{array}{r}-0.36 \\
(-2.4,1.6)\end{array}$ & & $\begin{array}{r}-0.03 \\
(-0.2,0.2)\end{array}$ & \\
\hline 16822119 & $\begin{array}{l}\text { Walker, } \\
2006\end{array}$ & $\mathrm{Ml}$ & $\begin{array}{r}47 \\
(3 \mathrm{mo})\end{array}$ & No & $\begin{array}{l}19.85 \\
(8.51)\end{array}$ & $\begin{array}{r}15.53 \\
(11.64)\end{array}$ & $\begin{array}{r}-4.33 \\
(-7.9,-0.7)\end{array}$ & $\begin{array}{r}-2.29 \\
(-7.1,2.5)\end{array}$ & $\begin{array}{r}-0.36 \\
(-0.7,-0.1)\end{array}$ & $\begin{array}{r}-0.19 \\
(-0.6,0.2)\end{array}$ \\
\hline 16822119 & $\begin{array}{l}\text { Walker, } \\
2006\end{array}$ & TAU & $\begin{array}{r}50 \\
(3 \mathrm{mo})\end{array}$ & No & $\begin{array}{l}18.41 \\
(8.47)\end{array}$ & $\begin{array}{l}16.38 \\
(10.3)\end{array}$ & $\begin{array}{r}-2.03 \\
(-5.2,1.2)\end{array}$ & & $\begin{array}{r}-0.17 \\
(-0.4,0.1)\end{array}$ & \\
\hline 22000326 & $\begin{array}{l}\text { Winters, } \\
2012\end{array}$ & $\mathrm{Ml}$ & $\begin{array}{r}256 \\
(6 \mathrm{mo})\end{array}$ & No & $\begin{array}{r}7.94 \\
(10.34)\end{array}$ & $\begin{array}{r}3.36 \\
(5.41)\end{array}$ & $\begin{array}{r}-4.59 \\
(-5.9,-3.3)\end{array}$ & $\begin{array}{r}0.58 \\
(-1.7,2.9)\end{array}$ & $\begin{array}{r}-0.46 \\
(-0.6,-0.3)\end{array}$ & $\begin{array}{r}0.06 \\
(-0.2,0.3)\end{array}$ \\
\hline 22000326 & $\begin{array}{l}\text { Winters, } \\
2012\end{array}$ & TAU & $\begin{array}{r}55 \\
(6 \mathrm{mo})\end{array}$ & No & $\begin{array}{l}10.13 \\
(6.03)\end{array}$ & $\begin{array}{r}4.97 \\
(6.03)\end{array}$ & $\begin{array}{r}-5.17 \\
(-7.1,-3.2)\end{array}$ & & $\begin{array}{r}-0.52 \\
(-0.7,-0.3)\end{array}$ & \\
\hline
\end{tabular}

Abbreviations: PMID = PubMed ID (or other ID), N=number randomized;SD = standard deviation;End mean = mean at End;MD = mean difference;NMD = net mean

difference;SMD = standardized mean difference; SNMD = standardized net mean difference; $\mathrm{MI}$ = motivational interviewing; Educ = psychoeducation; TAU = treatment as usual

Table F-5. Brief interventions, cannabis abstinence

\begin{tabular}{|c|c|c|c|c|c|c|}
\hline PMID & Citation & Intervention & $x$ & $\mathrm{~N}$ & $\begin{array}{l}\log \text { OR } \\
(95 \% \mathrm{Cl})\end{array}$ & $\begin{array}{l}\text { OR } \\
\text { (95\% Cl) }\end{array}$ \\
\hline 20053238 & $\begin{array}{r}\text { Bernstein, } \\
2009\end{array}$ & $\mathrm{Ml}$ & 6 & 42 & $\begin{array}{l}0.13 \\
(95 \% \mathrm{Cl}:-1.04,1.31)\end{array}$ & $\begin{array}{l}1.14 \\
\text { (95\% Cl: } 0.35,3.69)\end{array}$ \\
\hline 20053238 & $\begin{array}{r}\text { Bernstein, } \\
2009\end{array}$ & TAU & 7 & 55 & & \\
\hline 26362000 & $\begin{array}{r}\text { Brown, } \\
2015\end{array}$ & $\mathrm{Ml}$ & 16 & 69 & $\begin{array}{l}-0.55 \\
(95 \% \mathrm{Cl}:-1.29,0.2)\end{array}$ & $\begin{array}{l}0.58 \\
(95 \% \mathrm{Cl}: 0.27,1.22)\end{array}$ \\
\hline 26362000 & $\begin{array}{r}\text { Brown, } \\
2015\end{array}$ & TAU & 24 & 70 & & \\
\hline 14678061 & $\begin{array}{r}\text { McCambridge, } \\
2004\end{array}$ & $\mathrm{Ml}$ & 16 & 97 & $\begin{array}{l}1.35 \\
\text { (95\% Cl: } 0.21,2.49)\end{array}$ & $\begin{array}{l}3.85 \\
(95 \% \mathrm{Cl}: 1.23,12.03)\end{array}$ \\
\hline 14678061 & $\begin{array}{r}\text { McCambridge, } \\
2004\end{array}$ & TAU & 4 & 82 & & \\
\hline
\end{tabular}




\begin{tabular}{|c|c|c|c|c|c|c|}
\hline PMID & Citation & Intervention & $x$ & $\mathrm{~N}$ & $\begin{array}{l}\log \text { OR } \\
(95 \% \mathrm{Cl})\end{array}$ & $\begin{array}{l}\text { OR } \\
(95 \% \mathrm{Cl})\end{array}$ \\
\hline 18778385 & $\begin{array}{r}\text { McCambridge, } \\
2008\end{array}$ & Ml & 35 & 164 & $\begin{array}{l}0.35 \\
(95 \% \mathrm{Cl}:-0.21,0.91)\end{array}$ & $\begin{array}{l}1.42 \\
(95 \% \mathrm{Cl}: 0.81,2.49)\end{array}$ \\
\hline 18778385 & $\begin{array}{r}\text { McCambridge, } \\
2008\end{array}$ & Educ & 26 & 162 & & \\
\hline 29252011 & $\begin{array}{r}\text { Spirito, } \\
2017\end{array}$ & MI & 10 & 32 & $\begin{array}{l}1.51 \\
\text { (95\% Cl: } 0.11,2.92)\end{array}$ & $\begin{array}{l}4.55 \\
(95 \% \text { Cl: } 1.12,18.48)\end{array}$ \\
\hline 29252011 & $\begin{array}{r}\text { Spirito, } \\
2017\end{array}$ & Educ & 3 & 33 & & \\
\hline 22000326 & $\begin{array}{r}\text { Winters, } \\
2012\end{array}$ & $\mathrm{Ml}$ & 145 & 257 & $\begin{array}{l}0.77 \\
(95 \% \mathrm{Cl}: 0.17,1.36)\end{array}$ & $\begin{array}{l}2.16 \\
(95 \% \text { Cl: } 1.19,3.91)\end{array}$ \\
\hline 22000326 & $\begin{array}{r}\text { Winters, } \\
2012\end{array}$ & TAU & 21 & 56 & & \\
\hline
\end{tabular}

Abbreviations: PMID = PubMed ID (or other database ID), N=number of subjects; $\mathrm{x}=$ number abstinent; $\mathrm{N}=$ number of subjects; Log OR=log(odds ratio); OR=odds ratio; 95\% CI=95\% confidence interval; $\mathrm{MI}=$ motivational interviewing; Educ = psychoeducation; $\mathrm{TAU}=$ treatment as usual 
Table F-6. Brief interventions, substance use problem scales reported

\begin{tabular}{|c|c|c|c|c|c|}
\hline Score Name & Description & $\begin{array}{l}\text { Item } \\
\text { Number }\end{array}$ & $\begin{array}{l}\text { Score } \\
\text { Range }\end{array}$ & $\begin{array}{l}\text { Score } \\
\text { Direction }\end{array}$ & Score Source \\
\hline $\begin{array}{l}\text { Brief Young } \\
\text { Adult Alcohol } \\
\text { Consequences } \\
\text { Questionnaire } \\
\text { (BYAACQ) }\end{array}$ & $\begin{array}{l}\text { This scale can help assess alcohol problems among college } \\
\text { students, track changes in alcohol problems throughout college, and } \\
\text { measure the response to alcohol interventions. It consists of 24- } \\
\text { items and was derived from the 48-item Young Adult Alcohol } \\
\text { Consequences Questionnaire. The B-YAACQ has items that tap the } \\
\text { full range of the alcohol problems continuum from signs of excessive } \\
\text { drinking to symptoms consistent with alcohol abuse and alcohol } \\
\text { dependence. }\end{array}$ & 24 & $0-24$ & $\begin{array}{l}\text { higher score, } \\
\text { greater } \\
\text { problems }\end{array}$ & $\begin{array}{l}\text { Kahler CW, Strong DR, Read JP (2005) } \\
\text { Toward efficient and comprehensive } \\
\text { measurement of the alcohol problems } \\
\text { continuum in college students: the Brief } \\
\text { Young Adult Alcohol Consequences } \\
\text { Questionnaire. Alcohol Clin Exp Res } \\
\text { 29:1180-1189. }\end{array}$ \\
\hline $\begin{array}{l}\text { Cannabis } \\
\text { Problems } \\
\text { Identification } \\
\text { Test (CUPIT } \\
\text { (subscale } \\
\text { problems)) }\end{array}$ & $\begin{array}{l}\text { The CUPIT is a self-report questionnaire with two subscales } \\
\text { (Bashford et al., 2010). Ten items reflect impaired control over } \\
\text { cannabis use (subscale Impaired Control) with scores ranging } \\
\text { between } 0 \text { and } 58 \text {. An example of an item from the Impaired Control } \\
\text { subscale is: "Over the last } 3 \text { months, how often have you used } \\
\text { cannabis first thing in the morning?", with the following response } \\
\text { options: "never, once or twice, less than monthly, monthly, one day a } \\
\text { week, several days a week or daily/always". Six items reflect adverse } \\
\text { consequences of cannabis use (subscale Problems) with scores } \\
\text { ranging between } 0 \text { and } 24 \text {. An example of an item from the Problems } \\
\text { subscale is: "Over the last } 3 \text { months, did your use of cannabis ever } \\
\text { interfere with (get in the way of) your work at school, your job, or your } \\
\text { home life?", with the following response options: 'never, sometimes, } \\
\text { quite often, very often and always/all the time'. }\end{array}$ & 6 & $0-24$ & $\begin{array}{l}\text { higher score, } \\
\text { greater } \\
\text { problems }\end{array}$ & $\begin{array}{l}\text { Bashford, J., Flett, R., \& Copeland, J. } \\
\text { (2010). The Cannabis Use Problems } \\
\text { Identification Test (CUPIT): Development, } \\
\text { reliability, concurrent and predictive validity } \\
\text { among adolescents and adults. Addiction, } \\
\text { 105, 615-625. }\end{array}$ \\
\hline $\begin{array}{l}\text { Cannabis } \\
\text { Problems } \\
\text { Questionnaire } \\
\text { (CPQ) }\end{array}$ & & 22 & $0-22$ & & $\begin{array}{l}\text { Copeland J, Gilmour S, Gates P, Swift W. } \\
\text { The Cannabis Problems Questionnaire: } \\
\text { factor structure, reliability, and validity. Drug } \\
\text { Alcohol Depend [Internet] 2005;80(3):313-9. } \\
\text { Available from: } \\
\text { http://dx.doi.org/10.1016/j.drugalcdep.2005. } \\
04.009\end{array}$ \\
\hline $\begin{array}{l}\text { DSM-IV, alcohol } \\
\text { (DSM-IV, } \\
\text { alcohol) }\end{array}$ & $\begin{array}{l}\text { Two sets of questions based on DSM-IV criteria addressed whether } \\
\text { adolescents had experienced consequences due to alcohol or } \\
\text { marijuana use (Tucker et al., 2003). There were six items for alcohol } \\
\text { (e.g., missed school or work, passed out) and five for marijuana (e.g., } \\
\text { got into trouble at school or home, had difficulty concentrating). Both } \\
\text { scales average responses across items that are rated on a four-point } \\
\text { scale (never, one time, two times, three or more times) and are } \\
\text { reliable with adolescents ( } a=.77 \text { for marijuana and } a=.81 \text { for } \\
\text { alcohol). }\end{array}$ & 6 & $\begin{array}{l}\text { 4-point } \\
\text { scale }\end{array}$ & $\begin{array}{l}\text { higher score, } \\
\text { greater } \\
\text { problems }\end{array}$ & $\begin{array}{l}\text { Tucker, J. S., Orlando, M., \& Ellickson, P. L. } \\
\text { (2003). Patterns and correlates of binge } \\
\text { drinking trajectories from early adolescence } \\
\text { to young adulthood. Health Psychology, 22, } \\
79-87 \text {. }\end{array}$ \\
\hline
\end{tabular}




\begin{tabular}{|c|c|c|c|c|c|}
\hline Score Name & Description & $\begin{array}{l}\text { Item } \\
\text { Number }\end{array}$ & $\begin{array}{l}\text { Score } \\
\text { Range }\end{array}$ & $\begin{array}{l}\text { Score } \\
\text { Direction }\end{array}$ & Score Source \\
\hline $\begin{array}{l}\text { Global } \\
\text { Assessment of } \\
\text { Individual Needs } \\
\text { - Quick (GAIN-Q } \\
\text { (SPS)) }\end{array}$ & & & & $\begin{array}{l}\text { higher score, } \\
\text { greater } \\
\text { problems }\end{array}$ & $\begin{array}{l}\text { Dennis, M.; Scott, C.; Godley, M.; Funk, R. } \\
\text { Comparisons of Adolescents and Adults by } \\
\text { ASAM Profile Using GAIN Data from the } \\
\text { Drug Outcome Monitoring Study (DOMS): } \\
\text { Preliminary Data Tables. Bloomington, IL, } \\
\text { Chestnut Health Systems; } 1999 .\end{array}$ \\
\hline $\begin{array}{l}\text { Marijuana } \\
\text { Problem } \\
\text { Inventory (MPI) }\end{array}$ & $\begin{array}{l}\text { The Marijuana Problems Index (Johnson \& White, 1995) is a } 23 \text {-item } \\
\text { measure adapted from the Rutgers Alcohol Problem Index (White \& } \\
\text { LaBouvie, 1989) that assesses for a variety of marijuana-related } \\
\text { negative consequences. The MPI assesses the frequency of } \\
\text { problems on a rating scale of } 0 \text { (never) to } 4 \text { (more than } 10 \text { times) as a } \\
\text { result of marijuana use. The total MPI score for each participant was } \\
\text { computed by adding the } 23 \text { item scores. Alpha reliability coefficients } \\
\text { at baseline and follow-ups ranged from .86-. } 97 \text {. }\end{array}$ & 23 & $0-92$ & $\begin{array}{l}\text { higher score, } \\
\text { greater } \\
\text { problems }\end{array}$ & $\begin{array}{l}\text { V. Johnson, H.R. White. An investigation of } \\
\text { factors related to intoxicated driving } \\
\text { behaviors among youth Journal of Studies } \\
\text { on Alcohol, } 50 \text { (4) (1989), pp. 320-330 }\end{array}$ \\
\hline $\begin{array}{l}\text { Marijuana } \\
\text { Problem } \\
\text { Inventory (MPI) }\end{array}$ & $\begin{array}{l}\text { The Marijuana Problems Index (Johnson \& White, 1995) is a 23-item } \\
\text { measure adapted from the Rutgers Alcohol Problem Index (White \& } \\
\text { LaBouvie, 1989) that assesses for a variety of marijuana-related } \\
\text { negative consequences. The MPI assesses the frequency of } \\
\text { problems on a rating scale of } 0 \text { (never) to } 4 \text { (more than } 10 \text { times) as a } \\
\text { result of marijuana use. The total MPI score for each participant was } \\
\text { computed by adding the } 23 \text { item scores. Alpha reliability coefficients } \\
\text { at baseline and follow-ups ranged from .86-.97. }\end{array}$ & 23 & $0-92$ & $\begin{array}{l}\text { higher score, } \\
\text { greater } \\
\text { problems }\end{array}$ & $\begin{array}{l}\text { Johnson V, White HR. The relationship } \\
\text { between work-specific and generalized } \\
\text { stress and alcohol and marijuana use } \\
\text { among recent entrants to the labor force. } \\
\text { Journal of Drug Issues. 1995; 25(2):237- } \\
251 \text {. }\end{array}$ \\
\hline $\begin{array}{l}\text { Personal } \\
\text { Consequences } \\
\text { Scale (PEI-PCS) }\end{array}$ & $\begin{array}{l}\text { This 11-item self-report scale from the Personal Experience Inventory } \\
\text { (Henly \& Winters, 1988) focuses on negative consequences of } \\
\text { alcohol use and other drug involvement, including legal, health, motor } \\
\text { vehicle, social, and family ( .92; test-retest .87). Each item has a 4- } \\
\text { point response option (strongly disagree, disagree, agree, strongly } \\
\text { agree); scores range from } 11 \text { to } 44 \text {. The PCS was administered at } \\
\text { intake and the 6-months follow-up. }\end{array}$ & 11 & $11-44$ & $\begin{array}{l}\text { higher score, } \\
\text { greater } \\
\text { problems }\end{array}$ & $\begin{array}{l}\text { Henly, G. A., \& Winters, K. C. (1988). } \\
\text { Development of problem severity scales for } \\
\text { the assessment of adolescent alcohol and } \\
\text { drug abuse. International Journal of the } \\
\text { Addictions, } 23,65-85 .\end{array}$ \\
\hline $\begin{array}{l}\text { Personal } \\
\text { Experience } \\
\text { Inventory items } \\
\text { (PEI-PCS) }\end{array}$ & $\begin{array}{l}\text { This 11-item self-report scale from the Personal Experience Inventory } \\
\text { (Henly \& Winters, 1988) focuses on negative consequences of } \\
\text { alcohol and other drug involvement, including legal, health, motor } \\
\text { vehicle, social, and family ( } \alpha=.92 \text {, test-retest }=.87) \text {. Each item has } \\
\text { a 4-point response option (strongly disagree, disagree, agree, and } \\
\text { strongly agree); score range is 11-44. }\end{array}$ & 11 & $11-44$ & $\begin{array}{l}\text { higher score, } \\
\text { greater } \\
\text { problems }\end{array}$ & $\begin{array}{l}\text { Henly, G. A., \& Winters, K. C. (1988). } \\
\text { Development of problem severity scales for } \\
\text { the assessment of adolescent alcohol and } \\
\text { drug abuse. International Journal of the } \\
\text { Addictions, } 23,65-85 .\end{array}$ \\
\hline
\end{tabular}




\begin{tabular}{|c|c|c|c|c|c|}
\hline Score Name & Description & $\begin{array}{l}\text { Item } \\
\text { Number }\end{array}$ & $\begin{array}{l}\text { Score } \\
\text { Range }\end{array}$ & $\begin{array}{l}\text { Score } \\
\text { Direction }\end{array}$ & Score Source \\
\hline $\begin{array}{l}\text { Personal } \\
\text { Experiences } \\
\text { Inventory (PEI } \\
\text { (subscale)) }\end{array}$ & $\begin{array}{l}\text { PEI has two sections: chemical involvement problem (153 items) and } \\
\text { psychosocial problems ( } 147 \text { items), each with multiple subscales (see } \\
\text { Results). Scaling varies based on the section, including frequencies } \\
\text { and Likert ratings (e.g., Bstrongly agree^). Psychometric information } \\
\text { is in the PEI manual. Manual description: PEl is a } 276 \text {-items self- } \\
\text { report Questionnaire made to identify problems commonly associated } \\
\text { with adolescent substance abuse. PEl is designed to document the } \\
\text { onset, nature and degree of alcohol and other substance } \\
\text { involvement, and to identify the personal risk factors that may } \\
\text { precipitate or maintain substance abuse. Subscales: Substance use } \\
\text { problem severity ( } 10 \text { scales: } 94 \text { items). Substance use } \\
\text { frequency/onset: } 19 \text { items. Personal risk factors ( } 8 \text { scales: } 79 \text { items). } \\
\text { Environmental risk factors ( } 4 \text { scales: } 35 \text { items). Problem screens } \\
\text { such as school problems, family problems, and psychiatric disorders } \\
\text { (6 screens: } 31 \text { items). Validity indices ( } 5 \text { scales: } 70 \text { items). }\end{array}$ & 147 & NR & $\begin{array}{l}\text { higher score, } \\
\text { greater } \\
\text { problems }\end{array}$ & $\begin{array}{l}\text { Winters KC, Henly GA. Personal Experience } \\
\text { Inventory Test and Manual. Los Angeles: } \\
\text { Western Psychological Services; } 1989 .\end{array}$ \\
\hline $\begin{array}{l}\text { Revised } \\
\text { Behavior } \\
\text { Problems } \\
\text { Checklist } \\
\text { subscales' } \\
\text { composite } \\
\text { (RBPC } \\
\text { subscales) }\end{array}$ & $\begin{array}{l}\text { The Revised Behavior Problems Checklist (Quay \& Peterson, 1987) } \\
\text { Conduct Disorder and Socialized Aggression subscales were used to } \\
\text { create a composite score of parent reported adolescent behavior } \\
\text { problems. Internal consistency reliability was high at baseline and } \\
\text { follow-up (as > .90). }\end{array}$ & 39 & NR & $\begin{array}{l}\text { higher score, } \\
\text { greater } \\
\text { problems }\end{array}$ & $\begin{array}{l}\text { Quay, HC.; Peterson, DR. Manual for the } \\
\text { Revised Behavioral Problem Checklist. } \\
\text { Department of Psychology, University of } \\
\text { Miami; Coral Gables, FL: } 1987 .\end{array}$ \\
\hline $\begin{array}{l}\text { Risks and } \\
\text { Consequences } \\
\text { Questionnaire } \\
\text { (RCQ-M) }\end{array}$ & $\begin{array}{l}\text { The Risks and Consequences Questionnaire (RCQ) measures } \\
\text { problems associated with alcohol and marijuana use (missing school, } \\
\text { relationship difficulty, etc.). At baseline it covers } 12 \text { months pre- } \\
\text { incarceration and at } 3 \text { months after release it covers } 90 \text { days post- } \\
\text { incarceration. Alcohol (RCQ-A) and marijuana (RCQ-M) scales (11 } \\
\text { items, each) are scored according to whether events occurred } \\
\text { (yes/no). It is reliable and valid for use with incarcerated adolescents, } \\
\text { with Cronbach alpha ranging from } 0.72 \text { to } 0.83 \text { (Stein et al., 2010a). }\end{array}$ & 11 & NR & $\begin{array}{l}\text { higher score, } \\
\text { greater } \\
\text { problems }\end{array}$ & $\begin{array}{l}\text { Stein, L.A.R., Lebeau, R., Clair, M., Rossi, } \\
\text { J.S., Martin, R.M., Golembeske, C., 2010a. } \\
\text { Validation of a measure to assess alcohol- } \\
\text { and marijuana-related risks and } \\
\text { consequences among incarcerated } \\
\text { adolescents. Drug Alcohol Depend. 109, } \\
\text { 104-113. }\end{array}$ \\
\hline $\begin{array}{l}\text { Rutgers Alcohol } \\
\text { Problem Index } \\
\text { (RAPI) }\end{array}$ & $\begin{array}{l}\text { The original RAPI is a 23-item self-administered screening tool for } \\
\text { assessing adolescent problem drinking. It was developed in order to } \\
\text { create a conceptually sound, unidimensional, relatively brief, and } \\
\text { easily administered instrument to assess problem drinking in } \\
\text { adolescence. }\end{array}$ & 23 & $0-92$ & $\begin{array}{l}\text { higher score, } \\
\text { greater } \\
\text { problems }\end{array}$ & $\begin{array}{l}\text { Towards the assessment of adolescent } \\
\text { problem drinking. White HR, Labouvie EW. J } \\
\text { Stud Alcohol. } 1989 \text { Jan; 50(1):30-7 }\end{array}$ \\
\hline
\end{tabular}




\begin{tabular}{|c|c|c|c|c|c|}
\hline Score Name & Description & $\begin{array}{l}\text { Item } \\
\text { Number }\end{array}$ & $\begin{array}{l}\text { Score } \\
\text { Range }\end{array}$ & $\begin{array}{l}\text { Score } \\
\text { Direction }\end{array}$ & Score Source \\
\hline $\begin{array}{l}\text { Rutgers Alcohol } \\
\text { Problems Index I } \\
\text { Severity of } \\
\text { Dependence } \\
\text { Scale } \\
\text { (RAPI/SDS) }\end{array}$ & $\begin{array}{l}\text { The number of cannabis related consequences (CC) in the past } 3 \\
\text { months consisted of } 23 \text { items from the adapted version (Vandrey et } \\
\text { al., 2005) of the Rutgers Alcohol Problems Index (White and } \\
\text { Labouvie, 1989) and 5-items from the Severity of Dependence Scale } \\
\text { (Martin et al., 2006): interpersonal (e.g., had a fight, argument or bad } \\
\text { feelings with a friend), intrapersonal (e.g., missed out on other things } \\
\text { because you spent too much money on cannabis), and substance } \\
\text { use disorder symptoms (e.g., kept smoking when you promised } \\
\text { yourself not to) }(=0.95) \text {. }\end{array}$ & 28 & NR & $\begin{array}{l}\text { higher score, } \\
\text { greater } \\
\text { problems }\end{array}$ & $\begin{array}{l}\text { Vandrey, R., Budney, A.J., Kamon, J.L., } \\
\text { Stanger, C., 2005. Cannabis withdrawal in } \\
\text { adolescent treatment seekers. Drug Alcohol } \\
\text { Depend. 78, 205-210. / Martin, G., } \\
\text { Copeland, J., Gates, P., Gilmour, S., 2006. } \\
\text { The Severity of Dependence Scale (SDS) in } \\
\text { an adolescent population of cannabis users: } \\
\text { reliability, validity and diagnostic cut-off. } \\
\text { Drug Alcohol Depend. 83, 90-93. }\end{array}$ \\
\hline $\begin{array}{l}\text { Severity of } \\
\text { Dependence } \\
\text { Scale (SDS) }\end{array}$ & $\begin{array}{l}\text { Severity of dependence for heroin, cocaine and amphetamine was } \\
\text { measured by a Severity of Dependence Scale (SDS). The total SDS } \\
\text { score was derived from five items, each of which was scored on a } \\
\text { four-point scale (scored 0-3). Since severity of dependence can be } \\
\text { expected to vary over time, the SDS measures were requested for } \\
\text { recent drug use. The five items related to problems of dependence } \\
\text { experienced at any time in the last year and all items were completed } \\
\text { separately for heroin, cocaine and amphetamine. The items were: (1) } \\
\text { Did you think that your use of [named drug] was out of control? (2) } \\
\text { Did the prospect of missing a fix (or dose) or not chasing make you } \\
\text { anxious or worried? (3) Did you worry about your use of [named } \\
\text { drug]? (4) Did you wish you could stop? (5) How difficult did you find } \\
\text { it to stop or go without [named drug]? }\end{array}$ & 5 & $0-15$ & $\begin{array}{l}\text { higher score, } \\
\text { greater } \\
\text { problems }\end{array}$ & $\begin{array}{l}\text { Gossop, M., Griffiths, P., Powis, B., \& } \\
\text { Strang, J. (1992). Severity of dependence } \\
\text { and route of administration in heroin, } \\
\text { cocaine and amphetamines. British Journal } \\
\text { of Addiction, 87, 1527-1536. }\end{array}$ \\
\hline $\begin{array}{l}\text { Teen Addiction } \\
\text { Severity Index } \\
\text { (T-ASI) }\end{array}$ & $\begin{array}{l}\text { The Teen Addiction Severity Index (T-ASI) is a semistructured } \\
\text { interview (Kaminer et al., 1991) modified from the Addiction Severity } \\
\text { Index (McLellan et al., 1980) to fill the need for a reliable, valid, and } \\
\text { standardized instrument for evaluating the severity of adolescent } \\
\text { substance abuse and associated problem domains. The T-ASI was } \\
\text { found to have good psychometric properties (Kaminer et al., 1993). } \\
\text { The T-ASI problem domains include alcohol, substance use, school } \\
\text { or mployment, family, peer/social, legal, and psychiatric. Each } \\
\text { domain is scored as the mean of three scales (range: } 0 \text { to 4): a) } \\
\text { youth's perception of the importance of the problem; b) youth's } \\
\text { perception of the need for treatment for the problem; and c) rater's } \\
\text { perception of the seriousness of the problem. The T-ASI also } \\
\text { assesses the number of substances used, recency in controlled } \\
\text { environment, age of first alcohol use, and age of first substance use. }\end{array}$ & NR & NR & $\begin{array}{l}\text { higher score, } \\
\text { greater } \\
\text { problems }\end{array}$ & $\begin{array}{l}\text { Kaminer Y, Bukstein OG, Tarter TE (1991) } \\
\text { The Teen Addiction Severity Index: } \\
\text { Rationale and reliability. Int J Addict 26:219- } \\
226 .\end{array}$ \\
\hline
\end{tabular}




\begin{tabular}{|c|c|c|c|c|c|}
\hline Score Name & Description & $\begin{array}{l}\text { Item } \\
\text { Number }\end{array}$ & $\begin{array}{l}\text { Score } \\
\text { Range }\end{array}$ & $\begin{array}{l}\text { Score } \\
\text { Direction }\end{array}$ & Score Source \\
\hline $\begin{array}{l}\text { Brief Young } \\
\text { Adult Alcohol } \\
\text { Consequences } \\
\text { Questionnaire } \\
\text { (BYAACQ) }\end{array}$ & $\begin{array}{l}\text { This scale can help assess alcohol problems among college } \\
\text { students, track changes in alcohol problems throughout college, and } \\
\text { measure the response to alcohol interventions. It consists of 24- } \\
\text { items and was derived from the 48-item Young Adult Alcohol } \\
\text { Consequences Questionnaire. The B-YAACQ has items that tap the } \\
\text { full range of the alcohol problems continuum from signs of excessive } \\
\text { drinking to symptoms consistent with alcohol abuse and alcohol } \\
\text { dependence. }\end{array}$ & 24 & $0-24$ & $\begin{array}{l}\text { higher score, } \\
\text { greater } \\
\text { problems }\end{array}$ & $\begin{array}{l}\text { Kahler CW, Strong DR, Read JP (2005) } \\
\text { Toward efficient and comprehensive } \\
\text { measurement of the alcohol problems } \\
\text { continuum in college students: the Brief } \\
\text { Young Adult Alcohol Consequences } \\
\text { Questionnaire. Alcohol Clin Exp Res } \\
\text { 29:1180-1189. }\end{array}$ \\
\hline
\end{tabular}

Table F-7. Brief interventions, substance use problem scale outcomes and effects

\begin{tabular}{|c|c|c|c|c|c|c|c|c|}
\hline PMID & Citation & Intervention & $\begin{array}{r}\mathrm{N} \\
\text { (endtime) }\end{array}$ & Scale & $\begin{array}{r}\text { Baseline } \\
\text { Mean } \\
(\mathrm{SD})\end{array}$ & $\begin{array}{l}\text { End Mean } \\
\text { (SD) }\end{array}$ & $\begin{array}{r}\text { SMD } \\
(95 \% \mathrm{Cl})\end{array}$ & $\begin{array}{r}\text { SNMD } \\
(95 \% \mathrm{Cl})\end{array}$ \\
\hline 27801991 & $\begin{array}{l}\text { Arnaud, } \\
2017\end{array}$ & $\mathrm{Ml}$ & $\begin{array}{r}141 \\
(3 \mathrm{mo})\end{array}$ & Yes & $\begin{array}{l}11.26 \\
(7.39)\end{array}$ & $\begin{array}{r}4.45 \\
(4.85)\end{array}$ & $\begin{array}{r}-0.9 \\
(-1.1,-0.7)\end{array}$ & $\begin{array}{r}-0.15 \\
(-0.4,0.1)\end{array}$ \\
\hline 27801991 & $\begin{array}{l}\text { Arnaud, } \\
2017\end{array}$ & TAU & $\begin{array}{r}175 \\
(3 \mathrm{mo})\end{array}$ & Yes & $\begin{array}{r}9.72 \\
(7.02)\end{array}$ & $\begin{array}{r}4.05 \\
(4.66)\end{array}$ & $\begin{array}{r}-0.75 \\
(-0.9,-0.6)\end{array}$ & \\
\hline 29750362 & $\begin{array}{l}\text { Colby, } \\
2018\end{array}$ & $\mathrm{Ml}$ & $\begin{array}{r}83 \\
(3 \mathrm{mo})\end{array}$ & Yes & $\begin{array}{r}8.46 \\
(4.35)\end{array}$ & $\begin{array}{l}4.76 \\
(4.5)\end{array}$ & $\begin{array}{r}-0.7 \\
(-0.9,-0.5)\end{array}$ & $\begin{array}{r}-0.5 \\
(-0.8,-0.2)\end{array}$ \\
\hline 29750362 & $\begin{array}{l}\text { Colby, } \\
2018\end{array}$ & TAU & $\begin{array}{r}84 \\
(3 \mathrm{mo})\end{array}$ & Yes & $\begin{array}{r}6.99 \\
(4.48)\end{array}$ & $\begin{array}{r}5.97 \\
(4.09)\end{array}$ & $\begin{array}{r}-0.19 \\
(-0.4,0)\end{array}$ & \\
\hline 30138016 & $\begin{array}{l}\text { D'Amico, } \\
2018\end{array}$ & $\mathrm{Ml}$ & $\begin{array}{r}153 \\
(3 \mathrm{mo})\end{array}$ & Yes & $\begin{array}{r}6.59 \\
(14.17)\end{array}$ & $\begin{array}{r}2.17 \\
(5.05)\end{array}$ & $\begin{array}{r}-0.29 \\
(-0.4,-0.1)\end{array}$ & $\begin{array}{r}0 \\
(-0.2,0.2)\end{array}$ \\
\hline 30138016 & $\begin{array}{l}\text { D'Amico, } \\
2018\end{array}$ & TAU & $\begin{array}{r}141 \\
(3 \mathrm{mo})\end{array}$ & Yes & $\begin{array}{r}7.86 \\
(16.57)\end{array}$ & $\begin{array}{r}3.39 \\
(9.03)\end{array}$ & $\begin{array}{r}-0.29 \\
(-0.5,-0.1)\end{array}$ & \\
\hline 24969735 & $\begin{array}{l}\text { de Gee, } \\
2014\end{array}$ & $\mathrm{Ml}$ & $\begin{array}{r}58 \\
(3 \mathrm{mo}) \\
\end{array}$ & Yes & $\begin{array}{r}6.2 \\
(4.3) \\
\end{array}$ & $\begin{array}{r}6.2 \\
(3.8) \\
\end{array}$ & $\begin{array}{r}0 \\
(-0.3,0.3) \\
\end{array}$ & $\begin{array}{r}0 \\
(-0.36,0.36)\end{array}$ \\
\hline 24969735 & $\begin{array}{l}\text { de Gee, } \\
2014\end{array}$ & Educ & $\begin{array}{r}61 \\
(3 \mathrm{mo})\end{array}$ & Yes & $\begin{array}{r}5.7 \\
(3.7)\end{array}$ & $\begin{array}{r}5.7 \\
(3.7)\end{array}$ & $\begin{array}{r}0 \\
(-0.2,0.2)\end{array}$ & \\
\hline 17869051 & $\begin{array}{l}\text { Martin, } \\
2008\end{array}$ & $\mathrm{Ml}$ & $\begin{array}{r}20 \\
(3 \mathrm{mo})\end{array}$ & Yes & $\begin{array}{r}5.8 \\
(1.2)\end{array}$ & $\begin{array}{r}3.8 \\
(2.8)\end{array}$ & $\begin{array}{r}-0.78 \\
(-1.2,-0.3)\end{array}$ & $\begin{array}{r}-0.54 \\
(-1.2,0.1)\end{array}$ \\
\hline $\begin{array}{r}\text { CN- } \\
01953820 \\
\text { (cochrane) }\end{array}$ & Giles, 2019 & $\mathrm{Ml}$ & $\begin{array}{r}181 \\
(12 \mathrm{mo})\end{array}$ & Yes & $\begin{array}{r}8.1 \\
(9.9)\end{array}$ & $\begin{array}{r}4.5 \\
(5.3)\end{array}$ & $\begin{array}{r}-0.17 \\
(-0.15,-0.19)\end{array}$ & $\begin{array}{r}-0.07 \\
(-0.11,-0.03)\end{array}$ \\
\hline
\end{tabular}




\begin{tabular}{|c|c|c|c|c|c|c|c|c|}
\hline PMID & Citation & Intervention & $\begin{array}{r}\mathrm{N} \\
\text { (endtime) }\end{array}$ & Scale & $\begin{array}{r}\text { Baseline } \\
\text { Mean } \\
(\mathrm{SD})\end{array}$ & $\begin{array}{r}\text { End Mean } \\
\text { (SD) }\end{array}$ & $\begin{array}{r}\text { SMD } \\
(95 \% \mathrm{Cl})\end{array}$ & $\begin{array}{r}\text { SNMD } \\
(95 \% \mathrm{Cl})\end{array}$ \\
\hline $\begin{array}{r}\text { CN- } \\
01953820 \\
\text { (cochrane) }\end{array}$ & Giles, 2019 & TAU & $\begin{array}{r}197 \\
(12 \mathrm{mo})\end{array}$ & Yes & $\begin{array}{r}6.5 \\
(8.7)\end{array}$ & $\begin{array}{r}4.0 \\
(4.8)\end{array}$ & $\begin{array}{r}-0.10 \\
(-0.08,-0.12)\end{array}$ & \\
\hline 17869051 & $\begin{array}{l}\text { Martin, } \\
2008\end{array}$ & TAU & $\begin{array}{r}20 \\
(3 \mathrm{mo})\end{array}$ & Yes & $\begin{array}{r}4.8 \\
(2.1)\end{array}$ & $\begin{array}{l}4.2 \\
(2)\end{array}$ & $\begin{array}{r}-0.23 \\
(-0.7,0.2)\end{array}$ & \\
\hline 18778385 & $\begin{array}{l}\text { McCambridg } \\
\text { e, } \\
2008\end{array}$ & $\mathrm{Ml}$ & $\begin{array}{r}164 \\
(3 \mathrm{mo})\end{array}$ & Yes & $\begin{array}{r}6.5 \\
(4.3)\end{array}$ & $\begin{array}{r}5 \\
(4.1)\end{array}$ & $\begin{array}{r}-0.29 \\
(-0.4,-0.1)\end{array}$ & $\begin{array}{r}0.04 \\
(-0.18,0.26)\end{array}$ \\
\hline 18778385 & $\begin{array}{l}\text { McCambridg } \\
\text { e, } \\
2008\end{array}$ & Educ & $\begin{array}{r}162 \\
(3 \mathrm{mo})\end{array}$ & Yes & $\begin{array}{r}7 \\
(4)\end{array}$ & $\begin{array}{r}5.3 \\
(4.3)\end{array}$ & $\begin{array}{r}-0.33 \\
(-0.5,-0.2)\end{array}$ & \\
\hline 21688877 & $\begin{array}{l}\text { Walker, } \\
2011\end{array}$ & Ml & $\begin{array}{r}101 \\
(3 \mathrm{mo})\end{array}$ & Yes & $\begin{array}{r}18.47 \\
(13.47)\end{array}$ & $\begin{array}{r}14.68 \\
(10.39)\end{array}$ & $\begin{array}{r}-0.27 \\
(-0.5,-0.1)\end{array}$ & $\begin{array}{r}-0.08 \\
(-0.2,0.36)\end{array}$ \\
\hline 21688877 & $\begin{array}{l}\text { Walker, } \\
2011\end{array}$ & Educ & $\begin{array}{r}100 \\
(3 \mathrm{mo}) \\
\end{array}$ & Yes & $\begin{array}{r}19.13 \\
(12.31) \\
\end{array}$ & $\begin{array}{r}14.24 \\
(10.18) \\
\end{array}$ & $\begin{array}{r}-0.34 \\
(-0.5,-0.2)\end{array}$ & \\
\hline 22000326 & $\begin{array}{l}\text { Winters, } \\
2012\end{array}$ & $\mathrm{Ml}$ & $\begin{array}{r}257 \\
(6 \mathrm{mo})\end{array}$ & Yes & $\begin{array}{l}15.42 \\
(4.37)\end{array}$ & $\begin{array}{l}12.46 \\
(2.82)\end{array}$ & $\begin{array}{r}-0.64 \\
(-0.8,-0.5)\end{array}$ & $\begin{array}{r}-0.21 \\
(-0.5,0.1)\end{array}$ \\
\hline 22000326 & $\begin{array}{l}\text { Winters, } \\
2012\end{array}$ & TAU & $\begin{array}{r}56 \\
(6 \mathrm{mo})\end{array}$ & Yes & $\begin{array}{l}15.5 \\
(4.8)\end{array}$ & $\begin{array}{l}13.5 \\
(3.1)\end{array}$ & $\begin{array}{r}-0.43 \\
(-0.7,-0.1)\end{array}$ & \\
\hline 17563146 & $\begin{array}{l}\text { Winters, } \\
2007\end{array}$ & $\mathrm{Ml}$ & $\begin{array}{r}52 \\
(6 \mathrm{mo})\end{array}$ & Yes & $\begin{array}{r}15.25 \\
(1.5) \\
\end{array}$ & $\begin{array}{r}11.5 \\
(1.41)\end{array}$ & $\begin{array}{r}-1.85 \\
(-2.1,-1.6) \\
\end{array}$ & $\begin{array}{r}-1.65 \\
(-2.2,-1.1) \\
\end{array}$ \\
\hline 17563146 & $\begin{array}{l}\text { Winters, } \\
2007\end{array}$ & TAU & $\begin{array}{r}26 \\
(6 \mathrm{mo})\end{array}$ & Yes & $\begin{array}{r}14.3 \\
(2)\end{array}$ & $\begin{array}{l}13.9 \\
(2.1)\end{array}$ & $\begin{array}{r}-0.2 \\
(-0.7,0.3)\end{array}$ & \\
\hline
\end{tabular}

Abbreviations: PMID = PubMed ID (or other ID); N=number randomized;SD = standard deviation; End mean = mean at End; SMD = standardized mean difference; SNMD = standardized net mean difference (MI versus TAU or Educ); $\mathrm{MI}=$ motivational interviewing; Educ = psychoeducation; TAU = treatment as usual 


\section{Appendix G. Nonbrief Interventions: Detailed Results}

Table G-1. Nonbrief interventions, alcohol use days

\begin{tabular}{|c|c|c|c|c|c|c|c|c|}
\hline PMID & Citation & Intervention & $\begin{array}{r}\mathrm{N} \\
\text { (endtime) }\end{array}$ & Scale & $\begin{array}{r}\text { Baseline Mean } \\
\text { (SD) }\end{array}$ & $\begin{array}{r}\text { End Mean } \\
\text { (SD) }\end{array}$ & $\begin{array}{r}M D \\
(95 \% \mathrm{Cl})\end{array}$ & $\begin{array}{r}\text { SMD } \\
(95 \% \mathrm{Cl})\end{array}$ \\
\hline 18072842 & $\begin{array}{l}\text { Baer, } \\
2007\end{array}$ & $\mathrm{Ml}$ & $\begin{array}{r}75 \\
(3 \mathrm{mo})\end{array}$ & No & $\begin{array}{r}5.8 \\
(6.8)\end{array}$ & $\begin{array}{r}4.5 \\
(7.1)\end{array}$ & $\begin{array}{r}-1.3 \\
(-3.2,0.6)\end{array}$ & $\begin{array}{r}-0.17 \\
(-0.4,0.1)\end{array}$ \\
\hline 18072842 & $\begin{array}{l}\text { Baer, } \\
2007\end{array}$ & TAU & $\begin{array}{r}52 \\
(3 \mathrm{mo})\end{array}$ & No & $\begin{array}{r}4.8 \\
(4.8)\end{array}$ & $\begin{array}{r}2.9 \\
(6.2)\end{array}$ & $\begin{array}{r}-1.9 \\
(-3.8,0)\end{array}$ & $\begin{array}{r}-0.24 \\
(-0.5,0)\end{array}$ \\
\hline $\begin{array}{r}\text { CN-00917707 } \\
\text { (Cochrane) }\end{array}$ & $\begin{array}{l}\text { D'Amico, } \\
2013\end{array}$ & $\mathrm{Ml}$ & $\begin{array}{r}109 \\
(3 \mathrm{mo})\end{array}$ & No & $\begin{array}{r}2.65 \\
(1.72)\end{array}$ & $\begin{array}{r}2.8 \\
(1.6)\end{array}$ & $\begin{array}{r}0.15 \\
(-0.2,0.5)\end{array}$ & $\begin{array}{r}0.08 \\
(-0.1,0.3)\end{array}$ \\
\hline $\begin{array}{r}\text { CN-00917707 } \\
\text { (Cochrane) }\end{array}$ & $\begin{array}{l}\text { D'Amico, } \\
2013\end{array}$ & PeerGroup & $\begin{array}{r}78 \\
(3 \mathrm{mo})\end{array}$ & No & $\begin{array}{r}2.31 \\
(1.45)\end{array}$ & $\begin{array}{l}2.24 \\
(1.4)\end{array}$ & $\begin{array}{r}-0.07 \\
(-0.5,0.3)\end{array}$ & $\begin{array}{r}-0.04 \\
(-0.2,0.2)\end{array}$ \\
\hline 12127465 & $\begin{array}{l}\text { Godley, } \\
2002\end{array}$ & TAU & $\begin{array}{r}51 \\
(3 \mathrm{mo})\end{array}$ & No & $\begin{array}{r}3.3 \\
(6.07)\end{array}$ & $\begin{array}{r}2.7 \\
(6.07)\end{array}$ & $\begin{array}{r}-0.6 \\
(-2.6,1.4)\end{array}$ & $\begin{array}{r}-0.08 \\
(-0.4,0.2)\end{array}$ \\
\hline 12127465 & $\begin{array}{l}\text { Godley, } \\
2002\end{array}$ & $\mathrm{CBT}+\mathrm{ICM}$ & $\begin{array}{r}63 \\
(3 \mathrm{mo})\end{array}$ & No & $\begin{array}{r}4.2 \\
(7.42)\end{array}$ & $\begin{array}{r}1.5 \\
(3.37)\end{array}$ & $\begin{array}{r}-2.7 \\
(-4.5,-0.9)\end{array}$ & $\begin{array}{r}-0.37 \\
(-0.6,-0.1)\end{array}$ \\
\hline 20219293 & $\begin{array}{l}\text { Godley, } \\
2010\end{array}$ & TAU & $\begin{array}{r}80 \\
(3 \mathrm{mo})\end{array}$ & No & $\begin{array}{r}2.01 \\
(2.84)\end{array}$ & $\begin{array}{r}1.38 \\
(2.08)\end{array}$ & $\begin{array}{r}-0.63 \\
(-1.3,0)\end{array}$ & $\begin{array}{r}-0.25 \\
(-0.5,0)\end{array}$ \\
\hline 20219293 & $\begin{array}{l}\text { Godley, } \\
2010\end{array}$ & $\mathrm{CBT}+\mathrm{ICM}$ & $\begin{array}{r}80 \\
(3 \mathrm{mo}) \\
\end{array}$ & No & $\begin{array}{r}1.14 \\
(1.77) \\
\end{array}$ & $\begin{array}{r}1.11 \\
(1.74) \\
\end{array}$ & $\begin{array}{r}-0.03 \\
(-0.5,0.4) \\
\end{array}$ & $\begin{array}{r}-0.01 \\
(-0.2,0.2) \\
\end{array}$ \\
\hline 20219293 & $\begin{array}{l}\text { Godley, } \\
2010\end{array}$ & $\mathrm{CBT}+\mathrm{Ml}$ & $\begin{array}{r}79 \\
(3 \mathrm{mo})\end{array}$ & No & $\begin{array}{r}1.2 \\
(1.85)\end{array}$ & $\begin{array}{r}1.29 \\
(1.96)\end{array}$ & $\begin{array}{r}0.09 \\
(-0.4,0.6)\end{array}$ & $\begin{array}{r}0.04 \\
(-0.2,0.2)\end{array}$ \\
\hline 20219293 & $\begin{array}{l}\text { Godley, } \\
2010\end{array}$ & $\mathrm{CBT}+\mathrm{MI}+\mathrm{ICM}$ & $\begin{array}{r}81 \\
(3 \mathrm{mo})\end{array}$ & No & $\begin{array}{r}1.35 \\
(2.04)\end{array}$ & $\begin{array}{r}1.2 \\
(1.85)\end{array}$ & $\begin{array}{r}-0.15 \\
(-0.7,0.4)\end{array}$ & $\begin{array}{r}-0.06 \\
(-0.3,0.1)\end{array}$ \\
\hline 16551142 & $\begin{array}{l}\text { Henggeler, } \\
2006\end{array}$ & Fam+CM+PeerGroup & $\begin{array}{r}37 \\
(4 \mathrm{mo})\end{array}$ & No & $\begin{array}{r}2.62 \\
(5.43)\end{array}$ & $\begin{array}{r}0.14 \\
(0.33)\end{array}$ & $\begin{array}{r}-2.48 \\
(-4.2,-0.8)\end{array}$ & $\begin{array}{r}-0.78 \\
(-1.3,-0.2)\end{array}$ \\
\hline 16551142 & $\begin{array}{l}\text { Henggeler, } \\
2006\end{array}$ & Fam+PeerGroup & $\begin{array}{r}29 \\
(4 \mathrm{mo})\end{array}$ & No & $\begin{array}{r}0.43 \\
(1.11)\end{array}$ & $\begin{array}{l}0.13 \\
(0.3)\end{array}$ & $\begin{array}{r}-0.3 \\
(-0.7,0.1)\end{array}$ & $\begin{array}{r}-0.09 \\
(-0.2,0)\end{array}$ \\
\hline 16551142 & $\begin{array}{l}\text { Henggeler, } \\
2006\end{array}$ & PeerGroup & $\begin{array}{r}64 \\
(4 \mathrm{mo})\end{array}$ & No & $\begin{array}{r}0.52 \\
(0.87)\end{array}$ & $\begin{array}{r}0.64 \\
(1.98)\end{array}$ & $\begin{array}{r}0.13 \\
(-0.4,0.6)\end{array}$ & $\begin{array}{r}0.04 \\
(-0.1,0.2)\end{array}$ \\
\hline 18705691 & $\begin{array}{l}\text { Liddle, } \\
2008\end{array}$ & CBT & $\begin{array}{r}112 \\
(5 \mathrm{mo})\end{array}$ & No & $\begin{array}{r}27.41 \\
(15.65)\end{array}$ & $\begin{array}{r}27.39 \\
(19.71)\end{array}$ & $\begin{array}{r}-0.02 \\
(-4.1,4)\end{array}$ & $\begin{array}{r}0 \\
(-0.2,0.2)\end{array}$ \\
\hline 18705691 & $\begin{array}{l}\text { Liddle, } \\
2008\end{array}$ & Fam & $\begin{array}{r}112 \\
(5 \mathrm{mo})\end{array}$ & No & $\begin{array}{r}28.47 \\
(17.36)\end{array}$ & $\begin{array}{r}19.75 \\
(18.18)\end{array}$ & $\begin{array}{r}-8.72 \\
(-12.8,-4.7)\end{array}$ & $\begin{array}{r}-0.4 \\
(-0.6,-0.2)\end{array}$ \\
\hline
\end{tabular}




\begin{tabular}{|c|c|c|c|c|c|c|c|c|}
\hline PMID & Citation & Intervention & $\begin{array}{r}\mathrm{N} \\
\text { (endtime) }\end{array}$ & Scale & $\begin{array}{r}\text { Baseline Mean } \\
\text { (SD) }\end{array}$ & $\begin{array}{r}\text { End Mean } \\
\text { (SD) }\end{array}$ & $\begin{array}{r}M D \\
(95 \% \mathrm{Cl})\end{array}$ & $\begin{array}{r}\text { SMD } \\
(95 \% \mathrm{Cl})\end{array}$ \\
\hline 19522781 & $\begin{array}{l}\text { Slesnick, } \\
2009\end{array}$ & TAU & $\begin{array}{r}42 \\
(3 \mathrm{mo})\end{array}$ & No & $\begin{array}{l}5.1 \\
(3)\end{array}$ & $\begin{array}{l}2.7 \\
(3)\end{array}$ & $\begin{array}{r}-2.4 \\
(-3.5,-1.3)\end{array}$ & $\begin{array}{r}-0.36 \\
(-0.5,-0.2)\end{array}$ \\
\hline 19522781 & $\begin{array}{l}\text { Slesnick, } \\
2009\end{array}$ & Fam & $\begin{array}{r}77 \\
(3 \mathrm{mo})\end{array}$ & No & $\begin{array}{r}7.63 \\
(7.65)\end{array}$ & $\begin{array}{r}2.23 \\
(4.61)\end{array}$ & $\begin{array}{r}-5.4 \\
(-7.2,-3.6)\end{array}$ & $\begin{array}{r}-0.81 \\
(-1.1,-0.5)\end{array}$ \\
\hline 25736623 & $\begin{array}{l}\text { Slesnick, } \\
2015\end{array}$ & $\mathrm{Ml}$ & $\begin{array}{r}86 \\
(3 \mathrm{mo})\end{array}$ & No & $\begin{array}{r}5.01 \\
(7.18)\end{array}$ & $\begin{array}{r}2.55 \\
(4.22) \\
\end{array}$ & $\begin{array}{r}-2.46 \\
(-4,-0.9)\end{array}$ & $\begin{array}{r}-0.34 \\
(-0.6,-0.1) \\
\end{array}$ \\
\hline 25736623 & $\begin{array}{l}\text { Slesnick, } \\
2015\end{array}$ & CBT & $\begin{array}{r}93 \\
(3 \mathrm{mo})\end{array}$ & No & $\begin{array}{r}4.53 \\
(7.03)\end{array}$ & $\begin{array}{l}3.11 \\
(4.7)\end{array}$ & $\begin{array}{r}-1.42 \\
(-2.9,0.1)\end{array}$ & $\begin{array}{r}-0.2 \\
(-0.4,0)\end{array}$ \\
\hline 25736623 & $\begin{array}{l}\text { Slesnick, } \\
2015\end{array}$ & $\mathrm{ICM}$ & $\begin{array}{r}91 \\
(3 \mathrm{mo})\end{array}$ & No & $\begin{array}{r}3.73 \\
(5.51)\end{array}$ & $\begin{array}{r}3.04 \\
(5.51)\end{array}$ & $\begin{array}{r}-0.69 \\
(-2.1,0.7)\end{array}$ & $\begin{array}{r}-0.1 \\
(-0.3,0.1)\end{array}$ \\
\hline 24841864 & $\begin{array}{l}\text { Wagner, } \\
2014\end{array}$ & TAU & $\begin{array}{r}235 \\
(4 \mathrm{mo})\end{array}$ & No & $\begin{array}{r}2.28 \\
(2.56)\end{array}$ & $\begin{array}{r}1.61 \\
(1.89)\end{array}$ & $\begin{array}{r}-0.67 \\
(-1,-0.3)\end{array}$ & $\begin{array}{r}-0.24 \\
(-0.4,-0.1)\end{array}$ \\
\hline 24841864 & $\begin{array}{l}\text { Wagner, } \\
2014\end{array}$ & $\mathrm{CBT}+\mathrm{Ml}$ & $\begin{array}{r}279 \\
(4 \mathrm{mo}) \\
\end{array}$ & No & $\begin{array}{r}2.42 \\
(2.77) \\
\end{array}$ & $\begin{array}{r}1.01 \\
(1.62) \\
\end{array}$ & $\begin{array}{r}-1.41 \\
(-1.7,-1.1) \\
\end{array}$ & $\begin{array}{r}-0.5 \\
(-0.6,-0.4) \\
\end{array}$ \\
\hline 26992083 & $\begin{array}{l}\text { Henderson, } \\
2016\end{array}$ & TAU & $\begin{array}{r}63 \\
(3 \mathrm{mo})\end{array}$ & Yes & $\begin{array}{r}0.18 \\
(0.16)\end{array}$ & $\begin{array}{r}0.04 \\
(0.09)\end{array}$ & & $\begin{array}{r}-0.82 \\
(-1.1,-0.6)\end{array}$ \\
\hline 26992083 & $\begin{array}{l}\text { Henderson, } \\
2016\end{array}$ & $\mathrm{CBT}+\mathrm{ICM}$ & $\begin{array}{r}63 \\
(3 \mathrm{mo})\end{array}$ & Yes & $\begin{array}{r}0.23 \\
(0.18)\end{array}$ & $\begin{array}{r}0.04 \\
(0.09)\end{array}$ & & $\begin{array}{r}-1.12 \\
(-1.4,-0.9)\end{array}$ \\
\hline $\begin{array}{r}2017-00657-001 \\
\text { (psycINFO) }\end{array}$ & $\begin{array}{l}\text { Trudeau, } \\
2017\end{array}$ & TAU & $\begin{array}{r}69 \\
(3 \mathrm{mo})\end{array}$ & Yes & $\begin{array}{r}85.18 \\
(43.08)\end{array}$ & $\begin{array}{r}82 \\
(31.47)\end{array}$ & & $\begin{array}{r}-0.07 \\
(-0.3,0.2)\end{array}$ \\
\hline $\begin{array}{r}2017-00657-001 \\
(p s y c I N F O)\end{array}$ & $\begin{array}{l}\text { Trudeau, } \\
2017\end{array}$ & CBT & $\begin{array}{r}48 \\
(3 \mathrm{mo})\end{array}$ & Yes & $\begin{array}{l}103.58 \\
(43.19)\end{array}$ & $\begin{array}{r}81.59 \\
(31.65)\end{array}$ & & $\begin{array}{r}-0.49 \\
(-0.8,-0.2)\end{array}$ \\
\hline
\end{tabular}

Abbreviations: PMID = PubMed ID (or other ID), N=number randomized;SD = standard deviation;End mean = mean at End;MD = mean difference; SMD = standardized mean difference; $\mathrm{MI}=$ motivational interviewing; Fam = family therapy; $\mathrm{CBT}=$ cognitive behavioral therapy; PeerGroup = peer group therapy; Educ = psychoeducation; CM = contingency management; ICM = intensive case management; TAU = treatment as usual 
Table G-2. Nonbrief interventions, cannabis use days

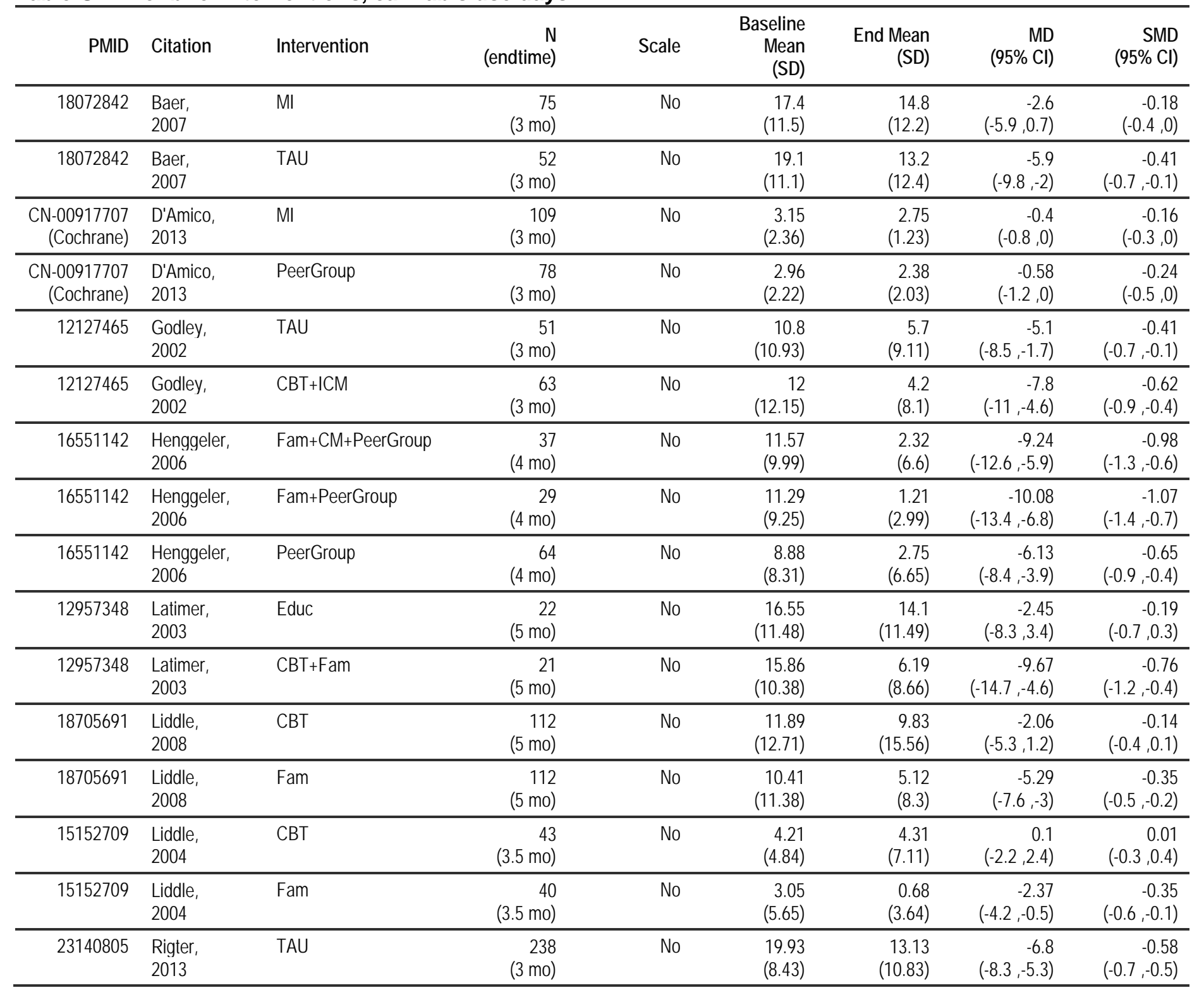




\begin{tabular}{|c|c|c|c|c|c|c|c|c|}
\hline PMID & Citation & Intervention & $\begin{array}{r}\mathrm{N} \\
\text { (endtime) }\end{array}$ & Scale & $\begin{array}{r}\text { Baseline } \\
\text { Mean } \\
\text { (SD) }\end{array}$ & $\begin{array}{r}\text { End Mean } \\
\text { (SD) }\end{array}$ & $\begin{array}{r}\text { MD } \\
(95 \% \mathrm{Cl})\end{array}$ & $\begin{array}{r}\text { SMD } \\
(95 \% \mathrm{Cl})\end{array}$ \\
\hline 23140805 & $\begin{array}{l}\text { Rigter, } \\
2013\end{array}$ & Fam & $\begin{array}{r}212 \\
(3 \mathrm{mo})\end{array}$ & No & $\begin{array}{r}20.5 \\
(8.47)\end{array}$ & $\begin{array}{r}15.07 \\
(10.07)\end{array}$ & $\begin{array}{r}-5.43 \\
(-7,-3.9)\end{array}$ & $\begin{array}{r}-0.47 \\
(-0.6,-0.3)\end{array}$ \\
\hline 26004659 & $\begin{array}{l}\text { Stanger, } \\
2015\end{array}$ & $\mathrm{CBT}+\mathrm{MI}$ & $\begin{array}{r}38 \\
(3 \mathrm{mo})\end{array}$ & No & $\begin{array}{l}1.3 \\
(1)\end{array}$ & $\begin{array}{r}1.2 \\
(0.9)\end{array}$ & $\begin{array}{r}-0.1 \\
(-0.5,0.3)\end{array}$ & $\begin{array}{r}-0.08 \\
(-0.4,0.2)\end{array}$ \\
\hline 26004659 & $\begin{array}{l}\text { Stanger, } \\
2015\end{array}$ & $\mathrm{CBT}+\mathrm{Ml}+\mathrm{CM}$ & $\begin{array}{r}80 \\
(3 \mathrm{mo})\end{array}$ & No & $\begin{array}{r}1.15 \\
(1.05)\end{array}$ & $\begin{array}{r}1.35 \\
(1.11)\end{array}$ & $\begin{array}{r}0.2 \\
(-0.1,0.5)\end{array}$ & $\begin{array}{r}0.16 \\
(-0.1,0.4)\end{array}$ \\
\hline $\begin{array}{r}\text { CN-01365355 } \\
\text { (Cochrane) }\end{array}$ & $\begin{array}{l}\text { Tolou-Shams, } \\
2017\end{array}$ & Educ & $\begin{array}{r}22 \\
(3 \mathrm{mo})\end{array}$ & No & $\begin{array}{r}5.12 \\
(8.26)\end{array}$ & $\begin{array}{r}6.48 \\
(10.63)\end{array}$ & $\begin{array}{r}1.36 \\
(-3.5,6.3)\end{array}$ & $\begin{array}{r}0.1 \\
(-0.3,0.5)\end{array}$ \\
\hline $\begin{array}{r}\text { CN-01365355 } \\
\text { (Cochrane) }\end{array}$ & $\begin{array}{l}\text { Tolou-Shams, } \\
2017\end{array}$ & $\mathrm{CBT}+\mathrm{Fam}$ & $\begin{array}{r}25 \\
(3 \mathrm{mo})\end{array}$ & No & $\begin{array}{r}12.21 \\
(13.38)\end{array}$ & $\begin{array}{r}8.75 \\
(12.38)\end{array}$ & $\begin{array}{r}-3.47 \\
(-9.7,2.7)\end{array}$ & $\begin{array}{r}-0.25 \\
(-0.7,0.2)\end{array}$ \\
\hline 11680557 & $\begin{array}{l}\text { Waldron, } \\
2001\end{array}$ & Educ & $\begin{array}{r}30 \\
(4 \mathrm{mo})\end{array}$ & No & $\begin{array}{l}19.86 \\
(8.11)\end{array}$ & $\begin{array}{r}16.72 \\
(10.46)\end{array}$ & $\begin{array}{r}-3.14 \\
(-7.3,1)\end{array}$ & $\begin{array}{r}-0.26 \\
(-0.6,0.1)\end{array}$ \\
\hline 11680557 & $\begin{array}{l}\text { Waldron, } \\
2001\end{array}$ & $\mathrm{CBT}+\mathrm{Ml}$ & $\begin{array}{r}31 \\
(4 \mathrm{mo})\end{array}$ & No & $\begin{array}{l}15.66 \\
(9.71)\end{array}$ & $\begin{array}{r}15.63 \\
(12.03)\end{array}$ & $\begin{array}{r}-0.03 \\
(-4.8,4.7)\end{array}$ & $\begin{array}{r}0 \\
(-0.4,0.4)\end{array}$ \\
\hline 11680557 & $\begin{array}{l}\text { Waldron, } \\
2001\end{array}$ & $\mathrm{CBT}+\mathrm{Ml}+\mathrm{Fam}$ & $\begin{array}{r}29 \\
(4 \mathrm{mo})\end{array}$ & No & $\begin{array}{r}17.02 \\
(10.49)\end{array}$ & $\begin{array}{r}11.42 \\
(10.95)\end{array}$ & $\begin{array}{r}-5.59 \\
(-10.4,-0.8)\end{array}$ & $\begin{array}{r}-0.46 \\
(-0.9,-0.1)\end{array}$ \\
\hline 11680557 & $\begin{array}{l}\text { Waldron, } \\
2001\end{array}$ & Fam & $\begin{array}{r}30 \\
(4 \mathrm{mo})\end{array}$ & No & $\begin{array}{l}16.46 \\
(9.81)\end{array}$ & $\begin{array}{r}7.49 \\
(8.09)\end{array}$ & $\begin{array}{r}-8.98 \\
(-12.9,-5)\end{array}$ & $\begin{array}{r}-0.74 \\
(-1.1,-0.4)\end{array}$ \\
\hline
\end{tabular}

Abbreviations: PMID = PubMed ID (or other ID), N=number randomized;SD = standard deviation;End mean = mean at End;MD = mean difference; SMD = standardized mean difference; $\mathrm{MI}=$ motivational interviewing; Fam = family therapy; $\mathrm{CBT}=$ cognitive behavioral therapy; PeerGroup = peer group therapy; Educ = psychoeducation; CM = contingency management; ICM = intensive case management; TAU = treatment as usual 
$\underline{\text { Table G-3. Nonbrief interventions, alcohol and other drug use days }}$

\begin{tabular}{|c|c|c|c|c|c|c|c|c|}
\hline PMID & Citation & Intervention & $\begin{array}{r}\mathrm{N} \\
\text { (endtime) }\end{array}$ & Scale & $\begin{array}{r}\text { Baseline } \\
\text { Mean } \\
(\mathrm{SD})\end{array}$ & $\begin{array}{r}\text { End Mean } \\
\text { (SD) }\end{array}$ & $\begin{array}{r}M D \\
(95 \% \mathrm{Cl})\end{array}$ & $\begin{array}{r}\text { SMD } \\
(95 \% \mathrm{Cl})\end{array}$ \\
\hline $\begin{array}{r}\mathrm{CN}-00241903 \\
\text { (Cochrane) }\end{array}$ & $\begin{array}{l}\text { Azrin, } \\
1994\end{array}$ & CBT & $\begin{array}{r}15 \\
(6 \mathrm{mo})\end{array}$ & No & $\begin{array}{r}6.6 \\
(7.4)\end{array}$ & $\begin{array}{r}2.3 \\
(2.9)\end{array}$ & $\begin{array}{r}-4.3 \\
(-8,-0.6)\end{array}$ & $\begin{array}{r}-0.58 \\
(-1.1,-0.1)\end{array}$ \\
\hline $\begin{array}{r}\mathrm{CN}-00241903 \\
\text { (Cochrane) }\end{array}$ & $\begin{array}{l}\text { Azrin, } \\
1994\end{array}$ & PeerGroup & $\begin{array}{r}11 \\
(6 \mathrm{mo})\end{array}$ & No & $\begin{array}{r}6.8 \\
(5.3)\end{array}$ & $\begin{array}{r}8.5 \\
(8.1)\end{array}$ & $\begin{array}{r}1.7 \\
(-3.3,6.7) \\
\end{array}$ & $\begin{array}{r}0.23 \\
(-0.4,0.9)\end{array}$ \\
\hline 25621927 & $\begin{array}{l}\text { Dakof, } \\
2015\end{array}$ & $\mathrm{CBT}+\mathrm{MI}$ & $\begin{array}{r}57 \\
(6 \mathrm{mo})\end{array}$ & No & $\begin{array}{r}22.27 \\
(13.94)\end{array}$ & $\begin{array}{r}7.72 \\
(13.96)\end{array}$ & $\begin{array}{r}-14.55 \\
(-19,-10.1)\end{array}$ & $\begin{array}{r}-0.9 \\
(-1.2,-0.6)\end{array}$ \\
\hline 25621927 & $\begin{array}{l}\text { Dakof, } \\
2015\end{array}$ & Fam & $\begin{array}{r}55 \\
(6 \mathrm{mo})\end{array}$ & No & $\begin{array}{r}20.62 \\
(14.42)\end{array}$ & $\begin{array}{r}4.96 \\
(10.54)\end{array}$ & $\begin{array}{r}-15.66 \\
(-19.8,-11.5)\end{array}$ & $\begin{array}{r}-0.97 \\
(-1.2,-0.7)\end{array}$ \\
\hline 20219293 & $\begin{array}{l}\text { Godley, } \\
2010\end{array}$ & TAU & $\begin{array}{r}80 \\
(3 \mathrm{mo})\end{array}$ & No & $\begin{array}{r}9.12 \\
(8.99)\end{array}$ & $\begin{array}{r}7.08 \\
(7.56)\end{array}$ & $\begin{array}{r}-2.04 \\
(-4.3,0.2)\end{array}$ & $\begin{array}{r}-0.24 \\
(-0.5,0)\end{array}$ \\
\hline 20219293 & $\begin{array}{l}\text { Godley, } \\
2010\end{array}$ & CBT+ICM & $\begin{array}{r}80 \\
(3 \mathrm{mo})\end{array}$ & No & $\begin{array}{r}7.68 \\
(7.98)\end{array}$ & $\begin{array}{r}4.11 \\
(5.09)\end{array}$ & $\begin{array}{r}-3.57 \\
(-5.4,-1.7) \\
\end{array}$ & $\begin{array}{r}-0.42 \\
(-0.6,-0.2) \\
\end{array}$ \\
\hline 20219293 & $\begin{array}{l}\text { Godley, } \\
2010\end{array}$ & $\mathrm{CBT}+\mathrm{MI}$ & $\begin{array}{r}79 \\
(3 \mathrm{mo})\end{array}$ & No & $\begin{array}{r}6.57 \\
(7.19)\end{array}$ & $\begin{array}{r}4.38 \\
(5.35)\end{array}$ & $\begin{array}{r}-2.19 \\
(-3.9,-0.5)\end{array}$ & $\begin{array}{r}-0.26 \\
(-0.5,-0.1)\end{array}$ \\
\hline 20219293 & $\begin{array}{l}\text { Godley, } \\
2010\end{array}$ & $\mathrm{CBT}+\mathrm{MI}+\mathrm{ICM}$ & $\begin{array}{r}81 \\
(3 \mathrm{mo}) \\
\end{array}$ & No & $\begin{array}{r}7.32 \\
(7.73)\end{array}$ & $\begin{array}{r}3.63 \\
(4.62) \\
\end{array}$ & $\begin{array}{r}-3.69 \\
(-5.4,-2) \\
\end{array}$ & $\begin{array}{r}-0.43 \\
(-0.6,-0.2) \\
\end{array}$ \\
\hline 25496283 & $\begin{array}{l}\text { Hogue, } \\
2015\end{array}$ & TAU & $\begin{array}{r}79 \\
(3 \mathrm{mo})\end{array}$ & No & $\begin{array}{r}6.2 \\
(9.2)\end{array}$ & $\begin{array}{r}6.8 \\
(8.7)\end{array}$ & $\begin{array}{r}0.6 \\
(-1.7,2.9)\end{array}$ & $\begin{array}{r}0.06 \\
(-0.2,0.3)\end{array}$ \\
\hline 25496283 & $\begin{array}{l}\text { Hogue, } \\
2015\end{array}$ & Fam & $\begin{array}{r}75 \\
(3 \mathrm{mo}) \\
\end{array}$ & No & $\begin{array}{r}6.6 \\
(8.5)\end{array}$ & $\begin{array}{r}8.9 \\
(9.9)\end{array}$ & $\begin{array}{r}2.3 \\
(-0.1,4.7) \\
\end{array}$ & $\begin{array}{r}0.22 \\
(0,0.5)\end{array}$ \\
\hline 29866383 & $\begin{array}{l}\text { Liddle, } \\
2018\end{array}$ & TAU & $\begin{array}{r}53 \\
(4 \mathrm{mo})\end{array}$ & No & $\begin{array}{r}27.57 \\
(18.01)\end{array}$ & $\begin{array}{r}5.07 \\
(10.7)\end{array}$ & $\begin{array}{r}-22.5 \\
(-27.4,-17.6) \\
\end{array}$ & $\begin{array}{r}-1.24 \\
(-1.5,-1) \\
\end{array}$ \\
\hline 29866383 & $\begin{array}{l}\text { Liddle, } \\
2018\end{array}$ & Fam & $\begin{array}{r}55 \\
(4 \mathrm{mo}) \\
\end{array}$ & No & $\begin{array}{r}33.4 \\
(19.05) \\
\end{array}$ & $\begin{array}{r}5.31 \\
(7.74)\end{array}$ & $\begin{array}{r}-28.09 \\
(-32.9,-23.2) \\
\end{array}$ & $\begin{array}{r}-1.55 \\
(-1.8,-1.3) \\
\end{array}$ \\
\hline 16989957 & $\begin{array}{l}\text { Slesnick, } \\
2007\end{array}$ & TAU & $\begin{array}{r}84 \\
(6 \mathrm{mo})\end{array}$ & No & $\begin{array}{r}18 \\
(10.2)\end{array}$ & $\begin{array}{r}15 \\
(10.8)\end{array}$ & $\begin{array}{r}-3 \\
(-5.8,-0.2)\end{array}$ & $\begin{array}{r}-0.23 \\
(-0.4,0)\end{array}$ \\
\hline 16989957 & $\begin{array}{l}\text { Slesnick, } \\
2007\end{array}$ & CBT & $\begin{array}{r}96 \\
(6 \mathrm{mo})\end{array}$ & No & $\begin{array}{l}20.1 \\
(9.9)\end{array}$ & $\begin{array}{r}12.9 \\
(11.1)\end{array}$ & $\begin{array}{r}-7.2 \\
(-9.8,-4.6) \\
\end{array}$ & $\begin{array}{r}-0.56 \\
(-0.8,-0.4) \\
\end{array}$ \\
\hline 25736623 & $\begin{array}{l}\text { Slesnick, } \\
2015\end{array}$ & Ml & $\begin{array}{r}86 \\
(3 \mathrm{mo}) \\
\end{array}$ & No & $\begin{array}{r}20.51 \\
(10.86) \\
\end{array}$ & $\begin{array}{r}13.7 \\
(13.03) \\
\end{array}$ & $\begin{array}{r}-6.81 \\
(-9.9,-3.7) \\
\end{array}$ & $\begin{array}{r}-0.47 \\
(-0.7,-0.3) \\
\end{array}$ \\
\hline 25736623 & $\begin{array}{l}\text { Slesnick, } \\
2015\end{array}$ & CBT & $\begin{array}{r}93 \\
(3 \mathrm{mo})\end{array}$ & No & $\begin{array}{l}17.63 \\
(11.8)\end{array}$ & $\begin{array}{r}16.08 \\
(12.23)\end{array}$ & $\begin{array}{r}-1.55 \\
(-4.5,1.4)\end{array}$ & $\begin{array}{r}-0.11 \\
(-0.3,0.1)\end{array}$ \\
\hline
\end{tabular}




\begin{tabular}{|c|c|c|c|c|c|c|c|c|}
\hline PMID & Citation & Intervention & $\begin{array}{r}\mathrm{N} \\
\text { (endtime) }\end{array}$ & Scale & $\begin{array}{r}\text { Baseline } \\
\text { Mean } \\
(\mathrm{SD})\end{array}$ & $\begin{array}{r}\text { End Mean } \\
\text { (SD) }\end{array}$ & $\begin{array}{r}M D \\
(95 \% \mathrm{Cl})\end{array}$ & $\begin{array}{r}\text { SMD } \\
(95 \% \mathrm{Cl})\end{array}$ \\
\hline 25736623 & $\begin{array}{l}\text { Slesnick, } \\
2015\end{array}$ & ICM & $\begin{array}{r}91 \\
(3 \mathrm{mo})\end{array}$ & No & $\begin{array}{l}16.64 \\
(10.4)\end{array}$ & $\begin{array}{l}14.81 \\
(12.2)\end{array}$ & $\begin{array}{r}-1.83 \\
(-4.7,1)\end{array}$ & $\begin{array}{r}-0.13 \\
(-0.3,0.1)\end{array}$ \\
\hline 9824170 & $\begin{array}{l}\text { Kaminer, } \\
1998\end{array}$ & CBT & $\begin{array}{r}16 \\
(3 \mathrm{mo})\end{array}$ & Yes & $\begin{array}{r}3.5 \\
(1.83)\end{array}$ & $\begin{array}{r}1.5 \\
(1.08)\end{array}$ & & $\begin{array}{r}-0.88 \\
(-1.3,-0.5)\end{array}$ \\
\hline 9824170 & $\begin{array}{l}\text { Kaminer, } \\
1998\end{array}$ & PeerGroup & $\begin{array}{r}16 \\
(3 \mathrm{mo})\end{array}$ & Yes & $\begin{array}{r}4.33 \\
(1.67)\end{array}$ & $\begin{array}{r}3.13 \\
(2.64)\end{array}$ & & $\begin{array}{r}-0.53 \\
(-1.1,0.1)\end{array}$ \\
\hline
\end{tabular}

Abbreviations: PMID = PubMed ID (or other ID); N=number randomized ;SD = standard deviation; End mean = mean at End; MD = mean difference; SMD = standardized mean difference; $\mathrm{MI}=$ motivational interviewing; Fam = family therapy; $\mathrm{CBT}$ = cognitive behavioral therapy; PeerGroup = peer group therapy; Educ = psychoeducation; CM = contingency management; ICM = intensive case management; TAU = treatment as usual

\section{Table G-4. Nonbrief interventions, illicit drug use days}

\begin{tabular}{|c|c|c|c|c|c|c|c|c|}
\hline PMID & Citation & Intervention & $\begin{array}{r}\mathrm{N} \\
\text { (endtime) }\end{array}$ & Scale & $\begin{array}{r}\text { Baseline } \\
\text { Mean } \\
(\mathrm{SD})\end{array}$ & $\begin{array}{l}\text { End Mean } \\
\text { (SD) }\end{array}$ & $\begin{array}{r}M D \\
(95 \% \mathrm{Cl})\end{array}$ & $\begin{array}{r}\text { SMD } \\
(95 \% \mathrm{Cl})\end{array}$ \\
\hline $\begin{array}{r}2002-13926- \\
001 \\
\text { (psycINFO) }\end{array}$ & $\begin{array}{l}\text { Azrin, } \\
2001\end{array}$ & CBT & $\begin{array}{r}27 \\
(6 \mathrm{mo})\end{array}$ & No & $\begin{array}{r}14.14 \\
(10.58)\end{array}$ & $\begin{array}{r}9.28 \\
(10.23)\end{array}$ & $\begin{array}{r}-4.86 \\
(-9.7,-0.1)\end{array}$ & $\begin{array}{r}-0.41 \\
(-0.8,0)\end{array}$ \\
\hline $\begin{array}{r}2002-13926- \\
001 \\
\text { (psycINFO) }\end{array}$ & $\begin{array}{l}\text { Azrin, } \\
2001\end{array}$ & Fam & $\begin{array}{r}29 \\
(6 \mathrm{mo})\end{array}$ & No & $\begin{array}{l}13.62 \\
(10.3)\end{array}$ & $\begin{array}{r}9 \\
(8.33)\end{array}$ & $\begin{array}{r}-4.62 \\
(-8.8,-0.4)\end{array}$ & $\begin{array}{r}-0.39 \\
(-0.7,0)\end{array}$ \\
\hline 21967492 & $\begin{array}{l}\text { Robbins, } \\
2011\end{array}$ & TAU & $\begin{array}{r}179 \\
(4 \mathrm{mo})\end{array}$ & No & $\begin{array}{r}3.21 \\
(1.61)\end{array}$ & $\begin{array}{r}2.14 \\
(1.25)\end{array}$ & $\begin{array}{r}-1.07 \\
(-1.3,-0.8)\end{array}$ & $\begin{array}{r}-0.63 \\
(-0.8,-0.5)\end{array}$ \\
\hline 21967492 & $\begin{array}{l}\text { Robbins, } \\
2011\end{array}$ & Fam & $\begin{array}{r}194 \\
(4 \mathrm{mo})\end{array}$ & No & $\begin{array}{r}3.21 \\
(1.79)\end{array}$ & $\begin{array}{r}1.07 \\
(1.25)\end{array}$ & $\begin{array}{r}-2.14 \\
(-2.4,-1.9)\end{array}$ & $\begin{array}{r}-1.26 \\
(-1.4,-1.1)\end{array}$ \\
\hline 19522781 & $\begin{array}{l}\text { Slesnick, } \\
2009\end{array}$ & TAU & $\begin{array}{r}34 \\
(3 \mathrm{mo})\end{array}$ & No & $\begin{array}{l}11.4 \\
(7.5)\end{array}$ & $\begin{array}{r}7.5 \\
(8.4)\end{array}$ & $\begin{array}{r}-3.9 \\
(-7,-0.8)\end{array}$ & $\begin{array}{r}-0.39 \\
(-0.7,-0.1)\end{array}$ \\
\hline 19522781 & $\begin{array}{l}\text { Slesnick, } \\
2009\end{array}$ & Fam & $\begin{array}{r}63 \\
(3 \mathrm{mo})\end{array}$ & No & $\begin{array}{r}12.9 \\
(9.29)\end{array}$ & $\begin{array}{r}7.24 \\
(8.39)\end{array}$ & $\begin{array}{r}-5.66 \\
(-8.2,-3.1)\end{array}$ & $\begin{array}{r}-0.57 \\
(-0.8,-0.3) \\
\end{array}$ \\
\hline 24841864 & $\begin{array}{l}\text { Wagner, } \\
2014\end{array}$ & TAU & $\begin{array}{r}235 \\
(4 \mathrm{mo})\end{array}$ & No & $\begin{array}{r}4.49 \\
(7.46)\end{array}$ & $\begin{array}{r}3.72 \\
(8.08)\end{array}$ & $\begin{array}{r}-0.77 \\
(-2,0.4)\end{array}$ & $\begin{array}{r}-0.09 \\
(-0.2,0)\end{array}$ \\
\hline 24841864 & $\begin{array}{l}\text { Wagner, } \\
2014\end{array}$ & $\mathrm{CBT}+\mathrm{MI}$ & $\begin{array}{r}279 \\
(4 \mathrm{mo})\end{array}$ & No & $\begin{array}{r}5.87 \\
(8.43)\end{array}$ & $\begin{array}{r}2.06 \\
(5.01)\end{array}$ & $\begin{array}{r}-3.81 \\
(-4.8,-2.8)\end{array}$ & $\begin{array}{r}-0.42 \\
(-0.5,-0.3)\end{array}$ \\
\hline 30556713 & $\begin{array}{l}\text { Zhang, } \\
2018\end{array}$ & MI & $\begin{array}{r}86 \\
(3 \mathrm{mo})\end{array}$ & No & $\begin{array}{r}20.45 \\
(10.86)\end{array}$ & $\begin{array}{r}13.7 \\
(12.97)\end{array}$ & $\begin{array}{r}-6.75 \\
(-9.9,-3.6)\end{array}$ & $\begin{array}{r}-0.47 \\
(-0.7,-0.3)\end{array}$ \\
\hline
\end{tabular}




\begin{tabular}{|c|c|c|c|c|c|c|c|c|}
\hline PMID & Citation & Intervention & $\begin{array}{r}\mathrm{N} \\
\text { (endtime) }\end{array}$ & Scale & $\begin{array}{r}\text { Baseline } \\
\text { Mean } \\
\text { (SD) }\end{array}$ & $\begin{array}{r}\text { End Mean } \\
\text { (SD) }\end{array}$ & $\begin{array}{r}M D \\
(95 \% \mathrm{Cl})\end{array}$ & $\begin{array}{r}\text { SMD } \\
(95 \% \mathrm{Cl})\end{array}$ \\
\hline 30556713 & $\begin{array}{l}\text { Zhang, } \\
2018\end{array}$ & CBT & $\begin{array}{r}93 \\
(3 \mathrm{mo})\end{array}$ & No & $\begin{array}{r}17.97 \\
(11.67)\end{array}$ & $\begin{array}{r}16.08 \\
(12.23)\end{array}$ & $\begin{array}{r}-1.89 \\
(-4.9,1.1)\end{array}$ & $\begin{array}{r}-0.13 \\
(-0.3,0.1)\end{array}$ \\
\hline 30556713 & $\begin{array}{l}\text { Zhang, } \\
2018\end{array}$ & $\mathrm{ICM}$ & $\begin{array}{r}91 \\
(3 \mathrm{mo})\end{array}$ & No & $\begin{array}{r}17.04 \\
(10.46)\end{array}$ & $\begin{array}{l}14.81 \\
(12.2)\end{array}$ & $\begin{array}{r}-2.23 \\
(-5.1,0.6)\end{array}$ & $\begin{array}{r}-0.16 \\
(-0.4,0)\end{array}$ \\
\hline 11727882 & $\begin{array}{l}\text { Liddle, } \\
2001\end{array}$ & Fam & $\begin{array}{r}100 \\
(5.5 \mathrm{mo})\end{array}$ & Yes & $\begin{array}{r}9.96 \\
(3.61)\end{array}$ & $\begin{array}{r}6.1 \\
(4.28)\end{array}$ & & $\begin{array}{r}-0.86 \\
(-1.1,-0.6)\end{array}$ \\
\hline 11727882 & $\begin{array}{l}\text { Liddle, } \\
2001\end{array}$ & PeerGroup & $\begin{array}{r}52 \\
(5.5 \mathrm{mo})\end{array}$ & Yes & $\begin{array}{r}8.83 \\
(2.76)\end{array}$ & $\begin{array}{r}7.33 \\
(3.41)\end{array}$ & & $\begin{array}{r}-0.33 \\
(-0.6,-0.1)\end{array}$ \\
\hline $\begin{array}{r}2017-00657- \\
001 \\
\text { (psycINFO) }\end{array}$ & $\begin{array}{l}\text { Trudeau, } \\
2017\end{array}$ & TAU & $\begin{array}{r}80 \\
(3 \mathrm{mo})\end{array}$ & Yes & $\begin{array}{r}56.77 \\
(14.75)\end{array}$ & $\begin{array}{r}59.08 \\
(12.01)\end{array}$ & & $\begin{array}{r}0.14 \\
(-0.1,0.4)\end{array}$ \\
\hline $\begin{array}{r}2017-00657- \\
001 \\
\text { (psycINFO) }\end{array}$ & $\begin{array}{l}\text { Trudeau, } \\
2017\end{array}$ & CBT & $\begin{array}{r}49 \\
(3 \mathrm{mo})\end{array}$ & Yes & $\begin{array}{r}57.78 \\
(15.96)\end{array}$ & $\begin{array}{r}57.13 \\
(11.38)\end{array}$ & & $\begin{array}{r}-0.04 \\
(-0.3,0.2)\end{array}$ \\
\hline
\end{tabular}

Abbreviations: PMID = PubMed ID (or other ID); N=number randomized ;SD = standard deviation; End mean = mean at End; MD = mean difference; SMD = standardized mean difference; $\mathrm{MI}=$ motivational interviewing; Fam = family therapy; $\mathrm{CBT}$ = cognitive behavioral therapy; PeerGroup = peer group therapy; Educ = psychoeducation; $\mathrm{CM}=$ contingency management; ICM = intensive case management; TAU = treatment as usual

\begin{tabular}{|c|c|c|c|c|c|c|c|c|c|c|}
\hline $\begin{array}{l}\text { Author } \\
\text { (Year) } \\
\text { PMID }\end{array}$ & Arm 1 & Arm 2 & Outcome & $\begin{array}{l}\text { Measure } \\
\text { ment }\end{array}$ & $\begin{array}{l}\text { Timepoint } \\
\text { (Months) }\end{array}$ & $\begin{array}{l}\text { Arm } 1 \mathrm{~N} \\
\text { Analyzed }\end{array}$ & $\begin{array}{l}\text { Arm } 1 \\
\text { Outcome }\end{array}$ & $\begin{array}{l}\text { Arm } 2 \mathrm{~N} \\
\text { Analyzed }\end{array}$ & $\begin{array}{l}\text { Arm } 2 \\
\text { Outcome }\end{array}$ & $\begin{array}{l}\text { Calculated } \\
\text { Effect (95\% } \\
\mathrm{Cl}\end{array}$ \\
\hline \multirow{2}{*}{$\begin{array}{l}\text { Kaminer } \\
1998 \\
139,140\end{array}$} & CBT (delivery group) & $\begin{array}{l}\text { Peer Group } \\
\text { (delivery group) }\end{array}$ & $\begin{array}{l}\text { School } \\
\text { Problems }\end{array}$ & T-ASI & 0 & 16 & $1.27(1.10)$ & 16 & $1.75(1.36)$ & - \\
\hline & & & $\begin{array}{l}\text { School } \\
\text { Problems }\end{array}$ & T-ASI & 3 & 16 & $1.22(0.97)$ & 16 & $1.71(1.6)$ & $\begin{array}{l}\text { Net Diff }-0.01 \\
(-0.9,0.88)\end{array}$ \\
\hline \multirow{3}{*}{$\begin{array}{l}\text { Kaminer } \\
2002 \\
141-143\end{array}$} & $\begin{array}{l}\text { Educational (delivery } \\
\text { group) }\end{array}$ & $\begin{array}{l}\text { CBT (delivery } \\
\text { group) }\end{array}$ & $\begin{array}{l}\text { School } \\
\text { Problems }\end{array}$ & T-ASI & 0 & 37 & $1.6(1.2)$ & 51 & $1.6(1.4)$ & - \\
\hline & & & $\begin{array}{l}\text { School } \\
\text { Problems }\end{array}$ & T-ASI & 3 & 37 & $1.6(1.5)$ & 51 & $1.3(1.4)$ & $\begin{array}{l}\text { Net Diff } 0.3(- \\
0.57,1.17)\end{array}$ \\
\hline & & & $\begin{array}{l}\text { School } \\
\text { Problems }\end{array}$ & T-ASI & 9 & 37 & $0.8(1.1)$ & 51 & $0.9(1.0)$ & $\begin{array}{l}\text { Net Diff }-0.1 \\
(-0.76,0.56)\end{array}$ \\
\hline
\end{tabular}




\begin{tabular}{|c|c|c|c|c|c|c|c|c|c|c|}
\hline $\begin{array}{l}\text { Author } \\
\text { (Year) } \\
\text { PMID }\end{array}$ & Arm 1 & Arm 2 & Outcome & $\begin{array}{l}\text { Measure } \\
\text { ment }\end{array}$ & $\begin{array}{l}\text { Timepoint } \\
\text { (Months) }\end{array}$ & $\begin{array}{l}\text { Arm } 1 \mathrm{~N} \\
\text { Analyzed }\end{array}$ & $\begin{array}{l}\text { Arm } 1 \\
\text { Outcome }\end{array}$ & $\begin{array}{l}\text { Arm } 2 \mathrm{~N} \\
\text { Analyzed }\end{array}$ & $\begin{array}{l}\text { Arm } 2 \\
\text { Outcome }\end{array}$ & $\begin{array}{l}\text { Calculated } \\
\text { Effect }(95 \% \\
\mathrm{Cl}\end{array}$ \\
\hline \multirow[t]{6}{*}{$\begin{array}{l}\text { Liddle } \\
2004 \\
152-154\end{array}$} & \multirow[t]{6}{*}{$\begin{array}{l}\text { CBT + Peer Group } \\
\text { (delivery group) }\end{array}$} & \multirow[t]{6}{*}{$\begin{array}{l}\text { Family Therapy } \\
\text { (ecological) }\end{array}$} & $\begin{array}{l}\text { School } \\
\text { problems - } \\
\text { Academic }\end{array}$ & $\begin{array}{l}\text { Adolesce } \\
\text { nt } \\
\text { interview }\end{array}$ & 0 & 39 & $5.38(1.19)$ & 41 & $5.71(1.31)$ & - \\
\hline & & & $\begin{array}{l}\text { School } \\
\text { problems - } \\
\text { Academic }\end{array}$ & $\begin{array}{l}\text { Adolesce } \\
\mathrm{nt} \\
\text { interview }\end{array}$ & 1.5 & 39 & $5.33(1.25)$ & 41 & $5.18(1.13)$ & $\begin{array}{l}\text { Net Diff } 0.48 \\
(-0.06,1.02)\end{array}$ \\
\hline & & & $\begin{array}{l}\text { School } \\
\text { problems - } \\
\text { Academic }\end{array}$ & $\begin{array}{l}\text { Adolesce } \\
\text { nt } \\
\text { interview }\end{array}$ & $3-4$ & 39 & $4.78(1.07)$ & 41 & $4.86(1.03)$ & $\begin{array}{l}\text { Net Diff } 0.25 \\
(-0.26,0.76)\end{array}$ \\
\hline & & & $\begin{array}{l}\text { School } \\
\text { problems - } \\
\text { Discipline }\end{array}$ & $\begin{array}{l}\text { Adolesce } \\
\mathrm{nt} \\
\text { interview }\end{array}$ & 0 & 39 & $8.18(1.87)$ & 41 & $8.37(1.81)$ & - \\
\hline & & & $\begin{array}{l}\text { School } \\
\text { problems - } \\
\text { Discipline }\end{array}$ & $\begin{array}{l}\text { Adolesce } \\
\text { nt } \\
\text { interview }\end{array}$ & 1.5 & 39 & $7.53(1.37)$ & 41 & 7.33 (1.53) & $\begin{array}{l}\text { Net Diff } 0.39 \\
(-0.35,1.13)\end{array}$ \\
\hline & & & $\begin{array}{l}\text { School } \\
\text { problems - } \\
\text { Discipline }\end{array}$ & $\begin{array}{l}\text { Adolesce } \\
\text { nt } \\
\text { interview }\end{array}$ & $3-4$ & 39 & $7.58(1.55)$ & 41 & 7.09 (1.29) & $\begin{array}{l}\text { Net Diff } 0.68 \\
(-0.05,1.41)\end{array}$ \\
\hline \multirow[t]{7}{*}{$\begin{array}{l}\text { Azrin } 2001 \\
2002-112\end{array}$} & \multirow[t]{7}{*}{$\begin{array}{l}\text { CBT (integrated } \\
\text { intervention) }\end{array}$} & \multirow{7}{*}{$\begin{array}{l}\text { Family Therapy } \\
\text { (behavioral; } \\
\text { integrated } \\
\text { intervention) }\end{array}$} & $\begin{array}{l}\text { School } \\
\text { performance }\end{array}$ & PHYS & 0 & 27 & $\begin{array}{l}23.70 \\
(31.88)\end{array}$ & 29 & $\begin{array}{l}20.06 \\
(25.50)\end{array}$ & - \\
\hline & & & $\begin{array}{l}\text { School } \\
\text { performance }\end{array}$ & PHYS & 6 & 27 & $\begin{array}{l}57.52 \\
(32.78)\end{array}$ & 29 & $\begin{array}{l}67.93 \\
(23.51)\end{array}$ & $\begin{array}{l}\text { Net Diff - } \\
14.05(- \\
29.17,1.07)\end{array}$ \\
\hline & & & $\begin{array}{l}\text { School } \\
\text { performance }\end{array}$ & PHYS & 12 & 27 & $\begin{array}{l}60.61 \\
(27.25)\end{array}$ & 29 & $\begin{array}{l}65.74 \\
(26.90\end{array}$ & $\begin{array}{l}\text { Net Diff -8.77 } \\
(-23.53,5.99)\end{array}$ \\
\hline & & & $\begin{array}{l}\text { School } \\
\text { performance }\end{array}$ & YHPS & 0 & 27 & $\begin{array}{l}33.78 \\
(7.80)\end{array}$ & 29 & $\begin{array}{l}32.32 \\
(8.01)\end{array}$ & - \\
\hline & & & $\begin{array}{l}\text { School } \\
\text { performance }\end{array}$ & YHPS & 6 & 27 & $\begin{array}{l}37.89 \\
(11.30)\end{array}$ & 29 & $\begin{array}{l}38.05 \\
(11.02)\end{array}$ & $\begin{array}{l}\text { Net Diff }-1.62 \\
(-6.83,3.59)\end{array}$ \\
\hline & & & $\begin{array}{l}\text { School } \\
\text { performance }\end{array}$ & YHPS & 12 & 27 & $\begin{array}{l}36.85 \\
(9.15)\end{array}$ & 29 & $\begin{array}{l}40.00 \\
(10.63)\end{array}$ & $\begin{array}{l}\text { Net Diff }-4.61 \\
(-9.36,0.14)\end{array}$ \\
\hline & & & $\begin{array}{l}\text { School } \\
\text { performance }\end{array}$ & LSS-A & 0 & 27 & $\begin{array}{l}65.35 \\
(31.89)\end{array}$ & 29 & $\begin{array}{l}63.60 \\
(30.61)\end{array}$ & - \\
\hline
\end{tabular}




\begin{tabular}{|c|c|c|c|c|c|c|c|c|c|c|}
\hline \multirow[t]{3}{*}{$\begin{array}{l}\text { Author } \\
\text { (Year) } \\
\text { PMID } \\
\end{array}$} & Arm 1 & Arm 2 & Outcome & $\begin{array}{l}\text { Measure } \\
\text { ment }\end{array}$ & $\begin{array}{l}\text { Timepoint } \\
\text { (Months) }\end{array}$ & $\begin{array}{l}\text { Arm } 1 \mathrm{~N} \\
\text { Analyzed }\end{array}$ & $\begin{array}{l}\text { Arm } 1 \\
\text { Outcome }\end{array}$ & $\begin{array}{l}\text { Arm } 2 \mathrm{~N} \\
\text { Analyzed }\end{array}$ & $\begin{array}{l}\text { Arm } 2 \\
\text { Outcome }\end{array}$ & $\begin{array}{l}\text { Calculated } \\
\text { Effect }(95 \% \\
\mathrm{Cl}\end{array}$ \\
\hline & & & $\begin{array}{l}\text { School } \\
\text { performance }\end{array}$ & LSS-A & 6 & 27 & $\begin{array}{l}87.41 \\
(17.45)\end{array}$ & 29 & $\begin{array}{l}78.28 \\
(23.77)\end{array}$ & $\begin{array}{l}\text { Net Diff 7.38 } \\
(-7.16,21.92)\end{array}$ \\
\hline & & & $\begin{array}{l}\text { School } \\
\text { performance }\end{array}$ & LSS-A & 12 & 27 & $\begin{array}{l}83.81 \\
(23.90)\end{array}$ & 29 & $\begin{array}{l}79.24 \\
(25.01)\end{array}$ & $\begin{array}{l}\text { Net Diff } 2.82 \\
(-12.12 \\
17.76)\end{array}$ \\
\hline \multirow[t]{4}{*}{$\begin{array}{l}\text { Schaeffer } \\
2013 \\
185\end{array}$} & $\begin{array}{l}\text { NOS + Apprentice } \\
\text { training }\end{array}$ & TAU & $\begin{array}{l}\text { Educational } \\
\text { attainment }\end{array}$ & $\begin{array}{l}\text { Enrollme } \\
\text { nt in a } \\
\text { GED } \\
\text { program }\end{array}$ & 0 & - & - & - & - & - \\
\hline & & & $\begin{array}{l}\text { Educational } \\
\text { attainment }\end{array}$ & $\begin{array}{l}\text { Enrollme } \\
\text { nt in a } \\
\text { GED } \\
\text { program }\end{array}$ & 30 & 50 & $50.0 \%$ & 47 & $26.1 \%$ & $\begin{array}{l}\text { OR* } 2.85 \\
(95 \% \mathrm{Cl}, \\
1.20 \text { to } 6.75)\end{array}$ \\
\hline & & & $\begin{array}{l}\text { Educational } \\
\text { attainment }\end{array}$ & $\begin{array}{l}\text { Graduatio } \\
\mathrm{n} \text { from } \\
\text { high } \\
\text { school }\end{array}$ & 0 & - & - & - & - & - \\
\hline & & & $\begin{array}{l}\text { Educational } \\
\text { attainment }\end{array}$ & $\begin{array}{l}\text { Graduatio } \\
\mathrm{n} \text { from } \\
\text { high } \\
\text { school }\end{array}$ & 30 & 50 & $14.0 \%$ & 47 & $23.4 \%$ & $\begin{array}{l}\text { OR } 0.53 \\
(95 \% \mathrm{Cl} \\
0.19,1.52)\end{array}$ \\
\hline
\end{tabular}

Abbreviations: CBT = cognitive behavioral therapy; CI = confidence interval; Diff = difference; GED = General Equivalency Diploma; LSS-A = Life Satisfaction Scale for

Adolescents; $\mathrm{N}=$ number randomized; Net Diff = difference between in Intervention and Control groups in the mean change from baseline (time 0); NOS = not otherwise

specified; OR = odds ratio; PHYS = Parent Happiness with Youth Scale; PMID = Pubmed ID; T-ASI = Teen Addiction Severity Index; TAU = treatment as usual; YHPS = Youth Happiness with Parent Scale.

Bold font indicates that the 95\% CI does not contain 1 for ORs or 0 for differences. 
Table G-6. Family-related outcomes with nonbrief behavioral interventions

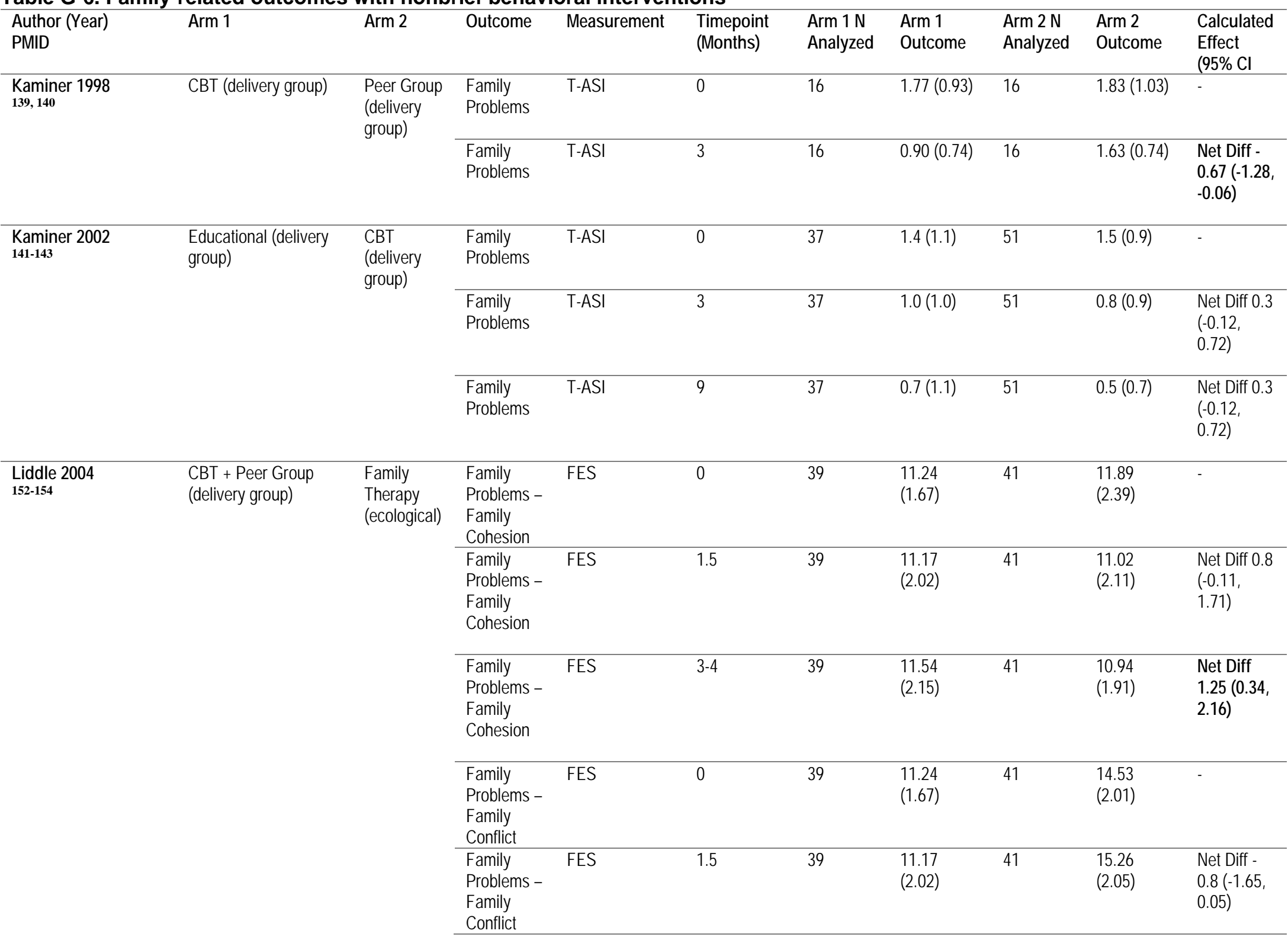




\begin{tabular}{|c|c|c|c|c|c|c|c|c|c|c|}
\hline $\begin{array}{l}\text { Author (Year) } \\
\text { PMID }\end{array}$ & Arm 1 & Arm 2 & Outcome & Measurement & $\begin{array}{l}\text { Timepoint } \\
\text { (Months) }\end{array}$ & $\begin{array}{l}\text { Arm } 1 \mathrm{~N} \\
\text { Analyzed }\end{array}$ & $\begin{array}{l}\text { Arm } 1 \\
\text { Outcome }\end{array}$ & $\begin{array}{l}\text { Arm } 2 \mathrm{~N} \\
\text { Analyzed }\end{array}$ & $\begin{array}{l}\text { Arm } 2 \\
\text { Outcome }\end{array}$ & $\begin{array}{l}\text { Calculated } \\
\text { Effect } \\
(95 \% \mathrm{Cl}\end{array}$ \\
\hline & & & $\begin{array}{l}\text { Family } \\
\text { Problems - } \\
\text { Family } \\
\text { Conflict }\end{array}$ & FES & $3-4$ & 39 & $\begin{array}{l}11.54 \\
(2.15)\end{array}$ & 41 & $\begin{array}{l}15.20 \\
(2.24)\end{array}$ & $\begin{array}{l}\text { Net Diff - } \\
0.37(-1.27 \\
0.53)\end{array}$ \\
\hline & & & $\begin{array}{l}\text { Positive } \\
\text { Family } \\
\text { Interactions }\end{array}$ & ADI & 0 & 43 & $\begin{array}{l}13.75 \\
(2.23)\end{array}$ & 40 & $\begin{array}{l}13.83 \\
(2.07)\end{array}$ & - \\
\hline & & & $\begin{array}{l}\text { Positive } \\
\text { Family } \\
\text { Interactions }\end{array}$ & ADI & 1.5 & 43 & $\begin{array}{l}15.03 \\
(2.04)\end{array}$ & 40 & $\begin{array}{l}14.36 \\
(2.34)\end{array}$ & $\begin{array}{l}\text { Net Diff } \\
0.75(-0.19, \\
1.69)\end{array}$ \\
\hline & & & $\begin{array}{l}\text { Positive } \\
\text { Family } \\
\text { Interactions }\end{array}$ & ADI & $3-4$ & 43 & $\begin{array}{l}15.17 \\
(2.02)\end{array}$ & 40 & $\begin{array}{l}14.26 \\
(2.18)\end{array}$ & $\begin{array}{l}\text { Net Diff } \\
0.99(0.07, \\
1.91)\end{array}$ \\
\hline & & & $\begin{array}{l}\text { Positive } \\
\text { Family } \\
\text { Interactions }\end{array}$ & ADI & 6 & 43 & $\begin{array}{l}15.07 \\
(2.28)\end{array}$ & 40 & $\begin{array}{l}14.85 \\
(2.36)\end{array}$ & $\begin{array}{l}\text { Net Diff } 0.3 \\
(-0.67 \\
1.27)\end{array}$ \\
\hline & & & $\begin{array}{l}\text { Positive } \\
\text { Family } \\
\text { Interactions }\end{array}$ & ADI & 12 & 43 & $\begin{array}{l}14.88 \\
(2.31)\end{array}$ & 40 & $\begin{array}{l}15.16 \\
(2.01)\end{array}$ & $\begin{array}{l}\text { Net Diff - } \\
0.2(-1.13 \text {, } \\
0.73)\end{array}$ \\
\hline & & & $\begin{array}{l}\text { Negative } \\
\text { Family } \\
\text { Interactions }\end{array}$ & ADI & 0 & 43 & $7.15(0.90)$ & 40 & $7.33(1.15)$ & - \\
\hline & & & $\begin{array}{l}\text { Negative } \\
\text { Family } \\
\text { Interactions }\end{array}$ & ADI & 1.5 & 43 & $7.30(0.88)$ & 40 & $7.46(0.75)$ & $\begin{array}{l}\text { Net Diff } \\
0.02(-0.39, \\
0.43)\end{array}$ \\
\hline & & & $\begin{array}{l}\text { Negative } \\
\text { Family } \\
\text { Interactions }\end{array}$ & ADI & $3-4$ & 43 & $7.60(0.65)$ & 40 & $7.38(0.74)$ & $\begin{array}{l}\text { Net Diff } \\
0.4(0.01 \\
0.79)\end{array}$ \\
\hline & & & $\begin{array}{l}\text { Negative } \\
\text { Family } \\
\text { Interactions }\end{array}$ & ADI & 6 & 43 & $7.61(0.60)$ & 40 & $7.43(0.78)$ & $\begin{array}{l}\text { Net Diff } \\
0.36(-0.03 \text {, } \\
0.75)\end{array}$ \\
\hline & & & $\begin{array}{l}\text { Negative } \\
\text { Family } \\
\text { Interactions }\end{array}$ & ADI & 12 & 43 & $7.69(0.52)$ & 40 & $7.53(0.72)$ & $\begin{array}{l}\text { Net Diff } \\
0.34(-0.05, \\
0.73)\end{array}$ \\
\hline $\begin{array}{l}\text { Santisteban } 2011 \\
183\end{array}$ & $\begin{array}{l}\mathrm{Ml}+\text { Educ + Fam } \\
\text { (culturally tailored) }\end{array}$ & $\begin{array}{l}\text { Fam } \\
\text { (structural) }\end{array}$ & $\begin{array}{l}\text { Family } \\
\text { problems - } \\
\text { Parent- } \\
\text { reported } \\
\text { composite }\end{array}$ & PPQ & 0 & 12 & $7.70(0.90)$ & 13 & 7.64 (1.28) & - \\
\hline
\end{tabular}




\begin{tabular}{|c|c|c|c|c|c|c|c|c|c|c|}
\hline $\begin{array}{l}\text { Author (Year) } \\
\text { PMID }\end{array}$ & Arm 1 & Arm 2 & Outcome & Measurement & $\begin{array}{l}\text { Timepoint } \\
\text { (Months) }\end{array}$ & $\begin{array}{l}\text { Arm } 1 \mathrm{~N} \\
\text { Analyzed }\end{array}$ & $\begin{array}{l}\text { Arm } 1 \\
\text { Outcome }\end{array}$ & $\begin{array}{l}\text { Arm } 2 \mathrm{~N} \\
\text { Analyzed }\end{array}$ & $\begin{array}{l}\text { Arm } 2 \\
\text { Outcome }\end{array}$ & $\begin{array}{l}\text { Calculated } \\
\text { Effect } \\
(95 \% \mathrm{Cl}\end{array}$ \\
\hline & & & $\begin{array}{l}\text { Family } \\
\text { problems - } \\
\text { Parent- } \\
\text { reported } \\
\text { composite }\end{array}$ & $\mathrm{PPQ}$ & 8 & 12 & $8.00(1.10)$ & 13 & 7.49 (1.14) & $\begin{array}{l}\text { Net Diff } \\
0.45(-0.43, \\
1.33)\end{array}$ \\
\hline & & & $\begin{array}{l}\text { Family } \\
\text { problems - } \\
\text { Parent- } \\
\text { reported } \\
\text { composite }\end{array}$ & PPQ & 0 & 12 & $6.08(2.09)$ & 13 & $6.26(1.52)$ & - \\
\hline & & & $\begin{array}{l}\text { Family } \\
\text { problems - } \\
\text { Parent- } \\
\text { reported } \\
\text { composite }\end{array}$ & PPQ & 8 & 12 & $7.45(1.54)$ & 13 & $5.89(1.89)$ & $\begin{array}{l}\text { Net Diff } \\
1.74(0.32, \\
3.16)\end{array}$ \\
\hline \multirow[t]{4}{*}{$\begin{array}{l}\text { Robbins } 2011 \\
172-179\end{array}$} & \multirow[t]{4}{*}{ Fam (structural) } & \multirow[t]{4}{*}{ TAU } & $\begin{array}{l}\text { Family } \\
\text { Functioning }\end{array}$ & $\begin{array}{l}\text { Composite of } \\
\text { PPQ and FES }\end{array}$ & 0 & 245 & $-0.03(1.01)$ & 235 & $0.04(0.99)$ & - \\
\hline & & & $\begin{array}{l}\text { Family } \\
\text { Functioning }\end{array}$ & $\begin{array}{l}\text { Composite of } \\
\text { PPQ and FES }\end{array}$ & 4 & 194 & $0.15(1.02)$ & 188 & $0.21(0.94)$ & $\begin{array}{l}\text { Net Diff } \\
0.01(-0.18, \\
0.2)\end{array}$ \\
\hline & & & $\begin{array}{l}\text { Family } \\
\text { Functioning }\end{array}$ & $\begin{array}{l}\text { Composite of } \\
\text { PPQ and FES }\end{array}$ & 8 & 169 & $0.31(0.96)$ & 164 & $0.25(0.97)$ & $\begin{array}{l}\text { Net Diff } \\
0.13(-0.06 \text {, } \\
0.32)\end{array}$ \\
\hline & & & $\begin{array}{l}\text { Family } \\
\text { Functioning }\end{array}$ & $\begin{array}{l}\text { Composite of } \\
\text { PPQ and FES }\end{array}$ & 12 & 169 & $0.35(0.96)$ & 158 & $0.14(0.99)$ & $\begin{array}{l}\text { Net Diff } \\
0.28(0.08 \text {, } \\
0.48)\end{array}$ \\
\hline
\end{tabular}

Abbreviations: ADI = Adolescent Diagnostic Interview; CBT = cognitive behavioral therapy; $\mathrm{CI}=$ confidence interval; FES = Family Environment Scale; MI = motivational interviewing; Net Diff = difference between in Intervention and Control groups in the mean change from baseline (time 0); N = number randomized; NOS = not otherwise specified; PMID = Pubmed ID; PPQ = Parenting Practices Questionnaire; OR = odds ratio; T-ASI = Teen Addiction Severity Index; TAU = treatment as usual.

Bold font indicates that the $95 \%$ CI does not contain 1 for ORs or 0 for differences 
Table G-7. Peer-related outcomes with nonbrief behavioral interventions

\begin{tabular}{|c|c|c|c|c|c|c|c|c|c|c|}
\hline $\begin{array}{l}\text { Author } \\
\text { (Year) } \\
\text { PMID }\end{array}$ & Arm 1 & Arm 2 & Outcome & Measurement & $\begin{array}{l}\text { Timepoint } \\
\text { (Months) }\end{array}$ & $\begin{array}{l}\text { Arm } 1 \mathrm{~N} \\
\text { Analyzed }\end{array}$ & $\begin{array}{l}\text { Arm } 1 \\
\text { Outcome }\end{array}$ & $\begin{array}{l}\text { Arm } 2 \mathrm{~N} \\
\text { Analyzed }\end{array}$ & $\begin{array}{l}\text { Arm } 2 \\
\text { Outcome }\end{array}$ & $\begin{array}{l}\text { Calculated } \\
\text { Effect }(95 \% \mathrm{Cl}\end{array}$ \\
\hline \multirow{2}{*}{$\begin{array}{l}\text { Kaminer } \\
1998 \\
\mathbf{1 3 9 , 1 4 0}\end{array}$} & CBT (delivery group) & $\begin{array}{l}\text { Peer Group (delivery } \\
\text { group) }\end{array}$ & $\begin{array}{l}\text { Peer } \\
\text { Problems }\end{array}$ & T-ASI & 0 & 16 & $1.08(1.31)$ & 16 & $1.58(0.79)$ & - \\
\hline & & & $\begin{array}{l}\text { Peer } \\
\text { Problems }\end{array}$ & T-ASI & 3 & 16 & $1.30(1.25)$ & 16 & $1.38(0.92)$ & $\begin{array}{l}\text { Net Diff } 0.42(- \\
0.34,1.18)\end{array}$ \\
\hline \multirow{3}{*}{$\begin{array}{l}\text { Kaminer } \\
2002 \\
\mathbf{1 4 1 - 1 4 3}\end{array}$} & $\begin{array}{l}\text { Educational (delivery } \\
\text { group) }\end{array}$ & CBT (delivery group) & $\begin{array}{l}\text { Peer } \\
\text { Problems }\end{array}$ & T-ASI & 0 & 37 & $0.5(0.8)$ & 51 & $0.6(0.7)$ & - \\
\hline & & & $\begin{array}{l}\text { Peer } \\
\text { Problems }\end{array}$ & T-ASI & 3 & 37 & $0.6(0.6)$ & 51 & $0.5(0.6)$ & $\begin{array}{l}\text { Net Diff } 0.2(- \\
0.09,0.49)\end{array}$ \\
\hline & & & $\begin{array}{l}\text { Peer } \\
\text { Problems }\end{array}$ & T-ASI & 9 & 37 & $0.5(0.5)$ & 51 & $0.5(0.8)$ & $\begin{array}{l}\text { Net Diff } 0.1(- \\
0.19,0.39)\end{array}$ \\
\hline \multirow[t]{5}{*}{$\begin{array}{l}\text { Liddle } \\
2004 \\
\text { 152-154 }\end{array}$} & $\begin{array}{l}\text { CBT + Peer Group } \\
\text { (delivery group) }\end{array}$ & $\begin{array}{l}\text { Family Therapy } \\
\text { (ecological) }\end{array}$ & $\begin{array}{l}\text { Peer } \\
\text { problems }\end{array}$ & $\begin{array}{l}\text { Affiliation with } \\
\text { delinquent } \\
\text { peers }\end{array}$ & 0 & 43 & $\begin{array}{l}100.26 \\
(15.45)\end{array}$ & 40 & $\begin{array}{l}99.07 \\
(15.90)\end{array}$ & - \\
\hline & & & $\begin{array}{l}\text { Peer } \\
\text { problems }\end{array}$ & $\begin{array}{l}\text { Affiliation with } \\
\text { delinquent } \\
\text { peers }\end{array}$ & 1.5 & 43 & $\begin{array}{l}114.67 \\
(14.11)\end{array}$ & 40 & $\begin{array}{l}112.28 \\
(12.38)\end{array}$ & $\begin{array}{l}\text { Net Diff } 1.2(- \\
5.1,7.5)\end{array}$ \\
\hline & & & $\begin{array}{l}\text { Peer } \\
\text { problems }\end{array}$ & $\begin{array}{l}\text { Affiliation with } \\
\text { delinquent } \\
\text { peers }\end{array}$ & $3-4$ & 43 & $\begin{array}{l}105.23 \\
(14.52)\end{array}$ & 40 & $\begin{array}{l}113.11 \\
(4.80)\end{array}$ & $\begin{array}{l}\text { Net Diff }-9.07 \text { (- } \\
15.34,-2.8)\end{array}$ \\
\hline & & & $\begin{array}{l}\text { Peer } \\
\text { problems }\end{array}$ & $\begin{array}{l}\text { Affiliation with } \\
\text { delinquent } \\
\text { peers }\end{array}$ & 6 & 43 & $\begin{array}{l}109.52 \\
(9.57)\end{array}$ & 40 & $\begin{array}{l}113.50 \\
(3.47)\end{array}$ & $\begin{array}{l}\text { Net Diff -5.17 (- } \\
11.21,0.87)\end{array}$ \\
\hline & & & $\begin{array}{l}\text { Peer } \\
\text { problems }\end{array}$ & $\begin{array}{l}\text { Affiliation with } \\
\text { delinquent } \\
\text { peers }\end{array}$ & 12 & 43 & $\begin{array}{l}106.27 \\
(20.14)\end{array}$ & 40 & $\begin{array}{l}112.56 \\
(8.13)\end{array}$ & $\begin{array}{l}\text { Net Diff }-7.48(- \\
14.41,-0.55)\end{array}$ \\
\hline
\end{tabular}

Abbreviations: CBT = cognitive behavioral therapy; $\mathrm{CI}=$ confidence interval; Diff $=$ difference; $\mathrm{N}=$ number randomized; Net Diff $=$ difference between in Intervention and Control groups in the mean change from baseline (time 0); NOS = not otherwise specified; OR = odds ratio; PMID = Pubmed ID; T-ASI = Teen Addiction Severity Index; TAU = treatment as usual. Bold font indicates that the $95 \%$ CI does not contain 1 for ORs or 0 for differences 
Table G-8. Mental health outcomes with nonbrief behavioral interventions

\begin{tabular}{|c|c|c|c|c|c|c|c|c|c|}
\hline $\begin{array}{l}\text { Author (Year) } \\
\text { PMID }\end{array}$ & Arm 1 & Arm 2 & Outcome & $\begin{array}{l}\text { Measurem } \\
\text { ent }\end{array}$ & $\begin{array}{l}\text { Timepoint } \\
\text { (Months) }\end{array}$ & $\begin{array}{l}\text { Arm } 1 \mathrm{~N} \\
\text { Analyzed }\end{array}$ & $\begin{array}{l}\text { Arm } 1 \\
\text { Outcome }\end{array}$ & $\begin{array}{l}\text { Arm } 2 \mathrm{~N} \\
\text { Analyzed }\end{array}$ & $\begin{array}{l}\text { Arm } 2 \\
\text { Outcome }\end{array}$ \\
\hline \multirow[t]{9}{*}{$\begin{array}{l}\text { Kaminer } 2008 \\
144-146\end{array}$} & \multirow[t]{3}{*}{$\begin{array}{l}\text { CBT + in-person } \\
\mathrm{MI}\end{array}$} & \multirow[t]{3}{*}{$\mathrm{CBT}+$ brief telephone MI } & $\begin{array}{l}\text { Suicidal } \\
\text { ideation }\end{array}$ & 0 & 38 & $12.2(9.0)$ & 43 & $8.8(6.9)$ & - \\
\hline & & & $\begin{array}{l}\text { Suicidal } \\
\text { ideation }\end{array}$ & 3 & 38 & $13.1(15.3)$ & 43 & $7.4(7.2)$ & $\begin{array}{l}\text { Net Diff } 2.3 \\
(-2.4,7.0)\end{array}$ \\
\hline & & & $\begin{array}{l}\text { Suicidal } \\
\text { ideation }\end{array}$ & 7 & 38 & $8.8(6.5)$ & 43 & $6.1(5.9)$ & $\begin{array}{l}\text { Net Diff }-0.7 \\
(-3.8,2.4)\end{array}$ \\
\hline & \multirow[t]{3}{*}{$\begin{array}{l}\text { CBT + in-person } \\
\text { MI }\end{array}$} & \multirow[t]{3}{*}{ TAU } & $\begin{array}{l}\text { Suicidal } \\
\text { ideation }\end{array}$ & 0 & 38 & $12.2(9.0)$ & 41 & $8.6(6.4)$ & - \\
\hline & & & $\begin{array}{l}\text { Suicidal } \\
\text { ideation }\end{array}$ & 3 & 38 & $13.1(15.3)$ & 41 & $10.6(7.0)$ & $\begin{array}{l}\text { Net Diff }-1.1 \\
(-5.8,3.6)\end{array}$ \\
\hline & & & $\begin{array}{l}\text { Suicidal } \\
\text { ideation }\end{array}$ & 7 & 38 & $8.8(6.5)$ & 41 & $7.0(7.7)$ & $\begin{array}{l}\text { Net Diff }-1.8 \\
(-5.2,1.6)\end{array}$ \\
\hline & \multirow[t]{3}{*}{$\begin{array}{l}\text { CBT + brief } \\
\text { telephone MI }\end{array}$} & \multirow[t]{3}{*}{ TAU } & $\begin{array}{l}\text { Suicidal } \\
\text { ideation }\end{array}$ & 0 & 43 & $8.8(6.9)$ & 41 & $8.6(6.4)$ & - \\
\hline & & & $\begin{array}{l}\text { Suicidal } \\
\text { ideation }\end{array}$ & 3 & 43 & $7.4(7.2)$ & 41 & $10.6(7.0)$ & $\begin{array}{l}\text { Net Diff }-3.4 \\
(-6.3,-0.5)\end{array}$ \\
\hline & & & $\begin{array}{l}\text { Suicidal } \\
\text { ideation }\end{array}$ & 7 & 43 & $6.1(5.9)$ & 41 & $7.0(7.7)$ & $\begin{array}{l}\text { Net Diff }-1.1 \\
(-4.0,1.8)\end{array}$ \\
\hline \multirow[t]{4}{*}{$\begin{array}{l}\text { Esposito-Smythers } 2011 \\
120\end{array}$} & \multirow[t]{4}{*}{$\begin{array}{l}\text { Family (integrated } \\
\text { intervention) }\end{array}$} & \multirow[t]{4}{*}{$\begin{array}{l}\text { TAU (delivery group, } \\
\text { integrated intervention) }\end{array}$} & Suicide attempt & 18 & 19 & $5 \%$ & 17 & $35 \%$ & $\begin{array}{l}\text { OR } 0.10 \\
(0.01,0.96)\end{array}$ \\
\hline & & & $\begin{array}{l}\text { Residential } \\
\text { placement }\end{array}$ & 18 & 19 & $3 \%$ & 17 & $21 \%$ & $\begin{array}{l}\text { OR } 0.10 \\
(0.01,2.19)\end{array}$ \\
\hline & & & Hospitalization & 18 & 19 & $16 \%$ & 17 & $53 \%$ & \\
\hline & & & $\begin{array}{l}\text { Partial } \\
\text { hospitalization }\end{array}$ & 18 & 19 & $5 \%$ & 17 & $24 \%$ & $\begin{array}{l}\text { OR } 0.18(0.02 \\
1.81)\end{array}$ \\
\hline
\end{tabular}

Abbreviations: CBT = cognitive behavioral therapy; CI = confidence interval; MI = motivational interviewing; $\mathrm{N}=$ number; Net Diff = difference between in Intervention and Control groups in the mean change from baseline (time 0); OR = odds ratio; PMID = Pubmed ID; TAU = treatment as usual. Bold font indicates that the 95\% CI does not contain 1 for ORs or 0 for differences. 
Table G-9. Physical health outcomes with nonbrief behavioral interventions

\begin{tabular}{|c|c|c|c|c|c|c|c|c|c|}
\hline $\begin{array}{l}\text { Author (Year) } \\
\text { PMID }\end{array}$ & Arm 1 & Arm 2 & Outcome & $\begin{array}{l}\text { Measure } \\
\text { ment }\end{array}$ & $\begin{array}{l}\text { Timepoint } \\
\text { (Months) }\end{array}$ & $\begin{array}{l}\text { Arm } 1 \mathrm{~N} \\
\text { Analyzed }\end{array}$ & $\begin{array}{l}\text { Arm } 1 \\
\text { Outcome }\end{array}$ & $\begin{array}{l}\text { Arm } 2 \mathrm{~N} \\
\text { Analyzed }\end{array}$ & Arm 2 Outcome \\
\hline \multirow[t]{8}{*}{$\begin{array}{l}\text { Rowe } 2016 \\
182\end{array}$} & \multirow[t]{8}{*}{$\begin{array}{l}\mathrm{CBT}+\mathrm{MI} \text { (integrated } \\
\text { intervention, parent) }\end{array}$} & \multirow[t]{8}{*}{ TAU } & $\begin{array}{l}\text { Testing positive for } \\
\text { STI }\end{array}$ & 0 & 76 & $9 \%$ & 78 & $12 \%$ & - \\
\hline & & & $\begin{array}{l}\text { Testing positive for } \\
\text { STI }\end{array}$ & 3 & 76 & $7 \%$ & 78 & $8 \%$ & $\begin{array}{l}\text { OR } 0.85(0.25 \\
2.89)\end{array}$ \\
\hline & & & $\begin{array}{l}\text { Testing positive for } \\
\text { STI }\end{array}$ & 6 & 76 & $4 \%$ & 78 & $6 \%$ & $\begin{array}{l}\text { OR } 0.60(0.14, \\
2.60)\end{array}$ \\
\hline & & & $\begin{array}{l}\text { Testing positive for } \\
\text { STI }\end{array}$ & 9 & 76 & $1 \%$ & 78 & $5 \%$ & $\begin{array}{l}\text { OR } 0.25(0.03, \\
2.26)\end{array}$ \\
\hline & & & $\begin{array}{l}\text { Testing positive for } \\
\text { STI }\end{array}$ & 18 & 76 & $3 \%$ & 78 & $5 \%$ & $\begin{array}{l}\text { OR } 0.50 \text { (0.09, } \\
2.81)\end{array}$ \\
\hline & & & $\begin{array}{l}\text { Testing positive for } \\
\text { STI }\end{array}$ & 24 & 76 & $5 \%$ & 78 & $5 \%$ & $\begin{array}{l}\text { OR } 1.03(0.25, \\
4.27)\end{array}$ \\
\hline & & & $\begin{array}{l}\text { Testing positive for } \\
\text { STI }\end{array}$ & 36 & 76 & $8 \%$ & 78 & $5 \%$ & $\begin{array}{l}\text { OR } 1.59 \text { (0.43, } \\
5.86)\end{array}$ \\
\hline & & & $\begin{array}{l}\text { Testing positive for } \\
\text { STI }\end{array}$ & 42 & 76 & $4 \%$ & 78 & $6 \%$ & $\begin{array}{l}\text { OR 0.60 (0.14, } \\
2.60)\end{array}$ \\
\hline \multirow[t]{2}{*}{$\begin{array}{l}\text { Esposito- } \\
\text { Smythers } 2011 \\
182\end{array}$} & \multirow[t]{2}{*}{$\begin{array}{l}\text { Fam (integrated } \\
\text { intervention) }\end{array}$} & \multirow[t]{2}{*}{$\begin{array}{l}\text { TAU (delivery group, } \\
\text { integrated } \\
\text { intervention) }\end{array}$} & $\begin{array}{l}\text { Emergency } \\
\text { department visit }\end{array}$ & 0 & - & - & - & - & - \\
\hline & & & $\begin{array}{l}\text { Emergency } \\
\text { department visit }\end{array}$ & 18 & 19 & $16 \%$ & 17 & $59 \%$ & $\begin{array}{l}\text { OR } 0.13(0.03 \text {, } \\
0.63)\end{array}$ \\
\hline
\end{tabular}

Abbreviations: $\mathrm{CBT}=$ cognitive behavioral therapy; $\mathrm{CI}=$ confidence interval; $\mathrm{MI}=$ motivational interviewing; $\mathrm{N}=$ number; OR = odds ratio; PMID = Pubmed ID; STI = sexually transmitted infection; TAU = treatment as usual.

Bold font indicates that the $95 \%$ CI does not contain 1 for ORs or 0 for differences. 
Table G-10. Arrests and convictions (legal outcomes) with nonbrief behavioral interventions

\begin{tabular}{|c|c|c|c|c|c|c|c|c|c|c|c|c|}
\hline $\begin{array}{l}\text { Author } \\
\text { (Year) } \\
\text { PMID }\end{array}$ & Arm 1 (N) & Arm 2 (N) & $\begin{array}{l}\text { Time } \\
\text { (mo) }\end{array}$ & Int. & Cont. & $\begin{array}{l}\text { Calc. Effect } \\
(95 \% \mathrm{Cl})\end{array}$ & $\begin{array}{l}\text { Convictio } \\
\text { ns for } \\
\text { Person } \\
\text { Crimes } \\
\text { Int. }\end{array}$ & $\begin{array}{l}\text { Convictio } \\
\text { ns for } \\
\text { Person } \\
\text { Crimes } \\
\text { Cont. }\end{array}$ & $\begin{array}{l}\text { Convictio } \\
\text { ns for } \\
\text { Person } \\
\text { Crimes } \\
\text { Calc. } \\
\text { Effect } \\
(95 \% \mathrm{Cl})\end{array}$ & $\begin{array}{l}\text { Convictio } \\
\text { ns for } \\
\text { Property } \\
\text { Crimes } \\
\text { Int. }\end{array}$ & $\begin{array}{l}\text { Conviction } \\
\text { s for } \\
\text { Property } \\
\text { Crimes } \\
\text { Cont. }\end{array}$ & $\begin{array}{l}\text { Conviction } \\
\text { s for } \\
\text { Property } \\
\text { Crimes } \\
\text { Calc. Effect } \\
(95 \% \text { Cl) }\end{array}$ \\
\hline \multirow[t]{6}{*}{$\begin{array}{l}\text { Henggeler } \\
2006 \\
\mathbf{1 3 4}\end{array}$} & $\begin{array}{l}\text { Fam (drug court + } \\
\text { multisystemic } \\
\text { therapy }+ \\
\text { contingency) }(\mathrm{N}=43)\end{array}$ & $\begin{array}{l}\text { Fam (drug court + } \\
\text { multisystemic } \\
\text { therapy) }(\mathrm{N}=38)\end{array}$ & 12 & $\begin{array}{l}1.28 \\
(1.44)\end{array}$ & $1.40(1.52)$ & $\begin{array}{l}\text { Mean Diff - } \\
0.12(-0.78 \\
0.54)\end{array}$ & - & - & - & - & - & - \\
\hline & $\begin{array}{l}\text { Fam (drug court + } \\
\text { multisystemic } \\
\text { therapy + } \\
\text { contingency) }(\mathrm{N}=43)\end{array}$ & $\begin{array}{l}\text { TAU (family court } \\
+ \text { community } \\
\text { services) }(\mathrm{N}=42)\end{array}$ & 12 & $\begin{array}{l}1.28 \\
(1.44)\end{array}$ & $1.00(1.15)$ & $\begin{array}{l}\text { Mean Diff } \\
0.28(-0.28 \\
0.84)\end{array}$ & - & - & - & - & - & - \\
\hline & $\begin{array}{l}\text { Fam (drug court + } \\
\text { multisystemic } \\
\text { therapy + } \\
\text { contingency) }(\mathrm{N}=43)\end{array}$ & $\begin{array}{l}\text { TAU (drug court + } \\
\text { community } \\
\text { services) }(\mathrm{N}=38)\end{array}$ & 12 & $\begin{array}{l}1.28 \\
(1.44)\end{array}$ & $1.45(1.35)$ & $\begin{array}{l}\text { Mean Diff - } \\
0.17(-0.79 \\
0.45)\end{array}$ & - & - & - & - & - & - \\
\hline & $\begin{array}{l}\text { Fam (drug court + } \\
\text { multisystemic } \\
\text { therapy) }(\mathrm{N}=38)\end{array}$ & $\begin{array}{l}\text { TAU (family court } \\
+ \text { community } \\
\text { services) }(\mathrm{N}=42)\end{array}$ & 12 & $\begin{array}{l}1.40 \\
(1.52)\end{array}$ & $1.00(1.15)$ & $\begin{array}{l}\text { Mean Diff } \\
0.40(-0.20 \\
1.00)\end{array}$ & - & - & - & - & - & - \\
\hline & $\begin{array}{l}\text { Fam (drug court + } \\
\text { multisystemic } \\
\text { therapy) }(\mathrm{N}=38)\end{array}$ & $\begin{array}{l}\text { TAU (drug court + } \\
\text { community } \\
\text { services) }(\mathrm{N}=38)\end{array}$ & 12 & $\begin{array}{l}1.40 \\
(1.52)\end{array}$ & $1.45(1.35)$ & $\begin{array}{l}\text { Mean Diff - } \\
0.05(-0.71 \\
0.61)\end{array}$ & - & - & - & - & - & - \\
\hline & $\begin{array}{l}\text { TAU (family court + } \\
\text { community services) } \\
(\mathrm{N}=42)\end{array}$ & $\begin{array}{l}\text { TAU (drug court + } \\
\text { community } \\
\text { services) }(\mathrm{N}=38)\end{array}$ & 12 & $\begin{array}{l}1.00 \\
(1.15)\end{array}$ & $1.45(1.35)$ & $\begin{array}{l}\text { Mean Diff - } \\
0.45(-1.00 \\
0.11)\end{array}$ & - & - & - & - & - & - \\
\hline \multirow[t]{3}{*}{$\begin{array}{l}\text { Dakof } \\
2015 \\
\mathbf{1 1 8}\end{array}$} & $\begin{array}{l}\mathrm{CBT}+\mathrm{Ml} \text { (delivery } \\
\text { group) }(\mathrm{N}=57)\end{array}$ & $\begin{array}{l}\text { Fam (ecological) } \\
(\mathrm{N}=55)\end{array}$ & 0 & $\begin{array}{l}2.11 \\
(1.18)\end{array}$ & $1.87(0.94)$ & - & - & - & - & - & - & - \\
\hline & & & 6 & $\begin{array}{l}0.32 \\
(0.69)\end{array}$ & $0.47(0.77)$ & $\begin{array}{l}\text { Net Diff }-0.39 \\
(-0.74,-0.04)\end{array}$ & - & - & - & - & - & - \\
\hline & & & 24 & $\begin{array}{l}1.19 \\
(1.54)\end{array}$ & $0.95(1.24)$ & $\begin{array}{l}\text { Net Diff } 0.00 \\
(-0.47,0.47)\end{array}$ & - & - & - & - & - & - \\
\hline $\begin{array}{l}\text { Azrin } \\
2001 \\
\mathbf{1 1 2}\end{array}$ & $\begin{array}{l}\text { CBT (integrated } \\
\text { intervention) }(\mathrm{N}=27)\end{array}$ & $\begin{array}{l}\text { Fam (behavioral; } \\
\text { integrated } \\
\text { intervention) } \\
(\mathrm{N}=29)\end{array}$ & 0 & $\begin{array}{l}0.84 \\
(1.02)\end{array}$ & $0.93(1.51)$ & - & - & - & - & - & - & - \\
\hline
\end{tabular}




\begin{tabular}{|c|c|c|c|c|c|c|c|c|c|c|c|c|}
\hline \multirow[t]{3}{*}{$\begin{array}{l}\text { Author } \\
\text { (Year) } \\
\text { PMID }\end{array}$} & Arm $1(\mathrm{~N})$ & Arm 2 (N) & $\begin{array}{l}\text { Time } \\
\text { (mo) }\end{array}$ & $\begin{array}{l}\text { Arrests } \\
\text { Int. }\end{array}$ & $\begin{array}{l}\text { Arrests } \\
\text { Cont. }\end{array}$ & $\begin{array}{l}\text { Calc. Effect } \\
(95 \% \mathrm{Cl})\end{array}$ & $\begin{array}{l}\text { Convictio } \\
\text { ns for } \\
\text { Person } \\
\text { Crimes } \\
\text { Int. }\end{array}$ & $\begin{array}{l}\text { Convictio } \\
\text { ns for } \\
\text { Person } \\
\text { Crimes } \\
\text { Cont. }\end{array}$ & $\begin{array}{l}\text { Convictio } \\
\text { ns for } \\
\text { Person } \\
\text { Crimes } \\
\text { Calc. } \\
\text { Effect } \\
(95 \% \mathrm{Cl})\end{array}$ & $\begin{array}{l}\text { Convictio } \\
\text { ns for } \\
\text { Property } \\
\text { Crimes } \\
\text { Int. }\end{array}$ & $\begin{array}{l}\text { Conviction } \\
\text { s for } \\
\text { Property } \\
\text { Crimes } \\
\text { Cont. }\end{array}$ & $\begin{array}{l}\text { Conviction } \\
\text { s for } \\
\text { Property } \\
\text { Crimes } \\
\text { Calc. Effect } \\
(95 \% \text { Cl) }\end{array}$ \\
\hline & & & 6 & $\begin{array}{l}0.42 \\
(0.82)\end{array}$ & $0.28(0.48)$ & $\begin{array}{l}\text { Net Diff } 0.23 \\
(-0.37,0.83)\end{array}$ & - & - & - & - & - & - \\
\hline & & & 12 & $\begin{array}{l}0.24 \\
(0.29)\end{array}$ & $0.51(0.59)$ & $\begin{array}{l}\text { Net Diff }-0.18 \\
(-0.77,0.41)\end{array}$ & - & - & - & - & - & - \\
\hline \multirow{2}{*}{$\begin{array}{l}\text { Henggeler } \\
2001 \\
\mathbf{1 2 9 - 1 3 3}\end{array}$} & $\begin{array}{l}\text { Fam (ecological) } \\
(\mathrm{N}=58)\end{array}$ & $\begin{array}{l}\text { TAU (delivery } \\
\text { group) ( } N=59 \text { ) }\end{array}$ & 11 & $40 \%$ & $53 \%$ & $\begin{array}{l}\text { OR } 0.76 \\
(0.51,1.13)\end{array}$ & - & - & - & - & - & - \\
\hline & & & 48 & - & - & - & $\begin{array}{l}0.15 \\
(0.43)\end{array}$ & $\begin{array}{l}0.57 \\
(1.80)\end{array}$ & $\begin{array}{l}\text { Mean Diff } \\
-0.42(- \\
0.90 \\
0.06)\end{array}$ & $0.19(0.43)$ & $0.20(0.61)$ & $\begin{array}{l}\text { Mean Diff - } \\
0.01(-0.20, \\
0.18)\end{array}$ \\
\hline $\begin{array}{l}\text { Esposito- } \\
\text { Smythers } \\
2011 \\
\mathbf{1 2 0}\end{array}$ & $\begin{array}{l}\text { Fam (structural) } \\
(\mathrm{N}=19)\end{array}$ & TAU (N=17) & 18 & $5 \%$ & $41 \%$ & $\begin{array}{l}\text { OR } 0.08 \\
(0.01,0.74)\end{array}$ & - & - & - & - & - & - \\
\hline
\end{tabular}

Abbreviations: CBT = cognitive behavioral therapy; CI = confidence interval; MI = motivational interviewing; $\mathrm{N}$ = number; OR = odds ratio; PMID = Pubmed ID; STI = sexually transmitted infection; TAU = treatment as usual.

Bold font indicates that the $\mathbf{9 5 \%}$ CI does not contain 1 for ORs or 0 for differences. 
Table G-11. Self-reported legal outcomes with nonbrief behavioral interventions

\begin{tabular}{|c|c|c|c|c|c|c|c|c|c|c|c|c|c|c|c|}
\hline $\begin{array}{l}\text { Author } \\
\text { (Year) } \\
\text { PMID }\end{array}$ & Arm 1 & Arm 2 & $\begin{array}{l}\text { Time } \\
\text { (Months) }\end{array}$ & $\begin{array}{l}\text { General } \\
\text { Del. } \\
\text { Int. }\end{array}$ & $\begin{array}{l}\text { General } \\
\text { Del. } \\
\text { Cont. }\end{array}$ & $\begin{array}{l}\text { General } \\
\text { Del. } \\
\text { Calc. } \\
\text { Effect } \\
(95 \% \mathrm{Cl})\end{array}$ & $\begin{array}{l}\text { Person } \\
\text { Crimes } \\
\text { Int. }\end{array}$ & $\begin{array}{l}\text { Person } \\
\text { Crimes } \\
\text { Cont. }\end{array}$ & $\begin{array}{l}\text { Person } \\
\text { Crimes } \\
\text { Calc. } \\
\text { Effect } \\
(95 \% \mathrm{Cl})\end{array}$ & $\begin{array}{l}\text { Property } \\
\text { Crimesl } \\
\text { Theft } \\
\text { Int. }\end{array}$ & $\begin{array}{l}\text { Property } \\
\text { Crimesl } \\
\text { Theft } \\
\text { Cont. }\end{array}$ & $\begin{array}{l}\text { Calc. Effect } \\
(95 \% \mathrm{Cl})\end{array}$ & $\begin{array}{l}\text { Status } \\
\text { Offense }\end{array}$ & $\begin{array}{l}\text { Status } \\
\text { Offense }\end{array}$ & $\begin{array}{l}\text { Status } \\
\text { Offense } \\
\text { Calc. } \\
\text { Effect } \\
(95 \% \mathrm{Cl})\end{array}$ \\
\hline \multirow[t]{5}{*}{$\begin{array}{l}\text { Liddle } \\
2004 \\
\text { 152-154 }\end{array}$} & $\begin{array}{l}\text { CBT + } \\
\text { Peer } \\
\text { Group } \\
\text { (delivery } \\
\text { group) } \\
(\mathrm{N}=43)\end{array}$ & $\begin{array}{l}\text { Family } \\
\text { Therap } \\
y \\
\text { (ecolog } \\
\text { ical) } \\
(\mathrm{N}=40)\end{array}$ & 0 & $51 \%$ & $48 \%$ & - & - & - & - & - & - & - & - & - & - \\
\hline & & & 1.5 & $33 \%$ & $10 \%$ & $\begin{array}{l}\text { OR } 4.4 \\
(1.3 \\
14.6) \\
\end{array}$ & - & - & - & - & - & - & - & - & - \\
\hline & & & 3 to 4 & $33 \%$ & $15 \%$ & $\begin{array}{l}\text { OR } 2.7 \\
(0.9,8.0)\end{array}$ & - & - & - & - & - & - & - & - & - \\
\hline & & & 6 & $30 \%$ & $28 \%$ & $\begin{array}{l}\text { OR 1.1 } \\
(0.4 .3 .0)\end{array}$ & - & - & - & - & - & - & - & - & - \\
\hline & & & 12 & $33 \%$ & $23 \%$ & $\begin{array}{l}\text { OR 1.7 } \\
(0.6,4.4)\end{array}$ & - & - & - & - & - & - & - & - & - \\
\hline \multirow[t]{4}{*}{$\begin{array}{l}\text { Hogue } \\
2015 \\
\mathbf{1 3 8}\end{array}$} & $\begin{array}{l}\text { Fam } \\
\text { (structur } \\
\text { al) } \\
(\mathrm{N}=104)\end{array}$ & $\begin{array}{l}\text { TAU } \\
(\mathrm{N}=101 \\
)^{-}\end{array}$ & 0 & 3.6 (3.1) & $3.9(2.8)$ & - & - & - & - & - & - & - & - & - & - \\
\hline & & & 3 & $3.5(2.5)$ & $3.1(2.6)$ & $\begin{array}{l}\text { Net Diff } \\
0.7(-0.1 \text {, } \\
1.5)\end{array}$ & - & - & - & - & - & - & - & - & - \\
\hline & & & 6 & $2.3(1.7)$ & $3.0(2.1)$ & $\begin{array}{l}\text { Net Diff - } \\
0.4(-1.1 \\
0.3)\end{array}$ & - & - & - & - & - & - & - & - & - \\
\hline & & & 12 & $2.5(2.5)$ & $2.7(2.6)$ & $\begin{array}{l}\text { Net Diff - } \\
0.1(-0.9 \\
0.7)\end{array}$ & - & - & - & - & - & - & - & - & - \\
\hline \multirow[t]{2}{*}{$\begin{array}{l}\text { Kaminer } \\
1998 \\
\mathbf{1 3 9}, \mathbf{1 4 0}\end{array}$} & $\begin{array}{l}\mathrm{CBT} \\
\text { (delivery } \\
\text { group) } \\
(\mathrm{N}=16)\end{array}$ & $\begin{array}{l}\text { Peer } \\
\text { Group } \\
\text { (deliver } \\
\text { y } \\
\text { group) } \\
(\mathrm{N}=16)\end{array}$ & 0 & $\begin{array}{l}1.33 \\
(1.15)\end{array}$ & $\begin{array}{l}0.75 \\
(1.22)\end{array}$ & - & - & - & - & - & - & - & - & - & - \\
\hline & & & 3 & $\begin{array}{l}0.90 \\
(1.20)\end{array}$ & $1(1.31)$ & $\begin{array}{l}\text { Net Diff - } \\
0.7(-1.5 \\
0.2)\end{array}$ & - & - & - & - & - & - & - & - & - \\
\hline
\end{tabular}




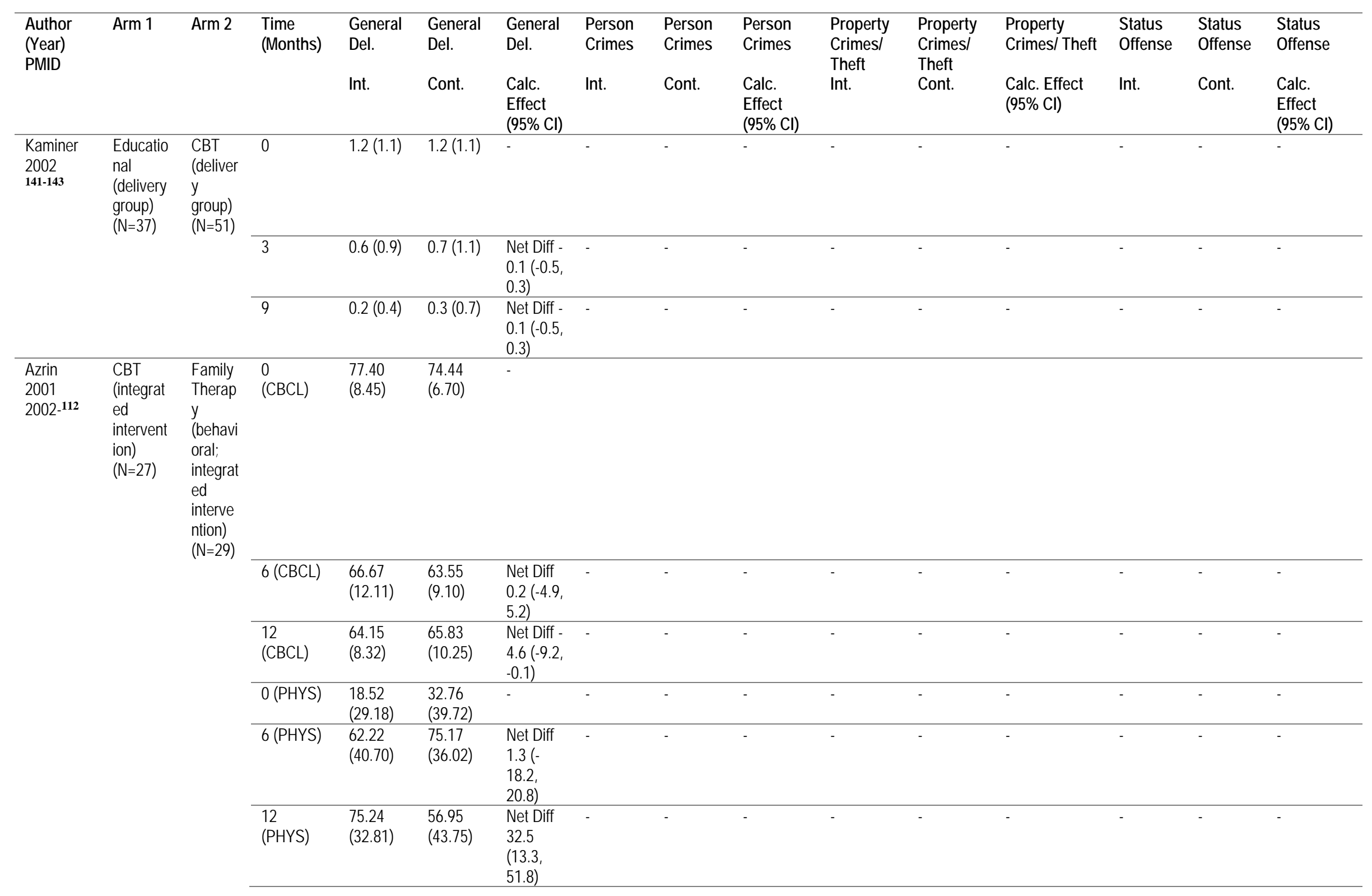




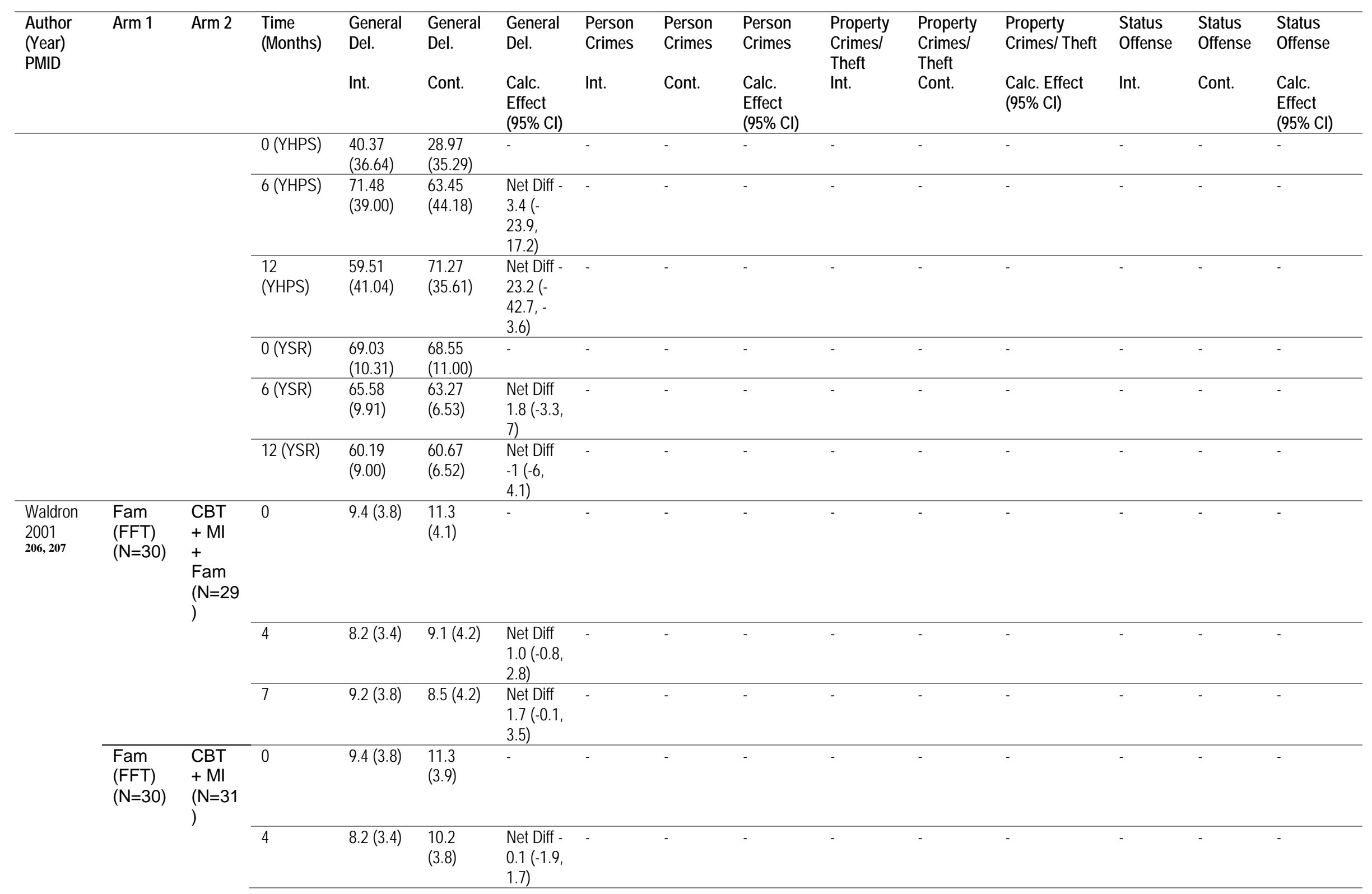




\begin{tabular}{|c|c|c|c|c|c|c|c|c|c|c|c|c|c|c|c|}
\hline \multirow[t]{11}{*}{$\begin{array}{l}\text { Author } \\
\text { (Year) } \\
\text { PMID }\end{array}$} & Arm 1 & Arm 2 & $\begin{array}{l}\text { Time } \\
\text { (Months) }\end{array}$ & $\begin{array}{l}\text { General } \\
\text { Del. } \\
\text { Int. }\end{array}$ & $\begin{array}{l}\text { General } \\
\text { Del. } \\
\text { Cont. }\end{array}$ & $\begin{array}{l}\text { General } \\
\text { Del. } \\
\text { Calc. } \\
\text { Effect } \\
(95 \% \mathrm{Cl})\end{array}$ & $\begin{array}{l}\text { Person } \\
\text { Crimes } \\
\text { Int. }\end{array}$ & $\begin{array}{l}\text { Person } \\
\text { Crimes } \\
\text { Cont. }\end{array}$ & $\begin{array}{l}\text { Person } \\
\text { Crimes } \\
\text { Calc. } \\
\text { Effect } \\
(95 \% \mathrm{Cl})\end{array}$ & $\begin{array}{l}\text { Property } \\
\text { Crimesl } \\
\text { Theft } \\
\text { Int. }\end{array}$ & $\begin{array}{l}\text { Property } \\
\text { Crimesl } \\
\text { Theft } \\
\text { Cont. }\end{array}$ & $\begin{array}{l}\text { Calc. Effect } \\
(95 \% \mathrm{Cl})\end{array}$ & $\begin{array}{l}\text { Status } \\
\text { Offense }\end{array}$ & $\begin{array}{l}\text { Status } \\
\text { Offense }\end{array}$ & $\begin{array}{l}\text { Status } \\
\text { Offense } \\
\text { Calc. } \\
\text { Effect } \\
(95 \% \mathrm{Cl})\end{array}$ \\
\hline & & & 7 & $9.2(3.8)$ & $\begin{array}{l}10.4 \\
(4.7)\end{array}$ & $\begin{array}{l}\text { Net Diff } \\
0.7(-1.1 \text {, } \\
2.5)\end{array}$ & - & - & - & - & - & - & - & - & - \\
\hline & $\begin{array}{l}\text { Fam } \\
(\mathrm{FFT}) \\
(\mathrm{N}=30)\end{array}$ & $\begin{array}{l}\text { Educ } \\
+ \text { Peer } \\
\text { Group } \\
(\mathrm{N}=30\end{array}$ & 0 & $9.4(3.8)$ & $\begin{array}{l}10.3 \\
(3.4)\end{array}$ & - & - & - & - & - & - & - & - & - & - \\
\hline & & & 4 & $8.2(3.4)$ & $9.5(3.5)$ & $\begin{array}{l}\text { Net Diff - } \\
0.4(-2 \\
1.2)\end{array}$ & - & - & - & - & - & - & - & - & - \\
\hline & & & 7 & $9.2(3.8)$ & $9.4(3.7)$ & $\begin{array}{l}\text { Net Diff } \\
0.7(-0.9 \text {, } \\
2.3)\end{array}$ & - & - & - & - & - & - & - & - & - \\
\hline & $\begin{array}{l}\mathrm{CBT}+ \\
\mathrm{Ml}+ \\
\mathrm{Fam} \\
(\mathrm{N}=29)\end{array}$ & $\begin{array}{l}\text { CBT } \\
+\mathrm{Ml} \\
(\mathrm{N}=31\end{array}$ & 0 & $\begin{array}{l}11.3 \\
(4.1)\end{array}$ & $\begin{array}{l}11.3 \\
(3.9)\end{array}$ & - & - & - & - & - & - & - & - & - & - \\
\hline & & & 4 & $9.1(4.2)$ & $\begin{array}{l}10.2 \\
(3.8)\end{array}$ & $\begin{array}{l}\text { Net Diff - } \\
1.1(-3.1 \\
0.9)\end{array}$ & - & - & - & - & - & - & - & - & - \\
\hline & & & 7 & $8.5(4.2)$ & $\begin{array}{l}10.4 \\
(4.7)\end{array}$ & $\begin{array}{l}\text { Net Diff - } \\
1.9(-4.1 \\
0.3)\end{array}$ & - & - & - & - & - & - & - & - & - \\
\hline & $\begin{array}{l}\mathrm{CBT}+ \\
\mathrm{Ml}+ \\
\mathrm{Fam} \\
(\mathrm{N}=29)\end{array}$ & $\begin{array}{l}\text { Educ } \\
+ \text { Peer } \\
\text { Group } \\
(\mathrm{N}=30\end{array}$ & 0 & $\begin{array}{l}11.3 \\
(4.1)\end{array}$ & $\begin{array}{l}10.3 \\
(3.4)\end{array}$ & - & - & - & - & - & - & - & - & - & - \\
\hline & & & 4 & $9.1(4.2)$ & $9.5(3.5)$ & $\begin{array}{l}\text { Net Diff - } \\
1.4(-3.4 \\
0.6)\end{array}$ & - & - & - & - & - & - & - & - & - \\
\hline & & & 7 & $8.5(4.2)$ & $9.4(3.7)$ & $\begin{array}{l}\text { Net Diff - } \\
1.9(-3.9, \\
0.1)\end{array}$ & - & - & - & - & - & - & - & - & - \\
\hline
\end{tabular}




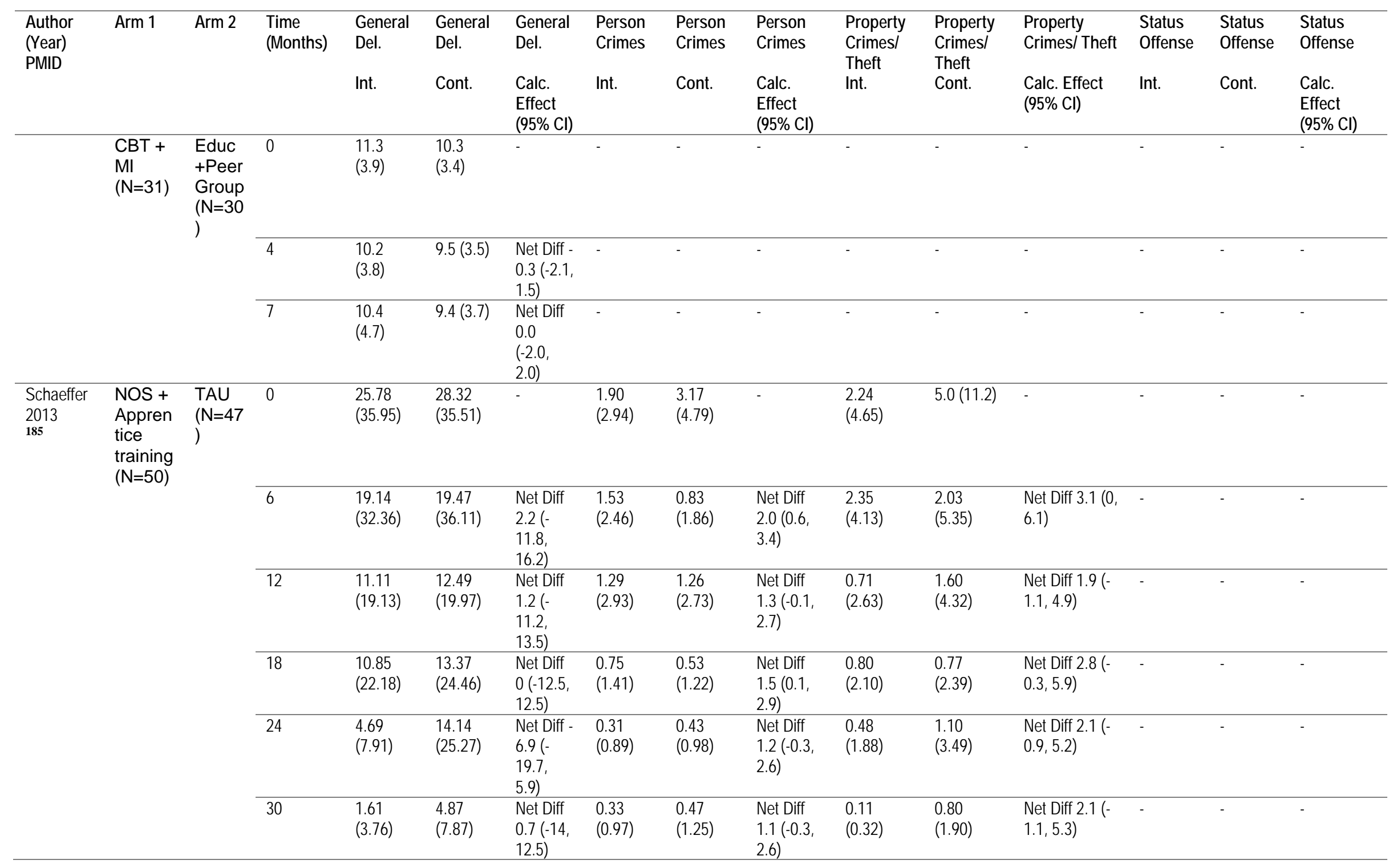




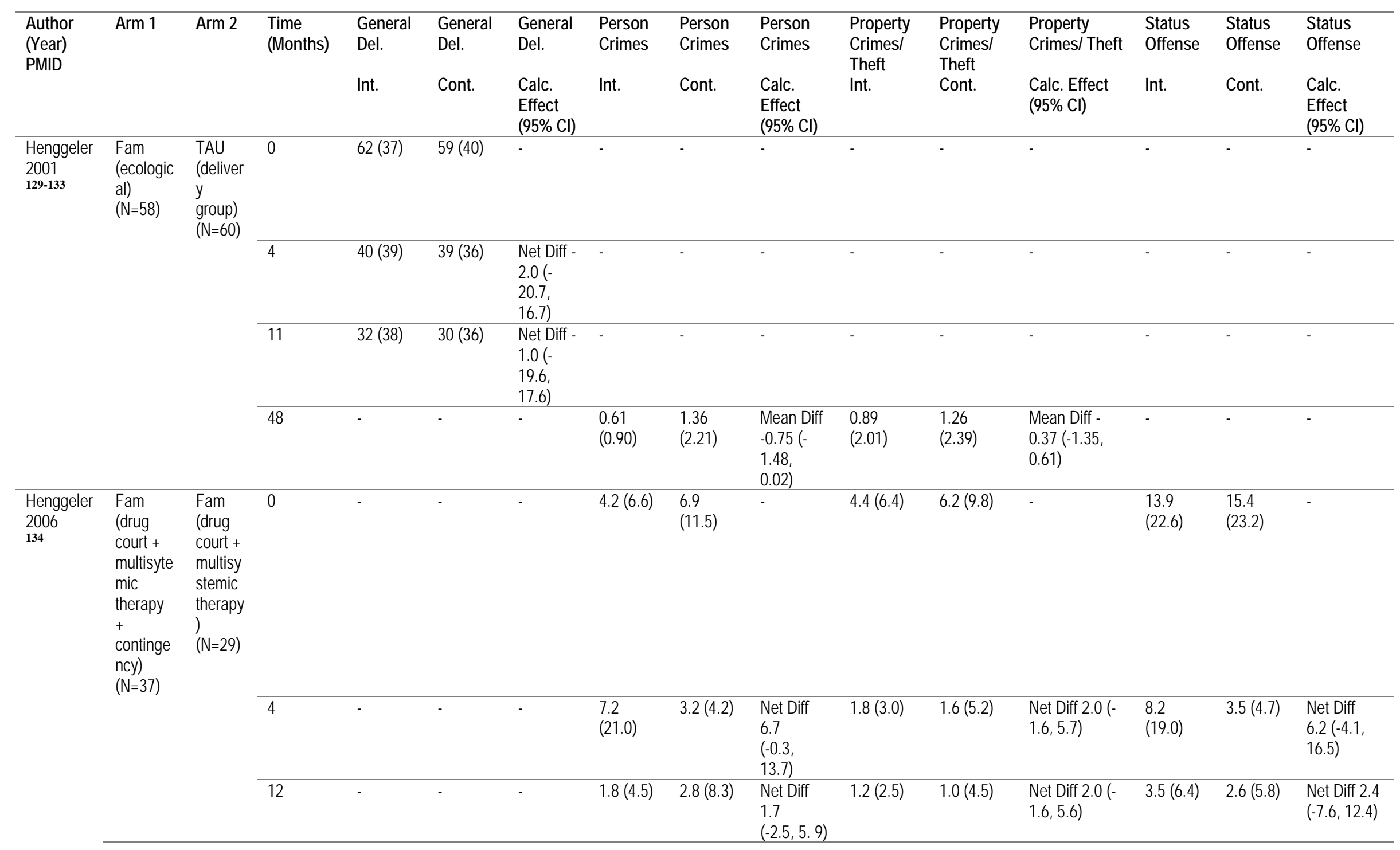




\begin{tabular}{|c|c|c|c|c|c|c|c|c|c|c|c|c|c|c|c|}
\hline \multirow[t]{7}{*}{$\begin{array}{l}\text { Author } \\
\text { (Year) } \\
\text { PMID }\end{array}$} & Arm 1 & Arm 2 & $\begin{array}{l}\text { Time } \\
\text { (Months) }\end{array}$ & $\begin{array}{l}\text { General } \\
\text { Del. } \\
\text { Int. }\end{array}$ & $\begin{array}{l}\text { General } \\
\text { Del. } \\
\text { Cont. }\end{array}$ & $\begin{array}{l}\text { General } \\
\text { Del. } \\
\text { Calc. } \\
\text { Effect } \\
(95 \% \mathrm{Cl})\end{array}$ & $\begin{array}{l}\text { Person } \\
\text { Crimes } \\
\text { Int. }\end{array}$ & $\begin{array}{l}\text { Person } \\
\text { Crimes } \\
\text { Cont. }\end{array}$ & $\begin{array}{l}\text { Person } \\
\text { Crimes } \\
\text { Calc. } \\
\text { Effect } \\
(95 \% \mathrm{Cl})\end{array}$ & $\begin{array}{l}\text { Property } \\
\text { Crimes/ } \\
\text { Theft } \\
\text { Int. }\end{array}$ & $\begin{array}{l}\text { Property } \\
\text { Crimes/ } \\
\text { Theft } \\
\text { Cont. }\end{array}$ & $\begin{array}{l}\text { Property } \\
\text { Crimes/ Theft } \\
\text { Calc. Effect } \\
(95 \% \mathrm{Cl})\end{array}$ & $\begin{array}{l}\text { Status } \\
\text { Offense } \\
\text { Int. }\end{array}$ & $\begin{array}{l}\text { Status } \\
\text { Offense } \\
\text { Cont. }\end{array}$ & $\begin{array}{l}\text { Status } \\
\text { Offense } \\
\text { Calc. } \\
\text { Effect } \\
(95 \% \mathrm{Cl})\end{array}$ \\
\hline & $\begin{array}{l}\begin{array}{l}\text { Fam } \\
\text { (drug }\end{array} \\
\text { court + } \\
\text { multisyte } \\
\text { mic } \\
\text { therapy } \\
+ \\
\text { continge } \\
\text { ncy) } \\
(\mathrm{N}=37)\end{array}$ & 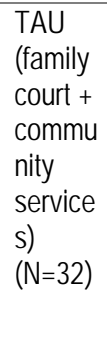 & 0 & - & - & - & $4.2(6.6)$ & $\begin{array}{l}9.5 \\
(17.5)\end{array}$ & - & $4.4(6.4)$ & $8.9(13.7)$ & - & $\begin{array}{l}13.9 \\
(22.6)\end{array}$ & $7.8(6.3)$ & - \\
\hline & & & 4 & - & - & - & $\begin{array}{l}7.2 \\
(21.0)\end{array}$ & $3.0(5.5)$ & $\begin{array}{l}\text { Net Diff } \\
9.5(1.27 \\
17.73)\end{array}$ & $1.8(3.0)$ & $2.7(5.4)$ & $\begin{array}{l}\text { Net Diff } 3.6 \text { (- } \\
0.9,8.1)\end{array}$ & $\begin{array}{l}8.2 \\
(19.0)\end{array}$ & $\begin{array}{l}6.9 \\
(11.0)\end{array}$ & $\begin{array}{l}\text { Net Diff - } \\
4.8(-12.34, \\
2.74)\end{array}$ \\
\hline & & & 12 & - & - & - & $1.8(4.5)$ & $\begin{array}{l}10.7 \\
(28.2)\end{array}$ & $\begin{array}{l}\text { Net Diff - } \\
3.6(- \\
12.77 \\
5.57)\end{array}$ & $1.2(2.5)$ & $2.5(5.7)$ & $\begin{array}{l}\text { Net Diff } 3.2(- \\
1.3,7.7)\end{array}$ & $3.5(6.4)$ & $\begin{array}{l}16.8 \\
(38.8)\end{array}$ & $\begin{array}{l}\text { Net Diff - } \\
19.4(-33.5 \text {, } \\
-5.3)\end{array}$ \\
\hline & $\begin{array}{l}\begin{array}{l}\text { Fam } \\
\text { (drug }\end{array} \\
\text { court + } \\
\text { multisyte } \\
\text { mic } \\
\text { therapy } \\
+ \\
\text { continge } \\
\text { ncy) } \\
(\mathrm{N}=37)\end{array}$ & $\begin{array}{l}\text { TAU } \\
\text { (drug } \\
\text { court + } \\
\text { commu } \\
\text { nity } \\
\text { service } \\
\text { s) } \\
(\mathrm{N}=29)\end{array}$ & 0 & - & - & - & $4.2(6.6)$ & $3.7(4.9)$ & - & $4.4(6.4)$ & $5.2(9.8)$ & - & $\begin{array}{l}13.9 \\
(22.6)\end{array}$ & $\begin{array}{l}8.6 \\
(10.6)\end{array}$ & - \\
\hline & & & 4 & - & - & - & $\begin{array}{l}7.2 \\
(21.0)\end{array}$ & $2.3(4.6)$ & $\begin{array}{l}\text { Net Diff } \\
4.4 \\
(-1.84 \\
10.64)\end{array}$ & $1.8(3.0)$ & $2.3(5.2)$ & $\begin{array}{l}\text { Net Diff 0.3 (- } \\
3.3,3.9)\end{array}$ & $\begin{array}{l}8.2 \\
(19.0)\end{array}$ & $4.4(7.4)$ & $\begin{array}{l}\text { Net Diff } \\
-1.5(-9.1 \\
6.1)\end{array}$ \\
\hline & & & 12 & - & - & - & $1.8(4.5)$ & $1.0(2.2)$ & $\begin{array}{l}\text { Net Diff } \\
0.3 \\
(-2.1,2.7)\end{array}$ & $1.2(2.5)$ & $1.9(7.2)$ & $\begin{array}{l}\text { Net Diff 0.1 (- } \\
3.5,3.7)\end{array}$ & $3.5(6.4)$ & $1.4(2.4)$ & $\begin{array}{l}\text { Net Diff - } \\
3.2(-10.59, \\
4.19)\end{array}$ \\
\hline
\end{tabular}




\begin{tabular}{|c|c|c|c|c|c|c|c|c|c|c|c|c|c|c|c|}
\hline \multirow[t]{8}{*}{$\begin{array}{l}\text { Author } \\
\text { (Year) } \\
\text { PMID }\end{array}$} & Arm 1 & Arm 2 & $\begin{array}{l}\text { Time } \\
\text { (Months) }\end{array}$ & $\begin{array}{l}\text { General } \\
\text { Del. } \\
\text { Int. }\end{array}$ & $\begin{array}{l}\text { General } \\
\text { Del. } \\
\text { Cont. }\end{array}$ & $\begin{array}{l}\text { General } \\
\text { Del. } \\
\text { Calc. } \\
\text { Effect } \\
(95 \% \mathrm{Cl})\end{array}$ & $\begin{array}{l}\text { Person } \\
\text { Crimes } \\
\text { Int. }\end{array}$ & $\begin{array}{l}\text { Person } \\
\text { Crimes } \\
\text { Cont. }\end{array}$ & $\begin{array}{l}\text { Person } \\
\text { Crimes } \\
\text { Calc. } \\
\text { Effect } \\
(95 \% \mathrm{Cl})\end{array}$ & $\begin{array}{l}\text { Property } \\
\text { Crimesl } \\
\text { Theft } \\
\text { Int. }\end{array}$ & $\begin{array}{l}\text { Property } \\
\text { Crimesl } \\
\text { Theft } \\
\text { Cont. }\end{array}$ & $\begin{array}{l}\text { Property } \\
\text { Crimes/ Theft } \\
\text { Calc. Effect } \\
(95 \% \mathrm{Cl})\end{array}$ & $\begin{array}{l}\text { Status } \\
\text { Offense } \\
\text { Int. }\end{array}$ & $\begin{array}{l}\text { Status } \\
\text { Offense } \\
\text { Cont. }\end{array}$ & $\begin{array}{l}\text { Status } \\
\text { Offense } \\
\text { Calc. } \\
\text { Effect } \\
(95 \% \mathrm{Cl})\end{array}$ \\
\hline & $\begin{array}{l}\begin{array}{l}\text { Fam } \\
\text { (drug }\end{array} \\
\text { court + } \\
\text { multisyst } \\
\text { emic } \\
\text { therapy) } \\
(\mathrm{N}=29)\end{array}$ & $\begin{array}{l}\text { TAU } \\
\text { (family } \\
\text { court + } \\
\text { commu } \\
\text { nity } \\
\text { service } \\
\text { s) } \\
(\mathrm{N}=32)\end{array}$ & 0 & - & - & - & $\begin{array}{l}6.9 \\
(11.5)\end{array}$ & $\begin{array}{l}9.5 \\
(17.5)\end{array}$ & - & $6.2(9.8)$ & $8.9(13.7)$ & - & $\begin{array}{l}15.4 \\
(23.2)\end{array}$ & $7.8(6.3)$ & - \\
\hline & & & 4 & - & - & - & $3.2(4.2)$ & $3.0(5.5)$ & $\begin{array}{l}\text { Net Diff } \\
2.8(-3.7 \\
9.3)\end{array}$ & $1.6(5.2)$ & $2.7(5.4)$ & $\begin{array}{l}\text { Net Diff } 2.0(- \\
3.2,7.2)\end{array}$ & $3.5(4.7)$ & $\begin{array}{l}6.9 \\
(11.0)\end{array}$ & $\begin{array}{l}\text { Net Diff }-11 \\
(-19.4,-2.6)\end{array}$ \\
\hline & & & 12 & - & - & - & $2.8(8.3)$ & $\begin{array}{l}10.7 \\
(28.2)\end{array}$ & $\begin{array}{l}\text { Net Diff - } \\
5.3(-14.6 \text {, } \\
4.0)\end{array}$ & $1.0(4.5)$ & $2.5(5.7)$ & $\begin{array}{l}\text { Net Diff } 1.2(- \\
4,6.4)\end{array}$ & $2.6(5.8)$ & $\begin{array}{l}16.8 \\
(38.8)\end{array}$ & $\begin{array}{l}\text { Net Diff - } \\
21.8(-36.4, \\
-7.2)\end{array}$ \\
\hline & $\begin{array}{l}\begin{array}{l}\text { Fam } \\
\text { (drug }\end{array} \\
\text { court + } \\
\text { multisyst } \\
\text { emic } \\
\text { therapy) } \\
(\mathrm{N}=29)\end{array}$ & $\begin{array}{l}\text { TAU } \\
\text { (drug } \\
\text { court + } \\
\text { commu } \\
\text { nity } \\
\text { service } \\
\text { s) } \\
(\mathrm{N}=29)\end{array}$ & 0 & - & - & - & $\begin{array}{l}6.9 \\
(11.5)\end{array}$ & $3.7(4.9)$ & - & $6.2(9.8)$ & $5.2(9.8)$ & - & $\begin{array}{l}15.4 \\
(23.2)\end{array}$ & $\begin{array}{l}8.6 \\
(10.6)\end{array}$ & - \\
\hline & & & 4 & - & - & - & $3.2(4.2)$ & $2.3(4.6)$ & $\begin{array}{l}\text { Net Diff - } \\
2.3(-6.4, \\
1.8)\end{array}$ & $1.6(5.2)$ & $2.3(5.2)$ & $\begin{array}{l}\text { Net Diff } \\
-1.7(-6.1,2.7)\end{array}$ & $3.5(4.7)$ & $4.4(7.4)$ & $\begin{array}{l}\text { Net Diff - } \\
7.7 \text { (-16.2, } \\
0.8)\end{array}$ \\
\hline & & & 12 & - & - & - & $2.8(8.3)$ & $1.0(2.2)$ & $\begin{array}{l}\text { Net Diff - } \\
1.4(-5.4, \\
2.6)\end{array}$ & $1.0(4.5)$ & $1.9(7.2)$ & $\begin{array}{l}\text { Net Diff } \\
-1.9(-6.4,2.6)\end{array}$ & $2.6(5.8)$ & $1.4(2.4)$ & $\begin{array}{l}\text { Net Diff - } \\
5.6(-14, \\
2.8)\end{array}$ \\
\hline & $\begin{array}{l}\text { TAU } \\
\text { (family } \\
\text { court + } \\
\text { commun } \\
\text { ity } \\
\text { services) } \\
(\mathrm{N}=32)\end{array}$ & $\begin{array}{l}\text { TAU } \\
\text { (drug } \\
\text { court + } \\
\text { commu } \\
\text { nity } \\
\text { service } \\
\text { s) } \\
(\mathrm{N}=29)\end{array}$ & 0 & - & - & - & $\begin{array}{l}9.5 \\
(17.5)\end{array}$ & $3.7(4.9)$ & - & 8.9 (13.7) & $5.2(9.8)$ & - & $7.8(6.3)$ & $\begin{array}{l}8.6 \\
(10.6)\end{array}$ & - \\
\hline
\end{tabular}




\begin{tabular}{|c|c|c|c|c|c|c|c|c|c|c|c|c|c|c|c|}
\hline $\begin{array}{l}\text { Author } \\
\text { (Year) } \\
\text { PMID }\end{array}$ & Arm 1 & Arm 2 & $\begin{array}{l}\text { Time } \\
\text { (Months) }\end{array}$ & $\begin{array}{l}\text { General } \\
\text { Del. } \\
\text { Int. }\end{array}$ & $\begin{array}{l}\text { General } \\
\text { Del. } \\
\text { Cont. }\end{array}$ & $\begin{array}{l}\text { General } \\
\text { Del. } \\
\text { Calc. } \\
\text { Effect } \\
(95 \% \mathrm{Cl})\end{array}$ & $\begin{array}{l}\text { Person } \\
\text { Crimes } \\
\text { Int. }\end{array}$ & $\begin{array}{l}\text { Person } \\
\text { Crimes } \\
\text { Cont. }\end{array}$ & $\begin{array}{l}\text { Person } \\
\text { Crimes } \\
\text { Calc. } \\
\text { Effect } \\
(95 \% \mathrm{Cl})\end{array}$ & $\begin{array}{l}\text { Property } \\
\text { Crimesl } \\
\text { Theft } \\
\text { Int. }\end{array}$ & $\begin{array}{l}\text { Property } \\
\text { Crimesl } \\
\text { Theft } \\
\text { Cont. }\end{array}$ & $\begin{array}{l}\text { Property } \\
\text { Crimes/ Theft } \\
\text { Calc. Effect } \\
(95 \% \mathrm{Cl})\end{array}$ & $\begin{array}{l}\text { Status } \\
\text { Offense } \\
\text { Int. }\end{array}$ & $\begin{array}{l}\text { Status } \\
\text { Offense } \\
\text { Cont. }\end{array}$ & $\begin{array}{l}\text { Calc. } \\
\text { Effect } \\
(95 \% \mathrm{Cl})\end{array}$ \\
\hline & & & 4 & - & - & - & $3.0(5.5)$ & $2.3(4.6)$ & $\begin{array}{l}\text { Net Diff - } \\
5.1(-10.7 \text {, } \\
0.5)\end{array}$ & $2.7(5.4)$ & $2.3(5.2)$ & $\begin{array}{l}\text { Net Diff } \\
-3.3(-8.5,1.9)\end{array}$ & $\begin{array}{l}6.9 \\
(11.0)\end{array}$ & $4.4(7.4)$ & $\begin{array}{l}\text { Net Diff 3.3 } \\
(-1.5,8.1)\end{array}$ \\
\hline & & & 12 & - & - & - & $\begin{array}{l}10.7 \\
(28.2)\end{array}$ & $1.0(2.2)$ & $\begin{array}{l}\text { Net Diff } \\
3.9(-4.8, \\
12.6)\end{array}$ & $2.5(5.7)$ & $1.9(7.2)$ & $\begin{array}{l}\text { Net Diff } \\
-3.1(-8.3,2.1)\end{array}$ & $\begin{array}{l}16.8 \\
(38.8)\end{array}$ & $1.4(2.4)$ & $\begin{array}{l}\text { Net Diff } \\
16.2(3.2 \\
29.2)\end{array}$ \\
\hline
\end{tabular}




\section{Appendix H. Risk of Bias Assessments}

\begin{tabular}{|c|c|c|c|c|c|c|c|c|c|c|c|c|c|}
\hline Study & 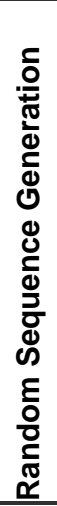 & 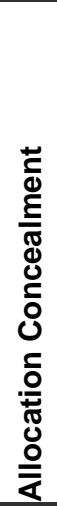 & 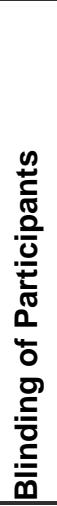 & 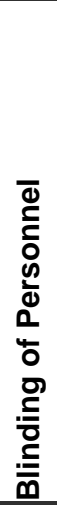 & 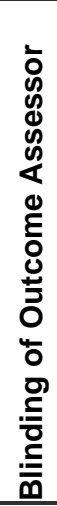 & 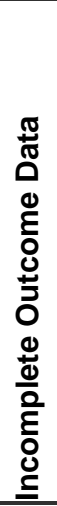 & 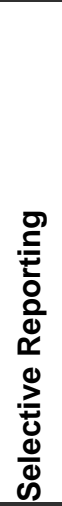 & 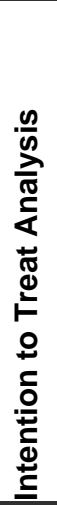 & 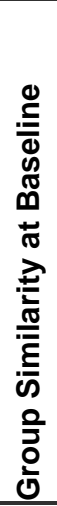 & 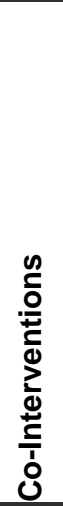 & 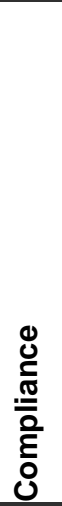 & 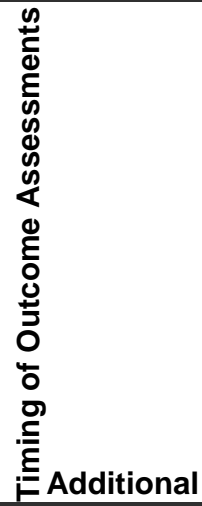 & Bias \\
\hline $\begin{array}{l}\text { Amini, } 1982 \\
\text { CN-00182281 } \\
\text { (Cochrane) }\end{array}$ & $\mathrm{U}$ & $U$ & $\mathrm{H}$ & $\mathrm{H}$ & U & $\mathrm{L}$ & $\mathrm{U}$ & $\mathrm{H}$ & L & $\mathrm{L}$ & $\mathrm{U}$ & $\mathrm{L}$ & No \\
\hline $\begin{array}{l}\text { Arnaud, } 2015 \\
\text { 2016-03749-004 } \\
\text { (psycINFO) }\end{array}$ & $\mathrm{L}$ & $\mathrm{L}$ & $\mathrm{H}$ & $U$ & $\mathrm{H}$ & $\mathrm{H}$ & U & $\mathrm{H}$ & $\mathrm{L}$ & L & $\mathrm{H}$ & $\mathrm{L}$ & No \\
\hline $\begin{array}{l}\text { Arnaud, } 2017 \\
27801991\end{array}$ & $U$ & U & $\mathrm{H}$ & $\mathrm{H}$ & U & $\mathrm{L}$ & U & $\mathrm{L}$ & $\mathrm{H}$ & L & U & $\mathrm{L}$ & No \\
\hline $\begin{array}{l}\text { Azrin, } 1994 \\
\text { CN-00241903 } \\
\text { (Cochrane) } \\
\end{array}$ & $\mathrm{H}$ & U & $\mathrm{H}$ & $\mathrm{H}$ & U & $\mathrm{L}$ & U & $\mathrm{H}$ & $L$ & L & $L$ & $\mathrm{~L}$ & No \\
\hline $\begin{array}{l}\text { Azrin, } 2001 \\
2002-13926-001 \\
\text { (psycINFO) }\end{array}$ & $\mathrm{L}$ & U & $\mathrm{H}$ & $\mathrm{H}$ & $\mathrm{L}$ & $\mathrm{H}$ & U & $\mathrm{H}$ & $\mathrm{H}$ & $\mathrm{H}$ & $\mathrm{H}$ & $\mathrm{H}$ & No \\
\hline $\begin{array}{l}\text { Baer, } 2007 \\
18072842 \\
\end{array}$ & $\mathrm{~L}$ & U & $\mathrm{H}$ & $\mathrm{H}$ & U & $\mathrm{H}$ & U & $\mathrm{H}$ & $\mathrm{L}$ & L & $\mathrm{H}$ & $\mathrm{L}$ & No \\
\hline $\begin{array}{l}\text { Bernstein, } 2009 \\
20053238\end{array}$ & L & $\mathrm{L}$ & $\mathrm{H}$ & $\mathrm{H}$ & $\mathrm{L}$ & $\mathrm{H}$ & U & $\mathrm{H}$ & L & L & U & $\mathrm{L}$ & No \\
\hline $\begin{array}{l}\text { Bernstein, } 2010 \\
20670329\end{array}$ & $L$ & $\mathrm{~L}$ & $\mathrm{H}$ & $\mathrm{H}$ & $L$ & $\mathrm{H}$ & $U$ & $\mathrm{H}$ & $\mathrm{L}$ & L & $U$ & $\mathrm{~L}$ & No \\
\hline $\begin{array}{l}\text { Braciszewski, } \\
2018 \\
132804409 \\
\text { (embase) }\end{array}$ & $\mathrm{H}$ & & $\mathrm{H}$ & $\mathrm{H}$ & $\mathrm{H}$ & $\mathrm{H}$ & $U$ & $\mathrm{~L}$ & $\mathrm{~L}$ & L & $\mathrm{L}$ & $\mathrm{L}$ & No \\
\hline $\begin{array}{l}\text { Brown, } 2015 \\
26362000 \\
\end{array}$ & $U$ & U & $\mathrm{H}$ & $\mathrm{H}$ & $\mathrm{L}$ & $\mathrm{H}$ & $U$ & $\mathrm{H}$ & $\mathrm{L}$ & $\mathrm{L}$ & $\mathrm{L}$ & $\mathrm{L}$ & No \\
\hline $\begin{array}{l}\text { Burrow-Sanchez, } \\
2012 \\
22866693 \\
\end{array}$ & $U$ & $U$ & $\mathrm{H}$ & $\mathrm{H}$ & $U$ & $\mathrm{H}$ & $U$ & $\mathrm{~L}$ & $U$ & $\mathrm{~L}$ & $\mathrm{~L}$ & $\mathrm{~L}$ & No \\
\hline $\begin{array}{l}\text { Burrow-Sanchez, } \\
2015 \\
25602465 \\
\end{array}$ & $\mathrm{~L}$ & $\mathrm{~L}$ & $L$ & $\mathrm{H}$ & $\mathrm{L}$ & $L$ & $U$ & $L$ & L & L & $\mathrm{H}$ & $L$ & No \\
\hline $\begin{array}{l}\text { Colby, } 2018 \\
29750362\end{array}$ & $\mathrm{~L}$ & $U$ & $\mathrm{H}$ & $\mathrm{H}$ & $L$ & $\mathrm{~L}$ & $U$ & $L$ & $\mathrm{H}$ & $\mathrm{L}$ & $U$ & $\mathrm{~L}$ & No \\
\hline $\begin{array}{l}\text { Cornelius, } 2009 \\
19321268 \\
\end{array}$ & $\mathrm{~L}$ & $\mathrm{~L}$ & $\mathrm{~L}$ & $\mathrm{~L}$ & $L$ & $\mathrm{~L}$ & $\mathrm{U}$ & $L$ & $\mathrm{H}$ & $\mathrm{H}$ & $\mathrm{U}$ & $L$ & No \\
\hline $\begin{array}{l}\text { Cornelius, } 2010 \\
20576364\end{array}$ & $\mathrm{~L}$ & $U$ & $\mathrm{~L}$ & $\mathrm{~L}$ & $\mathrm{~L}$ & $\mathrm{~L}$ & $\mathrm{U}$ & $L$ & $\mathrm{~L}$ & L & $U$ & $L$ & No \\
\hline $\begin{array}{l}\text { Cunningham, } \\
2015 \\
26347440\end{array}$ & $\mathrm{~L}$ & $U$ & $\mathrm{H}$ & $\mathrm{H}$ & $U$ & $\mathrm{~L}$ & $U$ & $\mathrm{~L}$ & $\mathrm{~L}$ & $\mathrm{~L}$ & $\mathrm{~L}$ & $L$ & No \\
\hline
\end{tabular}




\begin{tabular}{|c|c|c|c|c|c|c|c|c|c|c|c|c|c|}
\hline Study & 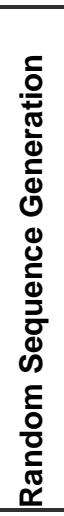 & 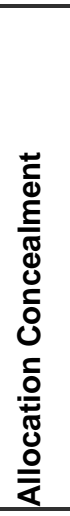 & 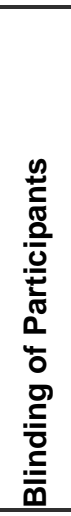 & 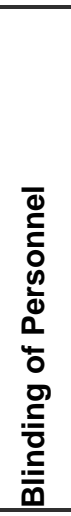 & 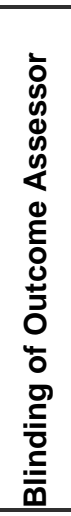 & 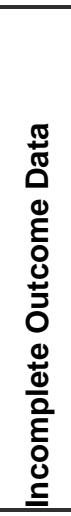 & 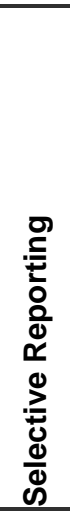 & 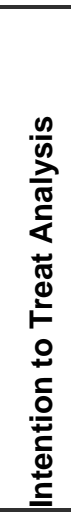 & 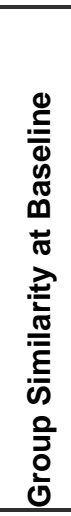 & 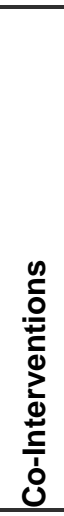 & 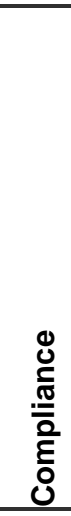 & 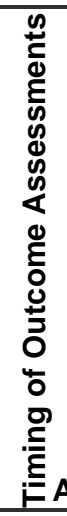 & Additional Bias \\
\hline $\begin{array}{l}\text { D'Amico, } 2008 \\
18037603\end{array}$ & $\mathrm{U}$ & $\mathrm{U}$ & $\mathrm{H}$ & $\mathrm{H}$ & $\mathrm{H}$ & $\mathrm{H}$ & $\mathrm{U}$ & $\mathrm{H}$ & U & $\mathrm{L}$ & $U$ & $\mathrm{H}$ & No \\
\hline $\begin{array}{l}\text { D'Amico, } 2013 \\
\text { CN-00917707 } \\
\text { (Cochrane) } \\
\end{array}$ & $U$ & $\mathrm{U}$ & $\mathrm{H}$ & $\mathrm{H}$ & 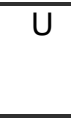 & $\mathrm{L}$ & $U$ & $\mathrm{H}$ & $\mathrm{H}$ & $\mathrm{L}$ & $\mathrm{L}$ & $\mathrm{L}$ & No \\
\hline 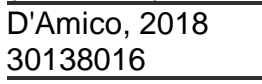 & L & $\mathrm{U}$ & $\mathrm{H}$ & $\mathrm{H}$ & $\mathrm{H}$ & L & $U$ & $\mathrm{~L}$ & $\mathrm{~L}$ & $L$ & $\mathrm{H}$ & $\mathrm{L}$ & No \\
\hline $\begin{array}{l}\text { Dakof, } 2015 \\
25621927 \\
\end{array}$ & $\bar{L}$ & $U$ & $\mathrm{H}$ & $\mathrm{H}$ & $\bar{U}$ & $\mathrm{H}$ & $\bar{U}$ & $\mathrm{~L}$ & L & $\mathrm{L}$ & $\mathrm{H}$ & 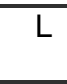 & No \\
\hline $\begin{array}{l}\text { de Gee, } 2014 \\
24969735\end{array}$ & $L$ & $U$ & $\mathrm{H}$ & $\mathrm{H}$ & $\mathrm{H}$ & $\mathrm{H}$ & $L$ & $\mathrm{~L}$ & $L$ & $\mathrm{H}$ & $\mathrm{L}$ & $L$ & No \\
\hline $\begin{array}{l}\text { De Sousa, } 2008 \\
\text { CN-00753784 } \\
\text { (Cochrane) }\end{array}$ & $L$ & $\mathrm{H}$ & $\mathrm{H}$ & $\mathrm{H}$ & $\mathrm{H}$ & $L$ & $\bar{U}$ & $\mathrm{~L}$ & $\mathrm{~L}$ & $\mathrm{~L}$ & $\mathrm{~L}$ & $\mathrm{~L}$ & No \\
\hline $\begin{array}{l}\text { De Sousa, } 2014 \\
\text { CN-01014147 } \\
\text { (Cochrane) }\end{array}$ & $L$ & $\mathrm{H}$ & $\mathrm{H}$ & $\mathrm{H}$ & $\mathrm{H}$ & $\mathrm{L}$ & $U$ & L & $L$ & $\mathrm{~L}$ & $L$ & $\mathrm{~L}$ & No \\
\hline $\begin{array}{l}\text { Delbelo, } 2017 \\
\text { NCT00550394 } \\
\text { (ctg) }\end{array}$ & $\bar{U}$ & $\mathrm{U}$ & $\mathrm{L}$ & $\mathrm{L}$ & $L$ & $\mathrm{H}$ & $U$ & $\mathrm{H}$ & $U$ & $L$ & $U$ & $\mathrm{~L}$ & No \\
\hline $\begin{array}{l}\text { Delbelo, } 2017 \\
\text { NCT00393978 } \\
\text { (ctg) }\end{array}$ & $L$ & $\mathrm{U}$ & $\mathrm{L}$ & $\mathrm{L}$ & $L$ & $\mathrm{H}$ & $U$ & $L$ & $U$ & $\mathrm{~L}$ & $U$ & $\mathrm{~L}$ & No \\
\hline $\begin{array}{l}\text { Dembo, 2014 } \\
\text { 2014-42452-005 } \\
\text { (psyclNFO) }\end{array}$ & $\bar{U}$ & $\mathrm{U}$ & $\mathrm{H}$ & $\mathrm{H}$ & $\bar{U}$ & $\bar{L}$ & $\bar{U}$ & $\bar{U}$ & $\bar{U}$ & L & $\mathrm{L}$ & $\mathrm{L}$ & $\overline{\text { No }}$ \\
\hline $\begin{array}{l}\text { Dennis, } 2004 \\
15501373 \text { (trial 1) }\end{array}$ & $\mathrm{L}$ & L & $\mathrm{H}$ & $\mathrm{H}$ & $L$ & $L$ & $U$ & $L$ & $\mathrm{~L}$ & $\mathrm{~L}$ & $U$ & $\mathrm{~L}$ & $\overline{\text { No }}$ \\
\hline $\begin{array}{l}\text { Dennis, } 2004 \\
15501373 \text { (trial 2) }\end{array}$ & L & $\mathrm{L}$ & $\mathrm{H}$ & $\mathrm{H}$ & $L$ & $L$ & $U$ & $L$ & L & $\mathrm{L}$ & $U$ & $\mathrm{~L}$ & No \\
\hline $\begin{array}{l}\text { Esposito- } \\
\text { Smythers, } 2011 \\
22004303\end{array}$ & L & $\bar{L}$ & $\mathrm{H}$ & $\mathrm{H}$ & $\mathrm{L}$ & L & $U$ & $\mathrm{H}$ & $\mathrm{L}$ & $\mathrm{L}$ & $\mathrm{H}$ & $\mathrm{L}$ & $\overline{\text { No }}$ \\
\hline $\begin{array}{l}\text { Figurelli, } 1994 \\
7862806\end{array}$ & $U$ & $U$ & $\mathrm{H}$ & $\mathrm{H}$ & $U$ & L & $U$ & $\mathrm{~L}$ & $U$ & $L$ & $\mathrm{~L}$ & $L$ & $\overline{\text { No }}$ \\
\hline $\begin{array}{l}\text { Findling, } 2009 \\
19298659\end{array}$ & $L$ & $\mathrm{~L}$ & $L$ & $\mathrm{H}$ & $L$ & $\mathrm{H}$ & $U$ & $L$ & $\mathrm{~L}$ & $\mathrm{~L}$ & $\mathrm{H}$ & $\mathrm{L}$ & No \\
\hline $\begin{array}{l}\text { Friedman, } 1989 \\
\text { CN-00496580 } \\
\text { (Cochrane) }\end{array}$ & $U$ & $U$ & $\mathrm{H}$ & $\mathrm{H}$ & $\mathrm{H}$ & $\mathrm{H}$ & $U$ & $\mathrm{H}$ & $\mathrm{H}$ & $L$ & $\mathrm{H}$ & $\mathrm{H}$ & $\begin{array}{l}\text { Yes: Parents of } \\
\text { older children } \\
\text { less participatory } \\
\text { (thus excluded). }\end{array}$ \\
\hline $\begin{array}{l}\text { Geller, } 1998 \\
9473913\end{array}$ & $U$ & $\mathrm{U}$ & $\mathrm{L}$ & $L$ & $L$ & $L$ & $U$ & $L$ & $\mathrm{~L}$ & $L$ & $\mathrm{~L}$ & $\mathrm{~L}$ & No \\
\hline $\begin{array}{l}\text { Giles, } 2019 \\
\text { CN-01953820 } \\
\text { (cochrane) }\end{array}$ & L & $\mathrm{H}$ & $\mathrm{H}$ & $\mathrm{H}$ & $\mathrm{H}$ & $\mathrm{L}$ & $U$ & L & L & L & $\mathrm{L}$ & $\mathrm{L}$ & No \\
\hline
\end{tabular}




\begin{tabular}{|c|c|c|c|c|c|c|c|c|c|c|c|c|c|}
\hline Study & 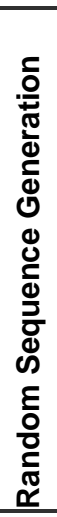 & 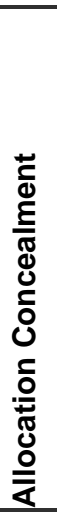 & 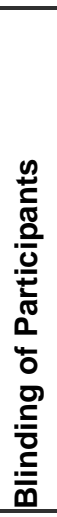 & 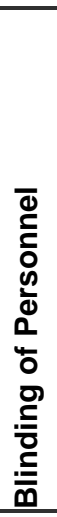 & 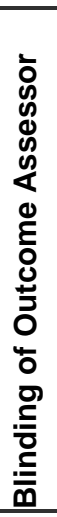 & 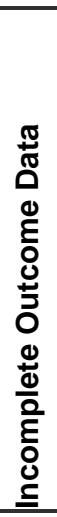 & 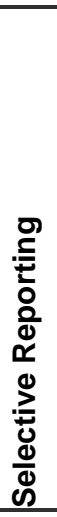 & 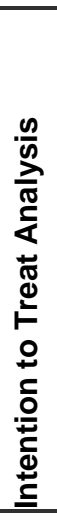 & 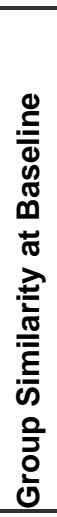 & 里 & 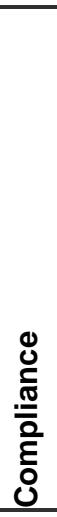 & 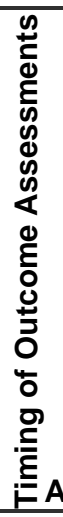 & $\begin{array}{l} \\
\text { Additional Bias } \\
\end{array}$ \\
\hline $\begin{array}{l}\text { Godley, } 2002 \\
12127465\end{array}$ & $\mathrm{~L}$ & $\mathrm{~L}$ & $\mathrm{H}$ & $\mathrm{H}$ & $\mathrm{L}$ & $\mathrm{L}$ & $\mathrm{U}$ & $\mathrm{L}$ & $\mathrm{L}$ & $\mathrm{L}$ & $\mathrm{H}$ & $\mathrm{L}$ & No \\
\hline $\begin{array}{l}\text { Godley, } 2010 \\
20219293\end{array}$ & $\mathrm{~L}$ & $\mathrm{H}$ & $\mathrm{H}$ & $\mathrm{H}$ & $L$ & $L$ & $U$ & $L$ & $L$ & $L$ & $U$ & $\mathrm{~L}$ & $\overline{\text { No }}$ \\
\hline $\begin{array}{l}\text { Godley, } 2019 \\
\text { CN-01745749 } \\
\text { (Cochrane) }\end{array}$ & $\mathrm{L}$ & $\mathrm{U}$ & $\mathrm{H}$ & $\mathrm{H}$ & $L$ & $\mathrm{~L}$ & $U$ & $L$ & $\mathrm{~L}$ & $L$ & L & $\mathrm{L}$ & $\overline{\text { No }}$ \\
\hline $\begin{array}{l}\text { Gonzalez, } 2015 \\
26454835\end{array}$ & $\mathrm{~L}$ & $U$ & $\mathrm{~L}$ & $\mathrm{~L}$ & $\mathrm{~L}$ & $\mathrm{H}$ & $U$ & L & $\mathrm{L}$ & L & L & $\mathrm{L}$ & $\overline{\text { No }}$ \\
\hline $\begin{array}{l}\text { Gray, } 2012 \\
22706327\end{array}$ & $U$ & $\mathrm{U}$ & $\mathrm{L}$ & $\mathrm{L}$ & $L$ & $\mathrm{~L}$ & $\mathrm{U}$ & L & $\mathrm{L}$ & $\mathrm{H}$ & $\mathrm{H}$ & $\mathrm{H}$ & $\overline{\text { No }}$ \\
\hline $\begin{array}{l}\text { Henderson, } 2016 \\
26992083\end{array}$ & $\bar{L}$ & $\mathrm{~L}$ & $\mathrm{H}$ & $\mathrm{H}$ & $\mathrm{U}$ & L & $U$ & L & $\mathrm{L}$ & L & $U$ & $\mathrm{~L}$ & No \\
\hline $\begin{array}{l}\text { Henggeler, } 1996 \\
8610836\end{array}$ & $U$ & $U$ & $\mathrm{H}$ & $\mathrm{H}$ & $U$ & $L$ & $U$ & $U$ & & $L$ & $U$ & $L$ & No \\
\hline $\begin{array}{l}\text { Henggeler, } 2006 \\
16551142 \\
\end{array}$ & $L$ & $\mathrm{~L}$ & $\mathrm{H}$ & $\mathrm{H}$ & $\mathrm{U}$ & $\mathrm{L}$ & $U$ & $\mathrm{~L}$ & $\mathrm{H}$ & L & $U$ & $\mathrm{~L}$ & $\mathrm{No}$ \\
\hline $\begin{array}{l}\text { Henggeler, } 2012 \\
22309470\end{array}$ & $U$ & $U$ & $\mathrm{H}$ & $\mathrm{H}$ & $\mathrm{H}$ & $\mathrm{L}$ & $U$ & $L$ & $L$ & $L$ & $L$ & $U$ & $\begin{array}{r}\text { Yes: } 2 \text { judicial } \\
\text { courts } \\
\text { randomized to } \\
\text { "usual services" } \\
\text { lost funding and } \\
\text { were replaced } \\
\text { with } 2 \text { others. }\end{array}$ \\
\hline $\begin{array}{l}\text { HJoanning, } 1992 \\
\text { CN-00631575 } \\
\text { (Cochrane) }\end{array}$ & $\mathrm{H}$ & $\mathrm{H}$ & $\mathrm{H}$ & $\mathrm{H}$ & $\mathrm{H}$ & $\mathrm{H}$ & $\mathrm{U}$ & $\mathrm{H}$ & $L$ & $L$ & L & & $\begin{array}{r}\text { Yes: 1. Family } \\
\text { members of } \\
\text { hose in AGT arm } \\
\text { vere substantially } \\
\text { less willing to } \\
\text { participate (or } \\
\text { have children } \\
\text { participate) than } \\
\text { other groups. } \\
\text { 2. Drug use } \\
\text { estimates relied } \\
\text { largely on adult } \\
\text { perceptions of } \\
\text { overall youth } \\
\text { behavior. }\end{array}$ \\
\hline $\begin{array}{l}\text { Hogue, } 2015 \\
25496283\end{array}$ & $L$ & $\mathrm{U}$ & $\mathrm{H}$ & $\mathrm{H}$ & $\mathrm{U}$ & $\mathrm{L}$ & $\mathrm{U}$ & $\mathrm{L}$ & $L$ & $L$ & $\mathrm{H}$ & $L$ & No \\
\hline $\begin{array}{l}\text { Kaminer, } 1998 \\
9824170\end{array}$ & $U$ & $\mathrm{U}$ & $\mathrm{H}$ & $\mathrm{H}$ & $\mathrm{L}$ & $\mathrm{H}$ & $U$ & $U$ & $\mathrm{~L}$ & L & $\mathrm{H}$ & $\mathrm{L}$ & $\mathrm{No}$ \\
\hline $\begin{array}{l}\text { Kaminer, } 2002 \\
12436013\end{array}$ & $\mathrm{U}$ & $\mathrm{U}$ & $\mathrm{H}$ & $\mathrm{H}$ & $\mathrm{U}$ & $\mathrm{H}$ & $U$ & $U$ & $L$ & $L$ & $\mathrm{H}$ & $\mathrm{L}$ & No \\
\hline $\begin{array}{l}\text { Kaminer, } 2008 \\
18978635\end{array}$ & $L$ & $\mathrm{U}$ & $\mathrm{H}$ & $\mathrm{H}$ & $\mathrm{U}$ & $\mathrm{L}$ & $U$ & $\mathrm{~L}$ & $L$ & $L$ & $\mathrm{~L}$ & $L$ & $\mathrm{No}$ \\
\hline
\end{tabular}




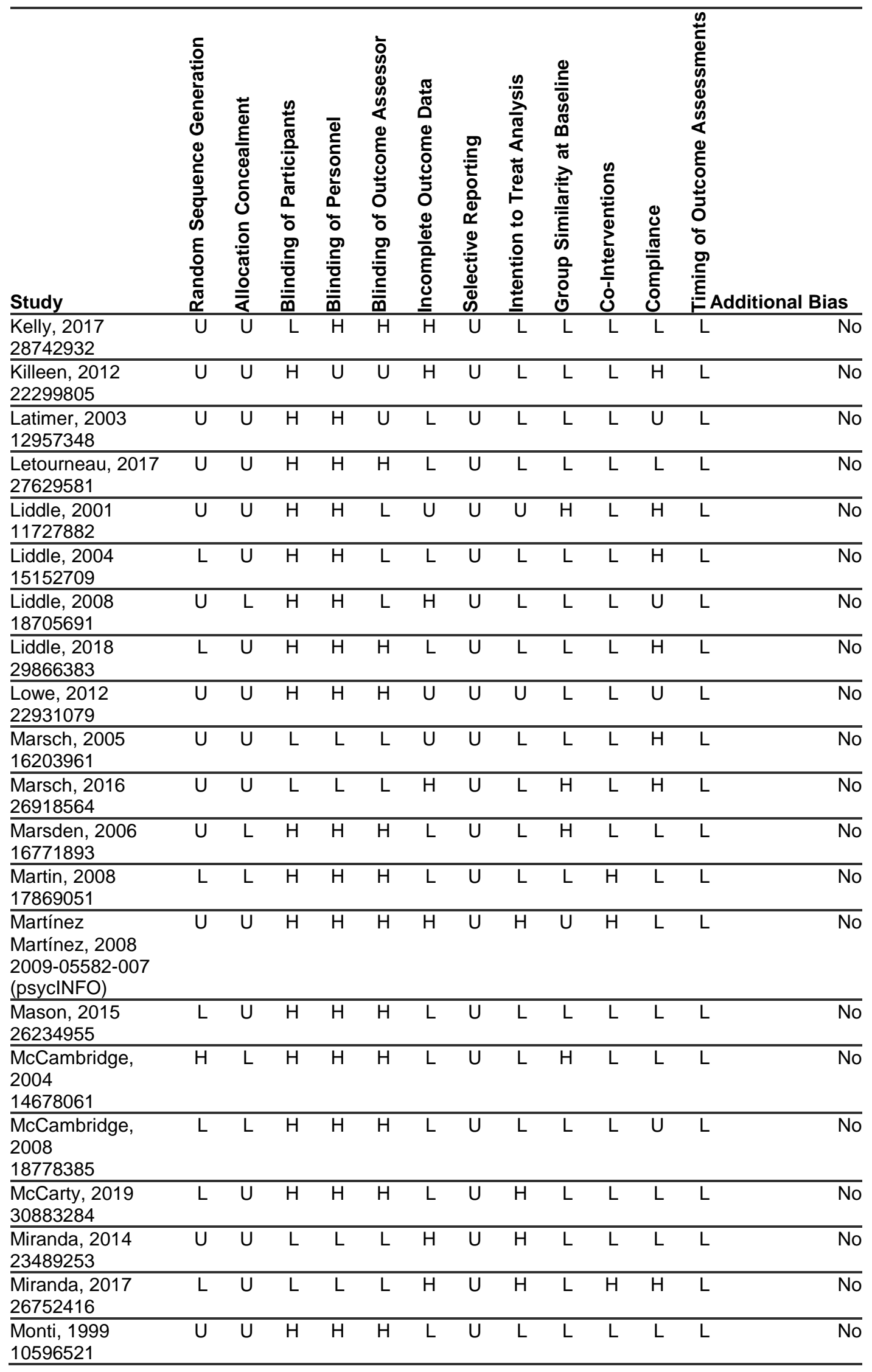




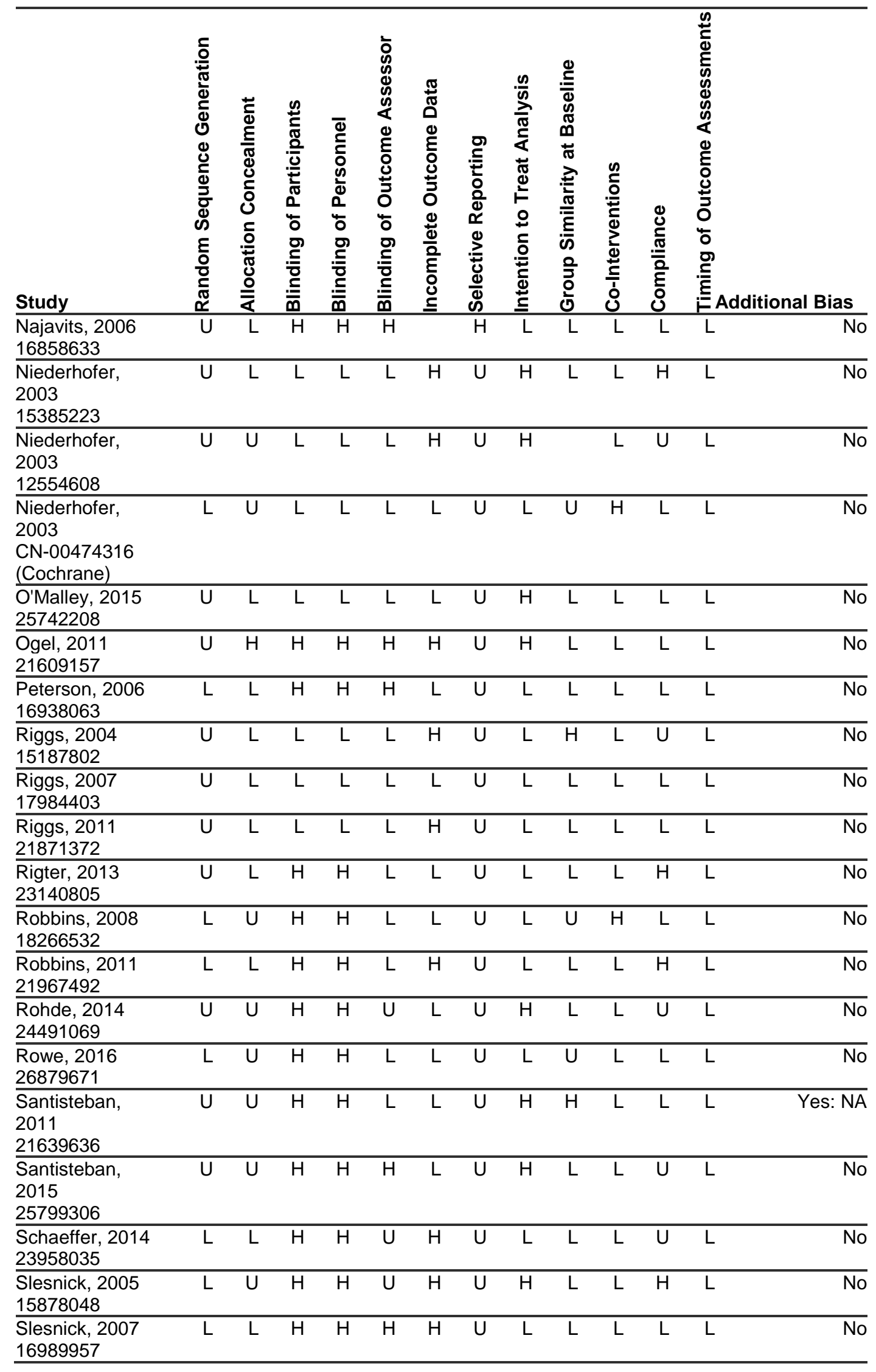




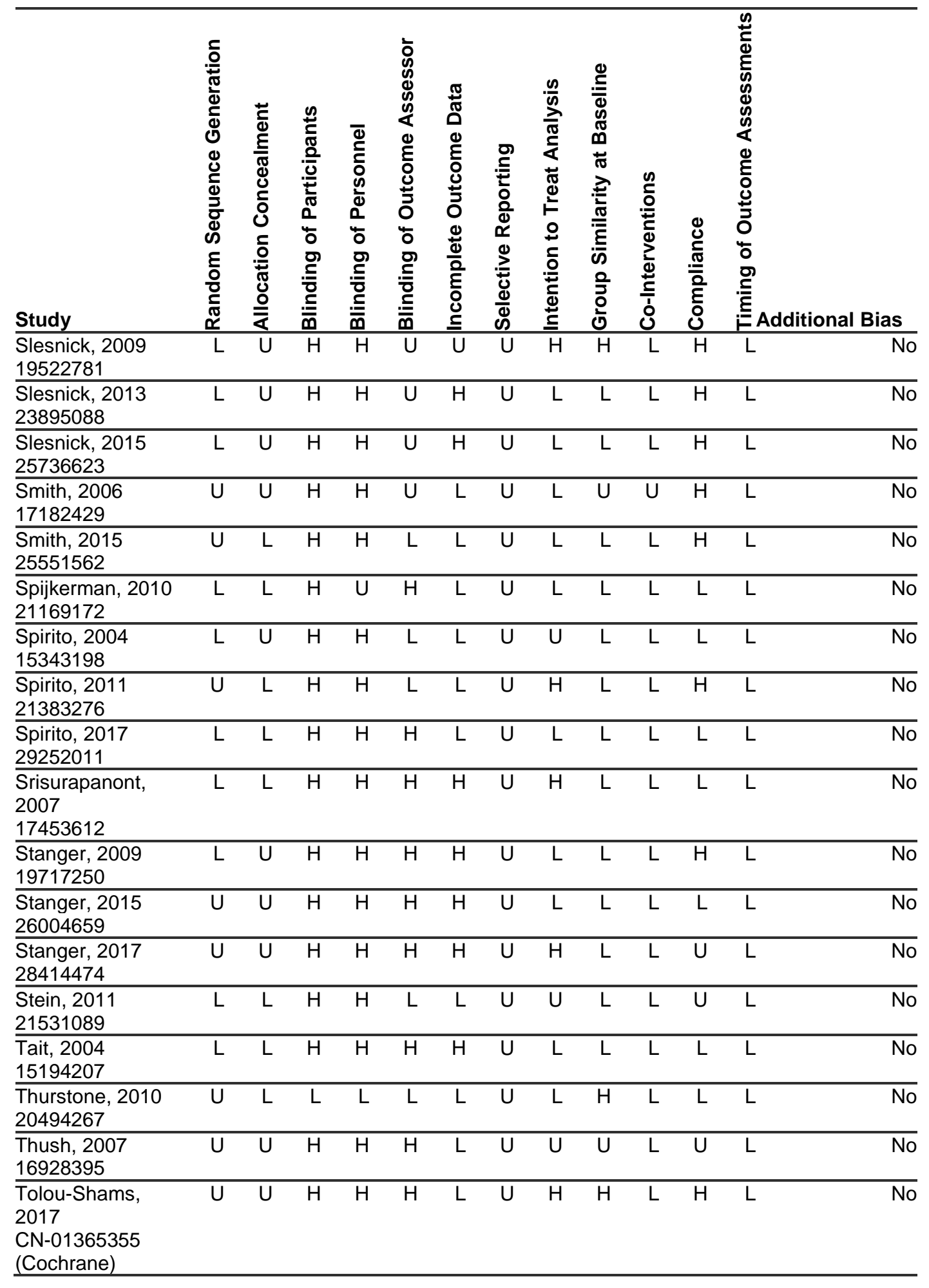




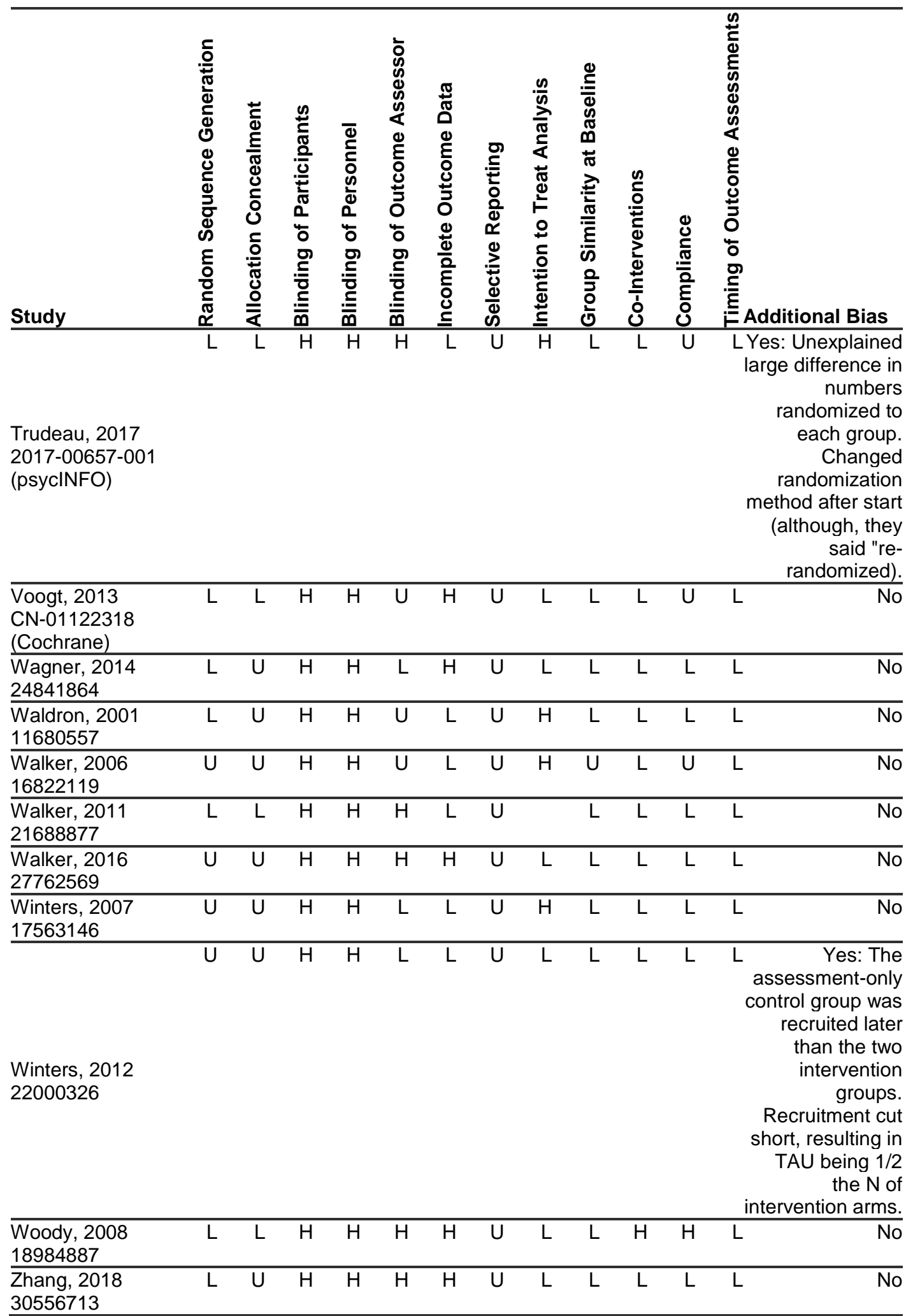

Random sequence generation (selection bias): Selection bias (biased allocation to interventions) due to inadequate generation of a randomized sequence; Allocation concealment (selection bias): Selection bias (biased allocation to interventions) due to inadequate concealment of allocations prior to assignment; Blinding of participants (performance bias): Performance bias due to knowledge of the allocated interventions by participants during the study; Blinding of personnel/ care providers (performance bias): Performance bias due to knowledge of the allocated interventions by personnel/care providers during the study; Blinding of 
outcome assessor (detection bias): Detection bias due to knowledge of the allocated interventions by outcome assessors; Incomplete outcome data (attrition bias): Attrition bias due to amount, nature or handling of incomplete outcome data; Intentionto-treat-analysis: Bias due to incomplete reporting and analysis according to group allocation; Group similarity at baseline (selection bias): Selection bias due to dissimilarity at baseline for the most important prognostic indicators; Co-interventions (performance bias): Performance bias because co-interventions were different across groups; Compliance (performance bias): Performance bias due to inappropriate compliance with interventions across groups; Timing of outcome assessments (detection bias): Detection bias because important outcomes were not measured at the same time across groups; Additional Bias: Bias due to problems not covered elsewhere in the table; Abbreviations: $\mathrm{H}=$ high risk of bias; $\mathrm{U}=$ unclear risk of bias; $\mathrm{L}=$ low risk of bias 


\title{
Appendix I. Technical Appendix
}

\author{
TA Trikalinos, DW Steele
}

\section{Network Meta-analysis Models}

We provide a detailed description of the models used in the main analysis.

\section{Notation}

Let $k=1, \ldots, K$ index studies and $(j=1, \ldots, J)$ index treatments in a network metaanalysis. For continuous outcomes, write $x_{k j}$ for the mean and $\sigma_{k j}^{2}$ for the conditional (sampling) variance of the responses with treatment $j$ in trial $k^{1}$

For dichotomous outcomes, write $r_{k j}$ for the number of people with events and $N_{k j}$ for the total number of people who received treatment $j$ in trial $k$.

Encode what treatment was assigned in an arm in a trial using the $(J-1)$-long row vector

$\mathbf{T}_{k j}= \begin{cases}\left(0, \ldots, 1_{[j]}, \ldots, 0\right) & \text { if } j<J \\ (0, \ldots, 0) & \text { if } j=J\end{cases}$

where has $1_{[j]}$ means ' 1 at the $j$-th position'. This can be used as a row in a design matrix encoded such that treatment $J$ is the reference treatment.

\section{Model}

Network meta-analysis is mathematically equivalent to a 2-level hierarchical model.

\section{Observational Part}

The first level (observational part) models the conditional distribution of data within each trial. For continuous outcomes, write:

$$
\begin{aligned}
& x_{k j} \sim N\left(\mu_{k j}, \sigma_{k j}^{2}\right) \\
& \mu_{k j}=\mathbf{T}_{k j} \boldsymbol{\delta}_{k}+\alpha_{k},
\end{aligned}
$$

where $\boldsymbol{\delta}_{k}=\left(\delta_{k 1}, \ldots, \delta_{k, J-1}\right)^{\prime}$ is a column vector of basic parameters, $\alpha_{k}$ is a study-specific intercept, and ' denotes transpose. The $\alpha_{k}$ can be interpreted as the mean of the

\footnotetext{
${ }^{1}$ If trial $k$ compares a strict subset of the $J$ treatments, say $j_{1}, j_{2}$, and $j_{3}, j \in\left\{j_{1}, j_{2}, j_{3}\right\}=$ $\mathcal{J}_{k} \subset\{1,2, \ldots, J\}=\mathcal{J}$.
} 
outcome under treatment $j$. Each $\delta_{k j}$ can be interpreted as the difference in the mean of the outcome between treatment $j<J$ and treatment $J$ in study $k$.

For dichotomous outcomes (2) and (3) become:

$$
\begin{array}{rlrl}
r_{k j} & \sim & & \operatorname{Bin}\left(\pi_{k j}, N_{k j}\right) \\
\mu_{k j} & := & & \operatorname{logit}\left(\pi_{k j}\right) \\
& = & \mathbf{T}_{k j} \boldsymbol{\delta}_{k}+\alpha_{k},
\end{array}
$$

where now the $\alpha_{k}$ can be interpreted as the log-odds of the probability of the outcome under treatment $J$. Each $\delta_{k j}$ can be interpreted as the difference in the log-odds of the probability of the outcome between treatment $j<J$ and treatment $J$ in study $k$.

The model in (3) (or in (6) for dichotomous outcomes) explicitly encodes a consistency assumption between direct and indirect effects. ${ }^{2}$

\section{Structural Part}

The structural part of the model prescribes how the study-specific parameters are related.

\section{The Intercepts $\alpha_{k}$}

We modeled the intercepts as fixed constants.

\section{The Treatment Effects $\boldsymbol{\delta}_{k}$}

We considered two variants.

1. Under an equal effect model

$$
\boldsymbol{\delta}_{k}=\boldsymbol{\delta},
$$

2. for all $k$. In this case the hierarchical model degenerates to a heteroskedastic regression model.

3. Under a random effects model, treatment-specific effects are modeled with a multivariate normal distribution

$$
\boldsymbol{\delta}_{k} \sim N(\boldsymbol{\delta}, \boldsymbol{\Sigma})
$$

\footnotetext{
${ }^{2}$ Model (3) reduces the number of treatment effects in the network's graph from $|\mathcal{E}| \geq$ $J-1$ to the $J-1$ in $\boldsymbol{\delta}_{k}$. All other effects are recovered as convex combinations of the elements of $\boldsymbol{\delta}_{k}$.
} 
4. where $\boldsymbol{\delta}=\left(\delta_{1}, \ldots, \delta_{J-1}\right)^{\prime}$ is a column vector of between-study means and covariance matrix

$$
\boldsymbol{\Sigma}=\tau^{2} \mathbf{\Omega}
$$

5. In (9), $\boldsymbol{\Omega}$ is a square correlation matrix. $\tau^{2}$ is a between-study variance parameter which we assume to be the same for all treatment effects. This homogeneity of variances assumption is commonly employed. The homogeneity of variances assumption together with (3) or (6) imply that $\boldsymbol{\Omega}$ has a compound-symmetry structure with all off-diagonal elements equal to 0.5 and all diagonal elements equal to $1 .^{3}$

\section{Comparison of Direct and Indirect Estimates of an Effect}

Consider a network with $J+1$ treatments, where treatment $j$ in the set of studies that include a comparison of interest (say, $j$ vs. $i$ ) is considered as $j^{*}$. Implementing the models previously described, the direct estimate for the comparison of interest is then the contrast between treatment $j^{*}$ and treatment $i$, and the indirect estimate is the contrast between treatment $j$ and treatment $i$. The consistency factor is then the contrast between treatment $j^{*}$ and $j$.

\section{Computation}

We fit models with gemtc, which uses rjags in R and JAGS for the Bayesian computation. Graph operations were done with igraph in $\mathrm{R}$.

\footnotetext{
${ }^{3}$ To show, start from (3) (equivalently, from (6) for dichotomous outcomes) and take variances.
} 